\section{Poster Session III}

December 7, 2016

Sponsorship Statement: Publication of this supplement is sponsored by the ACNP.

Presenter disclosures are found within the abstracts. Disclosure Format: Company Name (Financial Contributor), Type of Financial Disclosure, Funding Received for Self or Spouse.

Asterisks in the author lists indicate presenter of the abstract at the annual meeting.

\section{W1. Novel Pet Radioligands Show That COX-1 is Constitutively Expressed and That COX-2 is Induced by Inflammation in Rhesus Monkey Brain}

Stal Shrestha*, Mark Eldridge, Michelle Cortes, Prachi Singh, Min-Jeong Kim, Robert Gladding, Evan Gallagher, Megan Fredericks, Lora Weidner, Chad Brouwer, George Tye, Masahiro Fujita, Sami Zoghbi, Victor Pike, Robert Innis

National Institute of Mental Health, Bethesda, Maryland, United States

Background: Our laboratory recently developed two PET radioligands: 11C-PS13 for COX-1 and 11C-MC1 for COX-2, each of which potently and selectively inhibits the cognate enzyme in whole blood assays from monkey and human. Based on studies in peripheral organs, COX-1 is typically present at baseline (i.e., constitutively expressed) but not induced (i.e., upregulated) by inflammation. In contrast, COX-2 is minimally expressed at baseline in several peripheral tissues but markedly upregulated by inflammation at the level of both gene transcription and protein synthesis. In our presentation last year at the ACNP, we reported a) the pharmacological characterization of the COX-1 and COX-2 radioligands using in vitro enzymatic assays in monkey and human blood, and b) initial evaluation of these two radioligands in a healthy rhesus monkey brain. We showed that PS13 was potent and selective for COX-1 $(\mathrm{IC} 50=1 \mathrm{nM})$ compared to COX-2 (IC50>1,000 nM). Conversely, MC1 was potent and selective for COX-2 (IC50 = $3 \mathrm{nM}$ ) compared to COX-1 (IC50>1000 nM). Both 11C-PS13 and 11C-MC1 showed good uptake in monkey brain (peak concentrations of 3-5 SUV) and washed out relatively quickly (demonstrating that the binding was reversible, as expected). The purpose of this study was to determine whether a model on neuroinflammation (i.e., intracerebral injection of lipopolysaccharide (LPS)) would upregulate expression of COX-2 but not COX-1.

Methods: To induce transient inflammation, LPS (from Escherichia coli O26:B6) was injected into the right putamen of monkeys $(n=2)$. Prior to injection, we obtained a T1-weighted MRI to obtain anatomical information to guide the placement of the cannula. We injected a total dose of $10 \mu \mathrm{g}$ of LPS at a concentration of $1 \mu \mathrm{g} / \mu \mathrm{l}$, and an infusion rate of $0.5 \mu \mathrm{l} / \mathrm{min}$. Thus the entire dose was administered at a final volume of $10 \mu \mathrm{l}$ over a period of 20 minutes. The infusion cannula was left in place for an additional $10 \mathrm{~min}$ post-injection. This technique allows the LPS to diffuse $\sim 3 \mathrm{~mm}$ from the site of injection. About 180 $\mathrm{MBq}$ of each radioligand was injected intravenously into rhesus monkeys. Dynamic PET scans of brain were acquired for two hours pre- as well as post-LPS infusion. Standardized uptake value (SUV) was calculated from $30-90 \mathrm{~min}$, and was used as the outcome measure for calculating 1) specific uptake at baseline, and 2) per cent difference between pre- vs. post-inflammation. During the course of these studies, including the initial evaluation of the COX-1 and COX-2 radioligands in the brain $(n=13$ baseline scans with 11C-PS13; and $n=11$ baseline scans with 11C-MC1), we discovered that the excipient (Cremaphor) used to dissolve blocking drugs artificially altered the measurement of parent radioligand in plasma. For this reason, we used only brain SUV during the last $60 \mathrm{~min}(30-90 \mathrm{~min})$ as a measure of radioligand binding. We are seeking another solvent and will repeat these studies with full quantitation of distribution volume, which requires accurate measurement of the concentration of parent radioligand in plasma.

Results: 11C-PS13 showed specific uptake in healthy monkeys with $45 \%$ of the binding displaced by nonradioactive PS13 $(0.3 \mathrm{mg} / \mathrm{kg})$. However, compared to preinjection, there was no increase in uptake post-injection of LPS on either Day 1 or 3. On the contrary, 11C-MC1 showed no noticeable specific uptake in healthy monkeys (i.e. pre-injection). Most importantly, after post-injection of LPS the per cent specific uptake of the COX-2 radioligand was $41 \%$ on Day 3 , and $35 \%$ on Day 8 . Since both of the COX-1 and COX-2 radioligands are exploratory markers of neuroinflammation, we also confirmed the location of the edema using a T2-weighted MRI and inflammation using 11C-PBR28 radioligand for translocator protein (TSPO).

Conclusions: Our results suggest that COX-1 is constitutively expressed and that COX-2 is induced in monkey brain by intracerebral injection of the inflammagen, LPS. We used brain uptake of the radioligand to measure binding to COX, and these results need to be repeated with full quantitation that require serial measurements of the parent radioligand in plasma. If confirmed with these fully quantitative methods, $11 \mathrm{C}-\mathrm{MC} 1$ will be the first radioligand to show promise as a marker of COX-2, and 11C-PS13 will be the first direct-acting radioligand for COX-1, and not requiring delivery to brain via a lipophilic precursor, as in 11C-ketoprofen methyl ester. These radioligands could then be used to measure COX-1 and COX-2 in disorders thought to involve neuroinflammation and to measure the delivery to the brain of non-steroidal anti-inflammatory drugs (NSAIDs) as well as their selectivity for the two COX isoforms.

Keywords: Neuroinflammation, PET Imaging, COX-1 and COX-2.

Disclosure: Nothing to disclose. 


\section{W2. Circulating Levels of Oxytocin in Complicated Grief}

T.H. Eric Bui ${ }^{\star}$, Peter Rosencrans, Susanne Hoeppner, Rachel Ross, Elizabeth Hoge, Naomi Simon

Harvard Medical School, Boston, Massachusetts, United States

Background: Nearly 2.5 million deaths occur each year in the United States, leaving behind millions of bereaved loved ones. Although for the majority of bereaved individuals, lossrelated distress and disruption will recede over time, a subset will exhibit persisting and intense distress and impairment. This condition, complicated grief (CG), is characterized by debilitating symptoms that may persist years after the death (Shear et al. 2011). To date, little is known about the pathophysiology of CG, limiting development of targeted pharmacotherapy. Oxytocin, a neuropeptide produced in the hypothalamus, has been implicated in attachment and social behaviors (e.g., Theodoridou et al. 2009). CG may be conceptualized as a stress response to the loss of an important attachment figure (Simon 2012), and involves difficulty adjusting to the loss with persistent separation distress and protest with seeking of reunification. Despite the clear role of attachment in CG, no studies have examined the potential role of abnormalities in oxytocin in this condition. The present study thus aims to examine differences in plasma levels of oxytocin between bereaved individuals with CG, with major depressive disorder (MDD), and without any mental disorder. We hypothesized that plasma levels of oxytocin will be higher in individuals with CG compared to the other groups, reflecting an increased activation of the attachment system. In addition, we hypothesize that there will be a significant moderating effect of sex.

Methods: Participants were $N=47$ individuals with a primary diagnosis of CG $(\mathrm{M}(\mathrm{SD})$ age $=49.5(12.9) ; 70 \%$ women) and gender and age-matched (+/- 5 years) bereaved individuals with primary $\mathrm{MDD}(N=46 ; \mathrm{M}(\mathrm{SD})$ age $=49.3$ (13.3); 70\% women), and bereaved controls ( $N=47 ; \mathrm{M}(\mathrm{SD})$ age $=49.0(12.7) ; 70 \%$ women) evaluated by trained raters using the Structured Clinical Interview for DSM-IV (First et al., 1994) who participated in one of two prior studies that involved the completion of self-reported questionnaires and a blood sample collection. Blood was collected in EDTA tubes and immediately placed on ice, then centrifuged at $3,000 \mathrm{rpm}$ for $10 \mathrm{~min}$ at $4^{\circ} \mathrm{C}$. Plasma (citrated) was then transferred into $2 \mathrm{cc}$ plastic tubes, capped and frozen at $-80{ }^{\circ} \mathrm{C}$ until processing. Oxytocin immunoreactivity levels were quantified using a commercial oxytocin ELISA kit (Enzo Life Sciences, NY, USA). Thawed samples on ice were assayed according to manufacturer's instructions. The oxytocin assay had a sensitivity of $15.6 \mathrm{pg} / \mathrm{ml}$, and inter- and intraassay coefficient of variations below $16 \%$ in the present study. Participants completed self-report measures include the 19-item Inventory of Complicated Grief (ICG; total score ranges 0-76; Prigerson et al. 1995), the 10-item Perceived Stress Scale (PSS; total score ranges 0-40; Cohen et al. 1983). Results: CG symptom severity significantly differed across the three groups $(\mathrm{F}(2,130)=87.7, p<0.0001)$ with individuals with CG scoring significantly higher than controls $(\mathrm{t}=13.1, \quad p<0.001)$, and those with $\operatorname{MDD}(\mathrm{t}=8.58$, $p<0.001)$. Similarly, perceived stress significantly differed across the three groups $(\mathrm{F}(2,128)=101.4, p<0.0001)$, with individuals with CG significantly higher than controls $(\mathrm{t}=11.9, p<0.0001)$, but did not differ from those with $\operatorname{MDD}(\mathrm{t}=0.2, p=0.87)$. An analysis of variance with Sex and Group as between-subject factors, and circulating plasma oxytocin as the dependent variable, failed to show a significant effect of the Sex $\times$ Group interaction $(\mathrm{F}(2,134)=$ $0.77, p=0.47)$, and a main effect of $\operatorname{Sex}(F(1,134)=0.2$, $p=0.6)$. However, the main effect of Group was significant $(\mathrm{F}(2,134)=3.80, p<0.05)$. Post-hoc examination of specific contrasts showed that circulating plasma oxytocin in individuals with CG $(\mathrm{M}(\mathrm{SD})=1558.6(551.8) \mathrm{pg} / \mathrm{mL})$ significantly differed from MDD $(\mathrm{M}(\mathrm{SD})=1304.4(461.1) \mathrm{pg} / \mathrm{mL}$, $\mathrm{t}=2.52, p<0.05)$, and were marginally significantly different from controls $(\mathrm{M}(\mathrm{SD})=1373.0(438.7) \mathrm{pg} / \mathrm{mL}, \mathrm{t}=1.85$, $p=0.067)$. Finally, Pearson's correlation analyses failed to show a significant association between circulating plasma oxytocin and ICG or PSS scores.

Conclusions: Our findings suggest that circulating plasma oxytocin may be moderately elevated in individuals with complicated grief, suggesting that the oxytocin pathway may be involved in the pathophysiology of this condition. However, the reach of our results are limited by the fact that the relationship between peripheral and central levels of oxytocin are still unclear to date. Other limitations include the small sample size that precluded the examination of gender effects, and the lack of non-bereaved controls. Nonetheless, future studies confirming and furthering the implication of the oxytocin system in complicated grief are warranted.

Keywords: Oxytocin, Complicated Grief, Blood.

Disclosure: Nothing to disclose.

\section{W3. Shared Polygenic Risk for Neurodegenerative and Immune-Related Traits Reveals Pleiotropic Effects of Implicated Loci on Microglial Activation}

Daniel Felsky*, Nikolaos Pastopoulos, Julie Schneider, David Bennett, Philip De Jager

Centre for Addiction and Mental Health, Toronto, Canada

Background: Detailed investigations of polygenic risk burden for complex illness have been enabled by recent large-scale GWAS. In parallel, links between brain disorders and systemic inflammatory and vascular phenotypes have emerged. Here, we explored the relationship of polygenic risk scores for several major neurodegenerative phenotypes and diagnoses, as well as common vascular and inflammatory conditions, with late-life cognition and postmortem neuropathology in two large cohorts. By this we sought to evaluate the shared and independent contributions of common genetic variation to neuropathological mechanisms of cognitive decline.

Methods: $N=819$ subjects of Caucasian ancestry from the Religious Orders Study and Memory and Aging Project (ROS/MAP) with genomic, cognitive, and postmortem neuropathology data were included in the analyses. All subjects were assessed annually prior to death using a cognitive battery for five domains. Postmortem brain samples were collected and evaluated by trained neuropathologist for five measures of amyloid and tau deposition. Manually 
ascertained regional counts (four regions) of active microglia (at three stages) were available for a subset of $n=162$ brain samples. Eight genetic risk scores (GRS) were constructed based on published and unpublished data from major metaanalyses and consortia: Alzheimer's disease (AD; 21 SNPs), Parkinson's disease (PD; 32 SNPs), multiple sclerosis (MS; 200 SNPs), schizophrenia (SCZ; 102 SNPs), rheumatoid arthritis (RA; 76 SNPs), coronary artery disease (CAD; 63 SNPs), telomere length (TL; 8 SNPs), and hippocampal volume (HV; 20 SNPs). Linear models were used to test the associations between GRS and pathology or cognition, controlling for technical and demographic covariates, as well as APOE $\varepsilon 4$ status, and the top three principal components from EIGENSTRAT to control for population structure. GRS showing significant associations after FDR correction were carried forward into post-hoc tests of individual SNPs to locate the source of association signal. Variants associated with pathology and showing regional proximity to SNPs within other GRS were identified and characterized for effects on gene expression using the Broad Institute's GTEx portal.

Results: We noticed weak inverse correlations between $\mathrm{AD}$ and MS GRS (Spearman $\rho=-0.053, p=0.08$ ), and, as expected, the AD GRS was strongly associated with measures of classical $\mathrm{AD}$ pathology. After correction for multiple comparisons, the RA GRS was associated with active microglial counts in multiple brain regions, such that individuals with higher RA risk had lower counts of active microglia at all three stages in multiple brain regions (strongest association: ventral medial caudate, combined stages $2+3 ; \mathrm{t} 152=-3.78, p=2.3 \times 10-4)$. The CAD GRS was also significantly associated with stage 3 active microglia count in the inferior temporal cortex (t168=-2.59, $p=0.0016)$. For cognition, the AD GRS was associated with cognitive decline slope across all five domains (1.9x10$6>p<0.0053)$, and the SCZ GRS was associated with episodic memory $(p=0.0067)$, such that higher risk score indicated worse cognitive decline. Interestingly, the MS GRS showed a trend-level negative relationship with cognitive decline across all five domains. One of two variants driving the association of arthritis GRS with microglia count was within close proximity to a variant within the MS GRS: on Chr2, rs10175798 (arthritis; $4.8 \mathrm{~kb} 5$ ' of $\mathrm{LBH}$ ) is $22.8 \mathrm{~kb}$ from rs13414105 (MS; within $\mathrm{LBH}$, intronic). Although these variants are not in LD $(1000$ Genomes r2<0.2). rs13414105 is a cis-eQTL for LBH in heart (left ventricle GTEx $p=3 \times 10$ 6) and in brain (cortex GTEx $p=3.3 \times 10-4$ ), while rs10175798 has no effect on expression in any tissue.

Conclusions: We have shown that increased genetic burden for RA is associated with decreases in active microglial count, and that the mechanism of this effect may be independent of changes in $\mathrm{LBH}$ expression. We also show that risk for CAD and SCZ may be related to microglial activation and episodic memory decline, respectively. Genetic risk scores may provide insight into unique aspects of disease/trait manifestations as well as reveal pleiotropic effects of commonly implicated loci.

Keywords: Activated Microglia, Polygenic Risk Score, Neurodegenerative Disease, Inflammation, Postmortem Brain Tissue.

Disclosure: Nothing to disclose.
W4. Neurocognitive Sequela of Type II Diabetes: Evidence for Shared Genetic Etiology

David Glahn*, Emma Knowles, Samuel Mathias, Karen Hodgson, Rene L. Olvera, Peter T. Fox, Laura Almasy, Ravi Duggirala, Joanne Curran, John Blangero

\section{Yale University, Hartford, Connecticut, United States}

Background: Type 2 diabetes is associated with mild neurocognitive decrements in middle age and an almost two-fold increased risk for dementia in older adults. Yet, even individuals in the early stages of illness and those with subclinical forms of diabetes show decreased performance on tests of memory, processing speed, and executive functioning. Currently, the etiological roots of the association between cognitive decline and diabetes are unclear. Traditional models stress that metabolic dysregulation associated with diabetes, such as hypo- and hyper-glycemia, vascular risk factors, or micro- and macro-vascular complications, are at the root of the cognitive changes in the illness. However, more recent theories suggest the potential of a subtle cognitive predisposition associated with diabetes risk or that the relationship between cognitive decline and the manifestation of the illness is bidirectional. In either case, as both risk for type 2 diabetes and neurocognitive processing are heritable, it is possible that shared genetic factors (e.g. genes) influence the observed relationship. Here, we test for genetic correlation between neurocognitive performance and diabetes risk in a randomly ascertained longitudinally studied cohort of Mexican-Americans who are at high risk for diabetes. The San Antonio Family Study (SAFS) includes over $\sim 3,000$ individuals from $\sim 60$ large randomly ascertained pedigrees that we have followed for the past 25 years. Methods: Analyses were conducted in a subsample of 1910 SAFS individuals (60\% female, average age 42.08 years (S.D. 15.7 [18-97])) who have diabetes diagnostic information and a neurocognitive assessment. The neurocognitive assessment involved a comprehensive battery of clinical and experimental neuropsychological tests indexing attention, processing speed, executive functioning, working and longer-term memory, problem solving and intelligence. The diabetes assessment included self-report and fasting glucose measures. Using SOLAR, we assessed the phenotypic, genetic and environmental influences on 17 neurocognitive traits and self and laboratory measures of diabetes. To control for multiple comparisons, FRD corrections were applied.

Results: As previously reported, all of the neurocognitive measures were significantly heritable. 363 individuals reported a current diabetes diagnoses (19\% of the sample). Self-report diabetes $(\mathrm{h} 2=0.709, p=1.88 \times 10-16)$ and fasting glucose $(\mathrm{h} 2=0.301, p=1.00 \times 10-7)$ were likewise heritable. A negative genetic correlation between performance on the Penn Facial Memory test and diabetes status ( $\mathrm{rho}-\mathrm{g}=-0.373$, $p=0.0029$ ) was significant after correcting for multiple comparisons. In addition, a number of hits on an identical pairs continuous performance test ( $\mathrm{rho}-\mathrm{g}=-0.467, p=0.006$ ) trended towards significant.

Conclusions: We report evidence for pleitropy between facial memory and risk for diabetes, suggesting that shared genetic factors influence poorer decorative memory and increased risk for the illness. Our results extend prior observations of 
memory dysfunction in diabetes by indicating common genetic influences on these traits. Currently, we are working to localize and identify the genetic variants that drive this relationship. As the neurocognitive sequela of type 2 diabetes and increased rates of dementia represent a substantial public health burden, any insights into the biological mechanisms that drive this relationship are desperately needed.

Keywords: Memory, Type-2 Diabetes, Genetic Correlation. Disclosure: Nothing to disclose.

\section{W5. Adverse Childhood Experiences Moderates the Impact of Tryptophan Depletion on Executive System Function}

Sheila Shanmugan*, James Loughead, Wen Cao, Mary D. Sammel, Kosha Ruparel, Ruben Gur, C. Neill Epperson

University of Pennsylvania, Philadelphia, Pennsylvania, United States

Background: Executive dysfunction is present in several psychiatric disorders involving dysregulated serotonin signaling. However, studies using tryptophan depletion to probe serotonin's contribution to executive processes have yielded inconsistent results, in part due to confounding effects of age, sex, and hormonal status. We examined whether early life adversity contributes to differential responses to serotonergic manipulations. Using tryptophan depletion and fMRI allowed us to translate findings from rodent and nonhuman primate studies suggesting early life adversity has enduring effects on serotonergic function to human subjects. We hypothesized that early adversity would moderate the effects of tryptophan depletion on executive network activation during a working memory task.

Methods: Thirty-nine healthy, middle-aged women completed a letter n-back task during functional imaging in this double-blind, placebo-controlled, crossover study. History of early adversity was assessed using the Adverse Childhood Experiences questionnaire. Voxel-wise, wholebrain, repeated-measures ANOVA assessed whether adverse childhood experiences moderates the effect of tryptophan depletion at high and low levels of working memory load.

Results: Adverse childhood experiences significantly moderated the effect of tryptophan depletion on activation in brain regions part of 3 key networks involved in top-down cognitive control: the fronto-parietal control network, the cingulo-opercular control network, and the cerebellar error network. At high levels of working memory load, TD differentially impacted activation in the DLPFC and cerebellum depending on whether subjects had experienced early life adversity, while at low levels of working memory load, an interactive effect was observed in the dorsolateral prefrontal cortex, frontal pole, precuneus, and superior parietal lobule.

Conclusions: These findings provide novel evidence that early life adversity is a moderator of tryptophan depletion's effects on executive system function into adulthood and that individuals with high and low levels of early adversity respond differently to experimental manipulations of the serotonergic system.
Keywords: Executive Function, Serotonin, Adverse Childhood Events.

Disclosure: Nothing to disclose.

\section{W6. A Meta-Analysis With Focus on Developmental Stress and a Biologic Marker of Aging}

Mateus Levandowski*, Kathryn Ridout, Samuel Ridout, Lindsay Gantz, Kelly Goonan, Daniella Palermo, Lawrence Price, Audrey Tyrka

Pontifical Catholic University of Rio Grande do Sul, Porto Alegre, Brazil

Background: Early adversity increases lifetime risk for psychiatric and other medical disorders. Several recent studies have examined associations between early adversity and telomere length, a marker of cellular aging, providing insight into the biologic mechanisms underlying these associations. There is variability in the reported size and significance of this association. The objective of this study was to establish the relationship between early adversity and telomere length using meta-analytic techniques and to explore factors affecting this association.

Methods: A comprehensive search of PubMed/MEDLINE, PsycINFO, and Web of Science was conducted in June 2016. Included studies: 1) Examined the effects of early life adversity (in the form of abuse, neglect, caregiver loss through abandonment, death or separation, other adverse exposures, or socioeconomic status) occurring between the prenatal period and age 18 on human telomere length; 2) provided adequate description of the assessments used to determine early adversity exposure and telomere measurement; and 3) presented sufficient data to calculate effect sizes. Independent data extractors utilized a structured data abstraction form. Analyses were performed using Comprehensive Meta-Analysis Software (V2.2.064 Biostat, Englewood, New Jersey) with the standard meta-analysis function and random effects model. Method of moments random effects meta-regression and random effects subgroup moderator analyses were performed to examine potential sources of heterogeneity.

Results: Forty-one studies (cumulative $N=30,773$ ) met inclusion criteria. The association between early adversity and telomere length was significant (Cohen's d effect size = $-0.347 ; 95 \%$ CI, -0.456 to $-0.238, p<0.0001$ ). Sensitivity analyses revealed no outlier effects. Type and timing of adversity exposure significantly impacted the association with telomere length $(p<.0001$ and $p=.0025$, respectively). Subgroup analysis and meta-regression revealed that medication use, medical or psychiatric conditions, study design, methodological factors, age and smoking significantly affected the relationship.

Conclusions: Early adversity and telomere length are negatively related. This analysis suggests that early life adversity may have long-lasting consequences contributing to disease risk and biological aging. Further work is warranted to clarify the causality and reversibility of the observed association.

Keywords: Telomere, Meta-Analysis, Childhood Maltreatment, Early Life Stress.

Disclosure: Nothing to disclose. 
W7. Smaller Amygdala and Hippocampal Volume in Posttraumatic Stress Disorder From Multi-Site Investigation by Enigma and PGC Consortia

Rajendra Morey*, Mark Logue, Sanne van Rooij, Emily Dennis, Sarah Lancaster, Courtney Haswell, Lauren LeBois, Milissa Kaufman, Jonathan Wolff, Lauren O'Connor, Staci Gruber, Justin Baker, Sherry Winternitz, Kerry Ressler, Jim Lagopoulos, Elbert Geuze, Jennifer Stevens, Tanja Jovanovic, Miranda Olff, Laura Nawjin, Mirjam van Zuiden, Jessie Frijling, Saskia Koch, Richard Bryant, Mayuresh Korgaonkar, Mark Miller, Jasmeet Hayes, Jeffrey Spielberg, Erika Wolf, David Salat,

William Miberg, Regina McGlinchy, Katie McLaughlin

Duke University, Durham, North Carolina, United States

Background: The hippocampus and amygdala have been of particular interest to PTSD researchers. The hippocampus plays an essential role in memory encoding, contextual learning, and the contextual modulation of behavior, whereas the amygdala is critical to associative fear learning and fear expression. While numerous prior studies have found smaller hippocampal and amygdala volume in PTSD many other studies have been negative or shown larger amygdala volumes, in some cases reflecting small samples with insufficient statistical power.

Methods: The PGC-ENIGMA PTSD Working Group analyzed neuroimaging and clinical data from 1,868 subjects including 794 PTSD patients and 1,074 trauma-exposed controls contributed by 16 cohorts from five countries. We assessed the volumes of seven subcortical structures (nucleus accumbens, amygdala, caudate, hippocampus, pallidum, putamen and thalamus), lateral ventricle and total intracranial volume (ICV). Our study design implemented a standardized image-analysis pipeline based on FreeSurfer and quality control, established by the ENIGMA Consortium, at every site that provided individual subject data for meta-analysis.

Results: The amygdala and hippocampus, after adjusting for age, sex, and ICV, were smaller in subjects with current PTSD (amygdala, $\mathrm{D}=-0.11, \quad p=0.025 ;$ hippocampus, $\mathrm{D}=-0.17, p=0.00054)$. The ICV-unadjusted effect sizes for hippocampus and amygdala were larger (amygdala, $\mathrm{D}=-0.16, p=0.0058$; hippocampus, $\mathrm{D}=-0.22, p=0.000048$ ). In females, the negative association with hippocampal volume in the female-only participants had a large effect size $(\mathrm{D}=-0.31, p=0.00012)$, despite $1,100(>50 \%)$ fewer subjects than the full sample. The hippocampal volume association was non-significant in the male-only metaanalyses. Childhood trauma was associated with smaller amygdala volume $(\mathrm{D}=-0.16, p=0.0044)$ and hippocampal volume $(\mathrm{D}=-0.17, p=0.0031)$ in a model adjusting for age, sex, and ICV. The other subcortical regions did not show significant PTSD or trauma effects. Whereas we hypothesized that both amygdala and hippocampus volume would differ in PTSD, the hippocampal result was significant after imposing a Bonferrroni correction, which was not the case for the amygdala.

Conclusions: In the largest ever neuroimaging study of PTSD, we demonstrate that PTSD is associated with smaller hippocampus and amygdala volume. Our findings underscore the importance of these structures in PTSD that already have an established role in fear processing, episodic and contextual learning and memory, and processes related to PTSD symptomatology. Longitudinal studies investigating early interventions and treatments targeting the hippocampus and amygdala will help to advance the field of PTSD research.

Keywords: PTSD, Amygdala, Hippocampal Volume, Human Neuroimaging, Structural MRI.

Disclosure: Nothing to disclose.

\section{W8. Association of the COMT val158met Polymorphism With PTSD Risk: A Cross-Species Translational Study}

Jessica Deslauriers, Caroline Nievergelt, Adam Maihofer, Dewleen Baker, Mark Geyer, Victoria Risbrough*

University of California, San Diego, La Jolla, California, United States

Background: Posttraumatic stress disorder (PTSD) affects $7-8 \%$ of the American population while rates are up to $20 \%$ in military veterans. The val158met polymorphism in the cathechol-O-methyltransferase (COMT) gene has been associated with altered risk for neuropsychiatric disorders. Here we tested the hypothesis that the COMTval158met polymorphism modulates risk for development of PTSD. To test this hypothesis, we employed a cross-species translational approach. First we investigated the association of the COMTval158met polymorphism with PTSD diagnosis after combat deployment in Marines. Second, to confirm a potential causal role of the COMTval158met polymorphism in modifying response to extreme stress, we examined the sensitivity of mice "humanized" for the COMTval158met polymorphism to the predator stress model of PTSD.

Methods: Humans: The Marine Resiliency Studies I and II (MRSI: Jul 2008-May 2012; MRS-II: Oct 2011-Oct 2013) are longitudinal investigations in Marines $(N=2287)$ deployed to Afghanistan. As part of these investigations, Marines and Navy corpsmen underwent a psychiatric symptom assessment (clinician-administered PTSD scale, CAPS) for PTSD diagnosis prior to deployment and again 4-6 months after returning from deployment. With a multivariate logistic regression, we examined if COMTval158met genotype interacted with combat trauma during deployment (Combat Exposure Scale; CES) to modify PTSD symptoms post deployment. Ancestry, pre-deployment PTSD symptoms and trauma burden were used as covariates. Mice: We tested the response of mice "humanized" for the COMTval158met polymorphism (Risbrough et al., 2014) in the predator stress model of PTSD. Male Val/Val or Met/Met carriers were grouped into stress $(N=10-18$ per genotype) or control groups ( $N=8-15$ per genotype), with stressed groups being exposed to a cat for 10 minutes. Two protocols of predator stress were performed across two separate cohorts: "severe trauma" (physical contact allowed) and "mild trauma" (mouse in a cage during the exposure, no physical contact allowed). One week after the predator stress, generalized avoidance (open field; light-dark box) and avoidance of a "trauma cue" (cat odor) were evaluated over. A composite 
avoidance score (average of Z-scores across the 3 tests) was calculated for each animal.

Results: In Marine participants, Val carriers who endorsed high combat trauma had higher probability to develop PTSD symptoms after deployment compared to Met/Met carriers, as shown by a genotype $\mathrm{x}$ CES interaction (main effect of CES: odd ratio $2.10(1.36-3.26) ; p<.001$ and genotype $x$ CES interaction: odd ratio $0.44(0.19-1.01) ; p<.05)$. In mice, $\mathrm{Val} / \mathrm{Val}$ carriers exhibited increased avoidance behavior compared to Met/Met mice $(\mathrm{F}(1,38)=27.83 ; p<.001$ and $\mathrm{F}(1,51)=4.79 ; p<.05$, in severe and mild trauma experiments, respectively), suggesting baseline anxiety differences across Val and Met carriers. Severe and mild predator stress also significantly increased avoidance $(\mathrm{F}(1,38)=64.16$; $p<.001$ and $\mathrm{F}(1,51)=5.87 ; p<.05$, respectively), resulting in the greatest avoidance behavior being in the $\mathrm{Val} / \mathrm{Val}$ stressed mice.

Conclusions: These results suggest that in humans the COMTval158met polymorphism is associated with altered risk for PTSD in subjects exposed to high trauma, with Val carriers showing greater probability of developing PTSD compared to Met carriers. This association is confirmed in a humanized model of the COMTval158met polymorphism, supporting the involvement of this polymorphism in a double-hit model of PTSD. COMTval158met mice may be a useful model to understand the mechanisms underlying risk for development of PTSD-like symptoms.

Keywords: COMT Gene, PTSD, Trauma, Mice.

Disclosure: Nothing to disclose.

\section{W9. Response to and Recovery From Traumatic Stress in Rodents: A Behavioral and Neuroendocrine Characterization}

Kevin D. Cravedi, Daniel E. Altman, Chau T. Vuong, Sean R. Marcsisin, Jason C. Sousa, Nicole Moore*

Walter Reed Army Institute of Research, Silver Spring, Maryland, United States

Background: Rodent response to a traumatic stress (a potentially life-threatening stress event) includes measurable behavioral and neuroendocrine changes over time. Failure to return to baseline after the resolution of a stressor event may be an underlying factor in adverse long-term outcomes, such as post-traumatic stress disorder and other post-stressor psychological health concerns. To better understand the typical course of behavioral and neuroendocrine change following a potentially-life threatening event, we employed a rodent model to characterize the average response to two different stress events using a wide panel of behavioral and neuroendocrine markers.

Methods: Using adult male Sprague Dawley rats (75 days of age and older), we evaluated baseline measures followed by repeated post-stress evaluations to one-week post-stress exposure. Stressors included underwater trauma or predator stress, with a sham control. Behavioral changes evaluated include acoustic startle reflex and basic locomotor activity on the elevated plus maze. Neuroendocrine markers included corticosterone, epinephrine, norepinephrine, and prolactin. Maximal change from baseline, timecourse of recovery, and time to resolution of stress-induced change were measured.
Results: Categorically different stress exposures produced distinct stress response signatures, suggesting against a single universal behavioral or neuroendocrine response associated with a life-threatening stressor.

Conclusions: Determining specific outcomes associated with different types of stressors may have implications for therapeutic approaches to facilitate recovery.

Keywords: Acute Stress, Neuroendocrine Responses, Adaptive Behavior.

Disclosure: Supported by the Military Operational Medicine Research Program, US Army Medical Research and Materiel Command.

Material has been reviewed by the Walter Reed Army Institute of Research. There is no objection to its presentation and/or publication. The opinions or assertions contained herein are the private views of the author, and are not to be construed as official, or as reflecting true views of the Department of the Army or the Department of Defense. Research was conducted in compliance with the Animal Welfare Act and other Federal statutes and regulations relating to animals and experiments involving animals and adheres to principles stated in the Guide for the Care and Use of Laboratory Animals, NRC Publication, 2011 edition. All procedures were reviewed and approved by the WRAIR Institutional Animal Care and Use Committee, and performed in facilities accredited by the Association for Assessment and Accreditation of Laboratory Animal Care, International.

\section{W10. Heart Rate Variability Responses to a Standardized Virtual Reality Exposure in Veterans With PTSD}

Jorge Almeida*, Christopher Spofford, Mascha van 't Wout, William Unger, Noah Philip, Linda Carpenter, M. Tracie Shea

Brown University, Providence, Rhode Island, United States

Background: Virtual reality (VR) is growing in popularity for the treatment of posttraumatic stress disorder (PTSD). A growing evidence base supports the effectiveness of utilizing this technology for the benefit of PTSD patients and stimuli can be uniquely tailored to patient traumas to make exposures very specific. Combat Veterans within the Veterans Administration (VA) health system have perhaps benefitted the most from this innovation in treatment. However, to-date there has been little integration of heart rate variability psychophysiological data as a potential biomarker to differentiate PTSD patients from non-PTSD patients, and which might be used as an objective indicator of treatment effectiveness. Heart rate variability (HRV) refers to the variability in the R-R interval of the electrocardiogram. This is governed by the balance between sympathetic and parasympathetic outflow in the autonomic nervous system and can provide particularly relevant insights into a population such as individuals with PTSD, a group known to have hyperarousal and increased autonomic reactivity to stimuli. Several indices can be calculated from the electrocardiogram and used to characterize the sympatho-vagal balance. Briefly, time-domain indices and frequency-domain 
indices impart information regarding components of autonomic control. The purpose of the present study was to examine the HRV response to a series of non-personalized combat-related VR events as well as non-combat stimuli of increasing complexity (classroom scenes) in individuals with and without PTSD. We hypothesized that group differences in autonomic responsiveness would be reflected in the HRV as individuals with PTSD are expected to have a sympatho-vagal balance that demonstrates a sympathetic predominance.

Methods: 19 individuals with PTSD and 25 individuals without were recruited from the Providence, RI VA Medical Center patient population. All were required to have had combat experience during operations in Iraq or Afghanistan and PTSD patients were required to be engaged in therapeutic treatment. Participants with an abnormal electrocardiogram, those taking benzodiazepines, beta blockers and alpha-2 agonists were excluded. PTSD diagnosis was evaluated using the Clinician-Administered PTSD Scale and the Structured Clinical Interview of DSM Disorders. Virtual reality content was delivered with visual, auditory, haptic and olfactory stimuli in the combat scenarios, which increased progressively in intensity throughout six one-minute scenes. Classroom scenes included visual and auditory stimuli that increased in complexity similarly through a series of 6 oneminute scenes. A two-minute inter-stimulus interval separated all classroom or combat scenes and participants were randomized to receive either the battery of combat scenes or classroom scenes first to account for ordering effects. HRV was continuously acquired throughout the VR trial using a single-lead electrocardiogram (Biopac Systems Inc, Goleta, CA) and analyzed offline using specialized software (LabChart 8, ADI Instruments, Colorado Springs, CO). Baseline data were averaged across the 5-minute baseline period preceding the start of VR stimuli. Data from VR stimuli were averaged across the 1-minute periods of VR stimuli. Data were analyzed using SPSS software (IBM corporation, Armonk, NY) with a repeated measures ANOVA design and grouped according to patients with and without PTSD where appropriate. Bonferroni's correction for multiple comparisons was applied by the statistical program, which presents adjusted $p$-values and significance set at the $p=.05$ level. Low-frequency and high-frequency HRV are presented as normalized units (LFnu and HFnu, respectively).

Results: In the battery of classroom scenes there was a significant change in the LFnu $(p=.016)$ and HFnu $(p=.007)$, likely driven by an initial fall in LFnu from baseline to scene 1 and rise in HFnu from baseline to scene 1 . However, the main effect of group (PTSD and non-PTSD participants) was not significant $(p=.122$ for LFnu and $p=.105$ for HFnu). In the battery of combat scenes there was a similarly significant change in LFnu $(p=.034)$ and HFnu $(p=.038)$ and group was not significant $(p=.182$ for LFnu and $p=.142$ for HFnu).

Conclusions: These results indicate that even with short epochs such as the 1-minute scenes described here there is a detectable difference in the HRV indices of sympathetic (LFnu) and parasympathetic (HFnu) autonomic control. While the effect of group was not statistically significant there was a consistent qualitative difference where participants with PTSD demonstrated higher LFnu and lower
HFnu compared with participants that did not have PTSD. This may suggest a consistently higher sympathetic drive and lower parasympathetic drive in this group, as would be suggested by previous studies of hyperarousal and autonomic regulation in individuals with PTSD. The lack of group significance may be due to low power for assessment of HRV. HRV assessments typically require an epoch longer than 1-minute to reflect changes in autonomic regulation and therefore it is particularly striking that even with such short VR scenes differences were observed including the qualitative observation that individuals with PTSD demonstrate changes in sympathetic and parasympathetic regulation during VR stimuli that are consistent with a sympathetic predominance. Finally, respiratory sinus arrhythmia plays a central role in governing the HF measurements of HRV and respiratory rate/depth was not controlled for in the present study. These data support further studies designed to assess HRV responses to VR stimuli in PTSD patients with epochs that provide adequate time for the kinetics of HRV in order to more fully characterize HRV as a potential biomarker for the assessment and treatment of PTSD using VR technology. Keywords: Heart Rate Variability, PTSD, Biomarker, Psychophysiology.

Disclosure: Nothing to disclose.

\section{W11. Microglial Recruitment of Monocytes to the Brain Causes Stress-Induced Anxiety}

\section{Michael Weber*, Daniel McKim, Caroline Sawicki, Anzela Niraula, Jonathan Godbout, John Sheridan \\ Ohio State University College of Medicine, Columbus, Ohio, United States}

Background: Psychosocial stressors are associated with an increased prevalence of mental health complications, including anxiety and depression. Repeated social defeat (RSD) is a murine stress model that recapitulates many of the immune and behavioral outcomes of stress. For instance, RSD activates fear/stress neurocircuitry, accompanied by microglial activation, increased pro-inflammatory signaling and the promotion of anxiety. In addition, RSD promotes longterm "stress-sensitization" in which exposure to subthreshold stress 24 days after RSD causes re-establishment of anxiety. We have reported that a key part of RSD-induced anxiety is the trafficking of inflammatory Ly6Chi monocytes to fear and threat appraisal areas of the brain. We hypothesize that the site-specific trafficking of Ly6Chi monocytes to the brain is caused by active recruitment directed by resident microglia.

Methods: Mice were subjected to RSD. An aggressive male intruder CD-1 mouse was introduced into cages of established male cohorts ( 3 per cage) of C57BL/6 mice for 2 hours for six consecutive nights. To address the role of microglia, the colony-stimulating factor (CSF)-1 antagonist, PLX5622, was used to eliminate microglia. PLX5622 was formulated into standard rodent and mice were provided ad libitum access to PLX5622 or control diet for 14 days to deplete microglia.

Results: In the first experiment, microglia were eliminated prior to RSD. Elimination of microglia prior to RSD abrogated the recruitment of Ly6Chi monocytes to the 
brain. Moreover, microglia elimination and the absence of inflammatory monocytes prevented the induction of anxietylike behavior in response to RSD. Thus, microglia were necessary for the recruitment of monocytes to the brain and the development of anxiety during stress. In the second experiment, PLX5622 was used to study recall of anxiety following exposure to subthreshold stress 24 days after RSD. Exposure to sub-threshold stress 24 days after RSD caused Ly6Chi monocyte trafficking to the brain and re-established anxiety-like behavior. Elimination of microglia after RSD prevented the recruitment of Ly6Chi monocytes to the brain and anxiety-like behavior in RSD-sensitized mice.

Conclusions: Microglial activation during stress was critical for the development of anxiety via the recruitment of Ly6Chi monocytes to the brain. This understanding could lead to novel interventions to target myeloid cell trafficking and attenuate prolonged neurobehavioral complications associated with chronic stress.

Keywords: Acute and Chronic Stress, Microglia, Social Defeat, GC-Insensitive Monocytes, Anxiety.

Disclosure: Nothing to disclose.

\section{W12. Transdiagnostic Cognitive and Emotional Impairment in Mental Illness: Disrupted Neural Circuit Structure and Function}

Lisa McTeague*, David Carreon, Benjamin Rosenberg, Julia Huemer, Ying Jiang, Eickhoff Simon, Amit Etkin

\section{Stanford University, Stanford, California, United States}

Background: Historically, diagnostic categories have been conceptualized as discrete phenotypes. However, phenotypic comorbidity or symptom overlap abounds. Accumulating neurobiological findings (e.g., genetics, fMRI) highlight transdiagnostic commonalities. Recently we investigated whether a shared substrate might be revealed in regional gray matter volume (Goodkind et al., in 2015, JAMA Psych). Utilizing a meta-analysis of patient/control differences in voxel-based morphometry (VBM) across a range of diagnoses (bipolar and unipolar depression, anxiety, substance abuse/dependence, psychosis) we observed that gray matter volume was decreased in the bilateral insula and dorsal anterior cingulate across diagnoses, with the greatest loss in schizophrenia, the most impairing and chronic condition. Furthermore, we demonstrated that this network impairment predicted poor executive functioning/ cognitive control behavioral performance. To explore whether these nodes of decreased structural integrity might relate to observable signatures of aberrant brain function, we performed transdiagnostic meta-analyses of cognitive control as well as emotional functioning.

Methods: Studies on cognitive $(n=283)$ and emotional ( $n=252)$ functioning including patients across a range of Axis I diagnoses (bipolar and unipolar depression, anxiety, substance abuse/dependence, psychosis) and matched control participants were submitted to meta-analysis with Activation Likelihood Estimation. Cognitive/executive function or emotion provocation tasks during functional brain imaging that reported significant whole-brain, voxel-wise group differences in stereotaxic space were included. Metaanalysis utilized peak voxel coordinates for activation likelihood estimation of patterns reflecting patient hyperor hypo-reactivity in relation to control participants.

Results: During cognitive processing tasks, transdiagnostic patient hypoactivation was evident in anterior insula and dorsal anterior cingulate as well as left dorsolateral prefrontal cortex (dlPFC) and bilateral intraparietal sulci. The opposite pattern of patient hyperactivation emerged transdiagnostically in the mid-cingulate cortex/pre-supplementary motor area. Diagnostic-specific effects were observed unique to the left dlPFC due to pronounced hypoactivation in schizophrenia. During emotion processing, transdiagnostic effects emerged for patient hyperactivation in bilateral insula, left amygdala, and ventromedial prefrontal cortex, whereas dACC hyperactivation was specific to non-psychotic disorders. Notably, these results were not accounted for by age, medication usage, or specific task/domain. Considering regions of concordant dysfunction in structure and function (i.e., comparison across the VBM and two functional neuroimaging meta-analyses) revealed shared impairment particularly in right anterior insula extending to vlPFC.

Conclusions: Intact cognitive control and emotional functioning have characteristic patterns in functional neurocircuitry. Neuroimaging studies have shown that a "multiple demand" network forms a common functional substrate undergirding successful adaptation to diverse cognitive processing demands. This network comprises two subnetworks (i.e. frontal-parietal; cingular-opercular) also implicated in intact emotional reactivity and its regulation. The findings from these two large-scale meta-analyses of Axis I disorders suggest that cognitive and emotional perturbations amidst psychopathology are most pronounced within these networks promoting adaptive functioning and moreover, are evident across disorders (i.e., transdiagnostically). That is, cognitive disruptions are evident across both frontal-parietal and cingular-opercular networks whereas emotional reactivity aberrations are more pronounced in cingular-opercular nodes. Demonstrating an essential functional parallel to our prior structural findings, the cingular-opercular or salience network was highlighted as a particular common pathway to cognitive and emotional dyscontrol in psychopathology and potentially as a powerful common target for therapeutic intervention.

Keywords: Meta-Analysis, Research Domain Criteria (RDoC), Functional Neuroimaging, Cognitive Functioning, Emotional Reactivity.

Disclosure: Nothing to disclose.

W13. The Associative-Limbic Subthalamic Nucleus: Intrinsic Functional Connectivity and the Influence of Stimulation on Decisional Impulsivity in Obsessive Compulsive Disorder

Valerie Voon*, Fabien Droux, Laurel Morris, Stephan Charbades, Thierry Bougerol, Olivier David, Paul Krack, Mircea Polosan

\section{University of Cambridge, Cambridge, United Kingdom}

Background: Why do we make hasty decisions for short term gain? Rapid decision making with limited accumulation of evidence and delay discounting are forms of decisional impulsivity. The subthalamic nucleus (STN) is implicated in 
inhibitory function but its role in decisional impulsivity is less well-understood. The limbic-associative STN is of increasing interest in deep brain stimulation (DBS) for psychiatric disorders such as obsessive-compulsive disorder (OCD).

Methods: We assess OCD subjects who have undergone limbic-associative STN DBS and test the causal role of the STN. We test subjects on and off DBS in a counterbalanced order on two forms of decisional impulsivity: evidence accumulation during probabilistic uncertainty (Beads task) and delay discounting. In a separate study, we use resting state functional connectivity in 154 healthy controls to parcellate limbic, associative and motor cortical connectivity with subregions of the STN. We use a multi-echo sequence with independent components analysis, which has been shown to have enhanced signal-to-noise ratio, thus optimizing visualization of small subcortical structures. We further investigate correlations between decisional impulsivity measures in healthy controls and functional connectivity of limbic-associative and motor STN subregions.

Results: We show that stimulation of the STN in OCD patients is causally implicated in increasing decisional impulsivity with less accumulation of evidence during probabilistic uncertainty and in enhancing delay discounting. In a separate study in healthy controls, we show a dissociation in peak connectivity between anterior limbicassociative (ventral striatal, dorsolateral prefrontal cortex (BA 9/46), dorsal cingulate (BA 24)) and posterior motor (supplementary motor area (caudal BA 6) and M1 (BA 4)). Pre-SMA (rostral BA 6) and right inferior frontal cortex implicated in the 'stopping network' had peaks overlapping across anterior and posterior STN. Bilateral ventral striatum showed significant connectivity only to right anteromedial STN. We further show that evidence accumulation is associated with anterior associative-limbic STN (but not posterior motor STN) and right dorsolateral prefrontal functional connectivity in healthy controls, a region implicated in decision making under uncertainty.

Conclusions: STN stimulation shifts evidence accumulation in OCD subjects towards a functional less cautious style closer to that of healthy controls emphasizing its adaptive nature. Thus, subjects with OCD on STN stimulation may be less likely to check for evidence (e.g. checking that the stove is on) with no difference in subjective confidence (or doubt). We further replicate in humans using resting state functional connectivity, tracing studies conducted in non-human primates dissociating limbic, associative and motor frontal hyper-direct cortical connectivity with anterior and posterior sub-regions of the STN. We show lateralization of functional connectivity of ventral striatum consistent with previous observations of lateralization of emotionally evoked activity to right ventral STN. Together, our findings highlight specificity of the anterior associative-limbic STN in decisional impulsivity. Given increasing interest in the potential for STN DBS in psychiatric disorders and the neuropsychiatric symptoms of Parkinson's disease, these findings have clinical implications for behavioural symptoms and cognitive effects as a function of localization of STN stimulation.

Keywords: Subthalamic Nucleus, Deep Brain Stimulation, Impulsivity, Obsessive Compulsive Disorder, Resting State Functional Connectivity.

Disclosure: Nothing to disclose.
W14. Personality Changes in Refractory Patients With Obsessive-Compulsive Disorder Submitted to Gamma Ventral Capsulotomy

Raquel Paiva, Antonio C. Lopes, Marcelo Batistuzzo, Maria Eugenia de Mathis, Miguel Canteras, Benjamin Greenberg, Roseli Shavitt, Euripedes Miguel, Marcelo Hoexter*

University of Sao Paulo, Sao Paulo, Brazil

Background: Obsessive-compulsive disorder (OCD) is a chronic condition and has a lifetime prevalence of $2 \%$ to $3 \%$ in the general population. A small proportion of patients with OCD has severe and disabling symptoms, despite all available conventional treatments. For such individuals, psychiatric neurosurgical procedures are an alternative. In particular, gamma ventral capsulotomy (GVC) has shown promising results. However, to our knowledge, no prior studies have assessed personality changes in patients with refractory OCD after GVC. Thus, the aim of this study is to determine personality changes in subjects with OCD treated with GVC. We also sought to investigate whether or not these personality changes were associated with treatment response.

Methods: Pre- and 1-year postoperative assessments with the Revised NEO Personality Inventory (NEO PI-R) (Extraversion, Agreeableness, Conscientiousness, Neuroticism, Openness to Experience), the Temperament and Character Inventory (TCI) (Novelty seeking, Harm avoidance, Reward dependence, Persistence, Self-directedness, Cooperativeness, Self-transcendence) and the Yale-Brown Obsessive-Compulsive Scale (YBOCS) were obtained from refractory OCD patients submitted to GVC. Personality data were also collected from controls at baseline. Comparisons of means between groups were carried out using Mann Whitney test (two-tailed). For within-groups comparisons (pre vs. post), Wilcoxon signed-rank test were used (one-tailed). Correlations between changes in the severity of OCD and changes in personality scores were performed using the Pearson product-moment correlations (Persons' coefficient $=r$ ). A $p$ value of 0.05 was set as the significance threshold for the statistical analyses.

Results: Refractory OCD patients $(n=19)$ presented higher scores than controls $(n=28)$ in neuroticism $(p<0.001)$, conscientiousness $(p=0.02)$ and harm avoidance $(p<0.001)$ and lower scores in extraversion $(p<0.001)$, openness to experience $(p=0.04)$ and novelty seeking $(p=0.018)$. After GVC, OCD patients $(n=14)$ exhibited a significant decrease in the severity of obsessive-compulsive symptoms (mean \pm standard deviation pre- vs. post-treatment Y-BOCS: $32.6 \pm$ 2.6 vs. $19.4 \pm 11.9 ; p=0.002$ ). Neuroticism and persistence scores in OCD subjects significantly decreased after treatment ( $p=0.034$ and $p=0.032$, respectively; one-tailed). Changes in YBOCS scores were positively correlated with changes in persistence $(\mathrm{r}=0.55, p=0.04)$ and negatively correlated with changes in novelty seeking scores $(\mathrm{r}=-0.57$, $p=0.034$ ).

Conclusions: The present results suggest that GVC is not only a safe procedure in terms of personality functioning but actually may improve certain personality dimensions in patients with refractory OCD. Further personality studies 
with larger samples of refractory OCD patients and with longer follow-up periods are needed.

Keywords: Obsessive-Compulsive and Related Disorders, Neurosurgery, Personality.

Disclosure: Nothing to disclose.

\section{W15. Transcutaneous Auricular Vagus Nerve Stimulation (taVNS) Acutely Modulates Parasympathetic Tone as Measured by Decreases in Heart Rate in Healthy Individuals}

Bashar Badran*, Chloe Glusman, Christopher Austelle, Alan Badran, William DeVries, Jeffrey Borckardt, Mark George

Medical University of South Carolina, Charleston, South Carolina, United States

Background: Conventional (cervically implanted) vagus nerve stimulation (VNS) is FDA-approved for treating epilepsy or major depressive disorder. It has been suggested VNS can be administered non-invasively via electrical stimulation of the auricular branch of the vagus nerve that innervates the ear. This new form of VNS is called transcutaneous auricular vagus nerve stimulation (taVNS). Although early human trials demonstrate positive behavioral effects using taVNS, two issues are still debated: 1) whether taVNS truly stimulates the vagus system and its afferents 2 ) which taVNS parameters best modulate vagus system. Since the vagus nerve is highly involved in the parasympathetic nervous system, one can use physiological recordings during stimulation to determine whether taVNS has a vagus nerve mediated effect and determine optimal parameters. We have developed a novel taVNS stimulation system and tested the physiological effects of 9 different stimulation parameter combinations in order to systematically determine the optimal parameters. We hypothesized that taVNS would increase parasympathetic tone as measured by heart rate, skin temperature, and skin conductance. The parasympathetic effect size can then be used to rank optimal stimulation parameters.

Methods: 15 healthy participants (mean age $=26.5,7$ female) attended two separate experimental taVNS visits (one sham, one active; counterbalanced crossover design). Subjects laid supine with head elevated in a comfortable position while constant current taVNS was delivered to either the left tragus (active) or left earlobe (sham) using a Digitimer Ds7a stimulator and custom 3D-printed electrodes. Nine discrete stimulation parameter combinations were delivered of varying pulse width and frequency (pulse widths: $100 \mu \mathrm{s}, 200 \mu \mathrm{s}, 500 \mu \mathrm{s}$; frequencies: $1 \mathrm{~Hz}, 10 \mathrm{HZ}$, $25 \mathrm{~Hz}$ ) while keeping current constant (200\% perceptual threshold). Each stimulation parameter was tested for 60 seconds, flanked by a 90 second baseline period and a 180 second recovery period both of which no stimulation was delivered. Participants were asked to rate painfulness from 0 (no pain) to 10 (extreme pain) on a visual analog scale (VAS) after each stimulation period. Heart rate, skin temperature, and skin conductance were monitored live and recorded during the entire visit.

Results: Mean stimulation current varied for each pulse width (active range: $3-9.28 \mathrm{~mA}$, sham range: $1.98-6.56 \mathrm{~mA}$ ) and active taVNS was not significantly more painful than sham taVNS. Mean pain VAS scores, dependent on stimulation parameters, ranged from 0.2 to 0.83 (sham) and 0.57 to 1.94 (active). With respect to heart rate changes, there was no significant decrease in heart rate between all active and all sham stimulations in the overall group analysis. Upon individual parameter inspection, two of nine active taVNS parameters had significant decreases (versus sham taVNS) in heart rate during stimulation compared to baseline using a repeated measures ANOVA $(500 \mu \mathrm{s}, 10 \mathrm{~Hz}$ : 4.3BPM mean decrease, $P<0.01 ; 500 \mu$ s, $25 \mathrm{~Hz}: 1.1 \mathrm{BPM}$ mean decrease, $P<0.05)$. There were no unsafe drops in heart rate or adverse events during stimulation in any of the nine parameter sets. Using these data, we were able to rank parameters based on parasympathetic effect size in order from best (1) to worst (9) as follows: 1 . 500us, $25 \mathrm{~Hz} ; 2$. 500us, $10 \mathrm{~Hz}$; 3. 100us, $10 \mathrm{~Hz}$; 4. 200us, $1 \mathrm{~Hz}$; 5 . 100us, $25 \mathrm{~Hz}$; 6. $100 \mathrm{us}, 1 \mathrm{~Hz}$; 7. 200us, $10 \mathrm{~Hz} 8.500 \mathrm{us}, 1 \mathrm{~Hz} 9.200 \mathrm{us}, 25 \mathrm{~Hz}$. It is important to note only the first two of nine rankings had a significant condition effect, making the remaining seven rankings only mathematically different from each other.

Conclusions: taVNS effects, similar to other brain stimulation methodologies, are parameter (current, pulse width, and frequency) dependent. To our knowledge, these are the first data describing the acute physiological effects of taVNS and attempting to optimize parameters using parasympathetic tone as an indicator. We are now conducting a larger, headto-head challenge between the top two parameters to determine the true winning combination of pulse width and frequency that best modulate parasympathetic activity and will also determine their direct brain effects in the fMRI scanner.

Keywords: Brain Stimulation, Transcutaneous Auricular Vagus Nerve Stimulation (taVNS), Non-Pharmacological Therapy.

Disclosure: Nothing to disclose.

\section{W16. The Neural Basis of Impaired Safety Signaling in Obsessive Compulsive Disorder}

Annemieke Apergis-Schoute*, Claire Gillan, Naomi Fineberg, Emilio Fernandez-Egea, Barbara Sahakian, Trevor Robbins

\section{University of Cambridge, Cambridge, United Kingdom}

Background: In Obsessive Compulsive Disorder (OCD) lack of flexible control and heightened anxiety both contribute to the rigid compulsions and distressing obsessions that characterize this disorder. Neuroimaging studies have supported dysfunctioning of the cortico-striato-thalamocortical circuit to be central to OCD. Hyperactivation of the ventromedial prefrontal cortex (vmPFC) has been directly linked to the symptomatology of OCD and is mostly normalized by successful treatment. The vmPFC is known to play a multifaceted role in integrating affective evaluative processes while mediating flexible behavior and is implicated in fear learning and anxiety related disorders. We aimed to highlight this interaction in a sample of OCD patients without comorbidities by employing neuroimaging of Pavlovian fear reversal, a well-validated paradigm for 
studying flexible updating when the contingencies for a threatening (CS+) and safe stimulus (CS-) reverse.

Methods: We compared fear reversal learning in 43 OCD patients and 35 matched healthy controls by assessing threat expectancy with skin conductance responses (SCRs) and its neural correlates with functional magnetic resonance imaging. Our goals were to i) relate any differences in threat learning and reversal to group differences on the whole brain level corrected for multiple comparisons; ii) to assess the importance of the vmPFC in accurate threat reversal in OCD; and iii) to perform region of interest analyses (ROIs) for key areas in fear learning (amygdala and striatum) to investigate possible group differences. Learning was quantified as the difference between SCRs to the CS+ and CSduring acquisition and reversal, which were divided into early (first half) and late (second half) phases. The data analyses mainly used the contrast between the CS+ and CS-, which refers to the original CS+ and CS- during acquisition and the reversed CS+ and CS- during reversal.

Results: Discrimination between the threatening and safe stimulus was intact during initial conditioning in OCD patients $(N=43)$ compared to healthy controls $(N=35)$. In sharp contrast, patients failed to differentiate during reversal as indexed by skin conductance responses. Whole brain family wise error corrected comparisons revealed hyperactivation of the vmPFC in OCD during early conditioning for the CS+ versus CS- contrast $(P<0.001)$. This vmPFC hyperactivation predicted the degree of generalization in OCD patients during reversal whilst vmPFC safety signals for updating the CS- were non-existent throughout. The absence of such a safety signal in the vmPFC during both reversal and acquisition in OCD combined with otherwise intact learning, suggests that OCD patients acquire threat conditioning mainly by labeling the valence of the CS+, rather than additionally assigning a 'safe' label to the CS-. This account is further supported by the finding that, during early learning the vmPFC showed enhanced connectivity with the salience network, indicating that OCD patients could rely on the salience of the CS+ alone to acquire threat estimation during learning. Furthermore, vmPFC hyperactivation in OCD during early learning was correlated with CSresponding during early learning and early reversal. Previously it has been shown that the vmPFC has roles in extinction learning and retrieval, as well as in emotional regulation and (re)valuation and is implicated in impaired extinction learning in OCD. However, a critical role in safety signaling has hitherto received insufficient attention in anxiety related disorders. The differentiation failure during threat reversal in OCD patients was also reflected in their non-discriminative striatal responses during this stage, likely due to the absence of the vmPFC safety signal. Although anxiety is often considered central to OCD, suggesting a putative role for the amygdala, OCD patients and controls exhibited strikingly similar amygdala processing during acquisition and reversal learning, indicating that generalization was not due to amygdala impairment.

Conclusions: To summarize, our findings indicate that a hyperactive vmPFC combined with increased connectivity with areas involved in salience processing undermines accurate safety learning in OCD patients, resulting in inflexible threat beliefs. These results concerning vmPFC safety signaling can be informative for improving exposurebased therapies that rely on robust safety memories.

Keywords: OCD, vmPFC, Fear Conditioning, Functional MRI (fMRI).

Disclosure: Nothing to disclose.

\section{W17. Hippocampal-Dependent Pattern Separation and Completion of Complex Contextual Scenes}

Elizabeth Duval*, James Abelson, Israel Liberzon

University of Michigan Health System, Ann Arbor, Michigan, United States

Background: Pattern Separation (PS) and Pattern Completion (PC) are hippocampal dependent processes underlying the contextual encoding and retrieval of information. PS is the ability to encode new information as different from previously learned but similar information, while PC is the ability to recognize something as "known" based on previously learned information, even when only a portion of the learned information is available. These processes likely have relevance to fear learning, as encoding and retrieval of contextual information are crucial to disambiguate potential threat cues. Patients with PTSD show deficits in hippocampal-dependent aspects of fear learning as well as abnormalities in hippocampal structure and function, suggesting that memory processes like PS and PC may underlie PTSD symptoms. Further study of PS and PC abilities in fear learning paradigms may deepen understanding of neurocognitive deficits in PTSD. PS and PC have been assessed to date primarily using item/object identification tasks, but fear learning deficits in PTSD likely involve problems with separation and completion of patterns within complex environmental contexts. We sought to develop a novel task to assess PS and PC processes using complex scenes and confirm the hypothesized role of hippocampus in these processes on the new task.

Methods: Healthy adults completed two PS and PC tasks during fMRI. The established Mnemonic Similarities Task (MST; Stark et al., 2013) presents images of common objects during encoding and recall phases. During recall, participants see previously viewed objects, as well as distractors (never seen before) and lures (images that are similar to but different from encoded images). They have to indicate whether each item was "old", "similar" or "new". They then completed a Context Separation and Completion Task (CSC), currently under development in our lab, to assess PS and PC of complex scenes. This task uses degraded images of two previously learned scenes. Participants indicate which of the previously learned scenes they are viewing, or whether they are seeing a new scene (distractors). Difficulty is manipulated by scrambling pixels to varying degrees of degradation.

Results: Preliminary findings $(N=6$, data collection ongoing; $p<.01$, uncorrected) replicate previous reports of hippocampal activation during the well-established MST. We saw task-related hippocampal activation during both encoding $(30,-19,-14)$ and recall phases $(24,-26,-10$; all trials $>$ implicit baseline), as well as hippocampal activation associated specifically with PC $(24,-7,-20$; trials accurately remembered $>$ inaccurately remembered). The new CSC 
$\overline{S 466}$

task produced similar results, with hippocampal activation during PC (-30, -4, -20; trials accurately remembered $>$ inaccurately remembered).

Conclusions: Though preliminary, our results replicate previously reported hippocampal activity associated with PS and PC, and extend these findings to a task examining PS and PC using visually degraded complex scenes. We propose to further utilize both tasks in studies of PS and PC performance and underlying hippocampal function, to test hypotheses regarding hippocampal dependent memory deficits that may underlie fear learning abnormalities in PTSD.

Keywords: Memory Encoding and Retrieval, Functional MRI (fMRI), Context.

Disclosure: Nothing to disclose.

\section{W18. Stress Induces Relapse of OCD-Like Behaviors Following Recovery From Optogenetic OFC-VMS Hyperstimulation}

\section{Brandon Bizup, Jamie Pierson, Susanne Ahmari*}

University of Pittsburgh, Pittsburgh, Pennsylvania, United States

Background: Obsessive Compulsive Disorder (OCD) is a chronic, severe mental illness that affects $2-3 \%$ of people worldwide, yet the neural substrates of obsessions and compulsions remain unclear. To date, pathophysiologic models of OCD have primarily emphasized disruptions in balance between goal-directed and habit learning, but these models fail to capture an important aspect of the disorder: affective dysregulation. High levels of anxiety are extremely prevalent in OCD, and stress and anxiety can promote OCD relapse in individuals that are not currently symptomatic. However, despite the importance of the stress/anxiety axis in OCD, the mechanisms underlying the intersection of stress, anxiety, and OCD compulsions have not yet been investigated.

In previous work, we demonstrated that brief but repeated optogenetic hyperstimulation of projections from orbitofrontal cortex (OFC) to ventromedial striatum (VMS) leads to long-lasting perseverative grooming, a mouse behavior linked to OCD (Ahmari et al, Science, 2013). Increased grooming persisted for 2 weeks, and was correlated with an increase in evoked activity at OFC-striatal synapses. However, grooming levels decayed over time in the absence of stimulation, returning to baseline after 1 month. This unique longitudinal system of induction, persistence, and subsequent decay of OCD-like symptoms now allows us to examine the impact of stress/anxiety on relapse of OCD-like behaviors.

Methods: We injected EMX-Cre mice with Cre-inducible channelrhodopsin (AAV-diO-ChR2; $n=8$ ) or control virus (AAV-diO-YFP; $n=8$ ) in OFC, and implanted fiberoptic probes in VMS. After waiting 3 weeks to generate stable levels of viral expression, mice were stimulated 5 minutes/ day for 1 week (473nm laser, $10 \mathrm{~mW}$ power, $10 \mathrm{~Hz}, 10 \mathrm{msec}$ pulse width) to induce perseverative grooming. Stimulation was then stopped, and weekly grooming assessments were performed to monitor decay of the compulsive grooming phenotype in the absence of stimulation. After grooming returned to baseline levels, mice underwent an acute restraint stress (30 $\mathrm{min}$ ). Grooming behavior was then assessed 1 hour and 24 hours post-stress. Cortisol levels were obtained via cheek punch a) at baseline, b) after grooming induction, c) 30 minutes post-stress, and d) 24 hours post-stress. Animals were then sacrificed to verify viral expression/ fiberoptic placement. Data were analyzed using repeated-measures ANOVAs and post-hoc tests $(\alpha=0.05)$.

Results: One week of repeated OFC-VMS hyperstimulation ( 5 minutes/day) led to induction of perseverative grooming behavior $(p<.01)$. After stimulation was stopped, grooming remained significantly elevated for 1 week $(p<.01)$, but returned to baseline levels (i.e. indistinguishable from controls) after 4 weeks. 30- minute restraint stress was then administered. Stress led to an increase in grooming behavior in both ChR2+ animals $(p<.05)$ and controls; however, the grooming increase was larger in ChR2+ animals. Notably, the elevated grooming in $\mathrm{ChR} 2+$ mice was persistent 24 hours after stress $(p<.05)$, when control grooming levels had already returned to baseline. After all animals had returned to baseline, a second 30-minute restraint stress led to an increase in grooming that was persistent for 4 weeks in ChR2+ animals $(p<.05)$; throughout this time, controls remained indistinguishable from baseline. No differences in cortisol levels were observed in ChR2 vs controls throughout the experiment.

Conclusions: We have now developed a system which allows us to investigate the intersection between stress and relapse of OCD-like behaviors in mice. After induction of perseverative grooming via repeated OFC-VMS optogenetic stimulation, ChR2+ mice recovered to baseline grooming levels after 4 weeks. A single acute stressor after recovery then led to reinstatement of overgrooming behavior in ChR2 + mice that lasted for 24 hours. A second acute stressor led to prolonged (4 weeks) reinstatement of overgrooming behavior, suggesting a novel plasticity mechanism. ChR2+ and control animals showed no differences in cortisol levels immediately after stress or 24 hours later, suggesting that downstream circuit level changes mediated the differences in stress-induced grooming response in ChR2+ and control mice. Ongoing experiments are using activity mapping and in vivo calcium imaging to identify mechanisms underlying the stress-mediated relapse of OCD-like behavior.

Keywords: Obsessive-Compulsive Disorder (OCD), Optogenetics, Stress, Relapse, Ventral Striatum.

Disclosure: Nothing to disclose.

W19. Blockade of Oxytocin Receptors (OTR) in the Dorsolateral Bed Nucleus of the Stria Terminalis (BNST) Disrupts Acquisition, but not Consolidation, of cue Fear-Potentiated Startle

Mahsa Moaddab, Joanna Dabrowska*

Rosalind Franklin University, North Chicago, Illinois, United States

Background: In rodent and human studies, the bed nucleus of the stria terminalis (BNST) has emerged as a key brain region translating stress into sustained changes in anxiety. The BNST is necessary for expression of long-duration fear responses that resemble anxiety and it was shown to 
contribute to context conditioning. The BNST has one of the highest expression levels of oxytocin receptors (OTR) in the rodent brain, but their role in fear and anxiety is not known. Hence, the objective of the current study was to establish the role of OTR neurotransmission in the dorsolateral BNST (BNSTdl) in the formation of background anxiety and conditioned fear (cue and context). Here, we have used FPS paradigm, in which an ASR is significantly potentiated by a conditioning stimulus that was previously paired with a foot shock.

Methods: Male adult rats were bilaterally implanted with guide cannulas into the BNSTdl. Following post-operative recovery all rats were habituated to startle cabinets. On the next day rats were tested for an ASR and assigned into treatment groups based on average ASR. On the following day, in experiment 1 , we have measured the effect of OT (100 ng), selective OTR antagonist (OTA, $200 \mathrm{ng},(\mathrm{d}(\mathrm{CH} 2) 51$, Tyr (Me)2, Thr4, Orn8, des-Gly-NH29)-vasotocin), or ACSF infusion into the BNSTdl on baseline ASR (10 min before the testing). In experiment 2 and 3, we have employed FPS paradigm to test the effect of OT or OTA on acquisition or consolidation of conditioned fear, respectively. Rats were fearconditioned during training session, in which they received 10 presentations of a $3.7 \mathrm{~s}$ cue light (CS), each co-terminating with a $0.5 \mathrm{~s}$ foot-shock $(0.5 \mathrm{~mA})$. In experiment 2 , rats were infused with ACSF, OT, or OTA $10 \mathrm{~min}$ before the training session (fear acquisition), and in experiment 3,30 min after the completion of the training session (consolidation). Twenty-four hours later, rats were tested for the FPS expression; where they were exposed to 30 startle eliciting white-noise bursts. The session consisted of 10 baseline trials followed by additional 20 trials, with half presented in the presence of the cue light (CS) and the other half without the cue light (noise only, no CS), mixed in a pseudorandom order. The following parameters were analyzed in both experiments: context fear (pre- vs. post-training ASR), cue-fear (CS-trials vs. noise only trials), as well as background anxiety (posttraining ASR vs. noise only trials). Finally, to assess whether OTA might differently affect rats with low and high levels of baseline ASR, animals were divided into low and high responders based on their pre-training ASR (median split within each experimental group).

Results: In experiment 1 , neither OT nor OTA infusion into the BNSTdl affected the ASR $(P=0.88, n=21)$. In experiment 2, intra-BNSTdl administration of OTA, but not OT, blocked acquisition of cue-fear measured 24 hours later. Quantitative analysis revealed a significant main effect of TRIAL TYPE $(\mathrm{F}(1,37)=57.79, P<0.0001)$, but no main effect of TREATMENT $(\mathrm{F}(2,37)=1.12, P=0.33)$. However, there was a significant interaction between TRIAL TYPE and TREATMENT $(\mathrm{F}(2,37)=3.28, P=0.04$, twoway Anova, $n=40$ ). The post hoc comparisons revealed a significant increase in startle amplitude in light-noise trials compared to noise trials in ACSF-treated group ( $\mathrm{t}(13)=$ 5.52, $P<0.0001)$ and OT-treated group $(\mathrm{t}(12)=5.43, P<$ $0.0001)$, whereas there was no significant increase in OTAtreated rats $(\mathrm{t}(12)=2.25, P>0.05)$. Splitting rats into low and high responders revealed that in rats with low levels of ASR, quantitative group analysis showed a significant main effect of TRIAL TYPE $(\mathrm{F}(1,18)=38.08, P<0.0001)$, but no main effect of TREATMENT $(\mathrm{F}(2,18)=1.35, P=0.28)$ and a significant interaction between TRIAL TYPE and
TREATMENT $(\mathrm{F}(2,18)=5.66, P=0.01)$. While ACSFtreated animals $(\mathrm{t}(6)=5.23, P<0.001)$ and OT-treated animals $(\mathrm{t}(6)=4.61, P<0.001)$ exhibited a significantly potentiated startle response in light-noise trials, in OTAtreated rats the mean startle amplitude in light-noise trials was not different to that of noise trials $(\mathrm{t}(6)=0.83, P>$ 0.05). In contrast, in high responders, there was no main effect of TREATMENT $(P=0.64)$ and no interaction between TRIAL TYPE and TREATMENT $(P=0.55)$. These results suggest that OTA disrupts acquisition of cued fear only in low ASR responders. In regard to context fear, there was no significant effect of TRIAL TYPE or TREATMENT, or interaction between both. Although all groups demonstrated background anxiety (TRIAL TYPE, F $(1,38) 13.52$, $P=0.0007)$, there was no main effect of TREATMENT ( $\mathrm{F}(2$, $38)=1.279)$, or interaction between both $(\mathrm{F}(2,38)=1.437$, $P=0.2502)$. In experiment 3 , all animals showed robust cue fear learning (TRIAL TYPE; F $(1,19)=24.94, P<0.0001$ ). However, there was no effect of OT or OTA on cued fear, with no main effect of TREATMENT $(\mathrm{F}(2,19)=0.16, P=$ 0.84 ) and no interaction between TRIAL TYPE and TREATMENT $(\mathrm{F}(2,19)=0.50, P=0.61, n=22)$, suggesting that neither OT nor OTA affected consolidation of cued fear. Finally, mean shock reactivity during the fear conditioning session was not different between ACSF, OT and OTA groups $(P=0.99$, one-way ANOVA).

Conclusions: Blockade of OTR in the BNSTdl disrupts acquisition, but not consolidation, of the FPS (cued fear). In contrast, neither activation nor blockade of OTR in the BNSTdl affects acquisition or consolidation of context fear or background anxiety. We conclude that endogenous OTR neurotransmission in the BNSTdl is necessary for the acquisition of the cued fear-conditioning in rats with low, but not high baseline ASR.

Keywords: Oxytocin, bed Nucleus of the Stria Terminalis, Fear Conditioning, Anxiety, Fear-Potentiated Startle.

Disclosure: Nothing to disclose.

\section{W20. A Molecular Feed-Forward Inhibition Brake That Governs Remote Memory Re-Activation to Constrain Fear Generalization}

Nannan Guo, Marta Soden, Charlotte Herber, Antoine Besnard, Constance Cepko, Larry Zweifel, Amar Sahay*

Harvard Medical School, Boston, Massachusetts, United States

Background: Posttraumatic stress disorder (PTSD) is an anxiety disorder that is characterized by the presence of three major symptoms: persistence of fear memories, hyperarousal and increased avoidance behavior. With passage of time following trauma, PTSD patients show an increased fear response to a wide range of neutral cues, a phenomenon termed as "time dependent fear generalization", a feature also captured in numerous animal studies. This time dependent fear generalization is thought to result from loss of precision of memories over time, which at a neural level may be due to degradation of the underlying cortical representations. We, and others have recently hypothesized that circuit mechanisms modulating interference between 
memories in the hippocampus govern the stability of cognate remote cortical memory traces.

Methods: Here, we report identification of a molecular factor, Ablim3, with which we can selectively increase the number of excitatory inputs of dentate granule cells (DGCs) onto parvalbumin interneurons (PV-IN) without affecting DGC dendritic spine density and DG activity. By harnessing Ablim 3 to molecularly control feed forward inhibition in DG-CA3 circuit, we investigated how feed-forward inhibition dictates interference between memory traces in hippocampal-cortical networks to constrain time dependent fear generalization. To this end, we deployed viral expression systems, genetic ensemble tagging tools, viral synaptic tracing, optogenetics and behavioral assays.

Results: Viral down regulation of Ablim3 enhanced feed forward inhibition (FFI) in DG-CA3 circuit as assessed by anterograde synaptic tracing, viral labeling of neural connectivity, PV-IN activation and electrophysiological whole cell recordings from PV-INs and CA3 pyramidal neurons. Using genetic systems to indelibly tag context-specific neuronal ensembles we found that virally enhancing FFI in DG-CA3 circuit maintains precision of remote fear memories by promoting global remapping in CA3. Importantly, enhancing FFI in DG-CA3 constrained re-activation of CA3 and cortical ensembles encoding the fearful context when mice were placed in a neutral context at remote time points. Using optogenetics and genetic ensemble tagging tools we found that increasing feed-forward inhibition promoted stability of fear engrams at remote time points. We then extended these studies to identify alterations in FFI in aging and harnessed Ablim3 to reverse these changes and improve remote memory precision in aged mice.

Conclusions: Together, these studies demonstrate how decreasing memory interference in DG-CA3 causally impedes decontextualization of remote cortical memory traces. Further, our work identifies a novel molecular brake that maybe targeted for constraining time dependent fear generalization and improving memory precision in aging.

Keywords: Remote Memory, Engram, Inhibition, Dentate Gyrus, Fear Generalization.

Disclosure: US National Institutes of Health Biobehavioral Research Awards for Innovative New Scientists (BRAINS) 1-R01MH104175, NIH-NIA 1R01 AG048908-01A, Ellison Medical Foundation New Scholar in Aging, Whitehall Foundation, Ellison Family Funds, Inscopix Decode, Massachusetts Alzheimer's Disease Research Center/Harvard Neurodiscovery Center, and HSCI Development grants (AS).

W21. Normalization of Functional Connectivity Between Salience and Default Mode Networks is Associated With Symptom Reduction in Severe Obsessive-Compulsive Disorder

Brian Brennan*, Christine Perriello, Jason Elias, Jesse Crosby, Nathaniel Van Kirk, Jason Krompinger, James Hudson, Scott Rauch, Harrison Pope, Justin Baker

Harvard Medical School, Belmont, Massachusetts, United States

Background: Growing evidence suggests that abnormal functional connectivity, both within and between intrinsic brain networks, plays a central role in obsessive-compulsive disorder (OCD). However, findings from resting-state functional connectivity magnetic resonance imaging studies of OCD remain inconsistent. To expand upon prior crosssectional findings, we examined OCD patients pre- and posttreatment in a residential treatment program for OCD in order to assess measures of functional connectivity associated with overall symptom reduction. We assessed intranetwork and inter-network functional connectivity involving the default mode network (DMN), central executive network (CEN), and salience network (SN), using a seed-based analytical approach. We hypothesized that: 1) compared to non-OCD individuals, OCD patients would evidence pretreatment intra-network connectivity abnormalities within $\mathrm{SN}$ and inter-network abnormalities between SN and DMN; 2) among OCD patients, greater pre-treatment connectivity abnormalities would predict greater clinical improvement; and 3) pre-post improvement in these connectivity abnormalities would be associated with clinical improvement.

Methods: Fifty-two OCD participants received admission scans and 32 of these also received discharge scans. Fifty-two control individuals were scanned once. All imaging data were collected on 3-T Siemens Tim Trio scanners with a 12channel phased-array head coil. Imaging analyses were conducted using the CONN functional connectivity toolbox. Following spatial preprocessing, denoising of the data was performed using linear regression to adjust for unwanted physiological and motion effects. Regions of interest within DMN, SN, and CEN were created for subsequent analyses. The primary clinical outcome measure was the Yale-Brown Obsessive-Compulsive Scale (Y-BOCS). Significant clusters were defined using height threshold $P<.001$, cluster threshold $P<.05$ FDR-corrected, one-sided analyses unless otherwise noted.

Results: Compared to controls, OCD patients demonstrated significantly greater pre-treatment intra-network connectivity within SN and CEN as well as between midline core DMN regions, and significantly lesser inter-network connectivity between brain regions in DMN and regions in both the SN and CEN. Lesser pre-treatment connectivity between DMN and SN regions - specifically, between posterior cingulate cortex (PCC) and left inferior frontal gyrus (IFG) was associated with a greater decrease in Y-BOCS scores post-treatment. Correspondingly, post-treatment increases in connectivity between PCC and left IFG were associated with clinical improvement.

Conclusions: The above findings suggest that 1) OCD is characterized by large-scale inter-network dysconnectivity between DMN and both SN and CEN and 2) normalization of connectivity between DMN and $\mathrm{SN}$ may underlie symptom reduction. More specifically, functional connectivity between PCC and left IFG may have utility as a biomarker of treatment response in OCD. Neuromodulatory approaches aimed at enhancing connectivity between PCC and left IFG, and between DMN and SN brain regions more broadly, may have utility as treatments for OCD.

Keywords: Resting State Functional Connectivity, Obsessive Compulsive Disorder, Salience Network, Default Mode Network, Inferior Frontal Gyrus.

Disclosure: Rugen Therapeutics, consulting fees, Self; Eli Lilly, research grant support, Self; Transcept Pharmaceuticals, research grant support, Self; Genentech, consulting fees/ 
research support, Self; Shire, consulting fees/research support, Self; Sunovion, consulting fees/research support, Self; Pronutria, consulting fees, Self; APPI, royalties, Self; Oxford University Press, royalties, Self; Harvard Football Players Health Study, honoraria, Self; Valera Health, research support, Self.

\section{W22. Gonadal Hormones Alter Ethanol-Induced Memory Deficits in Adolescent Rats}

Jamel Travis, Keita Ishiwari, Ratna Sircar*

City College of New York, New York, New York, United States

Background: Earlier, our lab has reported that ethanol treatment in pre-pubertal adolescent male and female rats impairs memory acquisition. Whether ethanol affects memory function in post-pubertal rats when juvenile animals have become sexually is not known. In this study, we investigated the effects of hormonal manipulations of ethanol-induced changes in hippocampus-associated cognitive functioning in post-pubertal adolescent rats.

Methods: Post-pubertal adolescent male and female rats were administered a single injection of ethanol $(2 \mathrm{~g} / \mathrm{kg})$ intraperitoneally or equivalent volumes of deionized water. Some female rats were ovariectomized (OVXed) and given hormonal supplementation (estrogen and/or progesterone). Additional controls included sham-operated vehicle-treated animals. All rats were trained in the fear conditioning paradigm, and 24 hours later tested for contextual fear conditioning. Freezing during fear conditioning task performance was recorded.

Results: Acute ethanol treatment in intact post-pubertal female rats showed significant disruptions in hippocampusrelated contextual memory but not amygdala-associated cued fear memory. Post-pubertal male rats did not show any ethanol-induced memory deficit. There was significant effect of estrous cyclicity on ethanol-induced behavior in intact female rats. Exogenously administered estrogen with or without progesterone altered the sensitivity of ethanol-induced memory impairment in OVXed post-pubertal female rats.

Conclusions: Together, these data suggest that female gonadal hormones play an important role in modulating ethanol-induced memory impairment in post-pubertal animals.

Keywords: Adolescent Alcohol, Learning and Memory, Gonadal Hormones.

Disclosure: Nothing to disclose.

\section{W23. The "Strikingly Disproportionate Degree of Physical and Mental Energy" in Anorexia Nervosa (AN) Manifested as Restlessness and a Drive for Activity may Reflect a Disorder of Energy Homeostasis}

\section{Regina Casper ${ }^{\star}$}

Stanford University School of Medicine, Stanford, California, United States

Background: AN is a disorder of voluntary food restriction associated with severe pathological weight loss, yet no true loss of appetite. The genetic contribution to AN has been estimated in the range of $40-80 \%$. The etiology of AN remains obscure. Clinical observations that individuals remain active, sometimes overactive, and report no loss of energy suggest a disturbance in the mechanisms adjusting energy expenditure, because other conditions of caloric under-nutrition resulting in severe weight loss are generally associated with loss of energy, a slowing of movements as well as a decrease in spontaneous and self-initiated activity. We propose as a hypothesis that "physical and mental energy preserved in the presence of physical and physiological signs of starvation" might constitute a fundamental characteristic, a phenotype of AN determined by both genetic and environmental influences. The purpose of the presentation is to review and analyze studies that have measured daily energy expenditure and physical activity levels in AN and to discuss possible candidate genes involved in modulating energy levels in AN.

Methods: 1) Total energy expenditure (TEE) consists of the resting metabolic rate (RMR), the energy expended in activity, and the dietary-induced thermogenesis. TEE was measured in recent onset $\mathrm{AN}$ at body weights $30-40 \%$ below the normal range, using doubly labeled water (DLW), a gold standard method that provides estimates of habitual energy expenditure over a time period of 14 days.

2) Physical activity levels were recorded through different movement sensor sytems (such as actometers, accelerometers, actiheart) in hospitalized AN patients.

3) Genes regulating energy expenditure under physiological conditions which might constitute genetic risk factors for AN were reviewed.

Results: TEE: Five studies, conducted in outpatients and in hospitalized patients with AN diagnosed by DSM IV criteria and in controls which measured TEE by the DLW method reported lowered resting metabolic rates (RMR), but found TEE not to differ from healthy age-matched controls, whilst two studies conducted in chronic AN observed lower than normal TEE.

Physical activity: Ten studies using different methodologies to assess daily activity levels through movement sensor systems found that physical activity levels in patients hospitalized with AN were not different from normal weighed matched controls.

Genetic contribution: Genetic risk factors would point to variations in gene products that undergo changes in the process of the body's adaptations to caloric restriction and gene products that regulate energy balance and activity levels. For example, studies on leptin, an adipocyte-secreted hormone involved in energy homeostasis which acts as a signal from the periphery to the brain conveying information about the amount of energy available, have shown an inverse relationship between plasma leptin levels and symptoms of motor restlessness and physical activity in AN. Another potential candidate might be the fibronectin type III domain containing 5 (FNDC5) gene in human muscle with its product Irisin. Irisin appears to contribute to browning of subcutaneous white fat and might via a thermogenic response boost respiratory uncoupling. Other genomic alterations might involve missense mutations in the estrogen related receptor alpha (ESRRA) gene and the transcriptional repressor histone deacetylase 4 gene (HDAC4) that have been shown to segregate with AN. ESRRA participates in 
energy balance and metabolism and is upregulated by exercise and calorie restriction in peripheral tissues.

Conclusions: Experimental evidence that persons with $\mathrm{AN}$ at an average body mass index (BMI) of 14-16 are motorically as active as normal weighed healthy controls and that daily energy expended as activity is similar to energy spent by matched healthy controls would support the hypothesis that AN might be a disorder of energy homeostasis. The data would corroborate the notion that preserved energy levels observable in symptoms such as "restlessness" and "a drive for activity" constitute a phenotype of AN. Clinically, energy preserved in the presence of severe caloric under-nutrition might shed light on the patients' assertion that "there is nothing wrong" and that they feel "perfectly well". Very likely, gene-environment interactions contribute to abnormally preserved energy and activity levels in AN. If confirmed, the hypothesis would provide the opportunity to test gene variants and/or changes in gene expression associated with the " energy preserved" or "drive for activity" phenotype.

Keywords: Anorexia Nervosa, Drive for Activity, Energy Homeostasis.

Disclosure: Nothing to disclose.

\section{W24. Functional Connectivity Markers of Resilience in} Adolescents at Familial Risk for Depression

\section{Adina Fischer*, Catalina Camacho, Tiffany Ho, Susan Whitfield-Gabrieli, Ian Gotlib \\ Stanford University, Los Altos, California, United States}

Background: Major Depressive Disorder (MDD) is a prevalent and debilitating disorder, with first episodes that often emerge in adolescence. Parental MDD is a significant risk factor for the disorder; offspring are up to five times more likely to develop depression. Not all offspring of depressed parents, however, develop MDD (Gotlib \& Colich, 2014). In this context, adolescence is not only a time of stress and vulnerability for psychopathology, but also a neurodevelopmental period with potential for growth and positive development. Using resting-state functional magnetic resonance imaging (rsfMRI), which provides a functional measure of brain network coherence, researchers have identified network-level dysfunction in adolescents with MDD in the default mode network (DMN), salience network (SN), and affective network (AN) (Connely et al, 2013). Children at familial risk for MDD show connectivity anomalies in these networks (Chai et al., 2015). No studies, however, have examined neural markers of resilience by examining functional connectivity in adolescents at familial risk for MDD who do not develop depression. Identifying such markers of resilience could help to elucidate underlying protective or compensatory neural mechanisms and facilitate the development of more effective prevention and intervention approaches for adolescent depression. In this study, we used rsfMRI to examine differences in intrinsic connectivity in DMN, SN, and AN between highrisk adolescents who did and who did not subsequently develop MDD.

Methods: Seventy-six adolescent females (ages 10-14), including 39 daughters of mothers with recurrent episodes of depression (high risk) and 37 daughters of mothers with no known history of any Axis I disorder (low risk), were recruited as part of a larger longitudinal study. All daughters had no history of any Axis I disorder at the time of enrollment and returned at 18-month intervals to determine whether they met clinical criteria for Axis I disorders at any time in the past 18 months. They also completed the Childhood Depression Inventory (CDI) and Life Events Survey (LES) to assess the number of negative and positive life events that occurred within the past 18 months. A seedbased rsfMRI approach with rigorous motion correction was conducted in Conn (Whitfield-Gabrieli, 2012) to compare DMN (posterior cingulate cortex seed; PCC), SN (anterior cingulate cortex seed; ACC), and AN (amygdala seeds) connectivity in high-risk girls who subsequently experienced an episode of MDD and girls who remained resilient; we then compared connectivity in these two groups of participants with connectivity in low-risk controls who did not develop MDD. Finally, we examined the association between rsfMRI connectivity and positive and negative life events within each group.

Results: Seventeen of the 39 high-risk daughters (44\%) met criteria for MDD at some point since their entry into the study (but not at the time of this scan; "converted"), and 22 of the high-risk daughters (56\%) did not develop a depressive episode ("resilient"). These two groups of high-risk daughters did not differ significantly with respect to ethnicity, race, socioeconomic status, or severity of maternal depression. They also did not differ significantly with respect to age at the time of this scan $(18.78 \pm 2.6)$, years in study $(6 \pm 2$ years), or CDI score. There was no significant group difference between number of outliers in head motion or global signal intensity. Compared with both the converted and control daughters, resilient adolescents exhibited greater amygdala-based connectivity with orbitofrontal cortex and superior frontal gyrus. Compared with both the resilient and control adolescents, converted subjects exhibited greater PCC-based connectivity with ventral and subgenual ACC, as well as with the superior frontal gyrus. Finally, PCC-ventral ACC connectivity was negatively correlated with the number of positive life events experienced in the resilient group $(\mathrm{r} 21=0.62 ; p=0.002)$.

Conclusions: Adolescents at familial risk for depression who remained resilient demonstrated increased connectivity between the amygdala, a brain region involved in processing emotionally and motivationally salient stimuli that has been found to be hyperactive in MDD, and frontal regions that have been implicated in emotion regulation and executive control (Banks et al., 2007). These results are consistent with the formulation that greater amygdala-frontal connectivity is protective against depression, perhaps due to greater topdown control. Resilient adolescents also demonstrated less DMN connectivity than did adolescents who had experienced MDD; in addition, there was an inverse association in this group between DMN connectivity and the number of recent positive life events. Hyperconnectivity of the DMN has been implicated in negative self-focus, rumination, and depression severity (Hamilton et al., 2015). This brainbehavior correlation suggests that positive life experiences are protective, and are mediated by or instantiated through reduced DMN connectivity. Collectively, the present findings suggest that greater amygdala-frontal connectivity and 
reduced maladaptive DMN hyperconnectivity are neural markers of resilience against MDD.

Keywords: Major Depressive Disorder (MDD), Adolescence, Resilience, Resting State fMRI, Default Mode Network (DMN).

Disclosure: Nothing to disclose.

\section{W25. ER $\beta$ Attenuates ASD-Like Behavior by Regulating ER Stress Associated IRE-1/XBP-1 Pathway}

\section{Amanda Crider, Anthony Ahmed, Anilkumar Pillai* \\ Medical College of Georgia, Augusta, Georgia, United States}

Background: Autism spectrum disorder (ASD) is among the most devastating neurological disorders of childhood with a prevalence of about 1 in 68 children. ASDs are more prevalent in boys than in girls, with ratios of 3:1 for classic autism and 10:1 for Asperger syndrome, suggesting the possible role of sex hormones in the development of this disorder. Recent evidence points toward endoplasmic reticulum (ER) stress as a critical contributing factor to the pathophysiology of ASD. The "Unfolded Protein Response (UPR)" functions to restore ER homeostasis and help the cells adapt to ER stress conditions. However, when ER stress is prolonged or the degree of ER stress is too severe, UPR signaling can initiate deleterious effects on cellular functions. It remains unknown, however, whether direct modulation of this ER stress pathway is sufficient to control ASD-like behavior, and what is the mechanism involved.

Methods: We induced ER stress in mice by tunicamycin administration, and a battery of behavior tests related to ASD was performed. We used postmortem middle frontal gyrus samples from ASD and age and gender-matched control subjects to analyze the ER stress gene profile by real time PCR. Pharmacological agents and lentiviral particles were used to determine the role of ER stress pathway in ASD-like behavior in mice.

Results: We found significant significant increases in mRNA levels of ER stress markers in the postmortem middle frontal gyrus of ASD subjects. ER stress by tunicamycin treatment induced ER stress markers in mouse PFC and ASD-like behavior in male, but not in female mice. Ovariectomy induced changes in social and grooming behavior in mice. Tunicamycin treatment induced decrease in ER $\beta$ protein levels in mouse PFC. Treatment with PHTPP, a potent ER $\beta$ antagonist, induced ASD-like behavior in male mice. Inhibition of the IRE-1/XBP-1 pathway by pharmacological and lentiviral approaches blocked ER stress-induced ASDlike behavior. Moreover, ER $\beta$ agonist ERB-041 attenuated ER stress-induced ASD-like behavior in mice. Also, ERB-041 attenuated ER stress-induced XBP-1 splicing in mouse PFC. Conclusions: This is the first demonstration that ER stress associated IRE-1/XBP-1 pathway is involved in ASD-like behavior. Moreover, our findings on the role of ER $\beta 1$ in attenuating ASD-like behavior may shed more light into the mechanisms that regulate gender difference in ASD pathophysiology. This will help in the development of novel therapeutic targets for ASD and related neurodevelopmental disorders.

Keywords: ASD, Estrogen Receptor, ER Stress.

Disclosure: Nothing to disclose.
W26. Gonadal Steroids at Puberty Induce Maturation of Frontal Cortex Inhibitory Neurotransmission and Reversal Learning

David Piekarski*, Josiah Boivin, Wren Thomas,
Linda Wilbrecht

University of California, Berkeley, Berkeley, California, United States

Background: Development of the frontal cortex during adolescence is associated with significant remodeling of the frontal cortex and cognitive behavior. The onset of adolescence is triggered by the pubertal rise in circulating gonadal steroidsl and while it is clear that gonadal steroids at puberty trigger maturation of body and brain to support adult behavior, it is unknown to what extent they cause maturation of the frontal cortex. It is important to clarify the mechanisms causing adolescent frontal cortex development because this is a period of vulnerability to developing psychopathology and cognitive and behavioral disorders. We have advanced and delayed systemic exposure to gonadal steroids in peripubertal female mice using steroid injections or ovariectomy to determine if hormones impact adolescent development of inhibitory neurotransmission and cognitive flexibility. Using slice electrophysiology and behavior tests, our data suggest that miniature inhibitory post synaptic currents (mIPSCs), a measure of synaptic inhibition, increase in frequency and amplitude across adolescent development in the frontal cortex and that gonadal steroids organize the increase in mIPSC frequency.

Methods: Female C57Bl/6 mice, between 24 (prepubertal) and 55 (adolescent) days of age were used. Peripubertal exposure to gonadal steroids was manipulated either by ovariectomy or a hormone replacement paradigm in which mice received a single injection of estradiol followed 48 hours later by a single injection of progesterone while control mice received injection of oil vehicle. Whole cell patch clamp electrophysiology was conducted in acute slices to measure miniature inhibitory post synaptic currents (mIPSCs). In behavioral experiments, mice were treated with hormones or ovariectomy and cognitive reversal learning was assessed with a four-choice reversal learning foraging task.

Results: Electophysiology: mIPSCs were measured on layer $2 / 3$ pyramidal cells in the anterior cingulate cortex. mIPSC frequency increased from P24 (mid juvenile) to P40 (midadolescent ages; $\mathrm{t} 25=2.43 ; p<0.05)$. Treating prepubertal mice with estradiol and progesterone significantly increased mIPSC frequency $(\mathrm{t} 24=2.4 ; p<0.05)$. The developmental rise in mIPSC frequency was eliminated by prepubertal ovariectomy ( $\mathrm{t} 21=2.5 ; p<0.05)$; hormone replacement to prepubertally ovariectomized mice during adolescence trended toward rescuing the developmental rise in mIPSC frequency $(\mathrm{t} 20=2.07 ; p=0.051)$. Finally, postpubertal ovariectomy did not affect mIPSC frequency $(\mathrm{t} 24=0.1 ; p>0.05)$. Prepubertal hormone treatment did not alter mIPSC frequency in somatosensory cortex $(\mathrm{t} 23=0.38 ; p>0.05)$. Behavior: Prepubertal hormone exposure did not affect trials to reach criterion during the discrimination phase of the 4-choice reversal learning task but hormone exposed mice required significantly more trials to reach criterion in the reversal learning task $(\mathrm{F} 1,10=5.5 ; p<0.05)$. However, prepubertal ovariectomy did not affect learning in the 
discrimination or reversal phase when tested at P40 $(\mathrm{F} 1,9=0.09 ; p>0.05)$.

Conclusions: These data demonstrate that the onset of puberty and its contingent rise in circulating gonadal are both sufficient and necessary for the developmental rise in mISPC frequency. Further, hormones are sufficient but not necessary for the development of reversal learning on a fourchoice foraging task. These data may have broader implications for how puberty affects neuroplasticity, cognition, and risk for neuropsychiatric illness. Given that the age at puberty onset is advancing in the developed world and early puberty is associated with heightened risk for development of neuropsychiatric illness, it will be critical to better understand if the effects of pubertal steroids on frontal cortex affect risk for developing neuropsychiatric illnesses.

Keywords: Puberty, Inhibitory Neurotransmission, Reversal Learning.

Disclosure: Nothing to disclose.

\section{W27. Gaze Fixation Patterns in Children With Autism Spectrum Disorder and Typical Development Screening From the Japanese Community Based 5-Year-Old Children}

\section{Kazuhiko Nakamura*, Manabu Saito \\ Hirosaki University Graduate School of Medicine, Aomori, Japan}

Background: A number of studies have identified unique visual gaze patterns in individuals with autism spectrum disorder (ASD) using eye-tracking systems. Such gaze fixation patterns in individuals with ASD are considered to be associated with social attention. However, the previous studies show different results between ASD and typical development (TD). Using Gazefinder, an all-in-one eye-tracking system, we examined the gaze fixation patterns to specific objects in children with and without ASD screening from the Japanese community based 5-year-old children.

Methods: The children were all recruited from a population based group in the country of Hirosaki-city. We pursued the community health check-up with every 5-year-old children for three years in Hirosaki-city (2013-2015, N=3804). The number of children in the community health check-up were 2923. The total group encompassed 438 children of whom were also assessed at a follow-up. The assessment included an evaluation of the child's ASD diagnosis, based on parental interview according to DISCO and child cognitive assessment (WISC-IV) performed by a psychologist. The ASD diagnoses were based on clinical information in accordance with the DSM-5 criteria by a psychiatrist. The final group of 179 children consisted of 98 with ASD and 81 with TD. We measured the percentage of gaze fixation time allocated to particular objects depicted in movies. Gazefinder incorporates movies of human faces, biological motion, and people and geometric shapes, and it provides almost instantaneous data by automatically calculating the percentage gaze fixation time allocated by the participants among regions of interest in the movie. The total trial time is approximately $2 \mathrm{~min}$. The participant simply views a video monitor. We compared these percentages between the two groups (ASD and TD).
Results: Compared with the TD group, the ASD group showed significantly less gaze fixation time at locations of salient social information (the available percentage gaze fixation time: eyes in the movie of human faces without lip movement, mouth with blinking and talking, and people in the movie of people and geometry), and significantly increasing gaze fixation time of geometry in the movie of people and geometry, while there were no significant group wise differences in the responses to biological motion. However, the percentage gaze fixation times exhibited small effect sizes for the group difference. In ROC analysis in each area of interest, the significant AUCs were.643 (the available percentage gaze fixation time),.606 (eye region, silent),.597and.606 (mouth region, blinking and talking),.659 and.591 (people, same size and small size), and.594 (geometry, same size). AUCs were low accuracy $(<0.7)$.

Conclusions: These findings suggest that Gazefinder is potentially a valuable and easy-to-use tool for objectively measuring gaze patterns of children with ASD. Gaze fixation patterns can distinguish ASD and TD. Gazefinder may be a useful tool for screening ASD in a population based group. Keywords: Gaze Fixation, ASD, Children. Disclosure: Nothing to disclose.

\section{W28. Developmental Grey Matter Changes in Pediatric Posttraumatic Stress Disorder: A Longitudinal Study}

Sara Heyn, Christy Olson, Taylor Keding, Ryan Herringa*

University of Wisconsin School of Medicine and Public Health, Madison, Wisconsin, United States

Background: Childhood trauma is a worldwide public health concern that can lead to a number of psychosocial problems (De Bellis \& Zisk, 2014). By the age of 18 , nearly $5 \%$ of all children will be affected by posttraumatic stress disorder (PTSD). While prior studies have reported cross-sectional structural brain abnormalities among youth with PTSD (e.g. Morey et al., 2016; Keding \& Herringa, 2014), there are no reported whole brain studies examining longitudinal gray matter development in youth with PTSD compared to typically developing youth. Further, no reported studies have investigated how structural neurodevelopment is related to PTSD symptom change over time in affected youth. Such information is critical for identifying both adaptive and maladaptive neurodevelopmental trajectories in traumatized youth which could be used as potential targets of intervention. Methods: In a naturalistic longitudinal study, 22 medicationfree youth with PTSD (ages 8-18 years) and 20 nontraumatized healthy youth, matched on age and sex, were assessed with structured trauma and psychiatric interviews, and completed T1-weighted MRI scans at baseline and one year later. Parent/child reports of PTSD, anxiety, and depressive symptoms were obtained at both time points using standardized questionnaires and reduced to primary symptom dimensions using principal component analysis (Patriat et al, 2016). Grey matter images were processed using longitudinal segmentation and registration in the CAT12 toolbox in SPM12. Developmental differences in grey matter density between PTSD and healthy youth, as well as the relationship between symptom severity and grey matter density change 
among PTSD youth, were investigated using linear mixedeffects models. Voxel-wise analyses were covaried for age, sex, and total intracranial volume. Results were whole brain corrected using cluster-based thresholding (pFWE<.05).

Results: In the between-group analysis, both healthy and PTSD youth showed decreasing grey matter density with age (i.e. an age main effect) in widespread cortical areas, consistent with normative cortical thinning. However, a significant group by age interaction was present in the left dorsolateral prefrontal cortex (dlPFC, $\mathrm{k}=495)$. Here, dlPFC grey matter density decreased over time in healthy youth, a pattern that was absent in youth with PTSD. Within the PTSD group, symptom by time interactions emerged for multiple prefrontal-limbic regions implicated in regulating fear and emotional responses. Notably, a hyperarousal by time interaction was present in an overlapping cluster of the left dlPFC $(\mathrm{k}=1124)$. Here, gray matter density was positively associated with hyperarousal symptoms at time two, adjusted for time one density and symptom severity.

Conclusions: Together, these novel findings point to abnormal neurodevelopment of the dlPFC in youth with PTSD which is indicative of absent or delayed cortical thinning. Given the known role of the dIPFC in cognitive control and emotion regulation, this provides a potential neurobiological substrate of impaired fear and emotion regulation in youth afflicted with PTSD. Consistent with this notion, dlPFC density at time two was positively associated with hyperarousal symptoms in youth with PTSD. These findings offer potential biomarkers and therapeutic targets that could be employed in future studies to improve fear and emotion regulation in youth with PTSD.

Keywords: Pediatric PTSD, Neurodevelopment, Structural MRI, Dorsolateral Prefrontal Cortex.

Disclosure: Nothing to disclose.

\section{W29. Structural MRI as a Biomarker of Treatment Success in Neurodevelopmental Disorders}

Rylan Allemang-Grand, Leigh Spencer-Noakes, Jacob Ellegood, Brian Nieman, Jason Lerch*

University of Toronto, Toronto, Canada

Background: As our understanding of the etiology of Neurodevelopmental disorders (NDD) digs deeper into the biology, treatment interventions are becoming more specific, targeting cellular mechanisms and pathways. Although some interventions have been tested, current assays of treatment success are restricted to behavioural metrics, providing limited information of the brain. In order to accurately assay treatment success, non-invasive biomarkers that provide specific information regarding the neurobiological response to treatment are needed. In order to design and optimization biomarker development, mouse models of NDD can be utilized with the added benefits of environmental and genetic control. One such model is the inducible Mecp2 mouse model of Rett syndrome. In these mice, the Mecp2 gene is silent from birth leading to behavioural and neuronal impairments in early life $[1,2]$. However, at the time of the experimenters choosing, the functional Mecp2 gene can be reactivated leading to a restoration of homoeostasis and a structural / functional rescue of the cellular environment $[3,4]$. In this study, we wanted to determine whether structural MRI can serve as a biomarker with the ability to detect the cellular level changes that occur in the brain following reactivation of the functional Mecp2 gene.

Methods: In order to control the timing of Mecp2 reactivation, we crossed heterozygous Mecp2-STOP (Mecp2tm2Bird/J) females with Cre (CAG-cre/Esr1) males. Male WT, Cre, Mecp2-STOP and Mecp2/Cre pups were scanned in vivo with a Manganese-enhanced MRI protocol (MEMRI: T1-weighted gradient echo, $\mathrm{TR}=26 \mathrm{~ms}, \mathrm{TE}=5.37 \mathrm{~ms}, \mathrm{FOV}=$ $7.7 \times 2.0 \times 2.0 \mathrm{~cm} 3$, matrix $=770 \times 202 \times 202,100 \mathrm{um} 3$ isotropic resolution, $4 \mathrm{nt}, 1$ hour) at P50, followed by 4 weeks of treatment with oil or tamoxifen to reactivate Mecp2. At P80, a follow up MEMRI scan was conducted. The images were aligned using iterative linear and nonlinear registrations steps [5]. This process generates a consensus average of each individual brain and the deformations from this average. The Jacobian- determinants, which represents local volume changes, were then used in the statistical analyses. Multiple comparisons were controlled with the false-discovery rate. All experiments were approved by the Animal Care Committee of the Toronto Centre for Phenogenomics.

Results: At P50, Mecp2 silent mice had a 11\% decrease in brain volume compared to WT. Interestingly, Mecp2 reactivation increased total brain volume by $1.5 \%$ while the silent brain decreased by 3.8\% $(p<0.01)$. Furthermore, Mecp2 reactivation altered the growth of many regions (e.g. striatum, cerebellum and medulla), such that these areas acquired a trajectory similar to WT. However, these regions tend to atrophy within the group possessing a silent copy of Mecp2, leading to significant differences between these groups at P80 (10\% FDR-corrected). Although many cortical regions atrophied regardless of Mecp2 status, the extent of atrophy was reduced in the reactivated relative to silent group. Interestingly, Mecp2 reactivation in late adulthood also leads to volumetric rescue within many of the same regions as early reactivation group. However, the extent of volumetric growth is greater when reactivation occurs earlier in life.

Conclusions: Our results demonstrate that Mecp2 reactivation leads to a substantial neuroanatomical rescue across many brain regions involved in motor processing / control and respiration. This study demonstrates that structural MRI can be used as a biomarker of treatment success in mouse models of NDD and potentially also in humans due to the translation capacity of this method.

Keywords: Magnetic Resonance Imaging, Neurodevelopmental Disorders, Treatment.

Disclosure: Nothing to disclose.

\section{W30. Are Brain-Behavior Relationships for Internalizing and Externalizing Behaviors Similar Across Children With ASD, ADHD and OCD?}

Stephanie Ameis ${ }^{*}$, Evdokia Anagnostou, Jason Lerch, Grace Jacobs, Saba Shahab, Christopher Hammill, Aristotle Voineskos, Russell J. Schachar, Peter Szatmari, Paul Croarkin, Paul D. Arnold

Centre for Addiction and Mental Health, Toronto, Canada

Background: Externalizing (e.g. aggressive, noncompliant, oppositional) and internalizing (e.g., withdrawn, anxious, 
inhibited, depressed) behaviors are common among children with autism spectrum disorder (ASD), attention-deficit/ hyperactivity disorder (ADHD), and obsessive-compulsive disorder (OCD). Our recent study indicated that structural connectivity within an orbitofrontal-amygdalar network was associated with externalizing behaviors in healthy children. (Ameis, et. al, Biological Psychiatry, 2014). Here, we explored whether relationships between externalizing and internalizing behaviours and orbitofrontal-amygdalar circuitry may be present in children with either ASD, ADHD or OCD.

Methods: Structural imaging and behavioral measures were acquired in 125 children recruited through the Province of Ontario Neurodevelopmental Disorders Network (ADHD: $n=41$, Mean age $=9.4 \pm 2,7$ females; OCD: $n=22$, Mean age $=11.2 \pm 2.4 ;$ ASD: $n=62$, Mean age $=10.8 \pm 3.2 ; 12$ females). Cortical thickness measures across the cortex were derived from quality-controlled native MR images processed through the CIVET 1.1.12 pipeline. Average cortical thickness measures for each ROI within the AAL atlas were extracted. Regional volumes for amygdala were obtained using a validated, fully automatic label-fusion based segmentation method applied to MR images. Relationships between externalizing behaviors and internalizing (measured with the Child Behavior Checklist) and orbitofrontal cortex (OFC) thickness, amygdala volume, and orbito-amygdalar structural networks were examined using first-order linear fixedeffects models, after controlling for age, sex and total brain volume across children and adolescents with ASD, ADHD and OCD.

Results: Although no between-group differences in internalizing behaviors were found $(\mathrm{OCD}=65 \pm 9.2, \mathrm{ASD}=62.3$ $\pm 10.9, \mathrm{ADHD}=61.7 \pm 9.8, \mathrm{~F} 2,125=.8, p=0.44)$, externalizing behaviors differed between groups $(\mathrm{OCD}=55 \pm 10.3$, $\mathrm{ASD}=58 \pm 10.6, \quad \mathrm{ADHD}=62 \pm 8.9, \quad \mathrm{~F} 2,125=4.6, \quad p=.01)$, with significant differences found between OCD and ADHD participants. While no relationship between orbitoamygdalar coupling and either externalizing or internalizing behaviours was found across children with ADHD, ASD and OCD, a strong relationship between orbito-amygdalar coupling and externalizing behaviours was present in OCD children only in the right $\mathrm{OFC}(\mathrm{F} 1,16=8.8, p=0.009)$. A trend relationship was also shown between right OFC thickness and internalizing behaviors $(\mathrm{F} 1,121=2.7, p=0.1)$ across children with ASD, ADHD, and OCD. Finally, an inverse relationship between OFC thickness and externalizing behavior in OCD children only was found (left: $\mathrm{F} 1,16=8,1, p=0.01$, right: $\mathrm{F} 1,16=6.5, p=0.02)$. No significant associations between externalizing or internalizing behaviors and amygdala volume were found.

Conclusions: No cross-cutting relationship between orbitoamygdalar circuit structure and externalizing behaviors was found across children with ASD, ADHD and OCD. However, orbito-amygdalar covariance was related to externalizing behaviors in children with OCD only, as was OFC thickness. These relationships are very similar to those we have previously found in healthy controls children. The greater variability (clinical heterogeneity) in externalizing scores in children with ADHD and ASD, compared to OCD, suggest that efforts to improve clinical subtyping may be necessary for detection of biomarkers of externalizing behavior that cut across these disorders. Finally, the potential association of OFC thickness with internalizing behaviour across disorders deserves further investigation as data collection continues in our sample.

Keywords: Neurodevelopmental Disorders, Human Neuroimaging, Dimensional.

Disclosure: Nothing to disclose.

\section{W31. Altered Behavior, Sleep, and Epileptiform Activity in a Perinatal Immune Activation Mouse Model of Autism Spectrum Disorder (ASD)}

Galen Missig*, Abigail Alexander, Emery Mokler, James Robbins, Martha Sheets, Beate Finger, Christopher McDougle, William Carlezon

Harvard Medical School, Belmont, Massachusetts, United States

Background: Increasing evidence suggests a role for inflammatory processes in autism spectrum disorder (ASD). An immunological subtype of ASD may exist, considering that a subset of individuals with ASD show elevated inflammatory markers and neuroimmune responses, as well as epidemiological associations with familial autoimmune disorders. Previous research in mice has shown that immune activation during critical developmental periods can result in a phenotype that reproduces some of the core features of ASD. We have recently explored a "multiple hit" immune model, whereby mice are exposed to repeated perinatal immune activation.

Methods: In the multiple hit model, pregnant mice are injected with the viral mimic poly(I:C) (polyinosinic: polycytidylic acid) $(20 \mathrm{mg} / \mathrm{kg})$ on gestational day 12.5 in accordance with an established model of maternal immune activation. A subset of these offspring receives a second injection of LPS (lipopolysaccharide) $(10 \mathrm{mg} / \mathrm{kg})$ to induce a robust innate immune response on postnatal day 9. Preliminary data from our group shows that these regimens induce a state of ongoing immune activation in the brain that persists into adulthood, evidenced by a heightened level of multiple inflammatory markers. Here, we examined this two-hit model on behaviors related to three key domains of ASD: social, communication, and repetitive behavior. Additionally, we used electroencephalography (EEG) to assess two physiological measures that are commonly dysregulated in individuals with ASD: sleep and epileptiform activity. As part of a remote telemetry system, a batterypowered multi-lead transmitter was implanted subcutaneously at postnatal week 6 , enabling constant measurement of EEG, electromyography (EMG), generalized motor activity, and body temperature for a period of several weeks.

Results: We found that both prenatal and postnatal immune activation produce a complex behavioral phenotype that resembles ASD in multiple domains, and these effects were sex-dependent, as male mice were more affected in a number of measures. Overall, however, postnatal LPS treatment resulted in a more pronounced behavioral phenotype, and the effects of combined treatment were not greater than those of LPS alone. In the remote telemetry study, postnatal LPS resulted in several alterations in activity and sleep, qualitatively similar to the hyperactivity and sleep disturbances frequently observed in ASD. 
Considering that epilepsy is found in a higher percentage of individuals with ASD than in the general population, and there is evidence for increased epileptiform activity even in the absence of epilepsy, we examined EEG recordings from perinatal immune activated mice for the presence of epileptiform activity. This analysis revealed that a subset of the mice that received postnatal LPS displayed heightened levels of epileptiform activity, including the presence of spike-wave discharges, which were not seen in controls.

Conclusions: In summary, perinatal immune activation resulted in a sex-dependent behavioral phenotype that was accompanied by alterations in sleep and epileptiform activity that resemble key features of ASD, further supporting a potential immunological involvement in this condition.

Keywords: Autism Spectrum Disorder, Environmental Risk Factors, Immune Mechanisms.

Disclosure: Nothing to disclose.

\section{W32. The Janssen Autism Knowledge Engine (JAKE ${ }^{\mathrm{TM}}$ ): Results From a Clinical Validation Study of a Set of Tools and Technologies to Assess Potential Biomarkers for Autism Spectrum Disorder}

Seth Ness, Manyakov Nikolay, Abigail Bangerter, David Lewin, Shyla Jagannatha, Matthew Boice, Andrew Skalkin, Geraldine Dawson, Matthew Goodwin, Robert Hendren, Bennett Leventhal, Frederic Shic, Walter Cioccia, Gahan Pandina*

Johnson \& Johnson, Titusville, New Jersey, United States

Background: Autism spectrum disorder (ASD) is a neurodevelopmental disorder with a 1-2\% global prevalence. While behavioral therapies are a treatment mainstay, medications are used to treat associated symptoms and manage comorbid conditions. However, no medications are currently approved for core ASD symptoms. While biologic targets are emerging from genetic and neurobiologic studies, drug development has lagged, in part due to lack of treatment-sensitive outcome measures. Commonly used informant scales have limitations in that they can be subjective (observer report), designed for diagnosis, not sensitive to change, and may include terminology that is difficult for the lay person to understand. Objective measures of ASD symptoms have been sought to address these concerns. These range from passive, wearable devices that collect continuous data, such as wrist-based actigraphs, to sensors deployed in the lab (or home) that gather data on behavior and biology during computer-based visual and/or auditory challenge tasks, such as EEG or eye tracking.

We have designed a three-part system to optimize the collection of deep behavioral phenotyping and objective sensor-based data from individuals with ASD and their families that includes: 1. The JAKE ${ }^{\mathrm{TM}}$ Portal: a web-andmobile set of tools for use by caregivers and clinicians to log symptoms, track progress, and gather detailed medical information about individuals with ASD; 2. The JAKE ${ }^{\text {rм }}$ Biosensor Array: includes biosensors to assess physiological characteristics and behavior related to core ASD symptoms, with continuous, wearable biosensors that gather information daily, and a set of periodic biosensors that gather feedback during a battery of computer-administered tasks (quantifying reactions to/perception of social scenes and other relevant stimuli), and; 3.The $\mathrm{JAKE}^{\mathrm{rm}}$ Data Pipeline, a computational data processing pipeline that organizes, filters, and de-identifies data, alongside a robust set of data visualization and analytic tools.

Data will be presented from an interim analysis of a large clinical validation study with the JAKE ${ }^{\mathrm{TM}}$ system.

Methods: Participants with ASD (aged 6 years to adult) enrolled in an 8 to 10 week, prospective observational study using the $\mathrm{JAKE}^{\mathrm{TM}}$ system. After screening, participants were seen at clinical sites every four weeks (three visits), where standard rating scales and a battery of tasks were administered, and biosensor data collected. Sensor data were also collected in the home, and correlated with data collected via the JAKE portal. We report interim analyses of baseline relationships observed between web-and-mobile data and ASD-specific behaviors and symptoms, biosensor variables, and standard rating scales.

Results: A total of 136 participants with ASD (6-54 years, mean $=14.67+7.98, n=104$ male $)$ and 41 typically developing (TD) controls $(6-63$ years, mean $=16.27+13.18,27$ male $)$ were evaluated at baseline.

Differences between ASD and TD participants were noted on a variety of lab-based sensor outcomes. ASD participants showed lower EEG coherence during resting state eyes open (gamma, frontal vs. temporal, $p=0.032$ ) and eyes closed (gamma, frontal vs. parietal, $p=0.024$ ) tasks, higher ECG heart rate $(p<0.001)$, less attention to faces in social eyetracking (ET) tasks $(p=0.001)$, less ET preference for biological motion $(p<0.001)$, and atypical facial affect (according to pre-specified algorithms) when responding to funny videos (happy feature, $p<0.05$ ) as well as when asked to produce emotional faces (happy feature, $P=0.001$ ) compared to controls.

For a smaller subset of participants with ASD $(N=50)$, clinical data from continuous biosensors showed statistically significant correlations between accelerometer and reported sleep quality for ASD participants. Daily mood measures via the smart phone also correlated with in-clinic ASD symptoms, including negative mood and challenging behavior $(r=0.55, p<0.01)$ and high activity with repetitive behavior $(r=0.36, p<0.01)$.

Conclusions: Interim study results indicate the potential utility and sensitivity of the JAKE ${ }^{\mathrm{rm}}$ system for measuring differences in ASD symptoms and biology via subjective (report-based) and objective (sensor/challenge-task based) methods in individuals with ASD. These results support the potential for identifying biomarkers for stratification and measurement of change that may aid in developing and evaluating medications that treat the core and associated symptoms of ASD.

Keywords: Autism Spectrum Disorder, Eye-Tracking, Social Stimuli.

Disclosure: Johnson \& Johnson: Employee \& Stockholder, Self; Janssen Research \& Development: Consultant, Self; Akili, Inc, Consultant, Self; F. Hoffman LaRoche, Consultant, Self; Perkin Elmer: Consultant, Self; Guilford Press: Royalties, Self; Oxford University Press: Royalties, Self; National Institutes of Health: Grant Funding, Self; Illinois Children's Healthcare Foundation: Consultant, Self; Brain Research Foundation: Board Member, Self. 
W33. IQ-Based Developmental Phenotypes of Autism Spectrum Disorder in Early Childhood and Their Correlates

Marjorie Solomon*, Ana-Maria Iosif, Christine Nordahl, Lauren Libero, Deana Li, Simona Ghetti, Sally Ozonoff, Sally Rogers, David Amaral

University of California, Davis, Sacramento, California, United States

Background: Longitudinal Investigation of the developmental phenotypes of autism spectrum disorder (ASD) offers a promising means of identifying homogeneous pathophysiology-based subgroups. Drawing upon the recent California-based Autism Phenome Project (APP) longitudinal cohort, we investigate IQ change between ages 2 to 7 years, and whether there are cognitive phenotypes defined by IQ trajectory over this period. IQ is selected because it constitutes a great source of heterogeneity within the ASD phenotype, and is among the strongest predictors of adolescent and adult outcomes. Given their clinical significance, we also examine how adaptive functioning, problem behaviors, and the severity of autism symptoms are associated with IQ trajectory membership in children from the APP cohort during this early childhood period. More specifically, our objective are to: (1) examine cognitive development between ages 2 and 7 years in the APP cohort; (2) define ASD phenotypes derived from IQ-based developmental trajectories during this formative period; and (3) to investigate associations between adaptive functioning, problem behaviors, and autism severity, and IQ trajectory membership.

Methods: Participants were 97 individuals with ASD for whom complete IQ data was available at ages 2 and 7. Latent class modeling was used to identify cognitive developmental trajectories, using an adjusted DQ score derived from the Mullen Scales of Early Learning and the Differential Abilities Scale-II. After classifying the ASD participants into trajectories groups, we examined changes in IQ, adaptive functioning, problem behaviors, and autism severity from ages 2 to 7 using a repeated measures approach with correction for multiple comparisons. All core models included fixed effects for time, trajectory group, and the time by group interaction. Problem behaviors were assessed with the Externalizing scales score from the Achenbach System of Empirically Based Assessment (ASEBA), autism severity scores were assessed using the Autism Diagnostic Observation Schedule, Second Edition (ADOS-2), and adaptive functioning was measured using the total score from the Vineland Adaptive Behavior Scales, Second Edition (VABS-2).

Results: Three distinct trajectories of intellectual development were identified. The first consisted of individuals with IQs $\leq 77$ at both times (Greater challenges: $n=36 ; 37 \%$ ). The second was comprised of individuals with IQs $\geq 75$ at both times (Lesser challenges: $n=23 ; 24 \%$ ). A third group exhibited IQs $<82$ at T1, and had scores of $\geq 70$ at T3 with increases of $\geq 1$ standard deviation (Changers: $n=38$; $39 \%)$. Repeated measures analyses, revealed that the Lesser challenges group had significantly higher IQ scores than both the Changers (by 31 points) and the Greater challenges groups (by 39 points) at T1 (both $p<.001$ ). The $60 \%$ of the sample that was in either the Changers or Lesser challenges groups had significant improvements in IQ from T1 to T3 (30, and 10 points, respectively). On the VABS-2, at age 2, there were significant differences across trajectory groups, driven mainly by differences between the Greater challenges and the Lesser challenges groups $(t=4.23, p<.001)$. When examining group differences in VABS-2 score change between ages 2 and 7, the Lesser challenges group showed no change, while the Changers showed an improvement of 6 points $(\mathrm{t}=2.43, p<.02)$, and the Greater challenges group experienced a decline of 10 points $(t=4.5, p<.001)$. On the Externalizing scales of the ASEBA, there was no group difference at age 2, however the pattern of change over time differed across groups, such that the Changers experienced a significant decline in Externalizing symptoms $(t=4.32$, $p<.001)$, while the other groups did not. Autism severity scores changed significantly over time $(\mathrm{t}=4.24, p<.001)$, driven by a 2 point decrease in the Lesser Challenges group $(p<.001)$.

Conclusions: Conclusion: The analysis of trajectories of intellectual development suggests that a significant proportion of children with low early test results in the Changers group will undergo significant intellectual development by age 7, providing a hopeful message for families. Membership in the Changers group also was associated with showing reduced externalizing symptoms suggesting that high levels of such symptoms in early childhood are not necessarily associated with a poorer outcome by middle childhood, and that there is an association between reducing these symptoms and making significant strides in cognitive development, which also was accompanied by advances in adaptive functioning. However, despite the Changers strong cognitive, behavioral, and adaptive functioning development, only the Lesser Challenges group showed a significant age 2 to age 7 reduction in autism symptom severity, suggesting that different mechanisms may govern such symptoms. Our group also is conducting a middle childhood follow-up study using behavioral and neuroimaging assays of memory, executive control, language, academic performance, and anxiety to extend the investigation of ASD phenotypes into the understudied middle childhood period.

Keywords: Cognitive Functioning, Autism, Behaviors, Functioning.

Disclosure: Nothing to disclose.

\section{W34. Using Gene Expression Atlases in Animal Imaging: New Tools to Develop Observer Independent Hypotheses on Transcriptomic Changes}

Thomas Nickl-Jockschat*, Vinod Kumar Jangir, Nicola Grissom, Sarah McKee, Hannah Schoch, Nicole Bowman, Robbert Havekes, Manoj Kumar, Stephen Pickup, Harish Poptani, Teresa Reyes, Mike Hawrylycz, Ted Abel

\section{RWTH Aachen University, Aachen, Germany}

Background: Structural neuroimaging provides a viable path for translational psychiatric research in humans and animal models. We here report changes in grey matter and fibertract architecture in a 16p11.2 del/+ mouse model, a copy number variants (CNVs) exhibiting a high association with 
psychiatric and neurodevelopmental disorders such as autism spectrum disorder (ASD) and attention deficit hyperactivity disorder (ADHD). Since these disorders show a strong sex-bias with males about four times more often affected than females, we also investigated sex-specific effects of the genotype. Moreover, we used the Allen Mouse Brain Atlas to investigate, whether altered brain regions were associated with distinct gene expression patterns.

Methods: MRI was performed ex-vivo and the resulting images images were analyzed using Voxel-Based Morphometry for T1-weighted sequences and tract-based spatial statistics for DTI data sets. Moreover, all available in situ hybrdization (ISH) maps of the genes involved in the hemideletion were aligned to Waxholm space and clusters obtained by our TBSS approaches were analyzed to determine, which gene showed significant overexpression in those regions compared to the expression throughout the entire brain.

Results: We found pronounced sex-specific changes in male animals with increased FA in medial fiber tracts, especially in those proximate to the striatum. Moreover, we were able to identify three genes - TAOK2, MVP and SEZ6L2 - that show high expression patterns in brain regions with male-specific structural changes.

Conclusions: Our data suggest that translational structural imaging approaches in general and the use of gene expression atlases might help to develop observer-independent hypotheses regarding the molecular underpinnings of brain structure changes in psychiatric disorders. Moreover, it suggests that these changes might cause dysfunctional dendrite/neurite outgrowth, potentially mediated by the MAPK pathway.

Keywords: Genetic Mouse Model, MR Imaging, Gene Expression.

Disclosure: Nothing to disclose.

\section{W35. Parental Age and Offspring Psychopathology in the Philadelphia Neurodevelopmental Cohort}

Alison Merikangas*, Monica Calkins, Warren Bilker, Tyler Moore, Ruben Gur, Raquel Gur

University of Pennsylvania, Philadelphia, Pennsylvania, United States

Background: Increasing evidence implicates advanced paternal age in neuropsychiatric disorders in offspring, notably neurodevelopmental disorders, specifically schizophrenia and autism spectrum disorder (ASD). Advanced maternal age has also been associated with schizophrenia and other neurodevelopmental disorders, whereas younger maternal age has been linked with behavioral disorders in offspring. However, few studies have simultaneously considered the effect of co-parental age and the specificity of the associations with respect to different types of psychopathology. In addition, most prior studies have been conducted in clinical samples or registries that may reflect more severe forms of psychopathology.

The overall goal of this investigation is to examine the joint and independent associations between maternal and paternal age with offspring psychopathology in a community-based sample of adolescents from the greater Philadelphia area with emergent psychiatric symptoms and syndromes. The specific aims are to: 1) investigate the individual and joint influence of maternal and paternal age on offspring psychopathology; 2) examine the specificity of parental age at birth of offspring with respect to anxiety, behavior, mood, neurodevelopmental disorders (pervasive developmental disorders, PDD/ASD), and psychotic symptoms in youth; and 3) evaluate whether risk of particular disorders in offspring increases or decreases with parental age. Clarifying these associations may provide insight in the biological or psychosocial mechanisms that lead to child or adolescent psychopathology, and may provide insight into prevention. Methods: 8725 youths (age 8-21 years) from the Philadelphia Neurodevelopmental Cohort were included in the analyses (52\% female, mean age 14.2 years). Logistic regression models with parental age predicting offspring psychopathology were adjusted for sociodemographic factors (SES), and either co-parent age or comorbid disorders in youth as a test of specificity. Due to substantial missing data for paternal age, multiple imputation analysis by chained equations using fully conditional specification was completed.

Results: Parental Age:

Younger parental ages at offspring birth were generally associated with increased rates of offspring psychopathology (anxiety: $\mathrm{OR}=0.991, \quad p=0.045$; behavior: $\mathrm{OR}=0.978$, $p=<0.001$; psychosis: $\mathrm{OR}=0.967, p=0.005)$. After adjustment for comorbid disorders in offspring, there was also an association between older parental age and PDD/ASD $(\mathrm{OR}=1.035, p=0.028)$. Maternal Age: After adjustment for paternal age category, there were significant associations between younger maternal age with behavior $(\mathrm{OR}=0.970$, $p<0.001)$ and mood disorder $(\mathrm{OR}=0.976, p=0.001)$ symptoms. The effects of younger maternal age were specific to behavioral disorder symptoms $(\mathrm{OR}=0.976, p<0.001)$ and positive symptoms of psychosis $(\mathrm{OR}=0.976, p=0.029)$. Paternal Age: After adjustment for maternal age, older paternal age was associated with $\mathrm{PDD} / \mathrm{ASD}(\mathrm{OR}=1.032$, $p=0.040$ ), whereas younger paternal age was associated with behavior symptoms $(\mathrm{OR}=0.988, p=0.046)$ and positive symptoms of psychosis $(\mathrm{OR}=0.978, p=0.035)$. There was specificity of the association between increasing paternal age $\mathrm{PDD} / \mathrm{ASD}(\mathrm{OR}=1.032, p=0.015)$.

Conclusions: This work adds to the evidence regarding associations between parental age and psychopathology in offspring, and extends previous research based on adult clinical samples to a large, community-based sample earlier in the development of psychopathology. In addition to the finding that advancing paternal age is associated with PDD/ ASD, we also show that younger parental age is associated with psychopathology in youth, particularly behavioral disorders and psychotic symptoms. Differences in findings regarding maternal versus paternal effects, their specificity with respect to particular disorders in youth, and the directional influence of parental age highlight the complexity of associations between parental age and psychopathology. The generally weak effects also demonstrate that parental age is only one of a combination of genetic, biologic and psychosocial factors that contribute to psychopathology in youth.

Keywords: Parental age, Population-Based, Children and Adolescents, Psychopathology Patterns, Child Offspring.

Disclosure: Nothing to disclose. 
W36. Electric Field Induced by Repetitive Transcranial Magnetic Stimulation in Adolescents With Major Depressive Disorder: Comparison of Coil Localization Approaches

Zhi-De Deng*, Wesley Lim, Laura Haugen, John Port, Paul Croarkin

National Institute of Mental Health, Washington, District of Columbia, United States

Background: High frequency repetitive transcranial magnetic stimulation (rTMS) targeting the left dorsolateral prefrontal cortex is a promising treatment for adolescents with treatment-resistant major depressive disorder. Two most common approaches for localizing the DLPFC stimulation target are the so-called " $5 \mathrm{~cm}$ rule" and the Beam method for locating the F3 electroencephalogram site given by the International 10-20 system. Prior adult studies have shown that the $5 \mathrm{~cm}$ rule, in which the TMS coil is placed $5 \mathrm{~cm}$ forward from the abductor pollicis brevis (APB) activation hotspot in the motor cortex, misses the DLPFC target in more than a third of adult subjects. The Beam F3 site localization method may provide a more reasonable approximation to magnetic resonance imaging (MRI)guided neuronavigation for locating left DLPFC in a majority of adults. In our prior study with adolescents, the $5 \mathrm{~cm}$ rule and the Beam F3 method yield different locations that are on average $3.9 \mathrm{~cm}$ and $2.5 \mathrm{~cm}$ away from the MRI-derived DLPFC scalp target, respectively. However, little is known about the difference in TMS induced electric field strength between coil placements over the $5 \mathrm{~cm}$ and F3 locations. The goal of this study is to quantify the induced electric field at the DLPFC in a group of depressed adolescent patients.

Methods: This open-label study was conducted under an Investigational Device Exemption (\#G110091) from the United States Food and Drug Administration, and was approved by the Mayo Clinic Institutional Review Board. Ten patients between the age of 13.9 and 17.4 years participated after providing informed consent and assent. The patients met Diagnostic and Statistical Manual of Mental Disorder, 4 ed., criteria for a major depressive episode, had a baseline Children's Depression Rating Scale Resided total score $\geq 40$, and at least one prior failed antidepressant medication trial as defined by the Antidepressant Treatment History Form. Patients were custom fitted with a swim cap on which the APB motor hotspot, $5 \mathrm{~cm}$ site, and $\mathrm{F} 3$ site were marked with fiducial markers. T1-weighted structural MRI data was acquired on a GE 3- T DV750 scanner equipped with an eight channel head coil (true-axial fast 3D-SPGR sequence, repetition time $=12.6 \mathrm{~ms}$, echo time $=5.6 \mathrm{~ms}$, flip angle $=15$ degrees, field of view $=250250 \mathrm{~mm}$, slice $=1.5$ $\mathrm{mm}$, matrix $=512250$ pixels). The left DLPFC was determined by a MRI technician using the Medtronic Stealth navigation system, and a corresponding scalp projection was identified. Motor threshold was determined by visualization of movement technique at baseline. Thirty treatments were administered to the MRI-derived DLPFC scalp location at 5 days per week over 6-8 weeks. Anatomically realistic finite element head models were constructed from individual MRIs using SimNIBS 2.0.1. Each head model consists of five tissue compartments: skin, skull, cerebrospinal fluid, gray matter, and white matter, with assigned isotropic conductivities of
$0.465 \mathrm{~S} / \mathrm{m}, 0.010 \mathrm{~S} / \mathrm{m}, 1.654 \mathrm{~S} / \mathrm{m}, 0.276 \mathrm{~S} / \mathrm{m}$, and $0.126 \mathrm{~S} / \mathrm{m}$, respectively. Figure- 8 coil was centered on the APB, $5 \mathrm{~cm}$ and F3 sites, oriented 45 degrees toward midline. We evaluated the electric field strength at the primary motor cortex for the APB coil placement (with stimulation strength $=100 \%$ motor threshold), and at the DLPFC gray matter (middle frontal gyrus, middle frontal sulcus, and inferior frontal sulcus) for the $5 \mathrm{~cm}$ and $\mathrm{F} 3$ coil placements (stimulation strength $=120 \%$ motor threshold).

Results: Four female and six male depressed adolescents with a mean (standard deviation) age of 15.9 (1.1) years of age enrolled in the study, of which six responded to rTMS treatment. At motor threshold, the mean maximum induced electric field at the motor cortex is $124.9 \mathrm{~V} / \mathrm{m}$, which is comparable to the motor threshold level electric field strengths reported for adults. Using the $5 \mathrm{~cm}$ rule, at $120 \%$ motor threshold, the mean maximum induced electric field strengths at the middle frontal gyrus, middle frontal sulcus, and inferior frontal sulcus are $143.6 \mathrm{~V} / \mathrm{m}, 89.2 \mathrm{~V} / \mathrm{m}$, and $98.6 \mathrm{~V} / \mathrm{m}$, respectively. Using the Beam F3 method, at $120 \%$ motor threshold, the mean maximum induced electric field strengths at the middle frontal gyrus, middle frontal sulcus, and inferior frontal sulcus are $145.8 \mathrm{~V} / \mathrm{m}, 110.1 \mathrm{~V} / \mathrm{m}$, and $117.4 \mathrm{~V} / \mathrm{m}$, respectively. Of note, the electric field at the middle frontal sulcus is higher with the Beam F3 method compared to the $5 \mathrm{~cm}$ rule $(p<0.05)$. Both methods produce similar electric field strengths at the middle frontal gyrus and the inferior frontal sulcus.

Conclusions: Our electric field simulations suggest that the Beam F3 location method results in stronger stimulation of the middle frontal sulcus compared to the $5 \mathrm{~cm}$ rule, which might yield a differential treatment outcome in adolescents. A more in depth electric field analysis will include the MRIderived DLPFC scalp target for comparison. Region of interest analysis of the electric field distribution in combination with clinical outcome could yield predictive biomarker for patient selection and/or guide coil placement and dosing strategies in the adolescent patient population.

Keywords: Adolescent Depression, Repetitive Transcranial Magnetic Stimulation, Electric Field Modeling.

Disclosure: Nothing to disclose.

\section{W37. Investigation of Neurogranin Levels in Patients With Idiopathic Normal Pressure Hydrocephalus (iNPH) Using Two Independent Immunoassays}

Ina Tesseur, Darrel Pemberton, Peter van der Ark, Randy Slemmon, Maarten Timmers, Luc Janssens, Johannes Streffer, Luc Van Nueten, Maura Furey*, Sebastiaan Engelborghs, Mikko Hiltunen,

Tuomas Rauramaa, Kaj Blennow, Henrik Zetterberg, Roy Twyman, Ville Leinonen, Hartmuth Kolb

Janssen, San Diego, California, United States

Background: Neurogranin is a post-synaptic protein enriched in dendritic spines with an important function in long-term potentiation and memory consolidation. Mass spectrometry experiments suggest that neurogranin is secreted into CSF as a pool of C-terminal fragments. Recent evidence suggests that it holds promise as a biomarker for measuring synaptic degeneration in the brain. To date 
several studies show that neurogranin levels are upregulated in CSF in the pre-dementia stage of Alzheimer's disease, correlate well with markers of neurodegeneration such as Tau and Phospho-Tau and that increased levels can predict the rate of cognitive decline.

Methods: Lumbar CSF (CSF) and ventricular fluid (VF) were simultaneously collected at 0,3 and 6 months in 26 patients shunted due to iNPH with confirmed amyloid- $\square$ plaques in their frontal cortical brain tissue biopsies and 13 patients without confirmed amyloid- $\square$ pathology at the Kuopio University Hospital in Finland. All participating patients had an MMSE $\geq 20$ and a CDR $\leq 1$ before shunting. CSF and VF were analyzed for neurogranin with an in house immunoassay on the Meso Scale Discovery platform at the Neurochemistry Laboratory at Sahlgrenska University Hospital, Mölndal, Sweden and with the research grade neurogranin immunoassay from ADx NeuroSciences NV at the Reference Center for Biological Markers of Dementia (BIODEM), University of Antwerp, Belgium. In addition, all samples were analyzed with the INNOTEST ${ }^{\circledast}$ hTau and Phospho-Tau assays at the Neurochemistry laboratory. To account for inter-plate variability, all CSF or VF samples of one patient were run on the same plate. To account for inter-patient variability during biomarker stability calculations, values are expressed as a ratio towards 0 months per sample type.

Results: According to baseline $(63.2 \pm 13.7)$ and 6 months $(64.4 \pm 16.1)$ total CERAD scores, cognitive status remained stable. Absolute levels of neurogranin in iNPH patients with confirmed amyloid- $\square$ plaques were not significantly different from those patients without confirmed pathology whereas the overall levels of Tau and phospho-Tau were high in both groups. Neurogranin concentrations were stable in both groups over a period of 6 months, unless the patient received a recent shunt revision or adjustment $(N=4)$ or was affected by sleep apnea $(N=1)$ in addition to iNPH. Neurogranin levels in VF were on average $21 \%$ lower compared to lumbar CSF. 11 out of 85 CSF and 23 out of 105 VF samples measured below the limit of quantification (BLQ) in the in house immunoassay of the Neurochemistry Laboratory in Sweden $(\mathrm{LLoQ}=125 \mathrm{pg} / \mathrm{ml})$, yet all measured above the limit of quantitation in the immunoassay of $\mathrm{ADx}$ Neurosciences NV (LloQ $=20 \mathrm{pg} / \mathrm{ml})$. Neurogranin measured by the ADx immunoassay shows good correlation with Tau (Pearson $\mathrm{R}=0.69, P<0.0001$ ) and Phospho-Tau (Pearson $\mathrm{R}=0.79, P<0.0001$ ). In contrast the correlation with Tau (Pearson $\mathrm{R}=0.33, P<0.0001$ ) and Phospho-Tau (Pierson $\mathrm{R}=0.33, P<0.0001$ ) in the in house assay of the Neurochemistry Laboratory showed a lower Pearson R value. Conclusions: Longitudinal follow up of neurogranin over 6 months shows this biomarker is stable in a population of iNPH patients with or without concomitant amyloid- $\square$ pathology when measurable. The data also suggest no added value of measuring neurogranin in VF over lumbar CSF, and that neurogranin levels are generally lower in VF. It was surprising to observe a difference in correlation of neurogranin levels measured by the two immunoassays with Tau and Phospho-Tau, and we hypothesize that the two assays may detect a different fragment of neurogranin or a different pool of fragments. Although our results indicate that neurogranin holds promise as a marker of synaptic degeneration, the assays require further characterization and validation. Experiments are currently ongoing.
Keywords: Neurogranin, Amyloid, tau. Disclosure: Janssen Pharmaceuticals: Primary Employer, Self.

\section{W38. Effects of the Insulin Sensitizer Metformin in Alzheimer's Disease: Pilot Data From a Randomized Placebo-Controlled Crossover Study}

Steven Arnold ${ }^{*}$, Aaron Koenig, Dawn MechanicHamilton, Martha Combs, Anne Cappola, Long Xie, John Detre, David Wolk

Harvard Medical School, Charlestown, Massachusetts, United States

Background: To gauge metformin's CNS activity in $\mathrm{AD}$, we conducted a placebo-controlled crossover study of its effects on CSF, neuroimaging, and cognitive biomarkers.

Methods: Twenty non-diabetic subjects with MCI or mild $\mathrm{AD}$ were randomized to receive metformin then placebo for 8 weeks each or vice versa. CSF and neuroimaging (ASL-MRI) were collected for biomarker analyses, and cognitive testing was performed.

Results: Metformin was safe, well-tolerated, and measureable in CSF at an average steady-state concentration of $95.6 \mathrm{ng} /$ $\mathrm{ml}$. Metformin was associated with improved executive functioning, and trends suggested improvement in learning/ memory and attention. No significant changes in CBF were observed, though post-hoc completer analyses suggested an increase in orbitofrontal CBF with metformin exposure. No changes in CSF A $\beta$, t-tau, or p-tau levels were observed.

Conclusions: Metformin crosses the blood brain barrier, and exposure was associated with improvement on cognitive measures of interest in AD. Further study of these effects is warranted.

Keywords: Insulin Resistance, Metformin, Mild Cognitive Impairment due to $\mathrm{AD}$.

Disclosure: Nothing to disclose.

\section{W39. The Intergenerational Transmission of Early Deleterious Rearing Experiences on the HPA Axis: A Nonhuman Primate Epigenetic Model of HPA Axis Functioning}

Elizabeth Conradt, Elizabeth K. Woods, Melanie Schwandt, Stephen Lindell, Stephen Suomi, Christina Barr, J. Dee Higley

\section{Brigham Young University, Provo, Utah, United States}

Background: It is clear that early deleterious experiences increase the risk for affective psychopathology and aberrant HPA axis response to stress. Studies of children reared in deleterious backgrounds or following and maternal neglect have been modeled using nonhuman primates. Such studies have typically investigated early rearing without mothers in peer-only groups and have demonstrated that maternal absence has deleterious effects on the HPA Axis, but few studies have analyzed the effects of normal maternal variation on infant HPA Axis response and even fewer have investigated intergenerational transmission of such early experiences. 
Methods: Data were collected from 569 rhesus monkeys (Macaca mulatta), from which we assessed 276 motherinfant pairs, reared either with mothers or without adults in peer-only groups. We analyzed mother and infant cortisol and ACTH across 2 generations-females who would become mothers and the infants from these mothers. Mothers-The mothers of the infants tested were reared by their own mothers (MR), or without adults present in a peer-onlyreared condition (PR), or with limited daily contact with other peers (2-3 hours), but constant access to their terrycloth surrogate mother (SPR). Infants-All infants were reared by their mothers or in 31 cases, the infant was adopted by unrelated lactating female. Mother's plasma cortisol and ACTH were collected at the same time as their infants. Plasma cortisol and ACTH were collected monthly from infants starting on Day-14 and continuing monthly to Day150 of life, with each sample following a one-hour separation for testing. The effect of the mother's early rearing experience on her infant's plasma cortisol level was first tested using mixed design repeated measures analyses, using infants' ACTH and cortisol as dependent variables and mothers' early rearing experience as the independent variables. Regression was then used to correlate adult mothers' ACTH and cortisol levels with her infants' ACTH and cortisol levels.

Results: None of the mothers were abusive or showed outward signs of serious maternal behavior. Results showed that the infants' cortisol and ACTH levels were modulated by the mothers' early rearing, with infants from mothers who were reared as SPR subjects showing blunted cortisol and ACTH when compared to infants reared by their mothers. Adopted infants showed higher cortisol regardless of the mothers' early rearing experiences. Regressions showed that with maternal rearing condition controlled for, maternal cortisol and $\mathrm{ACTH}$ in response to stress was correlated with infant cortisol and ACTH response at across each of the time periods.

Conclusions: These data suggest that an infant's HPA axis is shaped by how their mother was raised, suggesting a maternal transgenerational transmission of early experiences. Mothers' HPA axis response shows a familial correlation with her offspring, independent of early maternal experience. Such data show transgenerational transmission of HPA functioning, even though the behaviors of the mothers were within what would be considered species norms for maternal care and suggest the potential to use a nonhuman primate model of epigenetic effects.

Keywords: Early Life Stress, Epigenetics, HPA Axis, Early Rearing, Intergenerational Transmission.

Disclosure: Nothing to disclose.

\section{W40. Altered Frontoparietal Thickness as a Trait or State Marker of Bulimia Nervosa: A Longitudinal Study Over Adolescence}

Marilyn Cyr*, Daniel Kopala-Sibley, Mihaela Stefan, Kate Terranova, Laura Berner, Rachel Marsh

Columbia University, New York, New York, United States

Background: Bulimia Nervosa (BN) typically begins in adolescence and is characterized by binge-eating and purging or other compensatory behaviors to avoid weight gain. Previous cross-sectional findings from adolescents and adults with $\mathrm{BN}$ suggest that self-regulatory disturbances underlie these impulsive and compulsive behaviors and may be due to functional (Marsh 2009; 2011) and anatomical (Marsh et al., 2015; He et al., 2016) alterations in frontoparietal cortices. In the present study, we used longitudinal MRI data from adolescents with and without $\mathrm{BN}$ to determine whether reduced thickness of frontal and parietal cortices arise early and persist over adolescence in $\mathrm{BN}$, contributing to the persistence of $\mathrm{BN}$ symptoms into young adulthood. More specifically, we tested whether reduced thickness of these regions constitute trait or state markers of $\mathrm{BN}$ and/or markers of the binge-eating and purging symptoms associated with the disorder.

Methods: Anatomical MRI scans were acquired at baseline from 33 adolescent females with $\mathrm{BN}(16.41+/-1.5$ years of age) and 28 age- and BMI-matched healthy control (HC) adolescents (16.13+/- 2 years). Additional MRI scans were acquired during follow-up assessments, each within 2-year intervals over adolescence. After quality control, first followup data were available from $27 \mathrm{BN}$ and 22 healthy control (HC) adolescents, and second follow-up data from $18 \mathrm{BN}$ and $12 \mathrm{HC}$ adolescents. Symptom severity was assessed in the $\mathrm{BN}$ group at each time point using the Eating Disorder Examination Questionnaire. Structural data were processed in Freesurfer; mean cortical thickness (CT) for each participant was extracted from Desikan Atlas parcels for inclusion in subsequent analyses.

The first set of analyses aimed to test the hypothesis that reduced CT within frontoparietal regions is a trait marker of $\mathrm{BN}$, independent of symptom improvement. Using growth curve analyses, we thus examined whether group status (BN vs. HC), time from baseline and their interaction predicted CT. In subsequent growth curve analyses, we further examined whether group status predicted CT after excluding $\mathrm{BN}$ adolescents who showed less than 50\% symptom improvement between their baseline and last assessments. The second set of analyses aimed to examine whether reduced $\mathrm{CT}$ in frontoparietal brain regions in the $\mathrm{BN}$ group constitute trait or state markers of binge-eating and compensatory behaviors. CT variables were thus decomposed into between-subject (i.e., mean CT across time points) and within-subject (i.e. individual deviation at each time point from one's mean CT) variables. Using multilevel regression analyses, we tested whether both between- and within-person variation in CT predicted the frequency of binge-eating and compensatory behaviors.

Results: In both groups, CT in frontoparietal regions significantly increased over time and the slope of change in CT over time did not differ across groups. However, both baseline CT and mean CT across the three time points was significantly reduced in $\mathrm{BN}$ relative to $\mathrm{HC}$ adolescents in bilateral frontal pole and left inferior frontal, paracentral and supramarginal gyri (p's<.05). Moreover, these group differences remained significant after excluding $\mathrm{BN}$ adolescents who did not show more than 50\% reduction in symptoms from baseline to their last assessment.

In the $\mathrm{BN}$ group, $\mathrm{CT}$ in frontoparietal regions predicted both between- and within-person variations in the frequency of binge-eating and compensatory behaviors. Specifically, on average across the three time points, greater CT in right 
inferior frontal, paracentral, and supramarginal gyri, as well as left frontal pole and rostral ACC predicted less frequent binge-eating episodes (p's <.05). Within-subject increases in CT (from each BN participant's mean CT) in right superior frontal gyrus, frontal pole, posterior cingulate cortex, and inferior and superior parietal lobes predicted decreases in the frequency of binge-eating episodes (from each participant's mean episodes frequency; p's $<.05$ ). Finally, increases in CT in right isthmus cingulate $(p=.01)$, as well as left anterior cingulate and right superior parietal lobe $(p ' s=.07)$, significantly (or marginally) predicted decreases in vomiting episodes.

Conclusions: CT in frontoparietal regions was reduced in $\mathrm{BN}$ relative to healthy adolescents, and these group differences remained stable over time, even when the severity of $\mathrm{BN}$ symptoms in the BN group decreased more than $50 \%$ relative to baseline levels. These reductions in CT may contribute to the development and persistence of $\mathrm{BN}$ and constitute trait markers of the disorder. In the $\mathrm{BN}$ group, within- and between-subject variation in the thickness of frontoparietal regions are associated with variation in symptoms severity, and thus may constitute both trait and state markers of the binge-eating and compensatory behaviors that characterize BN.

Keywords: Bulimia Nervosa, Adolescence, Magnetic Resonance Imaging, Longitudinal Imaging, Human Neuroimaging.

Disclosure: Nothing to disclose.

\section{W41. Reduced Taste Reward Prediction Error Signaling in Adolescent Anorexia Nervosa}

\author{
Marisa DeGuzman*, Megan Shott, Guido Frank \\ University of Colorado Anschutz Medical Campus, \\ AURORA, Colorado, United States
}

Background: Anorexia nervosa (AN) is a psychiatric disorder associated with severe underweight and increased mortality rate, yet its underlying neurobiology is not well understood. The dopamine system and brain reward circuits have been implicated in AN pathophysiology. Our lab has previously shown in adults with AN heightened brain response to unexpected taste reward receipt or omission. Here we tested if adolescents with AN would exhibit heightened brain response to salient stimuli compared to healthy adolescents during a taste reward paradigm.

Methods: We studied forty-two healthy control female adolescents (mean age $=15.2 \pm 2.5$ years, mean $\mathrm{BMI}=20.7 \pm$ $2.1 \mathrm{~kg} / \mathrm{m} 2$ ) and forty underweight female adolescents (mean age $=16.2 \pm 2.0$ years, mean $\mathrm{BMI}=15.8 \pm 0.7 \mathrm{~kg} / \mathrm{m} 2)$ diagnosed with $\mathrm{AN}$, and enrolled in a treatment program using blood oxygen level-dependent functional magnetic resonance imaging (fMRI).

During fMRI, participants learned to associate unique visual stimuli with taste stimuli (1M sucrose solution). When this learned association is unexpectedly violated, a prediction error (PE) is evoked. The PE signal has been linked with the dopamine function of brain reward circuits where dopaminergic neuron activity bursts if receiving unexpected reward and dips if the reward is unexpectedly omitted. All images were preprocessed using SPM8. The temporal difference algorithm was used to computationally model each participant's PE signals based on trial sequence and regressed with brain activation. First-level contrast images were analyzed using general linear models and three contrasts of interest were computed for each subject: unexpected reward receipt, unexpected reward omission, and expectation. Mean beta values were extracted from anatomical regions-of-interest (ROI) for the PE signal and these three contrasts. ROIs included insula, orbitofrontal cortex and ventral striatum. The data were found to be non-normally distributed using the Kolmogorov-Smirnov or Shapiro-Wilk test and rank transformation was used to normalize the data. The data were submitted to a general linear model (MANCOVA including age, medication use, and comorbid mood and anxiety disorders as covariates) to assess the effect of diagnosis on brain activity. Results were corrected for multiple ROI comparisons. Pearson's correlation analysis tested behavior-brain response relationships and significant correlations $(p<0.05)$ were false discovery rate corrected for multiple comparisons.

Results: AN showed lower PE signal compared to controls in the left inferior orbitofrontal cortex, right medial and middle orbitofrontal cortex, left posterior and ventral anterior insula. In response to unexpected receipt of taste reward, AN showed lower activation compared to controls in right medial orbitofrontal cortex and left middle orbitofrontal cortex. In response to the unexpected omission of taste reward, AN showed greater (less negative) activation than controls in the bilateral inferior orbitofrontal cortex, right middle orbitofrontal cortex, bilateral ventral anterior insula, left ventral striatum, and left rectus. In response to the expectation condition, AN showed lower activation in bilateral medial orbitofrontal cortex. A significant negative correlation was found in controls between sensitivity to reward and bilateral caudate head activity during unexpected omission. Harm avoidance was positively correlated in AN with both middle orbitofrontal cortex activity during unexpected receipt and with medial orbitofrontal cortex during expectation. Age was positively correlated in AN with bilateral medial orbitofrontal cortex PE signal.

Conclusions: These results suggest that the brain reward system in adolescent $\mathrm{AN}$ has reduced PE signaling. This was further explained by the hypo-responsive activity during unexpected receipt of reward and the less negative activation in AN compared to controls in response to unexpected omission of reward, where hypoactivation relative to baseline is expected. This provides additional evidence that the brain's dopaminergic reward circuit function is altered in $\mathrm{AN}$ and that the orbitofrontal cortex is associated with altered PE signaling in taste reward in AN. Importantly, while the ventral striatum is typically associated with $\mathrm{PE}$ coding, here the orbitofrontal cortex distinguished the AN group from controls across all conditions. This suggests that higher order cognition related brain regions are particularly involved in AN pathophysiology in this model. The OFC receives dopaminergic input from the midbrain, and contributes to dopamine regulation via direct connection to the ventral striatum. Reduced orbitofrontal activation therefore could alter normal taste reward processing in adolescent AN. Interestingly, this reduced $\mathrm{PE}$ signaling in $\mathrm{AN}$ is the opposite of previous findings in adult AN. Dopamine prediction error activity is heightened during adolescence compared to adults 
during normal development and reduced activation in this circuitry could be a developmental vulnerability in AN. Future studies will need to utilize longitudinal designs and explore the relationship between illness onset age and reward system function and development. In addition, this dichotomy emphasizes the need for careful consideration of age when studying brain reward function in AN.

Keywords: anorexia nervosa, reward prediction error, Functional MRI (fMRI), orbitofrontal cortex.

Disclosure: Nothing to disclose.

\section{W42. White Matter Connectivity Strength in Adolescents With Anorexia Nervosa Across Taste Reward Pathways and During Weight Recovery}

\section{Guido Frank*, Megan Shott, Marisa DeGuzman,} Bernadette Pivarunas

University of Colorado Anschutz Medical Campus, Aurora, Colorado, United States

Background: Anorexia nervosa (AN) is characterized by food restriction and severe underweight. Food intake regulation depends on the interaction of brain regions such as prefrontal and orbitofrontal cortex, insula, midbrain and ventral striatum, which integrate taste perception, food reward value, and cognitive-emotional associations with food. Alterations in those circuits could point toward vulnerabilities for reduced drive to eat and enabling severe weight loss. Previous structural brain research has found altered volumes in those regions and recently we found in adult AN when ill and after recovery greater white matter connectivity strength in the insula-prefrontal-orbitofrontalstriatal network. In this study we wanted to test whether white matter connectivity in adolescent AN is similarly increased and whether weight recovery is associated with normalization of those fibers.

Methods: We recruited 34 adolescents with AN (enrolled in a specialized eating disorder treatment program) and 33 healthy control adolescents (HC). Of those, 16 adolescents in each group completed two diffusion weighed brain imaging (DWI) sessions. For AN, scan 1 was within 1-2 weeks of admission for short term nutritional rehabilitation but still fulfilling diagnostic criteria. Scan 2 was at discharge from treatment. In HCs, scans were two cycle lengths apart. DWI was analyzed with FSL's Diffusion Toolbox 4.1.3 including correction for eddy current distortions and head motion. Probabilistic fiber tractography was computed for fiber paths between brain reward circuit regions using PROBTRACKX2 including modified Euler streamlining (10,000 sample tracts per seed voxel, 0.2 curvature threshold). Mean probability of streamlines for seed-target combinations was corrected for mean connection probability and normalized to control for size of seed and target regions. In total, 138 white matter tracts connecting seed and ipsilateral targets were studied. Connectivity data were extracted and analyzed using SPSS 23.0 (IBM-SPSS, Chicago, IL). The Shapiro-Wilk test for normality indicated that connectivity data were non-normally distributed (all $p$ values $<0.001$ ) and all data were ranked transformed testing the interaction between scan and group in a 2-way mixed ANOVA model.
Results: Groups were matched for age (AN 14.8 \pm 1.8 yrs; HC 16.1 $\pm 2.4 y r s, p<0.15$ ), body mass index (BMI, weight $\mathrm{kg}$ /height $\mathrm{m} 2$ ) was lower in $\mathrm{AN}$ at both time points (Scan 1: AN 16.5 \pm 1.5 ; HC 20.9 $\pm 2.2, p<0.001$; Scan 2: AN 18.9 \pm 1.3 ; HC 21.2 $\pm 2.2, p<0.002)$. Days between scans were more in AN $(56 \pm 12)$ compared to $\mathrm{HC}(44 \pm 17$, $p<0.02)$ and was included as a covariate in the model.

The multivariate analysis showed a significant effect for Scan x Group interaction (Wilk's Lambda $<0.001 ; p<0.014$ ). Regional Scan by Group differences existed for right basolateral nucleus of the amygdala (BLA) to anterior cingulate $(p<0.038$, partial $\eta 2=0.14)$, left posterior insula to prefrontal cortex (PFC) $(p<0.003$, partial $\eta 2=0.266)$, left posterior insula to medial orbitofrontal cortex (OFC) $(p<0.040,0.135)$, right posterior insula to $\mathrm{PFC}(p<0.027$, partial $\eta 2=0.158)$, left ventral anterior insula to caudate body $(p<0.038$, partial $\eta 2=0.140)$, left gyrus rectus to caudate head $(p<0.050$, partial $\eta 2=0.126)$. Connectivity from bilateral posterior insula to PFC and medial OFC, as well as from left ventral anterior insula to caudate body decreased in the AN group but increased in HC. From right BLA to the anterior cingulate and left gyrus rectus to head of caudate, connectivity increased from scan 1 to 2 in $\mathrm{AN}$, but decreased in HC.

There was a main effect for Group for right dorsal anterior insula to middle orbitofrontal cortex $(p<0.043$, partial $\eta 2=0.134)$, right posterior insula to middle orbitofrontal cortex $(p<0.042$, partial $\eta 2=0.135)$, right posterior insula to ventral striatum $(p<0.025$, partial $\eta 2=0.163)$, right ventral anterior insula to middle orbitofrontal cortex $(p<0.042$, partial $\eta 2=0.135$ ).

Post hoc paired samples t-tests did not show significant differences between scans for either group. T-tests for group differences separated by scan showed for scan 1 higher connectivity in AN versus $\mathrm{HC}$ from left dorsal anterior insula to ventral striatum $(p<0.022)$, but lower connectivity from left posterior ventromedial nucleus of the thalamus to the inferior frontal operculum; for scan 2, AN showed greater connectivity from left gyrus rectus to caudate body $(p<0.042)$ and head $(p<0.024)$ and from right posterior insula to gyrus rectus $(p<0.017)$, but lower connectivity from right posterior insula to ventral striatum $(p<0.040)$ and from left ventral anterior insula to caudate body $(p<0.030)$.

Conclusions: This study across a large set of white matter connections across the taste reward circuitry suggests that white matter connectivity is altered in adolescent AN and changes with weight restoration. Connections from insula to prefrontal and orbitofrontal cortex as well as caudate decreased in AN with weight restoration, while connection strength from the gyrus rectus to the caudate increased and was greater in AN. Previously, we have shown that gyrus rectus volume is increased in adolescent $\mathrm{AN}$, which could affect sensory specific satiety processing. Increase in connectivity from gyrus rectus to the striatum in adolescent AN during weight restoration further implicates this structure, this could be a trait vulnerability, and could alter dopaminergic taste reward processing in the striatum. Aside from replication in an expanded sample, this study suggests to test the hypothesis whether gyrus rectus signaling alters reward processing in adolescent AN. 
Keywords: anorexia nervosa, White Matter, Gyrus Rectus, Connectivity.

Disclosure: Nothing to disclose.

\section{W43. Integrating Multi-Omics Biomarkers and Postprandial Metabolism to Personalize Treatment Strategy for Anorexia Nervosa}

Pei-an Shih*, Blake Woodside, Katherine Halmi, Eileen Lam, Bruce German, Bruce D. Hammock, Jun Yang, Christophe Morisseau

University of California, San Diego, La Jolla, California, United States

Background: Anorexia Nervosa (AN) is a serious mental illness characterized by emaciation, an intense fear of gaining weight despite being underweight, and distorted body image. Few treatments reverse the core symptoms in AN such as profound aversion to food and food avoidance. Consequently, AN can have a chronic and relapsing course and the highest death rate of any psychiatric illness. Better understanding of the disease pathogenesis is needed in order to develop better treatments and improve AN outcomes. The hypothesis of this paper is that the pathogenesis and psychopathophysiology of AN can be better elucidated combining longitudinal phenotyping with multiple "omics" techniques, including exon sequencing, proteomics, lipidomic, and metabolomics.

Methods: This paper summarizes the key findings of a series of pilot studies in AN as well as study design, recruitment strategies, methods for biomarker and postprandial metabolism investigations, and planned outcomes in our NIHfunded multidisciplinary collaborative study.

Results: Exon sequencing data was analyzed in $1205 \mathrm{AN}$ and 1948 controls. Metabolomics, lipidomics, and proteomics data were collected in two independent convenience samples consisting of 75 subjects with eating disorders and 61 age-matched healthy controls. Study participants were female and the mean age was 22.9 (4.9 [SD]) years. These exploratory analyses indicated that Epoxide hydrolase 2 (EPHX2) genetic variations were significantly associated with AN and that activity of epoxide hydrolase $(\mathrm{sEH})$ was elevated in AN compared to controls. The polyunsaturated fatty acid (PUFA) and eicosanoids data revealed that Cytochromes P450 pathway was implicated in AN, and AN displayed a dysregulated baseline and postprandial metabolism of sEH-dependent omega-3 and omega-6 PUFA eicosanoids. These data suggest that dietary factors contribute to the burden of EPHX2-associated AN susceptibility and may affect disease outcomes.

Conclusions: The primary aim of our newly-funded NIH study is to establish EPHX2 multi-omics biomarkers, and test whether sEH-associated postprandial metabolism increases AN risk and affects treatment outcome through an $\omega-6$ rich breakfast challenge. Participants will include 100 ill AN patients, 100 recovered AN patients, and 100 age- and racematched healthy women. These data will allow us to investigate: 1) how genetic and dietary factors independently and synergistically contribute to AN risk and progression, and 2) if clinical severity and treatment response in AN are affected by the magnitude of $\mathrm{sEH}$ elevation and resulting eicosanoid dysregulation. Results of our study will 1) identify clinically relevant biomarkers, 2) unravel mechanistic functions of $\mathrm{sEH}$, and 3) delineate contributory roles of dietary PUFAs and Cytochrome P450 pathway eicosanoids for the purpose of developing novel AN treatments and improving disease prognosis.

Keywords: Pharmacogenetic Response, Gene Environment Interaction, Anorexia Nervosa, Fatty Acids.

Disclosure: Nothing to disclose.

\section{W44. Metabotropic Glutamate Receptor 5 Binding in Bulimia Nervosa: Preliminary Findings of a [11C] ABP688 Positron Emission Tomography Study}

Yoan Mihov*, Valerie Treyer, Funda Akkus, Gabriella Milos, Erika Toman, Anass Johayem, Simon Ametamey, Alfred Buck, Gregor Hasler

University of Bern, Bern, Switzerland

Background: Substance addiction is suggested to undergo three stages: a binge/intoxication stage, a withdrawal/ negative effect stage, and a preoccupation/anticipation stage (Koob \& Volkow, 2016). Substantial preclinical evidence implicates metabotropic glutamate receptor subtype 5 (mGluR5) in the binge/intoxication phase (Mihov \& Hasler, 2016). Furthermore, positron emission tomography (PET) studies in clinical samples show aberrant mGluR5 binding in substance addiction. In recent years, the phenotypical similarities between binge behavior in substance addiction and eating disorders have received increasing attention (Smith \& Robbins, 2013), suggesting a partially shared underlying pathophysiology, which likely involves mGluR5. To investigate mGluR5 in bulimia nervosa (BN) in vivo we designed a PET study with the mGluR5-specific radiotracer 3-(6-methyl-pyridin-2-ylethynyl)-cyclohex-2-enone-O-11Cmethyl-oxime ([11C]ABP688).

Methods: [11C]ABP688 PET was carried out in 15 female participants with $\mathrm{BN}$ and 14 healthy female controls. Due to the strong impact of smoking on mGluR5 shown in our previous work (Akkus et al., 2013) groups were matched for smoking status. This resulted in two groups, BN and controls, each comprising three subgroups (non-smokers, ex-smokers, and current smokers). Psychopathology in cases and controls was assessed by the Structured Clinical Interview for DSM-IV Axis I (SCID-I), Beck Anxiety Inventory (BAI), and Beck Depression Inventory (BDI). Participants with BN filled out the Eating Disorder Examination Questionnaire (EDE-Q) and the Eating Disorder Inventory (EDI-2).

We applied a bolus/infusion protocol, previously evaluated for PET with [11C]ABP688, which normalizes PET images to the cerebellar radioactivity concentration, allows reliable measurement of the relative distribution volume (DVR), and reduces potential bias due to arterial blood sampling needed for absolute quantification. With this protocol equilibrium between the tracer in tissue and blood is achieved $40 \mathrm{~min}$ after the start of radioligand infusion. A total of $600-800$ $\mathrm{MBq}$ of [11C]ABP688 in a 50- $\mathrm{mL}$ volume was administered using an infusion pump. Analyses were conducted with the PNEURO-Tool in PMOD and with SPSS. Groups were compared with Welch's t-tests; correlations were tested with 
Spearman's rho. All reported $p$-values refer to two-tailed tests, uncorrected for multiple comparisons. Descriptive statistics are reported as mean \pm standard error of the mean.

Results: Each group comprised five current smokers, two exsmokers, and seven (control sample) or eight (BN) nonsmokers. Both groups and their smoking subgroups were age-matched $(\mathrm{p} \geq 0.7)$. Persons with $\mathrm{BN}$ reported higher BAI scores $(13.3 \pm 2.5$ versus $4.9 \pm 1.1, p=0.006)$ and higher BDI scores $(18.9 \pm 3$ versus $2.3 \pm 0.5, p<0.001)$ than controls.

Analysis of PET data revealed significantly increased mGluR5 binding in $\mathrm{BN}(p=0.038)$ in the anterior cingulate gyrus (ACC) and non-signifcant trends $(0.05<p<0.1)$ for increased mGluR5 in $\mathrm{BN}$ in several other regions: the straight gyrus, medial orbital gyrus, pre-subgenual anterior cingulate, amygdala, lateral part of the anterior temporal lobe, posterior part of the superior temporal gyrus, middle and inferior temporal gyrus, fusiform gyrus, and nucleus accumbens.

Our previous work has shown a marked global reduction in mGluR5 binding in smokers (Akkus et al., 2013). Therefore, we carried out additional analyses within smoking and within non-smoking participants. The mGluR5 binding increase in the ACC in BN was significant in current smokers ( $p=0.037, n=5$ per group) but not in nonsmokers $(p=0.15, n=7$ or 8 per group). To test if this discrepancy reflects a systematic interaction between group and smoking an analysis of variance (ANOVA) was calculated with the fixed effects „sample “and „smoking “. Current and ex-smokers were clustered into one factor level (smoking, $n=7$ per group), whereas non-smokers constituted the other factor level. This resulted in a $2 \times 2$ ANOVA design, yielding a significant effect of sample $(\mathrm{F}=4.95 ; p=0.035)$, a significant effect of smoking $(\mathrm{F}=4.85 ; p=0.037)$, and a non-significant smoking-bysample interaction $(\mathrm{F}=0.113 ; p=0.74)$. Overall, these results confirm our previous findings of smoking-related decrease in mGluR5 binding and show increased mGluR5 binding in the ACC in smoking and non-smoking persons with BN.

We did not find significant correlations between ACC mGluR5 and any of the EDE-Q subscales within the entire $\mathrm{BN}$ sample or its smoking subgroups $(\mathrm{p} \geq 0.11)$. On the EDI-2, in non-smokers $(n=8)$, higher ACC mGluR5 binding was related to lower "drive for thinness "(rho= $-0.86 ; p=0.007)$. No significant correlations between BDI or BAI scores and mGluR5 binding in the ACC were found within the $\mathrm{BN}$ sample and its smoking subsamples ( $\mathrm{p} \geq .13$ ).

Conclusions: To our knowledge, this is the first in vivo study of mGluR5 in BN. Our preliminary findings suggest abnormal mGluR5 in BN. Together with consistent preclinical evidence that drugs targeting the mGluR5 reduce binging behavior in addiction, these results indicate that mGluR5 could be considered as a new pharmacological target in the treatment of $\mathrm{BN}$.

Keywords: mGluR5 Receptors, Bulimia Nervosa, PET Study. Disclosure: Nothing to disclose.
W45. Repeated Exposure to the Pairing of Salient Audiovisual Cues With Probabilistic Reward Delivery in a Rat Gambling Task may Lead to a Hypodopaminergic State: Implications for the Reward Deficiency Hypothesis of Addiction?

Catharine Winstanley ${ }^{\star}$, Jacqueline-Marie Ferland, Melanie Tremblay, Celine Hounjet, Cole Vonder Haar, David Lindenbach, Anthony Phillips, Wendy Adams

University of British Columbia, Vancouver, Canada

Background: Poor decision making, as measured by the Iowa Gambling Task (IGT), may be central to the addicted state, as is sensitivity to the motivational impact ("incentive salience") of reward-paired cues. Rats that make risky, maladaptive choices in a rodent IGT analogue (rat Gambling Task; rGT) are uniquely and adversely affected by cocaine self-administration (SA): their decisions become even riskier, and this correlates with enhanced cue-induced drug-seeking (Ferland and Winstanley 2016). Reward-paired cues that increase in salience with the win size significantly increase risk-preference on the rGT (Barrus and Winstanley 2016), and enhance the negative effects of cocaine SA on choice. Understanding the mechanism by which cues promote risky choice in the rGT could explain how cues promote bad decisions that facilitate addiction, thereby informing treatment strategies. Based on recent studies suggesting that repeated responding for uncertain outcomes, or repeated experience of a conditioned stimulus (CS) that predicts reward with maximal uncertainty (50\%), can sensitize the locomotor response to a psychostimulant drug, we hypothesized that persistent, elevated risky choice on the cued rGT results from a sensitization of the dopamine system, and an enhancement of the influence conditioned stimuli exert over behaviour. We therefore predicted that repeated performance of the cued rGT would sensitize the locomotor response to cocaine, accompanied by increased DA release in the nucleus accumbens (NAC). Similarly, we predicted that animals which showed higher levels of responding for a reward-paired cue (conditioned reinforcement (CRf)) would show a risk-preferring profile on the cued rGT.

Methods: Subjects were male and female Long Evans rats obtained from either Charles River or in-house breeding colonies. The rGT: In each 30-minute session, rats chose between 4 options that vary in the number of sugar pellets (1-4) that can be earned on win trials, but also the probability (0.1-0.6) and duration (5-40 s) of punishing time-out periods delivered on losses. "Safe" options associated with smaller per-trial gains yield the most reward per session, whereas "risky" options appear tempting due to the larger reward size, but result in less reward over time due to the longer and more frequent time-out punishments they are paired with. In the cued rGT, delivery of reward is accompanied by a $2 \mathrm{~s}$ audiovisual cue that increases in complexity with the reward size. Training continued for $\sim 35$ sessions until statistically stable choice is achieved. Locomotor sensitization: Prior to rGT training, we determined the locomotor response to an acute challenge with cocaine. Animals were placed in a novel arena. Following a $1 \mathrm{hr}$ habituation, all rats received an intraperitoneal (IP) injection of saline, followed $1 \mathrm{hr}$ later by $15 \mathrm{mg} / \mathrm{kg}$ cocaine. Locomotor activity was measured by camera using Ethovision. This process was then repeated 
after rats had learned the cued/uncued rGT. Microdialysis probes were then implanted into the NAC, and levels of DA at baseline, after saline administration, and after cocaine challenge was determined. Conditioned reinforcement: Animals completed a CRf training paradigm either before or after learning the cued rGT. In the first phase, animals acquired a conditioned association between a CS and delivery of reward. More specifically, reward was consistently delivered after presentation of a $5 \mathrm{~s}$ CS according to a variable-interval (VI) $60 \mathrm{~s}$ schedule across ten 60- min sessions. Animals had equal experience with a $5 \mathrm{~s}$ control stimulus that was never followed by food reward. In the subsequent $30 \mathrm{~min} \mathrm{CRf}$ test, responding on one lever (the conditioned response; CR) resulted in delivery of the CS, whereas responding on another delivered the control stimulus.

Results: As per our previous studies, animals performing the cued rGT made more risky choices on average than those performing the uncued task. Contrary to our expectations, we found that, while rats performing the uncued rGT exhibited significant locomotor sensitization to cocaine, this effect was blunted in rats trained on the cued rGT. Furthermore, animals trained on the cued task also had lower levels of accumbal DA at baseline, yet showed a comparable percentage increase in DA when challenged with cocaine. Finally, responding for CRf was negatively correlated with risky choice, such that animals showing the highest levels of CRf actually made the most optimal decisions.

Conclusions: These data suggest that repeated exposure to, or responding for, probabilistic rewards paired with audiovisual cues does not lead to a sensitization of the mesolimbic DA system, but instead leads to a hypodopaminergic state. This may render rats more vulnerable to the cognitive deficits resulting from cocaine SA. Furthermore, the ability of cues to bias rats towards risky choice cannot be attributed to increased sensitivity to CRf.

Keywords: Gambling, Dopamine, Microdialysis.

Disclosure: Nothing to disclose.

\section{W46. Sex-Dependent Modulation of Decision-Making in the Rat Gambling Task: Involvement of Brain Dopaminergic and Stress Systems}

Polymnia Georgiou*, Panos Zanos, Shambhu Bhat, Margaret McCarthy, Istvan Merchenthaler, Kathleen Tracy, Todd Gould

University of Maryland School of Medicine, Baltimore, Maryland, United States

Background: Problem or pathological gambling is characterized by endophenotypes including impaired decision-making, higher impulsivity and risk-taking behaviors. In humans, the prevalence of problem gambling is higher among men compared with women suggesting possible gender-related differences in several aspects of pathological gambling. Additionally, gender differences have been observed in the human Iowa Gambling Task (IGT), which involves learning to differentiate between advantageous and disadvantageous decks of cards. In the present study, we used a rat version of the gambling task ( $\mathrm{rGT}$ ) to assess whether there are biologically-based sex differences in decisionmaking and choice impulsivity.

Methods: Rats were trained to nose-poke for sucrose pellet rewards. As in the human IGT, four different options associated with different reward values and different probability and duration of punishing time-out periods were presented to male and female rats. The four different schedules consisted of the following rewarding/punishment probabilities: (i) $90 \%$ reward (1 pellet)/ $10 \%$ punishment ( 5 sec timeout); (ii) $80 \%$ reward (2 pellet)/ 20\% punishment (10 sec time-out); (iii) $50 \%$ reward (3 pellet)/ 50\% punishment (30 sec time-out); (iv) 40\% reward (4 pellet)/ 60\% punishment (40 sec time-out). The schedules were designed such that the options linked with larger rewards result in fewer pellets earned per unit time and the most optimal option was the 2-pellet schedule (P2). Rats typically learn to avoid risky options to maximize their earnings. To assess the underlying role of dopaminergic and stress systems in the performance of male/female rats in the rGT, rats were treated with a dopamine D2 receptor (D2R) antagonist (eticlopride) and agonist (quinpirole), a CRF1 receptor antagonist (antalarmin), as well as the $\alpha 2$ adrenergic receptor antagonist yohimbine, which acts as a pharmacological stressor. Quantitative PCR was used to assess for changes in mRNA levels of D2R and CRF1, in orbitofrontal cortex, medial prefrontal cortex, nucleus accumbens and amygdala.

Results: Female rats differentiated the most advantageous option of the rGT from the first session, whereas male rats differentiated this advantageous option following three training sessions. Conversely, male but not female rats improved their responding to the advantageous options as the task progressed. While administration of eticlopride induced a decrease in the choice of the most advantageous options in male but not female rats, administration of quinpirole induced a decrease in the choice of the most advantageous option in female but not male rats. Yohimbine dose-dependently decreased the P2 choice in female rats, whereas a decrease in the P2 choice was only observed at the highest dose in male rats. Administration of antalarmin dose-dependently increased responding to the P2 choice only in female rats. No effect was observed in male rats. Quantitative PCR experiments are ongoing, and will be reported.

Conclusions: This is the first study in rodents to show direct sex differences in decision-making and choice impulsivity. Our data also highlight differential, sex-dependent differences in short- and long-term decision-making performance. Keywords: Sex Differences, Gambling, Decision Making. Disclosure: Nothing to disclose.

\section{W47. Serotonin Control of Self-Control: 5-HT1B Receptor Modulation of Impulsivity}

Katherine Nautiyal*, Peter Balsam, Melanie Wall, Carlos Blanco, Rene Hen

\section{Columbia University, New York, New York, United States}

Background: Impulsivity is a core feature of many psychiatric disorders including substance use disorder, pathological gambling, attention deficit hyperactivity disorder, and personality disorders. Impulsive behavior is 
$\overline{S 486}$

modulated by serotonin but the mechanisms through which these effects occur are largely unknown. Given that polymorphisms in the serotonin $1 \mathrm{~B}$ receptor (5-HT1BR) are associated with increased impulsive behavior, we used mice with altered 5-HT1BR expression as a model of disordered impulsivity to better understand the underlying neural circuits.

Methods: We generated a transgenic mouse line (floxed tetO-htr1b) which allows for inducible and tissue-specific knockout of 5-HT1BRs. First, we explored the extent to which 5-HT1BR signaling influences different dimensions of impulsive behavior including impulsive action and impulsive choice using a number of different operant behavioral paradigms. We also used these data in an unsupervised factor analysis to examine the latent structure of impulsive behavior in mice and to determine how it is influenced by 5 HT1BR expression. Next, using tissue specific and inducible knockout lines, we investigated the circuit mechanisms through which 5-HT1BR modulates impulsivity. Lastly, using in vivo calcium imaging, the activity of 5 -HT1BR+ cells was assessed during impulsive behavior.

Results: The absence of 5-HT1BR expression increased impulsivity in tests of behavioral inhibition - the differential reinforcement of low-rate responding (DRL) and Go/No-Go operant paradigms. In tests of impulsive choice, another component of impulsive behavior characterized by intolerance to delay and increased risk-taking, there was no significant effect of 5-HT1BR knockout, allowing dissociation of these two types of self-control. Further analysis using an exploratory factor analysis revealed a good-fitting twofactor model to describe the behavioral data. The latent factors represented impulsive action and impulsive choice tasks as independent components, with 5-HT1BR expression and sex as significant predictors.

The increased impulsivity was mediated by adult, rather than developmental, expression of 5-HT1BR. Specifically, mice with only adult knockdown of 5-HT1BR displayed increased impulsive behavior, while adult rescue of the receptor following developmental knockdown restored the normal phenotype. Tissue-specific knockouts interestingly revealed that an absence of 5-HT1B autoreceptors did not significantly alter impulsivity in these tasks. However selective knockout of 5-HT1BRs from GABAergic cells throughout the brain was sufficient to recapitulate the impulsive phenotype. These results suggest that 5-HT1BRs affect impulsivity through modulation of inhibitory tone, rather than directly through alterations in serotonin levels.

Current studies are aimed at investigating the mechanisms underlying 5-HT1BR-dependent inhibitory control at the cellular level by monitoring changes in calcium-dependent fluorescence in GABAergic cells in the absence of 5-HT1BR, during behavioral tests of impulsivity.

Conclusions: Overall, our results point to a role for 5-HT1BR modulation of GABAergic signaling in the regulation of impulsivity, specifically through effects on behavioral inhibition. Furthermore, these effects are dependent on adult expression suggesting a potential avenue for pharmacological treatment for disorders with pathological impulsivity.

Keywords: Impulsivity, Serotonin 1b Receptor, Inhibitory Control, Calcium Imaging, Dorsal Striatum.

Disclosure: Nothing to disclose.
W48. Adult ADHD With Anxiety Disorder and Depression Comorbidity in a Clinical Trial Cohort

Michael Van Ameringen*, Beth Patterson, William Simpson, Jasmine Turna, Katrina Pullia

McMaster University, Hamilton, Canada

Background: Adult ADHD is highly comorbid with mood and anxiety disorders, with $85 \%$ of patients having at least one psychiatric comorbidity, and $60 \%$ having at least two. Results from the National Comorbidity Survey - Replication study revealed that when ADHD was used as the index disorder, social anxiety disorder (29.3\%), specific phobia (22.7\%), and PTSD (11.9\%) were the three most common DSM-IV anxiety disorders. Relatively high levels of comorbidity were also observed with mood disorders and ADHD (38.3\%). Although it has been hypothesized that anxiety may be a feature that is closely tied to the pathogenesis of the ADHD, it has also been proposed that ADHD with comorbid anxiety may represent a subtype of $\mathrm{ADHD}$ or that patients with $\mathrm{ADHD}$ and comorbid anxiety are phenotypically different from those with the pure disorder. The presence of anxiety comorbidity in adult ADHD has been associated with additive clinical effects, leading to more global impairment, poorer outcome, greater resistance to treatment and increased costs of illness. ADHD with comorbid anxiety disorders has also been associated with greater degrees of inattention. Stimulant medications are the first-line agents in the treatment of childhood ADHD and have demonstrated efficacy in adult ADHD. Few studies have examined the efficacy of ADHD treatments within these comorbid populations, as the bulk of the pharmacotherapy literature has examined the pure disorder. Only two randomized controlled trials have specifically examined adult ADHD with comorbidity. Adler and colleagues (2009) examined the use of atomoxetine in adult patients with ADHD and comorbid social anxiety disorder and found that patients treated with atomoxetine had significantly higher reductions in both $\mathrm{ADHD}(p<0.001)$ and social anxiety symptoms $(p<0.001)$, when compared to placebo. Rösler et al., 2010 examined the effects of extended release methylphenidate (MPH) on emotional symptoms of ADHD in a 24-week study. Extended-release MPH demonstrated improvements in emotional lability and dysregulation as well as improvements in obsessive compulsive disorder symptoms, as measured by a sub-scale. No change in anxiety, phobia or major depression symptoms were found. There have also been two studies using MPH compounds which have allowed mild comorbid depression or anxiety conditions. In one study, $9 \%$ of participants had a current comorbid condition and in the other, $80 \%$ had a lifetime mood disorder and $28 \%$ had a lifetime anxiety disorder. Both studies found that treatment response to MPH was not moderated by current or lifetime history of mood or anxiety disorders. Lisdexamfetamine dimesylate (LDX) is a central nervous system (CNS) stimulant with a unique chemical structure, and has been found to be efficacious in both child, adolescent and adult ADHD. Given the high degree of comorbidity between anxiety disorders and $\mathrm{ADHD}$, and the limited evidence to guide clinicians on treatment, we are conducting a prospective trial examining adult ADHD with comorbidity. As this study is ongoing, this poster will present a preliminary analysis of the impact of 
anxiety and mood comorbidity on Adult ADHD symptom severity and functional impairment.

Methods: In this study, we examined the relationship between ADHD, anxiety and mood symptom severity using the clinician-rated ADHD-Rating Scale (ADHD-RS), the Barkley Adult ADHD Rating Scale (BAARS-IV), with the Overall Anxiety Severity and Impairment Scale (OASIS), the Revised Padua Inventory, the Panic and Agoraphobia Scale (PAS), the GAD-7, the Social Phobia Inventory (SPIN), the Quick Inventory of Depressive Symptoms (QID-SR-16), and the the Clinical Global Impression-Severity Scale (CGI-S). In addition, we examined the relationship between ADHD symptom severity, anxiety and mood comorbidity and functional impairment using the Sheehan Disability Scale (SDS) and the Weiss Functional Impairment Rating Scale (WFIRS). The sample was comprised of adult ADHD participants entering a randomized, double-blind, 18-week cross-over trial of flexibly-dosed LDX in the treatment of Adults (aged 18-65) with ADHD and anxiety disorder or depression comorbidity, which is currently being conducted.

Results: Forty adult ADHD subjects were evaluated. The sample was $48 \%$ male, with a mean age of $33.6 \pm 11.4$ years, and mean scores on the ADHD-RS $=44.9 \pm 6.0$, CGI$\mathrm{S}=5.9 \pm 0.6, \mathrm{BAARS}=48.8 \pm 9.4$ suggestive of severe ADHD. Generalized Anxiety Disorder was the most common comorbid condition (92.5\%), followed by SAD (62.5\%) and OCD (37.5\%). No significant differences were found between males and females and no significant correlations were found with the ADHD-RS, in terms of symptom severity, comorbidity, ADHD sub-type or impairment. A positive correlation was found between inattentive symptoms (BAARS) and impairment: WFIRS $\mathrm{r}=0.35, p=0.03$; SDS $r=0.60, p<0.001$; while hyperactivity-impulsivity(BAARS) were positively correlated to ADHD severity: ADHD-RS $r=0.59, p<0.001$, CGI-S $r=0.57, p<0.001$

Conclusions: Similar to previous findings, comorbidity had little impact on ADHD severity. This finding is contrary to the anxiety and mood disorder literature where comorbidity tends to increase the severity of the index disorder and has a significant impact on functional impairment and treatment response. Unlike prior reports which have found that women with $\mathrm{ADHD}$ had higher anxiety severity and impairment than men, we did not find any differences found between genders. Inattention symptoms appear to have a stronger impact on impairment, while hyperactive-impulsive symptoms are stronger indicators of ADHD severity.

Keywords: ADHD, Psychiatric Comorbidity, Anxiety Disorders, Depression.

Disclosure: Shire Canada Inc: Investigator-Initiated Research Grant, Self.

W49. ADHD Symptoms in Youth are Differentially Associated With Polygenetic Risk and Neural Substrates if There is a History of Traumatic Brain Injury

Sonja Stojanovski, Daniel Felsky, Russell Schachar, Aristotle Voineskos, Anne Wheeler

SickKids Research Institute, Toronto, Canada

Background: Attention Deficit/Hyperactivity Disorder (ADHD) is both a risk factor for and major sequelae of traumatic brain injury (TBI) in youth. The objective of this study was to examine whether 1) genetic risk for $\mathrm{ADHD}$ and 2) altered brain structure; differentially predict ADHD symptoms in youth with and without a history of TBI.

Methods: In a large sample of youth (Philadelphia Neurodevelopmental Cohort) we investigated interactions between history of TBI and polygene scores (PGS) with ADHD symptoms $(\mathrm{TBI}=311$, NoTBI $=2269)$. We also examined interactions between history of TBI and neuroimaging phenotypes relevant to ADHD with ADHD symptoms, namely frontal white matter tract fractional anisotropy (FA) $(\mathrm{TBI}=121, \mathrm{NoTBI}=674)$ and subcortical volumes $(\mathrm{TBI}=$ 133, NoTBI $=754$ ).

Results: Youth with a history of TBI reported an increased number of ADHD symptoms compared to those without a history of TBI. PGS was associated with ADHD in those without a history of TBI but not in youth with a history of TBI. Common and unique neural substrates were associated with ADHD in those with and without a history of TBI. FA in the genu of the corpus callosum and left anterior corona radiate was positively associated with ADHD symptoms in youth without a history of TBI but negatively associated with symptoms in youth with a history of TBI. Volume in the bilateral Globus Pallidus was not associated with ADHD symptoms in youth without TBI but negatively associated with symptoms in youth with a history of TBI. Volume in the bilateral striatum and thalamus was negatively associated with the number ADHD symptoms in youth with and without history of TBI.

Conclusions: These results suggest that ADHD associated with TBI is a result of mechanical insult to similar and distinct neural pathways than those affected by genetic risk in developmental ADHD.

Keywords: ADHD, Polygenetic Risk Score, Human Neuroimaging, Traumatic Brain Injury.

Disclosure: Nothing to disclose.

W50. Comparison of the Implicit Association Test With Established Clinical Rating Scales in Suicide Risk Assessment: Baseline Data From the Better Resiliency Among Veterans and Non-Veterans With Omega-3 s (BRAVO) Study

Bryan Tolliver*, Bernadette Marriott, Joseph Hibbeln, Kathryn Magruder, Alicia Marzolf, Mark Hamner, Kelly Holes-Lewis, Therese Killeen, Constance Guille, James Fox

Medical University of South Carolina, Charleston, South Carolina, United States

Background: Suicide is the tenth leading cause of death in adults in the United States and recent data suggest that its incidence has risen significantly in the past decade. Rates of completed suicide are particularly high in U.S. military veterans as well as in civilians with recurrent mood disorders, posttraumatic stress disorder (PTSD), substance use disorders, and other Axis I psychiatric conditions. Despite advances in pharmacotherapy, psychotherapy, and brain stimulation treatments for depression, reliance on selfreport in assessment of risk for completed suicide continues 
to limit clinicians' ability to predict and prevent death by suicide. Novel strategies to identify behavioral markers of suicidality using objective neurocognitive tests such as the Implicit Association Test (IAT) have shown promise, but how such approaches relate to or compare with established clinical assessment instruments has received minimal study. The present analysis compares results of the IAT with those of established suicide risk assessment scales collected concomitantly at baseline upon entry to the BRAVO study, a 6-month randomized clinical trial of omega-3 highly unsaturated fatty acid supplementation in adults at high risk of suicide.

Methods: Participants were 125 adults $(n=62$ U.S. military veterans, $n=63$ civilians) age 18 or older recruited primarily from inpatient units and outpatient clinics at the MUSC Institute of Psychiatry and adjacent Charleston VAMC. The suicide/death IAT and established clinical rating scales (Beck Scale for Suicidal Ideation (BSS), Beck Hopelessness Scale (BHS), Beck Depression Inventory-II (BDI-II), and suicide module of the Mini International Neuropsychiatric Inventory (MINI)) were administered at study entry. Psychiatric diagnostic history and suicide attempt history were assessed using the MINI and the ColumbiaSuicide Severity Rating Scale (C-SSRS), respectively, during screening for study enrollment. Relationships among scores on the IAT and clinical rating scales were evaluated by correlational analysis and univariate analysis of variance (ANOVA).

Results: With the exception of significantly higher baseline BDI-II scores in female ( $n=46,37 \%$ of sample) than male subjects $(p<.05)$, baseline IAT and established rating scale scores did not significantly differ by sex, veteran status, or smoking status. Scores on the BHS and BDI-II were significantly lower in subjects with current alcohol dependence ( $n=47,38 \%$ of sample). Whereas BSS, BHS, and BDIII scores were all significantly higher in subjects with PTSD $(n=75,60 \%$ of sample; $p<.001)$, IAT results did not differ by PTSD status. All clinical rating scale scores correlated strongly $(\mathrm{R} 2=.22-.43, p<.0001)$ with the exception of the MINI suicide module (R2 =.014-.029, N.S.). IAT results were uncorrelated with scores on the BSS, BHS, or BDI-II and correlated only weakly with the MINI total suicide score $(\mathrm{R} 2=.047, p<.05)$. Subjects who reported at least one suicide attempt in the past 6 months had significantly higher scores on the BSS $(p<.006)$ and BDI-II $(p<.03)$ but not the BHS or IAT.

Conclusions: Results on the IAT were uncorrelated with scores from established clinical rating scales in this sample of adults at high risk of suicide. Whereas all established clinical rating scale scores were significantly higher in subjects with PTSD and BSS/BDI-II scores were higher in subjects with recent suicide attempt(s), no such association of these suicide risk factors with IAT scores was evident. Comparison of these assessments' relative ability to prospectively predict future suicidality (e.g. attempts, hospitalizations) await completion of the BRAVO study later in 2016.

Keywords: Suicide Assessment, Cognitive/Emotional Task Performance, Omega-3 Fatty Acid, Suicide.

Disclosure: Nothing to disclose.

\section{W51. Continuous Behavioral Data as a Depression Biomarker}

Hane Aung, Mark Matthews, Charles Forkish, Nosh Petigara, David Kupfer, Ellen Frank*, Tanzeem Choudhury

University of Pittsburgh School of Medicine, Pittsburgh, Pennsylvania, United States

Background: While the search for biomarkers for depression has focused largely on genetic and other biological variables, our group has focused on the potential of continuously monitored behavioral variables as important markers of mood episode onset. There is substantial evidence to suggest that periods of depression may be preceded by changes in physical activity and geo-locational movement. Such variables can be readily measured using GPS and accelerometer sensors within all commercial smartphones. Most important from the standpoint of their utility as predictors, these data can be acquired on a $24 / 7$ basis over long periods of time without active effort on participants' part. The smartphone can also serve as a platform for ecologically valid selfreporting that can be input simultaneously with the automatically acquired data.

Methods: Our collaboration with Dartmouth Hitchcock ImagineCare (ICDH), a comprehensive healthcare management enterprise, has provided the opportunity to examine such data from the mobile behavioral data collection platform we have developed within ICDH's ImagineCare app. The ImagineCare app has already been implemented in a cohort of over 3,500 patients with chronic medical disease on which a large number of other measures are also being collected. Within this ICDH data set, we are conducting two levels of analyses. The first is an understanding of associations between a wide range of variables constructed from the sensor data (e.g. time spent at primary location home - in a day, or distance traveled in a day) with selfreported scores of depression based on the PHQ2 and PHQ8. We are applying a bivariate regression analysis between each variable and the self-reported scores using generalized estimating equations (GEEs) that are designed to analyze longitudinal data and with no assumption of independence between variables. This informs the second stage of the analysis in which we are developing predictive models using supervised machine learning, specifically ensembles of decision trees models or Random Forests, to allow the system to eventually be independent of self-reporting and accurately estimate depression from the automatically-sensed data alone.

Results: We are in the early stages of analyzing the extraordinarily rich data set. To date, our analyses indicate that changes in sleep continuity may precede PHQ scores consistent with a major depressive episode by several weeks. Likewise, shifts to greater social isolation (i.e. fewer exits from home and less time spent away from home) appear to be early markers of depression onset. We anticipate that the more involved machine learning paradigms will yield models that may eliminate the need for self-reporting of mood symptoms.

Conclusions: Our initial analyses suggest the utility our mobile app as a feasible means of collecting behavioral biomarker data. The ubiquity of the smartphone and its 
almost continuous presence in our lives make it an ideal method for biomarker data collection as it requires little to no effort on the part of patients and one that requires no special equipment, that can be implemented at relatively low cost to health systems and research studies and that is entirely acceptable to patients.

Keywords: Biomarkers, Depression, Mobile Technology Disclosure: HealthRhythms, Inc.: Co-founder, Equity Holder, Self; HealthRhythms, Inc.: Co-founder, Equity Holder, Spouse.

\section{W52. Reduced Anterior Cingulate Cortex Gamma- Aminobutyric Acid in Adolescent Depression and Anhedonia: Replication in a Larger Sample}

Vilma Gabbay*, Kailyn Bradley, Reynolds Ostrover, Guoxin Kang, Xiangling Mao, Dikoma Shungu

Icahn School of Medicine at Mount Sinai, New York, New York, United States

Background: Major depression (MDD) is a devastating disorder across all age groups. While advances have been made into its neurobiological underpinnings, the major challenge has been the reliance on a categorical diagnosis of MDD that is based on clusters of symptoms, each with a distinct pathophysiology. Addressing this barrier, the NIMH launched the $\mathrm{RDoC}$ initiative to encourage investigations that utilize dimensional approaches to study specific behaviors and their underlying neuronal circuits. Our laboratory and others have examined anhedonia, a core symptom of MDD and a salient feature across other psychiatric conditions. Anhedonia reflects deficits in reward processes and has been documented as a predictor of adult psychopathology and associated with poor outcomes and suicidality. Biologically, reduced gamma-aminobutyric acid (GABA) levels have been repeatedly implicated in MDD, with a stronger effect associated with melancholic depression, known to be characterized by severe anhedonia. Previously, while utilizing both categorical and dimensional approaches to study the role of anterior cingulate cortex (ACC) GABA levels in adolescent MDD, we documented that decreased ACC GABA in adolescent MDD was driven by anhedonia severity. Here, we sought to replicate these findings in a larger sample of 80 psychotropic medicationfree adolescents and test the hypotheses that youth with MDD would exhibit reduced ACC GABA compared to healthy controls, and that this decrease would be specifically related to increased anhedonia severity. Our study of adolescents aimed to capture neurobiological changes early in the course of the illness prior to chronicity effects.

Methods: Forty-four youth with MDD (mean age $=16.31$, $\mathrm{SD}=2.64 ; 52 \%$ female) and 36 healthy controls (HC) with no history of mental illness (mean age $=15.80, \mathrm{SD}=2.12$; $64 \%$ female) were recruited (age range $=12-21$ years old) . All participants were medication naïve or free, except one, who had been on an antidepressant for less than one week prior to assessment. Participants were evaluated using the Kiddie-Schedule for Affective Disorders and SchizophreniaPresent and Lifetime. Depression severity was assessed using the clinician-rated Children's Depression Rating ScaleRevised (CDRSR) and the self-rated Beck Depression Inventory (BDI). Anhedonia was assessed using a combination of scores on the "loss of interest" and "loss of pleasure" questions from the BDI and the "difficulty having fun" question on the CDRSR; clinician- and self-rated assessments contributed equally to the final anhedonia measure. GABA/Water (W) and combined resonances of glutamate and glutamine (GLX)/W were measured in the ACC using proton magnetic resonance spectroscopy. Groups were compared using analysis of covariance, controlling for age and sex, as well as any factor that was significantly different $(p<.05)$ or approached being significantly different $(p<.10)$ between groups. Partial correlations were used to examine the relationships between anhedonia severity, depression severity, GABA/W, and GLX/W in the ACC in both the total sample and the group with MDD.

Results: Youth with MDD exhibited reduced GABA/W [mean $\mathrm{GABA} / \mathrm{W}=.00256 ; \mathrm{SD}=.00043$ ] compared to $\mathrm{HC}$ [mean GABA $/ \mathrm{W}=.00287 ; \mathrm{SD}=.00044 ; \mathrm{F}(1,72)=10.37$, $p=.002$ ] when controlling for age and sex, as well as ethnicity and cerebrospinal fluid volume in the ACC voxel of interest. There was no difference between groups in GLX/W $[F(1,72)=.006, p=.941]$. In the total sample, anhedonia severity $[\rho=-.29, p=.011]$, but not depression severity $[\rho=$ $-.19, p=.105]$, was correlated with GABA/W, when controlling for group (i.e., MDD vs. $\mathrm{HC}$ ). In just the group with $\mathrm{MDD}$, anhedonia severity and GABA/W were correlated, even when controlling for depression severity $[r=-.33$, $p=.031]$; depression severity and GABA/W were not directly correlated $[\mathrm{r}=-.22, p=.149]$. GLX/W was not correlated with anhedonia or depression severity in either the total sample or the group with MDD (ps>.10).

Conclusions: Depressed youth exhibited a reduction in ACC GABA compared to $\mathrm{HC}$, and GABA was specifically correlated with anhedonia but not depression severity in both the total sample and the group with MDD. These findings replicate our lab's prior results from a smaller sample showing a reduction in ACC GABA in anhedonic adolescents with MDD. This larger, more robust investigation confirms that dysfunction in GABAergic neurons, specifically within the prefrontal reward circuitry, may play a role early on in the pathogenesis of anhedonia.

Keywords: Cortical GABA, Adolescent Depression, Anhedonia, Research Domain Criteria (RDoC).

Disclosure: Nothing to disclose.

\section{W53. Age Moderates the Effect of Cortisol on the Prediction of Antidepressant Treatment Response}

Felipe Jain*, Victor Reus, Synthia Mellon, Elissa Epel, Christina Hough, Alexandra Morford,

Francesco Saverio Bersani, Owen Wolkowitz

The University of California, San Francisco Medical Center, San Francisco, California, United States

Background: Major Depressive Disorder (MDD) is a serious and prevalent condition resulting in high levels of morbidity and reduced quality of life. Although the hypothalamopituitary-adrenal axis (HPA axis) has been extensively studied in MDD, the relationship of HPA axis hormones such as cortisol to depression treatment outcome is unclear. Age and cortisol levels have previously been associated with depression symptoms. We hypothesized that age would 
moderate the effects of cortisol on depression treatment response.

Methods: 37 adult subjects (mean $38+/-12$ years old) with unmedicated MDD and free from other major medical illnesses were measured at baseline and post antidepressant (selective serotonin reuptake inhibitor) treatment for morning serum cortisol levels. Subject data were combined from two cohorts with identical protocols $(N=13$ in Cohort 1 and $N=22$ in Cohort 2.) Clinical outcome measures included the Quick Inventory of Depressive Symptoms (QIDS), the Life Orientation Test Optimism and Pessimism scales (LOT-opt and LOT-pess), and the Hamilton Depression Rating Scale 17-item (HDRS). Symptom change calculated as post- minus pre- treatment total scores. Cortisol levels were log transformed, and $\mathrm{z}$ scores calculated by cohort to facilitate comparison across assay batches from the two cohorts. A moderation analysis was conducted utilizing general linear models to predict absolute change in symptoms, with independent variables including Age, Cortisol, and Age X Cortisol interaction.

Results: Age moderated the effects of cortisol on the prediction of (a) change in QIDS (Main Effect $\mathrm{F}(3,31)=$ $8.8, \quad p<.0005$; Interaction term Beta $=-.39, \mathrm{SE}=.09$, $p<.0005)$, (b) change in LOT-pes (Main Effect $\mathrm{F}(3,33)=$ 4.67, $p<.01$; Interaction term Beta $=-.13, \mathrm{SE}=.04, p=.01)$, (c) change in LOT-opt (Main Effect $\mathrm{F}(3,6)=25.8, p<.001$; Interaction term Beta $=.12, \mathrm{SE}=.03, p=.01)$, and that there was a trend for the model to predict (d) change in HDRS $($ Main Effect $\mathrm{F}(3,33)=2.26, p=.10$; Interaction term Beta $=$ $-.17, \mathrm{SE}=.08, p=.06)$. Examination of the coefficients indicated that for all outcome measures, cortisol had a detrimental effect that was moderated by age: older subjects were less affected by elevated cortisol than younger subjects. Conclusions: The impact of morning cortisol on depression treatment outcome was age dependent and consistent across multiple measures. Elevated cortisol more markedly interfered with outcome in younger than older patients. Future research may address whether accounting for this relationship can help target HPA-axis modifying therapies. Our results should be prospectively confirmed in larger samples. Keywords: Major Depression Disorder, Cortisol, Age Effects, Antidepressant.

Disclosure: Nothing to disclose.

\section{W54. Deep Brain Stimulation to the Medial Forebrain Bundle in Treatment-Resistant Depression- A Placebo Controlled Clinical Study}

Thomas Schlaepfer*, Bettina Bewernick, Sarah Kayser, Sabrina Gippert, Christina Switala, Volker Coenen

\section{Johns Hopkins University, Freiburg, Germany}

Background: Several targets are currently under research for the treatment of otherwise treatment-resistant depression (TRD). Rapid and long-term antidepressant effects of DBS to the supero-lateral branch of the medial forebrain bundle (slMFB) have been demonstrated in our group in a pilot trial with eight patients. These findings have been replicated in four patients by colleagues in an interim analysis after 26 weeks. Consequently, a larger study with a staggered onset sham-controlled design was performed in order to assess putative placebo-effects and evaluate the antidepressant response on a larger sample.

Methods: Sixteen patients suffering from TRD were treated with DBS bilaterally to the slMFB. Patients were either stimulated with DBS immediately (group A) or with a delay of two months (group B) in a double blind staggered onset protocol.

Primary outcome criteria were the difference in antidepressant response between groups during sham phase as well as during long-term effect of unblinded $\mathrm{DB}$ stimulation. In addition, quality of life as measured with SF-36, global assessment of functioning (GAF) and cognitive performance were assessed.

Results: A significant difference in antidepressant effect compared to baseline was found in the first month for both groups. In the second month, only group A (immediately stimulated) showed a significant antidepressant response. A significant antidepressant effect for each month of DBS compared to baseline was found for the whole group (group A and group B) from month three (all patients stimulated) up to the end of the study (12 months). Main side effect was strabismus at higher stimulation currents. No change in cognition was identified; quality of life and GAF were improved.

Conclusions: This study demonstrates a significant difference in efficacy of sham and real stimulation in a larger sample. This confirms previous efficacy data from our group in a smaller sample of eight patients and data from colleagues on four patients. For three weeks, some of the shamstimulated patients showed a significant response which could possibly be explained by a transient placebo effect or a by direct effect of the electrode placement and test stimulation during surgery such as described in DBS for Parkinson's disease. In further studies, the initial placebo response could be studied in more details, e.g. by implementing a placebo phase later in the study protocol after stable stimulation parameters have been established.

Keywords: Deep Brain Stimulation, Therapy-Resistant Depression, Medial Forebrain Bundle.

Disclosure: Medtronic: Grant, Self.

\section{W55. Sex Differences in Emotion Processing and Regulation in Mood Disorders Across the Lifespan}

Emily Briceno, Lisanne Jenkins, Jon Stange, Leah Kling, Kristy Skerrett, Ghanshyam Pandey, Olusola Ajilore, Heide Klumpp, Pauline Maki, Sara Weisenbach, Scott Langenecker*

University of Illinois at Chicago, Chicago, Illinois, United States

Background: There is continued interest over what may render adults vulnerable to new onset depression, or worsening depression in middle and late adulthood. The lifespan risks for women and men are different, with a doubling of prevalence of depression after puberty, yet theoretical and empirical data have not clarified why women are at increased risk for MDD, and recurrence of MDD. On an even more intriguing level, emotion perception, a strength for women, is impaired in MDD, and perhaps more so in women in MDD. Furthermore, emotion regulation strategies 
are used to a greater extent in women relative to men. Recent work by our group suggests that interactions between age, sex, and disease are present in emotion processing. Finally, emotion regulation changes with age suggests that there may be a divergence in those who are healthy and those who experience mental illness, with the former group reporting improvement, and the latter group exhibiting evidence of continued difficulties. How might this puzzle be untangled to better assist and treat those who experience recurrent MDD. We contend that careful cross-sectional and longitudinal studies are needed across the adult lifespan to determine inflection points for any changes in emotion perception and regulation, whether they are different for men and women, and whether healthy controls show continued improvement or just decline at a slower level relative to those with mood disorders. We hypothesized sex (females $>$ males), age (younger $>$ older) changes in performance. We also hypothesized that amygdala activation would decrease with age in healthy individuals, but not in MDD. We expected that emotion regulation regions would differ by diagnosis, sex, and age. In particular, we expected higher activation in females, with greater increases with age, and an inverted pattern in females with MDD, and in the male groups compared to their opposite group and sex comparisons.

Methods: Cross-sectional data was collected in 370 healthy control women between the ages of 18 and 60 on the Facial Emotion Perception Test. They were 56\% female, with an average age of 31.7 (12.9) and average education of 15.3 years. Healthy controls were divided by age (bins of 18-24, $25-39,40-60)$, education ( $>16,16$ or less) and sex (cell Ns from 14-54). Overall accuracy and efficiency were computed and compared by sex and age. A subset of $\mathrm{HC}(N=52)$ also completed the FEPT during fMRI. In addition, a sample of females with $\operatorname{MDD}(N=53)$ were recruited and completed FEPT during fMRI. The model included regression of age, sex and diagnosis onto activation for all Faces minus animals, and each emotion related to neutral. Finally, a set of emotion regulation regions were identified from a metaanalysis (Frank et al., 2015), and used to define important ROIs for both emotion processing/reactivity (e.g., Amygdala; decreasing during reappraisal of negative emotional stimuli) and emotion regulation (e.g., inferior, middle frontal gyri; increasing during reappraisal of negative emotional stimuli). We investigated faces (identification) relative to animals (categorization), as well as fearful faces minus neutral faces. Results: As expected, in normative comparisons, younger, more educated females were the top performance in accuracy, in youngest and oldest high education groups. The sex effect was diminished in the lower education groups, and in both middle age groups. Age declines occur earlier in those with lower education levels, particularly females (in the middle age group). For fMRI, there was a decrease in amygdala activation with age in the healthy control group for all faces relative to animals, and in response to fearful faces relative to neutral faces. There was no difference between males and females in this decline and there was no decline with age in MDD. As expected, an age by sex by diagnosis interaction was present in the left inferior frontal gyrus, but not in the middle frontal gyrus for fearful minus neutral faces. Healthy females exhibited increased activation with age, whereas healthy males decreased activation with age.
Males and females with MDD did not show changes in activation with age.

Conclusions: This cross sectional set of data in emotion processing and with processing and regulation regions suggests that females exhibit the expected strength in emotion processing, and this most evident in early and late adulthood, and more so for those with higher levels of education. Activation differences show separation by emotion processing regions - age by disease effects, relative to emotion regulation regions (age by disease by sex interactions). Larger samples are needed, and likely longitudinal studies, to better understand these complex patterns of performance accuracy, regulation capacity, and activation changes. In addition, evaluating hormonal and related stress markers that could unequally effect the neural systems supporting emotion processing and regulation by age, sex, and disease are important for understanding mechanisms underlying any of these changes.

Keywords: Mood Disorders, Emotion Regulation, Functional MRI (fMRI), Sex Differences, Age Effects.

Disclosure: Nothing to disclose.

\section{W56. Neural Correlates in the Amydala of Extreme Responders to Auditory Pavlovian Threat Conditioning}

Lorenzo Diaz-Mataix*, Joseph E. LeDoux

New York University, New York, New York, United States

Background: In Pavlovian Auditory Threat Conditioning (PTC), an initially neutral conditioned acoustic stimulus (CS), after being associated with an aversive unconditioned stimulus (US), typically an electric shock, acquires the ability to induce conditioned responses (CR) such as freezing. The amygdala is essential for this form of associative learning and memory. The CS and the US information converge on cells of the lateral nucleus of the amygdala (LA). These cells undergo longlasting changes after conditioning, as shown by a potentiation single units (Quirk GJ. et al., 1995; Repa JC. et al. 2001, Maren S. 2000) and of the LA-Auditory Evoked Field Potentials (AEFP) (Diaz-Mataix L. et al., 2011). The central nucleus of the amygdala (CeA), which also shows a potentiated AEFP after conditioning (Duvarci S. et al., 2011), controls the expression of conditioned defensive responses, such as freezing behavior. Most of what is known about PTC and its underlying brain circuitry is based on experiments in which the data is analyzed after averaging individual values. However, there is great variability of individual conditioned responses. Using the central tendency and assuming population homogeneity masks neurobiological correlates of exaggerated or minimal reactivity in response to threatening stimulus in individuals. The hypothesis that drives the present experiments is that the neural activity in the LA and/or in CeA might differ in the different subpopulations.

Methods: Animals were habituated to unreinforced CS1 (5 KHz pips, $100 \mathrm{~ms}$ duration, delivered at $1 \mathrm{~Hz}$ during 30 seconds) in context A on two consecutive days (3 CS1 15 mins session / each day). 24 hours later, rats were conditioned by presenting 3 CS1 co-terminating with a footshock ( $1 \mathrm{~mA}, 1 \mathrm{~s})$ in context A. 2 days after conditioning long-term memory (LTM) was tested by presenting 10 CS1 in a modified context (B). On subsequent days, the animals were 
implanted with electrodes into the LA and CeA contralaterally. After at least one week of recovery, rats were conditioned following the same steps as before but changing the CS1 for another auditory stimulus (CS2; Frequency modulated sweeps $12.5 \mathrm{kHz}$ carrier frequency, $50 \mathrm{~Hz}$ modulation frequency, $2.5 \mathrm{kHz}$ modulation depth, $250 \mathrm{~ms}$ duration delivered at $1 \mathrm{~Hz}$ during 30 seconds) that does not cause generalization (Diaz-Mataix L. et al., 2001). Freezing during CS1 and CS2 was recorded for offline analysis. In the second part of the experiment the electrophysiological activity was recorded during every session in the two areas of study.

Results: The analysis of the individual freezing to the CS1 using data mining techniques, results in clearly different subpopulations in function of their CS-reactivity (GalatzerLevy I. R., 2015). This result confirms, with advanced mathematical and statistical tools, previous studies reporting the existence of a large percentage of rats exhibiting significantly higher or lower CS-reactivity (Bush DE. et. al., 2007; Yehuda R. and LeDoux JE. 2007; Kowansage KK. et al., 2013). In addition, extinction learning rate varies significantly when the animals are grouped according to their freezing level. Remarkably, although the animals exhibiting extreme freezing are approximately $40 \%$ of the population, our results suggest that the extinction learning curve of the whole population (including also the $40 \%$ ), is not different that the pattern of extinction expressed by the $60 \%$ regular freezers but has no similarity with the pattern of the remaining $40 \%$. The unit activity and the CS2 auditory-evoked field potentials in the LA and CeA are being analyzed to identify potential correlations between freezing phenotype and neural activity that could account for the aforementioned differences in the reactivity to the conditioned stimulus.

Conclusions: Many psychiatric problems (PTSD, Phobia, Generalized Anxiety) are characterized by over-responses to stimuli that trigger minimal reactions in healthy subjects. Our results comparing groups of animals exhibiting low, average and high responses will help to identify brain substrates responsible of the exaggerated responses exhibited by persons suffering the above mentioned disorders. On the other hand, the understanding of the neural correlates of the animals exhibiting weak responses might be related to resilience mechanisms.

This study has been supported by the $4 \mathrm{H}$ Foundation and funds from New York University.

Keywords: Individual Differences, Pavlovian Conditioning, Electrophysiology.

Disclosure: Nothing to disclose.

\section{W57. Imbalance Between Serotonin and Kynurenine Metabolism Associates With Impulsive and Suicidal Behavior in Bipolar Longitudinal Study Volunteers - Implications on Future NMDA Receptor-Based Treatment Studies in Depressive Illness}

Maria Juliana Orejarena*, Simon Evans, Shervin Assari, Robert Dantzer, Melvin McInnis, Alan Prossin

University of Texas Health Science Center at Houston, Houston, Texas, United States

Background: Activation of brain immune cells increases concentration of specific inflammatory cytokines both centrally (brain) and peripherally (plasma). The ensuing cascade of events subsequently impacts metabolism of tryptophan by inducing indolamine 2,3-dioxygenase, diverting production away from 5-hydroxytryptophan (5-HTP; a precursor to potent monoamine production) and toward harmful kynurenine metabolites including 3hydroxykynurenine (3-HK). 3-HK is a potent inducer of oxidative stress and neuronal apoptosis via production of harmful reactive oxygen species (ROS), end products capable of facilitating depressive symptoms. Shifting the balance from 5-HTP to 3 -HK production reduces concentration of euthymic mood sustaining monoamines (serotonin) and enhances production of depressogenic factors including 3$\mathrm{HK}$ and its metabolite (quinolinic acid), a potent NMDA receptor agonist. Initial evidence to date suggests an imbalance between 3-HK and 5-HTP may underlie the pathophysiology in refractory depressive episodes as well as suicidal/impulsive behaviors. Despite efforts, the impact of specific imbalance between these 2 factors in depressive syndromes remains unclear and its role in impulsive and suicidal behavior understudied. Initial and ongoing ketamine treatment studies in depressed individuals show that abrupt recovery is seen, but that substantial inter-individual variation burdens response to this promising, repurposed NMDA based strategy. By interfering with ketamine's NMDA blockade at inhibitory interneurons, imbalances between 3-HK and 5-HTP could usurp the protective benefits of ketamine at the neuronal level, helping to explain variation in response seen in treatment trials. Enhanced understanding of the clinical impact of balance (or imbalance) in the ratio of $3-\mathrm{HK} / 5-\mathrm{HTP}$ is essential in maximizing the clinical effectiveness of current/novel promising treatment strategies and in developing more effective alternate treatment strategies in debilitating depressive syndromes. This is particularly relevant in individuals suffering with debilitating bipolar depression. Here we investigate relationships between dysregulated serotoninkynurenine metabolism and impulsive, suicidal behavior in bipolar patients with varying degrees of neurotic coping styles in an attempt to pave the way to incorporating concepts of 3-HK/5-HTP imbalance into future studies of depressive syndromes.

Methods: We studied 60 individuals from the Prechter Bipolar Longitudinal Study of the University of Michigan Comprehensive Depression Center, selected based on willingness to provide plasma samples within a specified timeframe. Study subjects included forty volunteers with a diagnosis of Bipolar Disorder Type I (BP-I) (20 with high neuroticism and 20 with low neuroticism). High or low neuroticism was determined using a median split from the Neuroticism Factor of the NEO-PI amongst the BP-I group. Twenty healthy controls with Neuroticism Factor score falling within the lower median were included in the study. Amongst the 3 groups, study subjects were age matched with similarly proportioned sex distribution. Plasma samples obtained at time of study entry were stored at $-80 \mathrm{C}$ and subsequently used for metabolomics assessment (University of Michigan Metabolomics Lab) from which 3-HK and 5HTP concentrations were selected for analyses. Quantifying 3-HK from plasma samples is justified as a reasonable reflection of brain 3-HK owing to its ready transport across the blood brain barrier by LAT1. 
Results: 3-HK/5-HTP did not differ between the BP-I group and the healthy control group $(p>0.05)$. However, when looking at the clinical phenotypic level rather than diagnostic differentiation, volunteers with a prior suicide attempt had higher $3-\mathrm{HK} / 5$-HTP $(\mathrm{U} 39=2.5, p=0.011)$ and higher Neuroticism $(\mathrm{U} 39=2.6, p=0.007)$ than those without a prior suicide attempt, healthy controls included. Furthermore, individuals with High Neuroticism had higher 3-HK/ 5-HTP as compared to those with Low Neuroticism (U37 = 2.0, $p=0.043)$. 3-HK/5-HTP correlated with Neuroticism (rho $=0.33, p=0.043$ ) and measures of impulsivity including both the BIS-11 Total Score (rho $=0.46, p=$ 0.018 ) and individual BIS-11 component scores (Attentional: rho $=0.34, p=0.077$, marginally significant; Motor: rho $=$ $0.50, p=0.008$; Non Planning: rho $=0.36, p=0.052$ ). Within the BP-I group, those with a prior suicide attempt had higher $3-\mathrm{HK} / 5-\mathrm{HTP}(\mathrm{U} 27=2.2, p=0.025)$ but no difference in Neuroticism ( $p>0.05)$. Furthermore, 3-HK/5HTP correlated with BIS-11 scores including Total (rho= $0.57, p=0.017$ ), Motor Impulsiveness ( $r h o=0.63, p=0.005$ ), and Non-Planning Impulsiveness (rho $=0.38, p=0.089$; marginally significant), but not Attentional Impulsiveness $(p>0.05)$.

Conclusions: We identified positive relationships between 3HK/5-HTP and both impulsivity and history of suicide attempt(s) within BD-I volunteers, specifically those with highly Neurotic coping styles. These results suggest that combined regulation of both serotonergic and kynurenine pathways may be critical to modulating impulsive and suicidal behavior in BD-I, a severe psychiatric illness associated with substantial morbidity and mortality worldwide. Including 3-HK/5-HTP ratios as measures of treatment response in future candidate drug trials of ketamine may prove useful in determining optimal dose strategies, but more investigation is needed to validate our findings.

Keywords: NMDA, Bipolar Disorder, Kynurenine, Neuroticism.

Disclosure: Nothing to disclose.

\section{W58. Longer-Term Lurasidone Efficacy and Tolerability in an American Bipolar Disorder Specialty Clinic}

Terence Ketter*, Dennis Do, Anda Gershon, Alex Holsinger, Farnaz Hooshmand, Po Wang, Shefali Miller

Stanford University School of Medicine, Stanford, California, United States

Background: Lurasidone has been approved by the United States Food and Drug Administration as monotherapy and adjunctive therapy (added to lithium or valproate) for bipolar I depression. The longer-term efficacy and tolerability of lurasidone in more diverse bipolar disorder (BD) patients already taking multiple psychotropic medications remains to be established.

Methods: We naturalistically administered open lurasidone for up to 5.4 years to Stanford University Bipolar Disorder Clinic outpatients assessed with the Systematic Treatment Enhancement Program for BD (STEP-BD) Affective Disorders Evaluation, and monitored longitudinally with the STEP-BD Clinical Monitoring Form.
Results: 57 patients (30 BD I, 24 BD II, 3 BD NOS, mean \pm $\mathrm{SD}$ age $44.5 \pm 14.1$ years, $61.4 \%$ female) took lurasidone, combined with on average $3.1 \pm 1.4$ (in $74.6 \%$ added to at least 2 psychotropics, in only $3.5 \%$ as monotherapy) other psychotropic medications, started most often during syndromal $(56.1 \%)$ or subsyndromal $(21.1 \%)$ depression, but also during euthymia (22.8\%). Lurasidone was taken for $275 \pm 374$ (median 126) days, with a final dose of $56 \pm$ $33 \mathrm{mg} /$ day. Lurasidone tended to primarily relieve depressive symptoms and maintain euthymia. By final visit taking lurasidone, the syndromal depression rate decreased by under half to $33.3 \%$, the euthymia rate increased by over half to $38.6 \%$, whereas the subsyndromal depression rate was marginally increased unchanged at $24.6 \%$. In $15.8 \%$ of trials, lurasidone was continued on average $181 \pm 53$ days with no subsequent psychotropic added. In $7.0 \%$ of trials lurasidone was continued on average $200 \pm 76$ days, but had subsequent psychotropic added. Lurasidone was discontinued in $77.2 \%$ of trials, after on average $175 \pm 247$ (median 100.5) days, due to adverse effects in $47.4 \%$, most often due to central nervous (including akathisia in $10.5 \%$, sedation/somnolence in $8.8 \%$, and hypomania in $3.5 \%$ ) or gastrointestinal/metabolic (including nausea in $7.0 \%$ and weight gain in $5.3 \%$ ) problems, due to inefficacy in $19.3 \%$, and due to other reasons in $33.3 \%$. At least $7 \%$ weight gain was seen in $12.3 \%$ of patients. Aside from akathisia, sedation/somnolence, nausea, and weight gain, lurasidone was well tolerated.

Conclusions: In American BD specialty clinic outpatients, primarily (96.5\%) adjunctive longer-term lurasidone commonly yielded relief of syndromal depression and maintained euthymia, suggesting efficacy. However, approximately $47 \%$ discontinued lurasidone due to adverse effects, suggesting tolerability limitations in these challenging patients, three-quarters of whom were already taking at least two other psychotropic medications.

Keywords: Lurasidone, Bipolar Disorder, Efficacy and Tolerability.

Disclosure: Sunovion Pharmaceuticals: Research Grant, Advisory, Lecture Honoraria, Self; Merck \& Co., Inc.: Research Grant, Self; Acadia Pharmaceuticals: Advisory, Self; Allergan Pharmaceuticals: Advisory, Self; Navigen: Advisory, Self; American Psychiatric Publishing, Inc.: Royalties, Self; Janssen Pharmaceuticals: Employee, Stockholder, Spouse.

\section{W59. Intravenous Ketamine Treatment for Adolescents With Treatment-Resistant Depression: Clinical Effects and Impacts on Neural Structure}

Kathryn Cullen*, Bonnie Klimes-Dougan, Kelvin Lim, Patricia Carstedt, Lynn Eberly, Mark Roback, Sophia Albott, Palistha Amatya, Bryon Mueller

University of Minnesota Medical School, Minneapolis, Minnesota, United States

Background: Depression is a potentially severe disorder that often begins during adolescence. Standard treatments such as selective serotonin reuptake inhibitor medications and cognitive behavioral therapy are only effective in approximately two thirds of patients. About ten percent of adolescents with depression do not improve despite multiple 
treatment trials. Advancement in neuroscience-based treatment for depression would have a profound impact on public health by preventing negative outcomes and restoring healthy developmental trajectories. Ketamine is a glutamatergic agent that has shown promise in adults with treatment-resistant depression (TRD) but has not yet been tested in adolescents. Animal studies have shown that ketamine can lead to changes in neuronal architecture in both gray and white matter, which is one putative mechanism of its anti-depressive action. Although there have been limitations assessing white matter changes in humans, recent advances in diffusion imaging using high-resolution acquisition and multi-shell approaches may have promise as a sensitive measure of medication-induced microstructural changes that could reflect neural mechanisms.

Methods: In the current open-label pilot study, we tested the efficacy and mechanisms of ketamine as a treatment for TRD in adolescents. The study treatment consisted of a series of 6 intravenous ketamine infusions $(0.5 \mathrm{mg} / \mathrm{kg})$ over the course of two weeks. We measured depression severity using the Child Depression Rating Scale-Revised (CDRS-R) before the first and 24 hours after the last infusion. In addition, participants completed the Beck Depression Inventory (BDI) at baseline, endpoint, and before and after each infusion. We collected multi-shell diffusion imaging magnetic resonance imaging (dMRI) imaging before and after the set of infusions on a Siemens Prisma scanner with the following parameters: $1.5 \mathrm{~mm} 3$ voxel, 30 direction @ $\mathrm{b}=1000,1500,2000$, and $2500 \mathrm{~s} / \mathrm{mm} 2$ and $17 \mathrm{~b}=0 \mathrm{~s} / \mathrm{mm} 2$, multiband factor $=3$, both posterior-to-anterior and anterior-to-posterior phase encode acquisition. We applied the Neurite Orientation Dispersion and Density Imaging (NODDI) model to measure neurite density. We examined change in depression symptoms and in neurite density using paired t-tests implemented in SPSS version 22.

Results: To date, 8 (of planned $N=10$ ) adolescents have tolerated the ketamine infusions well with no serious side effects (the 8th participant is nearing completion of the protocol and not included in these analyses). Overall, there was a reduction in symptoms as measured by the CDRS-R $(p=0.047)$. Although BDI scores showed substantial reductions after each infusion (greatest mean drop in BDI was 10.5 points at first infusion), BDI scores tended to increase by the next assessment; overall drop from baseline to endpoint was $6.25(p=0.09)$. Neuroimaging data for one patient was excluded due to excessive motion. Preliminary (uncorrected) dMRI results suggested that ketamine treatment may lead to decreased neurite density in the left cingulate body.

Conclusions: Findings from this pilot study provide tentative evidence that serial ketamine infusions may be a useful short-term intervention for some adolescents with TRD. Placebo-controlled research is needed to confirm the clinical effects, and to explore whether long-term treatment may be tolerable and associated with prevention of relapse. Preliminary evidence suggests that ketamine treatment may impact white matter microstructure including neurite density, which could represent a mechanism of antidepressant action; however, a larger sample size is needed to confirm this preliminary finding.

Keywords: Adolescent Depression, Ketamine, White Matter. Disclosure: Nothing to disclose.
W60. C118335 (GR Modulator/MR Antagonist) and Imipramine Modulate Neuroendocrine Stress Responses Through Different Central Mechanisms in Males and Females

Matia Solomon*, Elizabeth Nguyen, Joshua Streicher, Valentina Ghisays, Jody Caldwell, Sarah Berman, Christina Estrada

\section{University of Cincinnati, Cincinnati, Ohio, United States}

Background: Aberrant glucocorticoid secretion is implicated in the pathophysiology of stress-related disorders (i.e., depression and anxiety). Glucocorticoids exert biological effects via mineralocorticoid (MR) and glucocorticoid (GR) receptors. Because of the dynamic interplay between $M R$ and GR on HPA axis regulation and emotionality, compounds targeting both receptors are of interest for stress-related pathology. Notably, women are more likely to suffer from depression and anxiety, perhaps due to sex differences in MR and GR function. Accordingly, genetic mouse models targeting forebrain GR and MR reveal sex differences in HPA axis function and behavior.

Methods: Here, we investigated the effects of C118335 (dual selective GR modulator/ MR antagonist) on neuroendocrine, behavioral and central (c-Fos) stress responses in male and female rats. Rats were treated for five days with C118335, imipramine (positive control), or vehicle and exposed to restraint and forced swim stress.

Results: C118335 dampened corticosterone responses to both stressors in males and females, without a concomitant antidepressant-like effect in the FST. Imipramine decreased corticosterone responses to restraint stress, but this antidepressant-like effect was independent of glucocorticoids in females and males. Despite similar neuroendocrine and behavioral phenotypes, C118335 differentially modulated c-Fos activity in males (increased infralimbic, decreased CA1) and females (increased paraventricular nucleus of hypothalamus). Imipramine decreased c-Fos expression in the basolateral amygdala, CA1, and CA3 in both sexes, but increased central amygdala c-Fos expression in females.

Conclusions: These data indicate sex differences in the efficacy of these compounds to modulate neuronal activity and suggest differential recruitment of neural circuits during stress exposure in males and females.

Keywords: Acute and Chronic Stress, Sex Differences, Antidepressants, HPA Axis, Glucocorticoid Receptor.

Disclosure: Corcept Therapeutics, Financial Support, Self.

\section{W61. Structural and Behavioral Correlates of Social Behavior in Depression-Like Phenotypes}

Carla Nasca*, Benedetta Bigio, Timothy Lau, Danielle Zelli, Bruce McEwen

\section{Rockefeller University, New York, New York, United States}

Background: Depression is a disabling disease affecting 1 out 6 people with an occurrence by sex that is twice as high in women as in men. Affected people tend to withdraw from normal life activities and social interactions among a variety of other symptoms. Interestingly, impairments in social 
interactions are more common in men with severe depression than in women. Yet, about half of affected people do not respond to current antidepressants, highlighting that there is an urgent need for novel medications. In a recent report, we discovered a previously unknown role of the medial amygdala (MeA) in social withdrawal in depression-like illness and the ability of a putative rapidly-acting antidepressant, acetyl-L-carnitine (LAC), to remediate retraction of dendrites of MeA stellate neurons and ameliorate dysfunctional social behavior that results from exposure to chronic restraint stress in male mice. As to whether atrophy of MeA stellate neurons may play a role in resistance to antidepressant treatment is unknown as well as it is unknown whether females experience the same MeA atrophy as males do. Toward such understanding, the heterozygous brain-derived neurotrophic factor (BDNF) Val66Met knock-in mouse that encodes the human BDNF variant represents one of the best animal models with depressive-like traits that are not rescued by common antidepressants.

Methods: Heterozygous male and female BDNF Val66Met mice (hets) were in-house bred and used for behavioral assessment (social avoidance and forced swim tests) of LAC efficacy on week 8 after screening for inherent susceptibility performed as previously described at the light dark test on week 7. LAC was orally administered for 3 days by dissolving the drug in the drinking water at a concentration of $0.3 \%$. Fresh brains were extracted and processed for morphological analysis following the FD Rapid GolgiStain protocol with few changes. When extracted, brains were coded for quantitative analysis by two blind observers. Both hemispheres were used to obtain $100-\mu \mathrm{m}$ sections. We used the parahippocampal formation, the optic tract and the endorhinal sulcus as anatomical references to localize the amygdala complex. To be selected for tracing, we established several criteria for Golgi-impregnated neurons: (1) have neuronal cell bodies located within the boundaries of the MeA, (2) no truncated dendrites are present on the neuron, (3) consistent staining along the entire length of the dendrites, (4) neurons had to be relatively isolated from other neurons to avoid interference during tracing with other dendrites.

Results: Susceptible heterozygous BDNF male hets, identified at the light-dark screening as the subset with high lightavoidance, display dysfunctional social interactions, a MeAdependent task, in the social avoidance test, along with impairments in hippocampal dependent tasks, such as reduced copying style (i.e.: high immobility time) in the forced swim test as compared to wild-type male mice. Antidepressant treatment with LAC, orally administered for 3 days, rapidly corrects hippocampal and MeA-dependent behavioral abnormalities in male BDNF hets with behavioral outcomes at the levels of wild-type male mice. We also find that LAC corrects retraction and decreased branching of dendrites of $\mathrm{MeA}$ stellate neurons in line with previous findings showing rapid behavioral and structural effects of LAC after exposure to chronic restraint stress. We find that female hets do not show retracted MeA stellate dendrites nor are bipolar dendrites in MeA affected in female hets, compared to WT females. In spite of such structural sexdifference in MeA dendritic tree, both sexes show behavioral impairments in hippocampal-dependent tasks that are corrected by LAC. Indeed, LAC orally administered for 3 days to both susceptible heterozygous BDNF male and female hets corrects depressive-like behaviors (immobility time) at the forced swim test. Ongoing studies are characterizing female hets for MeA-dependent tasks and both sexes for the gene expression profiles underlying MeA structural impairments. Conclusions: Our findings identify MeA stellate neurons as an important component in the responsiveness to nextgeneration antidepressant treatments, such as LAC, and show that LAC can rapidly promote antidepressant responses in both sexes. This may be useful as a model for translation to humans.

Keywords: Amygdala, Social Behavior, Depression, Sex Differences, Medial Amygdala.

Disclosure: Nothing to disclose.

\section{W62. Energy Regulation in Ventral Hippocampus and Metabolic States in Depressive-Like Phenotypes}

Benedetta Bigio*, Danielle Zelli, Aleksander Mathe, Vasco Sousa, Per Svenningsson, Bruce McEwen, Carla Nasca

\section{Rockefeller University, New York, New York,} United States

Background: Although the brain accounts for only $2 \%$ of body weight, it makes use of a large amount of the energy capacity of the body. Hence energy regulation in the CNS is crucial for a proper homeostasis in response to an allostatic overload, examples of which are depression and insulin resistance. It has also been proposed that energy alteration in the CNS might contribute to the dysfunction of signaling cascades observed in mood disorders, including depression, and to antidepressant actions. We recently found that agents that influence energy metabolism, such as the epigenetic molecule acetyl-L-carnitine (LAC), an activator of mitochondrial functions, exert rapid antidepressant-like effects. We found that LAC corrects features of depressive-like behaviors in male Flinders Sensitive Line (FSL) rats while correcting an insulin resistant-like state with systemic hyperglycemia and hyperinsulinemia and leading to changed gene expression profiles in a subregion of the ventral hippocampus, the ventral dentate gyrus (vDG) as compared to vehicle treated FSL rats. Interestingly, metabolic factors, including the insulin receptor (InsR) and glucose transporters (glut-4/12), in the vDG were identified as key players in predisposition to depression and LAC responsiveness in FSL rats, suggesting that energy regulation in the vDG may play a key role in treatment resistance in depression - a major health problem in humans. One of the better-known mouse models that displays resistance to conventional antidepressants is the BDNF loss of function humanized Val66Met mice.

Methods: We implemented a micro-dissection approach to screen global gene expression, using RNAseq, in the vDG of adult male hets that received oral administration of LAC or regular water. Screening for inherent susceptibility was performed as previously described using a modified version of the light-dark test. Behavioral assessments were carried out using the social interaction (SI) and forced swim (FS) tests after 3 days of treatment. Antidepressant treatment with LAC was administered orally at a concentration of $0.3 \%$. Briefly, RNAseq was performed using Illumina HiSeq2500 with three biological replicates for each condition. Statistical 
analyses for differentially expressed gene (DE) were performed with EdgeR; genes with more that 1 count per million $(\mathrm{cpm})$ in at least three samples were retained in the EdgeR analysis to control for low expressed genes. The following cut-off were used: FDR $<15 \%$ and FC $>1.3$. Heatmaps were generated with an in-house made script. Statistical overrepresentation for GOs was generated with Panther. Routinely blood assays for triglycerides, insulin and glucose were performed following the manufacture instructions after assessment of the specific dilution factors.

Results: We found that LAC exerts rapid antidepressant-like effects in male hets, which, we also find are resistant to common antidepressants in line with previous reports. Indeed, 3 days of LAC, and not 3 days of fluoxetine, oral administration, improved social interaction in male hets at the social avoidance test and improved copying style (e.g.: immobility time) at the forced swim test. While improving depressive-like traits, LAC reduced blood levels of triglycerides, glucose and insulin in male hets as compared to male hets that received water only, reinforcing a link between energy regulation and mood disorders. Centrally, male hets show reduced transcript and protein levels of the regulator of glutamate release mGlu2 (metabotropic glutamate receptor-2), a previously identified target for rapid antidepressant action, in the vDG that are corrected by LAC oral administration. The rapid action of LAC via central regulation of glutamate overflow is in line with previous findings showing a rapid action of ketamine, which also normalize glutamate hyperactivity. To further explore the contribution of energy-related pathways in vDG in treatment resistance in male hets we employed an RNA-seq analysis to identify the genes that are altered in hets and rapidly corrected by LAC. Ongoing pathway and GO analyses in treatment-resistant hets before and after LAC antidepressant treatment suggest changes in genes involved in glutamate signaling, NPY pathways and many energy-regulatory genes, paving the way for a better understanding of the role of central energy regulation in the vDG and metabolic states in depressive mood disorders.

Conclusions: These findings suggest that brain energy regulation in the vDG is an important factor to be considered for the development of better therapeutics and suggest that agents like LAC, by reducing glutamate overflow, could rapidly ameliorate depressive disorders and could also be considered for treatment of insulin-resistance in depressed subjects.

Keywords: Energy Homeostasis, Metabolic State, Fast-Acting Antidepressant, Dentate Gyrus, Insulin Resistance.

Disclosure: Nothing to disclose.

\section{W63. The National Pregnancy Registry for Atypical Antipsychotics: Effects of First Trimester Exposure to Aripiprazole and Quetiapine on Risk for Major Malformations}

Lee Cohen*, Adele Viguera, Marlene Freeman, Alexandra Sosinsky, Gina Savella, David Chitayat, Sonia Hernández-Díaz

Harvard Medical School, Boston, Massachusetts, United States

Background: Despite the widespread use of atypical antipsychotics in women of childbearing potential, reproductive safety data across these medicines is sparse. The National Pregnancy Registry for Atypical Antipsychotics (NPRAA) at Massachusetts General Hospital was established in 2008 to address this knowledge gap.

Methods: Eligible enrollees include pregnant women between 18 and 45 years of age. The exposed group is comprised of women who have taken one or more atypical antipsychotics during pregnancy; the comparison group is comprised of women who have not taken this class of medication during pregnancy. Three phone interviews are conducted: 1) baseline, proximate to the time of enrollment, 2) 7 months gestation, and 3) 3 months postpartum. Obstetric, labor and delivery, and pediatric medical records are obtained. Following receipt of medical records, relevant information is abstracted regarding primary and secondary outcomes including obstetrical, maternal, and neonatal outcomes. Potential major malformations are identified and relevant records are sent to a dysmorphologist blinded to drug exposure for adjudication.

Results: As of August 1 2016, 493 women in the exposed group and 262 women in the comparison group were enrolled $(N=755)$. The overall drop-out and lost to followup rate of subjects was $12 \%$. The proportion of study subjects for whom medical records were obtained was $86 \%$. A total of 489 women completed the study and were eligible for inclusion in the current analysis. Of 312 live births with first trimester exposure to atypical antipsychotics, four major malformations were confirmed. Of 138 lives births with first trimester exposure to quetiapine (Seroquel), one major malformation was confirmed. Of 96 live births with first trimester exposure to aripiprazole (Abilify), three major malformations were confirmed. Of the 177 control group live births, one major malformation was confirmed. The absolute risk of major malformations was $1.3 \%$ for infants exposed to any atypical antipsychotic during the first trimester and $0.6 \%$ for unexposed infants. The odds ratio for major malformations was $2.27(0.26,20.15)$ comparing exposed to unexposed infants, not reaching statistical significance.

Conclusions: The NPRAA offers a systematic way to collect prospective reproductive safety information which informs the care of women who may use atypical antipsychotics to sustain psychiatric well-being. This preliminary analysis indicates that these agents are not major teratogens but further information is needed to better estimate risk. The importance of pregnancy registries is underscored by recent FDA guidance (http://www.fda.gov/Drugs/Develop mentApprovalProcess/ Development Resources/ Labeling/ ucm093307.htm).

Keywords: Women's Mental Health, Pregnancy, Atypical Antipsychotics, Pharmacovigilance.

Disclosure: Alkermes: Research Support, Self; AstraZeneca: Research Support, Self; Bristol-Myers Squibb/Otsuka: Research, Self; Forest/Actavis: Research Support, Self; Ortho-McNeil Janssen: Research Support, Self; Sunovion Pharmaceuticals, Inc.: Research Support, Self; Cephalon, Inc.: Research Support, Self; National Institute on Aging: Research Support, Self; National Institutes of Health: Research Support, Self; National Institute of Mental Health: Research Support, Self; Takeda/Lundbeck: Research Support, Self; Noven Pharmaceuticals: Advisory/Consulting, Self; JDS Therapeutics LLC: Advisory/Consulting, Self. 
W64. Abnormal Brain Activation in the Right Ventrolateral Prefrontal-Amygdala Emotional Network to a Task of Attention and Emotion Regulation as a Marker of Prodromal Mood Disorder in Youth Bipolar Offspring

Fabiano Nery*, Wade Weber, Thomas Blom, Caleb Adler, Stephen Strakowski, Melissa DelBello

University of Cincinnati, Cincinnati, Ohio, United States

Background: The onset of bipolar disorder is typically during adolescence. Possible prodromal manifestations of bipolar disorder include depression, anxiety disorders, subthreshold manic/hypomanic symptoms, and affective lability. We used functional magnetic resonance (fMRI) to investigate brain activation in response to a task of attention and emotion regulation associated with familial risk to bipolar disorder and with progression to a mood disorder in youth offspring of bipolar I disorder parents.

Methods: 3D T1-weighted magnetic resonance images were obtained from 117 offspring of DSM-IV bipolar disorder type I patients (bipolar offspring; mean age \pm SD: $13.6 \pm 2.7$; females: $54.7 \%$ ) and 59 group-matched healthy offspring of healthy parents (healthy offspring; mean age \pm SD: $14.2 \pm 3.0$, females: $52.5 \%$ ). BD offspring were free of major mood or psychotic disorders. We used fMRI to study brain regional activation while subjects performed a continuous performance task with emotional and neutral distracters. Eventrelated region-of-interest analyses were performed to test the primary hypothesis.

Results: Bipolar offspring presented reduced activation to emotional distracters in the right ventrolateral prefrontal cortex $(p=0.04)$ and right anterior cingulate cortex $(p=0.03)$. There was reduced non-significant activation across additional brain areas, including left ventrolateral prefrontal cortex, left anterior cingulate cortex, bilateral striatum, and bilateral thalamus. There were no effects of group on activation to targets or neutral distracters. Secondary analysis comparing a subgroup of bipolar offspring who converted to a major mood episode after a period of follow up (converters, $n=17$ ) showed that at baseline, converters presented non-significant increased activation to emotional distracters across ventrolateral prefrontal cortex, anterior cingulate cortex, striatum, and thalamus. A 3-group comparison showed significant differences in activation to emotional distracters in right ventrolateral prefrontal cortex $(p=0.03)$, right anterior cingulate cortex $(p=0.05)$, right caudate $(p=0.04)$, and right putamen $(p=0.05)$. These statistical differences were likely driven by differences between non-converters and healthy offspring. At baseline, there were significant positive correlations between depressive symptom scores and activation to emotional distracters in the bilateral amygdala in the converters and significant negative correlations in the right ventrolateral prefrontal cortex in the non-converters.

Conclusions: Our results are consistent with a decreased "top-down" control of prefrontal areas over limbic areas in response to emotional stimuli in bipolar disorder. The reduced activation in right ventrolateral prefrontal cortex and right anterior cingulate cortex might be a marker of risk for developing mood disorders in youth at increased familial risk for bipolar disorder. We found preliminary evidence for a distinct pattern of regional brain activation at baseline for those bipolar offspring who developed their first mood episode during follow up. This increased activation might be an effect of prodromal disease, but it needs to be confirmed by a prospective examination.

Keywords: Bipolar Disorder, Functional MRI (fMRI), Atrisk, Attention, Emotion Regulation.

Disclosure: Eli Lilly \& Co: Employment, Spouse.

\section{W65. Systemic Inflammation in Depression With Increased and Decreased Appetite}

W. Kyle Simmons*, Kaiping Burrows, Jason Avery, Kent Teague, Wayne Drevets

Laureate Institute for Brain Research, Tulsa, Oklahoma, United States

Background: Although changes in appetite and weight are codified as diagnostic markers of major depressive disorder (MDD) in the DSM-V, their clinical presentation varies greatly among patients: some eat more when they become depressed, while others eat less. Two recent findings in the literature suggest that increased- and decreased-appetite depression phenotypes may be associated with different pathophysiological processes. First, latent class analyses of 16 depressive symptoms across multiple diagnostic inventories in the Netherlands Study of Depression and Anxiety (NESDA) dataset has repeatedly demonstrated that a severe atypical subtype of depression accompanied by increased appetite is associated with heightened systemic inflammation as measured by IL-6, CRP, and TNF-alpha (e.g., Lamers et al., 2016, Transl. Psychiatry; Lamers et al., 2013, Molec. Psychiatry). Second, using functional Magnetic Resonance Imaging (fMRI), our lab recently demonstrated differences in the neural response to food cues within reward and interoceptive neurocircuitry in two groups of depressed adults classified solely on their depression-related appetite changes (Simmons, et al., 2016). This finding is particularly interesting as systemic inflammation modulates the activity of both the reward system and the interoceptive regions of the insula identified in our study (e.g., Harrison, et al., 2015, Biological Psychiatry). It remains unclear, however, if evidence of systemic inflammation can be observed in groups of MDD subjects classified by their depression-related appetite changes alone, as was done in our fMRI study.

Methods: We recruited 18 unmedicated depressed adults reporting increased appetite and weight gain (MDD-increase), and 22 unmedicated depressed adults reporting decreased appetite and weight loss (MDD-decrease) within their current depressive episode. Additionally, 33 healthy non-depressed comparison subjects $(\mathrm{HC})$ were recruited. The depressed groups did not differ in age, body mass index, depression severity, anxiety severity, state or trait anxiety, or anhedonia. Using enzyme-linked immunosorbent assays (ELISA), we assayed blood plasma from the subjects to assess group differences in C-reactive protein (CRP), interleukin-1 receptor antagonist (IL-1RA), interleukin-6 (IL-6), and interleukin-10 (IL-10). All $p$-values reported below were corrected for multiple comparisons using Tukey's range test adjustment.

Results: The MDD-increase subjects exhibited significantly higher CRP than either the MDD-decrease $(p=0.003)$ or 
HC participants $(p=0.006)$. CRP levels did not differ, however, between MDD-decrease and HC subjects $(p=0.78)$. Similar group differences were observed for IL-1RA, with the MDD-increase subjects again exhibiting significantly higher IL-1RA than either the MDD-decrease $(p<0.001)$ or HC participants $(p<0.001)$. Unlike the CRP results however, MDD-decrease subjects also exhibited higher IL-1RA levels than HC participants $(p=0.04)$. The MDD-increase subjects had higher IL-10 compared to the HC participants $(p=0.04)$ but not compared to the MDD-decrease subjects $(p=0.31)$. Finally, there was a trend toward a group difference in IL-6, with MDD-increase subjects exhibiting marginally higher IL-6 than the HC participants $(p=0.095)$ but not the MDD-decrease subjects $(p=0.94)$.

Conclusions: Compared to the healthy controls and depressed subjects reporting decreased appetite, depressed subjects with increased appetite exhibited significantly greater systemic inflammation, as indexed by CRP, IL-1RA, and IL-10, with a trend toward higher IL-6 levels. Although there is a growing body of evidence that depression is associated with inflammation (e.g., see Miller \& Raison, 2015, Nature Reviews Immunology), the present findings lend support to the conclusion that systemic inflammation may be more strongly associated with some depressive phenotypes than others. Future research should assess whether depression-related appetite increases can be used as a clinical hallmark of depressed patients with elevated inflammation.

Keywords: Depression, Appetite, Inflammatory Markers. Disclosure: Nothing to disclose.

\section{W66. Seeking the Genetic Etiology of Domestic Behavior in House Cats and Tame Foxes as a Window Into Personality Disorders}

Carlos Driscoll*, Stephen Lindell, David Roberson, Joe Boland, Clay Stephens, Anna Kukekova, Meredith Yeager, Christina Barr

National Institute on Alcohol Abuse and Alcoholism, Rockville, Maryland, United States

Background: Personality and temperament are important predisposing factors for multiple psychopathologies. However, identifying and mapping the genes underling particular behaviors is difficult, in part due to a lack of experimentally appropriate models. We hypothesize that using the domestication of previously wild animals as a working analogy of personality may provide a way forward.

Domestication is a suite of heritable traits affecting behavior; domestic animals are those having undergone a process of selection relating to behavior and cognition, resulting in changes in allele frequencies. The systems that permitted initial domestication range from those involving fear and impulse control to those involving reward and sociality. Genetic factors that permitted - and were enriched - during domestication may overlap with those driving human temperament traits and risk for the psychiatric and alcohol use disorders.

Methods: We use three approaches to identify and map candidate genes underpinning domestic behavior in the house cat (Felis silvestris catus) and the domesticated red fox (Vulpes vulpes); first, an exome-based interrogation of tame-selected vs. aggression-selected farmed foxes; second, a WGS wildcat/domestic cat association study; and third, a WGS pedigree-based study using Asian leopard cat X domestic cat hybrids.

Exome sequence haplotypes from 12 tame-selected, 12 aggression-selected, 12 unselected but farm-raised, and 12 local Maryland, foxes were correlated with phenotype. Alleles having a behavioral effect are expected to have been enriched via selection, and foxes sharing such alleles will also share long haplotypes including these alleles, whether or not the alleles themselves were genotyped during the sequencing procedure.

Next, domestic cats $(N=5)$ are compared to their wild relatives $(N=31)$ through $10-15 \mathrm{X}$ WGS. Each group is interrogated for regions of homology segregating to behavioral phenotype.

Last, 20 hybrid offspring from domestic cat-Asian leopard cat (Prionailurus bengalensis) crosses, varying wildly in temperament, and their pure-bred ancestors, were WGS at 20-40X, mapped to the UCSC domestic cat reference sequence, and interrogated for regions of homology segregating to behavioral phenotype.

Results: Focusing on candidate genes relating to risk for alcohol use disorders (1536 marker Addictions Array, LNG). Here we identified functional/non-synonymous SNPs, some of which were predicted to be "damaging" or "potentially damaging" by in silico analysis (PolyPhen). For verification, a subset of nsSNPS in candidate genes of interest to psychiatric disorders were verified by Sanger sequencing in 2 additional domestic cats, 2 Asian leopard cats, and 10 first and second generation hybrids.

Conclusions: Animals that have been artificially selected for tameness/anxiolysis/food motivation/social cognition/aggression (i.e. domesticates) may be good models for how genetic variation contributes to human psychopathologies. We identified damaging nsSNPs in genes relevant to behavioral, psychiatric and neurodevelopmental disorders. Among them were those affecting anxious responding, reward, feeding, neuronal development, transcription control, and circadian rhythmicity, and in some instances, human genetic variation at these genes has been associated with psychiatric disorders and the addictions. Our results suggest polymorphism at these genes may contributing to individual differences in personality in humans and supports a role for targeting these systems to attenuate psychopathologies in select subjects.

Keywords: Personality, Domestication, Mood, Cat, Fox. Disclosure: Nothing to disclose.

\section{W67. A Brain-Gut-Derived Metabolite Association Network for Depressed Mood}

Jennifer Labus*, Arpana Gupta, Bruce Naliboff, Kirsten Tillisch, Emeran Mayer

University of California, Los Angeles, California, United States

Background: Gut microbiota can impact brain function and brain chemistry related to emotional and nociceptive behaviors, can directly affect the immune system, and can generate various neuroactive metabolites and neuro- 
transmitters (1-2). In addition to effects during early development, the bi-directional gut-brain-microbiome communication may be an ongoing dynamic process (3-4). Microbiota can initiate signaling mechanisms that affect the striatum (caudate nucleus, putamen, nucleus accumbens), amygdala, and hippocampus; regions involved in motor control, memory, and emotions (5-6). Tryptophan depletion has been associated with negative mood possibly through altering serotonin levels and decreasing brain-derived neurotrophic factor in the brain (7). Gut microbiota are known to regulate circulating concentrations of tryptophan critical for serotonin synthesis in the central nervous system (8-9). The aim of this study is to test association between gut microbial derived tryptophan metabolites, depression and brain regions known to be affected by microbiota signaling. Methods: A global metabolomics and bioinformatics platform (Metabolon, 617 Davis Drive, Durham NC) was used to characterize altered biochemical pathways that are evident in stool of 23 healthy controls (14 females). This proprietary platform is optimized to detect and identify biochemical metabolites of many different chemical classes. Whole brain structural, resting-state, and diffusion tensor magnetic resonance imaging data was acquired using a 3.0 T MRI scanner. Network analysis via graph theory was applied to test the network centrality or influence of the caudate nucleus, putamen, nucleus accumbens, amygdala, and hippocampus in terms of information flow in large-scale brain functional (resting-state) and anatomical (diffusion tensor imaging) networks. Degree Strength is a weighted measure of connectivity that reflects a brain region's involvement in the networkIt is computed as the sum of connections at a given brain region, factoring in the "weight" of each connection. The high resolution structural image was parceled into 165 regions based on Destrieux and Harvard-Oxford atlases. The anatomical network was constructed by performing deterministic fiber tractography in native space using the TrackVis. The final data matrix contained the absolute fiber count divided by the individual volumes of the two interconnected regions were used to construct the anatomical network. Region to region functional connectivity analysis was performed in the CONN toolbox. The function network matrix was comprised of $\mathrm{z}$ transformed $\mathrm{r}$ scores thresholded at $z>$.3. Spearman's correlations were used to calculate the association between the concentration of tryptophan metabolites, degree strength of brain regions of interests and depression symptoms as measured by the Hospital Anxiety and Depression Scale. Results were considered significant at $p<.05$. Cytoscape v. 2.8.3 was used to visualize and construct a brain-gut-derived metabolite interaction network for depression.

Results: Patients ranged from ages 18 to 44 (mean age $=26$ ). Anxiety and Depression scores as expected were below clinical levels but had adequate range for the analysis (range 0 to $13(\mathrm{~m}=4.6, \mathrm{sd}=3.3)$ for anxiety and 0 to 8 for depression $(\mathrm{m}=2, \mathrm{sd}=2.0)$.

Figure 1 displays the brain-gut derived metabolite association network for depression. Several metabolites involved in tryptophan metabolism were associated with brain regions having key roles in depression. Concentrations of tryptophan were positively correlated with indolelactate, known for its role in the degradation of tryptophan $(r=.62, p=.002)$. In turn, indolelactate along with indoleacetate, a microbiota derived metabolite known to positively affect intestinal health and reduce inflammation (10), and C-glucosyl tryptophan, negatively associated with birthweight and positively associated with age-related clinical traits, (11) were correlated with the anatomical strength of the left nucleus accumbens $(\mathrm{r}=-.42, p=.047 ; \mathrm{r}=.58, p=.004$; $\mathrm{r}=-.45, p=.03$, respectively). Depression was positively correlated anatomical strength of this nucleus accumbens region $(\mathrm{r}=.60, p=.002)$ as well as the functional degree strength of the left hippocampus $(r=.42, p=.048)$ and the anatomical. The function strength of the left hippocampus was correlated with 5-hydroxyindoleacetate, an indicator of serotonin synthesis $10(\mathrm{r}=.61, p=.002)$ and 3-methylindole, produced by tryptophan in the gut $(r=-.55, p=.006)$.

Conclusions: Network analysis indicated that gut-derived metabolite concentrations are associated with the global connectivity of regions involved in depression. This is consistent with the hypothesis that gut-derived metabolites can have a negative influence via these brain regions on mood. This preliminary analysis provides tentative support for idea that communication between the gut microbiome and brain is a dynamic ongoing process. Data-driven discovery methods such as pathway analysis are useful for generating future hypothesis and may provide new insights into the brain-gut-microbiome axis.

Keywords: Gut Microbiome, Brain, Depression.

Disclosure: Supported by NIH grants: P30 DK041301, R01 DK048351, P50DK64539, R01 HD076756, R21 HD086737.

\section{W68. Norepinephrine Transporter Gene Variant and Remission Success of Venlafaxine for Depression in Older Adults}

Daniel Mueller*, Victoria Marshe, Malgorzata Maciukiewicz, Soham Rej, Arun Tiwari, Etienne Sibille, Daniel M. Blumberger, Jordan Karp, Eric Lenze, Charles Reynolds, Benoit Mulsant, James Kennedy

University of Toronto, Toronto, Canada

Background: Late-life depression (LLD) is a disabling mental disorder affecting more than two

million older adults in the US. Unfortunately, more than 50\% of patients with LLD do not achieve remission with an antidepressant trial. In older adults, prolonged depressive symptoms are associated with greater caregiver burden, medical complications, functional impairment, and cognitive decline. Therefore, finding predictive biomarkers for antidepressant treatment success in older adults is imperative to achieve timely remission.

The primary objective of this study was to investigate five putatively functional variants of the norepinephrine transporter (SLC6A2, NET) and serotonin transporter (SLC6A4, SERT) genes and remission in depressed older adults treated with venlafaxine. For our secondary objective, we analysed 17 other variants in serotonergic system genes (HTR1A, HTR2A, HTR1B, TPH1, TPH2), potentially involved in the mechanism of action of venlafaxine.

Methods: Our sample included participants from the NIH-funded multi-site clinical trial IRLGRey (Incomplete Response in Late Life Depression: Getting to Remission; NCT00892047) study. This sample included older adults 
( $\geq 60$ years, $N=350$ ) with a DSM-IV-defined major depressive episode and a score of $\geq 15$ on the Montgomery-Asberg Depression Rating Scale (MADRS). Participants received protocolized treatment with open-label venlafaxine, up to $300 \mathrm{mg} /$ day for approximately 12 weeks. To avoid confounding effects of potential comorbid neurological abnormalities (e.g. early dementia symptomatology), participants were screened and excluded based on dementia diagnosis using DSM-IV criteria, as well as, a Folstein Mini-Mental State Examination (MMSE) score of $<24$ Each individual was genotyped for 22 polymorphisms in seven genes which were tested for association venlafaxine remission (MADRS $<10$ ) and changes in MADRS score during treatment.

Results: Our final sample included 350 participants of European $(N=311,88.9 \%)$, African $(N=33,9.4 \%)$, Asian $(N=5,1.4 \%)$ and admixed $(N=1,0.3 \%)$ ancestries. The sample consisted predominantly of females $(N=223,63.7 \%)$ with a mean +/- SD age of $68.6+/-7.0$ years. At baseline, participants were typically moderately depressed (mean +/$\mathrm{SD}$ MADRS $=26.6+/-5.6)$, and most had recurrent depression $(N=255,72.9 \%)$. On average, participants spent $94.6+/-18.4$ days in the treatment protocol and received a venlafaxine dose of $241.6+/-70.3 \mathrm{mg}$ with 179 (51.1\%) remitting and a mean $+/$ - SD percentage MADRS score change from baseline of $-50.3+/-37.4 \%$. After adjusting for multiple comparisons, NET variant rs2242446 was significantly associated with remission success $(\mathrm{OR}=1.67,95 \%$ C.I. $[1.13,2.42], p=0.009)$. Individuals with the $\mathrm{rs} 2242446 \mathrm{C} / \mathrm{C}$ genotype were more likely to be remitters $(73.1 \%$ remitters $)$ than those with either the $\mathrm{C} / \mathrm{T}(51.8 \%)$ or $\mathrm{T} / \mathrm{T}$ genotype (47.3\%). In addition, individuals with the $\mathrm{C} / \mathrm{C}$ genotypes had a shorter time to remission than those with the $\mathrm{C} / \mathrm{T}$ or $\mathrm{T} / \mathrm{T}$ genotypes, and showed a greater percentage MADRS score change from baseline. Given that the majority of individuals with the $\mathrm{C} / \mathrm{C}$ genotype received at least $150 \mathrm{mg} /$ day at the end of treatment $(92.3 \%, N=24)$, there is reasonable evidence suggesting that the NE transporter was being sufficiently inhibited to produce clinicially meaningful effects. In order to understand the functional effects of rs2242446 on the expression of the NET gene across different brain regions, we conducted in silico gene expression analyses using data from the UK Brain Expression Consortium (BRAINEAC.org). Using BRAINEAC data, we found that rs2242446 genotypes differentially impact NET gene expression within the hippocampus $(p=0.037)$, the thalamus $(p=0.037)$ and temporal cortex $(p=0.048)$. In these regions, indiviuals with the $\mathrm{C} / \mathrm{C}$ geneotype show significantly lower expression of NET, than either those with the $\mathrm{C} / \mathrm{T}$ or $\mathrm{T} / \mathrm{T}$ genotype. For the serotonin transporter, we saw no significant associations of rs25531/5- HTTLPR and 5-HTTVNTR genotypes with our outcomes of interest in any of the ethnic samples $(p>0.05)$. Similarly, none of the other serotonergic system genes showed any consistent significant associations across multiple outcomes.

Conclusions: We provided further evidence for the involvement of the NET gene in predicting remission to venlafaxine treatment in LLD. NET variant rs2242446 (-T182C) was significantly associated with greater and earlier treatment success in our sample of geriatric patients treated with venlafaxine. Our findings serve as a step towards predictive pharmacogenetic testing to deliver personalized medicine. However, since response to venlafaxine is likely influenced by additional factors, our findings warrant further investigation of NET to assess the clinical utility for pharmacogenetic testing in older adults. In particular, it will be important to determine how our findings can be integrated into currently available genetic algorithms.

Keywords: Late-Life Depression, Antidepressant, Pharmacogenomics, Serotonin and Norepinephrine Reuptake Inhibitor, Precision Medicine for Depression.

Disclosure: Nothing to disclose.

\section{W69. Cognitive Neurostimulation of the Dopamine System}

Kathryn Dickerson*, Jeff MacInnes, Nan-kuei Chen, R. Alison Adcock

Duke University, Durham, North Carolina, United States

Background: Dopamine is critical for human motivation, learning, and memory. Prior work implicates the dopamine system in a variety of volitional behaviors including motivation, valuation, effort allocation, and action contingency. Nearly all prior research of the dopaminergic midbrain has used extrinsic cues to elicit transient responses in midbrain activation, yet humans motivate themselves daily in the absence of direct external reinforcers. If volition is a primary function of the dopamine system, we reasoned humans could learn to use internally-generated representations to produce sustained activation within its source, the midbrain. We refer to activation of the brain using only thoughts and imagery as 'cognitive neurostimulation.' Cognitive neurostimulation of the dopaminergic midbrain would have applications for a) enhancing motivation, learning, and memory in healthy humans and b) translation to clinical populations with aberrant dopamine functioning (e.g., depression). The purpose of this experiment was to investigate if healthy individuals can learn to produce sustained activation of the midbrain, specifically the ventral tegmental area (VTA). We hypothesized a) individuals can learn to produce sustained activation of the VTA and b) real-time fMRI (rt-fMRI) neurofeedback will facilitate learning to produce sustained VTA activation. Secondary questions examined the ability of healthy individuals to learn to produce sustained activation of a related structure, the nucleus accumbens (NAcc), and to compare this to VTA activation.

Methods: Ninety-seven healthy adults participated in the experiment, and were randomly assigned to one of four groups: VTA Feedback Group, Attentional Control Group, NAcc Feedback Group, or Noise False Feedback (FF) Group. All groups completed a Pre-Test, three Training runs, and a Post-Test. All participants were instructed to try to increase motivation using personally relevant thoughts and imagery during Activate trials and to count backwards during baseline Count trials. The main difference across groups was the information displayed on a thermometer during Training runs. For the VTA Feedback group, the thermometer reflected mean BOLD signal within the VTA, updated $\sim 1$ second. Similarly, for the NAcc Feedback group the thermometer reflected mean BOLD signal from the NAcc, updated 1 second. The FF group received noise, false feedback they were instructed was veridical; however, the thermometer values were random. The Control group viewed a repeating 
pattern on the thermometer unrelated to brain activity. All groups were debriefed at the end of the study.

Results: We hypothesized the VTA Feedback group would learn to self-activate VTA BOLD signal using personally identified motivational thoughts and imagery. During a baseline Pre-Test (no neurofeedback), the groups did not differ in mean VTA activation. As predicted, during training, the VTA Feedback group elicited and sustained greater VTA activation than the Control group. Interestingly there were no differences in VTA activation between the VTA and FF groups or the FF and Control groups. The critical test came in the Post-Test, where we again asked participants to increase motivation and VTA BOLD signal. The Post-Test did not contain feedback, novel, or reward cues. In the Post-Test, the VTA Feedback group enhanced and sustained VTA activity greater than baseline, the Pre-Test, and both control groups (Control, FF). Lastly, the VTA Feedback group demonstrated increased functional connectivity with mesolimbic regions, reflecting plasticity after training. A complementary set of analyses was performed for the NAcc data. There were no group differences in NAcc activation during the baseline Pre-Test. Unlike the VTA data, during training no group significantly activated the NAcc above baseline. Likewise, there was no enhanced or sustained NAcc activation in any group in the Post-Test. Furthermore, there were no significant connectivity changes following NAcc neurofeedback training. To probe the VTA and NAcc groups further, we compared the feedback signal they received on a variety of properties including the mean signal, number of trials with positive feedback (mean $>0$ ), slope (i.e., increasing or decreasing over the trial), and signal variance (standard deviation, SD). Interestingly, there were no differences in mean signal or number of trials with positive feedback across groups. However, the NAcc group signal had significantly greater $\mathrm{SD}$ and more trials with a negative slope than the VTA group. These results suggest there may differences in the quality of the feedback the groups received, offering one explanation why the VTA Feedback group succeeded at learning to selfactivate the VTA while the NAcc group did not.

Conclusions: These results demonstrate two novel findings: learning and generalization after neurofeedback training of the midbrain, and the ability to sustain VTA activation in the absence of external reward cues. These findings endorse the use of rt-fMRI to align cognitive states with neurobiological responses.

Keywords: Brain, Dopmaine, Adaptive Behavior, Learning, Dopamine, Functional MRI (fMRI), Motivation, RealTime fMRI.

Disclosure: Nothing to disclose.

\section{W70. Control of Innate Anxiety and Learned Fear by Distinct Ventral Hippocampal Circuits}

Jessica Jimenez ${ }^{*}$, Katy Su, Alexander Goldberg, Victor Luna, Gokhan Ordek, Pengcheng Zhou, Stephanie Pena, Samantha Ong, Larry Zweifel, Liam Paninski, Rene Hen, Mazen Kheirbek

Columbia University, New York, New York, United States

Background: Mood and anxiety disorders are enormously complex illnesses for which effective therapeutic targets remain lacking. A repeatedly replicated finding in the field is that there exists a reduction of hippocampus (HPC) volume in mood and anxiety disorders. However, the subcortical circuits, cell-types, and mechanisms by which the HPC dynamically controls emotional behavior are not understood. Lesion and optogenetic manipulation studies have implicated the ventral hippocampus (vHPC) in anxiety-related behavior, however how emotional information is encoded within its circuits, cell types, and distinct output streams remains unknown. Principle neurons of the vHPC exhibit a rich heterogeneity of gene expression profiles and anatomical connectivity, with non-overlapping populations of neurons routing information to different cortical and subcortical targets implicated in reward, anxiety, fear, and aversion. Here, we have combined novel functional imaging tools and optogenetics to identify the mechanisms by which the vHPC contributes to innate anxiety and learned fear behaviors, and how these functions are represented across diverse limbic output streams.

Methods: We have used cell-type specific calcium imaging in vivo in freely behaving mice using miniaturized microscopes to visualize vHPC activity during anxiety-related behaviors. We expressed GCaMP6f in vHPC, and a gradient index lens was implanted above vCA1. We imaged the activity patterns of the same population of vCA1 neurons across multiple behavioral dimensions, including innately anxiogenic tasks (Open Field Test (OFT), Elevated Plus Maze (EPM), and Elevated Zero Maze (EZM)), innately rewarding tasks (novel object exploration and sucrose pellet consumption), and learned fear tasks (contextual fear conditioning (CFC)). For optogenetic manipulations, we virally expressed Arch or ChR2 opsins in vHPC and implanted a fiber optic either directly in vHPC or at vHPC terminal fields in the Basomedial Amygdala (BMA) or Lateral Hypothalamus (LHA).

Results: In our imaging experiments, we found that the majority of vHPC neurons exhibit heightened activity during exploration of innately anxiety-provoking environments, including the open arms of the EPM, center of the OFT, and open arms of the EZM. This enrichment for open-arm responsive cells in the EPM was not found in mice imaged in the dorsal hippocampus, a sub-structure of HPC thought to be more specialized in spatial navigation and contextual processing than in mood-related behaviors. We next asked how this increase in vHPC activity related to the anxietystate of individual subjects. In the EPM, we found that the magnitude of vHPC heightened open-arm calcium activity significantly correlated with the anxiety score of the animal. To further assess the connection between anxiety state and vHPC activity, we then pre-treated animals with an acute stressor prior to imaging in the EZM, and found that mice in high-anxiety behavioral states exhibited significantly greater increases in vHPC calcium activity upon exploration of the EZM open arms. We next investigated how vHPC processes learned fear environments in CFC tasks. We found that in contrast to vHPC responses in innately fearful contexts, reexposure to a previously fear-conditioned environment decreases overall activity within vHPC, while increasing correlated activity between cell pairs. To understand how these differential activity patterns in innate anxiety and learned fear tasks are represented across the cell population, we assessed the overlap between $\mathrm{vHPC}$ open-arm responsive 
cells, and highly-correlated CFC cells. We found these cell populations to be largely non-overlapping, and next assessed whether these innate and learned-fear responsive cells could be segregated into projection-specific cell populations. We first determined which vHPC limbic output streams are involved in mediating innate anxiety and learned fear behaviors, and employed optogenetic techniques in vHPC downstream terminal fields in two subcortical nuclei implicated in anxiety, fear, and behavioral responses to stress, the BMA and LHA. We found that modulation of vHPC-LHA terminals impacts innate anxiety and aversion, but not contextual fear conditioning, while vHPC-BMA terminal modulation impacts contextual fear memory but not innate anxiety behavior. Further, retrograde tracing studies in these terminal fields revealed that vHPC-LHA and vHPC-BMA projections represent largely non-overlapping cell populations. To determine how these projection-specific cell populations differentially process innate anxiety and learned fear behaviors, we utilized retrograde labeling techniques to selectively express the calcium indicator GCaMP6f in vHPC-LHA or vHPC-BMA projection neurons and imaged their activity in innate anxiety and contextual fear conditioning environments. Ongoing analysis is aimed at determining how these two populations of neurons differentially encode anxiogenic and fearful environments.

Conclusions: Our findings demonstrate a unique population-level activity signature for the encoding of anxiogenic environments within vHPC. Furthermore, differential recruitment of neuronal ensembles in innate and learned fear tasks suggests specific circuit mechanisms for encoding learned versus innately fearful environments. Our optogenetic studies indicate this specificity may lie in the divergent projections streams from the vHPC to the LHA and BMA. These results provide a functional blueprint of the neural activity, cell-types, and long-range circuits of the vHPC that generate innate and learned anxiety-related behaviors.

Keywords: Anxiety Circuitry, Ventral Hippocampus, Calcium Imaging, Fear Conditioning, Optogenetics.

Disclosure: Nothing to disclose.

W71. Selective Orexin-2 Receptor Antagonism Improves Mood in Major Depresive Disorder: A Randomized, Placebo-Controlled, Double-Blind Study

Justine Kent*, Peter Van der Ark, Ilse Van Hove, John Moyer, Remy Luthringer, Luc Van Nueten, Wayne Drevets, Peter De Boer

Johnson \& Johnson, Titusville, New Jersey, United States

Background: JNJ-42847922 (MIN-202) is a potent and selective antagonist of the human orexin-2 receptor $(\mathrm{OX} 2 \mathrm{R})(\mathrm{pKi}=8)$ that is being developed for the treatment of insomnia and major depressive disorder (MDD). Orexins promote arousal (wakefulness) and are hypothesized to play a role in excessive arousal (e.g. hypervigilance, anxiety, somatic tension, agitation and/or excessive rumination), including arousal persisting into the sleep period, which occurs in subsets of patients with mood disorders (Germain et al., 2004; Ho et al., 1996; Nofzinger et al., 2005). The intrinsic antidepressant activity of a selective OX2R antagonist has not yet been explored clinically; however, there is support for a role of OX2R antagonism in modulating depressive-like behaviors in rodent models of chronic stress, hypothesized to be related to normalizing glucocorticoid receptor sensitivity via the paraventricular nucleus (Nollet 2011, 2012, 2013) and/or attenuating orexin-induced increases in adrenocorticotropic hormone levels (Samson 2005). JNJ-42847922 therefore may have benefit in treating depression by attenuating hyperarousal during the sleep period and normalizing HPA axis hyperactivity.

Methods: This was a Phase $1 \mathrm{~b}$ multi-center, double-blind, diphenhydramine- and placebo-controlled study, consisting of a screening examination (day -28 to -1 ), a parallel group treatment phase of 10 days (women of child-bearing potential [WOCBP]) or 28 days (women not of childbearing potential (WONCBP) and men), and a 2-wk followup period. Subjects with a DSM-IV or 5 diagnosis of MDD, aged 18-64 years old, with a body mass index (BMI) between $18-30 \mathrm{~kg} / \mathrm{m} 2$, were enrolled. Subjects had a total score of $\geq 30$ on the Inventory of Depressive Symptomatology (IDS-C30) at screening and were either currently antidepressant-naive or treated with a maximum of 2 concurrent antidepressants, which were given at an optimal dose and for at least 4 weeks, but not longer than 24 weeks, with a suboptimal response. Subjects were randomized in a $2: 1: 1$ ratio to receive JNJ42847922 $20 \mathrm{mg}$, diphenhydramine $25 \mathrm{mg}$ or placebo at bedtime. Diphenhydramine was included as an experimental control, as it has well-established hypnotic effects, but no known antidepressant activity at the selected dose level. Due to limited reproductive toxicology data at the time of the study, the duration of treatment was limited to 10 consecutive days for WOCBP and 28 days for men and WONCBP. Symptoms of depression, sleep disruption, sleep quality, and rumination were evaluated using the 17-item Hamilton Depression Rating Scale (HDRS-17), IDS-C30, Quick Inventory of Depressive Symptoms (QIDS-SR16), polysomnography (PSG), subjective sleep measures, and the Ruminative Response Scale (RRS). For the HDRS-17, ICDSC30 and QIDS-SR16, summary statistics were produced excluding the insomnia items from the total score for each measure, to determine change in non-sleep related depression items.

Results: A total of 47 subjects (22 WOCBP, 25 men or WONCBP) were randomized (22 subjects to JNJ-42847922, 12 subjects to placebo and 13 subjects to diphenhydramine), all of whom completed the study. Most subjects were not currently treated with an antidepressant (10 of 47 subjects were taking an antidepressant; 9 were treated with an SSRI and one with duloxetine). Mean (SD) baseline depression scale scores were: HDRS-17 total score 19.0 (4.99), IDS-C30 total score 37.2 (6.91) and QIDS-SR16 total score 12.9 (3.43). The mean (SD) changes from baseline in HDRS-17 total score at Day 11 were: JNJ-42847922 (-5.5 [3.86]), placebo $(-3.6[4.03])$, and diphenhydramine $(-4.1$ [3.66]). On Day 28 $(N=24)$, the corresponding changes were: JNJ-42847922 (-9.9 [5.98]), placebo (-5.0 [5.37]) and diphenhydramine $(-6.6[5.62])$. There was a greater reduction $(p<0.05)$ in mean (SD) change from baseline in the adjusted HDRS-17 score (sleep items removed) at Day $11(N=47)$ in the JNJ-42847922 group (-4.5 [2.76]) compared with the placebo (-2.3 [3.03]) and the diphenhydramine (-2.3 [2.81]) groups. Scores on the HAM-D6, a subscale of the HDRS-17, lend 
support for an effect of JNJ-42847922 on the core symptoms of depression: mean (SD) change from baseline at Day 11 was significantly greater $(p<0.01)$ in the JNJ-42847922 group (-3.8 [2.22]) than in the placebo $(-1.5$ [2.15]) and diphenhydramine groups $(-1.8[(2.01])$.

JNJ-42847922-induced antidepressant efficacy was comparable in subjects who did, versus those who did not, receive concomitant antidepressant therapies. Notably, the antidepressant effect did not correlate with changes in PSGmeasures such as latency to persistent sleep or total sleep time, supporting an antidepressant effect independent of the compound's effect on sleep. Treatment with $20 \mathrm{mg}$ JNJ-42847922 daily up to 28 days was well-tolerated.

Conclusions: Overall, larger improvements in clinician-rated depression symptoms were observed for subjects treated with JNJ-42847922 compared with placebo and the experimental control, diphenhydramine. Efficacy of JNJ-42847922 in treating the symptoms of depression was manifest when sleep items were removed from the HDRS-17 and also on the HAM-D6 core depressive symptom subscale, suggesting that OX2R antagonism may have utility in the treatment of depression independent of effects on PSG-measured sleep parameters.

Keywords: Depression, Orexin Receptor Antagonist, Clinical Trial.

Disclosure: Johnson \& Johnson: Employee, Self.

\section{W72. Resting-State Functional Connectivity Dysfunction in Anhedonia as a Transdiagnostic Process: An RDoC Investigation}

Zoi Samara*, Andrea N. Goldstein-Piekarski, Trisha Suppes, Jerome Yesavage, Leanne Williams

Stanford University School of Medicine, Palo Alto, California, United States

Background: Anhedonia, the markedly diminished interest in pleasure or in the capacity to experience pleasure, cuts across many neuropsychiatric disorders including major depressive disorder, stress-related disorders, schizophrenia and substance use disorders and is often one of the most difficult to treat symptoms. Anhedonia's putative neural substrates involve the dopaminergic mesolimbic and mesocortical reward networks which comprise the ventral tegmental area (VTA), the ventral striatum and the prefrontal cortex, including the medial dorsal and ventral PFC and the orbitofrontal cortex. To develop more efficient treatments for anhedonia we need to characterize the dysfunctions of the reward network(s) that underlie significant loss of pleasure and positive affect independent of diagnostic labels. To address this issue, we examined MRI resting state functional connectivity aberrations associated with anhedonia in a clinical sample using a transdiagnostic approach.

Methods: Participants were drawn from a larger study for a preliminary analysis. In line with $\mathrm{RDoC}$ principles the study recruits all clients who "walk through the door" of a large community mental health clinic as well as the surrounding community, thus including participants from a broad spectrum of anxiety and mood-related symptoms. Twenty participants with high anhedonia as indexed by their score on the MASQ anhedonic subscale (10-items) and twenty participants with low MASQ anhedonia were assessed using functional MRI at $3 \mathrm{~T}$. Participants were unmedicated and studied at baseline using a standardized battery of functional brain imaging, structural brain imaging and self-report measures of anxiety and mood symptoms, and social and occupational functioning.

Results: We compared MRI resting state functional connectivity in a group of participants with high MASQ-anh $(\mathrm{m}=47.9 ; \mathrm{sd}=1.4)$ which differed significantly in their selfreported anhedonia symptoms $(\mathrm{t}=33.9 ; p<.0001)$ from a low MASQ-anh group $(\mathrm{m}=22.5$; sd $=3.1)$ while controlling for differences in mood and anxiety symptoms between the two groups. Seed-based functional connectivity analysis revealed that the high MASQ-anh group had significantly lower resting functional connectivity between the VTA and the left $(\mathrm{t}=3.67, \mathrm{pFWE}=.01)$ and right ventral striatum $(\mathrm{t}=4.01, \mathrm{pFWE}=.005)$. Exploratory whole-brain analyses further revealed that the high MASQ-anh group had increased connectivity between the left and right ventral striatum and the dorsomedial prefrontal region $(t=4.40$, puncorr $=.0001)$ that has been recently suggested as an optimized rTMS target for anhedonia.

Conclusions: Our RDoc approach highlights for the first time that the communication between two major nodes of the dopaminergic mesolimbic reward pathway is disrupted in anhedonia independently of the presence of mood and anxiety symptoms. Moreover, our observation that high anhedonia is associated with altered connectivity between the ventral striatum and the dorsomedial prefrontal cortex provide a putative mechanism of action of anhedoniaoptimized rTMS region.

Keywords: MRI, Connectivity, Anhedonia, RDoc.

Disclosure: Nothing to disclose.

\section{W73. Discovery of Functionally Selective Chaperone Ligands Capable of Facilitating the Secretion of Brain-Derived Neurotrophic Factor and Attenuating Stress-Induced Elevations in Nitric Oxide}

John Schetz ${ }^{\star}$, Dhwanil Dalwadi

University of North Texas Health Science Center, Fort Worth, Texas, United States

Background: Mood disorders cause a significant amount of suffering worldwide with depressive disorders constituting the majority of the mental health disease burden. Inadequate brain-derived neurotrophic factor (BDNF) signaling appears to be a key factor linked to major depression, and restoration of BDNF-mediated plasticity in the prefrontal cortex and hippocampus correlates with the actions of antidepressant drugs, such as serotonin and norepinephrine reuptake inhibitors and low dose ketamine. Evidence is mounting that depression and inflammation perpetuate one another. Psychosocial stressors, including interpersonal stress and social rejection, are accepted major risk factors for depression. Such experiences engage the hypothalamus-pituitaryadrenal gland (HPA) axis leading to an upregulation of proinflammatory cytokines, and larger cytokine responses to stressors are primed by depression. Excessive or chronic psychosocial stress can result in lasting impairment that may 
involve oxidative-nitrosative stress-induced loss or impairment to brain cells suggesting that reducing inflammation while promoting neurorestoration may be beneficial for treating depression. The Sigma-1 receptor (S1R) is a stress and ligand-regulated chaperone protein involved in neuroprotective, neurorestorative and neuroplastic responses. Here we explore S1R ligand chemotypes whose functionally selective molecular endpoints would suggest their utility in the treatment of depression.

Methods: The interaction strength of ligands with the cloned human S1R and the S1R density in different cell types were measured by radioligand binding. The ability of high-affinity $\mathrm{S} 1 \mathrm{R}$ ligands to modulate BDNF message or secretion was quantified by qPCR and in situ ELISA, respectively. The capacity of ligands to additionally attenuate stress-induced elevations in intracellular nitric oxide (NO) was monitored in real time by fast fluorescence imaging using an NOsensing dye.

Results: From a panel of structurally diverse ligands with a history of use in humans, three structural classes were identified as having a functional selectivity profile consistent with facilitating BDNF secretion from neuronal and glial cells with no change in BDNF mRNA. These same ligands also attenuated stress-induced elevations in intracellular NO. These responses were diminished by S1R antagonists demonstrating that they are mediated via the S1R. A focused structure-activity analysis for one of the identified S1Rinteracting chemotypes pinpointed a substructural feature essential for the BDNF-secreting phenotype.

Conclusions: Here we show that the S1R chaperone is capable of mediating BDNF secretion from brain cells and regulating nitric oxide levels when cells experience high levels of inflammatory stress. Utilizing a unique high throughput platform, orally-active, brain-penetrant small molecules with clinical histories were discovered having a BDNF-secreting functional selectivity profile mediated by the $\mathrm{S} 1 \mathrm{R}$ in neuronal and glial cells. These same compounds also dampened oxidative stress-induced surplus elevations in NO. When present in excess, as in the case of inflammation, nitric oxide and superoxide combine forming the brain-impairing reactive species peroxynitrite which further perpetuates inflammation. An innovative aspect of our strategy is targeting one receptor to interrupt the cycle of peroxynitrite generation by blocking unsafe elevations in nitric oxide under oxidative stress conditions, while simultaneously enhancing resilience to inflammatory brain insults and promoting neurorestoration and plasticity by facilitating the secretion of BDNF. Differential alkylation of a series of phenylalkylcarboxylesters demonstrates for the first time a structural basis for the BDNF-secreting functional selectivity mediated by the S1R. These studies may aid in the development of lead chemotypes for treating depressive disorders that are distinct from reuptake inhibitors and ionotropic receptor blockers. This work is also likely applicable to other disorders associated with insufficient BDNF signaling and inflammation including anxiety disorders, schizophrenia and neurodegenerative disorders such as Alzheimer's disease.

Keywords: Mood and Anxiety Disorders, Major Depressive Disorder (MDD), Antidepressant Agents, Brain-Derived Neurotrophic Factor, Drug Discovery - New Approaches. Disclosure: Nothing to disclose.
W74. Dextromethorphan/Quinidine Combination as a Novel Pharmacotherapeutic Approach in Patients With Treatment Resistant Depression: A Proof of Concept Clinical Trial

James Murrough*, Elizabeth Wade, Seharish Sayed, Gabby Ahle, Drew Kiraly, Alison Welch,

Katherine Collins, Laili Soleimani, Dan Iosifescu, Dennis Charney

Icahn School of Medicine at Mount Sinai, New York, New York, United States

Background: Currently available treatments for major depressive disorder (MDD) are only partially effective, with one third of patients not achieving adequate symptom control. For this group of patients suffering from treatmentresistant depression (TRD), novel treatments that act through non-monoaminergic mechanisms are urgently needed. Notably, prior studies have demonstrated that the glutamate $\mathrm{N}$-methyl-d-aspartate (NMDA) receptor antagonist ketamine displays rapid-acting and robust antidepressant effects, even in patients suffering from TRD. In the current study we endeavored to examine the effects of a combination of dextromethorphan (DM) and the CYP2D6 enzyme inhibitor quinidine (Q) in patients with TRD. Dextromethorphan modulates signaling at the NMDA receptor, in addition to other pharmacodynamics properties that include activity at sigma-1 receptors and at the serotonin transporter. Quinidine inhibits the hepatic metabolism of $\mathrm{DM}$, thereby increasing circulating concentrations of the latter. A combination product of DM $20 \mathrm{mg} / \mathrm{Q} 10 \mathrm{mg}$ given every 12 hours is approved for the treatment of pseudobulbar affect (PBA). In the current study, we tested the safety, tolerability and preliminary efficacy of DM/Q up to 45/10 mg every 12 hours in patients with TRD.

Methods: The current study consisted of an open-label proof of concept (POC) clinical trial of DM/Q for up to 10 weeks in patients with unipolar TRD, either as monotherapy or as adjunctive therapy. Patients initiated study drug at $20 / 10 \mathrm{mg}$ daily for 7 days, then increased to $20 / 10 \mathrm{mg}$ every 12 hours for 7 days, then increased to a maximum dose of $45 / 10 \mathrm{mg}$ every 12 hours if tolerated. The minimum required dose to remain in the study was $20 / 10 \mathrm{mg}$ every 12 hours. Eligibility criteria included men and women between the ages of 18 and 65 , a primary diagnosis of MDD as determined by the SCID, and a history of non-response to two or more prior adequate antidepressant trials as determined by Antidepressant Treatment History Form (ATFH) criteria. Safety and tolerability outcomes were assessed by discontinuation rate, frequency of adverse events [using the Patient Rated Inventory of Side Effects (PRISE)], and score change on the Columbia-Suicide Severity Rating Scale (C-SSRS), the Brief Psychiatric Rating Scale-Positive Subscale (BPRS+), and the Clinician Administered Dissociative States Scale (CADSS). Efficacy was assessed as change in MontgomeryAsberg Depression Rating Scale (MADRS) score. Additional efficacy and functional measures included the Quick Inventory of Depressive Symptomatology-Self Report (QIDS-SR), the Hamilton Anxiety Scale (HAM-A), and the Sheehan Disability Scale (SDS). All analyses were conducted on the intention to treat (ITT) sample, with last observation carried forward for missing data. 
Results: Twenty patients with TRD (11 women; mean age: $49.4 \pm 7.7$ years) were started on DM/Q therapy and represent the ITT sample. Six patients discontinued prior to study completion, yielding a completer sample of 14 patients (70\% retention). The most common adverse events were nausea and constipation, which each occurred in 3 patients. One serious adverse event occurred, representing hospitalization for insomnia and worsening depression three days after starting the study medication; this event was considered unlikely to be related to the study medication. There was no treatment-emergent suicidal ideation or changes in BPRS+ or CADSS score. MADRS score was reduced from baseline to the 10 -week primary outcome (mean change: $12.95 \pm 11.52, \mathrm{t}(\mathrm{df}=19)=5.04, p<.001)$, as was QIDS-SR score (mean change: $5.70 \pm 5.85, \mathrm{t}(\mathrm{df}=19)=$ $4.36, p<.001$ ). The overall response rate was $45 \%$ (as defined by a $50 \%$ decrease in MADRS score from baseline to primary outcome). HAM-A score was reduced from baseline to primary outcome (mean change: $5.70 \pm 5.85, \mathrm{t}(\mathrm{df}=19)=$ $4.36, p<.001)$. Disability lessened from baseline to primary outcome as measured by the SDS $(p<.001)$.

Conclusions: Herein we report acceptable tolerability and safety and preliminary efficacy of DM/Q up to $45 / 10 \mathrm{mg}$ administered every 12 hours in patients with TRD. Future larger placebo controlled randomized trials in this population are warranted.

Keywords: Glutamate, Major Depression Disorder, Treatment Resistant Depression, NMDA Antagonists, Dextromethorphan/Quinidine.

Disclosure: Nothing to disclose.

\section{W75. Why Depression Hurts: Initial Evidence for Neural Correlates of Emotional Allodynia}

Irina Strigo*, Arthur D (Bud) Craig

University of California, San Francisco, California, United States

Background: We have repeatedly found that major depressive disorder (MDD) is associated with a subjectively enhanced perception of unpleasantness in response to temperature stimuli that are not normally perceived as unpleasant; we termed this phenomenon emotional allodynia. Specifically, we found that individuals with MDD rated warm stimuli as quite unpleasant, even though they rated the stimuli as non-painful; the same stimuli were neither unpleasant nor painful for all healthy participants. In fact, the unpleasantness ratings of these stimuli were as high in MDD participants as the unpleasantness ratings of clearly painful heat stimuli were in healthy subjects. In control trials, we found that this emotional allodynia was not modulated by the attentional state of the subjects nor by acutely induced sadness (Strigo et al., 2008a,b; Strigo et al., 2010), which suggests that emotional allodynia is a stable characteristic of major depression, that is, a measurable manifestation of an emotional dysfunction that is inherent to this mental disorder. The current work aims to provide initial evidence regarding the neural correlates of emotional allodynia by using voxel-based linear mixed effects modeling of brain response to warm, non-painful temperature stimuli.
Methods: A total of thirty-six human subjects $(24 \mathrm{~F}, 12 \mathrm{M}$, $24.3 \pm 5.1$ years) gave written informed consent to participate in this study, which was approved by the University of California San Diego Human Research Protection Program. $19 / 36$ subjects suffered from MDD (based on DSM-IV criteria) and 17/36 subjects were never-depressed healthy adults. All subjects were examined with fMRI while they experienced non-painful warm $\left(38-40^{\circ} \mathrm{C}\right)$ temperature stimuli ( $9 \mathrm{~cm} 2$ thermode, Medoc TSA-II, Ramat-Yishai, Israel) applied to the left forearm. The current analyses specifically examined the relationship between emotional allodynia (EMA), depression severity (BDI-2), and insula activation during warmth using linear mixed effects modeling in the entire sample of 36 adults. The degree of emotional allodynia was calculated for each subject by calculating the affective bias scores for non-painful temperature stimuli (see (Strigo et al. 2008b). Twenty ratings of affective bias to warm, non-painful temperature stimuli were used to create the average emotional allodynia score for each subject. All subjects were unmedicated for at least 6 weeks prior to the imaging study (see (Strigo et al. 2008b, Strigo et al. 2008a).

Results: We found that depression severity, when controlling for emotional alllodynia, showed association predominantly with activation of the right insula, while emotional allodynia, when controlling for depression severity, showed association predominantly with activation of the left insula. Post-hoc examination of these effects showed that depression severity strongly and positively correlated with right insula activation, while emotional allodynia showed negative correlation with the left insula. In order to assess specificity of the insular asymmetry, homologous activation on the opposite sites were extracted. This analysis showed significant positive correlation between depression severity and right insula activation, which was specific to the right side $(Z=2.342$, $P=0.019, \mathrm{X} / \mathrm{Y} / \mathrm{Z}: 37 / 16 /-2)$. Conversely, negative association between emotional allodynia and the left insula activation was specific to the left side $(\mathrm{Z}=-2.263, P=0.023, \mathrm{X} / \mathrm{Y} / \mathrm{Z}$ : $-32 / 11 / 5)$. Furthermore, significant negative correlation was found between the degree of emotional allodynia and low frequency (parasympathetic) modulation of heart rate variability (RMSDD) $(r=-0.37, p<0.05)$.

Conclusions: Using linear mixed effects modeling of fMRI response to non-painful, warm stimuli, we examined the neural correlates of emotional allodynia, depression severity, and their interaction. Our results show hemispheric lateralization of activation in the insular cortex, whereby depression severity was positively associated with the increased right insula activation, while emotional allodynia (or, affective bias) was negatively associated with the left insula activation. Our results thus provide initial evidence for the neural substrates underlying increased emotional unpleasantness to warm, non-painful stimuli in depressed patients. Finally, considering prior and recent evidence supporting left hemisphere dominance in the control of parasympathetic outflow (Strigo and Craig, in press), these findings further confirmed the association between emotional pain and autonomic imbalance in MDD.

Keywords: Depression, Pain, BOLD Imaging, Insula, Interoception.

Disclosure: Nothing to disclose. 
W76. Serotonergic Influences on Anterior Cingulate and Insula are Influenced by Inflammation and are Associated With Subsequent Depression

Francis Lotrich*, Howard Aizenstein, Eva Szigethy

Western Psychiatric Institute and Clinic, Pittsburgh, Pennsylvania, United States

Background: How does inflammation cause depression, and why does it do this in only a minority of people? We have previously found that interferon-associated depression (a model of inflammatory-cytokine-associated depression) is associated with serotonin transporter polymorphisms. Using PET scans, increased transporter binding during inflammatory cytokine treatment was associated with the development of severe depression (whereas the transporter binding decreased in those who remained euthymic). We have also recently observed that kynurenine levels in people with inflammatory illness are cross-sectionally correlated with connectivity between the insula and BA25. We were thus interested in whether inflammation might functionally influence the serotonergic system in these areas, thereby influencing mood.

Methods: Before non-depressed adult subjects started treatment for hepatitis $\mathrm{C}$ with interferon-alpha (IFN-a), they were assessed using $3 \mathrm{~T}$ fMRI BOLD signals. Three sets of scans were obtained. The first set was prior to a serotonergic challenge. The second and third were during and immediately after a 30-minute intravenous infusion of $20 \mathrm{mg}$ citalopram. This infusion acutely blocks 5-HT reuptake, acutely increases synaptic 5-HT levels, and this has previously been found to increase the response of the amygdala to mathcing angry faces compared to matching neutral faces. Therefore, before, during, and after infusion, fMRI BOLD activity was assessed employing an angry faces matching task. These same three sets of scans were then repeated 3 weeks into IFN-a therapy. Those who developed MDD (SCID-IV defined; and quantified with the Beck Depression Inventory and Montgomery Asperg Depression Rating Scale) were compared with those who didn't. Planned comparisons used $p<0.05$ for significance ( $n=6$ with complete data), examining three regions of interest (amygdala, insula, anterior cingulate).

Results: We replicated the prior study that found that acute intravenous citalopram infusion accentuates the response of the amygdala to angry faces $(p<0.01)$. Conversely, both the insula and anterior cingulate (AC) decreased activity during angry face exposure - and this decrease was blunted by citalopram $(p<0.001)$. During IFN-a treatment in subjects who remained euthymic, all these effects of the 5-HT challenge disappeared (i.e. the citalopram challenge had no significant effect on fMRI activity). However, in subjects who developed inflammation-associated depression, the amygdala became hyper-responses to angry faces (while the AC and insula were no longer affected). The acute 5-HT challenge normalized all three brain areas (AC and insula decreased activity during angry faces, as they did before IFN treatment). Connectivity between the AC and insula was increased by the acute citalopram challenge prior to IFN-a treatment $(p<0.01)$. Conversely, AC-insula connectivity was decreased by the citalopram challenge during IFN-a treatment $(p<0.01)$.
Conclusions: This is preliminary evidence in a limited number of subjects that inflammation influences the role of 5-HT in three areas important for mood control. In fact, in the same individuals, the effect of a citalopram infusion on connectivity were sometimes entirely reversed by inflammation. Moreover, subjects who were 'resistant' to developing interferon-induced depression had very different changes than those who developed depression. Consistent with our prior PET findings, in those who remained euthymic (a decrease in transporters), the effect of a citalopram infusion was lost during IFN-a. And in those who developed depression (and had an increase in transporter levels), the acute 5-HT challenge decreased AC, insula, and amygdala activity during angry faces (the opposite of the 5-HT challenge prior to interferon treatment). These results support the hypothesis that emotional regulation can be influenced by inflammation via functional changes in the serotonergic system. Whether this pattern of results generalizes to other types of inflammation-associated depression or is unique to IFN-a will require further study.

Keywords: Depression, Cytokines, Inflammation, Functional Neuroimaging, Serotonin.

Disclosure: Nothing to disclose.

W77. Routine Screening for Fatty Acid Abnormalities in a Psychiatric Inpatient Facility: Results From the First 204 Patients

\section{Erik Messamore, Robert McNamara*}

University of Cincinnati College of Medicine, Cincinnati, Ohio, United States

Background: Deficits in long-chain omega-3 (LCn-3) fatty acids, including eicosapentaenoic acid (EPA) and docosahexaenoic acid (DHA), and increases in the arachidonic acid (AA)/EPA+DHA ratio, are associated with the pathophysiology of different psychiatric disorders. DHA is the most abundant omega-3 fatty acid in cortical membranes, and neuroimaging evidence suggests that blood DHA levels are associated with cortical structural and functional integrity. Prospective studies have found that a high blood AA/EPA $+\mathrm{DHA}$ ratio is a robust predictor of mood dysregulation during treatment with interferon-alpha in hepatitis $\mathrm{C}$ patients. Low blood EPA+DHA levels are also associated with increased cardiometabolic risk factors (e.g., CRP, triglycerides) and risk for cardiovascular-related mortality, a primary cause of premature excess mortality in patients with mood and psychotic disorders. Importantly, dietary supplementation with prescription and over-the-counter fish oil supplements correct EPA+DHA deficits in patients. Despite this body of evidence, however, LCn-3 fatty acid screening and treatment has been slow to impact conventional psychiatric training and practice. In a pilot study initiated mid-2014, whole blood fatty acid screening was implemented as part of routine laboratory assessments at a university-affiliated inpatient mental health center. Here we present data from the first 204 patients.

Methods: Whole blood was obtained from patients with a finger prick and spotted and dried onto an antioxidant-treated card. The card was mailed at ambient temperature and blood fatty acid composition determined by a CLIA-certified 
laboratory by gas-liquid chromatography (OmegaQuant, LLC, Sioux Falls, SD). This analysis determines 24 different fatty acids, including EPA and DHA, which are reported as a percentage of total fatty acids. Here we specifically focus on whole blood EPA, DHA, AA, and the AA/EPA+DHA ratio. Individual fatty acid levels were compared to U.S. population norms. Based on prospective evidence implicating EPA $+\mathrm{DHA}<4 \%$ of total fatty acid composition as a risk factor for cardiovascular-related mortality, we used $<4 \%$ to demarcate a 'state of deficiency' for categorical analyses.

Results: Patients $(n=204)$ had a mean age of $28.6+/-15.1$ years, were $55 \%$ female, and were predominantly diagnosed with treatment-refractory mood and anxiety disorders. No patient reported taking fish oil-based supplements. Male and female patients did not differ in EPA+DHA $(p=0.76)$, AA $(p=0.11)$, or the AA/EPA+DHA ratio $(p=0.48)$. Consistent with normative data, EPA+DHA was positively correlated with age $(\mathrm{r}=+0.28, p=0.0001)$. The DHA mean $(2.4 \%$ $+/-0.9 \%)$ was similar to population norms $(2.9 \%)$, whereas the EPA mean $(0.47 \%+/-0.2 \%)$ was $57 \%$ lower than population norms (1.1\%). The EPA+DHA mean $(2.9 \%$ $+/-1.1 \%$ ) was $34 \%$ lower than population norms (4.4\%). The majority $(85 \%)$ of patients exhibited whole blood EPA $+\mathrm{DHA}$ levels at $\leq 4 \%$ which is higher than population norms $(25 \%, p=0.0001)$. The AA mean $(11.4 \%+/-2.1 \%)$ was similar to population norms (10\%), and the mean AA/EPA+DHA ratio $(4.3+/-1.3)$ was $42 \%$ higher than population norms $(2.5 \%)$. In several notable cases, adjunctive treatment with prescription fish oil-based products resulted in robust increases in whole blood EPA+DHA levels.

Conclusions: These preliminary findings provide proof-ofconcept evidence that routine screening for, and treatment of, low blood EPA+DHA levels is feasible in routine psychiatric practice. Consistent with prior case-control studies, our initial results demonstrate that the majority of patients with mood and anxiety disorders exhibit very low blood EPA+DHA levels which places them at increased risk for cardiovascular-related morbidity and mortality. Analogous to routine cholesterol testing, this naturalistic study demonstrates that routine screening for fatty acid abnormalities (i.e., low EPA+DHA levels) is currently feasible and has the potential to improve long-term health outcomes for patients.

Keywords: Omega-3 Fatty Acid, Clinical Practice, Treatment. Disclosure: Nothing to disclose.

\section{W78. Ketamine Exerts NMDA Receptor Inhibition- Independent Antidepressant Actions via its Hydroxynorketamine Metabolites}

Panos Zanos*, Ruin Moaddel, Patric Morris, Polymnia Georgiou, Jonathan Fischell, Greg Elmer, Manickavasagom Alkondon, Peixiong Yuan, Heather Pribut, Nagendra Singh, Katina Sylvestre Dossou, Yuhong Fang, Xi-Ping Huang, Cheryl Mayo, Irving Wainer, Edson Albuquerque, Scott Thompson, Craig Thomas, Carlos Zarate Jr., Todd Gould

University of Maryland School of Medicine, Baltimore, Maryland, United States

Background: Major depressive disorder (MDD) affects approximately 16 percent of the world population at some point in their lives and is a leading cause of death. Despite a number of available monoaminergic-based antidepressants for MDD treatment, most patients require long-term administration (many weeks, if not months) to respond, and many patients never attain sustained remission of their symptoms. An exciting finding for the treatment of MDD is that the a single administration of the non-competitive glutamatergic N-methyl-D-aspartate receptor (NMDAR) antagonist, (R,S)-ketamine (ketamine) results in a rapid (within hours) and sustained (typically 1 week) improvement of core depressive symptoms including mood, anhedonia, and suicidal ideation. However, the widespread clinical use of ketamine is limited due to its abuse liability and capacity to produce dissociative effects. Ketamine's mechanism of antidepressant action has been hypothesized to be due to NMDAR inhibition; however, other NMDAR antagonists do not manifest similar actions. Ketamine is rapidly and stereospecifically metabolized to norketamine, dehydronorketamine, hydroxyketamine and the hydroxynorketamines (HNKs).

Methods: Using mouse tests of antidepressant efficacy, including the 1- and 24-hour forced swim test, noveltysuppressed feeding, learned helplessness, chronic corticosterone administration and chronic social defeat stress, we assessed the antidepressant and anti-anhedonic effects of ketamine's enantiomers and its hydroxynorketamine metabolites. We also performed in vitro field excitatory postsynaptic potential (fEPSP) measurements and western blots for AMPA receptor subunits (GluA1 and GluA2), brainderived neurotrophic factor (BDNF) and Eukaryotic Translation Elongation Factor 2 (eEF2). We have also conducted in vivo electroencephalogram measurements of gamma power as a measure of target engagement. Side effects of ketamine and its metabolites were also assessed.

Results: Our experiments reveal that NMDAR inhibition is not responsible for the unique antidepressant actions of ketamine since the (R)-ketamine enantiomer, which has $\sim 4$ fold lower affinity for the NMDAR, exerts superior antidepressant responses compared to (S)-ketamine in the forced-swim test, novelty-suppressed feeding and learned helplessness tests. In addition, MK-801 (another NMDAR channel blocker) does not exert the sustained effects observed following ketamine administration in the 24- hour forced-swim test and the chronic social defeat paradigm. We also demonstrated that production of the (2 S,6 S;2 R,6 R)hydroxynorketamine metabolite is essential for ketamine's sustained antidepressant effects, since blockade of ketamine's metabolism to this hydroxynorketamine metabolite abolished its antidepressant actions in the 24-hour forced-swim test and learned helplessness. We have also found that the (2 R,6 R)-HNK enantiomer exerts behavioral, electroencephalographic (enhanced gamma power oscillations), electrophysiological (increased hippocampal $\alpha$-amino-3-hydroxy-5methyl-4-isoxazole propionic acid (AMPA) receptormediated excitatory post-synaptic potentials) and cellular (increased synaptic AMPA receptor subunits GluA1 and GluA2) antidepressant and anti-anhedonic actions in vivo, which can explain the unique antidepressant actions of ketamine. Importantly these effects were prevented by pretreatment with the competitive AMPA receptor antagonist, NBQX, administered prior to, as well as 24 hours following, (2 R,6 R)-HNK administration, indicating the involvement of 
early and sustained AMPA receptor activation. This metabolite did not exert ketamine-associated discriminative properties (measured with the drug discrimination operant conditioning test) abuse potential (measured with intravenous self-administration), sensory dissociation (measured with pre-pulse inhibition) or locomotor stimulant side effects, indicating a safer side effect profile.

Conclusions: Our results indicate a novel mechanism underlying ketamine's unique antidepressant properties, which involves the required activity of a distinct metabolite and which is independent of NMDAR inhibition. Considering the lack of side effects, and the favorable physiochemical properties of hydroxynorketamines, these findings have relevance for the development of next generation, rapidacting antidepressants.

Keywords: Ketamine, Hydroxynorketamine, Major Depression Disorder, AMPA Receptors, Antidepressant.

Disclosure: University of Maryland Baltimore, Patent, Self.

\section{W79. Reward Prediction Errors Affect Episodic Memory:} Implications for Depression

\section{Nina Rouhani, Yael Niv* \\ Princeton University, Princeton, New Jersey, United States}

Background: How does reward feedback from the environment interact with the fidelity of episodic memories, and how are these processes affected by depression?

In reward learning, phasic dopaminergic signals track the degree to which actual outcomes are different from what was expected (a "reward prediction error" signal). These signals are critical for trial-and-error learning in the striatum. However, similar dopaminergic signals project to the hippocampus (Shohamy \& Adcock, 2010), suggesting that prediction errors might also influence the formation of episodic memories as well. In recent work, we have suggested that large prediction errors may serve to fractionate the stream of experience into separate memory traces (Gershman et al. 2014, 2015), potentially reducing interference between different remembered events.

Major Depressive Disorder (MDD) is characterized by both decreased behavioral impact of reinforcement and memory loss. A straightforward hypothesis is that anhedonia is due to or at least reflected in diminished encoding of prediction error signals. Indeed, MDD patients show weakened prediction error signaling in the striatum, correlated with the severity of anhedonia symptoms (Gradin et al., 2011). If prediction errors enhance episodic memory, such altered encoding of prediction errors may also explain symptoms of memory loss, especially for positively rewarding events.

Methods: Adopting a transdiagnostic approach, we tested many (hundreds) of participants using the Amazon Mechanical Turk platform. In several studies, we investigated whether learning in a high-risk environment, with frequent large prediction errors, gives rise to higher fidelity memory traces than learning in a low-risk environment. In our task, participants learned from trial and error which of two types of pictures, indoor or outdoor scenes, leads to higher rewards. Pictures were presented within different contexts ('rooms'), with each room associated with a different degree of outcome variance (risk) but matched in value. After being shown a trial-unique image, subjects estimated how much that type of scene (indoor or outdoor) was worth on average, and were then presented with the image along with a monetary outcome. After learning in both rooms, subjects completed a number of self-report inventories, including the inventory of depressive symptomology (IDS; http://www.idsqids.org/). Finally, they were subjected to a surprise recognition test in which they were shown half of the images they had seen in the rooms and a similar number of new foils, and were asked to determine whether each image is old or new.

Results: In each of several independent replications, subjects showed better learning performance in the low-risk room, although they optimally reduced their learning rate in the high variance environment. Conversely, the recognition test revealed that memory for high-risk scenes was better, and that this effect was driven by greater absolute (unsigned) prediction errors at reward outcome - the larger the prediction error (positive or negative), the better the item was remembered, across both rooms. Depressive symptomology (IDS) scores ( $N=136$ in this study) were inversely correlated with learning, as well as with memory accuracy, suggesting a weakened effect of objective prediction error magnitude on recognition memory, perhaps due to diminished neural encoding of prediction errors. This blunting was seen for both positive and negative prediction errors, in line with previous work that has not consistently seen asymmetric effects of depression on prediction error signaling. Conclusions: Our findings demonstrate that prediction error signaling affects memory formation and not only trial and error learning. They further suggest that blunted prediction error signaling in MDD might be related to memory-related symptoms. Future work in clinical populations will more directly investigate a potential asymmetric blunting of positive rather than negative prediction errors, or, alternatively, prediction errors due to receipt (or lack thereof) of rewards versus punishments.

Keywords: Reinforcement Learning, Episodic Memory, Reward Prediction Error, Depression, Computational Psychiatry.

Disclosure: Nothing to disclose.

\section{W80. Investigating Expression of Epistatic Interaction Networks in Bipolar Disorder in Human Dorsolateral Prefrontal Cortex}

Anandita Rajpurohit*, Carrie Wright, Richard E. Straub, Andrew E. Jaffe, Ran Tao, Fernando S. Goes, Peter P. Zandi, Thomas M. Hyde, Joel E. Kleinman, Daniel R. Weinberger, Joo Heon Shin

Lieber Institute for Brain Development, Baltimore, Maryland, United States

Background: Bipolar disorder (BD) is a common, highly heritable psychiatric illness whose genetic underpinnings remain unclear. Recent genome-wide association studies (GWAS) have identified multiple risk loci, but the complex genetic architecture of $\mathrm{BD}$ makes it necessary to evaluate findings in a broader biological context. Investigation of epistatic interactions between disease-associated genes has 
the potential to illuminate this area. Such interactions can be elucidated by the recently developed W-test, which identifies pair-wise epistasis effects, and which in a recent publication identified two interaction networks when applied to BD GWAS datasets (Wang, Maggie et al., Nuc. Acids Res., 2016). Further, gene set analysis, by grouping genes into gene sets based on shared properties, allows for incorporation of such biological knowledge to identify the modest regulations of related genes that potentially underlie complex disease. To explore whether the identified interaction networks are altered in $\mathrm{BD}$, we characterized their expression using RNA sequencing and gene set analysis of post-mortem brain tissue from patients and controls.

Methods: RNA sequencing data was evaluated for postmortem tissue samples from the dorsolateral prefrontal cortex (DLPFC) of 106 subjects (53 BD subjects and 53 matched control subjects). One control was selected for each BD subject, matching for age, gender, and ethnicity. All samples were processed according to established protocol (Jaffe et al., Nat. Neurosci., 2014).

Subjects were filtered for those with RNA integrity number (RIN) greater than 7, and gene abundance estimates were filtered for those with a normalized $\log 2$ (Reads Per Kilobase of transcript per Million mapped reads $(\mathrm{RPKM})+1$ ) expression value greater than 0.1 . Differential expression of the gene abundance estimates was analyzed using the Bioconductor package Limma in $\mathrm{R}$ and modeling for latent confounders using principal components (PCs). The following three gene set tests were then used to assess the expression of gene sets of interest across diagnosis groups. Gene Set Test is a permutation-based competitive gene set test. Correlation Adjusted Mean Rank (CAMERA) is a competitive gene set test that estimates and adjusts for intergene correlation within the data. Finally, ROAST is a selfcontained gene set test that uses rotation, rather than permutation, and thus similarly allows for correlation of genes.

Results: Differential expression analysis between BD subjects and controls of all Ensembl transcripts with normalized expression values of greater than 0.1 , modeling with twelve PCs, yielded 1837 differentially expressed genes. Following multiple testing correction using the Benjamini Hochberg method, no genes remained significantly differentially expressed.

We then performed gene set analysis based on interaction networks recently identified from two bipolar GWAS datasets. We first analyzed only those network genes replicated in both datasets: Gene Set 1 (Network 1) contained CSMD1, DPP10, SLIT3, and TMEM132D; Gene Set 2 (Network 2) contained A2BP1, MYO16, ELMO1, ACCN1, CNTNAP2, PARK2, and HNT. Analysis of these gene sets using the CAMERA global t-test based gene set test found Gene Set 1 to show a significant decrease in expression in BD patients $(p$ value $=0.003)$, with an inter-gene correlation of 0.139. This was confirmed using the rank-based CAMERA test $(p$ value $=0.012)$, the Gene Set Test (10000 simulations, $p$ value $=0.002)$, and the ROAST test (10000 rotations, $p$ value $<0.001)$. Gene Set 2 also showed decreased expression in $\mathrm{BD}$ patients using the ROAST test (10000 rotations, $p$ value $=0.005$ ).

We expanded our gene sets to include all genes identified in the interaction network. To Gene Set 1, we added CENPN,
NRXN3, PTPRT, and RTN4R. To Gene Set 2, we added NDST4, RTN4R, and MACROD2. Both Expanded Gene Sets showed a significant decrease in expression in BD patients using each of the gene set tests. For Expanded Gene Set 1, the decrease was shown to be significant using t-test based CAMERA test $(p$ value $=.005)$, the Gene Set Test $(10000$ simulations, $p$ value $=0.003)$, and the ROAST test $(10000$ rotations, $p$ value $<0.001)$. Similar results were found for Expanded Gene Set 2 for all three tests: the t-test based CAMERA test $(p$ value $=.008)$, the Gene Set Test $(10000$ simulations, $p$ value $=0.006)$, and the ROAST test $(10000$ rotations, $p$ value $=0.001$.

Conclusions: Our preliminary analysis suggests that two gene sets, each composing an epistatic interaction network, are significantly decreased in expression in $\mathrm{BD}$ subjects. These genes are known to be involved in a range of neuronal functions, including neurogenesis, axonal growth, and signal transduction, and they have been associated with autism and neurodegenerative disorders. We plan to further analyze these genes in additional datasets and in data generated from a RiboZero library preparation of samples from the same subjects. Our results may help elucidate the role of these genes in $\mathrm{BD}$ and provide a better understanding of the implications of epistasis in complex disease.

Keywords: Bipolar Disorder, Epistasis, Gene Set Analysis, RNA Sequencing.

Disclosure: Nothing to disclose.

\section{W81. Higher Trait Impulsivity is Associated With Lower Plasma Interleukin 6 Across Patients With Mood and Anxiety Disorders}

Marijn Lijffijt*, Tabish Iqbal, Sanjay Mathew, Alan Swann

\section{Baylor College of Medicine, Houston, Texas, United States}

Background: Patients with mood or anxiety disorders could have a sustained inflammatory state characterized by higher concentrations of pro-inflammatory cytokines, perhaps mediated by childhood trauma. Mood and anxiety disorders are also marked by elevated impulsivity, a predisposition to reactions before stimuli are fully analyzed and diminished ability to fit actions to environmental needs. Higher impulsivity could reflect a more severe illness-course in mood disorders. Research is limited, but suggests that impulsivity may be associated with higher levels of proinflammatory cytokines. This association may be mediated by childhood trauma.

Methods: We tested those hypotheses in medically healthy patients with a primary diagnosis of major depressive disorder (MDD) with treatment-resistant depression $(n=$ $140)$, symptomatic PTSD $(n=58)$, euthymic bipolar disorder $(n=33)$, or anxiety disorder $(n=8)$; patients did not meet criteria for current substance use disorders or a history of psychosis. Prior to enrollment in ongoing studies, patients were administered the SCID or MINI, completed the Barratt Impulsiveness Scale (BIS-11) and the Childhood Trauma Questionnaire (CTQ), and provided a blood sample for analysis of baseline C-reactive protein (CRP), interleukin 6 (IL-6), and tumor necrosis factor alpha (TNF- $\alpha$ ). General linear models investigated effects of BIS-11 and CTQ total 
scores, age, body mass index (BMI), group, sex, tobacco use, and history of a suicide attempt on CRP, IL-6, or TNF- $\alpha$. Sample sizes differed depending on the variables that were included.

Results: Plasma IL-6 concentration was associated with BIS-11 total score after controlling for the other independent variables $(\mathrm{F}[1,53]=8.38, p=.005)$. This association was due to a negative correlation between BIS-11 total score and IL-6 concentration after controlling for age and BMI $(r=-.35$, $p=.006, N=70)$. No other significant associations were found between impulsivity or childhood trauma and (other) cytokines. BIS-11 total score was also not associated with CTQ total score.

Conclusions: Instead of the expected positive relationship between BIS-11 and pro-inflammatory cytokines, we found a negative relationship, suggesting that in our sample of patients with mood or anxiety disorders, inflammation did not predispose to impulsivity or vice versa. Cytokine levels, impulsivity, and the relationship between impulsivity and IL-6 were not affected by CTQ scores, suggesting that in our sample self-reported childhood trauma did not predispose to inflammation or impulsivity in adults with a mood or anxiety disorder.

Keywords: Mood and Anxiety Disorders, Impulsivity, Immune System, Childhood Adversity.

Disclosure: Nothing to disclose.

\section{W82. SSRI Medication Status Mediates the Relationship Between Acyl Ghrelin and Food Reward Circuitry Activity in Women With Remitted Major Depressive Disorder}

Laura Holsen*, Hilal Cerit, Taryn Hye, Priyanka Moondra, Anne Klibanksi, Jill Goldstein

Harvard Medical School, Boston, Massachusetts, United States

Background: Use of antidepressants has been established to cause modest weight gain, with SSRIs conferring a higher propensity to increase weight compared to other medication classes. The mechanisms behind weight gain in individuals with major depressive disorder (MDD) taking antidepressants remain unresolved, although recent pre-clinical models suggest a potential connection between serotonin pathways and ghrelin, a peripherally-produced orexigenic hormone which acts centrally on mesolimbic regions to enhance the reward value of palatable foods. However, few studies have investigated of the effect of SSRIs on ghrelinergic signaling in individuals with MDD. The aim of the current study was to characterize the relationship between peripheral acyl (active) ghrelin and brain activity in SSRI-treated and unmedicated individuals with remitted MDD. We hypothesized that ghrelin would be associated with food-related reward activity in individuals taking SSRIs, indicating a potential mechanism through which SSRIs lead to weight gain.

Methods: Female participants [34 women with Major Depressive Disorder, in remission: 14 taking SSRI medications (Medicated group); 20 unmedicated (Unmedicated group); matched on age, handedness, and BMI] viewed highcalorie food, low-calorie food, and non-food (household objects) images while undergoing functional MRI (fMRI) scanning on a $3 \mathrm{~T}$ Siemens Trio MR scanner following a 14- hour fast. Blood samples were ascertained prior to the fMRI session (BL). Participants were then asked to consume a standardized meal containing $30 \%$ of the recommended daily caloric intake, with blood draws immediately prior to and 30,60, and 120 minutes following meal onset, and completed a bionutritionist-guided 24- hour recall of food consumed in the 24 hours prior to the visit. Appetite hormones (acyl ghrelin, leptin, glucose, insulin) were analyzed in duplicate with a commercial immunoassay kits. Participants also completed the Beck Depression Inventory (BDI), Spielberger State-Trait Anxiety Inventory (STAI), and appetite ratings using a visual analogue scale. Data analysis: fMRI data were analyzed in SPM8 (contrast of interest: highcalorie food $>$ non-food) using an independent t-test to compare rMDD and HC groups. Regions of interest, based on ghrelin receptor expression, included: ventral tegmental area (VTA), hypothalamus, nucleus accumbens (NAcc), amygdala, and hippocampus. Ghrelin levels were log transformed due to non-normal distribution. Area under the curve was calculated using the trapezoidal method. The standardized meal and free recall report were analyzed for total and macronutrient intake using the Nutritional Data System for Research.

Results: The Medicated group scored marginally higher on the BDI $[\mathrm{t}(32)=1.86, p=0.072]$ and on STAI Trait Anxiety $[\mathrm{t}(32)=2.04, p=0.050]$. There were no group differences on appetite ratings $(t=0.47-1.30, n . s$.$) . In the Medicated group,$ fasting BL ghrelin was positively associated with BOLD activity (high-calorie food $>$ non-food) in the right VTA $(\mathrm{r}=0.56, p=0.037)$, left hypothalamus $(\mathrm{r}=0.65, p=0.012)$, left amygdala $(r=0.74, p=0.002)$, and left hippocampus $(\mathrm{r}=0.63, p=0.016)$. These results held after controlling for BMI (VTA: $r=0.69, p=0.009$; hypothalamus: $r=0.65$, $p=0.017 ;$ amygdala: $\quad \mathrm{r}=0.74, \quad p=0.004 ;$ hippocampus: $\mathrm{r}=0.63, p=0.022$ ), BDI (VTA: $\mathrm{r}=0.66, p=0.015$; hypothalamus: $\mathrm{r}=0.69, \quad p=0.009$; amygdala: $\mathrm{r}=0.72, \quad p=0.005$; hippocampus: $\mathrm{r}=0.57, p=0.042$ ), and STAI Trait (VTA: $\mathrm{r}=0.52, p=0.071$; hypothalamus: $\mathrm{r}=0.62, p=0.025$; amygdala: $r=0.76, p=0.003$; hippocampus: $r=0.68, p=0.010$ ). Additionally, ghrelin AUC was positively associated with BOLD activity in the amygdala $(r=0.72, p=0.004)$ and hippocampus $(\mathrm{r}=0.63, p=0.016)$. Ghrelin (BL, AUC) was unrelated to BOLD activity in the Unmedicated group (I $\mathrm{rl}=0.03-0.27$, n.s.). Medicated $\mathrm{rMDD}$ women exhibited significantly higher postprandial acyl ghrelin compared to unmedicated rMDD women [ghrelin AUC; $\mathrm{t}(32)=2.26$, $p=0.031]$. Groups did not differ on other appetite hormone levels $(\mathrm{t}=0.052-1.24$, n.s.). Women in the Medicated group reported consuming slightly more calories in the 24 hours prior to the visit $[\mathrm{t}(32)=1.83, p=0.08]$ and during the standardized meal, consumed a higher proportion of calories from fat $[\mathrm{t}(31)=1.99, p=0.05]$ and a lower proportion of calories from carbohydrates $[\mathrm{t}(31)=1.99, p=0.55]$, compared to Unmedicated women.

Conclusions: Here we demonstrate that peripheral ghrelin is uniquely related to brain activity in response to palatable food images in individuals with rMDD who were taking SSRIs. In particular, fasting and postprandial ghrelin was positively associated with brain activity in the amygdala and hippocampus in SSRI-treated women, independent of BMI, current depressive symptoms, and trait anxiety. Moreover, 
SSRI-treated women exhibited elevated food intake, particularly high-fat food, in comparison to unmedicated women. These findings provide preliminary evidence that weight and appetite changes during SSRI treatment may be related to ghrelin signaling in neural systems related to food reward, and could inform the development of novel agents to counter these effects.

Keywords: Ghrelin, SSRI, Reward, Major Depression, Appetite.

Disclosure: Nothing to disclose.

\section{W83. A Preliminary Study of Behavioral Change Across} Time Following Social Rejection and Acceptance

David Hsu*, Anjali Sankar, Kristen Villalobos, Benjamin Sanford, Ashley Yttredahl, Erin McRobert, Alison Berent-Spillson

Stony Brook University Medical School, Stony Brook, New York, United States

Background: In a previous study we found that patients with major depressive disorder (MDD) showed overall reduced levels of endogenous opioid release in response to social rejection and acceptance by a desired dating partner, compared to healthy controls. It is possible that reduced opioid release mediated the observed prolonged negative emotions following rejection, and transient positive emotions following acceptance in patients with MDD. We have begun a new study to examine the potential mediating effect of opioid release on alleviating negative emotions and sustaining positive emotions during rejection and acceptance, respectively, in healthy individuals. We report here preliminary data for the behavioral change across time following social rejection and acceptance.

Methods: Ten healthy, romantically single young adults ( 5 women, mean age \pm s.d., $20 \pm 1.5$ years) rated online profiles of preferred-sex individuals that they would like the most. A few days later they were given feedback on a personal computer that they were liked (acceptance trial) or not liked (rejection trial) by their chosen profiles. Neutral trials consisted of a similar visual presentation with indication that others had not yet rated them. Self-reported changes in affect were recorded DURING feedback, and again five minutes AFTER feedback.

Results: Participants reported feeling more "sad and rejected" DURING rejection-neutral $(\mathrm{t} 8=3.9, P=0.005)$ and more "happy and accepted" DURING acceptanceneutral $(\mathrm{t} 7=2.1, P=0.07)$. Five minutes after the end of the feedback, these responses were significantly reduced for the measures "sad and rejected" (DURING[rejectionneutral] - AFTER[rejection-neutral], t8 $=4.9, P=0.001$ ), and "happy and accepted" (DURING[acceptance-neutral] AFTER[acceptance-neutral], $\mathrm{t} 7=3.7, P=0.008$ ).

Conclusions: Successful regulation of negative and positive emotions is essential for mental health. Preliminary data in healthy individuals showed a rapid return to a neutral emotional state following social rejection and acceptance. Studies are underway to relate these changes to endogenous opioid release using the selective mu-opioid receptor radiotracer $[11 \mathrm{C}]$ carfentanil with positron emission tomography (PET).
Keywords: Social Stimuli, Rejection, Endogenous Opioids, Mu-Opioid Receptors, Major Depressive Disorder (MDD). Disclosure: Nothing to disclose.

\section{W84. Sex Differences in the Association Between Aggression and Suicidal Behavior in US Veterans}

Erin McGlade*, Jennifer DiMuzio, Elliott Bueler, Margaret Legarreta, Deborah Yurgelun-Todd

University of Utah, Salt Lake City, Utah, United States

Background: Female veterans are the fastest growing demographic in the US Department of Veterans Affairs and approximately two million of the 21.9 million veterans in the US are female (National Center for Veterans Analysis and Statistics, 2016). Moreover, females are now permitted to serve in all combat roles in the military, making their experiences in the military much more diverse compared to previous generations. Female and male veterans report a host of psychiatric concerns such as mood disorders and suicidal behaviors with prior research showing a strong association between mood, aggression, and suicidal behavior (Gvion \& Apter, 2011). However, these symptoms may be expressed differently in females compared to males, making the study of sex differences in veterans crucial to the development and implementation of clinical treatment. The current study examined between-group sex differences as well as the association between mood symptoms, aggression, and suicide by sex.

Methods: Participants consisted of 205 veterans (female $n=$ 47 , male $n=158$ ) between the ages of 18 and 55. Participants completed well-validated measures of anxiety, depression, suicidal behaviors, and aggression including the Hamilton Depression Scale (HAM-D), Hamilton Anxiety Inventory (HAM-A), Buss-Perry Aggression Questionnaire (BPAQ), and Columbia Suicide Severity Rating Scales (CSSRS). Oneway ANOVA and correlations were analyzed in SPSS to examine between-group differences and within-group correlations for female versus male US veterans.

Results: Female and male veterans differed significantly on aggressive traits with males reporting higher aggression on every scale of the BPAQ. Males reported higher levels of physical aggression $(p=0.05$, males $=23.86$, females $=$ 20.37), verbal aggression $(p=0.001$, males $=14.32$, females $=12.10)$, anger $(p=0.04$, males $=16.37$, females $=$ $14.20)$, hostility $(p=0.03$, males $=19.70$, females $=17.00)$, and total aggression $(p=0.003$, males $=74.24$, females $=$ 63.53) compared to females. Interestingly, aggression was associated with lifetime suicidal behaviors including ideation and attempts differently by group. For males, lifetime suicidal behavior including ideation and attempts was correlated with physical aggression $(p=0.001, \mathrm{r}=0.26)$, verbal aggression $(p=0.04, \mathrm{r}=0.16)$, anger $(p<0.001, \mathrm{r}=$ $0.42)$, hostility $(p<0.001, r=0.46)$, and total aggression $(p<0.001, \mathrm{r}=0.44)$. However, for females, lifetime suicidal ideation was related only to hostility $(p=0.01, \mathrm{r}=0.37)$. For both males and females, lifetime suicidal ideation was correlated with symptoms of anxiety (HAM-A male $p<$ $0.001, \mathrm{r}=0.42$; female $p=0.002, \mathrm{r}=0.44)$ and depression (HAM-D male $p<0.001, \mathrm{r}=0.54$; female $p<0.001$, $r=0.55)$. There were no between-group differences in 
measures of suicidal behaviors or in anxious or depressive symptoms.

Conclusions: Results from the current study suggest important differences in the association between aggressive traits and suicidal behavior by sex with male aggressive traits related to suicidal behaviors, while female aggressive traits were not. Additionally, males and females differed significantly on ratings of aggressive traits but not on measures of suicidal behaviors, anxious, or depressive symptoms. Prior research has shown sex differences in functional connectivity for aggression and orbitofrontal (OFC) regions (McGlade, Rogowska, \& Yurgelun-Todd, 2015) and associations between other OFC mediated tasks (i.e., impulsiveness) and aggression (Archer \& Webb, 2006), suggesting unique neurobiological patterns may underlie the observed clinical differences. These data have significant clinical implications, as males with aggressive traits including urges to strike people, getting into lots of arguments, and trouble controlling one's temper may be more likely to also endorse suicidal ideation and attempts. Similarly, females who endorse high levels of hostility such as feeling like others are talking or laughing at them behind their backs, that others have been more unfairly fortunate, or feeling very bitter/jealous, may be more likely to endorse suicidal ideation or attempts. Additional studies are needed to further explore neurobiological differences underlying these sex differences.

Keywords: Sex Difference, Veterans, Suicide, Aggression. Disclosure: Nothing to disclose.

\section{W85. Effects of Nightly Sleep, Daily Physical Activity, and Shift Work on Daily Mood Under Stress in First-Year Residents}

Yu Fang, David Kalmbach, Todd Arnedt, Amy Cochran, Patricia Deldin, Adam Kaplin, Srijan Sen*

University of Michigan, Ann Arbor, Michigan, United States

Background: While short sleep duration, rotating shift schedules, and physical inactivity are recognized as important problems during residency, objective, real-time changes in these experiences and their relationships with mood are less understood. Here we seek to understand how the change of sleep duration, sleep-wake timing and physical activity interact with the fluctuation of mood during first-year residency.

Methods: Mood of 29 first-year residents from the Intern Health Study's 2014-15 and 2015-16 cohorts was recorded daily by mood 24/7. Objective, real-time assessment of sleep parameters and physical activity of the same participants were measured using accelerometry-based fitbit. Data collection ranged from pre-internship across the first six months of the intern year. Linear mixed models were applied to test the relationships between these measures.

Results: After internship started, first-year residents lost an average of 2.57 hours of sleep per week $(t=-2.52, p=.02)$, with average wake-time 1.42 hours earlier than preinternship baseline $(\mathrm{t}=-3.13, p<.01)$, and had reduced physical activity by $11 \%(\mathrm{t}=-2.76, p=.01)$. Correspondingly, mood decreased by $8 \%(\mathrm{t}=-3.57, p<.01)$. A feedback loop emerged, with a night of short sleep leading to poor mood the next day $(b=.12, p<.001)$, which, in turn, was associated with shorter sleep the next night $(b=.06, p=.04)$. Interns slept less and reported poorer mood when their sleep-wake timing during internship deviated from their personal preinternship baseline, irrespective of the deviation direction. Lastly, interns reported better mood on days with more physical activity $(\mathrm{b}=.03, p<.01)$.

Conclusions: Shift work, sleep deprivation, and physical inactivity confer a challenging environment for intern mental health. Physician mood, sleep duration, and physical activity substantially decrease in the first months of internship, and internship enforces markedly earlier wake times, which are not accommodated by commensurate changes in bedtime. Efforts to increase sleep opportunity, improve exercise-compatibility of the work environment, and design shift schedules allowing for adequate opportunity to resynchronize the circadian system hold promise to improve mood in this depression-vulnerable population.

Keywords: Acute and Chronic Stress, Depression, Residency Training.

Disclosure: Nothing to disclose.

\section{W86. Neurophysiological Mechanisms of Coping Strategies and Their Contribution to Stress Resilience}

Stanley Chen, Michael Vaysblat, Tony Fung, Alec Seidenberg, Ryan Chan, Jennifer Shmukler, Allyson Friedman*

\section{Hunter College, New York, New York, United States}

Background: Coping strategies, either active or passive, have been correlated with responsivity to stress and the development of depressive symptoms. Specifically, effective coping strategies can help build resilience to stress-induced depressive disorders. Given the gender disparity in the occurrence of depression, and variation of coping strategies between genders, it is of therapeutic interest to determine the neurophysiological basis for variable coping styles and how it may influence the differential incidence of stress susceptibility. Healthy mental functioning and resilience to stress induced depression is, in part, maintained by the activity of the brain's dopaminergic pathways, specifically from the dopamine (DA) neurons of the ventral tegmental area (VTA) in the mesolimbic reward circuitry. A critical component of this DA activity is burst firing, which provides salience to positive and negative stimuli. While multiple components regulate this burst firing, extrinsic inputs to the VTA are known to be activated in response to stress, specifically the bed nucleus of the stria terminalis (BNST). The BNST provides both excitatory and inhibitory influences by mediating local VTA GABAergic and dopaminergic neurons in the VTA. The BNST, which expresses sexually dimorphic components, receives direct inputs from the HPA axis and has opposing inputs into the reward center of the brain, making it poised to integrate information regarding sex, stress response and reward.

Methods: Following a defensive burying task, male and female (C57BL/6) mice were separated into groups based on whether they displayed active or passive behavioral coping strategies. This allows for the evaluation of the adaptive nature of response strategy. Behaviors of active burying and 
investigation of a predator odor are considered an active coping strategy, while immobility and avoidance of the stimuli is considered a passive coping strategy. We then evaluated their susceptibility to a 10 day repeated variable social stress (RVSS) model of depression. This is model is entirely based on social stress, and induces a long-lasting behavioral syndrome that includes social avoidance and anhedonia. To perform cell-type and circuit specific analyses, we utilize transgenic mouse lines in combination with retrograding viral markers to provide identification of projection and cell-type specific neurons during electrophysiological recordings.

Results: Coping strategy evaluation revealed a distribution of active copers and passive copers among the genders. Similar to the human population, we found that a significantly higher number of male mice are active copers as compared to female mice. Following the repeated variable social stress model, we found a correlation of active copers and resilience to RVSS in both genders. Using our cell- and projectionspecific technique, we were able to identify and record GABA neurons in the vBNST that project to the VTA. We found a decrease in excitability of these VTA-projecting GABAergic BNST neurons in active coping mice that are resilient to the RVSS paradigm. These results demonstrate that the BNST-VTA circuit may play a role in regulating the response to repeated social stress and can provide novel mechanistic insight into the neural circuits modulating coping style and stress sensitivity.

Conclusions: There is an increasing amount of clinical evidence demonstrating that active stress coping strategies can assist in the development of stress resilience. Importantly, turning passive strategies into more active coping strategies is the foundation for many successful cognitive behavioral-therapies. We have revealed differences in the ionic function of the vBNST GABAergic input to the VTA in active coping mice that may provide resilience to a social stress model of depression. Further investigations into the ionic mechanisms that underlie both passive and active coping styles can assist in identifying novel mechanistic therapeutic targets.

Keywords: Mood Disorders, Coping, Dopamine.

Disclosure: Nothing to disclose.

\section{W87. Sex Differences in the Peripheral Immune System in the Face of Depression}

\section{Ellen Amrock, Richard Shelton, Li Li*}

University of Alabama at Birmingham, Mountain Brook, Alabama, United States

Background: Recent findings suggest a relationship between inflammatory processes and the pathology of depression and suicidality. However, despite the fact that females are twice as likely as males to experience depression, it remains unclear for the role of gender in the link from inflammation to depression and suicide. Thus, the objective of this study was to determine whether inflammatory cytokines were associated with depression, and if females would show greater inflammatory processes that were associated with depressive symptoms.
Methods: The depressive severity was assessed using the Montgomery Åsberg Depression Rating Scale (MADRS) in each patient. Blood sample was collected from all participants for inflammatory factors measurement.

Results: Compared with depressed males, higher rates of suicidal thoughts and pessimism were present in depressed females although their MADRS scores were comparable. Among the cytokines measured, there were not significant differences in depressed males compared to male controls. However, several cytokines, including IL-6 and IL-8, were elevated in depressed females compared to female controls. IL-10 was lower in depressed females. Compared with depressed males, significant elevation in IL-6 was observed in depressed females. IL- 6 was positively correlated with both pessimism and suicidal thoughts in female patients.

Conclusions: Our results indicate that inflammatory processes are associated with depression in females but not males, compared to matched controls. These sex differences in the relationships between inflammatory factors and depression may be particularly important for understanding why depression is twice as likely to occur in females than in males.

Keywords: Sex Difference, Depression, Inflammation. Disclosure: Nothing to disclose.

\section{W88. Risk of Depressive Relapse in Women Undergoing Treatment for Infertility}

Marlene Freeman*, Hang Lee, Gina Savella, Samantha Marfurt, Shannon Murphy, Lee Cohen

Harvard Medical School, Boston, Massachusetts, United States

Background: Mood disorders are prevalent in women of reproductive age, and their course across infertility treatment and implications for clinical management have received little systematic study. The aims of the study were to delineate the predictors of depressive relapse in women with histories of depressive episodes (diagnoses of major depressive disorder or bipolar disorder) during infertility treatment and assess potential biomarkers of risk.

Methods: This was a prospective naturalistic investigation to assess the course of major depressive disorder (MDD) and bipolar depression across treatment for infertility. Participants included women planning or currently undergoing assisted reproductive technology (ART). Patients were prospectively followed for six months, during which they completed 7 study visits and were tracked with respect to: course of mood symptoms, antidepressant and other medication use, psychotherapy, perceived stress, partner support, use of gonadotropins or other hormonal interventions, and all infertility-based treatments and interventions. A subsample participated in the collection of biomarkers.

Results: $N=38$ women completed two or more study visits and were included in the analysis. Of the women in the study with a diagnosis of MDD $(N=25)$, the overall depressive relapse rate was $44 \%$. Among maintainers of antidepressants $(N=15)$, the relapse rate was $40.0 \%$, and among medication discontinuers $(N=10)$ the relapse rate was $50.0 \%$, and these rates were not significantly different. Of the women with diagnoses of bipolar disorder $(N=13)$, the overall relapse 
rate was $4 / 13(30.8 \%)$. Among the medication maintainers $(N=10), 40.0 \%$ relapsed, and among medication discontinuers $(N=3)$, none relapsed. Scores on the Perceived Stress Scale were correlated with relapse risk $(\mathrm{OR}=1.17$ [95\% CI: $1.08-1.26$ ], $p=0.0002)$. C-reactive protein was associated with depressive relapse $(\mathrm{OR}=1.92$ [95\% CI: 1.43-2.55], $p<0.0001$ ), although blood cortisol and interleukin-6 were not.

Conclusions: The risk of relapse among women with mood disorders undergoing infertility treatment is high. Stress and inflammation may contribute to relapse risk. Medication continuation does not necessarily confer adequate protection against relapse to depressive episodes in this context. There is a great need for additional clinical strategies for women with histories of depressive episodes undergoing ART.

Keywords: Women's Mental Health, Mood Disorders, Antidepressants, Infertility Treatment, Biomarkers.

Disclosure: JDS Therapeutics, LLC: Advisory Board, Self; Sunovion Pharmaceuticals Inc.: Advisory Board, Self; JNJ/ Jannsen Pharmaceutica: Independent Data Monitoring Committee, Self; GOED Newsletter: Medical Editing, Self; Takeda Pharmaceutical Company: Research Grant, Self; SAGE Therapeutics: Advisory Board, Self; JayMac Pharmaceuticals: LLC, Research Grant, Self.

\section{W89. Effects of Respiratory-Gated Auricular Vagal Nerve Stimulation in Major Depression}

Ronald Garcia*, Aileen Gabriel, Harlyn Aizley, Riccardo Barbieri, David Gitlin, Vitaly Napadow, Jill Goldstein

Brigham and Women's Hospital, Boston, Massachusetts, United States

Background: The comorbidity of major depressive disorder (MDD) and cardiovascular disease (CVD) will be the number one cause of disability worldwide by 2020 . Thus, understanding shared pathophysiology and development of novel interventions at the neuro-cardiac interface for regulation of mood and cardiac physiology in MDD is urgently needed. Transcutaneous vagus nerve stimulation (tVNS), a non-invasive technique which electrically stimulates the auricular branch of the vagus nerve (ABVN), has shown promising antidepressant effects and modulatory actions on cardiac autonomic physiology. However, responses to tVNS have varied substantially and thus skepticism regarding its efficacy remains. This may be in part due to individual variability in clinical presentation, but may also be due to lower sensitivity of current models of tVNS to vagal regulation. Interestingly, vagal afferent input and its regulatory actions on dorsal medullary nuclei (including nucleus tractus solitarius (NTS)) and higher brain areas are affected by rhythmical oscillations in respiration. In fact, NTS receives inhibitory drive from ventral respiratory group (VRG) medullary neurons during inhalation and firing facilitation during exhalation. Our group is testing whether tVNS gated to exhalation will be more effective in regulating vagal afferent input and thus optimize its modulation of neural circuitry upstream of NTS that is involved in mood and stress response regulation in MDD.
Methods: For preliminary analyses, we report here on the first 7 women with recurrent MDD who were currently experiencing an episode and were unmedicated (31.1 \pm 5.3 years). Functional MRI (fMRI) data were acquired on a Siemens MAGNETOM Skyra 3 T MRI scanner with a 32channel head coil $(\mathrm{TR}=1250 \mathrm{~ms}, \mathrm{TE}=33 \mathrm{~ms}$, slice thickness $2 \mathrm{~mm}$ ). Initially, subjects underwent a mild visual stress challenge task comprising presentation of blocks of negative valence/high arousal, neutral valence/low arousal, and fixation images adapted from the International Affective Picture System. The stress challenge preceded and followed a 20-minute session of respiratory-gated tVNS. Electrodes placed in vagal-innervated ear regions were used to administer electrical stimulation at $30 \mathrm{~Hz}(0.5 \mathrm{~s}$ duration) with current intensity set to achieve moderate (but not painful) sensation. Women were scanned twice within one week to assess modulatory acute effects of tVNS gated to exhalation vs gated to inhalation. fMRI brain activity in response to the task was calculated in a first-level GLM analysis (FEAT, FSL) using a regressor for negative valence/ high arousal images blocks convolved with the canonical hemodynamic response function (Double-Gamma). Results (i.e., parameter estimates and their variance) were transformed into standard space (MNI-152) and submitted to non-parametric permutation analysis (Randomise, FSL) to evaluate the effects of respiratory-gated tVNS on modulation of brain activity (two-sample paired t-test: POST vs. PRE respiratory-gated tVNS) followed by cluster-based correction for multiple comparisons $(p<0.05)$. An electrocardiogram was collected during the fMRI sessions and time intervals between successive $\mathrm{R}$ peaks were calculated. An adaptive point-process algorithm was applied to these recordings to compute variations in instantaneous estimates of the High Frequency component of heart rate variability (HF, 0.15 to $0.4 \mathrm{~Hz}$ ), an indicator of parasympathetic cardiac tone. In addition, a Beck Depression Inventory (BDI) was administered before and after each fMRI session. A paired Student's t-test (STATA 14, Stata Corporation, College Station, TX, USA) was used to evaluate the significant differences in HF values and BDI scores after respiratory-gated tVNS compared to baseline.

Results: Depressed women presented a significantly greater activation in anterior and mid-cingulate cortex, thalamus and anterior insula during the visual stress task following exhalatory-gated stimulation compared to the pre-tVNS task. No significant differences were observed in response to modulation with inhalatory-gated tVNS. In addition, a significant reduction was observed in BDI values after exhalatory-gated tVNS compared to baseline $(25.8 \pm 3.8$ vs $30.6 \pm 4.7, p=0.03$ ), while no differences were observed in response to inhalatory-gated stimulation $(27.6 \pm 4.1 \mathrm{vs}$ $29.6 \pm 6.2, p=0.14)$. Finally, a statistical trend toward significance was observed in normalized HF values after the administration of exhalatory-gated vagal stimulation $(0.54 \pm 0.27$ vs $0.32 \pm 0.25, p=0.056)$, with no apparent effects after inhalatory-gated tVNS $(0.43 \pm 0.32$ vs $0.34 \pm$ $0.28, p=0.27$ ).

Conclusions: Our results indicate a significant modulatory effect of exhalatory-gated tVNS in the cortical response to negative affective stimuli in women experiencing an episode of major depression. Specifically, exhalatory-gated vagal stimulation was significantly associated with increased 
response in cingulate cortex and anterior insula, reflecting an increased cortical control of arousal. Interestingly, we also found an acute reduction in mood symptomatology and a trending increase in vagal tone after the intervention, that will be tested further with increased sample size thus increased statistical power. However, even in this small initial sample, we demonstrated a significant effect of exhalatory- compared with inhalatory-gated vagal stimulation in mood and cardiovascular physiology regulation.

Keywords: Transcutaneous Auricular Vagus Nerve Stimulation (taVNS), Major Depression Disorder, Central Autonomic Network, Functional Neuroimaging, Heart Rate Variability.

Disclosure: Nothing to disclose.

\section{W90. Behavioral Characterization of Conditional Forebrain Knockout of Ankyrin-G and Mechanistic Characterization of the Interplay Between Ankyrin-G and Voltage Gated Calcium Channels}

Shanshan Zhu*, Zachary Cordner, Chi-Tso Chiu, Yan-Ning Zuo, Emmy Cao, Jiali Xiong, Ruoyun Xiong, Xiaofang Wang, Mikhail Pletnikov, Paul Jenkins, Kellie Tamashiro, Christopher Ross

Johns Hopkins University School of Medicine, Baltimore, Maryland, United States

Background: In Genome-Wide association study (GWAS), the ANK3 (Ankyrin-G) and CACNA1C (L-type voltage gated calcium channel alpha subunit, as well as other subunits) loci are among the strongest risk factors for bipolar disorder and schizophrenia. In CNS neurons, Ankyrin-G localizes to the axon initial segment (AIS) and node of Ranvier. AIS is a site for action potential initiation and propagation. Ankyrin-G functions by tethering other AIS components such as voltage gated sodium and potassium channels. In local cortical circuits that are believed to be altered in psychiatric disorders, chandelier cells are fast spiking interneurons that form axo-axonic inhibitory synapses on pyramidal cells AIS, forming GAT-1 and GAD67 positive cartridge-like structures wrapping around their AIS positively stained with Ankyrin-G. Previous studies from David Lewis's lab have found decreased Ankyrin-G label in postmortem schizophrenia cerebral cortex, suggesting loss-of-function effects. Voltage gated calcium channel opens upon membrane depolarization to let calcium ion into neurons. In vitro studies indicate Ankyrin-G and other AIS components are sensitive to calpain hydrolysis. And caplain is a cysteine protease whose activity is dependent on the presence of calcium inside the cell.

Methods: We established a forebrain-specific Ankyrin-G conditional knockout mouse model (CamKII-Cre X FloxAnkG exon 22-23), which deletes all major forms of Ankyrin-G. In our current model, CaMKII-Cre expression, and thus Ankyrin-G knockdown, begins in adulthood forebrain. We characterized behavior, examined the effects of psychiatric medications, and explored the cellular and molecular mechanisms, using double label immunofluorescence. In separate studies, we established an immunofluorescence based AIS quantification method for Ankyrin-G in primary neurons. By pharmacological modulation, we explored how voltage gated calcium channel may affect Ankyrin-G level at AIS of primary cultured neurons via quantifying Ankyrin-G immunofluoresce labeling at AIS of primary cultured neurons.

Results: Ankyrin-G knockout mice displayed increased motor activity in the open field apparatus, increased exploratory activity and less anxiety-like behavior in the elevated plus maze, but only mild cognitive deficit in the Y-maze, no PPI deficit and increased locomotor activity to methylphenidate, reminiscent of "manic"-like phase of affective disorder. Treatment with anti-mania agents, including chronic Lithium and Valproic Acid at therapeutic levels, ameliorated the hyperactivity. Mice showed "depression" like behavior after social defeat stress. The mice showed "cycle" - like switch between hyperactivity and "depression"-like behavior upon repeated social defeat. Heterozygous knockout mice displayed subtle behavioral alterations, but were more sensitive to sub-threshold social defeat. Sodium and potassium channel were diminished from AIS. Chandelier GABA interneuron cartridge structures on pyramidal neuron axon initial segment dramatically decreased in the Ankyrin - $\mathrm{G}$ KO mice. In the primary neuron experiments, Ankyrin-G immunolabeling at AIS was significantly decreased via calcium channel opener$\mathrm{KCl}+\mathrm{Bayk} 8644$ in a calcium ion dependent manner. This decrease could be rescued via calpain inhibitor. This result indicates a pathway calcium channel open $\rightarrow$ calpain active $\rightarrow$ Ankyrin-G degradation.

Conclusions: Our forebrain-specific Ankyrin-G conditional knockout mice may be useful as a model of aspects of the brain changes due to alterations at the ANK3 locus, associated with bipolar disorder and schizophrenia. The striking alterations of GABA-positive cartridges on the pyramidal axon initial segments suggest that, in this potential model of psychiatric disease, there can be rewiring of cortical microcircuitry even in adulthood. This mouse model may help elucidate psychiatric disease pathophysiology, facilitate development of tools to explore the mechanisms of psychiatric drugs, and make possible the testing of new therapeutic strategies for bipolar and schizophrenia. The interplay between calcium channels and Ankyrin-G may help to elucidate some overlapping phenotypes between schizophrenia and bipolar disorder, which may be caused by developmental and brain region specific dysregulation.

Keywords: Ankryin-G, Voltage-Gated Calcium Channel, Bipolar Disorder, Animal Model.

Disclosure: There are relevant financial interests to disclose. Poster Disclosure Janssen Pharmaceutical, this work is financially supported by Janssen Pharmaceutical, Self.

\section{W91. Lurasidone for the Treatment of Major Depressive Disorder With Mixed Features: Do Manic Symptoms Moderate Treatment Response?}

Joseph Goldberg*, Yongcai Mao, Cynthia Siu, Joyce Tsai, Andrei Pikalov, Joseph Calabrese, Anthony Loebel

Icahn School of Medicine at Mount Sinai, Norwalk, Connecticut, United States

Background: Manic symptoms below the threshold for hypomania (mixed features) are common in individuals with 
$\overline{S 516}$

major depressive disorder. This form of depression is often severe and associated with an increased risk for recurrence, suicide attempts, substance abuse, and functional disability. This post-hoc analysis evaluated whether the efficacy of lurasidone in major depressive disorder (MDD) with mixed features is moderated by the number and characteristics of manic symptoms present at study baseline.

Methods: Patients meeting DSM-IV-TR criteria for MDD who presented with two or three manic symptoms (consistent with the DSM-5 mixed features specifier) were randomly assigned to 6 weeks of double-blind treatment with either lurasidone $20-60 \mathrm{mg} / \mathrm{d}(N=109)$ or placebo $(N=100)$. Three non-specific mood symptoms (irritability, distractibility and aggressive behavior) were treated as manic symptoms for the purpose of this analysis; these were examined together with the seven DSM-5 specified manic symptoms. Finite mixture models were applied to identify latent class patterns of these 10 baseline manic symptoms. Changes from baseline in Montgomery-Åsberg Depression Rating Scale score (MADRS), Clinical Global Impressions severity score (CGI-S), and Young Mania Rating Scale score (YMRS) were evaluated using ANCOVA and a mixed model for repeated-measures analysis.

Results: At baseline, 32.5\% (68/209) patients had 2 or3 manic symptoms (mean MADRS $=33.0$ mean YMRS $=9.5$ ), $38.3 \%$ $(80 / 209)$ had 4 manic symptoms (mean MADRS $=33.6$ mean YMRS =10.6), and $29.2 \%(61 / 209)$ had $\geq 5$ manic symptoms (mean MADRS $=33.1$; mean YMRS $=12.1$ ). The most frequent $(>=40 \%)$ manic symptoms present at study entry were flight of ideas (66.5\%), irritability (56.9\%), pressured speech $(61.2 \%)$, distractibility (59.3\%), and decreased need for sleep (41.1\%). Three latent class profiles were identified based on all baseline manic symptoms: 105 (50.5\%) patients had manic symptom profile 1 (MIX 1) with mean MADRS 33.0, mean YMRS 9.2, mean number of manic symptoms $=3.8$ (dominated by flight of ideas and pressured speech and low prevalence of the other 5 core DSM-5 manic symptoms); 63 (30.3\%) patients had manic symptom profile 2 (MIX 2) with similar baseline mean MADRS and YMRS scores and lower number of manic symptoms; 40 patients had manic symptom profile 3 (MIX 3) with significantly higher severity scores in MADRS and YMRS and mean number of manic symptoms (all $p<0.05$, comparisons between the 3 profile groups). A significant moderating effect on change in YMRS score was observed for the symptom of "decreased need for sleep," with a greater lurasidone effect size (vs. placebo) found in patients without ( -7.4 vs. $-4.5, P<0.001)$ vs. with this symptom $(-6.1$ vs. $-5.8, p=\mathrm{NS}) \quad(p<0.05$ for moderating effect $)$. No significant moderating effects were found for the other nine manic symptoms for the week 6 changes in MADRS, YMRS and CGI-S scores.

Conclusions: In this post-hoc analysis of a placebocontrolled trial involving MDD patients with mixed features, absence of "decreased need for sleep" was found to be significantly associated with improvement in manic and depressive symptoms and to moderate the treatment effect on manic symptoms. Baseline manic symptom profiles can be identified in this patient population.

Keywords: Major Depressive Disorder (MDD), Lurasidone, Mixed Features, Mania.

Disclosure: Sunovion Pharmaceuticals, Consultant, Speakers Bureau, Self.
W92. Inflammation, Kynurenine Metabolism, and Depressed Mood: A Human Experimental Study

Hyong Jin $\mathrm{Cho}^{*}$, Jennifer Kruse, Naomi Eisenberger, Michael Irwin

University of California, Los Angeles, California, United States

Background: Kynurenine metabolism is increasingly recognized as a key neurochemical pathway in the link between inflammation and depression. Inflammatory mediators activate indoleamine 2,3 dioxygenase (IDO), an enzyme that metabolizes tryptophan (TRP) to kynurenine (KYN), hence increasing the ratio KYN/TRP. Further metabolic steps lead to an increase in quinolinic acid (QA), a potentially neurotoxic metabolite, and a decrease in kynurenic acid (KynA), a potentially neuroprotective compound. These changes are hypothesized to induce depression. However, the link between inflammation, kynurenine metabolism, and depression has never been examined in human experimental studies. This study examined, in healthy volunteers, 1) whether an experimentally administered inflammatory challenge (i.e., endotoxin) induces changes in kynurenine metabolism, 2) whether these changes correlate with inflammation-induced depressed mood, and 3) whether there are sex differences in these correlations.

Methods: In a randomized double-blind placebo-controlled design, participants aged 18-50 ( $N=115 ; 69$ females) were assigned to placebo or low-dose endotoxin ( $0.8 \mathrm{ng} / \mathrm{kg}$ of body weight, E. coli group O:113), which increases proinflammatory cytokine levels in a safe manner. Circulating proinflammatory cytokines (interleukin-6 [IL-6], tumor necrosis factor- $\alpha[$ TNF- $\alpha]$ ), circulating kynurenine metabolites (TRP, KYN, QA, KynA), and self-reported depressed mood (Profile of Mood States) were assessed over 6 hours, at baseline (T0), 2 hours (T2), and 6 hours (T6). The primary outcomes were IDO activity assessed using KYN/TRP and the putative neurotoxic index calculated as QA/KynA. For the correlation between IDO activity and depressed mood, baseline and T2 (when depressed mood peaked) were considered. Mixedeffects model regression was conducted.

Results: Participants exposed to endotoxin, compared with placebo, showed greater increases in IDO activity over time (KYN/TRP: $\mathrm{X}^{2}=95.3, \mathrm{df}=2, p<0.0001$ ) but not in neurotoxic index (QA/KynA: $\left.\mathrm{X}^{2}=3.4, \mathrm{df}=2, p=0.18\right)$. Among endotoxin-exposed participants $(N=61 ; 38$ females $)$, the correlation between IDO activity and depressed mood was highly significant (adjusted regression coefficient 90.2, $p=0.004)$. While IDO activity increased equally in females and males, the correlation between IDO activity and depressed mood was substantially stronger in females (adjusted regression coefficient 157.5, $P=0.001$ ) compared to males (adjusted regression coefficient 28.9, $P=0.34$ ), with a significant moderation (adjusted regression coefficient 123.7, $P=0.04)$. As reported by our previous study, participants exposed to endotoxin (vs. placebo) also showed greater increases in proinflammatory cytokines (IL-6: $\mathrm{X}^{2}=485.7, \mathrm{df}=2, \quad p<0.0001 ; \mathrm{TNF}-\alpha: \mathrm{X}^{2}=1050.4, \mathrm{df}=2$, $p<0.0001)$ and in depressed $\operatorname{mood}\left(\mathrm{X}^{2}=20.7, \mathrm{df}=2\right.$, $p<0.0001$ ); furthermore, females (vs. males) presented with greater depressive responses to endotoxin $\left(\mathrm{X}^{2}=19.1, \mathrm{df}=2\right.$, $p=0.0001)$. 
Conclusions: This study is the first to demonstrate a link between inflammation, kynurenine metabolism, and depression in humans using an experimental design. Inflammatory challenge increased IDO activity, which in turn was significantly correlated with inflammation-induced depressed mood. Previously reported sex differences in inflammationinduced depression appeared due in part to an augmented affective sensitivity to kynurenine metabolism changes in females.

Keywords: Inflammation, Kynurenine Metabolism, Depression, Sex Differences, Experimental Design.

Disclosure: Nothing to disclose.

\section{W93. Biobehavioral Prediction of Relapse in Major Depression: A Prospective, Multicenter, Observational Study}

Madhukar Trivedi*, Randall Morrison, Ella Daly, Jaskaran B. Singh, Maggie Fedgchin, Carol Jamieson, Kimberly Cooper, Wayne Drevets, Narayan Vaibhav A

University of Texas Southwestern Medical Center at Dallas, Dallas, Texas, United States

Background: The course of major depressive disorder (MDD) is often characterized by recurrent episodes. Furthermore, despite appropriate antidepressant treatment, many patients experience relapse following initial short-term improvement or remission. Considering the difficulty to accurately identify the onset of relapse, early identification of patients at high risk of impending relapse is imperative to initiate appropriate preventive measures. This observational study assesses a battery of behavioral and multi-modal biomarkers as precursors of relapse and also evaluates implications of methodological variability in defining relapse.

Methods: OBSERVEMDD is an ongoing, prospective, multicenter, longitudinal, observational study, designed to identify biobehavioral predictors of relapse in MDD patients. The patients, aged 18-64 years, with nonpsychotic, recurrent MDD according to the Diagnostic and Statistical Manual of Mental Disorders, Fifth Edition (DSM-5) criteria (with the most recent depressive episode occurring $\leq 24$ months before screening), who had responded to (within past 3 months) and continued to receive an oral antidepressant treatment regimen, with Montgomery-Asberg Depression Rating Scale (MADRS) total score of $\leq 14$ at screening, were enrolled. During the study, patients undergo weekly assessment of symptoms for depression (16-item Quick Inventory of Depressive Symptomatology-Self-Report [QIDS-SR16], anxiety (7-item Generalized Anxiety Disorder Scale [GAD-7]) and sleep (Medical Outcomes Study Sleep Scale-Revised [MOS Sleep-R]). Periodic assessments of health-related quality of life and functioning (EuroQol Group; 5-Dimension; 5-Level [EQ-5D-5 L] and 12-item World Health Organization Disability Assessment Schedule 2.0 [WHODAS 2.0]) were also performed. These assessments are conducted on a study-dedicated smartphone device. Speech and voice measurements are also collected at 2-week intervals. Clinician ratings (MADRS) and assessments of automatic cognitive processes (Depression - Implicit Association Test), as well as blood and saliva samples for biomarker assays are collected during clinic visits at 8-week intervals and at the time of relapse. The resulting dataset is intended to allow unique, predictive analyses of near-term relapse in a wellcharacterized sample of MDD patients.

To observe relapse in 150 patients, 330 patients were planned to be enrolled for prospective follow-up. The outcome of two definitions of relapse is reported in this interim analysis: Group 1, based on MADRS (requires two consecutive MADRS ratings of $\geq 22$ within approximately 1 to 2 weeks) and Group 2, based on the QIDS-SR16 (two successive QIDS-SR16 scores of $\geq 11$ required for 2 consecutive weeks). The recall period was 7 days for both MADRS and QIDS-SR16 scores.

Results: The study was initiated in December 2014 and as of July 2016, a total of 261 patients were enrolled. The mean (SD) age of enrolled patients was 42.8 (13.0) years and the majority of them were women (194 patients [74.3\%]). According to the relapse definition based on MADRS (Group 1), 21 (8.0\%) of 261 patients enrolled experienced relapse. Based on the QIDS-SR16 definition (Group 2), 47 (19.3\%) patients met criteria for relapse out of 244 patients assessed (17 patients with a baseline QIDS-SR16 score of $\geq 11$ were excluded) and 11 patients met both relapse criteria. The two groups of relapse patients did not differ significantly in their baseline characteristics (age, sex, body mass index, race, ethnicity, age of onset of MDD, duration of current episode and family history of depression). The mean (SD) age was similar between both groups (Group 1: 44.5 [11.4] years, Group 2: 42.8 [13.3] years). The majority of patients in both groups were women (Group 1: 18 [85.7\%]; Group 2: 39 [83.0\%]). The mean (SD) baseline MADRS total score of Group 1 was 7.4 (4.65) and Group 2 was 8.3 (3.71), while QIDS-SR16, total scores were 6.7 (4.33) for Group 1 and 6.9 (2.77) for Group 2. The mean (SD) duration of follow-up before the relapse event for Group 1 was 153.3 (97.2) days and Group 2 was 56.5 (66.8) days. Patients in both the groups were normal to mildly ill according to the Clinical Global Impression-Severity scale (CGI-S).

Further analyses comparing patients with relapse to those without relapse will be presented including biomarkers, speech and voice, patient-reported outcomes and cognitive measures. Additionally, predictive models of relapse will also be presented, in which variables predictive of impending relapse will be identified.

Conclusions: The variability in occurrence of relapse $(8.0 \%$ Group 1, MADRS-based and 19.3\% Group 2, QIDS-SR16based) observed in this study using two different definitions of relapse, highlights the necessity to better characterize this event and its predictors. Predictive models of relapse in early identification of patients at risk of near-term relapse thus identified can help initiate prompt preventive interventions. Keywords: MADRS, Major Depressive Disorder, relapse, predictors, QIDS-SR16.

Disclosure: Johnson \& Johnson, Janssen Research \& Development: Advisor/Consultant \& Research Support, Self; Alkermes Inc.: Advisor/Consultant, Self; Arcadia Pharmaceuticals Inc.: Advisor/Consultant, Self; AstraZeneca: Advisor/Consultant, Self; Medscape: Advisor/Consultant, Self; Eli Lilly \& Company: Advisor/Consultant, Self; Lundbeck: Advisor/Consultant, Self; Naurex: Advisor/Consultant, Self; Neuronetics: Advisor/Consultant, Self; Ostuka Pharmaceuticals: Advisor/Consultant, Self; Pfizer Inc.: Advisor/ 
Consultant, Self; Takeda Pharmaceuticals Inc.: Advisor/ Consultant, Self; SHIRE Development: Advisor/Consultant, Self.

\section{W94. Sphingosine-1-Phosphate 3 Receptors in the Medial Prefrontal Cortex Promote Behavioral Resilience to Chronic Stress}

Brian Corbett, Seema Bhatnagar*

University of Pennsylvania, Philadelphia, Pennsylvania, United States

Background: Repeated exposure to stress promotes the development of psychiatric disorders, including depression and posttraumatic stress disorder. However, not all stressed individuals go on to develop such psychiatric disorders. That is, some individuals are resilient to the effects of stress and others are vulnerable to its effects. Relatively little is known about the neural substrates that differentiate vulnerable from resilient individuals. In a paradigm of chronic social defeat in rats, we have identified subpopulations that are resilient or vulnerable to the neuroendocrine and behavioral effects of chronic social defeat. Rats that exhibit a short defeat latency (SL) following placement in the cage of an aggressive resident rat are vulnerable to the effects of stress whereas rats that exhibit longer defeat latencies (LL) are resilient to the effects of stress. SL rats display increased depressive-like and anxiety-like behaviors compared to LL rats. In the studies described here, our first goal was to identify a novel substrate mediating vulnerability or resilience. Using a PCR array approach, we determined that sphingosine-1phosphate-3 receptor (S1PR3) in the medial prefrontal cortex (mPFC) was elevated in resilient vs. vulnerable rats. S1PR3 is a G-protein-coupled receptor (GPCR) that reduces inflammation and, as an S1PR, has the ability to reduce neuronal activity through $\mathrm{Gi}$ coupling and activation of G-protein-coupled inwardly rectifying potassium channels. Based in these results, we hypothesized that elevated expression of this receptor in the mPFC promotes resilience. We tested this hypotheses using viral-mediated over-expression or knockdown of these receptors in rats undergoing chronic social defeat.

Methods: In separate experiments in adult male SpragueDawley rats, we used a viral vector to overexpress S1PR3 (AAV2-CMV-S1PR3-eGFP) in the mPFC or siRNA directed towards S1PR3 in the $\mathrm{mPFC}$ or appropriate control virus in rats undergoing defeat or no defeat. Rats were injected with virus specifically in the $\mathrm{mPFC}$ (Bregma $+3.2 \mathrm{~mm}$, midline, $4.4 \mathrm{~mm}$ ventral), allowed to recover (28 days for overexpression, 7 days for knockdown), and exposed to social defeat in the resident-intruder paradigm for 5 days. Then rats were tested in the social interaction test on day 6 and forced swim test on days 7-8. On day 9, rats were exposed to $30 \mathrm{~min}$ restraint and blood collected for measurement of hormones. Brains were collected and processed to confirm viral expression and to assess neural activity in regions downstream from the mPFC.

Results: S1PR3 overexpression in the mPFC increased latencies to be defeated, increased social interaction and reduced immobility in the forced swim test compared to controls indicating reductions in depressive-like and anxiety- like behavior compared to controls. Conversely, virally mediated S1PR3 knockdown decreased latencies to be defeated and increased depressive-like and anxiety-like behavior. Similar to resilient LL rats in our previous studies, rats overexpressing S1PR3 in the $\mathrm{mPFC}$ also displayed appropriate facilitated ACTH responses to novel restraint compared to controls. In contrast, S1PR3 knockdown rats displayed insufficient ACTH responses during restraint compared to controls. Neuronal activity in the mPFC was decreased in S1PR3-overexpressing rats and increased in S1PR3 knockdown rats. Furthermore, overexpression of S1PR3 in the mPFC decreased neuronal activity in response to restraint in regions downstream from the $\mathrm{mPFC}$, including the bed nucleus of the stria terminalis and paraventricular hypothalamus.

Conclusions: Based on these results, we propose that S1PR3s in the $\mathrm{mPFC}$ promote resilience to the adverse effects of stress by reducing activity in the $\mathrm{MPFC}$ and within its extended network. We are currently exploring whether reductions in immune/inflammatory processes by S1PR3 over-expression also contribute to resilience since the canonical effects of S1PRs and their endogenous ligands is on immune/inflammatory systems. We conclude that the S1PR3 are a novel class of receptors that promote behaviors and neuroendocrine responses characteristic of resilient individuals.

Keywords: Resilience, Drugs, G Protein Coupled Receptors, Medial Prefrontal Cortex.

Disclosure: Nothing to disclose.

\section{W95. Nitrous Oxide Alters Hippocampal Neurogenesis? A Potential Role for Treating Depression}

Farah Chamaa, Kareem Makkawi, Elie Al-Chaer, George Bikhazi, Ziad Nahas*, Wassim Abu-Kheir

American University of Beirut, Riad El-Solh, Beirut, Lebanon

Background: Treatment resistant major depression (TRMD) is common, accounts for the high morbidity and mortality, and underscores why the World Health Organization projects depression to rank second in the global burden of disease. Subsequently, the identification of distinct and more efficacious antidepressant strategies are highly needed. A novel treatment potential has relied on inhibiting the $\mathrm{N}$-methyl-D-aspartate glutamate receptors (NMDA) and been mostly investigated through the use of ketamine. Another antagonist with a different mechanism of action is nitrous oxide (N2O, laughing gas) (Nagele et al.) In this study, we aim to test for the effect of $\mathrm{N} 2 \mathrm{O}$ exposure on hippocampal neurogenesis in adult brain rats.

Methods: Adult Sprague-Dawley rats were exposed to onehour inhalation of nitrous oxide (70\% nitrous oxide/ $30 \%$ oxygen) or $30 \%$ oxygen only as sham. Rats were injected intraperitoneally with BrdU, a marker of stem/progenitor cells proliferation, right before and after the inhalation period. Twenty-four hours and 7 days after the last injection, their brains were perfused, fixed and cut at $40 \mu \mathrm{m}$ sections to collect the hippocampus using the fractionator method. The cuts were separated into rostral, intermediate and caudal regions of the hippocampus. 
Results: Preliminary data has shown that 1- hour exposure to $\mathrm{N} 2 \mathrm{O}$ was sufficient to induce a surge in BrdU-positive cells at the level of the hippocampus 7 days post inhalation period. While the rates of hippocampal neurogenesis were comparable between the nitrous oxide and the sham group at day 1 , proliferation levels increased by $40 \%$ at day 7 . The effect size was 0.7

Conclusions: This study is the first to investigate the possible influence of $\mathrm{N} 2 \mathrm{O}$ on hippocampal neurogenesis after one hour of inhalation. Future studies should investigate increased nitrous oxide exposures and their antidepressant behavioral correlates.

Keywords: Antidepressant, Neurogenesis, Nitrous Oxide, Anesthetic, Depression.

Disclosure: Nothing to disclose.

\section{W96. Inflammation and Response to ECT in Depression}

Jennifer Kruse*, Eliza Congdon, Stephanie Njau, Elizabeth Breen, Richard Olmstead, Katherine Narr, Randall Espinoza, Michael Irwin

University of California, Los Angeles, California, United States

Background: Inflammation is associated with depression and is implicated as a causal factor in some types of depression, including interferon- $\alpha$ induced depression, or depressive symptoms elicited by experimental administration of endotoxin. Those with an "inflammatory subtype" of depression may differ in response to treatment. Studies demonstrate that depressed patients with elevated inflammation may respond less robustly to antidepressant medications, but this has not been studied with regard to electroconvulsive therapy (ECT). ECT has been found to alter cytokine levels, yet no study has evaluated whether levels of inflammation may predict response to ECT. There is a need for biological markers that can better predict treatment response. The purpose of this study was to investigate whether baseline levels of cytokines may predict clinical outcome in depressed patients treated with ECT. Further, given evidence that ECT alters levels of cytokines, we secondarily aimed to evaluate whether changes in cytokines associate with clinical outcome.

Methods: Subjects were depressed patients ( $n=30,14$ males, 16 females; mean age 43) receiving ECT at the University of California, Los Angeles (UCLA) Resnick Neuropsychiatric Hospital. We administered the Hamilton (HAM-D-17) and Montgomery-Asberg Depression Rating Scales (MADRS), which established response to treatment, as well as the Quick Inventory of Depressive Symptomatology (QIDS) scale, and sampled plasma concentrations of the pro-inflammatory cytokines TNF- $\alpha$, interleukin (IL)-6, IL- 8 , and IL- $1 \beta$, and the anti-inflammatory cytokine IL-10, before ECT (T1), after the second ECT session (T2) and at the completion of the index treatment series (T3). Statistical analysis was pursued via set wise regression analysis to control for multiple predictor variables while avoiding over-fitting the model with too many predictors at once. Five sets of predictor variables were evaluated in individual regression analyses: demographic variables (age, gender, body mass index), depression chronicity variables (duration of current episode, duration of lifetime illness, age at first episode, age at depression diagnosis), baseline cytokines (TNF- $\alpha$, IL-6, IL-10 and IL-8), short term cytokine change (T2 - T1), and long term cytokine change (T3 - T1), with final (T3) MADRS and QIDS scores as the outcomes of interest. Any predictor variable from the first five sets that suggested the possibility of a contribution to clinical outcome $(p<0.15)$ was carried forward into a subsequent regression analysis. From that regression analysis, predictor variables that meaningfully contributed to the model (beta $>0.2$ ) were carried forward into a final regression, with the goal of constructing the most robust predictive model possible with the available data. In follow-up analyses, the General Linear Mixed Model (GLMM) was used to determine if change in cytokine levels differ in ECT responders versus non-responders, with time modeled as a repeated measure. Discriminant function analysis was also completed, to evaluate the extent to which predictor variables identified via regression analyses were able to predict responder subgroup.

Results: Baseline IL-6 levels, but not demographic variables, nor indices of depression chronicity, were shown to predict subsequent clinical outcome after ECT. In the regression analysis evaluating baseline cytokines as predictors of final MADRS and QIDS scores, higher baseline IL-6 was associated with lower final (T3) MADRS ( $p=0.05$; beta $=$ $-0.411)$ and QIDS $(p=0.041$; beta $=-0.415)$ scores. Variables with any suggestion of an effect $(p<0.15)$ on final MADRS or QIDS scores from the initial 5 sets of regression analyses included BMI ( $p=0.143$ for MADRS), baseline IL-6 ( $p=0.05$ for MADRS, $p=0.041$ for QIDS), short term IL-8 change ( $p=0.096$ for MADRS), and longer-term IL-10 change $(p=0.049$ for MADRS). These variables were together evaluated in a subsequent regression analysis as predictor variables for clinical outcome (final MADRS and QIDS scores). From this regression analysis, variables that meaningfully contributed to the model (beta values of $>0.2$ for any measure) were evaluated in a final regression analysis. Predictor variables in the final regression included baseline IL-6, short-term IL-8 change, and long-term IL-10 change. This regression model was significant with regard to predicting both final MADRS $(p=0.01)$ and QIDS $(p=0.043)$ scores. The only individual predictor that was significant in the final regression was baseline IL-6, for QIDS only $(p=0.043)$. A discriminant function analysis demonstrated that baseline IL-6, short-term IL-8 change, and longterm IL-10 change together were able to predict $73.3 \%$ of responders/non-responders correctly, with $79 \%$ sensitivity and $64 \%$ specificity. Follow-up analyses in treatment responders and non-responders showed that IL-6 levels were elevated in responders compared to non-responders $(p=0.005)$, and that IL-10 levels decreased in nonresponders over the course of treatment $(p=0.041)$.

Conclusions: Baseline IL- 6 was a statistically significant predictor of clinical outcome in depressed patients treated with ECT, whereas usual clinical predictors such as age and depression chronicity were not useful predictors. Interestingly, elevated IL-6 predicted improved clinical outcome, contrary to studies of antidepressant medications in which elevated inflammation generally predicts poorer outcome. This suggests that elevated inflammation may promote antidepressant response to ECT. Further, in exploratory analyses, a model incorporating baseline IL-6, short-term 
IL-8 change, and long-term IL-10 change predicted responder status with $79 \%$ sensitivity and $64 \%$ specificity. Inflammatory biomarkers may help guide more effective and targeted individualized treatment strategies for depression in the future.

Keywords: Inflammation, Depression, Electroconvulsive Therapy.

Disclosure: Nothing to disclose.

\section{W97. Effects of Repeated Ketamine Infusions on Suicidal Ideation in Treatment-Resistant Depression}

Jennifer L. Phillips, Sandhaya Norris, Jeanne Talbot, Abigail Ortiz, Pierre Blier*

University of Ottawa Institute of Mental Health Research, Ottawa, Canada

Background: Suicidal ideation (SI) is a common feature in patients with major depressive disorder (MDD), yet despite its prevalence, few treatments are available. Administration of subanaesthetic doses of intravenous ketamine in patients with treatment-resistant depression has been shown to elicit rapid, albeit transient suppression of SI and reduction in depressive symptoms. Repeated ketamine administration may prolong its anti-suicide and antidepressant effects. The objectives of this study were first, to determine whether repeated ketamine infusions could elicit decrease in SI even in patients who do not have an antidepressant response, and secondly, to determine whether changes in SI with repeated ketamine infusions can be detected using both clinician and patient-administered measures.

Methods: Twenty-six patients with treatment-resistant MDD (mean age 42.5 years, 52\% males) completed a randomized controlled trial of intravenous ketamine. Inclusion criteria required a primary diagnosis of MDD, evidence of treatment-resistance and a Montgomery-Åsberg Depression Rating Scale (MADRS) score $\geq 25$. Treatment resistance was defined as failure to respond to at least two antidepressant medications of different pharmacological classes plus two augmentation strategies in the current depressive episode. Patients received 7 subanaesthetic $(0.5 \mathrm{mg} / \mathrm{kg}$ over 40 minutes) intravenous ketamine infusions during the study. A single infusion was administered during the Phase I double-blind crossover with midazolam $(35 \mu \mathrm{g} / \mathrm{kg}$ over 40 minutes, used as an active placebo), and 6 open-label ketamine infusions were administered over a two-week period during Phase 2. The primary outcome measures were changes in SI from baseline (prior to the first infusion in Phase I) to follow-up (after administration of the 7 th ketamine infusion at the end of Phase II). SI was assessed using the suicide item of the clinician-administered MADRS (item 10, MADRS-SI), the self-administered Scale for Suicide Ideation (SSI5, summing screening items 1-5), and the Quick Inventory of Depressive Symptomatology-Self Report suicide item (item 12, QIDS-SI). Change scores for each measure were calculated as follow-up value minus baseline value.

Results: Overall, patients had significant decreases in SI scores from baseline to follow-up as assessed with all three suicide measures. Patients had an average reduction in MADRS-SI ratings of -2.0 points on a $0-6$ point scale during the study. A repeated measures analysis of covariance of baseline and follow-up MADRS-SI scores was significant when change scores in non-suicide-related MADRS items (MADRS items 1-9) were entered as a covariate $(\mathrm{F} 1,25=$ $12.87, p=0.001)$. This suggests that the anti-suicide effects of ketamine are not mediated by reduction in depressive symptoms. Both ketamine responders (defined as patients who achieved $\mathrm{a} \geq 50 \%$ reduction in total MADRS scores from baseline to follow-up; $n=15)$ and nonresponders $(n=$ 11) had significant decreases in MADRS-SI scores over follow-up (responders -2.4 points, $\mathrm{t} 14=7.86, p<0.001$; nonresponders -1.5 points, $\mathrm{t} 10=5.88, p<0.001)$. Patients also had significant reductions in SI scores with repeated ketamine treatment according to the self-administered SSI5 $(\mathrm{t} 24=4.40, p<0.001)$ and the QIDS-SI $(\mathrm{t} 25=4.81, p<$ 0.001). Pearson correlation analyses revealed significant positive correlations among the three suicide assessment measures (all significant at $p<0.001$ ).

Conclusions: In this preliminary study, repeated doses of ketamine decreased SI in patients with treatment-resistant depression. While ketamine treatment did not elicit an antidepressant response in all patients in this sample, the majority had a suppression of SI as assessed using both clinician and self-administered measures. Such findings suggest that ketamine has a direct effect on abating SI independent of its antidepressant action.

Keywords: Ketamine, Suicidal Ideation, Major Depressive Disorder (MDD).

Disclosure: Janssen: Consultant, Self; Janssen: Clinical Trial Participation, Self.

\section{W98. N6-Methyladenine is an Epigenetic Marker of Early Life Stress}

Stacey Kigar*, Liza Chang, Candace Guerrero, Jacqueline Sehring, Amelia Cuarenta, Laurie Parker, Anthony Auger, Vaishali Bakshi

National Institute of Mental Health, Bethesda, Maryland, United States

Background: 6-methyladenine (6mA) was characterized earlier this year for the first time in mammalian stem cells and a variety of cell lines, and there its function as an epigenetic modification was established. This work built upon a series of 2015 publications demonstrating the presence and epigenetic influence of $6 \mathrm{~mA}$ in a variety of multi-cellular species. These discoveries herald a major paradigm shift, as $6 \mathrm{~mA}$ was previously thought to exist exclusively in bacteria and some unicellular organisms. Given the environmentally-sensitive nature of this modification in bacteria, and data suggesting that $6 \mathrm{~mA}$ is enriched in adult brain tissue of Drosophila, we hypothesized that $6 \mathrm{~mA}$ might be present and modified by early life stress and biological sex in the mammalian brain. To our knowledge, this possibility has not been addressed elsewhere in the literature. We focused on 5-hydroxytryptamine (serotonin) receptor $2 \mathrm{a}(\mathrm{Htr} 2 \mathrm{a})$ expression given a known role for this receptor in the development of anxiogenic behavior, and focused on the effects of neonatal predator odor-induced stress on the juvenile rodent amygdala given this region's sensitivity to olfactory-based threat. 
Methods: We developed a variable predator odor exposure (POE) stress paradigm for neonatal Sprague Dawley rats to model the lasting effects of early life stress (ELS). Litters were culled to 5 males and 5 females on postnatal day 0 (P0), or the day of birth. On P1-3, animals were briefly (5 min) exposed to cat, adult male rat, and ferret odors, respectively. No further manipulations were performed until behavioral testing at postnatal day 32 (P32) in the elevated plus maze (EPM). On P33, animals were sacrificed by rapid decapitation and amygdalae were collected. Separate cohorts of unhandled P35 male/female rats were sacrificed to confirm sex differences found in the POE study. All procedures were approved by the University of Wisconsin-Madison Animal Care and Use Committee.

Amygdalae from both POE and a subset of the sex difference samples were homogenized and processed to collect DNA and RNA. RNA was converted to cDNA for RT-qPCR analysis; DNA was used for methylation sensitive or dependent restriction enzyme digests, MeDIP analysis, and HPLC-MS/MS analysis. The remaining sex difference samples were fixed and used for ChIP.

Results: By combining distinct behavioral measures from the EPM (head dips, time spent in the open arm, and time spent in stretch attenuated posture) into a composite $\mathrm{z}$-score, we report neonatal POE increases 'emotionality' in stressed adolescent females, but not in males. Furthermore, there is a sex difference between control females and males, where males exhibit higher emotionality scores independent of stress status. We also report that early life stress (ELS) resulted in detectable changes of $6 \mathrm{~mA}$ in females. Explicitly, we find evidence of $6 \mathrm{~mA}$ within the developing mammalian brain and data supporting its regulation across development by biological sex and stress. We observe that $6 \mathrm{~mA}$ abundance at a discrete location within the Htr2a promoter is inversely proportional to Htr2a mRNA transcription in the juvenile amygdala. Explicitly, we find that males, regardless of their stress status, have higher levels of $6 \mathrm{~mA}$ compared to control females at an Sp1 transcription factor binding site within the Htr2a promoter. Stressed females have male-typical levels of $6 \mathrm{~mA}$ at this site. We further confirmed this data by performing MeDIP and ChIP assays on separate cohorts of unhandled male and female rat amygdalae. We validated that the overall abundance of amygdalar $6 \mathrm{~mA}$ is within experimentally reasonable levels using HPLC-MS/MS. We note that while there were no overall sex differences in the amount of $6 \mathrm{~mA}$, there are sex differences in the amount of $5 \mathrm{mC}$ in the P33 amygdala.

Conclusions: This study is the first of its kind, to our knowledge, to demonstrate that a novel epigenetic phenomenon is sensitive to a psychosocial stressor, namely POEinduced ELS, and that this effect is sex-specific. These findings have major implications for our understanding of how gene transcription is regulated, and what systems of regulation go awry in the development of psychiatric disorders. Specifically, our data indicate the existence of adenine methylation within mammalian DNA as a novel epigenetic marker that is responsive to biological sex and early life stress. It will be exciting to determine the unexplored role of $6 \mathrm{~mA}$ on mammalian brain development and function, as well as its potential role in the etiology of psychiatric disorders.
Keywords: Amygdala, Early Life Stress, Sex Difference, DNA Methylation, Anxiety.

Disclosure: Nothing to disclose.

\section{W99. Identifying Clinically Relevant Subgroups in Major Depressive Disorder Using Resting-State Functional Connectivity: Results From the EMBARC Study}

Crystal Cooper*, Neil Salvalia, Phillip Agres, Micaela Chan, Liang Han, Fahd Alhazmi, Maurizio Fava, Ben Kurian, Patrick McGrath, Ramin Parsey, Myrna Weissman, Gagan Wig, Madhukar Trivedi

University of Texas Southwestern Medical Center, Dallas, Texas, United States

Background: Major Depressive Disorder (MDD) is a serious brain syndrome that causes considerable morbidity and mortality. A growing body of literature supports the concept that psychiatric illnesses like MDD are the result of neuropathology at the level of the brain circuit and not just regional differences (Andreasen 1997; Phillips, Drevets et al. 2003; Ressler and Mayberg 2007; Taylor and Liberzon 2007). This neuropathology can be revealed as altered connectivity between regions of the brain. Functional connectivity between brain regions can be assessed using functional magnetic resonance imaging (fMRI) during non-task (resting) periods (Biswal, Yetkin et al. 1995; Biswal, Van Kylen et al. 1997; Lowe, Mock et al. 1998; Xiong, Parsons et al. 1999; Cordes, Haughton et al. 2000; Greicius, Krasnow et al. 2003; Peltier, Polk et al. 2003; Beckmann, DeLuca et al. 2005). Connectivity measures from the baseline fMRI scans can provide an objective measure that is related to clinical features and even serve as a bioimarker for treatment outcome and prediction. However, in order for a clinical diagnostic test to be meaningful, the measured value must be able to classify one individual as belonging to a particular group of individuals as opposed to other groups. Such diagnostic tests based on brain circuits are unknown in MDD. The Establishing Moderators and Biosignatures of Antidepressant Response for Clinical Care (EMBARC) study (Trivedi et al. 2016) aims to identify biomarkers to predict treatment outcomes in MDD. Identifying candidate biomarkers will serve as a first step. To identify candidate biomarkers for resting fMRI, we implemented an iterative, data-driven, clustering method to classify patients into subgroups. Patterns of resting-state functional correlation (RSFC) in their large-scale brain networks served as inputs for this clustering approach. Then, we characterized the subgroups by their clinical and brain structural features.

Methods: Data consisted of 185 unmedicated MDD patients who consented and participated in the EMBARC study, a multisite, double-blind, placebo-controlled clinical trial of early-onset MDD (first episode before 30 years of age). Each participant was scanned at one of four sites. The resting fMRI sequence was approximately $6 \mathrm{~min}$ and included 180 volumes. A RSFC matrix was constructed for each participant by measuring the pair-wise correlations in the blood oxygen level-dependent time-series of putative neocortical areas (Chan et al., 2014) and subcortical regions of interest as defined by FreeSurfer. Patient-to-patient similarity was 
quantified in functional connectivity by comparing each patient's RSFC matrices to one another. Then, subgroups were established by iteratively finding individuals with high RSFC similarity to many others and assigning each patient to a single cluster with which their RSFC exhibited high similarity. Once the RSFC clusters were defined, a series of nonparametric tests were conducted to identify if these clusters contained significant clinical and brain structural features. Lastly, a leave-one-out prediction analysis was conducted to assess whether the MDD subgroups could be predicted simply and accurately for unsampled participants. Results: Patient clusters were reliably identified across a variety of brain parcellations, RSFC matrix thresholds, and participant samples. Critically, the RSFC distinct MDD subgroups classified also exhibited multivariate clinical and anatomical variability that might otherwise be indiscernible from the full sample. Subgroups reliably differed in depressive symptoms as measured by several clinical scales and in FreeSurfer-based estimates of brain structure. For instance, one RSFC MDD subgroup was characterized by high symptoms of anhedonia, a reduced ability to experience pleasure, with reduced ventral striatum size, while another subgroup was characterized by high levels of depression severity and longer current episode duration with increased amygdala and anterior cingulate size. The leave-one-out analysis revealed participants to exhibit relatively greater similarity to their own cluster than to other clusters and a 70\% prediction accuracy suggesting that unsampled participants may reliably be matched to the correct RSFC-defined subgroup.

Conclusions: There are number of treatments available for MDD, but no single treatment works for everyone. Clinicians currently have no way to determine which treatment option, if any, is most likely to get their patient better. Therefore, a method to identify brain biomarkers that serve as a diagnostic tool and help predict treatment response is critically needed. The current study aimed to identify clinically relevant subgroups in MDD based on RSFC. This data driven identification of RSFC-based clustering can identify subgroups of patients with multiple distinguishing clinical and anatomical features that correspond to group-based differences in RSFC across MDD sub-groups and compared to non-MDD healthy controls. These findings suggest that RSFC network patterns may be used to differentiate patients with MDD into clinically meaningful subgroups and could assist with biomarker based diagnoses and treatment prediction. Data from this study will be investigated as outcome predictors with the final EMBARC sample.

Keywords: Major Depressive Disorder (MDD), Resting State Functional Connectivity, Clustering, Classification, EMBARC. Disclosure: Nothing to disclose.

W100. Neonatal Maternal Separation Stress Elicits Lasting DNA Methylation Changes in the Hippocampus of Stress-Reactive Wistar-Kyoto Rats

Ilan Kerman*, Chelsea McCoy, Samir Rana, Sarah Clinton

Virginia Tech, Roanoke, Virginia, United States

Background: Adverse early-life experiences have long-term effects on the brain and biobehavioral responses to stress in many species. Although most work on early-life stress (ELS) highlights its harmful effects such as increasing risk for neuropsychiatric disorders, smoking, physical inactivity, obesity, and cardiovascular disease in humans, emerging evidence suggests a more nuanced view. The match/ mismatch hypothesis of disease states that ELS can have adaptive value to individuals facing later stressful experience. For instance, repeated childhood stress exposure confers protective neuroendocrine effects when adult offspring face stress. This suggests that repeated ELS elicits a predictive adaptive response (PAR) where past stressful experiences augment coping with future stress. PAR is thought to be strongest in stress-susceptible individuals and may be evolutionarily conserved by rapid cross-generational environmental changes. Our recent work lends further support to this theory. We exposed Wistar-Kyoto (WKY) offspring, a stress susceptible rat strain that exhibits behavioral abnormalities commonly associated with depression and anxiety disorders, to daily 3-hour maternal separation (MS-180) during the first two weeks of life. While MS-180 has frequently been used to demonstrate deleterious effects on adult rodent emotional behavior, we found that MS-180 elicits several adaptive effects in adult WKY offspring, including diminished behavioral despair, increased social exploration, and improved cardiovascular measures, such as decreased baseline heart rate (HR) and increased $\mathrm{HR}$ variability.

Methods: The present study exploited the WKY-MS model to identify neurobiological and epigenetic mechanisms that mediate a PAR to convey adaptive behavioral and physiological changes in adult MS-180-exposed WKY offspring. We hypothesized that MS-180 triggers DNA methylation changes in the brains of WKY offspring, which may contribute to their enhanced stress resilience in adulthood. To test this, we first examined global DNA methylation (5methylcytosine) levels in multiple brain regions of adult WKY rats that were exposed to MS-180 (or control condition). Because we found dramatic MS-180-induced hypermethylation in the WKY hippocampus, we then used next-generation sequencing to interrogate gene-specific methylation changes that occurred following MS-180 exposure. Our final experiment tested the hypothesis that enhancing DNA methylation in adult WKYs (by increasing dietary methyl donor content) would elicit adaptive behavioral and cardiovascular changes akin to what occurred following MS-180 exposure. Specifically, we predicted that feeding adult male WKYs a methyl-donor fortified diet would: (a) have anxiolytic and antidepressant effects in tests of anxiety- and depression-like behavior; and (b) elicit positive cardiovascular changes, including reduced baseline $\mathrm{HR}$ and greater $\mathrm{HR}$ variability, similar to what we previously observed in MS-180-exposed WKY offspring.

Results: MS exposure triggered dramatic DNA hypermethylation specifically in the hippocampus, but not within other brain regions. Next-generation sequencing methylome profiling revealed reduced methylation at intragenic sites within two key nodes of insulin signaling pathways: the insulin receptor (Insr) and one of its major downstream targets, mitogen activated protein kinase kinase kinase 5 (Map3k5). We then tested the hypothesis that enhancing DNA methylation in WKY rats would elicit adaptive changes akin to the effects of MS. Dietary methyl donor supplementation 
improved WKY rats' anxiety/depression-like behaviors and also improved cardiovascular measures, similar to previous observations following MS.

Conclusions: Overall these data suggest a potential molecular mechanism that mediates a predicted adaptive response, whereby ELS induces DNA methylation changes in the brain that may contribute to successful stress coping and adaptive physiological changes in adulthood.

Keywords: Epigenetics, Depression, Anxiety, Early Life Stress, Cardiovascular.

Disclosure: Nothing to disclose.

W101. Brain Insulin Signaling Mediates Antidepressant Response to Ketamine in Rodent Model of Tricyclic Antidepressant Resistance

Susannah Tye*, Joshua Price, Shari Sutor, William Bobo, Mark Frye

Mayo Clinic College of Medicine, Rochester, Minnesota, United States

Background: The mechanisms mediating antidepressant response to low-dose ketamine in treatment-resistant depression are not well understood. We have previously shown that activation of the insulin signaling pathway directly correlates with antidepressant response to ketamine in tricyclic antidepressant-resistant rats. The role of insulin secretion, insulin signaling and glucose uptake in ketamine's rapid antidepressant mechanism of action has not been explored. We aimed to address this issue in the current study.

Methods: Socially isolated male Wistar rats were pre-treated with saline $(0.9 \% / \mathrm{d} ; 14 \mathrm{~d})$ or adrenocorticotropic hormone-(124) $($ ACTH $100 \mu \mathrm{g} / \mathrm{d} ; 14 \mathrm{~d})$. Animals received ketamine hydrochloride $(10 \mathrm{mg} / \mathrm{kg})$ or vehicle saline $(0.9 \%) 1 \mathrm{hr}$ prior to behavioral tests [open field test (OFT) and forced swim test (FST)]. A group of ACTH-treated rats also received metformin $(200 \mathrm{mg} / \mathrm{kg})$ or metformin $(200 \mathrm{mg} / \mathrm{kg})+$ ketamine $(10 \mathrm{mg} / \mathrm{kg})$. All animals were euthanized $90 \mathrm{mins}$ following treatment. Brain, plasma and peripheral blood mononuclear cells (PBMCs) were collected and snap frozen on dry ice. A subset of animals receiving the same pretreatment were euthanized at the equivalent time point (90 mins following treatment), but did not undergo behavioral testing. Brain and PBMC tissue from these animals was harvested and cultured for subsequent insulin and glucose assays. Post-FST plasma glucose and insulin levels were quantified with a glucometer and high sensitivity insulin ELISA kit, in accordance with manufacturers instructions. Brain and PBMC glucose uptake was quantified using the Cayman glucose uptake assay. Total and phosphorylated levels of insulin signaling pathway proteins in prefrontal (PFC) brain and PBMC tissues was determined using standard western blot procedures.

Results: Results demonstrated that ketamine reduced FST immobility in a subset (50\%) of tricyclic antidepressantresistant animals $(p<0.05)$. Ketamine's antidepressant actions in the FST (latency to immobility and total immobility time) correlated with levels of phosphorylated insulin signaling proteins (Akt: $p<0.05$; mTOR: $p<0.01$; and GSK3: $p<0.01)$ in PFC and PBMC tissue of ACTH-treated animals, but not saline treated controls. Metformin treatment, alone or in combination with ketamine, exerted an antidepressant effect in ACTH pretreated $\operatorname{rats}(p<0.05)$. The effect of ketamine+ metformin on immobility time was not significantly different from that observed for the ketamine responsive animals. However, we did not observe the same divergence in response. That is, metformin, when administered together with ketamine, increased response rate relative to ketamine alone $(p<0.01)$. Correlations between brain insulin signaling pathway protein activation and blood glucose levels was also observed (mTOR: $p<0.05$; and GSK3: $p<0.05)$. Further, we demonstrated that changes in blood glucose and phosphorylated levels of mTOR and GSK3 in WBCs differentiated ACTH pre-treated ketamine responders from non-responders. Cell culture assays confirmed that ketamine acted directly on brain and PBMC tissue to increase glucose uptake and stimulate release of insulin (brain tissue only), concurrent with activation of insulin signaling pathway proteins.

Conclusions: These findings suggest the antidepressant-like effects of ketamine in treatment-resistant individuals is associated with direct modulation of insulin signaling and glucose uptake. Insulin action and glucose uptake may serve as critical co-regulators of antidepressant response to ketamine in individuals with impaired insulin signaling and metabolic capacity.

Keywords: Ketamine, Insulin, Antidepressant, Treatment Resistance.

Disclosure: Nothing to disclose.

W102. Ketamine Action in a Cellular Model System Suggest a Camp-Dependent and NMDA ReceptorIndependent Mechanism for its Rapid Antidepressant Action

\author{
Nathan Wray, Jeffrey Schappi, Mark Rasenick* \\ University of Illinois College of Medicine, Chicago, \\ Illinois, United States
}

Background: Previous studies have demonstrated that all extant classes of antidepressants increase coupling between the $G$ protein, $G \alpha$ s and adenylyl cyclase, resulting in persistent cAMP elevation. This effect requires sustained drug treatment and is observed after 3 days in cultured neural or glial cells or 3 weeks in rats. This is apparently due to Gos being released from constraints of a lipid raft environment to cholesterol-poor regions of the plasma membrane. Consistent with this, both peripheral tissue and postmortem brain from depressed human subjects show a greater proportion of Gos in lipid rafts. While most antidepressants require several weeks for a clinical effect, ketamine appears to alleviate depression within hours, only to relapse after several days.

Methods: C6 cells were treated with luM ketamine, harvested and lipid raft fractions prepared and the amount of Gos was assessed by Western blot. C6 cells expressing a stable fluorescent Gos fusion protein (GFP-Gos) were treated similarly with ketamine and the mobility of GFP-Gos was determined by Fluorescence Recovery after Photobleaching (FRAP) using a Zeiss 710 Meta confocal microscope. BDNF, 
CREB and p-CREB were also quantified after immunoblotting.

Results: In an effort to determine whether ketamine showed an antidepressant biosignature similar to other antidepressants, studies in cultured cells were initiated. It was hypothesized that ketamine would have an effect similar to antidepressants but along a shorter time course. Brief (15 minute) ketamine treatment evoked a biochemical hallmark (translocation of Gos from lipid rafts) like that seen after prolonged treatment with several species of drugs with established antidepressant activity. This is not mimicked by other NMDA antagonists, suggesting an additional site for ketamine action. The ketamine induced Gos translocation allows increased functional coupling of Gas and adenylyl cyclase to increase intracellular cyclic adenosine monophosphate (cAMP). Furthermore, increased intracellular cAMP mediates phosphorylation of cAMP response elementbinding protein (CREB), which increases BDNF levels. These events follow ketamine treatment, but do so after a delay of several hours. BDNF production was indeed dependent on cAMP as the cAMP antagonist Rp-cAMPS attenuated BDNF expression levels. Furthermore, BDNF expression was increased in primary astrocytes after ketamine treatment and attenuated by Rp-cAMPs.

Conclusions: These results reveal a novel antidepressant mechanism mediated by acute ketamine treatment in glial cells that may contribute to ketamine's antidepressant effect. Furthermore, the translocation of GFP-Gos produced by ketamine and all tested compounds with antidepressant activity (but not mood-stabilizers, antipsychotics or anxiolytics) might serve as a useful platform for identifying compounds with potential antidepressant activity and for predicting clinical response.

Keywords: cAMP Signaling, Antidepressant Agents, GPCR, BDNF, Lipid Rafts.

Disclosure: Nothing to disclose.

\section{W103. Using Diffusion Tensor Imaging to Effectively Target TMS to Deep Brain Structures}

Bruce Luber*, Zhi-De Deng, David Murphy, Simon Davis, Andrew Martella, Angel Peterchev, Sarah Lisanby

National Institute of Mental Health, Bethesda, Maryland, United States

Background: TMS has become a powerful tool to explore cortical function, and in parallel has proven promising in the development of therapies for various psychiatric and neurological disorders. Used in conjunction with brain imaging, precise methods of targeting have been developed which greatly increase the effect of TMS on neural processing. Unfortunately, the direct effects of TMS have been limited to 2-3 centimeters beneath the scalp with conventional stimulation coils, while other research such as deep brain stimulation has indicated that modulation of much deeper regions may be highly efficacious in treating psychiatric disorders. In this study, we sought to develop a novel paradigm to individualize TMS coil placement to noninvasively achieve activation of a specific deep brain target:
Brodmann Area 25 (BA25), which has been implicated as an important node in the neurocircuitry of depression.

Methods: Demonstrating that diffusion tensor imaging (DTI) tractography could be used to guide trans-synaptic activation of a deep brain target such as BA25 with cortical TMS required two separate MRI sessions after initial screening and consenting of 10 healthy adult participants. In the first MRI session, structural, functional, and DTI data sets were acquired. A noninvasively accessible cortical TMS target was then determined from analysis of the diffusion and anatomical imaging data. Using DTI, a target in the right frontal pole that demonstrated both anatomic and functional connectivity to right BA25 was found in each subject. The individual stimulation sites fell within a $5 \mathrm{~cm}$ diameter area on the forehead centered on those coordinates, with a mean distance from center of $10.1+/-8.1 \mathrm{~mm}$. In the second session, interleaved TMS/fMRI was performed using this target, measuring BOLD signal changes during application of single-pulse TMS at the diffusion tractography determined stimulation site. This was done in the scanner using a series of single TMS pulses applied to the right frontal pole. Pulses of four intensity levels-80\%, 100\%, 120\%, and 140\% of motor threshold-were delivered in a random and counterbalanced order.

Results: In nine of ten subjects, TMS to the individualized frontal pole sites resulted in significant BOLD activation of BA25, and this activation increased with TMS intensity. ROI analyses were performed for the contrasts of $100 \%, 120 \%$, and $140 \%$ conditions with the $80 \%$ baseline at four locations: the deep target right BA25 and its contralateral homologue, and the right frontal pole region that contained the TMS stimulation site, and a dorsal prefrontal cortical site that did not show connectivity with the seeded region. The deep target region in right hemisphere BA25 showed activation in the ROI for both supra-threshold stimulation levels $(120 \%$ and $140 \%$ ) relative to $80 \%$ that survived cluster correction for multiple comparisons ( $\mathrm{z}$-score threshold $\mathrm{z}>1.65$ and cluster significance $p<0.05$ ), showing an increase in activation in our BA25 centered region of interest. A smaller cluster in the corresponding left hemisphere BA25 ROI was also significantly activated at the $120 \%-80 \%$ contrast, with a cluster size of 6 voxels and a maximum z-score of 2.6. No significant activations were seen in the control ROI. A similar result was found when comparing the ROIs in contrasts of the $80 \%$, $100 \%, 120 \%$ and $140 \%$ TMS levels from rest. Increasing TMS intensity produced increased activation in the BA25 target ROI. The inverse was true beneath the stimulation site, where significant deactivation was seen that increased with TMS intensity. Beyond a group level analysis, we sought to confirm the stimulus intensity dependence of activation in our deep brain target within individual subjects. To achieve this, a multivariate covariance analysis was performed to test for a monotonically increasing activation resulting from stimulation at increasing $(80 \%, 120 \%$, and $140 \%)$ TMS intensities. A statistically significant pattern which included right $\mathrm{BA} 25$ was found ( $p<.03$ using permutation testing) based on the first three principal components in which 9 out of the 10 subjects showed monotonic increases in expression of the network.

Conclusions: In the present study DTI tractography beginning in a brain region too deep for effective direct TMS was used to find transsynaptically connected superficial cortical 
site that could be effectively stimulated. Then using interleaved fMRI/TMS, it was demonstrated that DTI-guided TMS could activate the deep target, and could do so in a dosage dependent manner. The present results suggest a new technique for brain stimulation technology that has all three of the following features: it is (1) noninvasive, (2) targetspecific and (3) deep, providing a means of studying brainbehavior relationships of brain networks that involve deep targets that were previously thought to be beyond reach. The successful activation of BA25 in a dosage-dependent manner represents an initial step towards using tractography-guided TMS to noninvasively target areas for therapy and for research no matter where they are situated in the brain.

Keywords: TMS Targeting, Interleaved TMS/fMRI, DTI, TMS.

Disclosure: Nothing to disclose.

\section{W104. An Inflammatory Story: Higher C-Reactive Protein Prospectively Associated With Greater Depression and Anxiety Severity in a Population-Based Study}

Joy Lin, Aoife O'Donovan*

University of California, San Francisco, California, United States

Background: Depression and anxiety have been linked with elevated inflammation in numerous clinical studies. Mounting evidence indicates that inflammation may actually provoke symptoms of both depression and anxiety through effects on the brain. However, activation of biological stress response systems may also promote inflammation in depressed and anxious individuals. A better understanding of the dynamic relationships of depression and anxiety with inflammatory markers over time may shed light on these causal pathways. Nonetheless, few studies have examined prospective associations of depression and anxiety with inflammation. In a population-based study, we examined if inflammation is associated with symptoms of depression and anxiety in both cross-sectional and longitudinal analyses.

Methods: Our sample included 18,603 individuals from the Health and Retirement Study, a population-based sample of Americans over the age of 50 (Mean age $=68 \pm 10,41 \%$ male). Depression was assessed using a nine-item version of the Center for Epidemiologic Studies Depression Scale; anxiety with a five-item version of the Beck Anxiety Inventory; and inflammation with high sensitivity C-reactive protein (hsCRP), which was log-transformed to normalize the data. Hierarchical linear regression was used to examine the relationship of hsCRP with depression and anxiety both cross-sectionally and longitudinally. All models were adjusted for age, gender, race, and education and for longitudinal models, we also adjusted for baseline depression, anxiety, or hsCRP as appropriate.

Results: Higher levels of depression and anxiety were associated with higher hsCRP in cross-sectional analyses. In longitudinal analyses, higher baseline hsCRP was associated with more severe depression two, four, and six years later adjusting for baseline depression, and more severe anxiety four years later adjusting for baseline anxiety. Moreover, both depression and anxiety predicted elevated
hsCRP in individuals two, four, and six years later, adjusting for baseline hsCRP. However, the change in hsCRP over the four-year time period was not significantly associated with increased symptoms of either depression or anxiety, and the change in depression and anxiety was not significantly associated with the change in levels of hsCRP over time.

Conclusions: Our study extends findings that link elevated systemic inflammation with depression and anxiety crosssectionally to a large, prospective, population-based sample of Americans in later life. Our results indicate that elevated inflammation predicts more severe depression and anxiety, and that higher levels of depression and anxiety predict higher levels of inflammation. Thus, depression and anxiety may have bidirectional recursive relationships with inflammation. Reducing either depression and anxiety or inflammation could have knock-on effects and improve overall health in depressed and anxious individuals.

Keywords: Depression, Anxiety, Inflammation.

Disclosure: Nothing to disclose.

\section{W105. Effect of Lurasidone Dose on Recurrence Prevention in Patients With Bipolar I Disorder: Post Hoc Analysis of a Placebo-Controlled Randomized Withdrawal Study}

Mark Frye*, Joyce Tsai, Yongcai Mao, Andrei Pikalov, Antony Loebel

\section{Mayo Clinic, Rochester, Minnesota, United States}

Background: Treatment guidelines for bipolar I disorder have focused on maintenance mood stabilization. Lurasidone is currently FDA approved for bipolar I depression. This post-hoc analysis evaluated the effect of dose on recurrence prevention of lurasidone, adjunctive to lithium (Li) or valproate (VPA) for the maintenance treatment of bipolar disorder.

Methods: Patients with a diagnosis of bipolar I disorder with $\geq 1$ manic, mixed manic, or depressed episode in the past 2 years, and with a Young Mania Rating Scale (YMRS) or Montgomery-Åsberg Depression Rating Scale (MADRS) total score $\geq 14$ (if on Li or VPA), or $\geq 18$ (if not on Li or VPA), received open-label treatment with lurasidone 20$80 \mathrm{mg} / \mathrm{d}$ adjunctive to Li or VPA for up to 20 weeks during an open-label stabilization phase. Patients who achieved predefined stability criteria were randomized to 28 weeks of double-blind treatment with lurasidone, $20-80 \mathrm{mg} / \mathrm{d}$, adjunctive to $\mathrm{Li}(N=104)$ or VPA $(N=134)$, or placebo adjunctive to $\mathrm{Li}(N=109)$ or VPA $(N=141)$. A Cox proportional hazards model was used to assess the hazard ratio at Week 28 of time to recurrence of any mood disorder (primary outcome), and time to recurrence of a depressive episode. Kaplan-Meier estimates of the probability of time to recurrence were also calculated. Separate analyses were performed for each modal dose of lurasidone during the open-label phase.

Results: A total of 496 patients met stabilization criteria and were randomized to adjunctive lurasidone $(N=246)$ vs. placebo $(N=250)$. The modal daily dose of lurasidone in the open-label phase was $20-40 \mathrm{mg}, 60 \mathrm{mg}$, and $80 \mathrm{mg}$ in $42.1 \%, 32.9 \%$, and $25.0 \%$ of patients, respectively. For the total intent-to-treat population, non-significantly fewer patients in the lurasidone 
group had recurrence of any mood event compared with the placebo group, with a hazard ratio of $0.71(95 \% \mathrm{CI}=0.49-1.04$; Cox model $P=0.078$; log-rank test $P=0.055)$, representing a risk reduction of $29 \%$. There appeared to be a dose-related increase in the recurrence prevention efficacy of lurasidone. For patients treated with a modal daily dose of $80 \mathrm{mg}$, the hazard ratio was significant for time to recurrence of any mood disorder, 0.35 (95\% CI =0.14-0.85; Cox model $P=0.020$; logrank test $P=0.017$ ), representing a risk reduction of $65 \%$; the hazard ratio was also significant for time to recurrence of a depressive episode, 0.18 (95\% CI $=0.04-0.81$; Cox model $P=0.025$; log-rank test $P=0.014$ ), representing a risk reduction of $82 \%$. For patients treated with a modal daily dose of $60 \mathrm{mg}$, the hazard ratio was non-significant for time to recurrence of any mood episode, 0.79 (95\% CI $=0.43-1.47)$, representing a risk reduction of $21 \%$; the hazard ratio was also non-significant for time to recurrence of a depressive episode, 0.70 (95\% $\mathrm{CI}=0.31-1.59$ ), representing a risk reduction of $30 \%$. For patients treated with a modal daily dose of $20-40 \mathrm{mg}$, the hazard ratios for time to recurrence of any mood disorder, or a depressive episode, were not different from placebo. For the secondary outcomes, time to all-cause discontinuation and time to recurrence based on severity measures (MADRS or YMRS $\geq 18$ or CGI-BP-S $\geq 4$ ), patients treated with a modal daily dose of lurasidone $80 \mathrm{mg}$ demonstrated a significant reduction in risk of recurrence of $67 \%$ (Cox model $P=0.024$ ) and $72.5 \%$ (Cox model $P=0.024$ ), respectively. Risk reduction was nonsignificant on these two secondary outcomes for both the $60 \mathrm{mg}$ and $20-40 \mathrm{mg}$ doses.

Conclusions: In this double-blind study of patients with a bipolar I diagnosis who had been stabilized on lurasidone plus lithium or valproate, 28 weeks of continued treatment with adjunctive lurasidone was found to have greater recurrence prevention efficacy as the daily dose was increased from $20 \mathrm{mg}$ to $80 \mathrm{mg}$. These results are particularly notable since this is a flexible dose study, where use of higher doses typically reflects dose escalation in response to inadequate treatment response.

Keywords: Bipolar Disorder, Atypical Antipsychotic Drug, Maintenance Treatment, Recurrence Risk, Lurasidone.

Disclosure: Sunovion Pharmaceuticals Inc: Sponsor of Clinical Trial and the Current Post-hoc Analysis, Self.

W106. Ketamine and Pramipexole Promote Plasticity in Human iPSC-Derived Dopaminergic Neurons via BDNF/ TrkB and mTOR Mediated Signalling: Possible Relevance for Treatment-Resistant Depression

Emilio Merlo Pich*, Ginetta Collo, Laura Cavalleri, Stefania Fedele, Cristiano Chiamulera, Mark J. Millan, PierFranco Spano

Takeda Development Center Europe, London, United Kingdom

Background: Emerging evidence of dysfunctional relationships between glutamate neurotransmission and neural plasticity has been recognised as pathologic feature in depression. Ketamine, a dissociative anaesthetic that blocks the N-methyl$\mathrm{D}$-aspartate (NMDA) receptor and engages $\alpha$-amino-3hydroxy-5-methyl-4-isoxazolepropionic acid (AMPA) receptor glutamate transmission [1], can induce rapid-onset antidepressant effects lasting 1 week after single infusion. The sustained effect was related to re-activation of neural plasticity in the frontocortical/hippocampal networks via $\mathrm{BDNF} / \mathrm{TrkB}$ and $\mathrm{mTOR}$ signalling, critical pathways involved in cell growth and plasticity [2]. However, improvement of anhedonia, known to be dependent on mesolimbic dopaminergic (DA) transmission, may not be easily explained. Since NMDA and AMPA receptors control DA transmission and ketamine increase dopamine release, neural plasticity in DA neurons may be implicated in the sustained antidepressant effects of ketamine. In previous works, using primary cultures of mouse mesencephalic DA neurons, we showed that substances that release DA, such as amphetamine and cocaine, can increase dendritic arborization and soma size [3,4]. These effects were mediated by the activation of presynaptic DA D3 receptor (D3R) whose signalling includes the mTOR pathway. Interestingly, also D3R-preferential DA agonists can induce similar effects [3], including pramipexole, currently investigated as adjunctive treatment in inadequate response to antidepressants [5].

To improve translational relevance, we used DA neurons differentiated from human inducible Pluripotent Stem Cells (hiPSCs) to study the mechanism of action of ketamine and pramipexole.

Methods: Human iPSCs were generated from healthy donors following the approval of the local ethics committee and differentiated into DA neurons [6]. DA neurons, cultured for 60-80 days, expressed differentiation markers, including tyrosine hydroxylase $(\mathrm{TH})$, dopamine transporter (DAT), NMDA subunit NR2B, AMPA subunit GluR2; they showed functional dopamine uptake measured with HPLC. Maximal dendrite length, number of primary dendrites and soma size of TH-positive neurons were measured using computerassisted morphometry 3 days after exposure to either ketamine, the AMPA positive modulator CX614, the NR2B antagonist Ro 25-6981 or pramipexole. Western blot and immunofluorescence analysis for p-p70S6 kinase and TH were performed within 2-60 min after challenge.

Results: Ketamine (0.01-1 $\mu \mathrm{M})$ dose-dependently increased dendritic arborisation and soma size. These effects were inhibited by pre-treatment with the AMPA antagonists NBQX $(10 \mu \mathrm{M})$ and GYKI52466 $(10 \mu \mathrm{M})$. Similar effects were produced by Ro 25-6981 $(1 \mu \mathrm{M})$ and CX614 $(10 \mu \mathrm{M})$. Ketamine increased phosphorylation of p70S6 kinase, located downstream of mTOR, $5 \mathrm{~min}$ after exposure, an effect blocked by the PI3K inhibitor LY294002 $(10 \mu \mathrm{M})$ and the mTORC-1 complex inhibitor rapamycin $(20 \mathrm{nM})$. Immunoneutralisation of BDNF and inhibition of TrkB signalling with K252a $(0.2 \mu \mathrm{M})$ and PP2 $(10 \mu \mathrm{M})$ also prevented the effects of both ketamine and CX614. Ketamine and CX614 effects were abolished by pretreatments with the D3 antagonists SB277011-A (50 nM) or S33084 (10 nM). Similar experiments were performed to study neural plasticity produced by pramipexole, whose effects was blocked by SB277011-A, S33084, LY294002, rapamycin, immunoneutralisation of BDNF and inhibition of TrkB signalling.

Conclusions: Ketamine and pramipexole both induce structural plasticity in human DA neurons, confirming preliminary data obtained in mouse DA neurons. Ketamine effects were mediated by AMPA receptor activation which, in turn, engaged $\mathrm{BDNF} / \mathrm{TrkB}$ signalling to drive neural plasticity. These effects depended on the co-activation of D3R whose 
intracellular signalling partially overlaps with the BDNF-TrkB pathway, triggering mTOR signalling. These data, in line with recent literature, suggest that plasticity in two complementary circuits, mesencephalic DA and frontocortical/hippocampal systems, may concur in determining the ketamine and pramipexole protracted antidepressant effects.

Keywords: Induced Pluripotent Stem Cells (iPSCs), AMPA Glutamate Receptors, Dopamine D3 Receptors, NMDA Glutamate Receptors.

Disclosure: Takeda: Full time employee, Self.

\section{W107. RDoC Working Memory Measures Related to Dimensions and Categories of Psychopathology and Disability}

Robert Bilder*, Jesse Rissman, Sandra Loo, Jean Baptiste Pochon, Julia Webster, Catherine Sugar, Holly Truong, Carrie Bearden, Michael Gitlin

Semel Institute for Neuroscience \& Human Behavior, Los Angeles, California, United States

Background: The NIMH Research Domains Criteria (RDoC) initiative has stimulated efforts to identify novel dimensions of brain and behavioral function in hopes that these may provide greater traction for understanding mental disorders and their treatments. A core question is how the $\mathrm{RDoC}$ domains relate to traditional measures of psychopathology as these are routinely assessed using categorical diagnostic or dimensional assessments of symptoms, and to what extent RDoC domain measures and clinical measures contribute to broad measures of disability.

Methods: To avoid biases associated with recruiting diagnostic groups, we examined two groups of individuals: (1) Care-Seeking (CS, $n=55)$ individuals from the community, recruited through clinics and clinical web-sites, if they were seeking help for the way they were thinking or feeling; and (2) Non-Care-Seeking (NCS, $n=50)$ individuals from the same community, who were not seeking care and had not done so within the year preceding enrollment. All participants had the same battery of clinical, cognitive, EEG and MRI assessments. Clinical assessments included MINI 7.0 neuropsychiatric interview, multiple symptom rating scales, and the WHODAS 2.0 disability assessment. The neurocognitive tests on working memory included both "complex multifactorial" measures (e.g., Digit Span, Letter-Number Sequencing, Symbol Span, Spatial Addition, Automated Operation Span) and "cognitively specific" measures (e.g., Lateralized Change Detection, Spatial Working Memory Capacity, Dot Pattern Expectancy, Switching Stroop Color Word, Recent Negatives, Delayed Face Recognition). Analyses focused on examining the effect sizes for associations of the neurocognitive measures with clinical measures.

Results: CS and NCS groups were well matched on age, sex, personal and parental education, but the CS group had lower personal and family income. The CS group had more categorical mental disorders than the NCS group, the most prominent of which were current Major Depressive Disorder in $31 \%$ of CS compared to $11 \%$ of NCS, and lifetime MDD in $77 \%$ of CS and $40 \%$ of NCS (Eta2 $=.06$ and.14, respectively). The CS group also showed higher scores on broad dimensional measures of symptoms (e.g., BPRS: mean +/- SD,
$\mathrm{CS}=43 / 10, \quad \mathrm{NCS}=34 / 8, \quad \mathrm{Eta} 2=.20)$. The neurocognitive measures were more consistently related to dimensional measures of psychopathology (e.g., BPRS total score) than to CS vs NCS group status, or to presence vs absence of categorical mood disorder. The strongest associations between cognitive and clinical measures had medium effect sizes $(\mathrm{R} 2=.21)$, and these were found more among the "complex multifactorial" tests (e.g., Spatial Addition [R2=.21], Symbol Search $[R 2=.10]$, Digit Span $[R 2=.07])$, but there were also nominally significant associations with "cognitively specific" measures (e.g., Lateralized Change Detection, capacity $[\mathrm{R} 2=.07]$, Switching Stroop [R2 =.07], Delayed Face Recognition $[R 2=.06])$. Stepwise regression models to explain WHODAS 2.0 scores from routine clinical and cognitive measures selected BPRS total, then a total score reflecting the "burden" of all possible diagnoses, and measures of WM capacity from the LCD test (total $\mathrm{R} 2=.62$; BPRS alone, $\mathrm{R} 2=.42$ ). Interestingly, the K10 scale alone did well explaining variance on the WHODAS $2.0(\mathrm{R} 2=.60)$, and after entering the K10, only the LCD capacity measure offered additional explanatory power (total R2 $=.69$ ).

Conclusions: In samples recruited without ascertainment biases based on diagnostic group, dimensional measures of psychopathology are better than diagnostic categories at distinguishing care-seeking behavior, and are more closely related to cognitive measures of the RDoC Working Memory domain. Consistent with prior research (Gold et al., 2012), we find that "complex polyfactorial" tests are more closely related to clinical dimensions and categories than are the more "cognitively specific" measures proposed to discern discrete neural systems functions in the RDoC initiative. To account for real world disability, the most "efficient" measures are also broad-based clinical instruments (and indeed the K10 accounts for more variance in disability than do any of the primary clinical measures, including diagnostic group). After accounting for that "general psychopathology" effect, however, specific measures of working memory may help to explain additional disability. The theoretical model on which our assessments was based, suggest that this may implicate local cortical circuit dysfunction, which we are now examining with convergent methods using EEG along with diffusion, structural, and functional MRI methods.

Keywords: Research Domain Criteria (RDoC), Working Memory, Neurocognition, Disability.

Disclosure: Johnson \& Johnson: Equity ownership, Self; Sunovion: Honoraria, Self; Lumos Labs, Inc.: Consultancy, Self; Snapchat, Consultancy, Self; ThinkNow, Inc.: Consultancy, Self.

\section{W108. High Importance of Religion or Spirituality may Generate Resilience That Prevents Hippocampus From Shrinking in Persons at High Familial Risk for Depression}

Zhishun Wang*, Jie Yang, Connie Connie Svob, Tingting Ji, Jonathan Posner, Virginia Warner, Ardesheer Talati, Myrna Weissman

Columbia University, New York, New York, United States

Background: Brain structure and grey matter integrity have been shown to be altered in persons who are suffering from, 
or at high familial risk to develop a major depressive disorder (MDD). In our longitudinal study, we investigated familial risk for MDD by performing structural MRI on the biological offspring of a three-generation cohort. One of our findings was a remarkable reduction in cortical thickness and subcortical volumes in the high risk group. Also, in an article published in PNAS reported that there was a significant atrophy in hippocampus in depressive individuals. Further, we found that the individuals who reported high religious importance also had thicker cortical thickness in the left and right parietal and occipital brain regions and showed resilience against interference-related neural activity in frontal brain regions. Building upon these findings, the aim of the present study was to conduct a more in-depth investigation of the effect of high religious/spiritual importance on subcortical brain structures in individuals at high familial risk for MDD.

Methods: 143 participants, 83 at high familial risk for MDD (33.12 \pm 12.69 years old; 37 males, 46 females), 60 at low familial risk for MDD (26.72 \pm 13.22 years old; 26 males, 34 females). At the time of MRI scanning, participants were asked to respond to the question, "How important to you is religion or spirituality?", with responses ranging from 1 (not important at all) to 4 (highly important). We dichotomized responses as either "high" $(\geq 3)$ importance $(N=23)$ or "low" $(<3)$ importance $(N=74)$. In this study, we compared the shapes of the subcortical brain structures of the individuals at high familial risk for MDD between those who reported high importance of religion or spirituality $(N=12)$ and those who did not $(N=51)$. We acquired a high-resolution $(1.17 \times 1.17 \times 1.2 \mathrm{~mm}) \mathrm{T} 1$-weighted anatomical image on a Siemens Sonata $1.5 \mathrm{~T}$ scanner using a 3D MPRAGE sequence, from each of the 143 participants. We performed subcortical shape analysis on these brain images using FSL toolbox run in a Linux environment. We first segmented each T1-weight anatomical image into 15 subcortical objects (brain stem, left \& right nucleus accumbens, left \& right putamen, left \& right caudate, left \& right palladium, left \& right thalamus, left \& right hippocampus, left \& right amygdala) using a model-based segmentation toolbox, FSL FIRST. We then performed general linear model (GLM)based group comparison between the high importance and low importance within the individuals at high familial risk for depression, while covarying for age and sex effect, on each of the 15 shape objects using FSL randomise procedure. We reported the group comparison results of the subcortical shape analyses with the significance at a $p$ value threshold $<0.005$ that was corrected for multiple comparisons by combining this $p$ value with a cluster extent threshold with a cluster size of 16 voxels, which was determined by a Monte Carlo simulation.

Results: Within the individuals at high familial risk for depression, those who reported religion or spirituality as lower importance or no religious importance had greater shape atrophy or shrinking in the right hippocampus within an area including 50 voxels at a center MNI coordinate [-19 -37 6], than those who reported highly religious importance. Conclusions: Extensive studies have reported that the hippocampus is involved in the actions of stress, depression and antidepressant treatment. Recent brain imaging studies have shown that the hippocampus undergoes selective shape shrinking in stress-related neuropsychiatric disorders such as depressive disorder. Our finding suggests that religion/spirituality may generate resilience against depression through the enhanced volume of the hippocampus, the subcortical structure that plays a critical role in learning, memory and emotion.

Keywords: Major Depression Disorder, Hippocampus Shrinking, Importance of Religion, Structural MRI, Subcortical Shape Analysis.

Disclosure: Nothing to disclose.

\section{W109. Remodeling of Structural Brain Network Topology in Maltreated Youth With and Without Posttraumatic Stress Disorder}

Delin Sun*, Courtney Haswell, Rajendra Morey, Michael De Bellis

Duke University Medical Center, Durham, North Carolina, United States

Background: Posttraumatic stress disorder (PTSD) is common, chronic, and associated with high psychiatric comorbidity in youth maltreatment. Changes in specific brain structures have been found in maltreated youth with PTSD (1). However, little is known about the brain structural network differences between maltreated youth with and without PTSD. Here, we investigated the cortical morphometric network based on structural inferences made between specific pairings of cortical regions that vary in tandem with respect to cortical thickness (2). The correlation strength has been speculated to increase between regions that are concurrently affected by the disease process (maybe due to loss of input from a directly affected region), and decrease between affected and non-affected regions (3). We hypothesized between-group differences of network centrality in cortical regions associated with emotion processing, social cognition and inhibitory control, particularly in anterior cingulate cortex (ACC), ventromedial prefrontal cortex (vmPFC) and inferior frontal gyrus (IFG).

Methods: Participants: Demographically matched maltreated youth with PTSD $(N=31)$, without PTSD $(N=32)$, and nonmaltreated healthy controls $(n=57)$ were recruited $(1,4)$. Participants underwent extensive assessments for strict inclusion/exclusion criteria. Maltreated participants were defined by a positive forensic investigation conducted by child protective services (CPS) that indicated physical and sexual abuse and/or neglect, and they were separated into PTSD and non-PTSD groups according to DSM-IV-TR and DSM-5.

MRI Acquisition and Network Analyses: All images were acquired using a Siemens Trio 3.0 Tesla MRI system (Trio, Siemens Medical Systems) scanner (3D, GRE (MPRAGE), axial, $\mathrm{TR}=1750 \mathrm{~ms}, \mathrm{TE}=1100 \mathrm{~ms}, \mathrm{FOV}=256 \mathrm{~mm}$, slice thickness $=1.0 \mathrm{~mm}$, flip $=20^{\circ}$, Bandwidth $(220 \mathrm{~Hz} /$ pixel $)=$ 256 (phase) $\times 256$ (frequency), number of excitations $=1$ ). Cortical thickness was assessed in 148 cortical regions (5) after regressing out the influences of gender, age, social economic status and IQ. Interregional partial correlations across participants that exceeded specified thresholds constituted connections in a cortical brain network. Four measures of network centrality, i.e. degree, betweenness, closeness and eigenvector centralities, were applied to 
characterize network topology and the importance of nodes with the network (6). Ten thousand times permutation tests were employed to investigate the between-group alternations in key centrality measures of cortical areas.

Results: Maltreated youth with PTSD demonstrated larger centrality in left subcallosal gyrus (in ACC) and right gyrus rectus (in vmPFC), and smaller centrality in right orbital part of IFG than maltreated youth without PTSD. Compared with healthy controls, maltreated youth without PTSD showed smaller centrality in left orbital part of IFG, while maltreated youth with PTSD showed smaller centrality in right orbital part of IFG. Between-group differences were also found in several other brain areas.

Conclusions: Maltreated youth with (versus without) PTSD showed altered network centrality for brain regions that are widely associated with social cognition, emotional processing, inhibition of unwanted memories, response inhibition, motion and somatosensory information processing. Further longitudinal work is needed to characterize vulnerability to chronic and persistent PTSD, and resilience to PTSD following maltreatment.

Keywords: Childhood Maltreatment, PTSD, Graph Theory, Cortical Thickness.

Disclosure: Nothing to disclose.

\section{W110. Neural Correlates of Implicit Emotion Processing in Pediatric Bipolar Disorder and Familial Risk of Bipolar Disorder}

Simone Haller*, Joel Stoddard, Kendra Hinton, Banafsheh Sharif-Askary, Kenneth Towbin, Daniel Pine, Melissa Brotman, Ellen Leibenluft

National Institute of Mental Health, Bethesda, Maryland, United States

Background: Bipolar disorder (BPD) is a highly heritable and impairing psychiatric condition. Fronto-limbic dysfunction during face-emotion processing has been suggested as a marker of genetic risk of BPD. Investigating whether aberrant responses to face-emotions in fronto-limbic networks manifest in both patients with BPD and their unaffected relatives is important in order to differentiate neural mechanisms of illness vs. risk.

Methods: Study groups comprised adolescents (12-21 years) with $\mathrm{BPD}(n=20)$, at familial risk of BPD (one first-degree relative with BDP; FS; $n=20$ ), or with no psychopathology (healthy volunteer; $\mathrm{HV} ; n=28$ ). Groups were matched on age, intelligence, and sex. During fMRI, participants judged the gender of happy, fearful, and angry face-emotions, each with three intensities of expression $(50 \%, 100 \%, 150 \%)$. In a preliminary analysis, whole-brain effects of Group, FaceEmotion, and Intensity were tested with a multivariate model, and the results were thresholded at voxelwise $p=.005$, whole-brain cluster corrected to $p<0.05(\mathrm{k}=75)$.

Results: A Group by Intensity interaction emerged in the left dlPFC. Post-hoc comparisons suggested that the interaction was driven by increased activation in BPD youths to high intensity expressions relative to $\mathrm{HV}(p=.05)$ and $\mathrm{FS}(p=.001)$ groups.

Conclusions: Youths with BPD showed increased activation of the dlPFC to high intensity emotional expressions relative to both healthy adolescents and adolescents at risk for BP.
This suggests that dlPFC dysfunction during implicit faceemotion processing is associated with $\mathrm{BP}$ itself, rather than with risk for the illness. This finding extends prior work on PFC-dependent cognitive functioning in BPD, perhaps indicating effortful disengagement from highly intense emotional displays to maintain task performance. Replication with larger samples is needed.

Keywords: BOLD Imaging, Bipolar Disorder, At-Risk, Emotion Processing.

Disclosure: Nothing to disclose.

\section{W111. Targeting the Brain Circuit With Protein: Protein} Interaction-Based Molecular Probes

Syed Ali, Zhiqing Liu, Miroslav Nenov, Federico Scala, Thomas James, Aditya Singh, Haiying Chen, Jia Zhou, Fernanda Laezza*

University of Texas Medical Branch, Galveston, Texas, United States

Background: Protein-protein interactions (PPI) within ion channel complexes fine-tune neuronal excitability and are emerging as links to the biology of psychiatric disorders. Their highly specific and flexible interfaces could make protein-channel interactions ideal targets for development of molecular probes that would provide the neuropharmacology community with the tools to interrogate circuit biology with unprecedented precision. In response to the urgent need for novel, powerful molecular probes, we have identified the PPI between the voltage-gated $\mathrm{Na}+$ (Nav) Nav1.6 channel and its accessory regulator protein, fibroblast growth factor 14 (FGF14) as a novel, functionally relevant regulator of neuronal excitability in the cortico-mesolimbic circuit. This PPI is also a downstream target of a glycogen synthase kinase 3 (GSK3) pathway that is aberrant responsive to depression and addiction. To interrogate the role of the FGF14:Nav1.6 complex in native conditions in the nucleus accumbens (NAc) circuit, we designed the ZL181 peptide-derivative mapped to the FGF14:Nav1.6 complex interface and showed it has in vitro-to-ex vivo activity. These data identify a new relevant probe for interrogating the FGF14:Nav1.6-C-tail complex as a new functionally/translationally relevant druggable target for medication development against disorders of the emotional domain.

Methods: We employed medicinal chemistry for peptide modification, the split-luciferase assay (LCA), surface plasmon resonance (SPR), patch-clamp electrophysiology in heterologous expression systems and ex vivo brain slices to synthesize and validate the activity of ZL181 in in vitro, incell and ex vivo preparations.

Results: We used a four amino-acid scaffold previously validated as an FGF14 dimer peptide inhibitor to generate a series of analogues with improved drug-like properties. Initial validations using the split-luciferase assay (LCA) led to the identification of ZL181 as a promising compound. With LCA, we showed that ZL181 inhibited the FGF14:Nav1.6-Ctail complex formation $(75 \pm 6 \%, n=9, p<0.001$, Student's t test) compared to control ( $0.5 \%$ DMSO, used as a vehicle) with an estimated IC50 $=63 \mu \mathrm{M}$. SPR studies showed higher binding affinity of ZL181 for FGF14 $(\mathrm{Kd}=13 \mu \mathrm{M})$ compared to the Nav1.6 C-tail $(\mathrm{Kd}=212 \mu \mathrm{M})$ and binding pockets confirmed 
by in silico docking. The functional activity of ZL181 was first evaluated in HEK293 cells stably expressing Nav1.6 using patch-clamp electrophysiology. We found that in cells pretreated with ZL181 $(20 \mu \mathrm{M})$, Nav1.6-mediated peak current density was significantly lower $(-20.9 \pm 3.4 \mathrm{pA} / \mathrm{pF}, n=12$, $p<0.05)$ compared to DMSO treated control $(-73.8 \pm 13.6$ $\mathrm{pA} / \mathrm{pF}, n=12)$ and the phenotype was even greater if FGF14GFP was transiently co-expressed $(-7.4 \pm 4.4 \mathrm{pA} / \mathrm{pF}, n=19$ in the presence of ZL181 compared to untreated FGF14-GFP control, $-18.1 \pm 3.8 \mathrm{pA} / \mathrm{pF}, n=20$ ). Furthermore, we found that ZL181 had no effect on the biophysical properties of Nav1.6, but prevented changes in the channel availability induced by FGF14-GFP expression ( $p>0.05$, one-way ANOVA, post-hoc Dunnett's test). Having validated the functional activity of ZL181 in cell lines, we then sought to use ZL181 as a probe to interrogate the role of the FGF14:Nav1.6 complex in medium spiny neurons (MSN) of the NAc. Patch-clamp electrophysiology studies in Fgf14+/+ animals indicated that ZL181 significantly reduced the number of evoked action potentials (AP) compared to the DMSO control group (the maximum number of evoked APs in DMSO was 18.4 \pm 1.3 , $n=8$ versus $6.9 \pm 2.3, n=10$ in ZL181 $p<0.005$ with Student t-test). In the same experimental groups, ZL181 significantly increased both AP voltage threshold $(-35.9 \pm 2.3 \mathrm{mV}$ for the DMSO group, $n=8$ versus $-27.8 \pm 2 \mathrm{mV}$ for ZL181, $n=10$; $p<0.05$ with Student t-test) and current threshold $(72.5 \pm$ $11.5 \mathrm{pA}$, DMSO group $n=8$ versus $135.8 \pm 12.5 \mathrm{pA}$ for ZL181, $n=10 ; p<0.01$ with Student's t-test) in MSNs compared to DMSO control. Importantly, ZL181 did not lead to changes in intrinsic excitability and AP threshold in MSNs derived from Fgf14-/- animals, suggesting that its activity depends upon the expression of FGF14.

Conclusions: We have designed and validated a new FGF14dependent molecular probe that can fine-tune neuronal excitability. This and other related probes will provide new research tools to study the biology of the reward circuit and advance treatment for a broad spectrum of psychiatric disorders of the affective and cognitive domain.

Keywords: Excitability, GSK3, Mood and Anxiety Disorders. Disclosure: Supported by: NIH Grant R01MH095995 (FL), John Sealy Memorial Endowment Funds (FL), R01 DA038446 (JZ), P30 DA028821 (JZ), T32 GM089657-04 NIGMS/NIH (SRA), NIH/NIEHS-T32ES007254 (TFJ).

\section{W112. No Free Lunch in Life: The Neural Basis of Conflict Resolution}

Thilo Deckersbach*, Samuel Zorowitz, Afsana Afzal, Kristen Ellard, Alik Widge, Anna Gilmour, Todd Herrington, Shaun Patel, Angelique Paulk, Emad Eskandar, Darin Dougherty

Massachusetts General Hospital, Charlestown, Massachusetts, United States

Background: Reward and punishment govern human behavior. Individuals seek to obtain rewards and to avoid punishment. By and large, studies have investigated the processes associated with reward and punishment separately. However, in daily life, obtaining rewards often comes with the likelihood of negative consequences (punishment). For behavior to be effective, humans need to resolve the conflict between these two determinants of behavior in most given situations. We have developed a novel paradigm that integrates reward and punishment to study the neurobiology of conflict in decision-making.

Methods: 20 healthy volunteers completed and AversionReward Conflict (ARC) paradigm during fMRI scanning. In the ARC paradigm participants chose between a safe option (pays $\$ 0.01$ ) and a risky option (pays between $\$ 0.05-\$ 1.00$ ) at the risk of receiving uncomfortable electrical stimulation. Risk levels were varied between three categories (10\%, 50\%, 90\% probability of shock). Thus, participants can avoid stimulation at the cost of foregoing reward. To predict the probability of each participant's decision for each trial we utilized a Bayesian hierarchical regression model that inferred a per-trial estimate of the probability of a subject's decision (risky vs. safe option) conflict as a function of the risk, level of reward and reaction time. Choice was modeled as a logistic regression on categorical risk $(10 \%, 50 \%, 90 \%)$ and continuous reward levels (\$0.06 - \$0.96). Reaction times were simultaneously used to predict likelihood-of-take through a parabolic regression, which assumed that reaction times increase as a participant approaches their point of maximum conflict (50\% probability of choosing the risky option, also called the decision boundary). FMRI data were analyzed using FsFAST. Outputs from the behavioral analyses, in particular the distance-fromdecision-boundary (point of maximum conflict), were used as parametric regressors in the fMRI analyses.

Results: fMRI analysis shows that activation in the dorsolateral prefrontal cortex (DLPFC) and the dorsal anterior cingulate cortex (dACC) increased as subjects approach their decision boundary (point of maximum conflict; $p<.001$, family wise error corrected). These activations reflect conflict detection and/or resolution as they were reflective of either approach (take) or avoidance (forego reward).

Conclusions: While most previous work in decision-making has focused on studying reward and punishment separately, in this novel paradigm we integrate both risk of punishment as well as monetary reward to study the neural mechanisms underlying conflict processing during decision-making. The increased DLPFC and dACC activation during trials near the individual's decision boundary suggests that DLPFC and dACC play a role in signaling and resolving the conflict that arises between choosing a reward coupled with electric stimulation or foregoing that reward to avoid an aversive experience.

Keywords: Reward-Based Decision-Making, ApproachAvoidance, Conflict Monitoring, Risk-Reward Paradigm. Disclosure: Nothing to disclose.

\section{W113. Hippocampal Serotonin Transporter Binding Inversely Correlates With Serum BDNF Levels in Healthy Humans}

Julia Sacher*, Matthias L. Schroeter, Pablo M. Rusjan, Jürgen Kratzsch, Julia Luthardt, Georg Becker, Marianne Patt, Osama Sabri, Arno Villringer, Swen Hesse

\section{Max Planck Institute, Leipzig, Germany}

Background: The hippocampus has a critical role in learning, memory and mood regulation. Furthermore, an extraordinary capacity for neural plasticity in this brain structure has been 
documented that is not limited to the developing brain. Evidence for adult neurogenesis in the hippocampus has been found as a result of exercise and environmental enrichment (Olson et al., 2006). Psychosocial and physiological stress (McEwen et al., 1999), as well as childhood adversity and depression (Opel et al., 2014) have been associated with neural atrophy in the adult hippocampus. One of the mechanisms discussed as a mediator for adult hippocampal neuroplasticity is via enhancement of serotonin (5-hydroxytryptamin, 5-HT) availability and an increase in brain-derived neurotrophic factor (BDNF). In the mature brain, BDNF is critical for synaptic plasticity and dendritic growth and can be regulated through serotonergic signaling and vice versa (Lesch and Waider, 2012; Martinowich and $\mathrm{Lu}, 2008$ ). However, a first report exploring both measures has not supported a strong relationship across a variety of serotonin transporter (5-HTT)rich regions (Klein et al., 2010). Here, we investigate 5-HTT availability in the hippocampus in healthy human subjects in vivo and BDNF-concentration in serum, a measure that currently seems to reflect the totality of circulating BDNF with less variability than plasma or whole blood (Polacchini et al., 2015). Given the key role of the 5-HTT for the management of reuptake and availability of endogenous 5-HTT in the synaptic cleft, we hypothesized hippocampal 5-HTT binding to inversely correlate with serum BDNF levels.

Methods: We used [11C]DASB Positron Emission Tomography (PET) imaging to determine 5-HTT density in vivo in 22 healthy human subjects. Hippocampal binding potential (BPnd) was estimated using the Logan graphical analysis with reference tissue (cerebellum cortex) and simplified reference tissue model 2 (SRTM2). BDNF concentration was measured in serum samples.

Results: We found an inverse correlation between 5-HTT BPnd and $\mathrm{BDNF}$ serum levels $(\mathrm{R} 2=0.51, p=0.0003)$ in the hippocampus in healthy human subjects (mean age: 37 years [SD: 9 years], 12 females, 10 males) for both analysis methods (Logan and SRTM2). A complementary study using the whole brain and whole genome wide atlas of the Allen Institute for Brain Sciences showed that BDNF expression in the hippocampal regions is significantly higher than whole brain expression.

Conclusions: Our findings support a region-specific inverse association between hippocampal 5-HTT density and peripheral serum BDNF levels. In the light of a previous negative report on 5-HTT binding levels in the caudate, putamen and thalamus, our results are the first to highlight the specific role of the hippocampus for this 5-HTT and BDNF relationship in humans in vivo. This finding is further supported by postmortem data from the human Allen Brain Atlas emphasizing the hippocampus as the brain region with highest BDNF expression across the whole brain. Our data argue for the suitability of serum BDNF as a peripheral indicator for 5-HTT availability in the hippocampus. Thus, monitoring individual serum BDNF levels could be useful for identifying heightened vulnerability to major depressive disorder (MDD) relapse, for example during main hormonal transition periods associated with heightened risk for MDD. Furthermore, this peripheral marker could be a valuable asset in studies testing selective 5-HT reuptake inhibitors to improve neural reorganization post-stroke.

Keywords: Hippocampus, Serotonin Transporter, PET Imaging, BDNF.

Disclosure: Nothing to disclose.
W114. Disruption of White Matter Tracts as Measured by Diffusion Tensor Imaging is Associated With Poor Antidepressant Response to Lurasidone in Bipolar Depression

Martin Lan*, Harry Rubin-Falcone, Fatima Motiwala, Jonathan Stewart, David Hellerstein, J. Mann, Patrick McGrath

Columbia University Medical Center, New York, New York, United States

Background: Bipolar disorder remains a significant public health problem. Patients spend the most time in the depressed phase and that phase is associated with the most morbidity and mortality. Treatment of bipolar depression is a trial and error process. White matter disruptions have been found in bipolar disorder. Previous reports suggest that white matter disruptions may be associated with resistance to medication treatment, but this has never been studied with a prospective study using an FDA approved medication.

Methods: 18 subjects with bipolar disorder who were in a major depressive episode and off of all medications were recruited. Magnetic resonance imaging was acquired using a 64 direction diffusion tensor imaging sequence on a Siemens $3 \mathrm{~T}$ scanner. Subjects were treated with eight weeks of lurasidone. Montgomerey Asberg Depression Rating Scale (MADRS) was measured on a weekly basis. Tract-Based Spatial Statistics was utilized to perform a regression analysis on the FA data using treatment outcome as assessed by percent change in MADRS as a regressor while controlling for age and sex, using a threshold of $\mathrm{p}$ (cluster corrected) $<0.05$. Results: FA was positively correlated with antidepressant treatment response in multiple regions of the mean FA skeleton. Regions of the cingulate bilaterally were correlated, as well as areas of the parietal and frontal lobes bilaterally. Conclusions: These data indicate that greater disruptions in the white matter tracts in bipolar disorder are associated with less antidepressant response to an acute treatment course of an FDA approved medication, lurasidone. White matter disruptions may therefore have clinical importance to the disorder. This may be a step to develop a tool to identify treatment resistant patients who would require more aggressive treatment options or safety monitoring.

Keywords: DTI, Bipolar Disorder, Atypical Antipsychotics, Depression.

Disclosure: Sunovian Pharmaceuticals: Salary Support, Self.

\section{W115. Connectome-Wide Analysis Reveals Common Dimensional Reward Deficits Across Mood and Psychotic Disorders}

Anup Sharma*, Daniel Wolf, Rastko Ciric, Joseph Kable, Tyler Moore, Simon Vandekar, Natalie Katchmar, Aylin Daldal, Kosha Ruparel, Christos Davatzikos, Mark Elliott, Monica Calkins, Russell Shinohara, Danielle Bassett, Theodore Satterthwaite

University of Pennsylvania, Philadelphia, Pennsylvania, United States

Background: Anhedonia is central to a number of psychiatric disorders and is associated with substantial disability. A 
dimensional conceptualization of anhedonia posits that symptom severity relates to a continuum of deficits among specific neural networks. Prior investigations of functional connectivity related to reward deficits have focused on casecontrol designs using seed-based analyses. Here, the authors explore the entire functional connectome in relation to reward deficits across a heterogeneous population of adults with psychiatric disorders.

Methods: Using a sample of 225 adults from five diagnostic groups (major depressive disorder, $n=32$; bipolar disorder, $n=50$; schizophrenia, $n=51$, psychosis risk, $n=39$; and normal controls, $n=53$ ), the authors conducted a connectome-wide analysis to examine the relationship between a dimensional measure of reward responsivity (reward sensitivity subscale of the Behavioral Activation Scale) and resting-state functional connectivity using multivariate distance-based matrix regression.

Results: This connectome-wide analysis identified foci of dysconnectivity associated with reward responsivity in the nucleus accumbens (NAc), default mode network (DMN) and cingulo-opercular network (CON). Follow-up analyses revealed dysconnectivity among specific large-scale functional networks and their connectivity with the NAc. Reward deficits were associated with DMN hyper-connectivity and diminished connectivity between DMN and CON. In addition, impaired reward responsivity was associated with decreased connectivity between the NAc and DMN and increased connectivity between the NAc and CON.

Conclusions: These results emphasize the centrality of the nucleus accumbens in the pathophysiology of reward deficits and suggest that dissociable patterns of connectivity among large-scale networks are critical to the neurobiology of reward dysfunction across clinical diagnostic categories.

Keywords: Functional Neuroimaging, Anhedonia, Reward Deficit, Resting State fMRI.

Disclosure: Nothing to disclose.

W116. Deep Brain Stimulation of Striatal White Matter Alters Top-Down Control Signals in Cingulate and Prefrontal Cortices

Alik Widge*, Samuel Zorowitz, Afsana Afzal, Kara Farnes, Angelique Paulk, Earl Miller, Thilo

Deckersbach, Sydney Cash, Emad Eskandard, Darin Dougherty

Harvard Medical School, Charlestown, Massachusetts, United States

Background: Deep brain stimulation (DBS) of the ventral capsule/ventral striatum (VC/VS) has open-label efficacy in obsessive-compulsive disorder (OCD) and major depressive disorder (MDD). Mixed results in recent controlled trials highlight how little is understood about this invasive therapy's mechanisms. We previously reported that VC/VS DBS improves' patients information-processing on cognitive control tasks. We now present results linking that behavior change to changes in oscillatory neural firing related to topdown control.

Methods: 14 patients with VC/VS DBS and severe MDD (two with comorbid OCD) performed the Multi Source Interference Task (MSIT) with DBS ON, then repeated the task after an hour with DBS OFF. We recorded 60-channel EEG during task performance, digitized electrode positions, and localized scalp EEG to cortical sources. A further four patients without psychiatric diagnoses completed a similar protocol in the epilepsy monitoring unit, in this case receiving intermittent stimulation through clinical electrodes passing through the internal capsule. In these patients, we assessed the behavioral effects of unilateral stimulation at multiple sites within the VC/VS.

Results: In psychiatric patients, MSIT reaction times were $7 \%$ faster with DBS ON $(t=-9.28, d f=3879, p<2.8 \mathrm{e}-20$, Wald test on GLM coefficients), even after controlling for within-block learning, repeated-measures testing, and confounding trial factors. Non-parametric regression of tasklinked EEG showed alpha and theta band changes in dorsal and rostral cingulate and bilateral DLPFC. All significant clusters $(p<0.05$ after FDR-adjustment) were $400-500 \mathrm{~ms}$ long, and showed an abrupt offset at the reaction time, indicating that the DBS effect was specific to task-induced oscillations. In epilepsy patients, VC/VS stimulation decreased reaction time up to $11 \%$, but with anatomic specificity. Right dorsal VC/VS stimulation produced a profound behavioral effect $(\mathrm{t}=-4.39, \mathrm{df}=1056, p<1.21 \mathrm{e}-5)$, while left ventral stimulation was ineffective $(t=0.22$, $\mathrm{df}=1056, p<0.83$ ).

Conclusions: Improved cognitive performance in humans receiving VC/VS stimulation is associated with changes in oscillatory phenomena previously linked to top-down executive control. This supports the hypothesis that DBS acts by modulating frontal structures through white matter pathways. A second set of experiments with acute stimulation suggest that the effect is linked to fibers passing through the right dorsal striatum, possibly those connecting thalamus to right dorsal cingulate. The anatomic specificity of the effect and task-driven electrophysiologic changes might be useable as biomarkers for closed-loop therapy titration to improve clinical response in MDD and OCD.

Keywords: Deep Brain Stimulation, Cognitive Control, Anterior Cingulate Cortex, Prefrontal Cortex, Neuromodulation.

Disclosure: Harvard Medical School: Patent filings, Self.

\section{W117. Alterations of Neuroligin-2 and Gabaergic Function in Rats Exposed to Chronic Mild Stress: Modulation by Chronic Treatment With Lurasidone}

Andrea Carlo Rossetti, Laura Rubini, Francesca Nirella, Giorgio Racagni, Raffaella Molteni, Marco Andrea $\mathrm{Riva}^{*}$

University of Milan, Milan, Italy

Background: In recent years, the concept of "synaptopathy" has extended from neurodegenerative disorders to psychiatric diseases. Indeed, alterations of synaptic structure and function act as a main determinant for mental illness and may represent an important target for therapeutic intervention. On these bases, aim of this study was to establish the potential impact of chronic stress on neuroligins, a family of postsynaptic type 1 transmembrane proteins that play a pivotal role in synaptogenesis but also in the control of the excitation/inhibition (E/I) balance in the CNS. Furthermore, 
we investigated the ability of lurasidone, an antipsychotic drug with a multi-receptor profile, to counteract the effects set in motion by chronic stress exposure.

Methods: Adult male Wistar rats were exposed to chronic mild stress (CMS) for 2 weeks and sucrose consumption was used to identify rats that were susceptible to the stressful manipulation. Control and CMS-susceptible rats were then randomized to receive chronic vehicle or the multi-receptor modulator lurasidone ( $3 \mathrm{mg} / \mathrm{kg} /$ day) for 5 more weeks, while continuing the stress procedure. 24 hours after the last drug administration, the animals were sacrificed and the brain regions of interest were rapidly dissected and stored for molecular analyses. Specifically, real time PCR and/or western blot analyses were used to investigate the expression of neuroligin (NLGN) 1 and 2, as well as a number of GABAergic markers, using $ß$-actin as internal standard.

Results: Exposure to CMS produced significant changes on neuroligins, with a specific reduction of NLGN2 levels in ventral and dorsal hippocampus, without affecting the expression of NLG-1. Interestingly, chronic treatment with lurasidone was able to normalize the reduced protein levels of NLGN-2 in the ventral hippocampus. Moreover, chronic drug treatment increased NLGN-2 in the dorsal hippocampus of control as well as of stressed rats. The changes of NLGN-2 levels were associated with altered expression of different GABAergic determinants. Indeed, CMS reduced the levels of the presynaptic marker GAD67 and VGAT in the ventral hippocampus as well as the protein levels of parvalbumin, which identifies a sub-group of GABAergic neurons, in dorsal hippocampus. Chronic administration of lurasidone was also able to normalize the stress-induced changes of GAD67 as well as of parvalbumin.

Conclusions: Our results provide novel evidence on the ability of lurasidone to interfere with molecular systems crucial for synaptic function, such as the neuroligins. In particular, we found that lurasidone was able to normalize the effects produced by CMS exposure on NLGN2 and on the GABAergic system. In a translational perspective the effects of lurasidone may be important to normalize synaptic mechanisms and neurotransmitter unbalances that contribute to specific functions that are deteriorated in stressrelated psychiatric disorders.

Keywords: GABAergic Interneurons, Hippocampus, Synaptic Function, Chronic Stress, Lurasidone.

Disclosure: Sumitomo Dainippon Pharma; Research Grant, Self.

W118. A Randomized, Double-Blind, PlaceboControlled Study of Brexpiprazole as Adjunctive Therapy in the Treatment of Adults With Major Depressive Disorder

Mary Hobart*, Aleksandar Skuban, Peter Zhang, Ye Yang, Carol Augustine, Claudette Brewer, Nanco Hefting, Raymond Sanchez, Robert McQuade

Otsuka Pharmaceutical Commercialization and Development, Inc., Princeton, New Jersey, United States

Background: Brexpiprazole is a serotonin-dopamine activity modulator that is a partial agonist at 5-HT1A and dopamine
D2 receptors, and an antagonist at 5-HT2A and noradrenaline alpha $1 \mathrm{~B} / 2 \mathrm{C}$ receptors, all at similar potency. Brexpiprazole was approved in 2015 in the United States for treatment of schizophrenia and for use as adjunctive treatment in MDD. The current study (NCT02196506) was designed to further assess the efficacy, tolerability and safety of brexpiprazole ( $2 \mathrm{mg} /$ day) as adjunctive therapy to an assigned open-label antidepressant therapy (ADT) in patients with MDD.

Methods: This study had 3 phases: a screening phase (7-28 days); a prospective phase: 8 -week, single-blind placebo plus an investigator-determined, open-label ADT; a randomized phase: 6-week, double-blind, adjunctive brexpiprazole $(2 \mathrm{mg} /$ day) vs. placebo in patients with an inadequate response to ADT. The primary efficacy endpoint was change from the end of the prospective phase to the end of the randomization phase [hereafter referred to as change from baseline] in Montgomery-Åsberg Depression Rating Scale (MADRS) total score. Efficacy analyses were conducted using mixed model repeated measures (MMRM), including treatment, visit, site and treatment-by-visit interaction as fixed effects and baseline score-by-visit as covariate. Tolerability and safety outcomes included treatment-emergent adverse events (TEAEs), suicidality, body weight, and laboratory tests including prolactin.

Results: A total of 394 patients were randomized and received treatment $(\mathrm{ADT}+$ placebo $=202 ; \mathrm{ADT}+$ brexpiprazole $=192$ ). MADRS total scores at randomization were 26.2 and 27.1 in the $\mathrm{ADT}+$ placebo and $\mathrm{ADT}+$ brexpiprazole groups, respectively. A total of 202 patients on ADT+placebo and 191 patients on $\mathrm{ADT}+$ brexpiprazole were included in the primary efficacy analysis; $97 \%$ on ADT + placebo and $92 \%$ on ADT+brexpiprazole completed the study. Statistically significant improvements for the primary endpoint, mean change from baseline in MADRS total score was demonstrated for patients receiving adjunctive brexpiprazole compared with adjunctive placebo (LS mean change: -10.4 vs $-8.1 ;-2.30$ [95\% CI $-3.97 ;-0.62] p=0.0074)$. Overall incidence of TEAEs was $60 \%$ and $50 \%$, in the ADT +brexpiprazole and $\mathrm{ADT}+$ placebo groups, respectively. Most frequent TEAEs (incidence $\geq 5 \%$ and greater than placebo) in the $\mathrm{ADT}+$ brexpiprazole group were akathisia $(8.3 \%$ vs $5.0 \%$ in the placebo group+ ADT group), restlessness (8.3\% vs $2.0 \%)$, weight increase $(5.2 \%$ vs $0.5 \%)$ and upper respiratory tract infection (5.2\% vs $5.0 \%$ ). There were no deaths, completed suicides or suicide attempts during the study. Four patients $(2.1 \%)$ in the $\mathrm{ADT}+$ brexpiprazole group and 1 patient $(0.5 \%)$ in the $\mathrm{ADT}+$ placebo group withdrew due to AEs. The LS mean weight gain was $1.6 \mathrm{~kg}$ in the ADT +brexpiprazole group vs $0.5 \mathrm{~kg}$ in the $\mathrm{ADT}+$ placebo group. An increase in body weight of $\geq 7 \%$ was seen at the last visit in $8 / 192(4.2 \%)$ brexpiprazole and $2 / 202(1.0 \%)$ placebo patients. The safety and tolerability profile of brexpiprazole was similar to that observed in the US registration studies and showed no unexpected side effects.

Conclusions: This study confirmed the efficacy and tolerability of brexpiprazole as adjunctive treatment in patients with MDD with inadequate response to ADTs.

Keywords: Brexpiprazole, Major Depressive Disorder (MDD), Adjunctive Treatment.

Disclosure: Otsuka: Employee, Self. 
W119. Association of Thalamic Hyperactivity With Treatment-Resistant Depression and Poor Response in Early Treatment for Major Depression: A Resting-State fMRI Study

Yasumasa Okamoto*, Takanao Yamamura, Go Okada, Masahiro Takamura, Naho Ichikawa, Chiyo Shibasaki, Atsuo Yoshiko, Hidehisa Yamashita, Shigeto

Yamawaki

\section{Hiroshima University, Minami-ku, Japan}

Background: A better understanding of the biological pathogenesis of treatment-resistant depression (TRD) is required to rapidly detect patients who are likely to develop treatment resistance, and to develop more effective therapeutics for these patients. One of the possible methods used to reveal the biological mechanisms underlying TRD is resting-state functional magnetic resonance imaging (fMRI). Several studies have suggested that patients with TRD have different resting-state spontaneous neural activity compared with patients with treatment-sensitive depression. In contrast, neuroimaging studies have also identified brain regions that are associated with responsiveness to treatment in patients with major depressive disorder (MDD). We therefore hypothesized that the spontaneous regional neural activity in patients with TRD would be associated with antidepressant treatment responsiveness in the early phase of treatment in patients with MDD. The purpose of this study was to identify brain regions in which spontaneous neural activity is not only altered in TRD but also associated with early treatment resistance in MDD.

Methods: Sixteen patients with TRD, 16 patients with earlyphase non-TRD and 26 healthy control (HC) subjects underwent resting-state functional magnetic resonance imaging. The non-TRD group included patients with MDD who were either untreated or treated with a single antidepressant at an insufficient dose and duration. Patients who were experiencing treatment-resistance according to the criteria established by European Medicines Agency guidelines (treatment-resistance level of at least stage 2, according to the Thase and Rush definition) were recruited.

To identify brain region differences in spontaneous neural activity between patients with and without TRD, we assessed fractional amplitude of low-frequency fluctuations (fALFF). We also calculated correlations between the percent change in the Hamilton Rating Scale for Depression (HRSD17) scores and fALFF values in brain regions with differing activity for patients with and without TRD. This study protocol was approved by the Ethics Committee of the Hiroshima University. Written informed consent was obtained from all the participants.

Results: Age, sex, age of onset and intelligence quotient were not statistically different among the three groups. Both groups of patients showed moderate severity of depressive symptoms and depression severity was not significantly different between the patients with TRD and non-TRD before selective serotonin reuptake inhibitor treatment. Most of the patients with TRD were chronically depressed (87.5\%). None of the patients had comorbid psychotic symptoms.

Compared with patients in the non-TRD group, patients with TRD showed increased fALFF values in the right inferior frontal gyrus (IFG), right middle occipital gyrus, right thalamus, right inferior parietal lobule (IPL) and vermis. In patients with non-TRD, only the right thalamus showed negative correlation between mean fALFF values and percent change in HRSD17 scores.

Conclusions: We combined cross-sectional comparisons of patients with TRD and non-TRD with a prospective followup of the non-TRD group. Through this approach, we reveal that spontaneous thalamic hyperactivity has a key role not only in patients with TRD, but also in the early phase of antidepressant treatment resistance among patients with MDD. In addition, we show that spontaneous activity in the right IFG, IPL and vermis may contribute to the specific neural substrates underlying TRD. These results suggest altered cognitive and emotion regulation neural circuits in patients with TRD.

Keywords: Treatment Resistant Depression, Resting State fMRI, Thalamus.

Disclosure: Nothing to disclose.

\section{W120. Apathy Mediates Cognitive Difficulties in Geriatric Depression}

Prabha Siddarth*, Helen Lavretsky

Semel Institute for Neuroscience \& Human Behavior, Los Angeles, California, United States

Background: Cognitive impairment associated with late-life depression can persist after remission of mood symptoms. Apathy is a common symptom of late-life depression and often leads to worse clinical outcomes. We examined if severity of apathy mediates cognitive difficulties in a cohort of older adults with major depression.

Methods: 138 older adults with major depression (54.4\% female; mean age $=69.7(7.4)$ years; mean education $=15.6$ (2.7) years) were recruited to participate in a treatment study. All participants received a comprehensive evaluation of depression (Hamilton Depression Rating Scale), apathy (Apathy Evaluation Scale), and cognition, as measured by a comprehensive neuropsychological battery. In this study, we focused our attention on two domains, namely memory (the California Verbal Learning Test-II [long delayed free recall] and the Rey-Osterrieth Complex Figure Test [30-minute delayed recall]), and cognitive control (the Trail Making Test, parts A and B, and the Stroop Color Trial and interference task [Golden version]). We first transformed raw scores to z-scores for each test score of interest for each participant (reversing z-scores for those variables where better performance was represented by lower values). These $\mathrm{z}$-scores were then averaged to produce cognitive domain scores. Data analyses included regression models, as well as mediation analyses using the PROCESS procedure of Hayes et al. We also explored whether the mediation effects were different in females and males. All analyses controlled for age, sex and educational level.

Results: Regression models indicated that increased apathy was significantly associated with more severe depression $(\mathrm{F}(1,133)=30.7, p<.0001)$ and worse performance in the cognitive control domain $(\mathrm{F}(1,133)=5.1, p=.02)$ but not memory $(\mathrm{F}(1,133)=0.6, p=.4)$. Worse cognitive control $(\mathrm{F}(1,133)=6.2, p=.01)$ but not memory $(\mathrm{F}(1,133)=1.1$, $p=.3)$ was also associated with increased depression scores. 
Mediation analyses revealed a significant indirect effect of cognitive control on depression through increased apathy scores $(-0.28(0.13), 95 \%$ CI $[-0.61,-0.08])$ with the mediator accounting for $37 \%$ of the total effect. Stratifying by sex, we found that females exhibited a significant indirect effect: $-0.36(0.20), 95 \%$ CI $[-0.92,-0.07])$ with the mediator accounting for $49 \%$ of the total effect, while there was no mediation by apathy on the relationship between depression and cognitive control in males.

Conclusions: The findings imply that increased apathy mediates the relationship between cognition and depression, especially in females. The identification of mediating effects may inform future treatment strategies and preventive interventions that can be focused on decreasing apathy to improve the cognitive outcomes of late-life depression.

Keywords: Late-Life Depression, Cognitive Impairments, Apathy.

Disclosure: Nothing to disclose.

\section{W121. Translational Profiling of CA3 Neurons in BDNF Va66Met Mice Exposed to Early Life Stress Reveals Molecular Pathways Associated With Susceptibility to Mood Disorders}

Jason Gray*, Joshua Kogan, Todd Rubin, Eric Schmidt, Bruce McEwen

Rockefeller University, New York, New York, United States

Background: Early life stress (ELS) has been associated with an increased risk of developing a mental health disorder later in life. Rodent models of ELS show impaired learning and memory in adulthood that is coincident with persistent changes in hypothalamic-pituitary adrenal (HPA) axis reactivity and neuronal morphology. Importantly, decreases in dendritic spine density in the CA3 neurons of the hippocampus (a region important for learning and memory) have been observed in adult mice exposed to bedding deprivation as pups.

Several genetic mutations have also been identified that can increase the risk of developing a mood disorder. Among them is a single nucleotide polymorphism in the brain derived neurotrophic factor (Bdnf) gene that results in the substitution of a methionine (Met) for a valine (Val) at position 66 . These mice have decreased activity-dependent secretion of BDNF and exhibit increased sensitivity to the effects of stress in adulthood. However, the effects of ELS on BDNF Val66Met mice remain unknown. In this study, the effects of ELS on the translational profile of CA3 pyramidal neurons were examined in the BDNF Val66Met mice. These results will reveal the similarities and differences underlying genetic and environmental manipulations that increase susceptibility to mood disorders.

Methods: Isolation of in vivo translating RNA fractions from a genetically homogenous population of CA3 pyramidal neurons was accomplished using transgenic mice expressing an EGFP fused to the L10a ribosomal subunit that is under the control of a cell-type specific promoter (Gprin3). These reporter mice were crossed with BDNF Val66Met allele carriers to generate double transgenic animals, which were then subjected to bedding and nesting deprivation from P2-P12 (Rice...Baram, 2008). Afterwards, mice were given standard housing conditions until 4 mos of age. Mice were rapidly decapitated and the hippocampus was dissected for RNA isolation by Translating Ribosomal Affinity Purification (TRAP). TRAP and unbound mRNA fractions were subjected to RNA-sequencing using an Illumina Hi-Seq 2500 to collect $100 \mathrm{bp}$ reads at a sequencing depth of $30 \mathrm{M}$ reads/ sample. Results were aligned against the mouse genome (mm10) and the numbers of reads for each transcript were normalized to obtain relative expression levels. Strand software (Agilent) was used to perform statistical analysis to identify differentially expressed genes, which were grouped into pathways using the DAVID online tool.

Results: Comparisons of the TRAP fractions from unstressed BDNF-Met/+ mice with unstressed wild type (WT) mice identified changes in the levels of 1,420 genes. Further, unstressed homozygous BDNF-Met/Met mice showed 3,736 genes changed when compared to unstressed WT mice. WT mice subjected to ELS compared with unstressed controls revealed that 5,939 genes remain changed even in adulthood. Interestingly, over half of the genes changed after ELS in WT mice were identical to those changed in the unstressed BDNF-Met/Met mice. Pathway analysis of the genes changed in Met allele carriers and mice exposed to ELS revealed common molecular mechanisms that might underlie the altered neuroanatomy and behavior, such as changes in the regulatory genes of the cytoskeleton, which are likely to be involved in neuronal remodeling. Slightly fewer genes $(5,078)$ were changed in response to ELS exposure in BDNF-Met/+ mice compared with unstressed BDNF-Met/+ mice.

Conclusions: The substantial overlap in gene pathways changed in BDNF Met allele carriers or WT mice exposed to ELS identifies common mechanisms of stress susceptibility derived from either genetic or environmental factors. Together, this data not only reveals mechanisms underlying mood disorder susceptibility in CA3 neurons, but also lays the groundwork for developing novel treatments to reverse the changes induced by ELS or resulting from a genetic predisposition to mood disorders.

Keywords: Hippocampus, Gene Environment Interactions, BDNF, Early Life Stress, RNA-seq.

Disclosure: Nothing to disclose.

\section{W122. Activation of Oxytocin Neural Circuits may Mediate Stress-Induced Deficits in Female Social Behavior}

\section{Natalia Duque-Wilckens ${ }^{\star}$, Brian Trainor}

University of California, Davis, Davis, California, United States

Background: Oxytocin (OT) is often considered to have prosocial and anxiolytic properties, but recent evidence suggests that the effects of OT are context and sex-specific. It has been proposed that OT increases the salience of social cues, which can explain why OT can either enhance or inhibit social behaviors. We recently discovered that social defeat stress induces hyperactivity in OT neurons in the medioventral bed nucleus of the stria terminalis (BNSTmv) and paraventricular nucleus, and that this effect is primarily observed in females but not males. These studies used the monogamous California mouse, which is one of the few species in which 
social defeat stress can be studied in both males and females. We also found that effects of intranasal infusions of OT (INOT) on social interaction behavior are sex specific. In mice naïve to defeat (control), a low dose of INOT has no effect on social interaction in males while it reduces social interaction in females, mirroring the effect of social defeat stress on this behavior. In stressed mice, INOT has no effect on females but increases social interaction in males. Here we conducted experiments aimed at testing the hypothesis that hyperactivity of OT neurons contributes to social withdrawal in females, and identifying receptors involved in this response as well as possible sites of action.

Methods: (1) To assess effects of OTR inhibition on behavior, control and stressed males and females were randomly assigned to receive an IP injection of saline or OTR antagonist (L-368,899, Sigma) $30 \mathrm{~min}$ before behavior testing. (2) To determine the effects of specific OT neurons suppression on behavior, control and stressed females received vivo morpholinos (GeneTools LLC, Philomath, OR) antisense or missense into BNSTmv. (3) Currently, we are using two different approaches to identify connections of OT neurons in BNSTmv. First, we are infusing a recombinant adeno-associated virus (gift of V. Grinevich) which selectively expresses Venus from an OT gene promoter into the BNSTmv. Second, we are injecting neuroanatomical tracers into predicted projection sites of the BNSTmv and staining for OT in the BNSTmv.

Results: (1) Control males and females showed high levels of social interaction. In females but not males, stress reduced social interaction. Interestingly, a single i.p. dose of a brain accessible OTR antagonist rapidly blocked the effects of stress on social interaction in females. In males the same dose reduced social interaction in both naïve and stressed males. (2) Injections of vivo morpholinos antisense into BNSTmv effectively reduced expression of OT neurons compared to injections of morpholino missense and anatomical controls. A correlational analysis of preliminary data shows a significant negative correlation between number of OT neurons in BNSTmv and social interaction behavior in stressed females. (3) Infusion of AAV OTpr-Venus is selectively expressed in OT neurons of California mice.

Conclusions: Stress-induced increase in OT neurons activity appears to increase the activation of OTR receptors which in turn drives females away from unfamiliar social contexts. These results suggest that the use of OTR antagonists could have unappreciated benefits as an anxiolytic agent in social contexts.

Keywords: Oxytocin, Social Anxiety, Bed Nucleus of the Stria Terminalis, Sex Differences, Social Defeat Stress.

Disclosure: Nothing to disclose.

\section{W123. Prediction of Clinical Depression Using Smartphone Sensory Data}

Jayesh Kamath*, Asma Farhan, Chaoqun Yue, Morillo Reynaldo, Shweta Ware, Jin Lu, Jinbo Bi, Alexander Russel, Bamis Athanosis, Bing Wang

University of Connecticut Health Center, Farmington, Connecticut, United States

Background: Depression questionnaires such as the Patient Health Questionnaire (PHQ-9) partially rely on behavioral data to diagnose and monitor clinical depression. Behavioral patterns can be detected by the large complement of sensors available on mobile phones including the global positioning systems (GPS) and accelerometers. Furthermore, mobile phones are ubiquitous, especially among college students. The objective of the present study is to investigate if sensory data collected from smart phones can predict depression symptomatology in a group of college participants.

Methods: A smartphone sensing app, called LifeRhythm was developed by the study team for both iOS and Android platforms. The app collected location and activity information via sensors available on the phones. A total of 88 college participants were recruited to install the app on their own smart phones over 8 month study period. At study initiation, all participants underwent assessment by a study clinician to determine their study eligibility. They were also assessed using a Diagnostic Statistical Manual-based interview and PHQ-9 evaluation to classify them into "depressed" or "nondepressed/control" category. Three sets of data were collected during participant's study participation: Data collected by the LifeRhythm app, PHQ-9 questionnaire completed electronically by the participant every two weeks on their smart phones, and clinical assessments conducted by the study clinician on a monthly basis (for participants in the "depressed" category). Behavioral features were developed and extracted from the GPS location and phone usage data. Results: Of the total 88 participants, 62 were iPhone users and 26 were Android phone users. A total of 18 participants were categorized as depressed and 70 were categorized as nondepressed controls. Three types of features were extracted from the data gathered by the LifeRhythm app: 1. Features based on raw GPS data- location variance, time spent in moving, total distance, average moving speed 2. Features based on location clusters- variability of time spent at different locations called entropy, normalized entropy (entropy divided by the number of unique clusters), and time spent at home 3. Features based on activity data i.e. stationary vs non-stationary states (e.g. walking, running). Correlational analyses were conducted between the smartphone data-based features and PHQ-9 scores. The preliminary analyses of the Android data indicate the entropy and number of unique locations showed a statistically significant correlation $(p<0.05)$ with the PHQ-9 scores. Differences were noted between the correlational analyses of the Android vs iPhone data with PHQ-9 scores. Differences were also noted between the data gathered between three sets of participants in the depressed category in relation to their PHQ-9 scores: participants with- consistently high PHQ-9 scores, with fluctuating PHQ-9 scores, and with consistently low PHQ-9 scores (in remission). Results for these will be presented and discussed in a descriptive/graphical manner.

Conclusions: This is the first study of its kind that collected smartphone based data together with clinical ground-truth to investigate depression over an extended period of time and on both iOS and Android platforms. Study results suggest that behavioral data collected using the LifeRhythm app can predict and monitor clinical depression with good accuracy. The multi-feature regression model and algorithm developed by our study team further improved accuracy compared to the single feature models. Diagnosis and monitoring of clinical depression may be significantly enhanced by combining smart phone-based behavioral data with standard clinical assessments and PHQ-9 scores. 
Keywords: Depression, Smartphone-Based App, Sensory Data, Mobile Health.

Disclosure: Nothing to disclose.

\section{W124. Neural Correlates of Empirically Derived Patterns of Psychiatric Symptoms in Youth}

Melissa Brotman*, Joel Stoddard, Katharina Kircanski, Susan Zhang, Argyris Stringaris, Daniel Pine,

Ellen Leibenluft

National Institute of Mental Health, Bethesda, Maryland, United States

Background: By conceptualizing domains of behavior transdiagnostically, the National Institute of Mental Health Research Domain Criteria (NIMH RDoC) initiative encourages studies examining the neural circuitry of dimensional traits that transcend traditional diagnostic boundaries. Among youth, symptoms of irritability, anxiety, depression, and inattention/hyperactivity all are prominent dimensions of psychopathology. However, there is considerable cooccurrence among these different types of symptoms, possibly reflecting shared neurobiological vulnerabilities.

Aggregating these symptom dimensions into naturallyoccurring clusters or 'symptom profiles' may account for such co-occurrence and provide empirically-derived alternatives to established DSM diagnoses. Such an approach may reveal neural signatures associated with specific combinations of symptoms. Here, we assess the neural correlates of multidimensional symptom classes derived through latent profile analysis (LPA). We hypothesize that different symptom profiles will be associated with distinct patterns of neural responses to stimuli depicting face emotions.

Methods: Two hundred youth aged 7 to 18 years with fMRI data were included in the LPA analysis. Parents and/or youth completed symptom measures assessing: depression, anxiety, irritability, and attention deficit hyperactivity disorder (ADHD). The fMRI task involved making implicit (i.e., gender) ratings of happy, angry, neutral, and fearful faces across a range of intensities.

Several classes of symptoms emerged, hereafter referred to as "LPA groups": (1) low levels of all symptoms (LOW); (2) high levels of all symptoms (HIGH); (3) high levels of irritability and $\mathrm{ADHD}$, with moderate-to-high anxiety and depression (ANX/DEP+ IRR/ADHD); (4) moderate-to-high anxiety and depression (ANX/DEP - IRR/ADHD).

fMRI analyses were conducted using AFNI. First, we modeled an omnibus three-way interaction to predict activation: LPA group $\mathrm{x}$ Face emotion $\mathrm{x}$ Emotion intensity. Follow-up analyses focused on the interactions of LPA group $\mathrm{x}$ Face emotion and LPA group $\mathrm{x}$ Emotion intensity. Analyses were thresholded voxelwise $p<0.005, \mathrm{~K}=83$.

Results: Accuracy and reaction time did not differ across LPA groups. Neither the three-way interaction nor the LPA group $\mathrm{x}$ Emotion intensity analysis yielded significant findings. Two regions emerged as significant in the LPA group $\mathrm{x}$ Face emotion activation analyses: right dorsolateral prefrontal cortex $($ dlPFC $)(\mathrm{k}=104$, Peak $x y z=46.2,18.8,31.2 ; \mathrm{F}(6$, $389.8)=5.17, p<0.001)$ and right fusiform gyrus $(\mathrm{k}=96$, Peak $\mathrm{xyz}=41.2,56.2,-16.2 ; \mathrm{F}(5.9,383.4)=5.11, p<0.001)$.
In the right dlPFC, the ANX/DEP - IRR/ADHD group exhibited hyperactivation in response fearful faces, relative to both the LOW group and ANX/DEP+ IRR/ADHD group (both ps $<0.05$ ). In contrast, the ANX/DEP+ IRR/ADHD group exhibited hypoactivation to fearful faces relative to the LOW symptom group $(p<0.05)$. Thus, ANX/DEP - IRR/ ADHD youth and ANX/DEP+ IRR/ADHD youth exhibited opposing patterns of dlPFC activation to fearful faces. The level of dIPFC activation to fearful faces in the HIGH group was in between those of the other groups, and did not differ significantly from either of them.

In the right fusiform gyrus, the HIGH group exhibited hypoactivation to fearful faces relative to both the LOW group and ANX/DEP - IRR/ADHD group (both ps $<0.05$ ). The level of fusiform gyrus activation in the ANX/DEP+ IRR/ADHD group was similar to that in the HIGH group, but did not differ significantly from the other groups.

Conclusions: In studies applying the $\mathrm{RDoC}$ framework to fMRI data, researchers typically examine associations between individual symptom dimensions and brain activity, and sometimes examine interactions between symptom dimensions. However, these studies are limited due to comorbidity, and hence collinearity, among symptom domains. In this study, we leveraged a large, well-phenotyped sample to integrate multiple symptom dimensions into classes, and then examined associations between these classes and brain activity during an implicit face emotion processing task.

The LPA groups exhibited differing patterns of activation to fearful faces in two regions: the dlPFC and the fusiform gyrus. The dIPFC mediates cognition, response inhibition, and motor control, and has been implicated in the pathophysiology of mood and anxiety disorders. Whereas ANX/DEP - IRR/ADHD showed hyperactivation to fearful faces, ANX/DEP+ IRR/ ADHD showed hypoactivation to fearful faces in the dlPFC. These results suggest that there are unique pathophysiological correlates of anxiety and depressive symptoms based on whether they are comorbid with irritability and ADHD. This pathophysiological distinction may have treatment implications. Similarly, activation in the fusiform gyrus differentiated groups. Here, youth with high levels of all symptoms showed hypoactivation to fearful faces, and were most similar ANX/ DEP+ IRR/ADHD. This finding suggests that the presence of high irritability and ADHD symptoms in youth is associated with unique pathophysiological markers. Additional work is needed to explore the stability and longitudinal clinical trajectories of these symptom classes, as well as corresponding neural correlates.

Keywords: Disruptive Mood Dysregulation Disorder, Anxiety, ADHD, Face Emotion Processing, fMRI.

Disclosure: Nothing to disclose.

\section{W125. A Model Psychopharmacology Curriculum for Teachers of Psychiatric Residents}

Ira Glick*, David Janowsky, Carl Salzman, Richard Shader

Stanford University School of Medicine, Stanford, California, United States

Background: This is the first psychopharmacology curriculum in America. It was developed by ACNP members in 
the early 1980 's. It gives a snapshot of what the field looked like as it developed in the last four decades.

Methods: An ACNP subcommittee developed the following curricula that includes the objective, overview, what and how to teach including a didactic program, development of individual or small-group supervision, development of psychopharmacology unites, utilization of reading lists, use of psychopharmacology-psychobiology journals and newsletters, representative basic psychopharmacology books, development of psychiatric neurobiology. It also included how to evaluate, how to integrate psychopharmacology into an ongoing residency curriculum, clinical psychopharmacology: relationship of research to training, and a proposed investigative psychiatry curriculum. The appendices include representative psychopharmacology curricula for various programs, lecture outlines, literature references, resident performance rating for psychopharmacology, and how to integrate psychopharmacology into an ongoing residency curriculum.

Results: The poster will include a table of contents and one of the twenty lecture outlines that was developed by ACNP members on psychopharmacologic treatment of psychiatric disorders.

Conclusions: At this point, this first version has evolved into nine editions now done electronically for teachers of 1) residents, 2) medical students, and 3) primary care physicians. It is now embedded in most US residency programs and varying amounts of incorporation into specific programs. The World Psychiatric Association (WPA) is distributing the residency version to about 100 third world countries.

Keywords: Psychopharmacology, Residency Training, Curriculum.

Disclosure: Janssen: Consultant, Self; Otsuka: Consultant, Research, Lecture Honorariums, Self; Forrest: Consultant, Self; Takeda: Consultant, Self; Teva: Consultant, Self; Sunovion: Consultant, Self; Envivo: Research, Self; Alkermes: Research, Self; Lilly: Research, Self; Neurex: Research, Self; Pfizer: Research, Self; Allergen: Research, Self; Johnson \& Johnson: Equity, Self.

\section{W126. NIMH IRP Translational Neuropsychopharmacology Initiative}

Janet Clark*, Carlos Zarate, Susan Amara

National Institute of Mental Health, Bethesda, Maryland, United States

Background: The need for novel therapies for central nervous system (CNS) disorders with improved efficacy, safety, and tolerability is unquestionably high, as it is widely recognized that, despite currently recognized treatments, CNS disorders are major contributors to the global burden of illness and incur high economic costs. Unfortunately, over the past two decades, CNS drug discovery has been, with a few exceptions, relatively unsuccessful in delivering new chemical entities especially for the treatment of psychiatric disorders. The National Institute of Mental Health (NIMH) has become increasingly aware of the reduced investment by pharmaceutical companies in the development of therapeutics for treating psychiatric disorders despite the unmet medical need. In response to the reduced efforts in the pharmaceutical industry towards psychiatric drug discovery the NIMH Intramural Research Program (IRP) is proposing to re-invigorate psychiatric drug discovery by facilitating and de-risking the discovery and development of novel treatments. Support for the discovery and development of new treatments for psychiatric disorders including target validation, biomarker development, IND enabling studies, and Phase I safety / tolerability and Phase II proof of concept studies are all in scope for this important NIMH IRP initiative.

Methods: After completing a confidentiality agreement, interested parties including academic groups, small and large pharma or biotech may apply by submitting a proposal including detailed information on: the psychiatric indication, therapeutic rationale, target engagement, current stage of development, a development plan, project milestones, intellectual property and patent landscape, future objectives and anticipated outcomes. In addition, a compound 'report card' with details on the chemical properties of the compound of interest is completed and submitted with each proposal.

Results: To steer this initiative the NIMH IRP has established the NIMH Translational Neuropsychopharmacology Task Force (TNTF). The TNTF is comprised of a panel of neuroscience drug discovery/development experts from the pharmaceutical industry and the NIH who prioritize, critically review, and recommend new proposals for support to the NIMH IRP Leadership. Supported proposals will either be sourced by NIMH IRP Principle Investigators and Staff or the desired work may be supported by outsourcing under the direction of the IRP. The NIMH IRP has experience in the clinical testing of novel therapies for the treatment of psychiatric diseases including generalized anxiety disorder (GAD), treatment resistant depression (TRD), bipolar disorder (BPD), perimenopausal depression (PMD). In addition, the NIMH IRP has extensive CNS imaging capabilities including PET and fMRI that are readily integrated into clinical studies to answer questions regarding target engagement or better understand disease pathology.

Conclusions: The NIMH is committed to engaging with the scientific community and reinvigorating the development of new therapies for the treatment of psychiatric disorders. The current reduction in psychiatric drug discovery and development in the pharmaceutical industry provides an opportunity for the NIMH IRP to contribute in an important way to de-risking novel therapeutics and engage industry in an effort to get more effective treatments to patients.

Keywords: Novel Therapeutics, Drug Discovery/Development, Neuropsychopharmacology.

Disclosure: Nothing to disclose.

W127. Using Artificial Intelligence on Mobile Devices to Measure and Maximize Medication Adherence in CNS Trials

Laura Shafner, Adam Hanina*, Amir Kalali

\section{AiCure, New York, New York, United States}

Background: Accounting for and minimizing subject nonadherence is critical to clinical trial design, particularly in CNS where high levels of nonadherence have been observed. Accurately monitoring and collecting drug adherence data 
can allow for a better understanding and interpretation of the outcomes of clinical trials. The majority of clinical trials use a combination of pill counts and self-reported data to measure drug adherence, despite the drawbacks of relying on these types of indirect measures. It is assumed that doses are taken, but the exact timing of these is often incomplete and imprecise. To evaluate the use of an artificial intelligence (AI) platform on mobile devices in measuring and increasing medication adherence compared with other adherence measures, this study collected data from three CNS trials conducted between 2012 and 2016 in subjects with schizophrenia and ADHD.

Methods: A total of 324 subjects were randomized across 30 US sites in three separate CNS trials. 164 subjects were monitored using the AI platform (50.6\%); subjects were assigned a device with the AI application downloaded or downloaded the AI application onto their personal device. Subjects were asked to use the application for each dosing administration. Subjects dosed once or twice daily with 2 pills or more per dose. The primary adherence measures for the studies were based on pharmacokinetic data, scheduled pill counts, and AI platform adherence data.

Results: Subjects used the AI application for an average of 8 weeks (ranging from 6 days to 24 weeks). The mean time to use the AI platform was 71.2 seconds per pill. The mean age was 40.8 and $66.6 \%$ of the subjects were male. The AI platform was used for a total of 9,240 subject days, with 30,740 collected adherence parameters of which 26,643 $(80.2 \%)$ were visual confirmations of medication ingestion. The average discontinuation rate was $26.9 \%$. The mean (SD) cumulative adherence based on the AI platform's visual confirmation of ingestion was $83.3 \%$, and $88.8 \%$ when including self-reported adherence on the device or over the phone, for subjects who completed the trials. The mean (SD) cumulative adherence based on pill count for subjects who completed the trials, ranged from $95 \%$ to $100 \%$.

Conclusions: Subjects monitored using the AI platform demonstrated consistently high cumulative adherence $(83.3 \%)$, as measured by visual confirmation of medication ingestion on the AI platform. Subjects were able to use the technology quickly and easily for up to 6 months in an ambulatory setting. While pill count data is typically unreliable and overestimates adherence, these studies showed that it may be more reliable in trials where it is combined with an artificial intelligence platform, particularly for those subjects who terminate early or for subjects who have high adherence rates based on the AI platform. Study coordinator interventions based on real-time data contributed to high rates of adherence and use of the technology did not increase the drop-out rate. Rapid detection of nonadherence (non-use or incorrect usage of the technology) suggests the AI platform could be used effectively in placebo lead-in periods as part of larger enrichment strategies. This study demonstrates the feasibility of using artificial intelligence platforms on smartphones to ensure high adherence, provide reliable adherence data, rapidly detect nonadherence, and predict future nonadherence in CNS trials.

Keywords: Artificial Intelligence, Medication Adherence, CNS Clinical Trials, Clinical Trial Design, Clinical Trial Methodology.

Disclosure: AiCure: Employee, Self; AiCure: Employee, Shareholder, Self.
W128. Is Religion a Protective Factor Against Suicidality?

Ahmad Hameed*, Amanda White, Michael Mitchell, Venkatesh Basappa Krishnamurthy, Eric Youngstrom, Roger Meyer, Alan Gelenberg

Penn State Milton S. Hershey Medical Center, Hershey, Pennsylvania, United States

Background: Psychiatrists in the past have struggled with trying to address a balance between mental health and religion. Now we are more openly talking about religion with our patients. Studies have suggested that religious involvement is associated with fewer negative mental health outcomes including suicidality. Suicidal behavior is more common among individuals who do not consider themselves as religious and do not have moral objections to suicide. However, few studies have examined suicidal ideation and have used a standardized suicide rating scales such as the Columbia Suicide Severity Rating Scale (C-SSRS) to study the effect of religion on suicidality.

Methods: Data were collected and analyzed as part of an original study comparing suicide rating scales in adult psychiatric inpatients $(n=199)$. The C-SSRS was used to assess suicidal ideation and behavior in patients' lifetime and past month. Three questions about religiosity from a Risk Assessment Measure (RAM) inquired about belief in God, attendance of religious services, and moral objections to suicide.

Results: To examine whether religiosity was related to outcomes on a standardized suicide rating scale, a secondary analysis was performed using chi-square tests. $\phi$ was calculated to determine the magnitude of possible relationships. A majority of patients believed in God $(86.9 \%, n=172)$ and believed suicide is immoral $(62.4 \%, n=123)$. A minority of patients regularly attended religious services $(38.7 \%, n=77)$. Suicidal ideation was not less prevalent among those who believed in God and who had moral objections to suicide. However, those who regularly attended religious services were less likely to indicate a past month history of passive $(\phi=-0.16 ; p<0.05)$ and active non-specific suicidal ideation $(\phi=-0.14 ; p<0.05)$. Patients who believed in God and who had moral objections to suicide were less likely to have lifetime and past month history of suicide attempt; $\phi s$ ranged from -0.17 to $-0.23 ; p<0.05$. They were also less likely to have lifetime history of suicidal behavior $(\phi=-0.15$, $\phi=-0.21 ; p<0.05)$. Past month history of suicidal behavior was less common among those who believe in God $(\phi=-$ $0.15 ; p<0.05)$ and those who regularly attend religious services $(\phi=-0.17 ; p<0.05)$. This relationship trended for those who had moral objections to suicide but just missed significance.

Conclusions: Our data supports the notion that religion is a protective factor against suicidality. We found that adult psychiatric inpatients who attended religious services exhibited less suicidal ideation. Those who believed in God, attended religious services and had moral objections to suicide exhibited less suicidal behavior. To the best of our knowledge, this study was unique in employing a standardized suicide rating scale (C-SSRS) to examine religiosity as a protective factor for suicidality among adult psychiatric inpatients. This study adds to the findings on religiosity and 
suicidal behavior observed in non-clinical samples and contributes to the limited literature on religiosity and suicidal ideation. When assessing for suicidal risk, clinicians should consider their patients' belief in God, attendance of religious services, and moral objections to suicide.

Keywords: Suicide, C_ SSRS, Religion.

Disclosure: Nothing to disclose.

\section{W129. Lisdexamfetamine Dimesylate (LD) Combined With Regular Release Stimulants in Adult Attention Deficit Hyperactivity Disorder (ADHD)}

Faruk Abuzzahab*, Kathy Abuzzahab, Ferial Kamsheh

Clinical Psychopharmacology Consultant, Minneapolis, Minnesota, United States

Background: Our group had previously presented a poster on LD in ADHD at the 4th World Congress on ADHD in Milan, Italy on June 8th, 2013. The same poster was presented at the Neuroscience Education Institute's Psychopharmacology Congress in Colorado Springs, CO, and was later published. $\mathrm{LD}$ is a unique prodrug long-acting stimulant combining lysine with dextroamphetamine. After oral administration, $\mathrm{LD}$ is rapidly absorbed from the gastrointestinal tract and converted by hydrolytic activity of red blood cells to dextroamphetamine, which is responsible for its activity. The chemical designation for lisdexamfetamine dimesylate is (2S)-2,6-diamino-N[(1-S)methyl-2-phenylethyl] hexanamide dimethanesulfonate (See figure 2). LD was initially approved in the USA for children 6-12 years of age at doses of $30 \mathrm{mg}, 50 \mathrm{mg}$, and $70 \mathrm{mg}$ a day, and later for adults at the same doses1. Our previous poster was on adults with ADHD needing higher doses of LD than recommended up to $280 \mathrm{mg} /$ day. One of the dilemmas of clinical pharmacology is that individuals who participate in research studies in pre-marketing are markedly different from those seen in clinics.

Methods: Outpatient charts of 50 adult patients, 29 males and 21 females, diagnosed with ADHD, were reviewed. They ranged in age between 18 and 71 with an average of 43 years \pm 12.92 and an average body mass index of $28 \mathrm{~kg} / \mathrm{m} 2 \pm$ 4.97. The 50 adult patients were split into two groups; one group with patients taking additional regular release central nervous system stimulants, and the other with patients who were not taking additional central nervous system stimulants. Results: 35 out of 50 patients (equivalent to $70 \%$ ) required additional regular release central nervous system stimulants, presumably because LD at the FDA approved dose of $70 \mathrm{mg}$ / day failed to fully control their ADHD symptoms (See Figure 1). There were no statistical differences in age or body mass index between the 35 people who required additional medications and the 15 people who did not require additional medications. This group of 15 adults with ADHD who did not require additional central nervous system stimulants was too small for statistical purposes to achieve meaningful results.

Conclusions: In this preliminary report, charts of fifty adults with $\mathrm{ADHD}$ receiving $\mathrm{LD}$ at $70 \mathrm{mg} /$ day were reviewed, and revealed that $70 \%$ required additional central nervous system stimulants. Outpatient charts indicate that the use of LD in doses higher than the maximum recommended ones in its package insert may or may not suggest that it is due to differences between the pre-marketing study populations and the ones seen in clinics.

Sponsored in part by Psychopharmacology Fund and University of Minnesota Medical Foundation, Minneapolis, MN.

Submitted to the American College of Neuropsychopharmacology in Hollywood, FL, December 2016.

Keywords: Lisdexamfetamine, Central Nervous System, ADHD.

Disclosure: Nothing to disclose.

\section{W130. Phospho-Tau and Amyloid Precursor Protein are Aberrantly Elevated in Mouse Cerebellum Following Repetitive Blast-Induced Mild Traumatic Brain Injury}

Garth Terry*, Kole Meeker, Marcella Cline, James Meabon, Murray Raskind, David Cook, Elaine Peskind

University of Washington, Seattle, Washington, United States

Background: There is growing concern that repetitive mild traumatic brain injury (mTBI) from either blast (e.g., battlefield-related explosions) or impact (e.g., contact sports such as American football or boxing) may increase the risk of developing neurodegenerative conditions that include $\mathrm{Alz}$ heimer's disease-related disorders and chronic traumatic encephalopathy (CTE). CTE is a chronic neurodegenerative tauopathy characterized by the accumulation of fibrillary tangles composed of hyper-phosphorylated tau, notable as deposits in neurons, neuronal processes, certain astrocytes, and perivascular domains of the central nervous system (CNS).

To address the possible role of disturbed phospho-tau regulation in the pathophysiology of $\mathrm{mTBI}$, we and other research groups have reported that blast- and impactinduced mTBI elevate the expression of a variety of phospho-tau species in rodents. For example, we previously reported that phospho-tau levels are aberrantly elevated in mouse hippocampus for at least 30 days after a single mild blast exposure, while under these same conditions phosphotau increases in cerebellum were found only at 24 hours post exposure (Huber, et al, 2013).

In this study we chose to focus on the prolonged effects of repetitive blast exposures on tau phosphorylation in the cerebellum because we have recently reported that the cerebellum is particularly vulnerable to chronic neuron loss due to repetitive blast exposure, giving rise to anatomical patterns of injury in the cerebellum that are consistent with FDG-PET neuroimaging findings Veterans with blast-related mTBI (Meabon, et al., 2016).

Methods: All experiments and procedures were approved by the VA IACUC. Using a helium-driven pneumatic shock tube (which simulates battlefield-relevant shock waves), 3-4 month-old male C57BL/6 J mice anesthetized with isoflurane were exposed to eight (two consecutive exposures per day) primary blast overpressures (BOP; 19 psi peak intensity) $(n=4)$ or identical sham condition without blast exposure $(n=6)$. At 30 days post blast or sham exposure mice were euthanized, cerebellum was dissected and used for Western 
blotting to examine resulting protein changes. Levels of phosphorylated tau (pTau396; 50-60kD) were normalized to total tau levels (Tau5; 50-60kD). Total levels of amyloid precursor protein (APP) $(22 \mathrm{C} 11 ; \sim 100 \mathrm{kD})$ were normalized to the protein gel loading control pyruvate kinase $(\mathrm{PK} ; \sim 60$ $\mathrm{kD})$. Statistical analysis was performed using two-tailed t-test comparing blast and sham exposed mice.

Results: Antibodies recognizing pTau396 were used to quantify phospho-tau to total tau (Tau5) ratios in cerebellar protein lysates from mice that had received 8 blast-exposures (twice a day for 4 days). Western blot analysis revealed that pTau/total tau ratios were elevated in cerebellum 30 days after the last blast exposure compared to sham controls $(p<0.05)$. Similarly, amyloid precursor protein (APP) was elevated in cerebellum 30 days after last blast exposure compared to sham controls $(p<0.01)$, indicative of axonal injury.

Conclusions: Phosphorylated tau (pTau) and amyloid precursor protein (APP) are both persistently elevated in mouse cerebellum 30 days after repetitive blast induced mild traumatic brain injury (mTBI). Extending upon our prior findings in cerebellum following a single blast exposure, these results show that repetitive injury can induce neurodegenerative biomarkers present for a long period of time. While the presence of pTau or APP in human cerebellum following mTBI is not fully understood, considering the current findings with the human neuroimaging findings we and others have previously reported may reflect abnormalities in tau and APP function in human cerebellum, an intriguing possibility that warrants in-depth investigation. Keywords: Mild Traumatic Brain Injury, Tau, Amyloid Precursor Protein.

Disclosure: Nothing to disclose.

W131. Stimulation of the Rat Lateral Septum Inhibits Ventral Tegmental Area GABAergic Interneurons and Concomitantly Increases Dopamine Extracellular Levels

Ignacio Vega, Hector Yarur, Katia Gysling*

Pontificia Universidad Catolica de Chile, Santiago, Chile

Background: Dopamine (DA) neurons of the ventral tegmental area (VTA) are crucial in motivated behavior by predicting the availability of reward and recognizing associated cues. Thus, the mechanisms that command the activity of VTA dopaminergic neurons are critical in the motivational process. Anatomical and pharmacological evidence shows that several brain regions such as the prefrontal cortex, lateral hypothalamus and rostral medial tegmentum regulate the activity of VTA dopaminergic neurons. However, there still scarce knowledge of how other brain regions such as the lateral septum (LS) modulate VTA activity, despite the fact that Olds and Milner reported in 1954 high positive reinforcement when rats self-stimulate their LS.

Methods: We performed in vivo dual-probe microdialysis to reveal the neurochemical relationship between LS and VTA. We stimulated the LS with high $\mathrm{K}+$ artificial cerebrospinal fluid and measured the extracellular levels of GABA and DA in the VTA.
Results: We observed a significant increase in the extracellular levels of GABA and DA in the VTA after a depolarizing stimulus in the LS. Intra VTA infusion of bicuculline, (GABA-A receptor antagonist) inhibited the increase in DA but not GABA VTA levels. We hypothesized that LS could be modulating the activity of GABAergic interneurons in VTA through GABA-A $\square 1$ receptors. To test this hypothesis we infused indiplon, a selective positive allosteric modulator of GABA-A $\square 1$ receptors expressed in VTA gabaergic neurons, and measured the response to the LS depolarizing stimulus. We observed a significantly higher increase in VTA DA extracellular levels induced by LS stimulation in the presence of indiplon.

Conclusions: Our data suggest that the depolarizing stimulus in the LS activates LS gabaergic neurons that synapse onto VTA gabaergic interneurons resulting in a disinhibition of VTA dopaminergic neurons. Further studies should address if this neural circuitry is involved in the positive reinforcement effects induced by LS stimulation.

Keywords: Lateral Septum, Dopamine, Ventral Tegmental Area (VTA), GABAergic Interneurons.

Disclosure: Nothing to disclose.

\section{W132. PI3K/AKT/mTOR Signaling and Differentiation of Human iPS Cells}

Alan Cross ${ }^{*}$, Amritha Jaishankar, Nicholas Brandon, Steve Wesolowski, Roland Burli, Dan Hoeppner, Suel-Kee Kim, Seungmae Seo, Joo Heon Shin, Carlo Colantuoni, Josh Chenoweth, Daniel Weinberger, Ron McKay

AstraZeneca Pharmaceuticals, Thornton, Pennsylvania, United States

Background: The molecular pathways regulating stem cell function in development also play key roles throughout life in both homeostasis and disease. We have developed a controlled experimental platform to interrogate human induced pluripotent stem (iPS) cells in early neural fate determination. Because pathways that confer risk for neurodevelopmental disorders such as $\mathrm{PI} 3 \mathrm{~K} / \mathrm{AKT} / \mathrm{mTOR}$ signaling regulate human iPS cells and continue to play an important role in differentiated neurons, we have used this paradigm to assess how compounds that target the PI3K/ AKT/mTOR pathway act on transitions in functional state as cells move towards a neuronal phenotype. In addition, we show how these cell systems can be used to further interrogate signaling dynamics and identify potential targets. Methods: Human pluripotent cells were grown as monolayer cultures. Differentiation towards the body is promoted by BMP4 treatment and neurectoderm fates are promoted by inhibition of BMP \& Activin signaling with NOGGIN \& the ALK4/5/7 inhibitor SB431542.

Differential sensitivity of discrete cell states was measured using dose-response analysis of patient-specific iPS cells in presence of PI3K inhibitors. Patient-specific iPS cells (Controls and Schizophrenics) were treated with Neuregulin1(NRG1) ligands. Phenotypic (Cell morphology) and biomarkers in this signaling pathway (phosphorylation of AKT/ S6 residues) were assayed in individual cells using HighContent Imaging in the presence of well-annotated drugs. 
RNA-seq analysis of patient-specific iPS cells in self-renewal and differentiation at daily time intervals was used to define stable transcriptional signatures of individual genomes as well as interrogate differentially-activated signaling pathways and AKT-isoform specific expression differences.

Results: 1-6 days following removal of the ROCK inhibitor, human iPSCs spontaneously formed an epithelial sheet in the monolayer cell culture system we have utilized. Initially, the pluripotency regulators POU5F1/OCT4 and NANOG were uniformly expressed; however, a clear spatial distinction in their expression rapidly emerged between cells on the edge and the core of the epithelium. This self-organization occurred with each passage of the cells.

Immunolocalization with specific antibodies and automated image analysis demonstrated preferential activation of the PI3K/AKT/mTOR signaling pathway by neuregulin $1 \beta$ (NRG1 $\beta$ ) acting through the ERBB2/ERBB3 receptor in the peripheral zone of the epithelium as early as Day1. These receptors were enriched in cells at the edge of the epithelial sheet that showed a rapid preferential response to addition of exogenous NRG1 $\beta$. Cells within $100 \mathrm{~mm}$ of the edge of the epithelial sheet rapidly activate mTORC2 monitored by phosphorylation of AKT and S6RP in response to NRG1 $\beta$. Cells in the edge and core zone were in distinct signaling states as demonstrated by a differential dose-response to the mTORC1/2 inhibitor AZD8055. The effects of distinct PI3K isoform inhibitors depended on the state of the iPS derived cells. Conclusions: The key signaling pathways regulating stem cells continue to have a major role in brain development, and dysregulation of these pathways contribute to risk for neurodevelopmental disorders. The AKT/mTOR signaling pathway regulates pluripotency and we have shown functional specificity of this pathway in a cellular model that recapitulates specification of cells that generate the brain. This was achieved by generating an assay platform using human iPS cells where high-content imaging was supported by next-generation RNA sequencing technologies

To date, pharmacogenomics studies using cellular models of disease to identify new targets have been reported to be variable. This may well be a consequence of the failure to consider cellular heterogeneity that exists even within common transformed cell lines. Our high-content approach will lead to a radically new understanding of how drugs interact with the dynamics of cell signaling and tissue assembly.

Keywords: Stem Cells, AKT1/ AKT3, Neurodevelopmental Disorders.

Disclosure: AstraZeneca: Employee, Self.

\section{W133. Whole Exome Sequencing Identifies a GABRA6 Variant That Predicts Alcohol Response and Consumption in Rhesus Macaques}

Christina Barr*, Carlos Driscoll, J. Dee Higley, Stephen Lindell, David Goldman, Qiaoping Yuan, Stephen Suomi

National Institute on Alcohol Abuse and Alcoholism, Bethesda, Maryland, United States

Background: We have been examining genetic factors that contribute to variation in the neurobiological systems that influence reward pathways, behavioral dyscontrol/impulsivity and vulnerability to stress and anxiety, as these systems may also influence alcohol use and addiction vulnerability in modern humans. In order to interrogate these systems, we wanted to sequence the exomes of rhesus macaque subjects that were selected based on variation in temperament. The major advantage of using Whole Exome Sequencing (WES) is that DNA extracted whole blood can be used without the need for extracting DNA from the tissue of interest. WES allows for the capture of large insertion/deletions and SNPs, and is best for either non-synonomous or frameshift mutations. While there are no commercially available reagents for performing WES in nonhuman primates, a human whole exome platform is available.

Methods: Exonic sequences were enriched using the Agilent SureSelect all exon capture array targeting $\sim 38 \mathrm{Mb}$ and were annotated using Ensemble genes, refGene and refSeq xenoRefGene for $\mathrm{H}$. sapiens \& $\mathrm{M}$. musculus from UCSC. Sequence alignment was performed using CASAVA, Recla ibration \& SNP calling using GATK, Annotations, filtering, counts and calculations using custom scripts, and visualizations using R. Missense variants' functionalities were inferred using Polyphen. PolyPhen posterior proabability scores associated with false positive rate (FPR) below 5\% are characterized as 'probably' damaging and those below $10 \%$ are determined to be 'possibly' damaging under the HumDiv classifier model. Focusing on candidate genes relating to risk for alcohol use disorders (1536 marker Addictions Array, LNG), we identified a number of non-synonymous SNPs, some of which were predicted to be "damaging" or "potentially damaging" by in silico analysis (PolyPhen). Among these were SNPs in the MPDZ, GAL, GABARA6, and CRHR2 genes. The latter encode the alpha- 6 subunit of the GABA-A receptor and the corticotropin releasing hormone- 2 receptor, respectively.

Results: The population of study included macaques raised in a relatively appropriate social environment (MR) and those in a nursery/peer reared setting (PR). Among these, we wanted to determine whether the rhGABRA6 Tyr28Phe polymorphism would predict alcohol response. Ethyl alcohol ethanol $(16.8 \%(\mathrm{v} / \mathrm{v})$ USP) was administered intravenously (2.2 grams $/ \mathrm{kg}$ or 2.0 grams $/ \mathrm{kg}$ for males and females, respectively) at a constant rate over 15 minutes as previously described. Following the IV ethanol infusions, animals were placed in a padded testing room. Animals were scored by 3 individuals for their intoxication ratings on a scale of 1-5. We found that animals carrying the $28 \mathrm{Phe}$ allele had higher intoxication scores when given a binge dose of ethanol. There was also a rearing (PR vs MR) by GABRA genotype interaction on alcohol self-administration. Carriers of this variant exhibited lower levels of early life stress induced alcohol consumption relative to homozygotes for the ancestral allele.

Conclusions: Studies in humans and rodents have demonstrated that variation at the GABRA6 gene predicts individual difference in alcohol response. Although little has been done to examine GABRA6 variation as it relates to alcohol abuse or dependence in human subjects, our data also support a role for this SNP in protecting against early stress-induced increases in alcohol intake. Peer reared macaques carrying the $28 \mathrm{Phe}$ allele did not exhibit 
upregulated alcohol self-administration as did their Tyr/Tyr counterparts, suggesting that damaging variation at this gene could serve a protective role for alcohol missuse in human subjects. Ethanol is a positive allosteric modulator of GABARA4 and GABARA6 conductivity. GABA-A receptors are ligand-gated channels, comprised of 5 subunits, most commonly 2 alpha, 2 beta and 1 gamma. The GABRA6 gene, which encodes the 6 subunit is confined in its expression to cerebellar granule cells, and, therefore, a role for functional variation at this gene in determining levels

Keywords: Rhesus, Alcohol, GABA.

Disclosure: Nothing to disclose.

\section{W134. The Atease Study: A Multicenter Randomized Clinical Trial of the Safety and Efficacy of TNX-102 Sl in the Treatment of Military-Related PTSD}

Gregory Sullivan*, Judith Gendreau, R. Michael Gendreau, Amy Schaberg, Bruce Daugherty, Heather Jividen, Ashild Peters, Perry Peters, Frank Weathers, Seth Lederman

Tonix Pharmaceuticals, Inc., New York, New York, United States

Background: Posttraumatic stress disorder (PTSD) is one of the most prevalent and disabling psychiatric disorders in military personnel deployed to Middle East combat theaters. Only two pharmacotherapies, both selective serotonin reuptake inhibitors (SSRIs), are FDA-approved for PTSD. One failed to show efficacy in Veterans and males with PTSD, and the other was never studied in predominantly military-related PTSD. Study of a serotonin-norepinephrine reuptake inhibitor (SNRI) in a large sample also had no effect on PTSD or disability in the combat subsample $(N=77)$ of a pooled analysis. These facts underscore the urgent unmet need for an evidence-based pharmacotherapy with a distinct mechanism of action for military-related PTSD.

TNX-102 SL is a proprietary formulation of cyclobenzaprine, which among tricyclics has a unique profile of potent binding and antagonist activity at 5-HT2A, alpha1-adrenergic, and H1-histaminergic receptors, presumed to result in improvement in sleep architecture and sympatholytic effects. TNX-102 SL was designed for bedtime sublingual administration and rapid transmucosal absorption, detectable in plasma in minutes. Avoidance of first-pass hepatic metabolism results in lower exposure to a long-lived active metabolite, norcyclobenzaprine. This double-blind placebocontrolled randomized clinical trial was designed to assess the safety and efficacy of TNX-102 SL in militaryrelated PTSD.

Methods: The 'AtEase Study' was a Phase 2, multicenter, 12week, double-blind placebo-controlled trial in adults meeting a DSM-5 diagnosis of PTSD, assessed by the ClinicianAdministered PTSD Scale for DSM-5 (CAPS-5). Patients were randomized to TNX-102 SL $2.8 \mathrm{mg}, 5.6 \mathrm{mg}(2 \times 2.8 \mathrm{mg}$ tablets), or placebo in a 2:1:2 ratio at 24 US sites. Eligible participants must have experienced PTSD Criterion A-qualifying trauma(s) during military service since 2001. Inclusion criteria: CAPS-5 score at baseline $\geq 29$; no antidepressant treatment in $>2$ months; willingness to be free of other psychotropics. Exclusions: severe suicide risk; moderate or severe traumatic brain injury (TBI); substance use disorders in prior 6 months; lifetime bipolar 1 or 2, psychotic disorders, and obsessive compulsive disorder. The primary efficacy endpoint was mean change from baseline (MCFB) in CAPS-5 severity score between TNX-102 SL $2.8 \mathrm{mg}$ and placebo analyzed via mixed-effects modeling repeated measures (MMRM). Secondary measures included: CAPS-5 symptom cluster scores; Clinical Global Impression - Improvement scale (CGI-I); PROMIS Sleep Disturbance instrument; Sheehan Disability Scale (SDS). CAPS-5 raters were Masters-level or above mental health clinicians who underwent a rigorous rater training and certification program. Patients were monitored for suicidality, depression severity, and adverse events.

Results: Between January-December, 2015, 245 participants were enrolled. The efficacy population comprised: placebo $N=92$; TNX-102 SL $2.8 \mathrm{mg} N=90$; and TNX-102 SL $5.6 \mathrm{mg} N=49$. MMRM with multiple imputation (MI) indicated the MCFB in CAPS-5 for the TNX-102 SL $2.8 \mathrm{mg}$ group was not significantly different from placebo at Week 12. In contrast, the TNX-102 SL $5.6 \mathrm{mg}$ group showed greater MCFB improvement in CAPS-5 than placebo at Week 12 $(p=0.031)$, with a mean (standard error) point difference from placebo of -5.0 (2.33). Analysis of CAPS-5 symptom clusters indicated the largest effects were on Arousal \& Reactivity, including significant improvement on the sleep disturbance item at all assessments. The TNX-102 SL $5.6 \mathrm{mg}$ arm also had a greater CGI-I responder rate, and produced greater improvement in the SDS work/school and social/ leisure domains.

Subsequent analyses indicated that efficacy results were more pronounced in subsets with more severe symptoms as evidenced by higher CAPS- 5 baseline scores. All subsequent analyses are based on baseline CAPS-5 $\geq 33$, with: Placebo $N=77$; TNX-102 SL $2.8 \mathrm{mg} N=70$; and TNX-102 SL $5.6 \mathrm{mg} N=38$. MMRM analysis demonstrated the TNX-102 SL $5.6 \mathrm{mg}$ group produced a -6.8 (2.69) point greater decrease in CAP-5 compared with placebo $(p=0.013)$, with an effect size (ES) of 0.53 . The Intrusion cluster $(p=0.026$, $\mathrm{ES}=0.46)$ and Arousal \& Reactivity $(p=0.012, \mathrm{ES}=0.52)$ also were significantly decreased, and there was a trend for a lower Mood/Cognitions cluster score $(p=0.065, \mathrm{ES}=0.39)$. The most frequent adverse event (AE) reported was transient oral hypoaesthesia at the administration site in $38 \%$ of TNX-102 SL treated patients compared to $2 \%$ on placebo. The TNX-102 SL $5.6 \mathrm{mg}$ group had minimally higher systemic AE rates for somnolence (16\%), dry mouth (16\%), headache $(12 \%)$, and sedation $(12 \%)$, yet there were no discontinuations in the group due to $\mathrm{AE}$ and a high completion rate of $84 \%$, suggesting they were tolerable.

Conclusions: TNX-102 SL at $5.6 \mathrm{mg}$ taken sublingually at bedtime for 12 weeks demonstrated a significant improvement in CAPS-5 over placebo. There were no drug-related serious AEs. The most common reported AEs are oral hypoaesthesia, somnolence, and dry mouth. This is the first multicenter registration-quality trial of any drug to demonstrate a treatment effect in a population with military-related PTSD. Early effects on sleep and hyperarousal are consistent with the mechanistic hypothesis that TNX-102 SL's primary actions on sleep architecture and autonomic balance may be critical to the observed treatment effect. A CAPS-5 threshold $\geq 33$ reveals robust effects of TNX-102 SL on military-related 
PTSD and will be used as the entry criteria for future Phase 3 PTSD trials.

${ }^{*}$ TNX-102 SL is an Investigational New Drug and has not been approved for any indication.

Keywords: Posttraumatic Stress Disorder, Randomized Clinical Trial, Cyclobenzaprine.

Disclosure: Tonix Pharmaceuticals, Inc: employee, Self.

\section{W135. Selective and State-Dependent Changes in CSF Abeta42 Levels in Cognitively-Intact Elderly With Late Life Major Depression}

Nunzio Pomara*, Jay Nierenberg, Davide Bruno, Chelsea Reichert, Ricardo Osorio, Antero Sarreal, Raymundo Hernando, Charles Marmar, Thomas Wisniewski, Henrik Zetterberg, Kaj Blennow

New York University School of Medicine, Orangeburg, New York, United States

Background: Numerous studies have linked depressive symptoms, or syndromal depression, to increased risk for Alzheimer's disease (AD) irrespective of its onset age. The neurobiological basis for this association, however, remains poorly understood. Studies which have examined biomarkers of $\mathrm{AD}$ in peripheral and central tissues in depression, including CSF Abeta42 (A $\beta 42)$ and brain amyloid using PET ligands, have provided conflicting results. These discrepant results may have been due to methodological differences across studies, including heterogeneity in study populations (e.g., inclusion of individuals with mild cognitive impairment) and the use of approaches for detecting depression (e.g., patients' self-ratings) that lack diagnostic specificity. In addition, only a few studies have employed structured interviews based on DSM diagnostic criteria or standardized pre-analytical and laboratory procedures for quantifying $\mathrm{A} \beta$. Finally, all of the existing studies have been limited to cross sectional comparisons based on a single $A \beta$ determination; thus, it is not known if these abnormalities persist over time and if depressive symptoms influence $A \beta$ levels. The current study was conducted to address the aforementioned limitations.

Methods: Our baseline sample consisted of 47 subjects aged 60 years and older; 28 with LMDD and 19 controls. Of the 47 older subjects, 31 were e4 non-carriers and 16 were e4 carriers. All subjects were cognitively-intact and had a) no evidence of dementia, b) a Mini-Mental State Exam score of at least 28, and c) no gross MRI abnormalities other than white matter hyperintensities. CSF collection was repeated after 3 years for 36 of these individuals: 19 with LLMD and 17 controls. We evaluated the effects of diagnosis and time on A $\beta 42$ levels with $2 \times 2$ repeated measures ANOVA.

Results: Longitudinal comparisons of controls and LLMD revealed that the LLMD group was significantly less depressed at follow up than at baseline, as determined by the HAM-D, whereas controls remained unchanged. There was a significant interaction between diagnosis and time on CSF A $\beta 42$ levels. Importantly, the reductions in depressive symptoms observed over time were significantly correlated with increases in CSF A $\beta 42$ levels, both in the entire cohort $(\mathrm{r}=-.451, p=.006)$ and within the LLMD group $(\mathrm{r}=-.547$, $p=.015)$, but not in the control group $(p=.809)$. The same

W136. Residual Halogenated Disinfection By-Products in Drinking Water are Inversely Correlated With Suicide Rates

Perry Renshaw*, Jacob Stephenson, Hana Sabic, Rebekah Huber

University of Utah, Salt Lake City, Utah, United States

Background: A recent study has suggested that an increased risk of suicide may be associated with the number of previous hospitalizations with infection (Lund-Sørensen et al. Increased risk of suicide death associated with hospitalization for infection. JAMA Psychiatry. 2016). These authors followed more than 7 million Danish citizens over 30 years. Individuals with 7 or more pathogenic infections had a $300 \%$ increased risk of suicide. Earlier work has suggested that increased inflammation, measured by CSF cytokine levels, is present in suicidal individuals (Lindqvist D, Janelidze S, Hagell P, et al. Interleukin-6 is elevated in the cerebrospinal fluid of suicide attempters and related to symptom severity. Biol Psychiatry. 2009;66(3):287-292.) To the extent that suicide rates are correlated with infection, public health measures to detect and eradicate microbial pathogens may also change suicide rates. In this study we focused on drinking water. In order to eliminate infectioncausing microorganisms, drinking water is commonly subjected to several different disinfection treatments, including chlorination, ozone treatment, chlorine dioxide, chloramines and ultraviolet light. When chlorine is added to water, it reacts with organic matter, resulting in the formation of potentially toxic chemical by-products (Richardson SD. The role of GC-MS and LC-MS in the discovery of drinking water disinfection by-products. Environmental Monitoring. 2002;4(1):1-9). These byproducts include trihalomethanes (THM) and haloacetic acids (HAA). THM have been associated with increased rates of bladder and colorectal cancer in humans (Backer et al., Household exposures to drinking water disinfection byproducts: whole blood trihalomethanes levels. J Expo Anal Environ Epidemiology. 2000: 10(4); 321-6). HAA has been classified by the EPA as a possible carcinogen based on evidence from animal studies (EPA 2002). 
Methods: To measure the effect of chlorination on drinking water, state data was gathered from the Environmental Working Group (EWG) on HAA and THM levels found in tap water reported in 2012 (Water treatment contaminants: forgotten toxics in American water, 2013). HAA and THM measurements were expressed in parts per billion (PPB) for all the largest water utilities $(n=201)$, which were averaged for each state. HAA and THM levels serve as a direct measure of chlorination used in drinking water.

Results: Suicide rates were negatively correlated with average state HAA $(n=44)$ and THM $(n=45)$ measurements $(\mathrm{r}=-$ $0.33, p=0.030 ; \mathrm{r}=-0.3, p=0.049)$. Median HAA levels were 22.8 (SD 8.63) PPB and median THM levels were 38.66 (SD 13.9) PPB. According to the EWG report (2013), allowed levels of THM are 80 PPB, and allowed levels of HAA are 60 PPB.

Conclusions: These data demonstrate that water chlorination (reflected in residual levels of HAA and THM) not only reduces the levels of microorganisms found in drinking water but may also decrease the rate of suicide. Further research is needed to identify those pathogens found in drinking water that are responsible for up-regulation of inflammatory responses and increasing suicidal ideation. For example, recent reports have suggested that infection with the parasite Toxoplasma gondii is positively correlated with suicide rates (Ling et al., Toxoplasma gondii seropositivity and suicide rates in Women. J Nerv Ment Dis 2011; 199(7): 440-444).

Keywords: Suicide, Infection, Serious Mental Illness. Disclosure: Nothing to disclose.

\section{W137. Effects of Weight Loss on Neuronal Response to Food Cues}

Kristina Legget*, Marc-Andre Cornier, Allison Hild, Jason Tregellas

University of Colorado School of Medicine, Aurora, Colorado, United States

Background: Understanding the impact of weight loss on neuronal responses to visual food cues in obese individuals may provide insight into mechanisms of weight loss and maintenance. The goal of the current study was to determine the effects of $10 \mathrm{~kg}$ weight loss on neuronal response to food cues in obese adults. Additionally, a preliminary examination of the effects of different weight-loss methods was performed, comparing weight-loss via caloric restriction and bariatric surgery.

Methods: Twenty-three obese adults (mean body mass index (BMI): $40.7 \pm 3.3 \mathrm{mg} / \mathrm{kg} 2$; mean age $40.0 \pm 11.3$ years) eligible for weight loss surgery (BMI $>40$ or $B M I>35$ with comorbid obesity-related conditions) completed the study. Neuronal response to visual food cues was assessed using fMRI in the fasted state and again in the acute fed state (30 min after a liquid breakfast meal) at baseline and after approximately $10 \mathrm{~kg}$ weight loss. Participants achieved weight loss through either diet (caloric restriction with meal replacement milkshakes; $N=15$ ) or bariatric surgery (gastric bypass or gastric band; $N=8$ ). Functional images were acquired with an echo-planar gradient-echo $\mathrm{T}^{*}$ Blood Oxygenation Level Dependent (BOLD) imaging contrast technique $(\mathrm{TR}=2000 \mathrm{~ms}, \mathrm{TE}=30 \mathrm{~ms}, \mathrm{FOV}=240 \mathrm{~mm} 2$, 642 matrix, 27 axial slices angled parallel to the planum sphenoidale, $2.6 \mathrm{~mm}$ thick, $1.4 \mathrm{~mm}$ gap). During fMRI scanning, participants viewed images of foods with high hedonic value, foods with neutral hedonic value, and nonfood objects. Two runs were performed, with each run consisting of a randomized block design with 6 blocks of pictures ( 4 stimuli for $4 \mathrm{~s}$ each per block) of each category. fMRI data were analyzed using SPM 8 software. Data were realigned, normalized, and smoothed with an $8 \mathrm{~mm}$ FWHM Gaussian kernel. The hemodynamic response was modeled with a double gamma function, without temporal derivatives, using the general linear model. A $128 \mathrm{~s}$ high pass filter was applied. The primary contrast of interest compared hedonic foods to nonfood objects. Comparisons across conditions were evaluated with directional contrasts (SPM t-contrasts) in the context of a repeated measures ANOVA. Following the primary analysis of weight loss effects, exploratory analyses compared diet-induced to surgery-induced weight loss.

Results: At baseline, in the fasted state, neuronal responses to visual food cues were observed in a network of regions, including the bilateral insula, somatosensory cortex, parietal cortex, posterior cingulate cortex, and visual cortex. Across all subjects, no significant differences in neuronal response were observed between fasted and fed states. Following $10 \mathrm{~kg}$ weight loss, a greater response was observed in the fasted state, particularly in the insula and visual cortex. A greater neuronal response to a meal (i.e., reduced response when fed compared to fasted) was observed following weight loss in posterior sensory processing and attention-related areas, such as visual cortex and parietal cortex, and areas known to be involved in processing visual food cues, such as the insula. Exploratory analyses comparing diet and surgery groups did not reveal weight-loss related differences in the fed state. In the fasted state, however, greater weight-loss related effects were observed in the diet compared to surgery group in visual cortex, posterior cingulate cortex, precuneus, parahippocampal gyrus, parietal cortex, and dorsolateral prefrontal cortex.

Conclusions: Across all subjects, greater neuronal response to hedonic food cues was observed in the fasted state following $10 \mathrm{~kg}$ weight loss compared to baseline. Additionally, weight loss was associated with greater responsivity to a meal (i.e., greater reduction in response to food cues when fed compared to fasted). This suggests a possible increase in sensitivity to energy balance following weight loss. Although no differences were observed in weight-loss related changes in responsivity in the fed state between diet and surgery groups, those in the diet group demonstrated greater response to visual food cues in the fasted state after weight loss. This suggests that when caloric restriction is used for weight loss, neuronal response to food cues may increase, potentially leading to an increased drive to eat hedonic foods that could contribute to weight regain. That this increased weight-loss related responsivity was not observed in the surgery group suggests that neuronal response to hedonic food cues may not increase in this group as weight decreases. It is possible that increased responsivity to food cues following diet-induced weight loss may make weight regain more likely than those who lose weight through surgical means. 
$\overline{\mathrm{S} 546}$

Keywords: Functional MRI (fMRI), Obesity, Diet, Bariatric Surgery, Weight Loss.

Disclosure: Nothing to disclose.

\section{W138. The Importance of Being Negative: A Serious Treatment of Non-Trivial Edges}

Lisanne Jenkins, Liang Zhan, Ouri Wolfson, Johnson Keiriz, Paul Thompson, Olusola Ajilore*, Moo Chung, Alex Leow

University of Illinois at Chicago, Chicago, Illinois, United States

Background: Understanding the modularity, i.e. community structure, of brain networks can inform the study of organization and mechanisms of brain function and dysfunction, thus potentially the treatment of neuropsychiatric diseases. Adapted from methods originally developed for the social sciences, existing network modularity approaches to fMRI-derived connectomes (such as Q optimization algorithms) yield variable results with suboptimal reproducibility. Many of these existing methods largely ignore negative negative BOLD-signal correlations in fMRI networks, only considering the right tail of the correlation histogram, ignoring potentially neurobiologically relevant information. We propose a new, reproducible approach that exploits how frequently the BOLD-signal correlation between two nodes is negative.

Methods: As an alternative to Q, we previously developed a graph distance (shortest path length) based modularity approach for the structural connectome. This path length associated community estimation technique (PLACE) is designed to extract global-to-local hierarchical modular structure in the form of bifurcating dendrograms. The PLACE benefit function is the YPL metric, defined at each bifurcation as the difference between the mean inter- and intra- modular graph distances. Thus, maximizing $\Psi \mathrm{PL}$ is equivalent to searching for a partition with stronger intracommunity integration and stronger between-community separation.

The new method described here, termed probabilityassociated community estimation (PACE), was developed to describe the modularity of a functional connectome, mathematically represented as an undirected graph $\mathrm{FC}(\mathrm{V}, \mathrm{E})$ where $\mathrm{V}$ is a set of vertices (i.e., nodes) and $\mathrm{E}$ is a set of edges (indexed by considering all pairs of vertices). Each edge of $\mathrm{E}$ is associated with a weight that can be either positive or negative. Given a collection of functional connectomes $S$ on the same set of nodes $\mathrm{V}$ (but having edges with different weights), we can define the aggregation graph $G(V, E)$. For each edge $\mathrm{E}$ connecting node $\mathrm{i}$ and node $\mathrm{j}$, we consider the probability of observing a negative value at this edge in $S$ (i.e., the BOLD signals of $\mathrm{i}$ and $\mathrm{j}$ are anti-correlated). Then, inspired by PLACE, given $\mathrm{C} 1, \mathrm{C} 2, \ldots, \mathrm{CN}$ that are $\mathrm{N}$ subsets (or communities) of $\mathrm{V}$, we define the mean intra-community edge positivity or negativity for the n-th community.

The intuition of PACE for fMRI connectomes is that edges that are most frequently anti-correlations should be placed across communities. Given a collection of functional connectomes $\mathrm{S}, \mathrm{PACE}$ identifies a natural number $\mathrm{N}$ and a partition of $\mathrm{V}$ that maximizes the PACE benefit function $\Psi P$.
To capture the hierarchical configuration of the functional connectome, we adopt a PLACE-like algorithm and computed global-to-local 4-level bifurcating trees.

We validated PACE on the Human Connectome Project (HCP) and the 1000 Functional Connectomes (F1000) resting state connectome datasets, by comparing PACE to six Q-based methods (five weighted and one binarized). We conducted 100 runs for each method, and quantified pairwise similarity between two modular structures using normalized mutual information.

Results: Q-based methods produced substantially variable modular structures across runs (the number of communities across runs was also variable). In contrast, PACE produced identical results up to the third level (i.e., 8 communities) for HCP and throughout all four levels for F1000. We demonstrate that negative correlations alone are sufficient in understanding resting-state fMRI connectome modularity, although PACE permits a dual formulation that leads to equivalent solutions regardless of whether one considers positive or negative edges.

Conclusions: Existing Q-based methods of defining modularity return variable results. In contrast, the novel PACE framework provided here returns consistent and reproducible modularity. When applied to the HCP dataset, we showed that PACE yielded similar results to existing methods, providing evidence for convergent validity. The superiority of PACE is evident in that correlations with highest probability of being negative are consistently placed between modules, and due to the equivalent dual forms, no arbitrary weighting factor is required to balance the influence between negative and positive correlations, as is currently employed in all Q modularity-based approaches. Future research using PACE is encouraged to further demonstrate the superiority of this approach to defining community structure, and the code will be made publically available.

Keywords: Functional MRI (fMRI), Connectome, Functional Neuroimaging, Large Scale Networks.

Disclosure: Nothing to disclose.

\section{W139. A Translational Electrophysiological Signature of Sustained Attention in Humans and Mice}

Kevin Spencer*, Kristen Richardson, Shogo Hirano, Brad Buran, Benjamin Eschle, Alexander Nakhnikian, Corey Puryear, Grace Francis, David Gerber, Margaret Levin

\section{Harvard Medical School, Boston, Massachusetts, United} States

Background: Progress in developing new therapies for cognitive symptoms of mental illness has been limited by a lack of preclinical measures that enable prediction of human clinical outcome. One approach to bridging this translational gap is to develop biomarkers that can serve as objective measures of cognitive functions in animals and humans. Combining electrophysiological recording with matching behavioral tasks across species could enable the direct assessment of conserved neural circuitry underlying cognitive functions. This approach could be applied to assess the effects of disease state and/or candidate therapeutic compounds on task performance and relevant neural circuitry as 
a basis for developing translational biomarkers with specific cognitive domain relevance.

Attentional functions are crucial components of higher order cognitive processes due to their role in regulating access to capacity limited processes, and due to their impairment in a variety of CNS disorders, such as schizophrenia, Alzheimer's disease, and attention deficit/hyperactivity disorder. Therefore, in this project, we aimed to identify translatable signatures of attentional functions in the scalp-recorded electroencephalogram (EEG) of humans and in the local field potentials (LFPs) of mice performing the 2-choice serial reaction time task (2-CSRT), a sustained attention task that was modified from the 5-CSRT that is commonly used in preclinical research.

Methods: Human methods: In the human 2-CSRT, healthy $(N=11)$ detected laterally-presented target stimuli while keeping their eyes focused on a central warning stimulus. The warning stimulus appeared $500 \mathrm{~ms}$ prior to target presentation. Target stimuli were presented at low contrast and with durations of 12,36 , or $106 \mathrm{~ms}$ to vary difficulty. Target detection was indicated by pressing a button corresponding to the visual field (left/right) of the target. The EEG was recorded from 71 scalp electrodes, and was average-referenced following artifact detection and removal with independent component analysis.

Mouse methods: 32 female $\mathrm{C} 57 \mathrm{Bl} / 6 \mathrm{~J}$ mice were implanted with 2 depth electrodes (4-microwire bundles) in the prelimbic (PrL) and parietal (Par) cortices with a reference screw electrode placed over cerebellum for continuous LFP recordings. In the mouse 2 -CSRT, animals were presented with 2 levers with target lights over each lever. After a $500 \mathrm{~ms}$ delay, one light was illuminated and animals were trained to depress the lever under the illuminated target. Target stimuli were presented with durations of $0.5,1$, or $10 \mathrm{~s}$ to vary difficulty.

Common analysis methods: Evoked power of oscillations in the EEG and LFP was computed using the Morlet wavelet transform for Correct and Incorrect trials. Behavioral measures and evoked power were statistically analyzed using repeated-measures ANOVA.

Results: In both species, accuracy increased (mice: $p<$ 0.0001; humans: $p<0.01$ ) and RT tended to decrease (mice: $p<0.0001$; humans: $p=0.066$ ) as target duration increased. In mice, target stimuli evoked oscillations at $0-50 \mathrm{~ms}$ from $\sim 20-45 \mathrm{~Hz}$ in PrL and from $\sim 20-90 \mathrm{~Hz}$ in Par. These oscillations were analyzed in 2 bands, beta $(15-30 \mathrm{~Hz})$ and gamma $(30-90 \mathrm{~Hz})$. Evoked power of these oscillations was increased in Correct trials relative to Incorrect trials for both frequencies and both regions (2-way RM ANOVA, performance [Correct and Incorrect] and time [-1 to 0.5 peristimulus onset] as factors; $\operatorname{PrL}$ beta: Perf $p<0.003$; PrL gamma: Perf X Time $p<5 \mathrm{e}-16$; Par beta: Perf X Time $p<$ 5e-16; Par Gamma: Perf $p<0.02$ ). Post hoc tests indicate significant differences between Correct and Incorrect evoked power occurred just after the onset of the target stimulus presentation (0-50 ms). All duration conditions were included in evoked power measures.

In humans, the warning stimulus evoked an oscillation at 106-206 ms from $25-43 \mathrm{~Hz}$ with maxima at frontal and parieto-occipital electrodes. Only the data from the $12 \mathrm{~ms}$ target duration condition were analyzed because there were not enough Incorrect trials for analysis in the other duration conditions. The evoked power of the warning stimulusevoked oscillation was greater on Correct compared to Incorrect trials at frontal $(p<0.01)$ and parieto-occipital electrodes $(p<0.001)$.

Conclusions: We developed a sustained attention task for mice and humans that showed similar behavioral functions across species. In the EEG and LFP data, we identified signatures of sustained attention in both species as well: oscillations spanning the upper beta and gamma bands that had greater power on trials in which target stimuli were detected rather than missed. These oscillations were found in the mouse analog of the prefrontal and posterior parietal cortex, and at electrodes over the prefrontal, parietal, and occipital cortex in humans. While these oscillations were evoked by different types of stimuli (warning stimuli in humans, targets in mice), we suggest that in both cases the effect of attention on these oscillations reflected a similar engagement of attentional circuitry. Because of the characteristics shared by these oscillations across species spectrum, anatomy, and response to task manipulations we propose that these oscillations reflect a shared attentional mechanism in mice and humans. Our findings suggest that cross-species electrophysiological assays of attentional and other cognitive functions can be developed and used to identify translatable biomarkers of neural circuit functions. Keywords: Electrophysiology, Mouse, Human, Attention. Disclosure: Galenea Inc.: Employee, Self.

\section{W140. Effect of Acute Alcohol on Dopamine Transmission Assessed by [11C]-(+)-PHNO}

Thulasi Thiruchselvam*, Alan Wilson, Isabelle Boileau, Bernard Le Foll

\section{Centre for Addiction and Mental Health, Toronto, Canada}

Background: Previous positron emission tomography (PET) studies exploring the effect of acute alcohol on dopamine (DA) levels have yielded inconsistent results, with only some studies suggesting increased synaptic DA levels after an alcohol challenge. The D2/D3 agonist radiotracer, [11C](+)-propyl-hexahydro-naphtho-oxazin ([11C]-(+)-PHNO), has greater sensitivity to synaptic DA fluctuation than previously used antagonist radiotracers and is in principle more suitable for imaging alcohol-induced changes in DA. Its high affinity for the D3 receptor also enables measuring changes in D3-rich brain areas which have previously been unexplored. The aim of this study was to investigate whether alcohol reduces [11C]-(+)-PHNO binding in the striatum and in D3- rich extra-striatal areas.

Methods: Eight healthy drinkers underwent two [11C](+)-PHNO PET scans following alcohol $(\sim 1 \mathrm{ml} / \mathrm{kg}$ of body weight of $95 \%$ alcohol) and placebo in a randomized, blind, crossover design. [11C]-(+)-PHNO binding in the striatum and in the extra-striatal regions (substantia nigra, ventral pallidum and globus pallidus) were compared between the two scans.

Results: Acute alcohol administration did not significantly reduce [11C]-(+)-PHNO binding in either the striatum or the D3-rich areas outside of the striatum. However, greater percent change in $[11 \mathrm{C}]-(+)-\mathrm{PHNO}$ binding $(\triangle \mathrm{BPND})$ 
between scans was related to lower blood alcohol levels in participants.

Conclusions: Using the agonist radiotracer, [11C](+)-PHNO, our findings suggest that alcohol is not associated with robust changes in tracer binding in striatal or extra-striatal regions. However, we found that changes in [11C]-(+)-PHNO binding following acute alcohol is dependent on blood alcohol levels. Findings suggest that increases in DA may occur at lower stimulating doses. The effect of lower doses of alcohol on DA transmission warrant further investigation.

Keywords: Dopamine (D2, D3) Receptors, PET Imaging, Alcohol Exposure.

Disclosure: Nothing to disclose.

\section{W141. Psychological Resilience and the Gut Microbiome}

Emeran Mayer*, Arpana Gupta, Bruce Naliboff, Kirsten Tillisch, Jennifer Labus, Lisa Kilpatrick

University of California, Los Angeles, California, United States

Background: Resilience to stress is a complex multidimensional construct which refers to an individual's adaptive response to adversity. This response can reflect the ability to "bounce back" after a stressful situation, or the ability to not develop psychiatric or medical disease despite physical or psychological stress exposure. Despite the considerable clinical implications, the biological mechanisms of resilience are not well understood. The human microbiome is now understood to have a profound influence on health and disease, beyond immediate digestive issues, and a perspective of humans as superorganisms, comprised of a complex ecological system, has emerged. Stress, and specifically the neurotransmitter norepinephrine (NE), have been shown to alter gut microbial composition and metabolic activity. Furthermore, modulation of the gut microbiota has been shown to lead to changes in hypothalamic-pituitary-adrenal (HPA) axis responses, brain responses to emotional stimuli, affective behavior, and emotional traits. Thus, the gut microbiota may play an important role in resilience. We aimed to examine the relationship between psychological resilience and microbiota diversity/metabolic activity.

Methods: In 19 healthy individuals (11 female; mean age, 26.6 years), resilience was assessed using the Brief Resilience Scale (BRS) and Connor-Davidson Resilience Scale (CDRISC). The participants also provided stool samples. Stool samples were analyzed using $16 \mathrm{~S}$ ribosomal RNA sequencing and the phylogenetic diversity (whole tree) of the gut microbiota was determined. Stool samples were also analyzed using an integrated analytic platform for metabolomics (Metabolon, 617 Davis Drive, Durham NC). Pearson correlations between microbiota diversity and resilience scores were calculated. In addition, a multivariate partial least squares (PLS) analysis was utilized to examine the relationship between resilience scores and serum metabolites. Metabolite significance was evaluated using bootstrap sampling.

Results: BRS and CD-RISC scores were positively correlated with the phylogenetic diversity of the gut microbiota $(r=$ $0.62,0.50 ; p=0.005,0.03$, respectively). PLS identified a metabolomic profile highly correlated with both BRS and CD-RISC scores $(r=0.81,0.72$, respectively; $p=0.015)$. Metabolites positively associated with resilience included delta- and gamma-trocopherol (vitamin E), and adenosine5-monophosphate. Metabolites negatively associated with resilience were more numerous and included galactose, cholesterol, sphingosine, hippurate, and putrescine.

Conclusions: The results support a relationship between the microbiome and psychological resilience. Furthermore, the metabolomic results are consistent with previous animal studies showing the importance of vitamin $\mathrm{E}$ function in mitigating anxiety and sensitivity to stressful situations. In addition, metabolites negatively associated with resilience have been previously shown to be involved in oxidative stress, accelerated senescence, anxiety, and depression.

Keywords: Resilience, Metabolites, Oxidative Stress. Disclosure: Nothing to disclose.

\section{W142. Prediction of Response to Cognitive-Behavioral Therapy in Obsessive-Compulsive Disorder: A Multivariate Analysis of Resting State Functional Connectivity}

Jamie Feusner*, Nicco Reggente, Teena Moody, Francesca Morfini, Jesse Rissman, Joseph O'Neill

Semel Institute for Neuroscience \& Human Behavior, Los Angeles, California, United States

Background: Cognitive-behavioral therapy (CBT) is an effective treatment for reducing symptoms of obsessivecompulsive disorder (OCD). Although many with OCD will benefit from CBT, the response still varies significantly between individuals. In addition, specialized CBT for OCD has limited availability, can be an expensive treatment, and by its nature is stressful and often time-consuming. This underscores the importance of developing reliable predictors of response to treatment to help with clinical decisionmaking. Although several studies have examined clinical and neurobiological features pre-treatment that are correlated with response to treatment, only one has examined functional connectivity as a predictor, and none have applied multivariate approaches. We used a multivariate pattern recognition approach applied to resting state functional connectivity pre-CBT in order to make predictive inferences on the individual patient level, as to their degree of response to treatment. In addition, we applied the same approaches to pre-treatment symptomatology in order to further elucidate mechanisms of functional connectivity associated with obsessions and compulsions, in a data-driven manner.

Methods: We acquired resting state functional magnetic resonance image BOLD data in 25 medicated and unmedicated adults (mean age $32.96 \pm 9.40 ; 12 \mathrm{M} / 13 \mathrm{~F}$ ) with OCD before 4 weeks of intensive daily exposure and response prevention, a form of CBT. Core OCD symptomatology was measured using the Yale-Brown Obsessive Compulsive Scale (YBOCS). Image preprocessing included parcellation of the brain into 264 regions of interest, each belonging to one of 14 functional networks previously derived from meta-analyses of functional studies. We computed a pairwise Pearsoncorrelation matrix for each mean time course resulting in a 264 x 264 matrix containing the pairwise functional 
connectivity values ( $r$-values) across all ROIs. Matrix cells corresponding to each functional network were identified to create feature sets. We implemented a leave-one-patient-out cross-validation to assess the predictive power of our feature sets in regards to our behavioral measures of interest: change in YBOCS scores from pre- to post-CBT. Specifically, we built a least absolute shrinkage and selection operator (LASSO) regression model on $\mathrm{n}-1$ patients using their feature sets. We correlated the predicted values with the actual values in order to yield a multiple R2 as a measure of our model's feature-dependent predictivity. Additionally, we applied the same analysis to the pre-CBT (baseline) YBOCS scores.

Results: OCD participants showed significant clinical symptom improvements pre- to post-CBT. Pre-CBT, mean YBOCS scores were $24.20 \pm 4.31$ (range 18-28). Post-CBT, mean YBOCS scores were $13.64 \pm 4.00$ (range 8-23). Mean decrease in YBOCS scores were $10.56 \pm 4.43$ ( $\mathrm{t} 24=11.93$, $P<.001$; 95\% bootstrapped CI: 8.96-12.32), representing a range of $11.1 \%$ to $68 \%$ improvement. Connectivity strength in the ventral attention network (including middle frontal gyrus, inferior frontal gyrus, and temporo-parietal junction) predicted reduction of YBOCS scores pre- to post-CBT $(\mathrm{R} 2=.18, P=.01)$. Connectivity strength in the cinguloopercular network (including dorsal anterior cingulate, central opercular cortex, insula, and superior frontal gyrus) at baseline was predictive of baseline severity of YBOCS scores $(\mathrm{R} 2=.35, P=.0009)$.

Conclusions: This represents the first study in OCD to use multivariate pattern recognition to determine neurobiological markers predictive of response to treatment. Although all participants showed improvements in OCD symptoms, there was a wide range of response. The predictive ability of individual differences in connectivity strength in the ventral attention network may reflect the importance of inherent capability to attend to perceptually-driven stimuli in the environment (perhaps also reflecting degree of internal distraction from obsessive thoughts) in response to $\mathrm{CBT}$ treatment. In addition, findings suggest an important role of the cingulo-opercular network in predicting severity of obsessions and compulsions, which may be a function of ability to maintain control over behaviors and thought patterns in the face of emotional arousal. Results have clinical implications for identifying individual OCD patients who will maximally benefit from treatment with intensive CBT, and have implications for further understanding the pathophysiology of OCD.

Keywords: Obsessive Compulsive Disorder, CognitiveBehavioral Therapy, Resting State Functional Connectivity, Machine Learning, Multivariate.

Disclosure: Nothing to disclose.

\section{W143. Neuromodulation for Intractable Self-Injurious Behavior Associated With Autism Spectrum Disorder: A Translational Study}

Andrew Chang, Sanmit Adhikari, Alec Stepanian, Jerome Chung, Victoria Berges, Gene Fridman, Jay Baraban, Irving Reti*

Johns Hopkins University, Baltimore, Maryland, United States
Background: About a quarter of individuals with an autism spectrum disorder (ASD) display self-injurious behavior (SIB) ranging from head banging to self-directed biting and punching. Sometimes, these behaviors are extreme and unresponsive to pharmacological and behavioral therapies. We have found electroconvulsive therapy (ECT) can produce life-changing results, with more than $90 \%$ suppression of SIB frequency. However, these patients typically require frequent maintenance ECT (mECT), as often as every 5 days, to sustain the improvement gained during the acute course. Long-term consequences of such frequent mECT started as early as childhood in some cases are unknown. Accordingly, there is a need for the development of alternative forms of chronic stimulation for these patients.

Methods: To evaluate the feasibility of deep brain stimulation (DBS) for SIB associated with ASD, we have utilized two genetically distinct mouse models demonstrating stereotypic excessive self-grooming, namely the Viaat-Mecp2-/y and Shank3B-/- lines. In preliminary studies, we found that a single ECS significantly suppresses the excessive selfgrooming of Viaat-Mecp2-/y mice, similar to the positive effect of ECT observed in the clinic. In order to test whether DBS could also suppress excessive self-grooming, we targeted the subthalamic nucleus (STN) due to its central role in response inhibition, regulating output nuclei from the basal ganglia. Moreover, STN-DBS is effective in suppressing repetitive stereotyped behaviors in both monkeys and humans suffering with severe obsessive compulsive disorder. We delivered bilateral monopolar high frequency stimulation (HFS) for up to 3 hours daily over 3 days. Mice were either knockouts or controls, and received either active or sham stimulation.

Results: We found that STN-HFS significantly suppressed excessive self-grooming in both genetic lines. Suppression occurs both acutely when stimulation is switched on, and persists for several days after HFS is stopped. This effect was not explained by a change in locomotor activity which was unaffected by STN-HFS. Likewise, social interaction deficits were not corrected by STN-HFS. Finally, HFS in a control brain region, the visual association area $\mathrm{V} 2$ of the visual cortex, had no effect on excessive self-grooming, indicating the therapeutic effect of STN-HFS is not due to non-specific stimulation of the brain. It is unknown whether STN neurons or nearby fibers of passage projecting from the cortex mediate either the acute or persistent responses to STN-HFS. Accordingly, we have embarked on an optogenetics-based study to learn more about the pathways mediating the HFS response. In order to guide our selection of stimulation parameters for the behavioral experiments, we are presently conducting electrophysiological recordings triggered by optogenetic activation at the STN and in cortical regions that send fibers of passage adjacent to the STN.

Conclusions: We are exploring the feasibility of DBS for SIB associated with ASD as an alternative to long-term frequent mECT, and our data suggest STN-DBS could be a candidate site for clinical trials. However, focal brain stimulation engages neural elements that form part of distinct, parallelorganized, functional basal ganglia-thalamocortical circuits. Accordingly, we are employing optogenetic techniques to learn more about the circuitry engaged by STN-HFS. Such studies might provide clues about brain circuitry which 
could potentially also be harnessed by less invasive techniques such as epidural cortical stimulation as well as non-invasive, nonconvulsive neuromodulatory techniques such as transcranial magnetic stimulation and transcranial direct current stimulation.

Keywords: Autism Spectrum Disorder, Electroconvulsive, Deep Brain Stimulation, Self-Injurious Behavior, Stereotypy. Disclosure: Nothing to disclose.

\section{W144. Genetic Variation in CYP2D6 and CYP2C8 is Associated With Differences in Human Brain Expression}

Mellissa Giegerich*, Greer Prettyman, Gianluca Ursini, Joo Heon Shin, Andrew Jaffe, Thomas M. Hyde, Joel Kleinman, Daniel R. Weinberger, Kristin Bigos

Lieber Institute for Brain Development, Baltimore, Maryland, United States

Background: The cytochrome P450 (CYP450) family of genes greatly affects xenobiotic metabolism, particularly in the liver and gut. Many of these isoforms, however, are also expressed in the brain. The effects of genetic variation in CYP450s on peripheral drug metabolism have been studied extensively, while the impact on brain expression is unknown. One of the roles of CYP450s in brain involves the regulation of neurosteroids, and therefore genetic variation in CYP450s may result in differences in human brain function.

Methods: We aimed to test the effects of genetic variation in CYP450s on gene expression in post-mortem human brain. We chose 28 single nucleotide polymorphisms (SNPs) that were associated with differential peripheral drug metabolism of antipsychotic, and we verified whether these SNPs were associated with gene expression using an eQTLs catalogue generated from the Lieber Institute post-mortem brain collection. Specifically, mRNA expression was measured using RNAseq in the dorsolateral prefrontal cortex (dlPFC) of approximately 400 human adult brain specimens. We identified expression quantitative trait loci (eQTLs) and quantified the data across five convergent feature summarizations including gene, exon, junction, transcript, and expressed region. The eQTL models tested for additive genetic effects on expression while adjusting for sex, ancestry (multidimensional scaling components), expression heterogeneity (principal components), and diagnosis. Significant eQTLs (at p(FDR-corrected $<0.01$ ) were replicated in two independent sets of dlPFC samples, including the CommonMind Consortium (same allelic direction and $p<0.01$ ) and the GTEx project (same allelic direction).

Results: Of the 28 SNPs selected for their effects on peripheral drug metabolism, 5 SNPs were significantly associated with gene expression in CYP2C8 (rs1934952 and rs6583967) and CYP2D6 (rs3892097, rs1135840, and rs16947). The variants rs3892097 and rs1135840 were significantly associated with expression of CYP2D6 (gene level) in the dlPFC, while the missense mutation rs16947 was associated with CYP2D6 expression at the exon level. More specifically, the number of $\mathrm{T}$ alleles of rs3892097 was associated with higher expression of CYP2D6 $(p=1.3141 \mathrm{e}-$ 38 , beta $=0.32817$ ), and the number of C alleles of rs1135840 was associated with lower expression of CYP2D6 $(p=2.7701 \mathrm{e}-37$, beta $=-0.21548)$. The number of $\mathrm{A}$ alleles of rs16947 was associated with higher expression of an exon within CYP2D6 $(p=7.6149 \mathrm{e}-12$, beta $=0.11537)$. The variants in CYP2C8 were both significantly associated with CYP2C8 transcripts. Specifically, the number of T alleles of rs1934952 was associated with higher expression of a CYP2C8 transcript (ENST00000527420, $p=8.4437 \mathrm{e}-12$, beta $=0.032498$ ), and the number of $C$ alleles of rs6583967 was associated with higher expression of that same transcript $(p=2.0950 \mathrm{e}-19$, beta $=0.044120)$.

Conclusions: CYP450s, which act as monooxygenase enzymes, metabolize drugs and other endogenous compounds in the brain. These genes are expressed differently in the brain than they are in the liver and gut. Little is known about their function in the brain, but these results show that CYP2D6 and CYP2C8 are highly expressed in the dorsolateral prefrontal cortex. Further research is necessary to understand the mechanism behind how these SNPs alter brain function, and functional magnetic resonance imaging could be a tool by which these effects can be explored.

Keywords: RNA Sequencing, Genetics, Postmortem Human Brain, Cytochrome P450s.

Disclosure: Nothing to disclose.

\section{W145. Genetic Variation in Cytochrome P450s Predicts Patterns of Functional Activation During Performance of Cognitive and Emotional Tasks}

Greer Prettyman*, Mellissa Giegerich, Venkata S. Mattay, Daniel R. Weinberger, Kristin Bigos

Lieber Institute for Brain Development, Baltimore, Maryland, United States

Background: The cytochrome P450 (CYP450) family of enzymes plays an important role in the metabolism of drugs and endogenous molecules. The role of these enzymes in the liver has been studied extensively, but despite their documented presence in the human brain, little is known about their effect on the brain. CYP2D6 in particular has been of interest due to its high genetic variability and role in metabolism of psychotropic drugs, including the atypical antipsychotic risperidone. CYP2D6 is highly expressed in the brain and has been linked to personality differences and increased suicide risk. CYP2D6 activity scores have previously been shown to predict neural activation during working memory and emotional face matching tasks. We hypothesize that single nucleotide polymorphisms (SNPs) in CYP2D6 as well as other CYP450 genes will predict different patterns of neural activation during performance of various cognitive and emotional tasks.

Methods: We examined the effects of SNPs in CYP450 genes on neural activation patterns during cognitive and emotional tasks. CYP450 SNPs were selected based on significant prediction of drug clearance of one of five antipsychotics in the Clinical Antipsychotic Trials of Intervention Effectiveness trials. Functional MRI data was collected in healthy participants who completed cognitive tasks for working memory (N-back, $n=457$ ), emotional scene encoding $(n=251)$, emotional face matching $(n=321)$ and cognitive control (flanker, $n=225$ ) as part of the Clinical Brain Disorders Branch NIMH dataset. CYP450 genotypes were 
analyzed as a linear regression in SPM12 for each fMRI task, and activation differences were considered significant at a threshold of $p<0.001$ uncorrected, and an extent of 10 voxels at the whole brain level. Genotype groups were balanced for age, sex, IQ, years of education, and task performance.

Results: Of the 28 CYP450 SNPs tested, 20 SNPs predicted differences in functional activation during one or more cognitive tasks. Of note, carriers of CYP2D6 missense mutation rs1135840 showed an increase in activation of the middle temporal gyrus during the $\mathrm{N}$-back working memory task $(\mathrm{T}=4.37, \mathrm{PFWE}=0.033, \mathrm{KE}=114)$ and a decrease in activation of the superior parietal lobule $(\mathrm{T}=3.63, \mathrm{PFWE}=$ $0.360, \mathrm{KE}=10$ ) during an emotional memory task. Carriers of the $\mathrm{T}$ allele in rs3892097, a diagnostic for the nonfunctional CYP2D6*4 haplotype, showed a decrease in activation of the superior temporal gyrus during the N-back working memory task $(\mathrm{T}=4.01, \mathrm{PFWE}=0.119, \mathrm{KE}=20)$. Carriers of the missense mutation rs16974, a known mutation in the CYP2D6* 2 extensive metabolizer haplotype, showed decreased activation in the temporal lobe during the $\mathrm{N}$-back working memory task $(\mathrm{T}=3.52, \mathrm{PFWE}=0.462, \mathrm{KE}=$ 14). Minor allele carriers also showed decreased activation in the superior temporal gyrus while discriminating incongruent vs. congruent arrows in a flanker cognitive control task $(\mathrm{T}=4.01, \mathrm{PFWE}=0.142, \mathrm{KE}=10)$ and a decrease in activation of the inferior parietal lobule during a go/no-go inhibition portion of the task $(\mathrm{T}=3.93, \mathrm{PFWE}=0.196, \mathrm{KE}=$ 17). Variation in other CYP450 genes including CYP3A4, CYP3A43, CYP2E1, CYP2C8, CYP2C9, CYP2C19, and CYP1A1 also predicted differential patterns of functional activity during cognitive and emotional tasks.

Conclusions: While the cognitive and emotional implications of these imaging phenotypes are not well understood, the different patterns of brain activity that are associated with genetic variation in CYP450 genes indicate a role of these enzymes on brain function. Differences in expression of mRNA of these CYP genes have been observed and may be driving the differences in neural activation for carriers of minor alleles. Ultimately, understanding the role of CYP450 enzymes in the brain could lead to better predictions about cognitive changes resulting from drugs and endogenous substrates metabolized by these enzymes.

Keywords: Functional Neuroimaging, Imaging Genetics, Cytochrome P450s.

Disclosure: Nothing to disclose.

\section{W146. Convergence and Divergence of Anorexia Nervosa and Cancer Cachexia}

McKenna Williams*, Blake Woodside, Ruth Patterson, Katherine Ann Halmi, Pei-an (Betty) Shih

University of California, San Diego, Valencia, California, United States

Background: Anorexia nervosa and cancer cachexia are both severely debilitating, with high incidences of morbidity and mortality. Interestingly, while they have distinct etiologies, they share a strikingly similar magnitude of weight loss as the disease progresses. In this study, we aim to identify the convergence and divergence of anorexia nervosa, a major psychiatric illness characterized by a progressive 'wasting phenotype' and aberrant metabolism, and cancer cachexia, a 'wasting syndrome' commonly observed in late stage cancer patients that is characterized by catabolic state and skeletal muscle wasting. Examining these two disorders in parallel provides a more comprehensive understanding of their unique and shared characteristics, and builds a foundation of knowledge that can ground new, testable hypotheses for further research and ultimately advance treatment strategies. Methods: A systematic literature search was conducted using the MEDLINE/PubMed database. English-language, peerreviewed articles were selected for analysis based on their relevance to three pre-selected domains: body composition, inflammation, and treatment. The following keywords were employed to target articles relating to the aforementioned domains: cachexia, anorexia nervosa, anorexia-cachexia, inflammation, cytokines, nutrition, treatment, weight loss, fat mass, lean body mass, mortality, and BMI.

Results: A total of 98 studies were selected for analysis. In studies involving patients with anorexia nervosa $(n=54)$, the mean age was $19.75 \pm 4.75(\mathrm{SD})$ and the mean BMI upon admission to a treatment program was $15.26 \pm 2.14$ (SD) ( $n=2,740,94.19 \%$ female). In comparison, patients with cancer cachexia had a mean age of $61.25 \pm 5.97$ and mean BMI of $21.34 \pm 2.24(n=1,819,33.24 \%$ female $)$. Anorexia nervosa results in loss of fat mass often with the retention of lean mass and muscle strength due in part to patients' excessive exercising (observed in up to $80 \%$ of patients), while cancer cachexia leads to skeletal muscle wasting resulting in loss of both fat and lean body mass alongside the loss of muscle strength. While the two diseases share "anorexia", a loss of appetite for food, this loss is driven by a behaviorally self-induced starvation in patients with anorexia nervosa in comparison to the tumoral-associated or treatment-induced loss of appetite in patients with cancer cachexia. Beyond the phenotypic and behavioral domains, aberrant inflammatory markers, specifically proinflammatory cytokines including TNF-a, IL-6, and IL-1B, were associated with both disorders. Surprisingly, the pattern of inflammatory marker associations in AN was varied across studies. Out of ten studies selected as relating to inflammation in anorexia nervosa, five studies reported higher circulating levels of TNF-a or IL-6. Three studies reported no difference in circulating levels of TNF-a, IL-6, or IL-1B between patients with anorexia nervosa and healthy controls, while two studies reported lower levels of TNF-a, IL-6, and IL-1B. One study reported lower levels of TNF-a and IL-6 but higher levels of IL-1B. By contrast, a higher level of IL-6 is consistently observed in cancer cachexia, resulting in it being recommended as a potential diagnostic criteria for cancer cachexia. Other inflammation markers including C-reactive protein (CRP), TNF-a, and IL-1 have also been established as hallmark features of the cancer cachexia syndrome. While both anorexia nervosa and cancer cachexia can lead to death, up to $20 \%$ of anorexia nervosa patients die if untreated, whereas $20 \%$ of cancer death is directly attributed to cachexia. No effective pharmacological treatment is available for either of these disorders.

Conclusions: The relatively high prevalence and mortality rates of anorexia nervosa and cancer cachexia urgently demand more studies to improve our understanding of the underlying mechanisms of each disorder. Due to the anosognosic nature of anorexia nervosa and a lack of 
effective diagnostic biomarker for cancer cachexia, both disorders are often underdiagnosed and undertreated until the disorder progresses to an advanced state. Anorexia nervosa and cancer cachexia share evidence of aberrant systemic inflammation, yet there is a degree of contradicting evidence regarding the presentation of inflammatory markers in anorexia nervosa. Given the significant convergence and divergence of these two wasting disorders, we propose that a comparative study utilizing the state of the art multiomics technology may pave the way to enable a systematic investigation to better understand the similarity and differences in molecular mechanisms between these two forms of wasting disorders. These comparative studies in turn will enable the development of more sensitive and specific biomarkers for each disorder, and pave the foundation needed for future research to advance treatment development for patients.

Keywords: Cachexia, Anorexia Nervosa, Inflammation. Disclosure: Nothing to disclose.

\section{W147. Automated Quantification Using FDG PET of Cold-Inducible Brown Adipose Tissue in Fibromyalgia Syndrome}

Jose Pardo*, Joel Lee, Robert Larson, Paul Thuras, Alice Larson

University of Minnesota, Minneapolis, Minnesota, United States

Background: Increasing recognition of the importance of brown adipose tissue (BAT) motivates the development of reproducible and quantitative methods for measuring it. PET has become the principal method to non-invasively detect BAT in humans. However, improvements in quantitation will drive further clinical application. One disorder hypothesized to involve dysregulation in thermoregulation and the processing of pain involving BAT is fibromyalgia syndrome (FMS; Larson et al 2014). This report describes a novel approach to measuring cold-inducible, BAT activity (ci-BAT) semi-quantitatively and reliably with minimal operator intervention.

Methods: ci-BAT was measured with [18]F-fluorodeoxyglucose PET/CT to test whether FMS patients have altered BAT activation compared to normal controls. Subjects had an overnight fast followed by either of two early morning (8 AM) breakfasts on two different days: 1) high fat, low carbohydrate, protein sparing (HFLCPS) diet to decrease activity in BAT via the Randall cycle; or 2) low fat, low carbohydrate (LFLC) diet to increase FDG uptake. Three and a half hours later (to permit release of free fatty acids from the high fat breakfast), the subject was clothed in a light hospital gown and rested on a recliner in a temperaturecontrolled [warm, $21^{\circ} \mathrm{C}\left(75^{\circ} \mathrm{F}\right)$ for HFLCPS condition; or cool, $17^{\circ} \mathrm{C}\left(62^{\circ} \mathrm{F}\right)$ for LFLC condition] room for 3 hours. Each PET scan was attenuation corrected with the associated CT scan. The field of interest for this work sampled from the head to thorax just below the clavicles and required two bed positions. Data were corrected for decay, electronic deadtime, randoms, and scatter. PET and CT scans for each session were merged using FLIRT (FSL, Oxford, UK). To account for differences in dose, body composition, tracer delivery, and to serve as an internal standard, the FDG data were normalized through proportional scaling to whole brain glucose uptake (1000 counts) which remains relatively constant under physiological conditions. Threshold parameters to optimally separate ci-BAT from non-ci-BAT were developed based on the distribution of the pixel-wise parametric data from each merged PET/CT scan for each study session occurring on different days.

Results: The algorithm and scripts are available upon request. Pertinent details follow. A bounding box was used to isolate the head. A histogram of the extracerebral voxel intensities of all CT scans was generated. The threshold for all fat was set from 887 to 999 HU ( FWHM) disregarding the subtle differences in attenuation between white and brown adipose tissue. This threshold generated a template for all fat that was merged subsequently with the coregistered PET scan for each individual session on different days (warm, cool). Histograms of voxel activity in bins of differing radioactivity were used to isolate $\mathrm{BAT}$ at $21^{\circ} \mathrm{C}\left(75^{\circ} \mathrm{F} ; \mathrm{A}\right)$ and at $17^{\circ} \mathrm{C}\left(62^{\circ} \mathrm{F}\right.$; B). Subtracting studies in the warm condition from those done in the cool condition showed a rightward shift in distributions. A threshold of 2,745 counts excluded $95 \%$ of all fat voxels as active under conditions designed to turn off BAT (HFLCPS, warmth; i.e., false positive). Histograms of activity (FDG uptake) within the template of fat were generated for each temperature (cold, warm) and subject group (control, patient). Box and whisker plots highlighted the range of data. BAT uptake at warm temperature was the same across groups and showed good reliability. There was considerable heterogeneity in the cold condition across both groups, but FMS patients showed a trend-level decrease in uptake despite a large outlier that was not removed from the data [Wilcoxon Mann-Whitney test: $\mathrm{n} 1=10 ; \mathrm{n} 2=12 ; \mathrm{U}=86.0 ; \mathrm{p}($ two tail $)=0.09$ ]. Of note, the volume of ci-BAT was not significantly different across groups.

Conclusions: The novel protocol described herein appears reliable and sensitive to the effects of cold on BAT activity. It can be readily implemented and will improve the measurement of ci-BAT activity beyond what is currently available. For the first time, the protocol provided preliminary evidence for dysregulation in cold-inducible BAT activation in fibromyalgia syndrome. The method will improve translation of preclinical models to humans including the search for drugs and other interventions with the potential to modulate BAT activity

Keywords: Metabolism, Adipose Tissue, Chronic Pain, Obesity, Positron Emission Tomography.

Disclosure: Nothing to disclose.

\section{W148. Functional Characterization of Ankyrin Loss-Of-Function Mutations Associated With Autism Spectrum Disorder}

Jacob Garza*, Tracey Petryshen

Harvard Medical School, Boston, Massachusetts, United States

Background: Autism spectrum disorder (ASD) is a developmental neurological disorder characterized by abnormal cognition, inhibition of social behaviors and onset of focused 
or isolated behaviors. Although the underlying causes are not clearly known, recent large-scale genomic studies have identified candidate genes involved in synaptic transmission and brain development that are highly associated with ASD patients. Among the risk genes identified, ankyrin-2 (ANK2) and ankryin-3 (ANK3) have been found in large-scale exome sequencing studies. Loss-of-function missense mutations were shown to be significantly enriched in ASD patients. ANK2 and ANK3 encode membrane associated scaffolding proteins with integral roles in protein localization and axon guidance and growth. Thus, any disruption to ankyrin function may have critical consequences for synaptic function and neurotransmission. The goal of this project is to functionally characterize the de novo loss of function mutations in ankyrins and to identify how dysfunction of ANK2 and ANK3 contributes to the pathogenesis of ASD. Methods: A novel CRISPR/Cas9-based approach was applied in which ANK2 or ANK3 were transcriptionally repressed in a mouse neural cell model. Mouse Neuro-2a cells were transfected with expression plasmids for a catalytically inactive CAS9 protein tethered to a Krüppel associated box domain (KRAB) transcriptional repressor, and a second plasmid that expresses a single guide RNA (sgRNA) to direct the repressor to a specific site upstream of the ANK2 or ANK3 transcriptional start site. sgRNA were designed using the CRISPR Design Tool (crispr.mit.edu) from MIT. Fourteen sgRNA and one non-targeting control sgRNA were designed with an NGG protospacer motif and screened for efficiency of transcriptional repression. Once transfected, the cells underwent positive selection with zeocin and puromycin for 3 days and subsequently used for protein and RNA extraction. Ankyrin transcript levels were measured using qPCR to validate the transcriptional repression. Since microtubule instability is linked to psychiatric illnesses such as ASD, and ankyrins are known to interact with microtubules, tubulin polymerization using fractionated protein lysates for soluble and polymerized alpha-tubulin were measured. Furthermore, expression of the microtubule end-binding protein 3 (EB3) was evaluated using Western blot.

Results: Multiple sgRNA were designed to target ankyrins for transcriptional repression. As the level of transcriptional repression is optimal when the sgRNA is located within $200 \mathrm{bp}$ upstream of the transcriptional start site, 14 sgRNA were designed at various locations within $200 \mathrm{bp}$ upstream of the transcriptional start site within ANK3 exon 1b, which encodes brain-specific isoforms (ANK3b). When compared to the control non-targeting sgRNA, four sgRNA were able to facilitate transcriptional repression of ANK3b, however three of these also had off-target effects that decreased expression of other ANK3 isoforms. A single sgRNA was shown to have specificity for ANK3b and was located 139 bp upstream from the transcriptional start site. ANK3b expression was measured using qPCR and was reduced by $54 \%(p=0.03)$ after transcriptional repression. In order to evaluate how suppression of ANK3b affects microtubule stability, the ratio of soluble: polymerized alpha-tubulin was measured. Repression of ANK3b increased the ratio of soluble: polymerized tubulin by $58 \%(p=0.02)$, indicating decreased microtubule stability after transcriptional repression. EB3 binds to plusends of the microtubule and its expression can provide information on the state of microtubule activity. In light of this, the protein expression of EB3 was evaluated after transcriptional repression of $\mathrm{ANK} 3 \mathrm{~b}$, and was increased by $20 \%(p=0.02)$, further supporting decreased microtubule stability.

Conclusions: Taken together, these results indicate that transcriptional repression of ankyrin-3 induces destabilization of microtubules, which may lead to neuronal dysfunction associated with neuropsychiatric illnesses such as ASD. These data will provide a better understanding of the genetic factors that may underlie the pathogenesis of ASD.

Keywords: ASD, Ankyrin, Microtubule, CRISPR.

Disclosure: Nothing to disclose.

\section{W149. Multimodal Mapping of Cortical Connectivity Before and After Corpus Callosotomy}

Miklos Argyelan*, Erin Yeagle, Victor Du, Jose Herrero, Pierre Megevand, Zoltan Klimaj, Laszlo Entz, David Groppe, Anil Malhotra, Ashesh Mehta

Hofstra Northwell School of Medicine, Glen Oaks, New York, United States

Background: The functional specialization of the cerebral cortex depends in a large part on its connectivity. While connectivity has traditionally been studied in humans with resting state functional MRI (rsfMRI) and/or diffusion tensor imaging (DTI), it may also be revealed by intracranial EEG (iEEG), which has a finer temporal resolution. A number of studies have investigated the correspondence between iEEG and MRI-based metrics of connectivity; however, the correspondence of these measures when connectivity is disrupted has yet to be examined.

Corpus callosotomy, one treatment for intractable epilepsy, massively alters connectivity by severing the major fiber bundle connecting the cerebral hemispheres. This operation's disruption of inter-hemispheric connectivity has been documented using resting fMRI in a single patient (Johnston et al., 2008). To explore whether similar effects would be identified in electrophysiological measures of resting connectivity, we monitored a patients' intracranial EEG, at rest before and after undergoing anterior callosotomy, and compared connectivity to pre- and post-callosotomy rsfMRI and DTI.

Methods: Bilateral, symmetric depth electrodes implanted in epilepsy patient: frontal, parietal, temporal and insular cortices (220 electrodes). This was followed by anterior corpus callosotomy with electrodes intact (laser ablation: electrodes held in place with skull bolts during surgery). We conducted iEEG monitoring (6 min), rsfMRI (6 min), DTI before and after callosotomy.

We analyzed intra and inter-hemispherical rsfMRI connectivity (BOLD signal correlation) change and corresponding change in the white matter microstructure in the corpus callosum. Corpus callosum was parcellated along the anterior-posterior and ventral-dorsal axis to yield 6 subareas for localizing electrodes relative to callosotomy. BOLD time courses were extracted from seeds in implanted electrode locations. In addition, we analyzed the bandlimited power in high gamma range $(50-150 \mathrm{~Hz})$ filtered to extract slow (.1-1 Hz) oscillations (Keller et al. 2014). EEG 
connectivity was calculated by correlating slow oscillations across all possible electrode pairs.

Results: Overall rsfmRI and iEEG connectivity measures decreased across hemispheres after callosotomy, while intrahemispheric connectivity did not change significantly. DTIparcellated FA, resting fMRI, and resting iEEG showed comparable decrease of interhemispheric connectivity in the 6 sub-regions, except in the most ventral and anterior region, where compared to fMRI/FA, iEEG showed increased breakdown of connectivity in frontal lobes.

Conclusions: Our results indicate that severing structural connectivity leads to decreased functional connectivity. The measured decrease of the functional connectivity is proportional to the decrease in structural connectivity in a quantifiable way. Interestingly these changes also correlate with connectivity measured with iEEG across all regions, expect the most ventral anterior region. This close similarity is especially intriguing given the significantly different temporal resolutions of these measures.

Keywords: fMRI Resting State, Intracranial EEG, DTI, Callosotomy, Longitudinal Imaging.

Disclosure: Nothing to disclose.

W150. Clathrin Nanoparticles Efficiently Deliver BDNF to the Hippocampus, Reverse BDNF Deficits and Improve Cell Survival and Proliferation in a Gt-Tg Mouse Model of HIV

Gordana Vitaliano*, Christopher Adam, Juan Fonseca Guzman, Jay McLaughlin, Marc Kaufman, Franco Vitaliano

Harvard Medical School, Belmont, Massachusetts, United States

Background: Human immunodeficiency virus (HIV) associated neurocognitive disorder (HAND) represents a major chronic health problem in the US and abroad. MRI studies consistently show a decrease in the size of the hippocampus and deficits in learning and memory in patients with HAND. Advances in treatment of HAND and other neurodegenerative disorders have been made by administering brain derived neurotrophic factor (BDNF) directly to the CNS, or by using drugs that can increase BDNF indirectly. BDNF promotes neuronal plasticity and restores brain functions. However, BDNF cannot easily cross an intact blood brain barrier (BBB), diffuse within the brain, and it is unstable in the blood or when delivered orally. The goal of this effort is to use an existing clathrin nanotechnology (Vitaliano at al. 2012) to produce stable BDNF-nanoparticles that can bypass the $\mathrm{BBB}$ intranasally, target tropomyosin receptor kinase $\mathrm{B}$ (TrkB) receptor rich brain regions, and reverse neurotoxic effects of HIV transactivator of transcription (tat) protein in a mouse model relevant to HAND.

Methods: A nanocarrier was constructed out of clathrin, a natural protein the transports molecules into cells. One molecule of BDNF was conjugated to the clathrin heavy chain via polyethylene glycol (PEG). Nanoparticle (NP) size and uniformity were determined by electron microscopy and SDS-PAGE. Test subjects, GT-tg bigenic mice (tat + ) were treated daily at 9 AM over 7 days with doxycycline (dox, $100 \mathrm{mg} / \mathrm{kg} / \mathrm{d}$ i.p.) to express neurotoxic tat protein. Control animals (tat-) received only saline. BDNF-nanoparticles $(0.3 \mathrm{mg} / \mathrm{kg}$ of BDNF with $2.4 \mathrm{mg} / \mathrm{kg}$ of clathrin), BDNF alone $(0.3 \mathrm{mg} / \mathrm{kg})$, clathrin alone $(2.4 \mathrm{mg} / \mathrm{kg})$, or vehicle (saline, $40 \mu \mathrm{l}$ ) was delivered intranasally at $2 \mathrm{PM}$, after daily dox or saline administration. For immunohistochemical studies, animals also received bromodeoxyuridine (BrdU $50 \mathrm{mg} / \mathrm{kg}$, every $12 \mathrm{~h}$ i.p.) on the $1 \mathrm{st}$ and 2 nd day of dox or saline administration. Animals were sacrificed and perfused at 7 PM on the 7th day of dox or saline administration. Cell proliferation was determined with a Ki67 antibody and survival of newborn cells with a BrdU antibody. For Western Blot (WB) analyses, an additional group of animals were sacrificed at $7 \mathrm{PM}$ on the 4th day of dox or saline administration. Hippocampal tissues were removed, weighed, dissolved and BDNF concentrations and signaling were analyzed.

Results: Microscopic and WB analyses of hippocampal brain regions confirmed delivery of BDNF-NPs. BDNF nanoparticles significantly improved cell survival and proliferation in GT-tg mouse hippocampus. BrdU+ cell densities (ANOVA, $\mathrm{F}(2,10)=21.79, \quad p<0.0002)$ and Ki67+ cell densities $(\mathrm{F}(2,10)=12.35, p<0.002)$ doubled in the granule cell layer of dentate gyrus in tat+ animals that received BDNF-NPs, compared to tat+ and tat- animals that did not receive nanoparticles. Furthermore, BDNF-NPs reversed BDNF deficits in tat+ animals by significantly increasing hippocampal levels of mature-BDNF $(\mathrm{F}(4,17)=5, p<0.007)$ and pro-BDNF $(\mathrm{F}(4,17)=4.4, \quad p<0.01)$. Mature-BDNF levels were significantly higher in tat+ animals that received NPs, compared to tat+ animals that received saline, or clathrin, or BDNF without clathrin, or tat- animals that received only saline. Finally, BDNF-NPs activated the Akt signaling pathway by significantly increasing hippocampal levels of pAKT $(\mathrm{F}(4,13)=5, \quad p<0.01)$ and Akt $(\mathrm{F}(4,18)=6.5$, $p<0.002)$ in GT-tg mice that received nanoparticles.

Conclusions: Our results demonstrate that engineered clathrin nanoparticles enabled BDNF to effectively bypass a BBB intranasally, and double mature-BDNF levels in the hippocampus. BDNF-NPs significantly enhanced hippocampal cell proliferation and survival and reversed neurodegenerative effects of tat protein in a mouse model of HAND. Hence, clathrin appears to provide a highly efficient nanoplatform for delivery of BDNF to the CNS. This nanotechnology may be able to enhance neuronal plasticity and restore brain functions more quickly and completely than existing treatment methods, while using much lower therapeutic drug doses and with fewer side effects. The development of a stable, targeted molecular nanoprobe may also provide a major new tool for research of molecular abnormalities in neurodegenerative disorders. This novel nanotechnology may serve as the basis for a next generation drug-delivery system that can specifically target relevant brain systems (Vitaliano 2016), and as it can also efficiently deliver imaging probes (Vitaliano at al. 2012) it may have utility as an imaging agent to enhance diagnosis and monitor progression of the disease.

Keywords: Neurotechnology, BDNF Delivery, Clathrin Nanoparticles, HIV Associated Neurocognitive Disorder, GT-tg Mouse Model.

Disclosure: ExQor Technologies, Inc: Equity Ownership/ patents, Self. 
W151. The Putative KCC2 Activating Small Molecule CLP257 Does not Have any Target Engagement or Functional Activity at KCC2 but Does Potentiates GABAA Receptor Activity

Ross Cardarelli, Karen Jones, Lucie Pisella, Lisa McWilliams, David Baker, Matthew Burnham, Heike Wobst, Qi Wang, Igor Medyna, Tarek Deeb, Stephen Moss, Nicholas Brandon*

AstraZeneca Pharmaceuticals, Lexington, Massachusetts, United States

Background: Fast synaptic inhibition in the human nervous system is mediated by GABAA and glycine neurotransmitter receptors which form ion channels permeable to anions including $\mathrm{Cl}-$. The ability of these receptors to suppress neuronal activity is largely dependent on membrane hyperpolarization enabled by low [Cl-]i. Neurons express a specialized $\mathrm{K}+/ \mathrm{Cl}-$ cotransporter (KCC2) that persistently extrudes $\mathrm{Cl}-$, thereby maintaining the inhibitory tone of GABAA and glycine transmission over time. Pharmacological inhibition of KCC2 with recently identified small molecules demonstrates that acute loss of KCC2 function leads to pain hypersensitivity and epileptiform activity. Importantly, human and animal studies revealed that KCC2 activity is dysregulated in multiple disorders including schizophrenia, chronic pain and seizure-related disorders, and recent genetic studies have shown that KCC2 mutations are associated with epilepsy. Compounds which increase KCC2 function could have utility in a range of diseases where there are few treatment options. A recent published small molecule screen performed elsewhere on the NG108-15 neuronal cell line identified CLP257 as a small molecule activator of KCC2. Further testing indicated that CLP257 alleviated hypersensitivity to pain in mice, but in contrast, it aggravated epileptiform activity in vitro. We therefore sought to further characterize the mechanism by which CLP257 exerts its efficacy.

Methods: We evaluated the effects of CLP-257 on the function of KCC2 in a range of cell line and neurons using a range of complementary techniques including thallium-flux assays, perforated patch-clamp electrophysiology, biochemical analysis of KCC2 subcellular localization, phosphorylation status and chloride imaging using SuperClomeleon to detect $\mathrm{Cl}-$-sensitive fluorescence and back-scattering inferometry to measure binding of the compound to KCC2. Results: In contrast with the original report, CLP257 did not enhance chloride (Cl-) extrusion in NG108-15 cells as measured by both $\mathrm{Cl}$ - imaging and perforated patch-clamp recordings. However, a prominent Cl- leak was detected in NG108-15 cells, which confounds the previous interpretation of the data. Furthermore, KCC2 protein was not detectable by immunoblotting in NG108-15 cells. Similar to the lack of activity in NG108-15 cells, CLP257 did not increase the function of KCC2 when over-expressed in HEK293 cells, as measured with both gramicidin perforatedpatch clamp recordings and thallium flux assays. Measurements using back-scattering interferometry further indicated that CLP257 did not directly bind to KCC2, whereas the KCC2 inhibitor VU0463271 exhibited nanomolar affinity. In contrast, secondary pharmacology screens revealed that CLP257 potently inhibited MAO-B function in the nanomolar range, and exhibited binding affinity to PPAR gamma, $\bullet$ adenosine transporters, and 5-HT1A receptors at relevant micromolar concentrations. Finally, we demonstrated that CLP257 displaces the GABAA channel blocker TBPS and functionally acts as a positive allosteric modulator of native GABAA receptor currents, which may provide an explanation for the activity of CLP257 in models of pain.

Conclusions: Our study indicates that CLP257 is not a direct modulator of KCC2 function and so the search continues for the first direct binding modulator of the transporter. We will discuss our own screening strategies in light of the data we obtained with CLP-257. The literature continues to suggest that a KCC2 activating compound could have efficacy in a range of neurological and psychiatric disorders.

Keywords: kcc2, Drug Discovery - New Approaches, GABAA Receptors, Pharmacology.

Disclosure: AstraZeneca: Full Time Employee, Self.

\section{W152. A Proof of Concept Trial of Comt Inhibition to Ameliorate Cognitive and Behavioral Sequelae of Traumatic Brain Injury: Preliminary Data From the Open Label Phase of the Spl Tolcapone Project}

Robert Schloesser*, Margo Lauterbach, Emily Berich, Alexander Maclay, Elizabeth Postell, Andrew Jaffe, Daniel Weinberger

Sheppard Pratt-Lieber Research Institute, Baltimore, Maryland, United States

Background: Patients with a history of traumatic brain injury (TBI) often experience chronic problems with cognition, mood, anxiety and behavioral control. There are no FDA-approved treatments for many symptoms and if available, treatments are frequently suboptimal. There is some evidence that psychotropic medications targeting dopaminergic signaling improve these symptoms. The enzyme Catechol-O-methyl transferase (COMT) catabolizes dopamine, and is highly expressed in the prefrontal cortex of the brain. While dopamine can be metabolized through several pathways, COMT constitutes the main mechanism to inactivate prefrontal cortical dopamine. Tolcapone is a brain penetrable COMT inhibitor that increases dopaminergic transmission in the prefrontal cortex. Tolcapone is FDAapproved for the treatment of Parkinson's disorder, but it carries a black-box warning of risk for hepatic failure. In studies with human subjects without brain disorders, tolcapone has been shown to modulate prefrontal cortical dopamine tone, which is associated with increased working memory performance and improved inhibitory control. Furthermore, a number of small clinical studies showed improvements in mood, cognition and impulse control. We are conducting an open-label, proof of concept clinical trial to identify responders and response characteristics of patients with a history of remote (at least 12 months) TBI and neuropsychiatric symptoms treated with tolcapone.

Methods: Study design: Open-label 2-week trial of tolcapone (200 mg TID) for patients with a history of TBI and neuropsychiatric and cognitive complaints.

Recruitment/Cohort: We are enrolling patients from the Neuropsychiatry Program (NP) at Sheppard Pratt Health 
System's specialty clinics for patients with TBI and concussion. Goal for recruitment is a minimum of 60 patients. We are presenting data on the first 10 patients $(n=10)$.

Cohort characterization: We are obtaining sociodemographic information as well as medical and psychiatric history including information from prior clinical imaging. NINDS/ FITBIR core, basic and several supplemental common data elements (CDE) are utilized for data harmonization purposes. Outcome measures: We are utilizing a comprehensive battery of outcomes measures, including standardized patient reported outcome (PRO) measurements instruments and cognitive testing.

PROs include: Traumatic Brain Injury - Quality of Life (TBIQol) - a computer adaptive measurement tool utilizing item response theory and testing 20 domains including emotional health (depression, anxiety, anger, emotional and behavioral dyscontrol), cognition (general cognitive function, communication/comprehension, executive function), physical health (headache pain, fatigue, mobility) and social participation (independence, ability to participate in social activities, satisfaction with social roles and activities) (Tulsky, 2015). Frontal Systems Behavior Scale (FrSBe), State-Trait Anxiety questionnaire (STAI), Geriatric Depression Scale 15 (GDS-15).

Cognition: NIH toolbox cognition tests, Hopkins Verbal Learning Test (HVLT), Brief Visuospatial Memory Test (BVMT), Trail Making Test.

Results: 9 patients completed the study to date. All patients tolerated the study medication well with few complaints. Reported side effects were mild-moderate in all cases, and included fatigue, diarrhea, and insomnia.

PROs and Proxy reported outcomes: TBI-QoL: Patients reported improvements in several measures within the emotional and cognitive domains. Group level statistics reveal significant improvement in some but not all domains; depression ratings as well as ratings of general cognition, executive function and comprehension showed significant improvements $(p<0.05)$. Patients and proxy (typically family members or group home staff) reported improvements in apathy and executive dysfunction in the FrSBe and this result was significant $(p<0.05)$.

Cognition: BVMT improved with tolcapone treatment and this effect was significant on a group level. NIH toolbox cognitive measures showed trends towards improved functioning in particular in the List Sorting and Flanker tasks. Conclusions: We are presenting study design and preliminary data from an ongoing open-label proof of concept/ responder identification trial. The design of the study is an open-label proof of concept study of 2 weeks treatment with tolcapone utilizing a battery of modern PRO measures and cognitive testing including the complete NIH toolbox cognitive domains that are part of FITBIR CDEs. Information from this trial will be utilized for the design and cohort selection of a follow up randomized controlled trial that will include modalities to examine target engagement including functional MRI imaging and electroencephalography. Given the open-label nature and the low number of participants that have completed the study to date, results are preliminary and should be considered with caution. Several patients reported improvements in a number of cognitive and emotional domains and some had improved cognitive performance in particular in a test for working memory and processing speed.

Keywords: Tolcapone, COMT, Clinical Trial, Traumatic Brain Injury, Neuropsychiatry.

Disclosure: Nothing to disclose.

\section{W153. fMRI Measured Motor Representation in Congenital Mirror Movement Individuals With DCC Haplotype}

Daniel Vosberg*, Amanda Chalupa, Natalia Jaworska, Sylvia Cox, Kevin Larcher, Dominique Allard, France Durand, Alain Dagher, Chawki Benkelfat, Myriam Srour, Ridha Joober, Franco Lepore, Guy Rouleau, Hugo Theoret, Cecilia Flores, Marco Leyton

McGill University, Montreal, Canada

Background: Members of a Québec family are haploinsufficient for the axon guidance molecule receptor gene, DCC. Sixty-five percent of carriers develop mirror movements (MM), involuntary contralateral responses that accompany voluntary movements. Based on rodent studies indicating that reduced DCC disrupts decussation of commissural axons, we hypothesized that individuals with MM would exhibit bilateral motor representations.

Methods: Brain activity was measured using 3-Tesla fMRI following voluntary right-hand movements vs. rest in 11 DCC mutation carriers with MM and 37 controls, comprising 7 DCC mutation carriers without MM, 12 relatives without the DCC mutation, and 18 unrelated healthy controls. Regions of interest (ROIs) comprised the contralaterally innervated primary motor cortex (M1) and the ipsilaterally innervated cerebellum. The fMRI data were preprocessed using SPM12, and mean beta weights were extracted from the ROIs using MarsBaR.

Results: Right-hand movements activated the left motor cortices and right cerebellum in all groups. In participants with MM, relative to all controls, right hand movements induced significantly greater activation in the ipsilateral right M1 $(p=0.025)$ and a pattern of greater activation in the contralateral left cerebellum $(p=0.12)$. Whereas all control groups exhibited a mean deactivation in the right $M 1$, the MM group exhibited a mean activation. Furthermore, there were trends of group $\mathrm{x}$ hemisphere interactions in M1 $(p=$ $0.055)$ and cerebellum $(p=0.069)$. Finally, the MM group exhibited significantly smaller interhemispheric (left-right) beta-weight differences in M1 $(p=0.02)$ and cerebellum $(p=$ 0.004 ), indicative of more bilateral motor representations.

Conclusions: Identifying clear gene-related effects on human brain anatomy and function has proven challenging. The present study identifies the first neuroimaging evidence that a mutation to the axon guidance molecule receptor gene, DCC, affects neural decussation in humans.

Keywords: Axon Guidance Molecule Genes, Neurocircuitry, Neurodevelopment.

Disclosure: Nothing to disclose. 
W154. Distinct Hippocampal Functional Connectivity Between Temporal Lobe and Juvenile Myoclonic Epilepsy

\section{Kelvin Lim*, Jazmin Camchong, Thomas Henry}

University of Minnesota, Minneapolis, Minnesota, United States

Background: Mesial temporal lobe epilepsy (mTLE) and juvenile myoclonic epilepsy (JME) are the most and second most prevalent specific epilepsy syndromes in adults and older children, respectively. These syndromes feature distinct sets of interictal cognitive, affective and other neuropsychiatric impairments. Disturbed learning and memory more often involve attentional deficits in JME, but more often involve delayed recall deficits in mTLE, for example. The range of learning and memory performance varies greatly across groups of mTLE patients and groups of JME patients, as is also the case for other interictal impairments across mTLE and JME groups. Functional imaging correlates of these interictal dysfunctions might elucidate the overall pathophysiology of mTLE and JME group differences in interictal dysfunctions. Individual patient imaging data might provide biomarkers linked to specific interictal dysfunctions that are useful in therapeutic trials and patient care.

Methods: We recruited left mTLE (TLE; $N=5$; Age $\mathrm{M}=46.2$, $\mathrm{SD}=13.44 ; 2$ females) and JME (JME; $N=6$; Age $\mathrm{M}=30.33$, $\mathrm{SD}=8.26$; 5 females) patients from a large tertiary epilepsy clinic (University of Minnesota Comprehensive Epilepsy Center, MINCEP). Clinical histories and examinations, and structural imaging and electroencephalographic (EEG) data provided definitive syndromic classifications. In addition to characteristic seizure and epilepsy risk factor profiles for their syndromes, the left mTLE patients had interictal and ictal epileptiform EEG activities limited to the left temporal lobe, and had left hippocampal sclerosis or normal structural MRI (without extratemporal lesions), while JME patients had interictal and ictal generalized fast spike-wave and polyspikewave EEG discharges and had normal brain structural MRI in each case. Rest fMRI data were collected with a 3Tesla unit using Human Connectome Project parameters and preprocessed with the following: dropped first 3 TRs (to account for magnet stabilization); motion correction; brain extraction; spatial smoothing (FWHM 4); band-pass temporal filtering (high-pass only 0.005); detrending; ICA denoising and registration to standard space. We compared resting functional connectivity (FC) between mTLE and JME patients. With seed-based FC measures, we examined left and right hippocampal FC differences between TLE and JME.

Results: When examining FC differences between patients with MTLE and JME, we found significant group differences in FC of left hippocampus. Patients with mTLE had significantly lower FC than patients with JME between left hippocampus and (i) left inferior frontal gyrus (BA 46 and 10); (ii) bilateral middle frontal gyrus (BA 9); (iii) left cerebellum; and (iv) right visual cortex (middle occipital gyrus and cuneus). There were no significant group differences in $\mathrm{FC}$ of right hippocampus.

Conclusions: Findings from this preliminary study suggest that there are significant differences in hippocampal functional connectivity between the two most common types of epilepsy, mTLE and JME. Observed differences were lateralized, patients with left mTLE showed more disruption in resting state synchrony between left hippocampus and regions known to mediate executive function and sensory processing. We did not find differences in FC of the right hippocampus between groups. Further studies with larger age- and gender-matched sample sizes need to be conducted to confirm robustness of current findings.

Keywords: Epilepsy, BOLD Imaging, Large Scale Networks. Disclosure: Nothing to disclose.

\section{W155. Regional Serotonin Transporter Distribution in the Brains of Active and Recently Retired NFL Players: A Study Using [11C]DASB PET}

Jennifer Coughlin*, Yuchuan Wang, Yong Du, Melin Vranesic, Matthew Peters, Akira Sawa, Gwenn Smith, Martin Pomper

Johns Hopkins University School of Medicine, Baltimore, Maryland, United States

Background: There is rising concern that former National Football League (NFL) players may experience onset of cognitive, affective, and motor impairments linked to history of sports-related head injury. Recently we showed that brains of elderly former NFL players have higher binding of [11C] DPA-713 to translocator protein $18 \mathrm{kDa}$ (TSPO), a marker of brain injury and repair, compared to healthy controls. We also found higher [11C]DPA-713 binding in brains of young, active and recently retired NFL players compared to matched controls. Since accumulating evidence suggests a link between brain inflammation and depressive illness, we sought to use [11C]DASB PET neuroimaging in the same population of young NFL players to test for parallel changes in the serotonin transporter (SERT) distribution and symptoms of depression compared to controls.

Methods: All participants completed a structured psychiatric research interview, including assessment of depressive symptoms using the 17-item Hamilton Depression scale (HAM-D). Regional distribution of SERT was quantified using [11C]DASB PET-based neuroimaging data from 13 well-characterized active or recently retired NFL players and 15 age-matched, athletic, healthy controls. Recent retirement was defined as within 12 years from last NFL play. Regional [11C]DASB non-displaceable binding potential (BPND) was estimated using the simplified reference tissue model from each subject's 90- min dynamic PET data. Regions of interest included several cortical, subcortical, and brainstem regions. Bonferroni correction for multiple comparisons was applied to statistical analyses.

Results: One of the NFL players scored a 14 on the HAM-D, consistent with moderately severe depressive symptoms, and one player scored an 11 on the HAM-D, indicating mild depressive symptomatology. All other players and all of the controls were without apparent depressive symptoms (HAM-D $<8$ ). In spite of higher binding of [11C]DPA-713 to TSPO in these players compared to controls, there was no difference in binding of [11C]DASB to SERT in all brain regions between the two cohorts. 
Conclusions: Our findings from this study suggest lack of change in SERT distribution in brains of young NFL players. The distribution of SERT may be unaffected by history of NFL-related brain injury, at least within the initial years after cessation play. Further analyses will evaluate the relationship between SERT and cognitive impairment, other behavioral symptoms, and binding of [11C]DPA-713 to TSPO within this population.

Keywords: TBI, TSPO, SERT, PET Imaging.

Disclosure: Nothing to disclose.

W156. Germline Truncation of Psychiatry Risk Gene Transcription Factor 4 Disrupts Cell Type-Specific Pathways in the Neural Transcriptome

BaDoi Phan, Stephanie Cereceo Page, Morganne Campbell, JooHeon Shin, Andrew Jaffe, Brady Maher*

Lieber Institute for Brain Development, Baltimore, Maryland, United States

Background: Haploinsufficiency of transcription factor 4 (TCF4) causes the autism spectrum disorder (ASD) PittHopkins Syndrome (PTHS). PTHS presents with intellectual disability, failure to acquire language, deficits in motor learning, and gastrointestinal abnormalities. To understand TCF4's role in neuronal development, we investigated dysregulation in the transcriptome of a germline TCF4 mutated mouse, a genetic model for PTHS.

Methods: The PTHS mouse carries a heterozygous germline truncation of TCF4 basic helix-loop-helix domain to inhibit DNA binding and gene regulatory activity. Tissue was microdissected from mouse cortex and flash frozen. TCF4 mRNA and protein levels were quantified in TCF4+/tr mice and wildtype littermates from embryonic day 12 (E12) through postnatal day 42 (P42), $N=3$ per condition. Six samples each from both genotypes at P1, P21, and P42 were sequenced with $150 \mathrm{bp}$ paired-end reads at 50 million reads per sample. Reads were aligned to the mm10 mouse reference genome, and differential expression (DE) determined at the gene, exon, and junction levels co-varying for age and un-modeled batch effects[8]-[10]. DE features were used in subsequent gene ontology enrichment, Ingenuity Pathway analysis, and cell type-specific expression analysis(CSEA). Lastly, we analyzed differentially expressed genes across the TCF4 truncation model of PTHS and two other ASD mouse models, MeCP2 knockout and homozygous Pten mutation.

Results: TCF4 expression peaks during mouse perinatal cortical developmental, similar to expression pattern in rat and human. In our PTHS mouse model, TCF4+/tr mice show decreased full-length Tcf4 expression $(p<0.001)$ and TCF4 protein $(p<0.01)$ during cortical development compared to wildtype littermates. RNAseq analysis reveals 10 differentially expressed genes that reach genome wide significance $(N=36, \mathrm{FDR}<0.05)$. DE genes enriched for myelination, oligodendrocyte development, axonal guidance, immune processes, and glutamate receptor signaling (adjusted $p<0.05$ ). Using CSEA, DE genes were highly specific to oligodendrocytes, oligodendrocyte progenitors, astrocytes, and Pnoc+ neurons (adjusted $p<0.05$ ). When compared to other models of ASD, 29 genes were found disrupted across the 3 mouse models of ASD $(F D R<0.05)$. These genes enriched most in immune cells and oligodendrocytes (adjusted $p<0.05$ ) and were involved in similar pathways such as immune signaling and axonal guidance (adjusted $p<0.001$ ). Conclusions: In this study, we apply RNA sequencing analysis to investigate gene regulation by TCF4 in a mouse model of PTHS. This genome-wide approach identified cell type-specific genes and signaling pathways that are disrupted and thus provide novel insights into potential pathophysiological mechanisms underlying PTHS. Furthermore, we were able to combine transcriptome analysis of multiple syndromic models of ASD, and show similar pathways are disrupted across these models, suggesting common celltypes and signaling pathways may be underlying a various forms of syndromic autism.

Keywords: Autism Spectrum Disorders, RNA Sequencing, Genetic Mouse Model.

Disclosure: Nothing to disclose.

\section{W157. Gaining Insight Into a Lack of Insight: Quantifying Anosognosia in an Open-Label Trial on the Effects of Increasing Cortical Dopamine on Cognitive and Behavioral Dysfunction in Patients With Traumatic Brain Injury}

Mark Kvarta*, Emily Berich, Elizabeth Postell, Alexander Maclay, Andrew Jaffe, Daniel Weinberger, Margo Lauterbach, Robert Schloesser

University of Maryland School of Medicine, Baltimore, Maryland, United States

Background: Many patients with traumatic brain injury (TBI) have lasting deficits in cognitive, social, and interpersonal domains of their lives. Anosognosia, or a deficit of self-awareness or insight into one's own symptoms or disability, contributes to poor outcomes because of unrealistic treatment goals, discord between patient and loved ones, and resistance to seek neuro-rehabilitation. Furthermore, anosognosia confounds clinical and research assessments based on patient self-report. Despite the prevalence of anosognosia after TBI, there is no universally accepted method to detect it or to quantify its severity. It is unclear whether there are any interventions that may improve anosognosia, as it is a rarely measured target. The goal of this study is to characterize anosognostic patients in a clinically relevant neuropsychiatric study population and to compare response to intervention modulating cortical dopamine levels with the rest of the study population, testing the hypothesis that anosognostic patients will report inferior outcomes because of lack of insight.

Methods: 18 patients were enrolled for an ongoing openlabel trial investigating the effects of tolcapone, a catechol-Omethyl transferase inhibitor, on cognitive and behavioral dysfunction in chronic TBI patients, at least one year after head injury. Patients were recruited from the Neuropsychiatry Program at the Sheppard Pratt Health System. We examined disparities in self- and caretaker-reports on the Frontal Systems Behavioral Scale (FrSBe) to identify anosognosia, and also compared self-assessments with objective cognitive and emotional function. Performance on both the NIH Toolbox for the Assessment of Neurological and Behavioral Function and the Brief Visuospatial Memory 
Test (BVMT) were assessed. All patients completed FrSBe self-rating assessments, rating their own abilities in three domains-apathy, disinhibition, and executive dysfunction -before and after head injury. For 9 patients, a caretaker completed a family rating assessment. Scores were then normalized to established t-scores. Criteria for impaired selfawareness of impairment were (1) an informer-report of at least 1 standard deviation greater of impairment ( $\mathrm{t}$-score $=$ 10) than patient self-report in at least one FrSBe domain and (2) an increase in FrSBe rating of impairment by informerreport post-injury compared to pre-injury. A $\Delta \mathrm{t}$-score was calculated by subtracting FrSBe t-scores generated from selfreport from those generated by informer-report for each subdomain, with larger $\Delta t$-scores suggesting greater disparity in self-awareness. All subjects were administered the Traumatic Brain Injury Quality of Life (TBI-QOL) survey before and after treatment. Patients were assessed before and after 2 weeks of treatment with tolcapone $200 \mathrm{mg}$ TID.

Results: 4 out of 9 patients met the criteria for anosognosia (ANOS), while 5 patients remained in the non-anosognostic (NON) group. All four of the ANOS patients met the criteria for the apathy subdomain of the FrSBe, three patients for executive dysfunction, and one patient for disinhibition. For the ANOS group, $\Delta \mathrm{t}$-apathy was $22.5 \pm 5.7 \mathrm{SEM}$ after injury, compared to $-7.4 \pm 7.8 \mathrm{SEM}$ for the NON patients $(p=0.022$, Student's t-test); apathy scores were significantly higher when rated by the informer $(85.3 \pm 13.5)$ than the patient $(62.8 \pm 8.0, p=0.029$ paired t-test) for the ANOS group but not for the NON group $(70.2 \pm 9.4$ informer vs. $77.6 \pm 5.8$ self, $p=0.40$ ). There was a difference between the groups in $\Delta \mathrm{t}$-disinhibition $(12.8 \pm 11.4$ ANOS vs. $-6.4 \pm 3.7 \mathrm{NON}$, $p=0.12)$ and $\Delta \mathrm{t}$-executive dysfunction $(9.3 \pm 5.8$ ANOS vs. $-10 \pm 6.7 \mathrm{NON}, p=0.100$ ), and a significant difference in executive dysfunction self-report $(61.3 \pm 6.0$ ANOS vs. $75 \pm 1.3 \mathrm{NON}, p=0.015)$. In multiple subdomains of TBIQOL, there was a trend for improvement in the NON group after two weeks of tolcapone treatment (cognition-general measures $p=0.015$, communication $p=0.077$, executive function $p=0.097$, grief $p=0.074$ ), but not the ANOS group $(p>0.1)$. There was no difference between groups on most NIH toolbox domains before or after treatment with tolcapone; however, ANOS patients improved on the "flanker" task for inhibitory control and attention after tolcapone treatment (group ${ }^{\star}$ treatment interaction $2 \times 2$ mixed ANOVA $\mathrm{F}(1,19)=7.35, p=0.027)$ while the NON group did not. On the BVMT, tolcapone improved total recall across all patients (t-score $50.0 \pm 4.6$ post- vs. $41.8 \pm 4.2$ pre-treatment; $\mathrm{F}(1,19)=12.7, p=0.007)$, with no group ${ }^{\star}$ treatment interaction $(p=0.37)$.

Conclusions: Tolcapone improves several subjective and objective measures of cognitive and behavioral function in TBI patients. A subset of chronic TBI patients with anosognosia can be identified by large disparities in selfvs. family-rated questionnaires of cognitive and behavioral function. These patients may represent a specific neuropathology characterized by differential response to treatment, as illustrated by an improvement in inhibitory control after tolcapone that was specific to the ANOS group. Assessing anosognostic patients with by self-report alone is likely to be fraught with confound. Quantifying anosognosia should help both clinicians and researchers utilizing selfreports, as well as inform realistic neurorehabilitation goals.
The ability to quantify, and thus objectively study and target, anosognosia in this subset of TBI patients should lead towards better individualized care.

Keywords: Insight, Traumatic Brain Injury, Tolcapone, Disinhibition, Apathy.

Disclosure: Nothing to disclose.

\section{W158. Brain-Wide Coupling Between Glucose Metabolism and Neuroglial Activity Predicts Agreeableness in Human Personality}

Ehsan Shokri Kojori`, Dardo Tomasi, Corinde Wiers, Elizabeth Cabrera, Elsa Lindgren, Gregg Miller, Veronica Ramirez, Sukru Demiral, Min Guo, Jizheng Zhao, Lori Talagala, Christopher Wong, Sunny Kim, Gene-Jack Wang, Nora Volkow

National Institute on Alcohol Abuse and Alcoholism, Bethesda, Maryland, United States

Background: Although there is an agreement in the literature that neuroglial demand and metabolic supply in the brain are proportional, compelling evidence suggests that regional glucose metabolism may vary independent of immediate neuroglial activity; for example, as a function of nonoxidative glycolysis (Goyal et al., 2015). We hypothesized that abundance of different metabolic pathways could be influenced by genetic factors that may also influence behavioral characteristics such as personality traits. We characterized metabolic coupling (MC) in the brain by studying the association between brain glucose metabolism and brain activity at rest and assessed the extent to which MC is important for predicting human personality traits.

Methods: Participants $(n=28$, age $=36.6 \pm 12.7$ years, 10 females) underwent resting-state functional magnetic resonance imaging (fMRI) sessions in two separate days each lasting $15 \mathrm{~min}$ (eyes open with no fixation cross) using a 3.0 T Siemens Prisma scanner with a 20 channel coil (TR = $1.5 \mathrm{sec}, 3-\mathrm{mm}$ isotropic, flip angle $=70 \mathrm{deg}$ ). The fMRI data were processed using Human Connectome pipeline for motion and EPI distortion correction, and normalization to the MNI template (2-mm isotropic). Measures of local brain activity were estimated using two independent indices of functional connectivity density ( $F C D, r=0.6$ with scrubbing, $0.01-0.1 \mathrm{~Hz}$ ) and fractional amplitude of low frequency fluctuations (fALFF, 0.01-0.1 Hz). The average of log-FCD and ALFF maps from the two sessions were computed to index resting brain activity. Participants also underwent PET (HRRT; 1.2-mm isotropic) for $75 \mathrm{~min}$ with FDG (10 mCi) tracer in list-mode (eyes open with no fixation cross). Quantification of glucose metabolic rates (GMR) was performed with the Sokoloff model on the last $40 \mathrm{~min}$ of PET-FDG data (Lumped constant $=0.52$ ). Metabolic images were normalized to MNI template (2-mm isotropic) using the fMRI transformation parameters. For each participant, MC was computed as the z-transformed correlation between brain activity (indexed by FCD or ALFF) and metabolism across voxels. The Revised NEO Personality Inventory (NEO PI-R) was collected $(n=27)$ to characterize neuroticism, extraversion, openness to experience, agreeableness, and conscientiousness traits. 
Results: There was a strong coupling between brain activity and metabolism in the gray matter within individuals for both log-FCD $(\mathrm{MC}=0.29 \pm 0.06, p<0.0001)$ or fALFF $(\mathrm{MC}=0.32 \pm 0.06, p<0.0001)$ activity measures. We found $\mathrm{MC}$ was correlated only with the agreeableness factor for logFCD $(r=-0.50, p=0.007$, two-tailed $)$ or fALFF $(r=-0.47$, $p=0.014$, two-tailed). The findings indicated that MC and behavioral associations with $\mathrm{MC}$, were comparable for FCD and fALFF brain activity metrics. In addition, we assessed the extent to which there was regional specificity in the association between agreeableness and glucose metabolism and brain activity measures. While both GMR and FCD showed negative correlations with agreeableness in superior frontal gyrus (BA 10), there were also negative correlations in right postcentral gyrus with GMR and negative correlations in fusiform gyrus, right inferior frontal and right precentral gyri with FCD $(p<0.01, \mathrm{k}>70)$. When we characterized the extent to which brain regions showed simultaneously high or low GMR and FCD (i.e., variations in regional power), we found bilateral hippocampus clusters were associated with agreeableness $(p<0.01, \mathrm{k}>70)$. Specifically, higher regional power in hippocampus was associated with higher agreeableness scores.

Conclusions: Here we show that higher metabolic diversity in the brain (inversely related to MC), is associated with higher agreeableness scores. There is evidence that variations in cannabinoid receptor $1(\mathrm{CB} 1)$ gene $(\mathrm{CNR} 1)$ is associated with individual differences in agreeableness (Juhasz et al., 2009). Though glucose metabolism in the central nervous system is regulated differently than the rest of the body, it is noteworthy that polymorphisms in CNR1 gene are associated with metabolic syndrome (Yu et al., 2013). Comparable cortical regions were identified in GMR and FCD maps for the associations with agreeableness. Yet, it was through regional power analysis that we found associations between bilateral hippocampus and agreeableness. At the same time, brain hippocampus is among regions with highest CNR1 expression levels. Our findings indicate that variations in CNR1 may contribute into regional variations in metabolic pathways in the brain. Future analysis will involve characterizing variation in CNR1 in relation to individual differences in agreeableness score in our sample. Finally, we provide evidence that measures of brain glucose metabolism and activity when combined together reveal unique information about characteristics of human behavior. Keywords: Glucose Metabolism, Graph Theory, Functional MRI (fMRI), Big Five Personality Factors, Cannabinoid Receptor.

Disclosure: Nothing to disclose.

\section{W159. Is There a Relation Between Striatal Dopamine} Release, Cortical Thickness, and Novelty Seeking?

Natalia Jaworska, Sylvia Cox, Kevin Casey, Isabelle Boileau, Mariya Cherkasova, Kevin Larcher, Alain Dagher, Chawki Benkelfat, Marco Leyton*

McGill University, Montreal, Canada

Background: Novelty seeking (NS) and impulsive personality traits have been proposed to reflect an interplay between cortical and limbic systems, including the ventral striatum.
Although neuroimaging studies have provided some evidence to support this (Leyton et al 2002; Schilling et al 2012; Casey et al 2013), most have small sample sizes and many report surprisingly large effects given the limitations of trying to relate a snapshot of brain function/structure to an entity as complex as personality. As such, the current work examined associations between striatal dopamine (DA) release, cortical thickness, and NS in the largest sample of adults to date, to the best of our knowledge.

Methods: Fifty-two healthy adults (45M/7 F; $23.8 \pm 4.9$ years old) underwent two positron emission tomography (PET) scans with [11C]raclopride (specific for striatal D2/3 receptors), one at baseline/placebo and another with a d-amphetamine $(0.3 \mathrm{mg} / \mathrm{kg}$, p.o.) challenge. Structural magnetic resonance image (MRI) scans were acquired, as were Tridimensional Personality Questionnaire (TPQ) data. Amphetamine-induced changes in [11C]raclopride BPND values $(\triangle \mathrm{BPND})$ were examined in the sensorimotor (SMST), associative (AST) and ventral limbic striatum (LST). Cortical thickness (CT) measures, adjusted for whole brain volume, were extracted from the dorsolateral prefrontal (DLPFC), sensorimotor (SM) and ventromedial prefrontal/ limbic cortex (vmPFC).

Results: As expected, BPND values were significantly lower in the amphetamine vs. no-drug sessions, with the largest effect evident in the LST. When comparing low vs. high LST $\triangle$ BPND groups (median split), higher NS2 (impulsiveness) scores were found in the high LST $\triangle$ BPND group (one-sided t-test: $p=0.04$ ). Partial correlations (controlling for age and gender) yielded a negative relation between ASTS $\triangle$ BPND and SM CT $(r=-0.38, p=0.008)$; trends for inverse relations existed between $\triangle B P N D$ values in the LST and SM CT, and between AST $\triangle$ BPND values and DLPFC CT. Overall, LST $\triangle \mathrm{BPND}$ was weakly related to SM CT.

Conclusions: These data replicate previously reported associations between novelty-seeking related impulsiveness and cortical thickness with DA release in the limbic striatum in a relatively large sample. The findings add to the plausibility of these associations while suggesting that the effects are likely weaker than has been proposed.

Keywords: Novelty Seeking, Impulsivity, Dopamine, Cortical Thickness.

Disclosure: Nothing to disclose.

\section{W160. Add-On Aspirin is not More Effective Than Placebo for Positive Symptoms in Schizophrenia Patients With High Plasma CRP Levels}

Mark Weiser*, Linda Levi, Shimon Burshtein, Liliana Fodoreanu, Roxana Chiriță, Ghiorghe Talău, Diana Cirjaliu, Robert Yolken, John Davis, Michael Davidson

\section{Sheba Medical Center, Ramat Gan, Israel}

Background: Studies have hypothesized that schizophrenia has an immunological basis, and several trials have indicated that aspirin and other NSAIDs might be efficacious in ameliorating symptoms of schizophrenia. In a post hoc analysis of data from an RCT which administered add-on aspirin or placebo to patients with schizophrenia already treated with antipsychotics, we found that patients with high levels of CRP, perhaps reflecting high levels of inflammation, 
had improved on aspirin vs placebo, compared to patients with lower levels of CRP. On the basis of this post-hoc analysis, we then performed a prospective RCT administering add-on aspirin to schizophrenia patients with high plasma CRP levels, with an a priori hypothesis that aspirin would be most effective in these patients.

Methods: Both RCTs included schizophrenia or schizoaffective patients. Inclusion criteria in the first study were 4 (moderate) or above on CGI-S and 4 (moderate) score on two of the following four PANSS items: delusions, hallucinatory behaviors, conceptual disorganization or suspiciousness/ persecution, and/or a acore of 18 or above on the PANSS negative symptom subscale. The duration of both studies was 16 weeks. The primary outcome measure in the first study was changes in total PANSS scores, and the secondary outcome measures included PANSS subscales and cognition. The first study, on which a post-hoc analysis was performed, was a multi-center, $N=200$ trial comparing aspirin (500 mg/bid) or placebo added on to anti-psychotics. The second trial included only patients with high plasma CRP levels ( $>3850 \mathrm{ng} / \mathrm{ml}$ ), who received add-on aspirin $500 \mathrm{mg} /$ bid $(n=80)$ or placebo $(n=80)$.

Results: In the first RCT, although overall there was no difference between add-on aspirin and placebo, a post-hoc analysis showed that patients with high CRP (>3850 ng/ml) were significantly more likely to have improvements in their mean PANSS positive scores $(\mathrm{ES}=0.61, p=0.03)$. In the second RCT, the mean age of patients was 41.5, mean duration of illness was 9 years, mean PANSS total score at baseline was 101. Analysis showed no significant differences between aspirin and placebo in the change in total PANSS scores $(p=0.89)$, PANSS positive score $(p=0.91)$, PANSS negative score $(p=0.86)$, PANSS general psychopathology score $(p=0.8)$, or cognition.

Conclusions: The results of a post-hoc analysis of an RCT indicated that a subgroup of patients with high levels of CRP showed significant improvements in positive symptoms when receiving add-on aspirin, perhaps reflecting inhibition of COX-1 or COX-2, or other biological effects, both inflammatory and non-inflammatory of aspirin. However, the second RCT, which a-priori randomized patients with high baseline CRP to add-on aspirin or placebo, showed no difference between drug and placebo. We conclude that aspirin is not efficacious in the treatment of schizophrenia. Keywords: Inflammation, Schizophrenia, Aspirin, Symptoms.

Disclosure: Nothing to disclose.

\section{W161. Dissecting the Complex Architecture of Schizophrenia: Transforming Data Into Actionable Knowledge}

Igor Zwir*, Javier Arnedo, Alberto Mesa, Daniel Mamah, Gabriel A. de Erausquin, C.R. Cloninger

Washington University School of Medicine, St Louis, Missouri, United States

Background: Patients with mental disorders sharing the same diagnosis may share few symptoms in common, vary widely in severity, and respond differently to treatments. Studies of schizophrenia have been plagued by weak and inconsistent findings, most likely as a result of the clinical and etiologic heterogeneity resulting from describing individuals just as having the disorder (cases) or not (controls). Yet, no available methodology allowed subclassification of people with a complex disorder such as schizophrenia into evidence-based subtypes having distinct genetic causes, symptoms, and treatments. Furthermore, most studies of complex disorders focused on single sources of data (clinical, genetics, or neuroimages), eliminating the possibility of having complementary perspectives of the patients. Here, we propose the application of novel person-centered methods to approach the problem of identifying subtypes of schizophrenia using an integrative assessment (phenomics).

Methods: We developed a data-driven, agnostic, machine learning method termed PGMRA. This approach can support different types of data including genotypes, clinical descriptions and images; it performs an unsupervised coclustering of subjects in each domain of knowledge. PGMRA identifies sets of interacting SNPs that cluster within subgroups of individuals (i.e., SNP sets) regardless of clinical status, employing a Generalized Factorization Method combined with Non-Negative Matrix Factorization to identify candidates for functional clusters. Subjects and/or SNPs may be present in more than one set. PGMRA examines the risk of a disorder for each SNP set by incorporating a posteriori subject status (e.g., case, control, relative). Statistical significance of the association of SNP sets disease status is calculated using the Sequence Kernel Association Test. The method behaves in a similar way for phenotypic sets regardless of their genetic background. Phenomic measurements include clinical descriptions, endophenotypes or neuroimaging, and each domain of information is analyzed separately. PGMRA identifies natural genotypic-phenotypic associations that are organized into networks which characterize disease architecture and map risk. PGMRA is a web server application at http://phop. ugr.es/fenogeno.

Results: We hypothesized that schizophrenia is a group of heritable disorders caused by a moderate number of separate genotypic networks associated with distinct clinical syndromes. To test this hypothesis, we reanalyzed data from the Molecular Genetics of Schizophrenia (MGS) study, and replicated the findings in 3 independent samples. We also identified numerous SNP sets in 12 additional studies of schizophrenia where the detailed phenotype was not reported. PGMRA analysis of MGS revealed 17 disjoint, non-overlapping, genotypic (multilocus) networks of SNP sets. The association of the SNP sets with schizophrenia risk and phenotype was stronger than that of their constituent (individual) SNPs (2E-17 $>p$-value $>1 \mathrm{E}-03)$. Networks mapped to $>96$ gene loci; each set of genes related to known functions and many were known associated with schizophrenia. Unbiased matching of genotypic and phenotypic sets uncovered many-to-many relations with high-level coincidence between genotypic and phenotypic sets $(1 \mathrm{E}-05<$ $p$-value $<7 \mathrm{E}-13)$. Genotype-phenotype architecture differentiates at least eight classes of schizophrenia each with distinct gene organization. Microarray analysis of transcriptome-gene expression data from lymphoblastoid cells from the same subjects revealed subsets of genes that were differentially expressed in the uncovered subtypes of schizophrenia. 
We also investigated diffusion tensor-images brain images from 47 subjects with schizophrenia and 36 healthy subjects using the same approach. We identified eight homogeneous and statistically significant sets of patients sharing particular sets of voxels with low fractional anisotropy, as well as distinct clinical pictures. Incorporating 43 bipolar patients into the analysis allowed us to successfully differentiate these patients from patients with schizophrenia and to find possible comorbidities among these disorders.

Conclusions: Tracing the network connections reveals complex pathways of hererogeneity and pleitotropy, and consequently, a distributed architecture of schizophrenia. These findings open the door for learning to treat each person based on specific causes and pathways.

Keywords: Schizophrenia; Technology, Genetic Architecture, Phenotypic Screening, Diffusion Tensor Imaging, Machine Learning.

Disclosure: Nothing to disclose.

\section{W162. Free Water Imaging Along the Psychosis Spectrum}

Amanda Lyall*, Ofer Pasternak, Carissa Tuozzo, Lisa Valerie Bitzan, Zachary Fitzgerald, Henry Dumke, Dominick Newell, Toshiaki Onitsuka, Yoji Hirano, Timothy Crow, Anthony James, Katherine Karlsgodt, Delbert Robinson, Joey Trampush, Juan Gallego, Philip Szeszko, Maurizio Fava, Anil Malhotra, Martha Shenton, Marek Kubicki

Harvard Medical School, Boston, Massachusetts, United States

Background: Free Water (FW) Imaging is a novel diffusion MRI method that is able to separately identify changes affecting the extracellular space from those that originate around neuronal tissue. Previous FW imaging studies of patients with schizophrenia (SZ) have identified increased extracellular water volume in early stages of SZ. In chronic SZ stages, this increase was diminished, and replaced by widespread decreased in tissue anisotropy (FAt). In this work, we aim to confirm these previous findings in schizophrenia subjects and, for the first time, apply FW Imaging to a population of individuals with chronic bipolar disorder (BP). We set to identify similarities and differences in FW Imaging between the first episode and chronic SZ and chronic BP that will provide a better understanding of possible distinct biological pathologies along the psychosis spectrum.

Methods: Three $3 \mathrm{~T}$ DWI datasets were independently acquired, and analyzed using the same method: 1) The first-episode SZ population was collected at Zucker Hillside Hospital, Glen Oaks, NY and consisted 63 first-episode SZ patients and 70 matched healthy controls (HC); 2) the chronic SZ population was collected at Kyushu University, Japan and consisted of 19 chronic SZ patients and $21 \mathrm{HC} ; 3$ ) the chronic BP population was collected at Oxford University and consisted of 17 chronic BP and 28 HC. For all three datasets, we utilized Tract Based Spatial Statistics (TBSS). FW Imaging was used to deconstruct the diffusion signal into extracellular FW map and a fractional anisotropy of the tissue (FA-t) map (Pasternak et al., 2009). These maps were projected onto the white matter "skeleton" and groups were compared using nonparametric permutation-based tests with a threshold-free cluster enhancement and familywise error correction $(p<0.05)$ controlling for age and gender.

Results: Our results demonstrate significant and widespread increases in FW, with only limited decreased in FA-t in the first-episode SZ population, and widespread reductions in FA-t with no significant increases in FW in the chronic SZ population. Additionally, the chronic BP population demonstrated significant increases in FW, with no reductions in FA-t.

Conclusions: This study was able to independently replicate previous Free Water Imaging results in separate populations of first-episode and chronic schizophrenia subjects. More importantly, for the first time, we are able to show that chronic BP displays similar white matter changes to those found in early stages of SZ. Since increases in FW in SZ-F have been previously attributed to a possible neuroinflammatory response, our study is the first to provide preliminary evidence that neuroinflammation may also be a prominent pathology in chronic BP. At the same time, chronic BP appears to differ from chronic SZ, as chronic SZ appears to be characterized by additional structural damage. This study offers previously unknown information about the differences in white matter changes across the psychosis spectrum and provides a promising avenue to investigate more specific biological differences in severe psychiatric disease.

Keywords: Human Neuroimaging, Schizophrenia, Bipolar Disorder.

Disclosure: Nothing to disclose.

W163. Neurometabolite Levels in Antipsychotic Naïve/ Free Patients With Schizophrenia: A Meta-Analysis of 1H-MRS Studies

Yusuke Iwata*, Shinichiro Nakajima, Eric Plitman, Yukiko Mihashi, Jun Ku Chung, Philip Gerretsen, Masaru Mimura, Ariel Graff-Guerrero

Centre for Addiction and Mental Health, Toronto, Canada

Background: Studies using proton magnetic resonance spectroscopy (1H-MRS) have reported altered neurometabolite levels in patients with schizophrenia. It has been repeatedly noted that levels of glutamatergic neurometabolite are elevated in patients within the early stages of schizophrenia. On the other hand, N-acetylaspartate (NAA) levels are typically found to be lower in patients with schizophrenia. Regarding other neurometabolites, results seem to be less consistent. Importantly, previous studies have suggested that the influence of antipsychotic (AP) administration is a possible confounding factor that may affect neurometabolite level assessment. Thus, the aim of the present study was to examine neurometabolite levels in APnaïve/free patients with schizophrenia through a metaanalysis.

Methods: A literature search was conducted using Embase, Medline, and PsycINFO to identify studies that compared neurometabolite levels in AP-naïve/free patients with schizophrenia to healthy controls (last search: June 2016). 
Eight neurometabolites, glutamate, glutamine, glutamate +glutamine (Glx), NAA, choline, creatine, myo-inositol, and $\gamma$-Aminobutyric acid (GABA), and 7 brain regions, medial prefrontal cortex, dorsolateral prefrontal cortex, frontal white matter [FWM], occipital lobe, basal ganglia, hippocampus/ medial temporal cortex, and thalamus [Thal], were examined. Standardized mean differences (SMDs) were calculated to assess neurometabolite level differences between groups. Analyses were performed when 2 or more studies were available. Moderator analyses were also performed to examine the influences of study characteristics on the differences in neurometabolite levels between groups. Subgroup analyses were performed for the following categorical characteristics: (a) studies only with AP-naïve subjects and (b) MRI magnetic field strength $(<3 \mathrm{~T}$ or $\geq 3 \mathrm{~T})$. Metaregression analyses were conducted for the following continuous characteristics: (a) age, (b) gender proportion, and (c) duration of untreated psychosis (DUP). Metaregression was performed if at least 5 datasets including neurometabolite levels and characteristics data were available in order to minimize the effect by chance. Owing to the number of comparisons, the significance level was set at a $p$ value of $<0.05 /$ the number of performed primary analyses. Results: Twenty-two studies were included in the analysis. Sixteen studies $(72.7 \%)$ included only AP-naive patients with schizophrenia, whereas 5 studies $(22.7 \%)$ included both AP-naive and AP-free patients with schizophrenia. One study $(4.5 \%)$ included only AP-free patients with schizophrenia. The total number of subjects was 1142 (AP-naïve schizophrenia $=461$ [40.4\%], AP-free schizophrenia $=108$ [9.5\%], and $\mathrm{HC}=573$ [50.2\%]). The age of the subjects was $26.3 \pm 4.1$ years old, the proportion of males was $66.7 \pm 13.0$ $\%$, and the DUP was $1.5 \pm 1.2$ years. PANSS total scores were $77.7 \pm 26.1$, indicating moderate illness severity.

Out of 56 potential comparisons ( 7 brain regions and 8 neurometabolites), a total of 29 analyses were performed (6 brain regions for Cho, Glx, and NAA, 3 for $\mathrm{Cr}$ and $\mathrm{mI}, 2$ for GABA and Glu, and 1 for Gln). Thus, we considered a $P$ value less than $0.0017(0.05 / 29)$ to be significant.

The results of the primary analysis showed lower thalamic NAA levels in the patient group ( 3 studies, $n=174, \mathrm{SMD}=$ $-0.56, P=0.0005)$. No differences were identified for other neurometabolites. In the subgroup analysis only examining AP-naïve studies, there were no differences between groups. In the subgroup analysis examining magnetic field strength $(<3 \mathrm{~T}$ or $\geq 3 \mathrm{~T}$ ), lower NAA levels were observed in the $<$ $3 \mathrm{~T}$ subgroup within the FWM (2 studies, $n=134, \mathrm{SMD}=$ $-0.65, P=0.0004)$. In the meta-regression analysis, no relationships were found between SMD of neurometabolite levels and subjects characteristics (i.e., age, gender, and DUP). Conclusions: This study extends previously reported metaanalysis findings demonstrating a decrease of NAA levels in patients with schizophrenia. Given the previous reported decrease in NAA of chronic schizophrenia, the present finding may suggest that NAA is decreased in schizophrenia throughout their illness course. On the other hand, previously reported alterations of glutamatergic neurometabolite levels were not observed in the present study. The limitation of this study is the small number of included subjects and studies. Overall, future studies should continue to study neurometabolite levels in AP-naïve patients with schizophrenia to further elucidate the underlying pathophysiology of the illness and to facilitate the development of potential treatments.

Keywords: $1 \mathrm{H}$ MRS, Schizophrenia, N-acetylaspartate. Disclosure: Nothing to disclose.

\section{W164. Brain Metabolism-Based Circuitry and Video- Based Behavioral Assays to Predict Novel Therapeutics for Schizophrenia, Depression, and Bipolar: Utility of 14C-2-Deoxyglucose Autoradiography and Smartcube in Mice}

Andrew Giovanni*, Sharon Engel, Carrie Bowen, Ryan Terry-Lorenzo, Larry Hardy, Phil Jones, Taleen Hanania, Vadim Alexandrov, Mohammed Qutaish, Marc Seaman, Paige Czarnecki, Jenna M. Sullivan

Sunovion Pharmaceuticals, Inc., Marlborough, Massachusetts, United States

Background: Human brain circuitry is fundamental to etiologies of neuropsychiatric diseases and the neuropharmacology of psychiatric drugs. Brain regions of Interest (ROI's) and circuits are defined that relate to clinical brain imaging data in psychological conditions. A reverse translational approach was used to examine mouse brain based on 14C-2-deoxyglucose (14C-2DG) uptake for the clinical therapeutics, Ketamine, Desipramine, Haloperidol, or Lithium. Our investigational compounds have in vitro profiles that differentiate them from the mechanism(s) of action associated with the reference drugs. The goal was to determine how our investigational lead compounds affected key regions of interest (ROI's) and neuronal circuits compared with the mimicked psychiatric drugs.

Methods: Eighty C57BL/6 mice were separated into 10 groups of 8 animals. All animal studies were approved by the Institutional Animal Care and Use Committee and were conducted in accordance with the National Institutes of Health Guide for the Care and Use of Laboratory Animals. After an overnight fast, each mouse was administered vehicle or test article (one of nine test compounds or reference drugs) while awake via intraperitoneal injection. Fifteen minutes after test article administration, each awake mouse was administered 14C-2DG intravenously. Mice were euthanized at 45 minutes post-injection of $14 \mathrm{C}-2 \mathrm{DG}$, and their brains were harvested. Sagittal $30-\mu \mathrm{m}$ thick sections of the 80 brain halves were produced using a cryomacrotome. High resolution optical images were acquired prior to each section being taken from the block. Sections were exposed to phosphor imaging plates to measure radioactivity in the tissue and produce autoradioluminograms.

Results: The test results revealed significant differences in the magnitude and polarity of glucose uptake in ROI's and neuronal circuitry across the multiple treatment groups. Striking similarities were evident in the effects of test compounds and corresponding reference drugs, particular with Ketamine and Lithium. A test compound resembled Haloperidol behaviorally, despite having no significant affinity for dopamine D2 receptors. These results provide evidence that behavioral outcomes in the mouse SmartCube assay for the different reference drugs and our investigational compounds can predict important changes in neuronal circuitry identified via $14 \mathrm{C}-2 \mathrm{DG}$ uptake in mouse brain. 
These approaches will enable systematic identification of candidate novel therapies to treat patients who are insufficiently helped by mechanisms available within the existing approved neuropsychopharmacopoeia.

Conclusions: These results provide evidence that behavioral outcomes in the mouse SmartCube assay for the different reference drugs and our investigational compounds can predict important changes in neuronal circuitry identified via 14C-2DG uptake in mouse brain. These approaches will enable systematic identification of candidate novel therapies to treat patients who are insufficiently helped by mechanisms available within the existing approved neuropsychopharmacopoeia.

Keywords: Neurocircuitry, Imaging, 2-Deoxyglucose.

Disclosure: Nothing to disclose.

W165. Dimensional Investigation of Reward Expectancy and Responsiveness Across Schizophrenia and Healthy Controls

Susana Da Silva, Sarah Saperia, Ishraq Siddiqui, Gagan

Fervaha, Ofer Agid, Zafiris Daskalakis, Arun

Ravindran, Aristotle Voineskos, Gary Remington, George Foussias*

Centre for Addiction and Mental Health, Toronto, Canada

Background: Motivational deficits are a prominent feature of schizophrenia, and are linked to the significant functional impairment experienced by individuals with the illness. Within the construct of motivation, reward expectancy (i.e., "wanting") and responsiveness (i.e., "liking") have received considerable attention in schizophrenia, although with comparisons to healthy individuals often yielding contradicting conclusions in the context of subjective reports. Most efforts to date to investigate such reward expectancy and responsiveness have conducted categorical comparisons between clinical and healthy control groups. While at times there have been differences identified, it remains unclear whether these are driven by diagnosis or the severity of motivation deficits experienced between groups. This study sought to investigate the experience of reward expectancy and responsiveness across individuals with a range of motivation impairments in order to ascertain the influence of motivation and diagnosis on the experience of reward.

Methods: One hundred and forty-six participants took part in this study (74 healthy controls and 72 stable adult outpatients with schizophrenia). All participants completed clinical and neurocognitive evaluations, including the Apathy Evaluation Scale (AES) for clinical motivation deficits, the Brief Assessment of Cognition in Schizophrenia (BACS) for neurocognitive function, and the Temporal Experience of Pleasure Scale (TEPS) for reward expectancy (anticipatory pleasure) and reward responsiveness (consummatory pleasure). Data analysis consisted of multivariate analysis of variance and hierarchical multiple regression to evaluate the influence of differing levels of motivation deficits, both as a continuous measure and across tertiles of motivation impairment, on the experience of reward.

Results: Across the entire sample, both clinical and nonclinical groups exhibited an overlapping and broad range of motivation deficits, with the overall severity of motivation deficits (AES) significantly predicting both reward expectancy $(\mathrm{r}=-0.36, p<.01)$, and reward responsiveness $(\mathrm{r}=$ $-.40, p<.01)$. Other clinical symptoms and cognitive deficits did not offer any additional predictive value for either facet of reward experience. Multivariate analysis revealed a significant effect of increasing severity of motivation deficits on overall reward experience $(\mathrm{F}=3.0, p=.02)$ and a significant motivation level by diagnosis interaction $(\mathrm{F}=3.1, p=.02)$, but no overall effect of diagnosis. Posthoc analyses revealed a significant effect of motivation level for both reward expectancy $(\mathrm{F}=4.0, p=.02)$ and reward responsiveness $(\mathrm{F}=5.1, p<.008)$, driven by significant impairments across both facets of reward experience only in those with severe motivation deficits. Interestingly, there was also a significant motivation level by diagnosis interaction only in reward expectancy $(\mathrm{F}=5.2, p=.007)$, driven by participants with schizophrenia with more severe motivation deficits.

Conclusions: Across a large sample of individuals with schizophrenia and healthy controls, the results of this study suggest that impairments in reward expectancy and responsiveness to reward are present specifically in those individuals with severe motivation deficits, with a diagnosis of schizophrenia influencing only impairments in reward expectancy but not reward responsiveness. These findings may help reconcile discrepancies in previous studies of reward experience comparing healthy controls and individuals with schizophrenia. Going forward, investigations in enriched samples of individuals with severe motivation deficits may advance our understanding of the behavioral and neurobiological underpinnings of impaired reward experience, and offer insights for novel treatment strategies for these deficits.

Keywords: Reward System, Motivation, Negative Symptoms, Schizophrenia.

Disclosure: Nothing to disclose.

\section{W166. Elevated Vascular Endothelial Markers in Schizophrenia}

Lisa Eyler*, Tanya Nguyen, Sheena Dev, A'verria Martin, Barton Palmer, Dilip Jeste

University of California, San Diego, La Jolla, California, United States

Background: Persons with schizophrenia suffer increased rates of medical comorbidities, particularly from conditions typically associated with aging, such as cardiovascular disease. Levels of vascular endothelial growth factor (VEGF) and adhesion molecules such as ICAM-1 and VCAM-1 have been related to aging processes and to neurodegeneration, but less is known about their role in schizophrenia, including their relationship to cognition.

Methods: We examined baseline data from an ongoing prospective longitudinal study of aging in schizophrenia. Outpatients with schizophrenia or schizoaffective disorder $(n=133)$ and healthy comparison participants (HC; $n=113$ ), who were matched on age and gender distribution, had fasting blood samples drawn that were assayed for levels of VEGF, ICAM-1, and VCAM-1. The participants were also 
well-characterized in terms of psychopathology, medical comorbidity, measures of physical function, and cognition. We examined group differences in the vascular endothelial biomarkers, and for those that differed significantly, also examined the clinical, cognitive, and physical health features that were related to their levels separately in each group. Biomarker levels were log transformed and non-parametric tests of differences in group means and correlations were employed.

Results: As expected, patients with schizophrenia had worse medical co-morbidity, physical function, higher body mass index (BMI), and worse cognitive function compared to the HC group. Levels of VEGF $(p=.015)$ and ICAM-1 $(p=.003)$, but not VCAM-1 $(p=.77)$ were elevated in the schizophrenia group compared to the HC group. Within the HC group, VEGF level was higher in older individuals, smokers, and those with worse overall physical comorbidity, higher systolic blood pressure, and higher Framingham risk scores for both cardiovascular disease (CVD) and coronary heart disease (CHD); whereas ICAM-1 levels were significantly related to cigarette smoking, BMI, systolic blood pressure, and Framingham risk for CHD. Within the schizophrenia group, higher levels of VEGF were associated with more severe depressive symptoms, worse total physical morbidity, systolic and diastolic blood pressure, and Framingham risk for CVD; ICAM-1 elevations were most prominent in those schizophrenia patients who had more severe depressive symptoms, higher diastolic blood pressure, and Framingham risk for CHD. Interestingly, worse performance on tasks of executive functioning was related to higher VEGF levels in HCs but not in the schizophrenia group, and at a trend level for ICAM-1 in both groups. Duration of illness or age of onset of schizophrenia, antipsychotic exposure, severity of psychotic symptoms, and BMI were not related to either elevated biomarker in schizophrenia.

Conclusions: Schizophrenia patients had elevation of biomarkers often linked to aging and neurodegeneration. Interestingly, in this cross-sectional analysis, we found these elevations in both younger and older patients, and in those with shorter and longer duration of illness. Persons with and without schizophrenia who had poor vascular health, hypertension, and were smokers, were most at risk for higher levels of these vascular endothelial markers; metabolic risk, as indexed by BMI, was not an important risk factor in schizophrenia. Consistent with their known role in neurodegeneration, elevations in VEGF and perhaps ICAM-1 were related to executive dysfunction, suggesting that reducing vascular risk in schizophrenia may be neuroprotective and improve cognitive functioning.

Keywords: Schizophrenia, Cardiovascular Disorders, Cell Adhesion Molecules.

Disclosure: Nothing to disclose.

\section{W167. Task Specific Disruptions in Theta Oscillations} During Working Memory in People With Schizophrenia

John Ragland ${ }^{*}$, Liang-Tien Hsieh, Jovian Lam, Sarah White, Cameron Carter, Tyler Lesh, Tara Niendam, Charan Ranganath

University of California, Davis, Sacramento, California, United States
Background: People with schizophrenia (SZ) have marked deficits in working memory (WM) that contribute to impairments in other cognitive domains and relate to poor functional outcomes. Previous brain and behavior research utilized fMRI to link WM to functioning of fronto-parietal cognitive control networks, and demonstrated that WM impairments in $\mathrm{SZ}$ are accompanied by dysfunction in these same control networks. More recently, electrophysiological (EEG) studies in nonhuman primates (Reinhart et al., 2012), suggested that coordinated low-frequency neural oscillations may underlie WM maintenance in these same brain regions. The goal of the current study was to utilize EEG to examine the impact of SZ on low-frequency oscillations during performance of a WM task that previously associated task performance with increased power of theta and alpha oscillations during WM maintenance (Hsieh et al., 2011). Because these theta oscillations were associated with a more anterior source (midline frontal) than alpha oscillations, which were associated with more posterior sources (parietal), we predicted that patients would show a specific impairment in theta power.

Methods: EEG data were acquired on 17 people with schizophrenia (SZ) and 16 demographically matched healthy controls (HC) using a 64-channel Neuroscan SynAmps-2 system while participants performed the Temporal Order and Item WM task (Hsieh et al., 2011). During WM trials, participants viewed 4 sequential fractal images and, after a $3000 \mathrm{~ms}$ delay, were presented with either two previous images and asked to indicate which was more recent (order task), or were presented with one new and one old item and asked to indicate which was previously studied (item task). Perceptual characteristics of task stimuli were selected to match task difficulty. Time-frequency analysis focused on delay periods for correct trials only. Baseline corrected data were analyzed using the EEGLAB toolbox in Matlab, and corrected for ocular artifacts using independent components analysis (ICA). Oscillatory power was computed by convolving single-trial epochs from each scalp electrode with six cycle complex Morlet wavelets, the results of which were binned into two frequency bands (theta, $3-8 \mathrm{~Hz}$; alpha, 8$10 \mathrm{~Hz}$ ) based on our a priori predictions. Data were averaged across all electrodes for each frequency band and task condition. Analysis of variance and t-tests were used to identify task effects and group differences; and Pearson's $\mathrm{r}$ identified any significant correlations between EEG power and task performance and clinical measures. Significance level was set at $p<.05$, two tailed, for these planned contrasts. Results: WM performance (percent correct) was less accurate in patients with SZ relative to $\mathrm{HC}$ across both temporal order and item WM tasks $[\mathrm{F}(1,31)=14.9, p<.001]$. Although performance was higher in the item task $[\mathrm{F}$ $(1,32)=7.5, \mathrm{p}, .01]$, there were no group by task interactions $[\mathrm{F}(1,31)=1.4, \mathrm{~ns}]$. Participants responded more quickly on item tasks than order tasks $[\mathrm{F}(1,31)=12.9, p<.01]$, but there were no group $[\mathrm{F}(1,31)=.34$, ns], or group by task interactions $[\mathrm{F}(1,32)=1.4, \mathrm{~ns}]$. As predicted, the overall ANOVA showed significantly reduced EEG power in SZ patients relative to the $\mathrm{HC}$ group in theta-band $[\mathrm{F}$ $(1,31)=4.2, p<.05]$, but not in alpha-band activity [F $(1,31)=1.4, \mathrm{~ns}]$. Although there was not a significant task by group interaction $[\mathrm{F}(1,31)=.94$, ns], we performed an exploratory comparison of task conditions for each 
frequency band, and found that there was a specific reduction in theta power in patients for the temporal order WM task $[\mathrm{t}(31)=2.2, p<.05]$ but not for the item WM task condition $[\mathrm{t}(31)=1.2, \mathrm{~ns}]$. For exploratory purposes, we also examined the topography of the group differences by averaging the electrodes into 9 clusters (left, mid and right frontal, central and posterior) and observed that group differences in theta power appeared most prominent in frontal electrode sites. Correlational analyses did not reveal any significant relationships between EEG power, task performance and clinical symptoms in the current sample. Conclusions: This preliminary study suggests that reduced low-frequency EEG theta oscillations during WM maintenance are a candidate mechanism for impaired task performance and associated deficits in the functioning of fronto-parietal control networks. Although this initial sample was inadequately powered to demonstrate significant interaction effects, exploratory analysis suggested that these EEG deficits might be task-specific and frequency-specific. In particular, it appeared that patients were most impaired in the theta band, specifically for the temporal order WM condition - which places the greatest demand on higherorder context maintenance processing. Moreover, examination of topography suggested that these theta deficits may be maximal over frontal electrode sites. These results converge with $\mathrm{fMRI}$ findings in suggesting that disrupted functioning of fronto-parietal cognitive control networks plays a central role in WM dysfunction in people with schizophrenia.

Keywords: Electrophysiology, Brain Imaging, Working Memory.

Disclosure: Nothing to disclose.

W168. Visually-Guided Hippocampal-Dependent Spatial Navigation by C57BL/6 J Mice is Sensitive to Acute Serotonin 2 A Receptor Agonism

Robert Stackman*, Gongliang Zhang, David Cinalli Jr

Florida Atlantic University, Boca Raton, Florida, United States

Background: As serotonin 5-HT2A receptor (5-HT2AR) agonists, serotonergic psychedelic drugs induce perceptual and visual hallucinations by increasing neuronal excitability and altering visual-evoked neuronal responses. The present study was designed to examine whether the perceptual alterations induced by a 5-HT2AR psychedelic drug would affect the integrity of hippocampal-dependent, visually guided spatial navigation.

Methods: Male C57BL/6 J mice received drug-free place navigation training in the hidden platform Morris water maze task. Once efficient and stable performance was achieved, mice received a systemic injection of the phenylalkylamine hallucinogen, TCB-2 (1.0 mg/kg, i.p.), a selective agonist of 5-HT2ARs, or vehicle. Mice subsequently received a platform-less probe test of spatial memory retention, or a test of the dependence of platform search behavior on the distal and proximal cues present in the testing environment. The influence of TCB-2 and vehicle were tested in a separate cohort of mice on the performance of the visible platform water maze task, as a method of assessing motivation to perform and the ability of the mice to use local cues to guide platform search behavior. To assess the influence of TCB-2 on in vivo hippocampal neurophysiological responses that may support spatial navigation, CA1 place cells were recorded before, during and after acute TCB-2 treatment. Results: Acute $1 \mathrm{mg} / \mathrm{kg}$ TCB-2 delayed the expression of goal-directed swimming responses by mice during a probe test in the hidden platform Morris water maze. Interestingly, TCB-2 did not affect the efficiency of the swim path or the proper use of distal visual cues during the probe test. TCB-2 did not affect visual cue approach behavior in the visible platform water maze, suggesting that the 5-HT2AR agonist exerted a task-specific retrieval impairment. Hippocampal place cell recordings revealed that systemic TCB-2 did not affect location-specific firing of CA1 neurons in a familiar environment, or the remapping of place cells when the mice explored a novel environment. However, TCB-2 impaired the long-term stability of place fields for the novel environment initially encoded under the influence of TCB-2. Conclusions: Systemic administration of a hallucinogenic 5HT2AR agonist delays the retrieval of spatial memory, but does not impair the use of visual cues to guide goal-directed spatial behavior. Electrophysiological data indicate that activation of 5-HT2ARs does not impair the coding and retrieval of spatial information as represented by locationspecific firing of CA1 neurons, but impairs the long-term stability of new formed place fields of CA1 neurons. These results suggest that hippocampal serotonergic activity and in particular 5-HT2AR activation influences the retrieval of hippocampal spatial memory. This retrieval delay may reflect the the 5-HT2AR agonist drug-induced altered perception of the visual cues which is necessary to orient the mouse and plan appropriate goal-directed spatial navigation.

Keywords: Memory Encoding and Retrieval, Hippocampal Function, Hallucinogens, Spatial Navigation.

Disclosure: Nothing to disclose.

\section{W169. Contribution of Impaired Motion Processing to Affect Recognition Deficits in Schizophrenia}

Antigona Martinez ${ }^{\star}$, Pablo Gaspar, Matthew Hoptman, Gaurav Patel, Cheryl Corcoran, Daniel Javitt

Nathan S. Kline Institute for Psychiatric Research, Orangeburg, New York, United States

Background: One important component of social functioning is the ability to rapidly and accurately perceive and respond to facial expressions. Behaviorally, impaired face emotion recognition (FER) has been reported numerous in patients with schizophrenia (Sz) along with abnormal activation within several key components of the face processing system including the posterior superior temporal sulcus (pSTS) (Pinkham, Hopfinger et al. 2008). In addition, abnormalities at sensory processing levels have been reported in Sz patients using psychophysical and neurophysiological methods (reviewed in Javitt 2009). These sensory processing impairments are most prominent in response to stimuli that bias functioning of the magnocellular visual pathway (Javitt 2009).

One crucial visual process that depends on intact functioning of the magnocellular pathway is motion perception (Maunsell, Nealey et al. 1990). In visual cortex, motion processing is 
mediated by the middle temporal area (MT+) (Zeki 1974) which is dominated by input from the magnocellular pathway (Merigan and Maunsell 1993). In Sz, deficits in motion processing are well established (Chen 2011), however, the contribution of these deficits, to impaired FER has not been evaluated. One limitation has been the use of static photographs of faces. These stimuli do not contain the dynamic facial information that is displayed in human social interactions and which are critical for communicating changes in affective states. Moreover, static stimuli do not capture the full range of neural processes involved in naturalistic face processing and thus may not tap into deficits associated with dysfunctional visual motion processing.

Here, we investigate the interplay between motion processing and dynamic face emotion perception in Sz using task-based and resting-state functional MRI (fMRI). We focused on activation and functional connectivity between two regions, motion-selective area MT+ and the pSTS which has been identified as critical for processes underlying the perception of social signals contained in dynamic faces (Sato, Kochiyama et al. 2004).

Methods: Subjects were 28 patients with diagnosed $\mathrm{Sz}$ and 21 similar-age healthy controls (HC). Stimuli were unique video clips of faces dynamically expressing one of five emotions (happy, sad, afraid, angry or neutral). For each video, single frames representing the emotion were extracted and used as corresponding static stimuli. To ascertain FER accuracy, a forced-choice behavioral version of the task was administered. Motion perception was assessed behaviorally by estimating thresholds for coherent motion detection. Additionally, in all subjects, motion selective brain areas were identified with fMRI using low contrast expanding/contracting rings as described elsewhere (Tootell, Reppas et al. 1995). Finally, resting-state functional connectivity maps were generated individually using seeds placed in MT+. Mean connectivity strength ( $\mathrm{Z}$ values) was then extracted for voxels within the pSTS and correlated with activation measures of each face emotion condition.

Results: Compared to $\mathrm{HC}$ subjects, $\mathrm{Sz}$ patients were significantly less accurate at discriminating facial emotions and these deficits were largest for dynamic vs. static faces. During fMRI scanning, dynamic emotional faces elicited robust activation in pSTS that was significantly reduced in patients and was correlated with patients' impaired performance in the FER task. Similarly, Sz patients showed significant deficits in behavioral and fMRI measures of simple motion processing. These deficits in motion processing were correlated with impaired activation of pSTS to dynamic emotional faces. Finally, connectivity strength between pSTS and MT+ was significantly reduced in $\mathrm{Sz}$ patients and, furthermore, this reduced connectivity was highly correlated with impaired activation of the pSTS to dynamic emotional faces.

Conclusions: These findings extend previous reports of impaired face emotion processing in $\mathrm{Sz}$ by using naturalistic videos of dynamic faces and linking these deficits both to impaired activation of the pSTS region and deficits in lowlevel motion processing within magnocellular visual areas. Further, the findings suggest that altered functional connectivity between motion-sensitive area MT+ and pSTS is, at least partly, responsible for affect recognition in $\mathrm{Sz}$ in support of the view that MT+ conveys dynamic facial information, such as gaze shifts or expressions, to pSTS (O'Toole, Roark et al. 2002). Finally, these results suggest that sensory processing deficits represent a critical component of the generalized social cognition impairments associated with schizophrenia.

Keywords: Face Emotion Processing, Schizophrenia, Sensory Processing.

Disclosure: Nothing to disclose.

\section{W170. Corollary Discharge Dysfunction During Vocalization in Individuals at Clinical High-Risk for Psychosis and in Patients Early in the Course of Schizophrenia}

Daniel Mathalon*, Brian Roach, Jamie Ferrie, Rachel Loewy, Barbara Stuart, Veronica Perez, Judith Ford

University of California, San Francisco, San Francisco, California, United States

Background: Animals have evolved neural systems that allow unconscious predictions about sensations resulting from their own actions. These action-based predictions are enacted via the corollary discharge mechanism originating in motor systems. In the case of vocalization, this prediction mechanism attenuates the cortical responses to these sounds and tags them as self-generated. We previously found attenuated speech-related suppression of auditory cortex in psychotic patients and a trend in individuals at clinical highrisk (CHR) for psychosis. Here we report data from a larger sample of healthy controls (HC), schizophrenia patients early in the course of their illness (ESZ), and CHR individuals.

Methods: Event-related potentials (ERP) were recorded from ESZ ( $n=84$, average age $=21.9$ years), CHR $(n=71$, average age $=19.4$ years $)$, and $\mathrm{HC}(n=103$, average age $=22.6$ years $)$ during a vocalization paradigm. The auditory N1 ERP component was elicited by vocalizations (phoneme "Ah") as subjects produced them (Talk) and heard them played back (Listen). To control for the effects of normal brain maturation and aging, N1 amplitude Talk-Listen difference scores from electrode $\mathrm{Cz}$ were regressed on age in the $\mathrm{HC}$ group, and the resulting regression equation was used to calculate age-corrected N1 Talk-Listen difference z-scores for all groups, including the CHR and ESZ groups.

Results: The Talk-Listen N1 suppression effect significantly increased with age in $\mathrm{HC}$ and $\mathrm{CHR}$ individuals over the age range from 12-36 years, but significantly decreased with age in the ESZ patients. The ESZ $(p=.0003)$ and CHR $(p=.04)$ groups showed significantly smaller age-corrected N1 suppression $\mathrm{Z}$-scores relative to $\mathrm{HC}$, but did not differ from each other $(p=.43)$. N1 during Listen was reduced in SZ $(p=.003)$, but not in $\operatorname{CHR}(p=.18)$, relative to $\mathrm{HC}$, consistent with prior studies showing the auditory N1 in response to sounds to be reduced in schizophrenia. CHR participants with more unusual thought content showed less N1 suppression $(r=-0.39, p<.001)$, but no significant clinical correlations emerged in the ESZ patients.

Conclusions: Suppression of N1 in response to self-produced vocalizations relative to the $\mathrm{N} 1$ elicited by listening to playback of these vocalizations increased with age from early adolescence to adulthood, consistent with enhancement of 
the effect with brain maturation. In contrast, the Talk-Listen N1 suppression deficit in the ESZ worsened with age in ESZ patients, consistent with a progressive pathophysiological process over the illness course in schizophrenia. Controlling for effects of normal aging, dysfunction of this putative corollary discharge mechanism during vocalization is evident in schizophrenia patients early in their illness, as well as prior to psycohsis onset in individuals at clinical high risk for psychosis, particularly those CHR individuals with more severe unusual thought content. Whether this dysfunction predicts conversion to psychosis in CHR individuals will be addressed in future longitudinal clinical studies.

Keywords: Auditory Deficits in Schizophrenia, Corollary Discharge, Schizophrenia Prodrome, ERP.

Disclosure: Nothing to disclose.

W171. Differential Pharmacological Manipulation of the Mesolimbic Network Through Cortical and Hippocampal Input Streams With Psychotropic Compounds: Effect of Subunit-Selective NMDA Receptor Blockers

Bernat Kocsis*, Benjamin Pittman-Polletta, Kun $\mathrm{Hu}$

Harvard Medical School, Boston, Massachusetts, United States

Background: (1) N-methyl-D-aspartate receptor (NMDA-R) hypofunction is one of the leading hypotheses of schizophrenic pathophysiology. NMDA-R antagonists elicit psychotic symptoms in human and schizophrenia-relevant signs in rodents, including alterations in oscillatory activity of cortical and hippocampal networks. (2) NMDA-Rs are composed of several subunits which make different contribution to these effects. Non-selective NMDA-R blockade induces strong aberrant gamma activity which, as we have shown recently (Kocsis, 2012), primarily depends on NMDA-Rs containing the NR2A subunit. (3) Narrowband high frequency oscillations (HFOs, $\sim 140 \mathrm{~Hz}$ ) are a recently discovered form of rhythmic activity potentiated by administration of hallucinogenic drugs including ketamine and LSD. They are most likely generated by subcortical networks in the mesolimbic system, e.g. the nucleus accumbens and the ventral tegmental area, which receive inputs from both hippocampus and frontal cortex. (4) HFOs are commonly observed coupled to low-frequency oscillations, most notably theta $(\sim 8 \mathrm{~Hz})$, and theta-HFO coupling has been related to increased cognitive control, which in turn is related to hippocampal (as opposed to cortical) dominance of mesolimbic input streams. (5) NMDA-R antagonism is associated with potentiation of HFOs, along with altered hippocampal and cortical activity, and altered basal ganglia and mesolimbic function. (6) To probe the relationship between hippocampal and cortical rhythms and low-frequency coupling of HFOs, we examined the effects of subtypespecific NMDA-R antagonism on low-frequency modulation of $\mathrm{HFO}$ amplitude.

Methods: Six rats were injected i/p with the NR2A-preferring antagonist NVP-AAM077 (20 mg/kg; Novartis), the NR2Bspecific antagonist Ro25-6985 (10 mg/kg; Tocris), and the non-specific NMDA-R antagonist MK-801 (0.2 mg/kg; Tocris). For each injection, local field potentials were recorded from frontal and occipital cortex and from hippocampus CA1 region from $3 \mathrm{~h}$ prior to $16 \mathrm{~h}$ following injection. For each site, in each experiment, spectral analyses were performed using the Thompson multitaper method, and phase-amplitude coupling (PAC) was assessed using wavelet convolution and an inverse-entropy index (PittmanPolletta, 2014). PAC quantifies the relationship between oscillatory activities of neuronal networks at different frequencies in which the amplitude of a high-frequency rhythm (e.g. gamma and HFO) depends statistically on the phase of a concurrent low-frequency rhythm (6-12 $\mathrm{Hz}$ theta and $1-5 \mathrm{~Hz}$ delta). Band power and PAC summed within theta-gamma, theta-HFO, and delta-HFO ranges after drug injections was statistically compared to saline injection.

Results: We found a markedly different signature of crossfrequency coupling following NR2A-preferring antagonism as compared to nonspecific antagonism of the NMDA-Rs. Both MK-801 and NVP-AAM077 administration resulted in prolonged periods ( 4-6 hours) of wakefulness and hyperactivity associated with aberrant high gamma activity, according to previous reports (Kocsis, 2012). During these periods, HFO power and amplitude modulation also increased dramatically in both conditions, however, MK-801 and NVP-AAM077 induced primarily theta-HFO and delta-HFO PAC, respectively. Following MK-801 injection, comodulograms revealed dramatic increases in thetaHFO PAC in all brain regions relative to saline injection. These increases were largest in frontal cortex, followed by occipital cortex, and then by CA1. Following NVP-AAM077 injection, the largest observed effect was a delayed increase in delta-HFO PAC at all sites, relative to saline. In the 1st hour, theta-HFO PAC was increased in occipital cortex and CA1; but by the 3rd-4th hour post-injection, delta-HFO PAC dominated the comodulograms in all three regions and was increased relative to saline injection while theta-HFO PAC had decreased relative to saline in all three regions. Over the same period that HFO phase-coupling switched from theta to delta frequencies, spectral analysis revealed a switch from the relative dominance of CA1 theta power to frontal delta power. Injection of Ro25-6985 predominantly affecting oscillations in rapid eye movement sleep increased HFO and theta-HFO coupling in this theta-dominated state.

Conclusions: These results suggest that low-frequency modulation of HFO amplitude may be an indicator of cortical vs. hippocampal control of mesolimbic circuits. Our results also suggest that subtype-specific NMDAR blockade produce distinct effects on mesoscale brain dynamics, perhaps resulting in subtype-specific behavioral alterations related to schizophrenia. While theta rhythm is associated with hippocampal circuitry in cognitive function, taskrelated neuronal activity in prefrontal cortex (PFC) is organized by a delta-range $(4 \mathrm{~Hz})$ oscillation (Fujisawa \& Buszaki). Both PFC and hippocampus project to the mesolimbic system and compete for dominance of mesolimbic inputs. Since HFO activity predominantly reflects mesolimbic population activity, its phase modulation may reflect the strength of inputs to the mesolimbic system. In turn, these inputs may be frequency specific, with delta rhythmic inputs coming from cortical networks and theta rhythmic inputs coming primarily from hippocampal networks. 
Keywords: NMDA Antagonists, High Frequency Oscillations, Cortico-Mesolimbic Couling.

Disclosure: Nothing to disclose.

\section{W172. Dynamic, Cell Type-Specific Cis-Regulatory Element Use in the Developing Human Frontal Cortex}

Amanda Price*, Joo Heon Shin, Ran Tao, Thomas M. Hyde, Joel E. Kleinman, Andrew E. Jaffe, Daniel R. Weinberger

Lieber Institute for Brain Development, Baltimore, Maryland, United States

Background: Although schizophrenia is a highly heritable psychiatric disorder, the underlying etiology is complex and still inadequately defined. Current evidence suggests that cisregulatory DNA elements such as enhancers may play an underappreciated role; for instance, most of the single nucleotide polymorphisms (SNPs) marking the genomic loci associated with schizophrenia risk recently identified by the Psychiatric Genomics Consortium fall within non-coding regions (Nature, 2014). Because the origins of schizophrenia are thought to be neurodevelopmentally influenced, cataloguing dynamic enhancer use over cortical development in human post-mortem brain tissue, particularly in a cell typespecific context, has the potential to illuminate currently unknown mechanisms underlying schizophrenia risk by identifying putative non-coding regulatory sequences critical to normal neuronal and glial developmental trajectories and the time windows in which they act.

Methods: To assess cis-regulatory element use over human cortical development, frozen human dorsolateral prefrontal cortex (DLPFC) from donors aged several months after birth to 23 years $(N=17)$ was analyzed for regions of open chromatin. These samples were dissected as previously described (Jaffe et al, Nat Neuro, 2015). Enriched neuronal and non-neuronal populations were isolated using NeuN antibody labeling and fluorescence-activated sorting of nuclei purified from the homogenate tissue. Regions of open chromatin in these cell populations were profiled using the Assay for Transposase-Accessible Chromatin with highthroughput sequencing (ATAC-seq). The resulting sequencing data was aligned using BWA. Duplicate reads were removed using Picard MarkDuplicates, and enriched peaks were called using MACS. Peaks were merged and coverage at each peak calculated using the bedtools suite. Putative enhancers were defined as peaks whose center was at least $2.5 \mathrm{~kb}$ away from a known transcription start site (TSS). Identifying overlapping peaks was done using the GenomicRanges and GenomicFeatures Bioconductor packages. Differential peak enrichment was assessed using the DESeq2 package. Gene Ontology analysis was performed using the ClusterProfiler package. Age-related analyses were conducted comparing the samples younger than $(N=14)$ versus older than $(N=20)$ age ten years.

Results: Sequencing was performed on the same libraries twice: first on a HiSeq 2000, and subsequently on a HiSeq 3000 , both using single end 50 base pair reads. Correlation of peak magnitude between each duplicate sample was high $(\mathrm{R} 2=0.67)$, so the aligned reads were merged to increase the coverage, and peaks were called using MACS. The mean sample depth of aligned reads after duplicate removal was 94 million reads, with very low proportions of reads aligning to the mitochondrial genome ( $0.04 \%$ of total aligned reads).

Merging the MACS output for the samples identified 144,643 peaks across all 24 chromosomes. Of these peaks, $31.48 \%$ overlap accessible regions identified in brain tissue using DNAseI-seq data from the ENCODE Project. 13.22\% of peaks fall within coding sequence, and $13.66 \%$ overlap promoters (defined as 2000 bases upstream and 200 bases downstream of a known TSS). Principal component analysis showed that the samples segregate by cell type, with the first principal component representing $66 \%$ of the variance explained. Indeed, 72,760 of the peaks were significantly differentially enriched by cell type (i.e., neuronal and glial cells, FDR $<0.05$ ). Performing gene ontology enrichment analysis of the genes closest to these significantly different peaks revealed many terms associated with neuronal function and maturation in the peaks preferentially present in neurons compared to glia, such as "neuron projection development" $(p<1.27 \mathrm{e}-39)$. Likewise, terms such as "glial cell differentiation" were overrepresented in peaks upregulated in non-neuronal samples $(p<7.01 \mathrm{e}-18)$. Fewer age effects on chromatin accessibility were detected; 21 peaks were significantly differentially enriched between the older and younger samples in our cohort.

Using a cutoff of 2.5 kilobases from the nearest TSS to the center of each peak, 121,365 peaks could be categorized as putative enhancers, including 16 of the age-regulated and 60376 of the cell type-regulated peaks. Ongoing analyses including paired genotype and gene expression data will further characterize these potential regulatory sequences.

Conclusions: Profiling the accessible chromatin landscape in different cell populations across human cortical development reveals the majority of differences to be related to cell type, while still identifying several age-related chromatin remodeling events. Our ongoing analysis will include a more indepth focus on genomic loci associated with risk for schizophrenia such as those identified by the Schizophrenia Working Group of the Psychiatric Genomics Consortium to explore the potential influence of non-coding DNA sequence in the development of this disease.

Keywords: ATAC-seq, Schizophrenia, Brain Development, Epigenetic Regulation, Chromatin.

Disclosure: Nothing to disclose.

\section{W173. Schizophrenia Polygenic Risk Score Predicts Antipsychotic Treatment Response in Patients With First Episode Psychosis}

Jianping Zhang*, Delbert Robinson, Jin Yu, Wolfgang Fleischhacker, Rene Kahn, John Kane, Anil Malhotra, Todd Lencz

Hofstra Northwell School of Medicine, Glen Oaks, New York, United States

Background: The genetic basis of antipsychotic drug efficacy is likely polygenic in nature. Genetic risks of schizophrenia may also be related to antipsychotic drug response. The Psychiatric Genomics Consortium (PGC) genome-wide association study (GWAS) provided evidence of association with schizophrenia risk for many single nucleotide 
polymorphism (SNP) across the genome. The present study examined whether polygenic risk scores (PRS) based on the PGC GWAS are predictive of antipsychotic efficacy in three cohorts of patients with first episode of psychosis.

Methods: Three clinical trial cohorts with genomic data and antipsychotic efficacy data available at baseline and 3-month follow-up were included in the present study. 1) European First Episode Schizophrenia Trial (EUFEST) has 141 firstepisode schizophrenia patients (age $=25.6 \pm 5.2$ years; male $=$ $60 \%$; all Caucasian) randomized to amisulpride, haloperidol, olanzapine, quetiapine or ziprasidone treatment. 2) Zucker Hillside Hospital First Episode Schizophrenia Clinical Trial (ZHH-FE) has 77 first-episode schizophrenia patients (age = $23.0 \pm 4.9$ years; male $=75 \%$; mixed ethnicity) randomized to risperidone or olanzapine. 3) The clinical trial conducted as part of the Center for Intervention Development and Applied Research at ZHH (CIDAR) has 100 patients with their first episode psychosis (age $=21.5 \pm 5.1$ years; male $=$ $75 \%$; mixed ethnicity) randomized to risperidone or aripiprazole treatment. DNA was extracted from peripheral lymphocytes and genotyping was performed using the Illumina Omni-1Quad array (EUFEST and ZHH-FE) or Illumina Infinium HumanOmniExpressExome array platform (CIDAR). All genomic data underwent standard quality control procedure. SNP imputation was conducted by IMPUTE2 against the 1000 Genomes and GRCh37/hg19. PRS was computed based on the results of the PGC schizophrenia GWAS using the PRSice software for the three cohorts separately. SNPs with a $p$-value less or equal to 0.01 in the GWAS were included in the calculation of PRS. The number of SNPs included was 8,903, 7,736, and 8,110 for EUFEST, ZHH-FE, and CIDAR, respectively. Symptom measure was the total score of the Positive and Negative Symptoms Scale (PANSS) for EUFEST or the Brief Psychiatric Rating Scale (BPRS) for ZHH-FE and CIDAR. Hierarchical linear regression was performed on the 3month symptom score with the PRS as the predictor while controlling for age, sex, and baseline symptom score. Genomic principal component scores were also covaried to control for population stratification for ZHH-FE and CIDAR due to their mixed ethnicity samples. Due to small sample sizes from each study, meta-analytic approach was used to combine the samples using partial correlation coefficient as the effect size measure.

Results: Combining the three cohorts in a meta-analysis, PRS was significantly predictive of 3-month symptom scores, pooled partial $r=0.165, n=318, p=0.003$. Higher PRS was associated with higher symptom scores at 3-month followup, suggestive of less improvement in treatment. Among individual cohorts, PRS significantly predicted 3-month BPRS total scores in the ZHH-FE cohort, beta $=0.68$, partial $r=0.293, p=0.013$, explaining additional $8.1 \%$ of total variance. In the EUFEST cohort, the finding was similar, beta $=0.19$, partial $r=0.212, p=0.012$, explaining additional $3.5 \%$ of total variance. However, PRS did not significantly predict 3-month symptom scores in the CIDAR cohort, beta $=-0.013$, partial $\mathrm{r}=-0.005, p>0.50$, explaining essentially no additional variance. The overall results remained significant when only European ancestry individuals were included in the analysis.

Conclusions: These findings suggest that polygenic schizophrenia risk scores may also be related to antipsychotic drug response. Patients with higher polygenic risk scores tended to have less improvement with antipsychotic drug treatment. Further analysis is needed to elucidate a more defined genomic profile for antipsychotic drug response.

Keywords: Pharmacogenetic Response, Antipsychotic, FirstEpisode Psychosis, Polygenetic Risk Score.

Disclosure: Genomind, Inc.: Grant support, Self.

\section{W174. No Evidence for Degeneration of Prefrontal Functioning After First Episode of Schizophrenia}

Tara Niendam*, Stefania Ashby, Pooja Patel, AnaMaria Iosif, Emilio Ferrer, Tyler Lesh, Marjorie Solomon, Ragland Dan, Cameron Carter

University of California, Davis, Sacramento, California, United States

Background: A large body of research supports the neurodevelopmental hypothesis of schizophrenia, whereby early CNS insults in the prenatal period and/or deviations in the course of child and adolescent brain development are believed to lead to the onset of psychosis in late adolescence or early adulthood. Based on this theory, cognitive impairments and associated brain changes are predicted to be present early in the premorbid period, worsen in the window proximal to psychosis onset, and then remain relatively stable. In contrast, the neurodegeneration hypothesis suggests that schizophrenia is associated with ongoing deterioration in brain structure and functioning after illness onset. Using fMRI and an established measure of cognitive control (AX-CPT), we examined the longitudinal pattern of prefrontal network activity over the course of the first two years of psychotic illness. We hypothesized that individuals who have experienced their first episode of a schizophreniaspectrum disorder (SZ) would show poor performance (relative to healthy controls) in conjunction with reduced dorsolateral prefrontal cortex (DLPFC) activation under conditions of high cognitive control. However, we predicted that individuals with SZ would show a stable pattern of performance and brain activity that was comparable to $\mathrm{HC}$ individuals over follow up, demonstrating that prefrontal functioning is already impaired at psychosis onset and does not deteriorate further over time.

Methods: One-hundred eighty participants ages 12-25 were studied, including 93 individuals with SZ (mean duration of untreated psychosis $4.9+/-4.5$ months) and 93 healthy controls (HC). Participants from the UC Davis Early Psychosis Programs were identified using the Structured Clinical Interview for DSM-IV (SCID-I/P). Participants completed the AX-CPT during fMRI on a 1.5 Tesla scanner at baseline and follow up. D'-context was used as the primary measure of participants' ability to utilize context to guide response selection on the AX-CPT. Functional MRI data were processed using SPM8 and focused on between-groups contrasts of the cue phase of high control (B cue) versus low control (A cue) trials, representing proactive control processes. Whole-brain voxel-wise and a priori DLPFC region of interest analyses were performed. Differences between groups in longitudinal patterns of behavioral performance (d'context) and brain activation (bilateral a priori DLPFC ROIs) were assessed using mixed-effects 
regression models in SAS. The impact of gender, duration of untreated psychosis (DUP), treatment with antipsychotic medication, clinical symptoms and global functioning on performance and brain activation were also examined.

Results: Consistent with previous findings with the AX-CPT, at baseline, individuals with SZ show impaired performance (d'context, $p<.05$ ) and reduced activation in the DLPFC and parietal cortex $(p<.05$ FWE) when compared to HCs under conditions of high cognitive control. Longitudinal analyses reveal improved d'context performance for individuals with SZ over follow up in a manner that paralleled the trajectory of typical development of HC individuals $(p<.001)$. While individuals with SZ overall show reduced bilateral DLPFC activation under high control conditions when compared to HC individuals, their pattern of DLPFC activation was stable over follow up and paralleled the pattern of HCs. Use of antipsychotic medication was associated with better performance at baseline $(p<.05)$, but no change in the trajectory of performance over follow up. Severity of clinical symptoms as well as poor global functioning were associated with the level of cognitive impairment at baseline, but did not impact the subsequent trajectory of improvement. No effect of DUP was observed on performance. Clinical course, DUP, medication use and functioning did not impact DLPFC brain activation at baseline or over follow up. Analyses of DLPFC connectivity will also be presented.

Conclusions: Individuals with first episode SZ show impaired cognitive control associated with reduced DLPFC activation when compared to HCs at baseline, with a pattern of improving performance and stable brain activation over follow up in a manner consistent with HCs. While clinical factors reduced and medication improved baseline performance, they did not affect the trajectory of performance for individuals with SZ over time. Consistent with the neurodevelopmental hypothesis, these findings demonstrate that prefrontal impairment is present at psychosis onset and does not deteriorate over time. In contrast, improved performance for individuals with $\mathrm{SZ}$ over time suggests they continue to benefit from ongoing brain maturation, during the critical period of development from adolescent into young adulthood, in a manner comparable to same-aged $\mathrm{HC}$ peers. Further, while clinical and functional factors have an impact at initial presentation, the course of clinical and functional outcome is not associated with the pattern of prefrontal cognition over time.

Keywords: First Episode Schizophrenia, Longitudinal, Functional MRI (fMRI), Cognition, Cognitive Control.

Disclosure: Nothing to disclose.

\section{W175. Database Application for Analysis of Post-Mortem Toxicology Reports}

Robin Kramer*, Saron Belay, Jose Apud, Stefano Marenco, Barbara Lipska

National Institute of Mental Health, Bethesda, Maryland, United States

Background: The Human Brain Collection Core (HBCC) is a branch under the National Institute of Mental Health (NIMH) that specializes in collecting human brain tissues, the extraction of RNA and DNA from these tissues, and distribution of these samples to various collaborators within $\mathrm{NIH}$ and extramural in order to facilitate the study of mental dysfunctions. With the consent of the next-of-kin of the decedent, HBCC obtains postmortem brain tissue from various Medical Examiner's Offices. Donated brains are dissected and RNA and DNA is extracted from particular brain regions. Specimens are used in molecular and genetic studies on mental disorders. To ensure quality and scientific integrity, the brain tissue is examined for neuropathological changes, RNA and DNA samples are tested for RNA and DNA quality and the donors' blood is tested for toxicology. The goals of the project are to design databases that implement, and curate the vast amount of collected data: demographic, medical, quality control as well as the toxicology results. In this project, we focus on the toxicological data and hypothesize that due to the large variety of compounds being prescribed, there will not be a prevalent drug detected across all donors within a diagnostic group.

Methods: HBCC uses two database applications, Specimen App and Phenotype App, developed in Visual Basic and MSSQL. These applications facilitate tracking of laboratory samples and clinical health records for the purposes of designing and conducting experiments. HBCC contracts with National Medical Services (NMS) to test donors' blood samples and produce a toxicology report. The toxicology report provides quantitative and qualitative data on various compounds detected in a donor's blood. A newly developed feature in Phenotype App categorizes the compounds in the Toxicology report following the Anatomical Therapeutic Chemical (ATC) classification system. The ATC classification system has five levels and categorizes drugs according to first their anatomical target and then their therapeutic, pharmacological, and chemical properties. The compounds tested in the NMS Forensics Panel were matched to DrugBank drug identifiers. The tests were then grouped by the ATC annotations.

Results: Detection is limited to drugs used near the time of death because the toxicology was performed on postmortem blood. According to the results of our analysis, the most commonly used drugs among cohorts were antipsychotics (schizophrenia), psychostimulants and antiepileptics (bipolar disorder), drugs used in addictive disorders (substance abuse), and psychostimulants (depression disorders). Donors with bipolar disorder had a high detection of antiepileptic drugs, specifically clonazepam, used often as a sedative/ antianxiety drug. Donors with substance abuse were positive for drugs used in addictive disorders which include illegal and recreational drugs.

Conclusions: ATC classification system provides a finer grain modeling and a consistent method for associating compounds that were present in the donors' blood with drugs at the time of death. The pattern of drug use in different cohorts is very important in experimental design. Accordingly, ATC codes allow for easier data analysis and pattern recognition of compounds detected in specific diagnostic groups. While drug categories vary between cases, individual compounds were widely varied within the diagnostic groups. With the exception of an antiepileptic/ anxiolytic drug clonazepam, no individual prevalence was observed. The toxicology feature has already proven useful for quality control on both diagnosis and laboratory 
procedures. The design and implementation of the new Toxicology feature within our Phenotype app was successful and we will be procuring the remainder of the toxicology data from NMS to expand the feature for the entire HBCC collection.

Keywords: Postmortem, Brain Tissue, Toxicology.

Disclosure: Nothing to disclose.

\section{W176. Abnormal Protein Insolubility in Brains From a Subset of Patients With Schizophrenia}

Leslie Nucifora, Matthew MacDonald, Brian Lee, Matt Peters, Alexis Norris, YeeWen Wu, Jonathan Pevsner, Russell Margolis, Robert Sweet, Christopher Ross, Akira Sawa, Frederick Nucifora*

Johns Hopkins University School of Medicine, Baltimore, Maryland, United States

Background: Schizophrenia affects $1 \%$ of the population and is characterized by positive and negative symptoms, as well as cognitive impairment. While candidate genes have been identified, the mechanisms of schizophrenia are still unknown, and are likely to be diverse. However, there may be subsets of schizophrenia that can be grouped by common pathophysiology in which some genetic and etiological risk factors converge into a small number of pathways. Changes in stress related cascades, including oxidative stress, can disrupt protein quality controls or modify proteins resulting in abnormal protein insolubility and ubiquitin processing. Protein insolubility has been implicated in many disorders, including cancer, cardiac and pulmonary disease, muscle diseases and neurodegenerative disorders. Furthermore, ubiquitin is a marker for protein insolubility in the neurodegenerative disorders, since it is identified in protein aggregates and in fact may be a signal leading to protein insolubility. However, there has been relatively little information to date on protein insolubility in brains of patients with mental illness. Disrupted In Schizophrenia 1 (DISC1) has been reported to occur more frequently in the insoluble fraction of post mortem brain samples from individuals with depression, bipolar disorder, and schizophrenia than in controls. In addition, we have shown that a mutation in Neuronal PAS Domain Protein 3 (NPAS3) that segregates in a small family with schizophrenia leads to NPAS3 protein insolubility and disrupts the transcriptional activity of NPAS3. Based on these data, we propose a broader concept of protein conformational abnormalities as a common mechanism in some forms of schizophrenia. Therefore, we sought to understand the extent to which abnormal insoluble proteins and markers of protein insolubility are present in a subset of patients with mental illness and what pathways are disrupted by protein insolubility.

Methods: Autopsy brains provided by the University of Pittsburgh and olfactory neurons obtained from patients through the Johns Hopkins School of Medicine Schizophrenia Center were subjected to cold Sarkosyl fractionation, using a series of detergents and gradients to fractionate proteins into soluble and insoluble fractions. All pellet samples were analyzed by SDS-PAGE analysis followed by Coomassie/Silver staining and Western blot analysis to quantify levels of total homogenate protein compared to total insoluble protein in the final pellet. Western blots were probed with a polyclonal ubiquitin antibody to determine levels of ubiquitination in each of the final pellet samples. Image J software was used to quantify the protein signal in the Coomassie/silver stained gels and Western blots. All available demographic information was used to determine any relationship between markers of protein insolubility and specific demographics. For Mass Spectometry experiments, the insoluble pellets were solubilized and digested by trypsin using the FASP method, and analyzed by LC-MS/MS on a Orbitrap Velos ${ }^{\mathrm{TM}}$ Hybrid Ion Trap-Orbitrap Mass Spectrometer (Thermo Scientific) with a nanoACQUITY UPLC system (Waters) and a picochip column (New Objective). Results were analyzed using Ingenuity Pathway Analysis.

Results: We demonstrate in both human autopsy brains and olfactory neurons, that a subset of patients with schizophrenia have increased markers of abnormal proteins, including increased protein insolubility and ubiquitination. The results cannot be explained by demographic differences, including $\mathrm{pH}$, post mortem delay, and antipsychotic exposure. Using mass spectrometry, we also demonstrate that the insoluble fraction contains proteins related to the protein ubiquitination pathway, unfolded protein response, oxidative stress and pathways related Huntington's disease, Alzheimer's disease and Amyotrophic Lateral Sclerosis Signaling. In addition, we identified proteins related to the 108 Schizophrenia associated loci in the insoluble fraction.

Conclusions: This study provides evidence of abnormal proteins in a subset of patients with schizophrenia. This would provide a novel mechanism, possibly through dysregulation of protein quality control, to explore the pathogenesis of these illnesses, and potentially a unifying pathogenesis that can group subtypes of mental illnesses at the molecular level. Correlating this molecular biomarker with specific clinical manifestations may lead to clinicalpathological associations defining a subset of patients and clarifying psychiatric nosology. Further exploration of the mechanisms leading to abnormal insoluble proteins could lead to new therapeutic targets, and utilizing the clinicalpathological correlations identified could lead to precision medicine with medications directed specifically to this subtype of mental illness.

Keywords: Insoluble Proteins, Autopsy Brains, Schizophrenia, Ubiquitination, Oxidative Stress.

Disclosure: Nothing to disclose.

\section{W177. Impact of Fetal-Expressed Genes on Cortical Thickness in Young Adults: Implications for the "Two-Hit" Model of Schizophrenia}

Joshua Roffman*, Thomas Soare, Hamdi Eryilmaz, Anais Rodriguez-Thompson, Phil Lee, Avram Holmes, Jordan Smoller, Randy Buckner

Massachusetts General Hospital, Charlestown, Massachusetts, United States

Background: Cortical thickness is reduced in first episode schizophrenia patients, potentially reflecting aberrant synaptic pruning in the years preceding illness. Cortical thickness is also strongly heritable, and its reduction in unaffected-first degree relatives suggests that it may index genetic loading for 
schizophrenia. While schizophrenia typically presents in young adulthood, it is thought to arise from subtle abnormalities in early brain development. Recent work has elaborated the temporal dynamics of gene expression within postmortem human brain tissue, revealing dramatic differences between fetal and postnatal gene expression patterns. Here, we grouped genes by their developmental expression profiles and weighed their relative influence on cortical thickness in young adults. Thus, we examined whether cortical thickness variation during a critical age period for schizophrenia risk reflects primarily the effects of contemporaneously expressed genes, or, alternatively, an echo of genes that are expressed predominantly in fetal life.

Methods: DNA and standardized $3 \mathrm{~T}$ structural MRI scans were obtained from 1,318 unrelated healthy individuals of European descent, age 18-35, through the Brain Genomics Superstruct Project (GSP). Freesurfer v5.0 was used to obtain mean cortical thickness within the bilateral dorsolateral prefrontal cortex (DLPFC, Brodmann areas 9 and 46) and across the remainder of the cortical mantle for each GSP participant. Using genotype data from the Illumina Infinium OMNI 1 quad chip, single nucleotide polymorphisms (SNPs) were pruned for linkage disequilibrium and for annotation to single brain-expressed genes, resulting in coverage of 6,583 genes. Then, using BrainCloud data obtained from DLPFC tissue, we rank-ordered these genes based on the ratio of their expression in second trimester fetal samples $(n=38)$ versus young adult samples, age 18-35 $(n=95)$. Using this ranking, genes were divided equally into ten deciles (decile $1=$ highest fetal: adult expression ratio; middle deciles $=$ constitutive expression; decile $10=$ lowest fetal: adult expression ratio). Using 10-fold cross-validation, polygenic scores were calculated for each GSP participant to determine the net contributions (R2) of genetic variants within each of the ten temporal expression deciles to cortical thickness, after controlling for population stratification markers, age, sex, head coil (12 vs. 32 channel) and scanner console. Polygenic models included SNPs at various significance thresholds $(\mathrm{PT}=1.0,0.5,0.4,0.3,0.2,0.1)$ per standard practice.

Results: For the DLPFC, decile 1 variants predicted variation in cortical thickness at all SNP inclusion thresholds ( $\mathrm{PT}=$ $1.0, p=.00037 ; \mathrm{PT}=.5, p=.000053 ; \mathrm{PT}=.4, p=.00013$; $\mathrm{PT}=.3, p=.00014 ; \mathrm{PT}=.2, p=.0027 ; \mathrm{PT}=.1, p=.038)$. No other decile significantly predicted DLPFC thickness. In contrast, decile 10 variants most strongly predicted variation in cortical thickness across the remainder of the cortical mantle $(\mathrm{PT}=1.0, p=.0065)$. Exploratory analysis of regionsof-interest outside the DLPFC confirmed that decile 1 variants exert a relatively specific effect on DLPFC thickness. Analysis of a second publicly-available dataset of adult samples (Allen Brain Atlas) confirmed robust correlations $(p<10-10)$ of decile 1 gene expression between the prefrontal cortex and ten other cortical regions, suggesting that regionspecific effects of fetal-expressed genes on cortical thickness do not reflect local gene expression differences during adulthood.

Conclusions: Genes that are preferentially expressed during fetal development confer a sustained, pronounced, and relatively unique effect on cortical thickness within the DLPFC, a region that has been consistently implicated in brain imaging and postmortem analyses of schizophrenia patients. Ongoing work is exploring potential mechanisms that link fetal gene expression to variance in cortical thickness, including analysis of DNA methylation in fetal and young adult brain tissue. This work may ultimately help connect two long-hypothesized periods of schizophrenia vulnerability - fetal development and late adolescence through a common mechanism, and may point to new avenues for early intervention.

Keywords: Cortical Thickness, Gene Expression, Schizophrenia, Dorsolateral Prefrontal Cortex.

Disclosure: Nothing to disclose.

\section{W178. Transcranial Direct-Current Stimulation (tDCS) in Treatment Refractory Auditory Hallucinations: Preliminary Findings}

Jean-Pierre Lindenmayer*, Tania Sultana, Amandeep Kaur, Anzalee Khan, Benedicto Parker

New York University, New York, New York, United States

Background: The successful use of transcranial direct current stimulation (tDCS) has been reported in the treatment of patients with treatment resistant auditory hallucinations with reduction in several open label case reports and more recently in a RCT in thirty patients with schizophrenia and medication-refractory auditory verbal hallucinations. tDCS delivers weak, electric direct currents over the scalp as a minimally invasive neurostimulation treatment with minimal side effects. The effects of tDCS on cortical excitability can be explained by neuronal membrane polarization shifts (subthreshold depolarization or hyperpolarization of resting membrane potential) and modifications of NMDA receptor efficacy, which may result in prolonged synaptic efficacy changes. The aim of our study was to replicate the above findings of the efficacy of tDCS on treatment refractory auditory verbal hallucinations, overall psychopathology and additionally on neurocognitive functions in patients with chronic schizophrenia.

Methods: Twenty-four hospitalized inpatient subjects with DSM-V schizophrenia or schizoaffective disorder were randomized to sham tDCS vs. active tDCS in a randomized double-blind design. tDCS twice daily for 20 minutes was administered for 4 consecutive weeks during an existing standard inpatient psychiatric rehabilitative treatment. The anode was placed on the left temporal-parietal junction over a point midway between F3 and FP1 and the cathode on the left dorsolateral prefrontal cortex over a point midway between T3 and P3 at a stimulation level set at $2 \mathrm{~mA}$ for 20 minutes for each session. Subjects were required to display treatment refractory auditory verbal hallucinations, defined as the persistence of daily auditory hallucinations without remission over 5 years, despite two adequate antipsychotic medication trials and adequate dosages. Most patients were on clozapine maintained on their current antipsychotic treatment throughout the study period. The Auditory Hallucination Rating Scale (AHRS), PANSS, MCCB MATRICS, and CGI-S/I were administered prior to tDCS and after the intervention. Data were analyzed using GLM Repeated Measures ANOVA.

Results: Eighteen subjects completed the study; nine subjects were randomized to sham tDCS vs. nine to active tDCS. 4 subjects terminated early, 2 subjects could not cooperate with 
the procedure. Subjects were primarily males $(88.88 \%)$ with a mean PANSS score at baseline of $78.96(\mathrm{SD}=7.54)$. There was significantly greater improvement $(\mathrm{F}(1,16)=6.983, p=$ 0.018 ) in overall PANSS total score after active tDCS (change from Baseline $=-7.11$ ) than after sham treatment (change from Baseline $=-2.22$ ). No significant difference was observed for the AHRS total score between groups $(\mathrm{F}(1,12)=.247, p=$ 0.110 ), however, there was a significant reduction for the combined sample in AHRS total score $(F(1,16)=15.666$, $p=.002)$. No significant differences were observed in MCCB $\mathrm{T}$ score change and in change of PANSS Item P3 Hallucinatory Behavior score between groups. Overall, 4 weeks of active tDCS stimulation was well tolerated.

Conclusions: Our preliminary results did not replicate previous results of $\mathrm{tDCS}$ in subjects with treatment refractory auditory hallucinations. There was no effect of 4 weeks of tDCS on treatment-refractory auditory hallucinations and on cognitive functions in subjects with chronic schizophrenia. However, low intensity tDCS stimulation was associated with a reduction of overall psychopathology and may prove a viable augmentation intervention for treatment refractory patients, though important limitations should be noted, such as the small sample size. Additional data collection for increased sample size is underway.

Keywords: Auditory Hallucinations, Schizophrenia, Neuromodulation.

Disclosure: Nothing to disclose.

\section{W179. Large-Scale Investigation of Schizophrenia Genetic Risk Factors in Cognition and Brain Structure in the Genus Consortium Collection}

Tracey Petryshen*, Gabriella Blokland, - GENUS Consortium

Center for Human Genetic Research, MGH, Boston, Massachusetts, United States

Background: Genome-wide association studies (GWAS) have identified genetic variants and biological pathways that contribute to the development of schizophrenia. Elucidating the role of these genetic risk factors in brain dysfunction underlying schizophrenia is essential for understanding the molecular mechanisms of disease and identifying novel treatment targets. The Genetics of Endophenotypes of Neurofunction to Understand Schizophrenia (GENUS) Consortium aims to clarify the disease-relevant function of schizophrenia genetic risk factors by examining their relationships with cognition and brain structure in a large schizophrenia sample collection.

Methods: Sixteen international research groups contributed a total of 4896 schizophrenia patients, 3331 healthy controls, and 804 familial high-risk subjects (unaffected relatives) with clinical, genotype, and neuropsychological data, as well as structural MRI data in 3,071 subjects and DTI data in 1,541 subjects. Phenotype processing and quality assurance protocols were developed and validated to maximize comparability of data across sites. Cognitive data were harmonized across samples by pooling control data and fitting a linear regression model (covarying for age, age ${ }^{\wedge} 2$, sex, and age-sex interactions), followed by calculating standardized residuals relative to controls. Structural MRI scans were processed using FreeSurfer v5.3 with manual or automated brain masking. To select robust phenotypes for genetic analyses, literature reviews were performed to identify cognitive and brain structural traits with moderate to high heritability, reliability, and case-control differences. ANOVA with Tukey's HSD posthoc comparisons was applied to each phenotype to confirm case-control differences in our sample. Genome-wide SNP data from each site were subjected to quality control procedures using Plink software and imputed to the 1000 Genomes Phase III reference panel using a standardized pipeline. Multidimensional scaling was performed to identify population ancestry. Genetic association analyses of 108 schizophrenia GWAS SNPs, polygenic risk scores, and biological pathways were performed by linear regression using Plink and $\mathrm{R}$ within each sample (covarying for age, age $\wedge 2$, sex, age-sex interactions, and 10 multidimensional scaling components), followed by inverse variance weighted meta-analysis across samples.

Results: Multidimensional scaling of SNP data determined that the sample ancestry is $79 \%$ European-derived, $8 \%$ African-derived, $8 \%$ East Asian, 3\% Latino, and 2\% other. The male: female proportion is 60:40. We selected 9 cognitive phenotypes for analyses that have moderate to high heritability according to literature meta-analysis $\left(\mathrm{h}^{\wedge} 2=28\right.$ $62 \%$; average $43 \%$ ): Spearman's 'g' (general cognitive ability) and 8 cognitive domains (processing speed, attention/ vigilance, verbal working memory, non-verbal working memory, verbal learning, visual learning, reasoning/problem solving, visuospatial ability). Cognitive phenotypes were significantly impaired in schizophrenia patients compared to controls in our sample (effect sizes -0.52 to $-1.12 ; p<0.001$ ), with the exception of reasoning/problem solving. Thirteen brain structure phenotypes were selected for analyses: volume of 9 regions (superior temporal gyrus, Heschl's gyrus, inferior and middle frontal gyri, anterior cingulate, inferior parietal lobule, insula, hippocampus, and amygdala), as well as 4 global volumes (total cortical gray matter, total brain, intracranial, and lateral ventricles). As expected, lateral ventricle volume was significantly larger in schizophrenia patients compared to controls, and volume of the 12 other regional and global phenotypes was significantly lower in patients $(p<0.0001)$. Genetic analyses of 108 schizophrenia GWAS SNPs detected significant associations (study-wide $p<5.3 \mathrm{E}-05$ ) between: Spearman's ' $\mathrm{g}$ ' and rs9922678 in the GRIN2A glutamate receptor gene, rs2905426, and rs4391122; visuospatial ability and rs1501357 in the HCN1 potassium channel gene and rs11210892; verbal learning and rs11210892. Polygenic risk for schizophrenia was associated with 6 of 9 cognitive phenotypes (all except verbal working memory, visual learning, and reasoning/problem solving) and explained $0.5-1.5 \%$ phenotypic variance $(p<0.0001)$.

Conclusions: Our analyses in a large sample collection confirmed significant abnormalities in cognition and brain structure in schizophrenia patients compared to controls. Association of schizophrenia genetic risk factors with cognitive phenotypes suggests putative neuromolecular mechanisms underlying schizophrenia pathophysiology.

Keywords: Schizophrenia Genetics, Cognition, Structural MRI, SNP, Gene Association.

Disclosure: Nothing to disclose. 
W180. Investigating Heterogeneity in Response to Antipsychotic Treatment in Schizophrenia

Anthony Ahmed*, David Dodell-Feder, Andriana Ilnicki, Brielle Marino

Weill Cornell Medical College, White Plains, New York, United States

Background: The CATIE trial is one of the most influential studies of the comparative efficacy of antipsychotic medications to date. It compared several antipsychotic medications and concluded that all antipsychotics compared in Phase 1 are equal in their efficacy and tolerability. It remains unclear however if there are separable patterns of antipsychotic response that may suggest a drug-by-patient effect. Latent variable modeling allows researchers to test for individual differences in developmental trajectories that may underlie psychiatric data. Latent growth curve (LGC) modeling and growth mixture models (GMM) are particularly informative for investigating rate of change in longitudinal data and repeated measures data with several time points. The goal of the presentation is to illustrate the use of latent variable modeling to investigate differential patterns of antipsychotic response in the CATIE trial.

Methods: Participants were 1,460 respondents with schizophrenia drawn from the CATIE trial that completed measures of psychosis, cognition, and psychosocial functioning. Participants were administered the Positive and Negative Syndrome Scale (PANSS), neurocognitive measures, and the Quality of Life Interview at baseline and several follow-up periods. First, we submitted participant scores to Latent Growth Curve Analysis (LGCA) to examine changes in psychotic symptoms, neurocognition, and functioning during the study from baseline to each followup period. Next, we used Latent Class Growth Modeling (LCGM) and Growth Mixture Modeling (GMM) to investigate the presence of sub-populations within the data that may differ in their trajectory of symptom improvement. We tested models with predictors including age, sex, marital status, ethnicity, education, illness duration, CGI severity, and medication type. We evaluated the fit of latent variable models using appropriate fit indices-Comparative Curve Fit Index (CFI), Tucker Lewis Fit Index (TLI), Akaike Information Criteria (AIC), Bayesian Information Criteria (BIC), Sample Size Adjusted BIC (SSA-BIC), Lo-Mendell Rubin Test (LMR) and the Bootstrap Likelihood Ratio Test (BLRT). Results: A linear function proved to be the best model of change for both the PANSS, psychosocial function, and the neurocognition scores when fit indices such as the comparative fit index (CFI) and information criteria are examined. Age, race, years of education, and years of present ill/ treatment were significant predictors of change in positive and negative symptoms, whereas education level and ethnicity were significant predictors of change in neurocognition and functional status during antipsychotic treatment. Next we addressed the question of whether latent subgroups are apparent in change trajectory of outcome measures. In both the LCGM and the GMM, the AIC, BIC, and SSA-BIC best supported the three- class model. Similarly, the LMR and the BLRT also supported the three class model with very low probability that a 1 or 2-class model would have produced the data $(p<0.0001)$.
The information criteria indices were however lower for the 3-class GMM than the 3-class LCGM, suggesting that a three-subgroup pattern of change in which there exists variability within subgroups best explain the pattern of antipsychotic response in PANSS scores.

For the neurocogniton data, the LCGM tended to favor the 4class model when the information criteria and the BLRT are examined. In contrast, the GMM favored a 2-class model when the information criteria, LMR, and the BLRT are examined. The two-class GMM was a better fit to the neurocognition data compared to the four-class LCGM. Mixture modeling of functioning data is pending.

Conclusions: Latent trajectories are apparent in the pattern of response to antipsychotic medications in Phase 1 of the CATIE trial when PANSS and neurocognition scores are considered. Age, race, education, and illness duration but not antipsychotic type predicted treatment response within unveiled subgroups. This is partly consistent with the conclusion drawn by the original CATIE investigators. Latent variable models are adaptable to studying repeated measures data when collected longitudinally at known time points.

Keywords: Growth Mixture Modeling, Treatment Response, Antipsychotic Treatment, Neurocognition.

Disclosure: Nothing to disclose.

\section{W181. Elevated TNFo Levels in Cerebrospinal Fluid of Patients With Schizophrenia}

Juan Gallego*, Christopher Morell, Monika Malhotra, Robert McNamara, Todd Lencz, Anil Malhotra

Weill Cornell Medical College, White Plains, New York, United States

Background: Elevated levels of pro-inflammatory cytokines have provided evidence in support of the inflammatory hypothesis of schizophrenia. Most studies of cytokines in schizophrenia have reported cytokine levels in peripheral blood and some used cerebrospinal fluid (CSF). However, the number of studies investigating cytokines in CSF in schizophrenia is still very small and those studies typically have small sample sizes. Of the cytokines studied, IL-6 and IL- 8 have been most commonly measured and reported but abnormalities in other cytokines, such as $\mathrm{TNF} \alpha$, have not been reported in the CSF in schizophrenia literature. Our aim was to study a panel of cytokines, including $\mathrm{TNF} \alpha$, in cerebrospinal fluid of a decently large sample of patients with schizophrenia and healthy volunteers. In addition, we examined correlations between these cytokines and psychiatric symptoms.

Methods: Thirty-three patients with schizophrenia-spectrum disorders and 23 healthy volunteers underwent a lumbar puncture. 15-25 cc of CSF were obtained from each subject. CSF cytokine (IL-1 $\beta$, IL-2, IL-4, IL-6, IL-8, TNF $\alpha$ ) concentrations were determined in duplicate by enzyme-linked immunosorbent assay (ELISA) and a high-sensitivity MilliplexTM Multiplex kit (HSTCMAG-28SK-06, Millipore, Billerica, MA) per manufacturer's instructions. In patients, psychiatric symptoms were assessed using the Brief Psychiatric Rating Scale - Anchored version (BPRS-A). Comparisons in cytokine levels between groups were performed 
using either t-tests for normally distributed variables or Wilcoxon rank-sum tests for non-normally distributed variables. Correlations were computed using the Pearson correlation coefficient.

Results: The mean age was 36.6 years $(\mathrm{SD}=11.7)$ in patients and 38.1 years $(S D=10.1)$ in controls. $24 / 33(72.3 \%)$ of the patients and $14 / 23(60.1 \%)$ of the healthy volunteers were male. Mean total BPRS score in patients was $28.6(\mathrm{SD}=9.0)$. All patients were on treatment with a variety of firstgeneration and/or second-generation antipsychotic medications. Mean TNF $\alpha$ values were elevated in patients $(6.47 \mathrm{pg} /$ $\mathrm{ml}[\mathrm{SD}=3.1])$ compared to healthy volunteers $(3.76 \mathrm{pg} / \mathrm{ml}$ $[\mathrm{SD}=2.5], p=0.001)$. There were no statistically significant differences in levels between patients and controls in IL-2 ( $\mathrm{mean}=5.46 \mathrm{pg} / \mathrm{ml}[\mathrm{SD}=1.9]$ vs. $4.82 \mathrm{pg} / \mathrm{ml}[\mathrm{SD}=2.0]$, IL-6 (9.31 pg/ml[2.6] vs. $8.9 \mathrm{pg} / \mathrm{ml}[2.9])$ and IL-8 (49.44 pg/ml [11.2] vs. $47.21 \mathrm{pg} / \mathrm{ml}[12.2]$. Levels of IL-1 $\beta$ and IL-4 were not detected in more than $30 \%$ of the CSF samples; therefore these cytokines were not entered in the analysis. Correlational analysis showed that TNF $\alpha$ was significantly correlated with the conceptual disorganization item on the BPRS-A $(\mathrm{r}=0.39, p=0.04)$. No other correlations were statistically significant.

Conclusions: $\mathrm{TNF} \alpha$, a pro-inflammatory cytokine and a key participant in the acute phase response of the inflammatory cascade, is elevated in CSF of patients with schizophrenia providing support to the inflammatory hypothesis in schizophrenia. Future studies should focus not only on IL- 6 but also in TNF $\alpha$ to further understand the role of proinflammatory in schizophrenia.

Keywords: Cerebrospinal Fluid, Cytokines, Schizophrenia. Disclosure: Nothing to disclose.

\section{W182. Amphetamine Enhances Gains in Auditory Discrimination Training in Adult Schizophrenia Patients: Assessing Tolerability and Predictive Biomarkers}

Neal Swerdlow*, Melissa Tarasenko, Savita Bhakta, Jo Talledo, Erica Hughes, Alexis Alvarez, Sophia Vinogradov, Gregory Light

University of California, San Diego, La Jolla, California, United States

Background: Functional disability in schizophrenia (SZ) reflects underlying neurocognitive deficits. Antipsychotics (APs) blunt acute psychosis, but their impact on negative symptoms or neurocognitive deficits are less dramatic. Efforts at remediating cognitive deficits using targeted cognitive training (TCT) generally show efficacy at the group level, yet almost half of all patients demonstrate virtually no cognitive enhancement after prolonged TCT treatment. We proposed pairing drugs with specific cognitive interventions, as a way to enhance SZ patients' ability to benefit from that intervention (Pharmacologically Augmented Cognitive Therapies ("PACTs")), similar to the use of pro-extinction drugs to enhance the response to cognitive therapies in anxiety disorders. We previously reported preliminary evidence from a small sample (9 SZ patients) that the pro-attention drug, d-amphetamine (AMPH, $10 \mathrm{mg}$ po), enhanced performance in AP-medicated SZ patients on a TCT task - Posit Science "Sound Sweeps" - which is a component of a TCT program known to enhance neurocognition in SZ patients. Here, we report results of a more substantive sample $(n=60)$, focusing on the ability of AMPH to enhance TCT learning, as well as evidence for tolerability of AMPH and predictors of AMPH sensitivity in APmedicated SZ patients.

Methods: Carefully screened and characterized HS $(n=35$; $\mathrm{M}: \mathrm{F}=25: 11$; age mean, range $=31.6 \mathrm{y} ; 20-55)$ and patients with a diagnosis of SZ $(n=25 ; \mathrm{M}: \mathrm{F}=13: 12$; age mean, range $=39.2 \mathrm{y} ; 23-55$ ) participated in this study. Subjects were tested three times with 1 week between tests: first in a screening session (no pill administered), and next in a double-blind order-balanced crossover design (test days 2 and 3), comparing PBO vs. $10 \mathrm{mg}$ of AMPH. On each test day, 1 hour of Sound Sweeps training was bracketed by brief (2-4 min) pre- and post-training assessments of auditory processing speed (APS). Training consisted of a speeded auditory time-order judgment task of two successive frequency modulation (FM) sweeps (Posit Science "Sound Sweeps" exercise). On test days 2 and 3, pre-training assessments began 210 min post-pill administration, when $10 \mathrm{mg}$ AMPH is known to be bioactive. Autonomic and subjective measures were collected throughout test days 2 and 3. On test day 1, subjects completed measures of neurocognition (MATRICS Consensus Cognitive Battery) and neurophysiological measures of information processing (event-related potential measures of mismatch negativity and P3a, and prepulse inhibition (PI) of startle).

Results: Baseline (screening and pre-training) performance (APS, trials completed) was impaired in SZ patients vs. HS (all p's $<0.01-0.0001$ ). Auditory system "learning" (APS post- vs. pre-training) in patients was enhanced by $\mathrm{AMPH}$ (main effect of diagnosis: ns; main effect of AMPH: $p<0.002$; diagnosis $\mathrm{x}$ drug interaction: $p<0.03$; main effect of AMPH in patients: $p<0.02$ ). In general, subjective effects of AMPH were mild and more pronounced in HS vs. patients, and included reduced drowsiness (HS) and increased "happiness" (165 min post-pill). Neither HS nor patients correctly identified the active drug at greater than chance levels. Ratings of "anxious" were increased in HS but modestly reduced in patients, particularly non-smokers. No patients reported significant discomfort; reports of even mild discomfort during testing (e.g. "feeling warm") were rare (5 reports out of 50 tests: 3 during placebo tests, 2 during active drug tests). AMPH increased heart rate (HR), systolic and diastolic blood pressure (sBP, dBP) in HS. By contrast, AMPH-induced autonomic activation in patients overall was not statistically significant, though the subgroup of patients with the lowest baseline levels of $\mathrm{HR}$, sBP and $\mathrm{dBP}$ did exhibit activating effects of AMPH. Age, weight, and smoking status did not impact autonomic sensitivity to AMPH in patients. Modest predictors of greater sensitivity to AMPH-enhanced APS learning included higher levels of PPI $(p<0.025)$ and shorter (faster) P3a latency $(p<0.035)$.

Conclusions: While TCT is efficacious for enhancing cognition and function in SZ patients at a group level, individual patient response to this time- and labor-intensive intervention varies considerably. Thus, treatments that augment and accelerate TCT-induced benefits will be of great value. We previously reported preliminary evidence from 9 patients that the pro-attentional drug, $\mathrm{AMPH}$, 
enhances initial APS learning in SZ patients; here we extend this evidence to a sample of 25 patients and 35 HS. That this form of "automatic" learning is enhanced in chronically ill AP-medicated SZ patients provides a "proof of concept" for the PACT approach. We did not successfully identify robust biomarkers predicting sensitivity to initial pro-learning effects of AMPH in SZ patients, though higher levels of PPI and faster P3a latency had modest predictive value. While there may or may not be reasons for caution regarding the use of a low dose of AMPH for a 30-session PACT regimen in AP-medicated, our experience is that $\mathrm{AMPH}$ was very well tolerated in patients - at least as well-, and perhaps better-tolerated than in HS. This evidence is consistent with reports of both single-dose challenges and RCTs of dailyadministered AMPH in AP-medicated SZ patients. These results carry the limitations of a single active-dose, single time-point, cross-over design, and should be followed by studies to identify optimal parameters for AMPH efficacy in this PACT model that can be applied to [longer-term trials/ therapeutic "doses"] of cognitive training effectiveness.

Keywords: Amphetamine, Cognitive Training, Schizophrenia, Biomarkers.

Disclosure: This work was supported by the NIMH R01MH059803 and MH094320; Brain \& Behavior Research Foundation (NARSAD) (GL, SB); Department of Veterans Affairs VISN-22 Mental Illness Research, Education, and Clinical Center (MIRECC) (GL); and Sydney R. Baer, Jr. Foundation.

\section{W183. Accelerated Biological Aging in Schizophrenia?}

Dilip Jeste*, A'verria Martin, Owen Wolkowitz, Barton Palmer

University of California, San Diego, La Jolla, California, United States

Background: Schizophrenia is associated with increased medical comorbidity and a much shorter life-span than the general population. Persons with schizophrenia have a 2 to 12 times higher mortality rate than age-comparable samples without serious mental illnesses. Two thirds of the excess deaths in schizophrenia are from "natural causes" such as cardiac and metabolic disorders. Together these findings suggest that schizophrenia may be associated with accelerated biological aging.

Methods: The protocol was reviewed and approved by the UC San Diego Human Research Protections Program; participants provided a written informed consent. Employing a structured multi-cohort longitudinal design, we developed an age-and gender-stratified cohort of outpatients with schizophrenia or schizoaffective disorder $(n=140)$ and healthy comparison subjects (HCs; $n=120)$. These participants were evaluated clinically using standardized rating scales for psychopathology, physical health, medical comorbidity, medication use, cognitive performance, and everyday functioning. Additionally, fasting blood was examined for a panel of selected biomarkers including systemic measures of inflammatory process (high-sensitivity C-reactive protein or hs-CRP), metabolic dysregulation (Homeostatic Model Assessment of Insulin Resistance; HOMA-IR), and oxidative stress (F2-isoprostanes). These biomarkers were selected because of their known association with biological aging. We assessed differences between schizophrenia and HC groups on clinical measures as well as the specified biomarkers of aging and their correlates.

Results: The present report focuses on baseline data from an ongoing prospective longitudinal study. The schizophrenia and HC groups were similar in mean age (48 years) and gender distribution (50\% women). Mean age of onset of schizophrenia was 23 years, and mean duration of illness was 25 years. The individuals with schizophrenia had, as expected, significantly worse psychopathology, cognitive performance, everyday functioning, and lifestyle (physical activities, sleep, and nutrition) than HCs. Past history of smoking was more common in schizophrenia than in the $\mathrm{HC}$ group ( $76 \%$ vs. $32 \%$ ), and $91 \%$ of the patients were on antipsychotic medications; of these, $81 \%$ were on atypical antipsychotics, $6 \%$ on typical, and $13 \%$ on both. The daily doses were generally in the low to moderate range. In terms of physical health, the schizophrenia group had higher prevalence of obesity than the HCs (55\% vs. $23 \%$ ), as well as higher levels of hypertension (53\% vs. $25 \%$ ), heart disease ( $24 \%$ vs. $6 \%$ ), and diabetes ( $23 \%$ vs. $5 \%$ ). The schizophrenia group also had significantly worse mean values on body mass index (BMI), waist-to-hip ratio, lipid panel, hemoglobin A1C, Framingham cardiovascular disease risk score, and number and severity of comorbid medical illnesses, using standardized published rating scales (all differences with $p<0.001)$. In terms of biomarkers, the schizophrenia group had significantly "worse" values (consistent with older biological age) than HCs on the three biomarkers of focus: hs-CRP, HOMA-IR, and F2-isoprostanes (all with $p<0.001$ ). Inter-correlations among these biomarkers were small $(\mathrm{r}<.3)$ except for that between hs-CRP and HOMA-IR $(r=.37)$. Most of these biomarker levels did not correlate significantly with measures of psychopathology or antipsychotic use. Among physical health measures, the strongest correlations of most biomarkers were with BMI $(r=.3$ to.6) and that of HOMA-IR with Framingham cardiovascular disease risk score $(r=.41)$.

Conclusions: We found abnormally high levels of three biomarkers that are known to be associated with biological aging. This finding is consistent with the hypothesis of accelerated biological aging in schizophrenia, but is limited by cross-sectional nature of the data available to this point. Keywords: Schizophrenia, Aging, Inflammation.

Disclosure: Nothing to disclose.

\section{W184. Peripheral Glucose Metabolism and Brain Bioenergetics Measured by 31P Magnetic Resonance Spectroscopy in Unaffected Siblings of Patients With Psychotic Disorders}

Virginie Anne Chouinard*, Fei Du, Sang-Young Kim, Chiara Dalla Man, Claudio Cobelli, Aaron Cypess, Linda Valeri, Kyle Ryan, Guy Chouinard, David C. Henderson, Bruce M. Cohen, Dost Ongur

Harvard Medical School, Belmont, Massachusetts, United States

Background: Increasing evidence suggests abnormal bioenergetic status both in brain and periphery in psychotic 
disorders. Patients with psychotic disorders are at high risk for glucose metabolism abnormalities, including type 2 diabetes, while there is converging evidence for impaired brain energy metabolism in psychotic disorders. The present study examined peripheral glucose metabolism and aspects of brain bioenergetics in unaffected siblings of patients with first episode psychosis, compared to patients and healthy individuals.

Methods: 22 unaffected siblings, 17 first episode psychosis patients and 19 healthy unrelated controls were evaluated using an oral glucose tolerance test, with hemoglobin A1c measurement. Insulin sensitivity was estimated using the oral minimal model method. Brain $31 \mathrm{P}$ magnetic resonance spectroscopy magnetization transfer (31 P-MT-MRS) was performed in all subjects. Brain parenchymal $\mathrm{pH}$ and measures of oxidative state (ratio of nicotinamide adenine dinucleotide $(\mathrm{NAD}+)$ and its reduced form $(\mathrm{NADH})$ ) were estimated from $31 \mathrm{P}-\mathrm{MT}-\mathrm{MRS}$ in the frontal lobe. $31 \mathrm{P}-\mathrm{MT}$-MRS acquisitions were obtained with a $4 \mathrm{~T}$ wholebody scanner interfaced with a Varian INOVA console, using a custom-designed prefrontal surface coil tuned to the $31 \mathrm{P}$ resonance frequency for collection of $31 \mathrm{P}-\mathrm{MT}$ MRS data.

Results: The mean (SD) of body mass index $(\mathrm{kg} / \mathrm{m} 2)$ was 25.8 (3.6) for patients, 24.1 (3.3) for siblings and 22.5 (3.2) for controls $(\mathrm{F}=4.32, p=0.02)$. Insulin sensitivity significantly differed among groups $(\mathrm{F}=3.40, p=0.04)$ (patients $<$ siblings $<$ controls) with differences between patients and controls $(p=0.02)$, and siblings and controls $(p=0.05)$. Correlation coefficient between insulin sensitivity and NAD $+/ \mathrm{NADH}$ approached, but did not attain statistical significance in controls $(r=-0.47, p=0.10)$, and there were no significant associations between insulin sensitivity and NAD $+/ \mathrm{NADH}$ in patients or siblings. Correlation coefficients between hemoglobin $\mathrm{Alc}$ and $\mathrm{NAD}+\mathrm{NADH}$ approached, but did not attain statistical significance in patients $(r=0.42$, $p=0.09)$ and siblings $(r=-0.40, p=0.10)$, and were significant in controls $(r=-0.48, p=0.04)$.

Conclusions: Patients and unaffected, untreated, siblings showed decreased insulin sensitivity compared to controls, suggesting abnormal glucose metabolism or a primary insulin signaling pathway abnormality related to risk and expression of psychosis. Furthermore, bioenergetic measures suggest possible dynamic relationships between peripheral glucose metabolism and specific bioenergetic markers in the brain.

Keywords: Psychotic Disorders, Unaffected Relatives, Glucose Metabolism, $31 \mathrm{P}$ Magnetic Resonance Spectroscopy, Bioenergetics.

Disclosure: Nothing to disclose.

\section{W185. Dysregulation of Markers of White Matter Integrity in the Postmortem Cingulum Bundle in Schizophrenia: Relation to Treatment Status}

\section{Rosalinda Roberts*, Kirsten Schoonover}

University of Alabama, Birmingham, Alabama, United States

Background: White matter in the brain is comprised of axonal projections between brain regions and forms the basis for connectivity in the brain. People with schizophrenia exhibit faulty synchronization in neural activity and abnormal connectivity termed "dysconnectivity" in many white matter tracts. The mechanisms behind white matter integrity deficits could be due to many things including abnormalities in myelin and/or cytoskeletal proteins.

Methods: We report here on the cingulum bundle (CB), a major white matter fasciculus that connects several gray matter components of the limbic cortex. This study used Western blot analysis to compare protein levels of myelin basic protein (MBP), neurofilament heavy chain (NEFH), the autophagosome marker LC3, and the microtubule marker $\alpha$ tubulin in postmortem human CB in schizophrenia subjects $(n=16)$ and matched controls $(n=13)$. Additionally, the schizophrenia group was subdivided by treatment status: off$(n=8)$ or on-medication $(n=8)$. The control and schizophrenia groups were equally matched for age (49 vs. $46 \mathrm{yrs}$ ), race $(3 \mathrm{AA} / 10 \mathrm{C}$ vs. $7 \mathrm{AA} / 9 \mathrm{C})$, sex ( $3 \mathrm{~F} / 10 \mathrm{M}$ vs $4 \mathrm{~F} / 12 \mathrm{M})$, postmortem interval (16.7 vs. $17.6 \mathrm{hrs})$ and $\mathrm{pH}(6.51$ vs. 6.81).

Results: There were no significant differences between age, race, sex, PMI or $\mathrm{pH}$ between controls and patients. The combined schizophrenia group did not exhibit any significant changes in any of the measured proteins. However, correlational analyses revealed a strong negative relationship between $\alpha$-tubulin and MBP $(r=-0.905, p=0.00002)$ and $\alpha$ tubulin and LC3 $(r=-0.680, p=0.011)$ in controls. This correlation was absent in schizophrenia subjects $(r=0.474$, $p=0.064$ and $r=0.094, p=0.729$, respectively), and significantly different from controls as revealed by coefficient comparison (CC: $p<0.0003$ and $p=0.028$, respectively). No correlation was observed between $\mathrm{NFH}$ and $\alpha$-tubulin in control subjects $(r=0.248, p=0.415)$; however, a negative correlation was observed in schizophrenia subjects $(r=-$ 0.556, $p=0.025$ ) that was significantly different from controls as revealed by coefficient comparison (CC: $p=0.037$ ).

When dividing the schizophrenia cohort by treatment status, $\alpha$-tubulin levels were different across groups (ANOVA, $p=0.042$ ); posthoc analysis with LSD tests revealed elevation of $\alpha$-tubulin protein levels in off-medication subjects compared to their medicated counterparts (an increase of $17.4 \%, p=0.018$ ) and to controls (an increase of $12.8 \%$, $p=0.042$ ). Protein levels of $\alpha$-tubulin were similar between controls and medicated patients. Correlation analysis revealed no relationship between $\alpha$-tubulin and MBP or $\alpha$ tubulin and LC3 in on-medication subjects $(r=0.159$, $p=0.707$ and $\mathrm{r}=0.347, p=0.399$, respectively) or offmedication subjects $(r=0.561, p=0.148$ and $r=0.409$, $p=0.314$, respectively). No correlation was observed between $\mathrm{NFH}$ and $\alpha$-tubulin in control subjects $(\mathrm{r}=0.248, p=0.415)$; however, a negative correlation was observed in onmedication schizophrenia subjects $(r=-0.825, p=0.012)$ that was significantly different from controls as revealed by coefficient comparison (CC: $p=0.009$ ).

Conclusions: In controls LC3 and MBP are both negatively correlated with $\alpha$-tubulin. This relationship is absent in the schizophrenia group as a whole and in the subgroups divided by treatment status. NEFH is not correlated with $\alpha$-tubulin in controls but is negatively correlated in the schizophrenia group; this pattern is driven by the treated subset of patients, perhaps suggesting a medication effect. These data suggest significant dysregulation of the relationship between $\alpha$ - 
tubulin and the observed markers of white matter integrity in schizophrenia and with relation to treatment status.

Keywords: Psychosis, Axons, Myelin, Antipsychotic Medication.

Disclosure: Nothing to disclose.

\section{W186. Relationship Between Executive Function and Frontoparietal Structural and Functional Connectivity in Early Psychosis: An MRI Connectome Study}

Susan Conroy*, Tom Hummer, Michael Francis, Matthew Yung, Joaquin Goni, Alan Breier

Indiana University School of Medicine, Indianapolis, Indiana, United States

Background: Structural and functional brain connectivity derangements are thought to contribute to the neurocognitive symptoms of psychotic disorders, including executive dysfunction. The process by which brain alterations may develop in early psychosis, and their relationship to cognition, is poorly understood. Individual whole-brain connectome modeling based on imaging methods could elucidate the mechanisms by which this occurs. This study utilized structural and functional connectomic measures, focusing on the frontoparietal network, which is known to subserve executive function.

Methods: 43 patients with early psychosis, within 3 years of first presentation (mean age 23.1, 84\% male), and 32 control participants (mean age 23.4; 81\% male) underwent 3 T MRI, including diffusion weighted imaging (DWI) to model individual structural connectivity, and resting state fMRI to model functional connectivity. Fractional anisotropy (FA), a measure of white matter integrity, and fMRI temporal correlations were used to compare frontoparietal network properties. Cognitive assessment utilized the Brief Assessment of Cognition in Schizophrenia (BACS), focusing on the Tower of London task which measures executive function. Results: As expected, participants with early psychosis had lower scores in both global cognition (BACS T-score 27.1 \pm 14.6 vs. $48.3 \pm 9.9$ for controls; $p<0.001)$ and executive function scores (Tower of London T-score $41.4 \pm 15.2$ vs. $51.5 \pm 9.4$ for controls; $p=0.001)$. Frontoparietal withinnetwork structural and functional connectivity measures did not differ between groups. Frontoparietal within-network structural, but not functional, connectivity was significantly correlated with executive function, but not overall cognitive scores, in both patients $(r=0.358 ; p=0.018)$ and controls $(r=0.480 ; p=0.005)$.

Conclusions: Structural, but not functional, connectivity measures within the frontoparietal network are associated with the lower executive function observed in patients with early psychosis. This cognition-connectivity relationship in this network is specific to the executive function domain. Future longitudinal analyses may shed light on the trajectory of this relationship as illness progresses.

Keywords: Connectome, Early Psychosis, Executive Function, Human Neuroimaging.

Disclosure: Nothing to disclose.
W187. Evidence for Shared Genetic Risk Between Schizophrenia and Smoking Behaviors: Findings From the Psychiatric Genomics Consortium

Roseann Peterson, Tim Bigdeli, Stephan Ripke, Silviu-Alin Bacanu, Pablo Gejman, Douglas Levinson, Dan Rujescu, Marcella Rietschel, Daniel Weinberger, Qingqin Li, Richard Straub, James Walters, Michael O'Donovan, Brian Mowry, Roel Ophoff, Ole Andreassen, Tonu Esko, Tracey Petryshen, Kenneth Kendler, Psychiatric Genomics Consortium, Ayman Fanous*

SUNY Downstate Medical Center, Brooklyn, New York, United States

Background: Currently, $17 \%$ of US adults and upwards of $60 \%$ of those with schizophrenia (SCZ) spectrum disorders smoke tobacco regularly, and several lines of evidence support a shared etiological basis for smoking and SCZ. Notably, the nicotinic acetylcholine receptor gene cluster that has been shown to influence heaviness of smoking in the general population was also reported as one of the 108 SCZ-risk loci identified in the Psychiatric Genomics Consortium (PGC) study of SCZ.

Methods: We investigated the genetic relationship between SCZ and smoking using polygenic risk scores constructed from results from the Tobacco and Genetics (TAG) meta-analyses of genome-wide association studies (GWAS) of smoking behaviors. The availability of subject-level smoking data in the PGC-SCZ facilitated analyses of smoking initiation (SI) and cigarettes-per-day (CPD), including association with TAGbased scores, and exploratory SCZ case-only GWAS.

Results: Phenotypic associations between SCZ symptom factors and smoking behavior were examined and the positive symptom factor score showed a significant positive association with SI $(P=3.21 \times 10-5)$. Polygenic scores based on TAG results for SI significantly predicted SCZ case status in the full PGC cohort (R2 $=0.0015, P=8.11 \times 10-15)$, as did scores based on results for CPD $(\mathrm{R} 2=0.0005, P=4.18 \times 10$ $6)$. These scores also significantly predicted SI $(R 2=0.0047$, $P=6.29 \times 10-5)$ and $\mathrm{CPD}(\mathrm{R} 2=0.0007, P=0.0067)$, respectively, among SCZ patients. In the replication phase of the cases-only GWAS of SI, we identified a genome-wide significant association upstream of TMEM106B on chromosome 7 ( $r$ 148253479; $P=3.18 \times 10-8$ ).

Conclusions: We provide evidence of a partially shared genetic basis for SCZ and smoking behaviors. Preliminary case-only results highlight novel SCZ specific genetic liability for smoking quantity. Future research needs to address mechanisms underlying the associations between these traits to aid both SCZ and smoking treatment and prevention efforts.

Keywords: Smoking, Schizophrenia, Genome-Wide Association.

Disclosure: Nothing to disclose. 
W188. Evaluation of miRNA and isomiRNA Expression in Schizophrenia Using Small RNA Sequencing of Postomortem Dorsolateral Prefrontal Cortex Brain Tissue

Carrie Wright*, Joo Heon Shin, Anindita Rajpurohit, Courtney Williams, Andrew Jaffe, Nicholas Brandon, Thomas M. Hyde, Joel E. Kleinman, Alan Cross, Daniel R. Weinberger

Lieber Institute for Brain Development, Baltimore, Maryland, United States

Background: MicroRNAs are small regulatory RNAs that individually modulate the expression of many genes. miRNAs participate in the regulation of nearly every biological process and are highly implicated in a wide variety of disease. IsomiRNA (IsomiRs) are miRNAs with slight variation in length and sequence from that of the canonically described microRNAs, due to altered miRNA biogenesis, genetic variants, posttranscriptional editing, and exonuclease degradation. Such changes in the sequence of miRNAs, can lead to drastic functional alterations by shifting the repertoire of binding partners, therefore leading to potentially significant downstream consequences. These slightly altered miRNAs are now known to be functional and associated with various disease states, however characterization remains limited. Alterations in miRNA expression and the expression and function of miRNA biogenesis related enzymes has previously been identified in schizophrenia. To explore whether the magnitude or diversity of isomiR production is altered in schizophrenia, we evaluated miRNA and isomiRNA expression in postmortem brain tissue of cases and healthy controls. This is to our knowledge, the first characterization of isomiRNA expression in schizophrenia. This work was performed as part of the AstraZeneca Postdoc Program training for Carrie Wright.

Methods: We evaluated RNA sequencing data of postmortem dorsolateral prefrontal cortex (DLPFC) samples from 10 subjects ( 5 subjects with schizophrenia and 5 control subjects matched for age, gender, ethnicity, and RIN) using the NextFlex small RNA sequencing library preparation, with 50ng of starting total RNA. Fifty base pair single-end sequencing was run on the HiSeq 2000, using the Illumina Real Time Analysis (RTA) module to perform image analysis and base calling, and the BCL Converter (CASAVA v1.8.2) to generate the sequence reads. Sequencing depth was over 1-2 million reads per sample. Bowtie in combination with miRanalyzer and Isomirage were used to align the reads to known miRNAs and to known isomiRs, respectively. The influence of diagnosis status on miRNA and isomiR abundance was then evaluated using a linear model regression covarying for principal components to capture latent sources of variation. Abundance estimates were normalized using log2 (Reads Per Kilobase of transcript per Million mapped reads (to either all miRNAs or all known isomiRNAs) (RPKM)+1) and filtered to include only those with expression values greater than 1. Multiple testing correction was performed using the Benjamini Hochberg method. The expression of individual miRNAs and isomiRs, as well as the overall magnitude and diversity of isomiR expression was then evaluated for group differences between cases and controls.
Results: Following multiple testing correction, no miRNAs were found to be significantly differentially expressed between cases and controls (18 were differentially expressed before correction). Additionally, no individual isomiRs were found to be differentially expressed (84 were differentially expressed before correction). Finally, no differences were found when evaluating the overall magnitude or diversity of isomiR expression.

Conclusions: This preliminary study suggests that despite the association of altered miRNA biogenesis in schizophrenia, no significant alterations in isomiR expression was detected. However, our sample size was very small and limited in power. We plan to further analyze miRNA and isomiR expression using a total of 100 schizophrenia subjects and 100 control subjects. This subsequent analysis should be adequately powered to evaluate global differences in isomiR expression across the diagnosis groups. We also hope to access an independent sample for replication purposes. Our results will further clarify the role of miRNA and isomiR expression in schizophrenia.

Keywords: Schizophrenia, isomiRNA, miRNA, RNA Sequencing.

Disclosure: There are relevant financial interests to disclose. Poster Disclosure AstraZeneca, Postdoc Program, Self; AstraZeneca, Employee, Alan Cross, AstraZeneca, Employee, Nicholas Brandon.

\section{W189. Single-Dose Memantine Normalizes Evoked Gamma Power and Phase Synchronization Deficits in Patients With Schizophrenia}

Gregory Light*, Wen Zhang, Yash Joshi, Savita Bhakta, Jo Talledo, Neal Swerdlow

University of California, San Diego, La Jolla, California, United States

Background: Patients with schizophrenia (SZ) have disruptions in NMDA receptor-mediated oscillations in the gamma frequency band $(30-80 \mathrm{~Hz})$, which are thought to underlie a broad range of cognitive processes. Evoked power and phase synchronization of gamma frequency oscillations in response to auditory steady state stimulation (ASSR) are translational biomarkers of NMDA receptor functioning that are associated with cognitive functioning with promising applications for screening early sensitivity to procognitive therapeutics. We previously reported that a single dose of memantine, a low-to-moderate affinity NMDA receptor antagonist that is FDA-approved to treat Alzheimer's disease, enhanced prepulse inhibition of startle and mismatch negativity in SZ patients. Here, we describe the effects of a single dose of memantine on evoked power and phase synchronization of ASSR obtained in our previous study.

Methods: SZ patients $(n=18 ; \mathrm{M}: \mathrm{F}=11: 7$; mean age $=37.7 \mathrm{y}$; range:21-48) and healthy comparison subjects $(n=14$; M: $\mathrm{F}=10: 4$; age $=26.6 \mathrm{y}$; range:19-36) completed two test days separated by one week. On each test day, subjects received either memantine $20 \mathrm{mg}$ (p.o.) or placebo in a double blind, randomized, counterbalanced, within-subject cross-over design. The ASSR paradigm was used to measure evoked gamma power and phase locking in response to $40 \mathrm{~Hz}$ click trains. 
Results: Patients with SZ had reduced evoked gamma power and phase locking, consistent with previous reports (main effect of group - Power: $F=5.89$, df 1,30, $p<0.025$; Phase locking: $\mathrm{F}=12.47$, df $1,30, p<0.0015)$. Memantine enhanced evoked power and phase locking in both SZ and healthy comparison subjects (drug effect - Power: $F=6.40$, df 1,30, $p<0.02$; Phase locking: $\mathrm{F}=8.49$, df $1,30, p<0.007)$. Memantine effects on both gamma power and phase locking were also significant when groups were matched for age (p's $<$ 0.005 and 0.0008 , respectively). Memantine-associated enhancement of gamma power and phase locking in SZ patients correlated negatively with age ( $p$ 's $<0.0015$ and 0.005, respectively). Significant relationships were also identified in patients between memantine effects on gamma power and phase locking $(r=0.74, p<0.0005)$, gamma power and mismatch negativity $(\mathrm{r}=0.54, p<0.025)$, and phase locking and prepulse inhibition of startle $(r=-0.59, p<0.027)$.

Conclusions: Single-dose memantine exposure normalizes deficits in cortical oscillatory dynamics known to be associated with cognitive dysfunction in SZ. Ongoing studies will determine whether these acute memantine-induced changes predict enduring pro-cognitive or otherwise clinically beneficial effects in SZ patients. These results support the use of gamma band ASSR parameters as translational pharmacodynamic endpoints in early-stage clinical trials.

Keywords: Gamma Oscillation; Clinical Symptom Type and Severity, Memantine, Cognitive Enhancement, NMDA Receptors, MMN, ERP, Auditory, Training.

Disclosure: Nothing to disclose.

\section{W190. Computational Psychiatry V. the Generalized Deficit: Probabilistic Reversal Learning Impairments in Psychosis are not Mechanistically Specific}

Angus MacDonald*, Edward Patzelt, Zeb KurthNelson, Deanna Barch, Cameron Carter, James Gold, John Ragland, Steven Silverstein

\section{University of Minnesota, Minneapolis, Minnesota,} United States

Background: Probabilistic reversal learning (pRL, Cools et al., 2002) paradigms have been used extensively in patients and in animal models to measure reward learning and, in particular, the construct of rule generation and selection. The negative symptoms of psychosis may reflect the kind of maladaptive reward learning that is measured by such tasks (Gold, Waltz, Prentice, Morris, \& Heerey, 2008). Recent studies of $\mathrm{pRL}$ in people with schizophrenia show impaired performance, but this impairment does not appear to be specific to reversal of the reward contingency and little work has explored whether this impairment is observed across psychotic diagnoses. Meanwhile, computational learning algorithms have become increasingly sensitive to patterns of responding during such learning tasks, allowing the examination individual rule selection and learning parameters (Sutton \& Barto, 1998). The current study was designed to evaluate how these parameter estimates differed across people with schizophrenia (SZ), schizoaffective disorder (SA) and bipolar disorder (BP), and to examine their relationship to symptom factors measured across these disorders.
Methods: As part of a cognitive battery in the multisite CNTRaCS consortium, 225 participants completed the pRL. Among these, SZ $(n=65)$, SA $(n=54), \mathrm{BP} \quad(n=50)$ and controls $(n=56)$ were demographically similar in terms of sex, age, racial and ethnic composition. In the pRL, participants selected one of two abstract images using a button press, after which they were told their choice was either correct or not. The task was probabilistic because one of the items was positively reinforced $80 \%$ of the time (and $20 \%$ of the time they were falsely told it was wrong), whereas the alternative item had the opposite contingency. The task involved reversal learning because after the participant met the initial acquisition criterion of selecting the positively reinforced item on 10 trials, the reinforcement contingencies changed in favor of the alternative item. The current version of the task was modified so that after initial acquisition the likelihood of false feedback dropped to $10 \%$. Each of three blocks had a different item pair and ended after the 10-trial criterion was met for the initial acquisition and two subsequent reversals. In addition to performance metrics, we compared computational models of individual decisions using up to four parameters, including rule selection, exploration, attentional lapses, and response bias.

Results: In terms of performance metrics, a group $\mathrm{x}$ stage interaction occurred because all psychosis patient groups were more impaired for the initial acquisition than controls. Among the $96 \%$ of all participants who completed the acquisition stage, there were no group differences in trials to obtain subsequent criteria, nor were there group differences in the number of perseverations or false-feedback switches. Patients' difficulty on the task derived from an excess of win-switch trials, or spontaneous switches (Kruskal-Wallis $\chi 2(3)=12.71$, $p<0.001$, SZ, SA, BP $>$ C). While the number of trials to completion was unrelated to symptoms as measured by the Brief Psychiatric Rating Scale (BPRS), the number of spontaneous switches was positively correlated with the BPRS total score (rho $=.20, p=.017$ ), but not symptom factor scores. In terms of computational modeling, 32 participants were removed (12 SZ, $8 \mathrm{SA}, 8 \mathrm{BP}, 4$ controls, group differences ns) because models did not predict their choices better than a null distribution. The two-parameter model, including rule selection and exploration, fit the remaining 193 participants' data most parsimoniously. While none of the psychosis groups differed from controls in rule selection, all psychosis groups differed from controls for exploration and did not differ from each other. The exploration parameter correlated with negative and positive symptom factor scores, and with the BPRS total score (rho $=.24, p=.005)$. To understand the nature of this exploration, we then divided win-switch trials based on whether the motor response was repeated or changed (e.g. left-left v. left-right). Most of the difference in spontaneous switches was associated with motor repetition responses $\left(\mathrm{K}-\mathrm{W} \chi 2(3)=8.53, p=0.037^{\star}\right)$, and there was no evidence of greater exploratory activity in psychosis patients when adjusting spontaneous switches for motor repetition responses $(\mathrm{K}-\mathrm{W} \chi 2(3)=1.01, p=0.8)$.

Conclusions: Consistent with previous research, we found patients with schizophrenia to be impaired on pRL. This impairment was not specific to schizophrenia and predominated in all patients in the initial acquisition stage. A computational model of rule selection and exploration predicted participant performance and showed patients' rule 
selection capacity was unimpaired whereas they appeared to explore reward contingencies more than controls. However, this behavioral anomaly appeared to be largely due to patients' tendency to repeat the previous motor response, distinct from the attentional lapse parameter. Motor repetition is rarely included in computational analyses and its source is underspecified. These findings provide an important caution about the impact of generalized performance deficits on computational models of learning developed in healthy populations: the interpretation of such parameters can be grossly misleading.

Keywords: Reversal Learning, Reward-Based Decision-Making, Computational Psychiatry, Computational Modeling. Disclosure: Nothing to disclose.

\section{W191. Impaired Visual Cortical Processing of Affective} Facial Information in Schizophrenia

\section{Yue Chen*, Steve Maher, Tor Ekstrom}

Harvard Medical School, Belmont, Massachusetts, United States

Background: Facial emotion perception impairment in schizophrenia is currently viewed as abnormal affective processing. Facial emotion perception also relies on visual processing. Yet, visual cortical processing of facial emotion is not well understood in this disorder.

Methods: We measured perceptual thresholds for detecting facial fear and happiness in patients $(n=23)$ and controls $(n=23)$, and adjusted emotion intensity of facial stimuli (via morphing between images of neutral and emotive expressions) for each subject. We then evaluated activations of the visual cortex and amygdala during the performance of perceptually-equated facial emotion detection tasks.

Results: Patients had significantly lower fear- and happinessinduced activations in the visual cortex and amygdala. Activations between the visual cortex and amygdala were largely correlated, but the correlations in patients occurred abnormally early in response time course during fear perception.

Conclusions: In schizophrenia, visual processing of facial emotion is deficient and visual and affective processing of negative facial emotion may be prematurely associated.

Keywords: Automated Computational Language Speech Faces, Emotion Perception, Schizophrenia.

Disclosure: Nothing to disclose.

\section{W192. MIN-101 Improves Sleep in Patients Suffering From Schizophrenia: A Randomized, Placebo- Controlled, Double Blind Study}

Jean-Yves Schaffhauser, Nadine Noel, Corinne Staner, Antoine Viola, Jay Saoud, Michael Davidson, David Kupfer*, Remy Luthringer

University of Pittsburgh School of Medicine, Park City, Utah, United States

Background: Schizophrenia is associated with abnormalities in circadian rhythms and sleep (particularly insomnia symptoms). Several recent studies point to strong links between circadian rhythms, dopamine dysregulation and psychosis, suggesting that improvement of circadian disturbance may be a useful therapeutic target in ameliorating symptoms of patients suffering from schizophrenia.

MIN-101 is a novel cyclic amido derivative, which is being developed to address unmet medical needs in schizophrenia, particularly negative symptoms. In vivo functional studies have established that MIN-101 is an antagonist at both 5HT2 A and sigma2 receptors.

The results reported here are based on objective sleep assessment using polysomnographic recordings carried out after 2 weeks of treatment with MIN-101 in schizophrenic patients suffering from an acute psychotic relapse.

Methods: This was a multi-center, inpatient and ambulatory, Phase 2A, double-blind, randomized, placebo-controlled, proof of concept study of MIN-101 in 96 patients with DSM-IV diagnosis of schizophrenia, and had a score of at least 60 on the Positive and Negative Syndrome Scale (PANSS) total score.

Patients were randomly assigned to receive MIN-101 or placebo in a ratio of 1:1. After a washout of previous treatments followed by a placebo run-in period of 3 days, patients received either MIN-101 $32 \mathrm{mg}$ BID or placebo for a treatment duration of 3 months. Over the first 6 days of treatment MIN-101 was titrated up to $32 \mathrm{mg}$ BID for the duration of the study.

Polysomnographic recordings were carried out at baseline and after two weeks of treatment in a sub-set of patients.

Results: After two weeks of treatment, MIN-101 showed effects on sleep characterized by improvements in sleep induction, sleep maintenance and Slow Wave Sleep (SWS) ultradian and circadian distribution. Compared to placebo, MIN-101 shortened the duration of sleep latency, and normalizing the SWS ultradian distribution over the night with a significant increase $(p \leq 0.024)$ observed during the first third of the night and a significant decrease $(p \leq 0.019)$ during the last third of the night.

Conclusions: This phase $2 \mathrm{~A}$ trial demonstrated that MIN-101 is a potent psychotropic compound with earlyonset positive functional effects that include sleep induction and shift in SWS ultradian distribution during the night, which are two key sleep parameters that are disturbed in schizophrenic patients. These effects on sleep parameters may contribute to clinical improvements in psychotic symptoms.

Keywords: Sleep Disturbance, Schizophrenia, Randomized Trial.

Disclosure: Minerva Neurosciences: Director, Self.

\section{W193. Alterations of Regulatory RNA in the Dorsolateral Prefrontal Cortex Contribute to Risk for Schizophrenia}

Mads Hauberg, Claudia Giambartolomei, Ariella Cohain, John Fullard, Panos Roussos*

Icahn School of Medicine at Mount Sinai, New York, New York, United States

Background: The most recent Psychiatric Genomic Consortium GWAS in schizophrenia (SCZ) reported $>100$ susceptibility loci, which are predominantly found in non- 
coding, cis regulatory regions. Functional understanding of non-coding disease-associated loci is an important next step towards the development of testable hypotheses regarding biological processes that may be involved in the pathogenesis of SCZ. We have developed the CommonMind consortium to generate and analyze molecular data from human postmortem brain samples including RNA sequencing and epigenome data. Conventionally, RNA sequencing data is used to quantify gene expression. However, transcription also takes place at many non-coding, regulatory sequences. In this study, we used this phenomenon to examine how disease and genetic variants alter enhancer function. More specifically, we applied RNA sequencing in combination with enhancer identification by the assay for transposaseaccessible chromatin with sequencing (ATAC-seq) and cap analysis of gene expression (CAGE) to postmortem brain samples in order to examine how disease and genetic variants alter enhancer function.

Methods: Enhancer sequences were predicted using cell typespecific (neuronal and non-neuronal) ATAC-seq profiles in human postmortem brain tissue and CAGE data from the FANTOM5 consortium. High-density quantitative trait loci (QTL), differential expression and coexpression network analysis for Ensembl and regulatory transcripts was conducted in 537 human post-mortem samples (258 SCZ samples and 279 controls) from the dorsolateral prefrontal cortex (DLPFC, BA9/46) as part of the CommonMind Consortium (CMC, www.synapse.org/cmc). After QC steps, 1,387 regulatory and 21,312 Ensembl transcripts were robustly expressed and subsequently used for downstream analysis.

Results: Differential expression was detected with 112 regulatory and 1,617 Ensembl transcripts in the DLPFC at an FDR of 5\%. Significant regulatory and Ensembl transcripts in the same locus ( $100 \mathrm{~kb}$ window) change in the same direction in SCZ (sign test: $p=8 \times 10-7$ ). Gene coexpression analysis identified a neuronal subnetwork of 23 regulatory and 727 Ensembl transcripts subserving functions related to synaptic transmission in the DLPFC that is significantly perturbed in SCZ and is highly enriched for SCZ genetic signal. QTL analysis detected 928 regulatory and 15,642 Ensembl transcripts with significant eQTLs at FDR 5\%. Conditional interference analysis identifies multiple Ensembl transcripts coordinated by regulatory RNAs. Colocalization analysis with SCZ GWAS risk variants identified 1 regulatory and 18 Ensembl transcripts that were significant after Bonferroni correction.

Conclusions: The analysis presented here has two fundamental goals, to describe differences in gene expression of regulatory RNA and the mechanisms that underlie genetic risk. Our findings point to a functional link between SCZ susceptibility loci and regulation of gene expression affecting transcripts clustered in specific subnetworks.

Keywords: Postmortem Brain Tissue, Next Generation Sequencing, Gene Expression, Neuronal Epigenome.

Disclosure: Nothing to disclose.
W194. Lack of Imaging Evidence for Impaired Internalization in Schizophrenia

Jodi Weinstein*, Elsmarieke van de Giessen, Rachel Rosengard, Najate Ojeil, Xiaoyan Xu, Roberto Gil, Lawrence Kegeles, Mark Slifstein, Anissa Abi-Dargham

\section{Columbia University, New York, New York, United States}

Background: We have previously shown that PET imaging of dopamine-D2 receptor (D2) radiotracers with psychostimulant challenge can be used to probe magnitude of D2 internalization due to agonist exposure, based on imaging the "late" phase of the displacement of D2 radiotracer binding when the dopamine (DA) pulse has subsided and the continued reduced radiotracer binding reflects receptor trafficking. In parallel, recent genetic, molecular and postmortem studies suggest that D2 trafficking may be impaired in patients with schizophrenia (SZ). Here we used the "late" phase imaging post challenge paradigm to test the hypothesis that D2 trafficking is impaired in SZ. We used PET imaging in SZ and matched healthy controls (HC). We hypothesized that SZ will show blunted late phase displacement compared to HC.

Methods: We imaged $10 \mathrm{SZ}$ and $9 \mathrm{HC}$ with PET and [11C] raclopride at baseline and at 2 time points $(3 \mathrm{hr}$ and $5 \mathrm{hr}$ or $5 \mathrm{hr}$ and $8-10 \mathrm{hr})$ after amphetamine challenge $(0.5 \mathrm{mg} / \mathrm{kg}$ $\mathrm{PO})$. For each scan, time activity curves for the striatum and each of its subregions were fitted using a simplified reference tissue model with cerebellum as reference to determine the binding potential relative to the non-displaceable compartment (BPND) and the percent reduction of BPND from baseline $(\triangle \mathrm{BPND})$ in each region of interest (ROI) at each time point after amphetamine. To test the hypothesis that the time course of return of BPND to baseline after DA surge differed between SZ and HC, we implemented a linear model for each ROI with $\triangle B P N D$ as dependent variable, time postamphetamine as repeated measure, and time postamphetamine and diagnostic group as fixed effects.

Results: The model did not show significant effects of diagnostic group or interaction of diagnostic group * time post-amphetamine on whole striatum $\triangle \mathrm{BPND}(\mathrm{F}=1.38$, $p=0.26$ and $\mathrm{F}=0.51, p=0.61$, respectively; similar results for striatal subregions). Time post-amphetamine significantly affected $\triangle B P N D$ in the striatum $(\mathrm{F}=3.58, p=0.05)$, especially sensorimotor striatum $(F=9.33, p=0.002)$, consistent with return of binding potential to baseline in both diagnostic groups.

Conclusions: We observed a similar pattern of return of binding potential to baseline as a function of time in SZ and HC. This study suggests that striatal D2 trafficking is not abnormal in SZ, at least within the limitations on this measure imposed by our imaging paradigm.

Keywords: Dopamine (D2, D3) Receptors, Schizophrenia, Dopamine, Antipsychotics, PET Imaging, Striatum, Receptor Internalization, Trafficking.

Disclosure: Nothing to disclose. 
W195. Comt (Val/Met) Genotype Effects on Gene Expression and Imaging Phenotypes in the Context of Polygenic Risk for Schizophrenia

Eugenia Radulescu*, Gianluca Ursini, Andrew Jaffe, Qiang Chen, Joo Heon Shin, Karen F. Berman, Joseph Callicott, Venkata Mattay, Daniel R. Weinberger

Lieber Institute for Brain Development, Baltimore, Maryland, United States

Background: Previous studies with candidate genes for schizophrenia produced conflicting results. Likewise, with few exceptions, most of the candidate genes failed to show association with schizophrenia in genome-wide association studies (GWAS). A number of possible explanations have been proffered, including biological factors like the polygenic nature of the illness, genetic epistasis, diverse environmental influences as well as the insinuation that the findings are false. Catechol-O-Methyl Transferase (COMT) is one of the classical candidate genes that demonstrated variable results in previous studies. Therefore, we selected it as an illustrative example to explore the importance of genetic background when studying the individual contribution of genes to risk for schizophrenia. In the current study, the associations of the functional COMT genotype, rs4680 (Val/Met), was examined in relation to the polygenic risk for schizophrenia measured as genetic risk profile score (RPS) based on the 108 PGC loci reported in the latest GWAS (Ripke et al 2014). The study hypothesis was that effects of COMT Val/Met genotype on two dorso-lateral prefrontal cortex (DLPFC) related phenotypes - specifically COMT gene expression in postmortem DLPFC and BOLD response during fMRIdepend on polygenic risk load of schizophrenia. To account for confounders like disease status, treatment, smoking, substance abuse, and precarious general health, functional MRI phenotypes and COMT gene expression were measured in healthy individuals, in both postmortem brain from and in living subject with no history of schizophrenia or relevant medical conditions.

Methods: a) Association of COMT Val/Met genotype and polygenic risk profile score (RPS) on COMT gene expression at the exon-level. Expression of COMT transcripts was measured from RNA-Seq in postmortem DLPFC $(N=99$ samples, all Caucasians, age $>13$ years, RIN $>6)$. For these samples, genotype (rs4680 Val/Met) and GWAS data were available. RPSs for the analyzed samples were computed from variants within the 108 PGC loci associated with schizophrenia risk (i.e. RPS level S1). Linear regression models implemented in statistical packages of 'R' software were used to test for Val/Met genotype, RPS effects and Val/Met $x$ RPS interaction effects on COMT transcripts. Five genomic principal components and ten gene expression principal components were used as covariates of non-interest to control for population ancestry, respectively expression heterogeneity, RNA quality and batch effects.

b) COMT Val/Met genotype and polygenic RPS associations with neuroimaging intermediate phenotypes. 232 healthy volunteers genotyped for COMT rs4680 Val/Met, with available GWAS data used for RPS calculation participated in an fMRI working memory study with the N-back working memory paradigm). All subjects had a performance accuracy $\geq 70 \%$ during the 2-back condition). Individual firstlevel GLM contrast images (2-back and 0-back) were analyzed within the SPM12 software (http://www.fil.ion.ucl. ac.uk/spm/software/spm12/) by using a flexible factorial design with Val/Met genotype as between-subject factor and RPS as a covariate of interest. Interaction Val/Met x RPS was also modelled. 'Batch effect' accounting for genotyping variability was used as covariate of noninterest.

Results: a) Association of COMT Val/Met and RPS with COMT expression. Val/Met genotype and RPS1 were associated in sum with the transcriptional regulation of 15/27 COMT unique exons. The number of 'risk' (Val) alleles was associated with lower expression for two exons showing a COMT genotype effect $(p=0.005)$. Likewise, for the exons showing an RPS effect (all 15; $p>0.0001$ and $<0.05$ ), an inverse relationship was observed between the 'risk' burden and transcript expression, with higher 'risk' profile associated with lower expression. No interaction Val/Met $x$ RPS was found on gene expression. b) Association of COMT Val/ Met and RPS with the n-back fMRI intermediate phenotype. When only the COMT effect was modelled, without controlling for the RPS, the COMT Val/Met effect within a pre-specified region of interest (ROI) encompassing right DLPFC was not statistically significant after multiple comparisons correction. However, when including in the model RPS1 and the interaction term COMT Val/Met x RPS, a significant main effect of $\mathrm{Val} / \mathrm{Met}$ on the R-DLPFC (GG $>A G>A A$ ) was found (MNI: $x=48, y=42, z=22$, BA46; $\mathrm{Z}$ score $=3.82, p<0.001$ after small volume correction within the pre-defined ROI). This effect is consistent with the previously found association between the load for COMT Val allele and a higher activation of DLPFC, suggestive of a more inefficient engagement of the DLPFC during cognitive processes involving working memory. There was no interaction effect of COMT Val/Met $x$ RPS within the DLPFC ROI.

Conclusions: The preliminary results of this study suggest independent effects of $\mathrm{rs} 4680 \mathrm{Val} / \mathrm{Met}$ and risk profiles scores on COMT gene expression and BOLD response during a working memory fMRI experiment. The association of COMT expression with RPS was particularly striking, and the directionality suggests that great genomic risk results in reduced COMT activity, which might in principle attenuate a previously proposed risk effect of relatively excess COMT activity on cortical function. This may be a possible explanation for the lack of association of COMT with schizophrenia in large GWAS studies: in the context of independent effects, higher RPS counteracts the COMT Val effect, which is more COMT activity.

A future thorough investigation of the COMT contribution to schizophrenia in relation with other genetic and environmental factors is therefore still warranted.

Keywords: COMT, Schizophrenia, Gene Expression, fMRI. Disclosure: Nothing to disclose. 
W196. Pattern Separation Deficits in First Episode Psychosis and a Ketamine Model Suggests Dentate Gyrus Pathology Linked to NMDA-Receptor Hypofunction

Nina Kraguljac*, Matthew Carle, Michael Frölich, Steve Tran, David White, Adrienne Lahti

University of Alabama at Birmingham, Birmingham, Alabama, United States

Background: Converging evidence from structural neuroimaging and post-mortem studies suggests that hippocampal subfields are differentially affected in schizophrenia. Recent studies report dentate gyrus dysfunction in chronic patients but the underlying mechanisms remain to be elucidated. Here we sought examine if this deficit is already present in first episode psychosis (FEP), and if N-methyl-d-aspartate (NMDA) receptor hypofunction, a putative central pathophysiological mechanism in schizophrenia, experimentally induced by ketamine, would result in a similar abnormality. Methods: We used a hippocampal memory task involving two phases, an incidental encoding phase during which subjects were asked to classify pictures of everyday objects as 'indoor' or 'outdoor' item, and a recognition phase where they were presented novel pictures, pictures that were exactly the same as presented before, and pictures that were similar, but not exactly the same as shown during encoding. Subjects were asked to indicate whether objects were new, similar, or old compared to those presented prior. We used this task in two settings: (1) a group of $23 \mathrm{FEP}$ and 23 matched healthy volunteers (HV), and (2) a group of $19 \mathrm{HV}$ before and during a ketamine challenge $(0.27 \mathrm{mg} / \mathrm{kg}$ over 10 minutes, then $0.25 \mathrm{mg} / \mathrm{kg} /$ hour for 50 minutes, $0.01 \mathrm{ml} / \mathrm{s}$ ). We calculated response bias corrected pattern separation and pattern completion scores. The Brief Psychiatric Rating Scale (BPRS) and its positive and negative subscales were used to assess symptom severity. Cognitive function was characterized using the Repeatable Battery for the Assessment of Neuropsychological Status (RBANS). For statistical analyses, we conducted independent sample and paired sample t-tests as appropriate. In an exploratory fashion, we also examined the relationships between pattern completion/ pattern separation performance and symptom severity as well as ketamine levels.

Results: We reported a deficit in pattern separation performance in FEP compared to $\mathrm{HV}(\mathrm{t}=2.16 ; p=.04)$ and in HV during the ketamine challenge compared to baseline $(t=3.57, p=0.003)$. Pattern completion scores did not differ between groups or across conditions. Exploratory analyses suggested a significant correlation between pattern separation scores and RBANS total scores $(r=0.44 ; p=$ 0.004 ), but not positive symptoms, or ketamine serum levels. Conclusions: As hypothesized, we observed a deficit in pattern separation, but not pattern completion in both datasets, implicating dentate gyrus involvement in the illness. Our findings extend prior studies reporting pattern separation abnormalities in chronic schizophrenia, and suggest a tentative mechanistic link between dentate gyrus dysfunction in first episode psychosis, cognition, and NMDA receptor hypofunction on GABAergic interneurons.

Keywords: First Episode Schizophrenia, Glutamate, Hippocampus.

Disclosure: Nothing to disclose.
W197. Neural Correlates of Self-Referential Memory in Schizophrenia

Amy Jimenez ${ }^{\star}$, Junghee Lee, Ana Ceci Myers, Jonathan K. Wynn, William P. Horan, Michael F. Green

Greater Los Angeles VA Healthcare System, Los Angeles, California, United States

Background: Background: Social information, especially information related to the self, enjoys a special processing status relative to non-social information. One advantage of this privileged status is enhanced memory for self-referential information, known as the self-reference memory (SRM) effect. Self-processing is reliably associated with activation in medial and ventrolateral prefrontal cortex (mPFC and vlPFC, respectively), temporo-parietal junction (TPJ) and posterior cingulate/precuneus (PCC/PC). Studies of the SRM effect show selective engagement of mPFC during "self" relative to other semantic processing conditions during encoding. Previous work in our lab indicated that, behaviorally, patients with schizophrenia fail to benefit from this memory boost. However, the neural correlates of this deficit are not known. The current study utilized a SRM task to examine 1) behavioral data from the retrieval phase to determine whether the patient sample would demonstrate a SRM effect, and 2) fMRI data at encoding to examine the neural correlates of self-oriented processing.

Methods: 18 clinically stable schizophrenia outpatients and 16 demographically matched healthy controls completed a self-referential recognition memory paradigm modified for event-related fMRI. During encoding, trait adjectives were judged in terms of their structural features ("case" condition), social desirability ("other" condition), or as selfreferential ("self" condition). Following a 12-minute delay comprised of distractor tasks, memory for trait adjectives was tested during an unexpected yes-no recognition test. Using a region-of-interest (ROI) approach, we examined regional BOLD activation within MPFC, vlPFC, TPJ, and PCC/PC. Right and left hemisphere ROIs were examined separately. ROI masks ( $9 \mathrm{~mm}$ diameter spheres) were created based on coordinates from prior literature. To assess within and between groups effects, beta weights were extracted from each ROI and analyzed with repeated-measures ANOVA (rmANOVA).

Results: There were no behavioral differences between groups during encoding in terms of self or social desirability judgments, or case accuracy. During retrieval, both groups demonstrated better recognition discriminability (d-prime) for adjectives from the "self" and "other" conditions compared to the "case" condition; d-prime scores were greater for the "self" condition compared to the "other" condition at the trend level. fMRI data obtained during encoding indicated that both patients and controls had greater activation during the "self" condition relative to the "other" and "case" condition in right and left mPFC, left vlPFC, and left TPJ, with no significant group by condition interactions. Activity in left PCC/PC was greater during "self" and "other" relative to the "case" condition in both groups. There were no group differences in activation except for a main effect of group in left TPJ (where patients were decreased compared to controls). 
Conclusions: Conclusions: We examined BOLD activity within a priori ROIs associated with self-referential memory in schizophrenia. The groups demonstrated comparable behavioral performance during the encoding and retrieval phases, despite prior literature indicating a deficit in SRM in patients. ROI BOLD activity indicated several regions sensitive to the "self" vs "other" manipulation, including mPFC, as expected. However, patients and controls showed similar activation levels in most ROIs during the encoding phase of this task, with no significant group by condition interactions. Contrary to our predictions, activity within $\mathrm{mPFC}$ did not discriminate between groups during selfprocessing. The only region to demonstrate between groups differences was left TPJ, which was decreased in patients across task conditions during encoding. In conclusion, the current, relatively small study failed to find group differences between patients with schizophrenia and controls in selfreferential memory processing.

Keywords: Schizophrenia, Self-Other Processing, Social Cognition, fMRI.

Disclosure: Nothing to disclose.

\section{W198. The Neurophysiological Mechanisms of Oxytocin During a False Belief Task in Individuals With Schizophrenia: An fMRI Study}

Lize DeCoster, Lisa Lin, Sophia Vinogradov, Daniel Mathalon, Joshua Woolley*

San Francisco Veterans Affairs Medical Center, San Francisco, California, United States

Background: The social cognitive deficits of schizophrenia, including difficulties in the ability to make high-level inferences about one's own and other people's mental states (i.e., Theory of Mind, ToM; Premarck \& Woodruff, 1978) independently predict worse clinical, functional, and occupational outcomes more strongly than positive symptoms and non-social cognitive deficits. Impaired ToM in schizophrenia has been well documented, indicating that areas such as the right temporo-parietal junction (rTPJ) and medial prefrontal cortex (mPFC) underlie these deficits (Lee et al., 2014). In a recent study, we demonstrated that the administration of oxytocin (OT) selectively improves high-level social cognition such as ToM in individuals with schizophrenia. These and other findings indicate that OT may be a useful treatment for their social deficits. In the current functional magnetic resonance imaging (fMRI) study, we explored the neurophysiological mechanisms of OT's prosocial effects in schizophrenia. Specifically, the current study investigated whether OT increases activity in brain areas related to ToM deficits in this patient group during a well-validated ToM task. Furthermore, we examined whether this increase in activation was related to behavioral improvement.

Methods: In the current study, 16 men with a chronic psychotic disorder ( $\mathrm{M}$ age: 32.07 years, SD: 9.16 years) and 20 age-matched controls ( $M$ age: 26.45 years, SD: 5.40 years) were administered intranasal oxytocin (40 IU) in a randomized, double-blind, placebo-controlled, cross-over fMRI study. All patients met diagnostic criteria for schizophrenia or schizoaffective disorder according to the Structured Clinical Interview for the DSM-IV (SCID). Healthy controls had no Axis I DSM IV disorder within the last year and no lifetime history of psychosis based on their SCID. For the two testing appointments, participants completed the false belief task as described in Lee et al., 2011 in the scanner. In this task, individuals are presented with two kinds of written short stories and are subsequently prompted to answer "True" or "False" to a statement about the story. In the false belief condition (e.g., "The morning of the high school dance, Sarah placed her shoes under her dresser and then went shopping. Later, her sister put the shoes under Sarah's bed."), participants make inferences about the agent in the story's false belief of reality in order to give a correct answer to the statement (e.g. "When Sarah returns, she looks for her shoes under her dresser."). In the false photograph condition (e.g. "When the photograph was taken, the boy was two yards away from the camera. The Eiffel tower was about 600 yards away."), participants make a true-false determination about a statement that does not involve an agent's false belief (e.g. "In the picture, the boy appears much smaller than the Eiffel tower."), but requires similar complex reasoning abilities.

Results: Individuals with schizophrenia were significantly less accurate during the false belief condition compared to the false photograph condition, $\mathrm{t}(15)=2.11, p<.05$, while healthy controls showed no significant difference, $\mathrm{t}(19)=.15$, $p=.88$. Furthermore, individuals with schizophrenia were significantly less accurate than healthy controls in the false belief condition, $\mathrm{t}(34)=2.57, p<.05$. When administered OT, accuracy during the false belief condition was significantly better for patients with schizophrenia compared to placebo administration, $\mathrm{t}(15)=2.41, p<.05$. Individuals with schizophrenia, showed hypo-activation in the rTPJ (50, $-41,28, z=5.11$ ), compared to healthy controls for the contrast false belief $>$ false photograph $(p<.001$, clustercorrected). Additionally, OT increased activation in this region in individuals with schizophrenia $(p<.001$, clustercorrected), compared to placebo. Finally, in schizophrenia, the OT-induced change in rTPJ activation was positively correlated with the OT-induced change in accuracy on the false belief task, $\mathrm{r}=.53, p<.05$.

Conclusions: Research on the effectiveness of OT for social cognitive deficits in schizophrenia has been mixed (e.g. Lee et al., 2013). These inconsistent findings in conjunction with concerns regarding questions of optimal dosages, have led to caution in translating this research to clinical practice. It has been argued that to improve the rigor and reproducibility of clinical trials of OT, studies must demonstrate target engagement (Insel, 2016). Target engagement is defined as evidence of activation of a proposed mechanism at a clinically effective dose. Therefore, in order to determine if OT administration can be an effective adjunctive treatment for schizophrenia, a measure of target engagement needs to be developed and linked to clinical outcomes. Our preliminary data indicates that OT increases activation in a neural network including the rTPJ in individuals with schizophrenia during a ToM task. Furthermore, this change in activation is correlated with significant behavioral improvements on the ToM task, suggesting that increased rTPJ activity after OT administration leads to improved behavioral ToM abilities. These findings suggest that changes in rTPJ and ToM performance are appropriate measures of neural and behavioral target engagement of OT in 
schizophrenia, and provide critical insights into the use of $\mathrm{OT}$ as an effective treatment for social deficits in this patient population.

Keywords: First Episode Schizophrenia, Oxytocin, Social Cognition, Functional MRI (fMRI).

Disclosure: Nothing to disclose.

\section{W199. Differential Association of Anandamide Levels With Binocular Depth Inversion Illusion Scores in Schizophrenia}

Anne R. Reuter, Juliane K. Mueller, Jan Malte Bumb, Cathrin Rohleder, Franziska Pahlisch, Franziska

Hanke, Dagmar Koethe, Emanuel Schwarz, F. Markus Leweke*

\section{University of Heidelberg, Mannheim, Germany}

Background: Binocular depth inversion illusion (BDII) represents a naturally occurring illusion of visual perception that involves higher-order visual and cognitive processes. Its impairment has been repeatedly linked to psychotic conditions (including delta-9-tetrahydrocannabinol induced altered states of consciousness) and identified as a potential marker for at risk mental states. The endogenous cannabinoid system (ECS) plays a critical role for regulation of various neurophysiological processes involved in perception and cognition. The endogenous cannabinoid N-arachidonoylethanolamide (anandamide) is a key component of the ECS and although it is assumed that it plays a pivotal role in cognition and in the pathophysiology of schizophrenia, little is known about the impact of anandamide levels on BDII alterations. Therefore, we explored associations between BDII and serum or cerebrospinal fluid (CSF) anandamide levels.

Methods: After obtaining written informed consent, we performed the binocular depth inversion illusion test (BDIIT) and collected blood and cerebrospinal fluid (CSF) in 28 first-episode antipsychotic-naïve schizophrenia patients (SZ) and 81 healthy controls (HC). The BDIIT was performed as previously described (Leweke et al. 2009). The endocannabinoids anandamide and 2-arachidonoyl-snglycerol as well as the related fatty acid ethanolamides palmitoylethanolamide and oleoylethanolamide were quantified by LC/MS-MS (Schreiber et al. 2007). Multiple linear regression analysis using gender, age, body mass index and former cannabis use as covariates, was applied to investigate interactions between diagnosis and anandamide levels on BDII test scores as well as for associations between anandamide and BDII test scores.

Results: CSF anandamide levels were significantly higher in SZ compared to HC, while serum anandamide showed no difference. BDII total and faces scores were significantly elevated in SZ compared to HC, indicating altered depth perception in SZ. BDII total and faces scores were strongly associated in $\mathrm{SZ}(\mathrm{rs}=0.99, P<0.001$, Spearman test). Anandamide levels were not correlated between serum and CSF in HCs $(r s=0.02, P=0.877)$.

Associations between serum anandamide and BDII were significantly different between SZ and HC (BDIItotal: PFWE $=0.014$; BDIIfaces: PFWE $=0.006$, multiple linear regression). A significant interaction term resulted from a SZ specific negative association between serum anandamide and BDII (BDIItotal: $P=0.006$, beta $=-0.62$; BDIIfaces: $P=$ 0.005 , beta $=-0.66$; multiple linear regression). For CSF anandamide, no significant interaction between BDII and diagnosis was found (BDIItotal: PFWE $>0.99$; BDIIfaces: PFWE $>0.99$, multiple linear regression).

Conclusions: The present study investigated for the first time the associations of serum and CSF anandamide levels and BDII scores in schizophrenia. BDIIT represents a test of impaired ability to mentally invert hollow (concave) objects to familiar convex percepts. BDII represents a sensitive measure of perceptual alterations in ultra-high risk individuals for schizophrenia as well as acute psychotic states including schizophrenia (Koethe et al. 2009). We found serum specific association differences of anandamide levels and BDII scores between schizophrenia patients and controls. Higher anandamide levels in CSF have been suggested protective in schizophrenia (Leweke, 2012). Here higher anandamide levels in serum were associated with lower BDII scores reflecting an increased ability for unconscious mental inversion of hollow objects, a regular mode of perception. Therefore, our results may suggest that the ECS system has a disease intrinsic role for modulating visual perception and associated higher-order cognitive function in schizophrenia.

Interestingly, in our study, this association was observed for serum anandamide not CSF levels. The latter reflect more temporal and limbic as well as basal ganglia sources while frontal cortical areas may drain more directly to the bloodstream via the dural venous sinuses providing a potential explanation for this phenomenon. Thus, this finding may be due to other brain areas preferentially involved in this perceptual alteration in SZ.

Keywords: Antipsychotic-Naïve First-Episode Schizophrenia, Visual Perception, Endocannabinoids, Anandamide, Humans.

Disclosure: Nothing to disclose.

\section{W200. CACNB4 Overexpression Linked to Deep Layer 3 Small Spine Loss in Schizophrenia}

Matthew MacDonald*, Jamil Alhassan, Jason Newman, Michelle Richard, Hong Gu, Allan Sampson, Kenneth Fish, Peter Penzes, Zachary Wills, David Lewis, Robert Sweet

\section{University of Pittsburgh Medical Center, Pittsburgh, Pennsylvania, United States}

Background: Deceased density of dendritic spines in adult schizophrenia $(\mathrm{Sz})$ subjects has been hypothesized to result from increased pruning of excess synapses in adolescence. In vivo imaging studies have confirmed that synaptic pruning is largely driven by the loss of large/mature synapses. Thus, increased pruning throughout adolescence would likely result in a deficit of large spines in adulthood. Recently, we reported that only the smallest spines are lost in deep layer 3 primary auditory cortex of $\mathrm{Sz}$, while larger spines are retained. Genetic studies have implicated postsynaptic density signaling proteins and calcium channels in $\mathrm{Sz}$ pathology and these proteins are essential for spinogenesis 
and plasticity. In the present study we searched for protein alterations in these networks linked to small spine loss.

Methods: Left and right hemisphere primary auditory cortex tissue from 20 pairs of $\mathrm{Sz}$ and matched control subjects was obtained. Tissue from the left hemisphere was utilized for immunohistochemistry while tissue from the right hemisphere was utilized for targeted mass spectrometry. Deep layer 3 spine density and volume was assessed in 20 pairs of $\mathrm{Sz}$ and controls in an initial and replication cohort (12 and 8 pairs) by immunohistochemistry-confocal microscopy. The two cohorts were processed and imaged separately. Targeted mass spectrometry was used to quantify postsynaptic density and voltage-gated calcium channel (VGCC) protein expression in a subset of these subjects. Primary cortical neuronal cultures were prepared from embryonic day 17 rats and transfected with CACNB4/MYC and/or GFP at day in vitro 12. Dendritic spine density, size, and type were recorded in StereoInvestigator.

Results: Levels of the tryptic peptide ALFDFLK, found in the $\mathrm{Sz}$ risk gene and VGCC subunit CACNB4, inversely correlated with the density of smaller, but not larger, spines across all subjects. Consistent with this observation, CACNB4 overexpression resulted in a lower density of smaller spines, but no change in density of larger spines or filopodia, in primary cortical neuronal cultures.

Conclusions: Our finding that primary auditory cortex layer 3 spine loss in $\mathrm{Sz}$ is limited to smaller spines strongly suggests that adult spine deficits result from a failure to generate and/or stabilize new/transient spines. This finding requires a rethinking of the over pruning hypothesis and should spur a more in-depth investigation of the mechanisms that govern spinogenesis, spine stabilization, and their role in Sz. As a first step, we have identified one such mechanism, increased CACNB4 expression, which appears to contribute to the loss of small spines in $\mathrm{Sz}$ and which could serve as a target for the development of novel $\mathrm{Sz}$ therapeutics. CACNB4 modulates the trafficking and biophysical properties of the alpha pore forming subunits. While the association of common and rare alleles of several VGCC subunits with Sz risk is well established, a consensus on whether they result in a loss or gain of function, and on how they may exert an effect on synapses is currently lacking. As CACNB4 interacts with and regulates multiple VGCC subunits implicated in Sz, it could act as a hub or common pathway by which VGCC genetic risk factors depress the formation and/or stabilization of small spinesCurrently, we are engaged in studies to determine the impact of CACNB4 overexpression on synaptic calcium signaling with the goal of elucidating the mechanism of small spine loss.

Keywords: Schizophrenia, Dendritic Spine, Proteomics, Postmortem Brain Tissue, Calcium.

Disclosure: Nothing to disclose.

\section{W201. Deciphering Schizophrenia Risk and Multi-Genetics of Motivation Neural Circuitry}

Qiang Chen, Ray Lefco, Kristin Nicodemus, Richard Straub, Daniel Weinberger, Caroline Zink ${ }^{*}$

Lieber Institute for Brain Development, Baltimore, Maryland, United States
Background: Lack of motivation (i.e., avolition) is a disruptive symptom of schizophrenia that is often resistant to current medications. Identifying genetic components of human motivation-related neural circuitry could clarify inter-individual differences and suggest pharmacological targets to alter such brain activity. Traditional use of imaging genetics, however, has tended to test the effects of one, or just a few, single nucleotide polymorphism(s) (SNPs) at a time, without accounting for genetic background and/or multigenetic/epistatic influences. The objective of the study was to determine specific multi-genetic influences on neural activity underlying incentive motivation in humans, as well as the associations between such activity and components of schizophrenia polygenic risk.

Methods: We created an fMRI task to specifically measure motivation-related midbrain-striatal activity in 86 healthy Caucasian adults. We used Random Forest (RF) multiple regression to determine multi-genetic influences on motivation-evoked neural activity and to rank genetic variations based on their predictive importance. Genes of interest were separated into 4 gene-sets: dopamine, glutamate, GABA, and acetylcholine (due to known roles in midbrain-striatum), each comprised of common, independent, putatively functional SNPs within synthesis/packaging, receptors, and clearance/degradation genes. Using the randomForest package in $\mathrm{R}$ software, separate $\mathrm{RF}$ regressions were conducted on each gene-set. Each RF regression was run 1000 times to get a stable distribution of variance explained by variables and average of importance measures for each SNP. Furthermore, to identify specific components of additive GWAS schizophrenia polygenic risk that predict motivation-related brain activity, we regressed incentive motivation neural activity on schizophrenia polygenetic risk scores created from the different gene sets. For each research participant, a schizophrenia "deconvolved" polygenic risk score was determined from SNPs in each of the four neurotransmitter gene set separately, as well as an overall GWAS polygenic risk score for comparison. The polygenic risk scores were defined as the weighted sum of the imputation risk allele probability for each index SNP picked by PLINK clumping in each gene-set. Weights were the natural $\log$ of the odds ratio obtained from the PGC2 schizophrenia GWAS study. To test the correlation between each score and motivation-related neural activity, polygenic risk scores were entered into a 2nd level multiple regression using SPM software, with age, gender, and IQ as covariates of non-interest. Both positive and negative correlations were evaluated in midbrain and striatum.

Results: A significant amount of variance (3.44\%, empirical $p=0.001)$ in average motivation-related ventral striatal activity was predicted by multi-genetic/epistatic effects in the dopamine signaling gene-set. Furthermore, SNPs in genes that code for dopamine synthesis/packaging proteins (e.g., VMAT, DDC, TH) had the most predictive influence. A significant amount of variance $(3.66 \%$, empirical $p=0.00)$ in midbrain activity (SN/VTA region) was predicted by multigenetic/epistatic effects in the GABA signaling gene-set. Overall additive GWAS schizophrenia risk (determined using various thresholds) did not predict midbrain or striatal activation to incentive motivation. We found, however, that GABA schizophrenia polygenic risk was associated with decreased motivation-related activity in the SN/VTA region 
of the midbrain $(p<0.01)$. Interestingly, the significance and extent of the negative correlation between schizophrenia polygenic risk scores from the GABA gene-set and midbrain motivation-related activity increased when polygenic risk scores were created a subset of the GABA gene-set SNPs corresponding to the most influential on motivation-evoked activity from the Random Forest analysis $(t=2.64->3.52$; cluster size $=27->152$ ).

Conclusions: These findings implicate dopamine and GABA proteins/genes that can be targeted in future studies to manipulate motivation-related neural activity levels and perhaps more effectively treat avolition in mental illness.

Keywords: Incentive Motivation, Dopamine, GABA, fMRI/ Imaging Genetics.

Disclosure: Nothing to disclose.

W202. Effects and Interaction of Delta-9Tetrahydrocannabidiol and Cannabidiol on Psychopathology, Neurocognition, and Endocannabinoids in Serum of Healthy Volunteers: Influence on Psychopathology

Juliane K. Mueller*, Anne R. Reuter, Bettina Lange, Axel Schaefer, Franziska Hanke, Franziska Pahlisch, Carola Schaefer, Anna-Maria Schmidt, Timo Woelfl, Frank Enning, Heike Tost, Andreas Meyer-Lindenberg, Dagmar Koethe, Cathrin Rohleder, Markus Leweke

Central Institute of Mental Health, Mannheim, Germany

Background: Frequent cannabis use has been identified as a relevant environmental risk factor for the development of psychosis and schizophrenia. Concerning genetical influences and cannabis Caspi et al. (2005) reported that carriers of the COMT allele valine158 (VV) are more likely to develop psychotic symptoms and schizophreniform disorders following frequent cannabis use than carriers of two copies of the methionine allele. $\Delta 9$-tetrahydrocannabinol ( $\Delta 9$-THC), the psychoactive substance of the Cannabis sativa plant, is known to transiently induce psychotic symptoms and cognitive impairment, while cannabidiol (CBD), a second component of the cannabis sativa plant, seems to have an antipsychotic effect. The underlying neurobiological principles to the well-known alterations of conscious experience reported after ingestion of different cannabinoids still aren't completely understood. We present first data of our study investigating the interaction of genetic (COMTgenotype) and environmental risk factors (phytocannabinoids) for schizophrenia on psychopathological alterations in healthy volunteers.

Methods: This study was registered (ClinicalTrials.gov ID: NCT02487381) and approved by the local Ethics Committee and the Federal Institute for Drugs and Medical Devices (BfArM, EudraCT-No. 2012-004427-20). We used a complex experimental approach using a randomized, double-blind, placebo-controlled Phase I clinical trial design administering $\Delta 9$-THC (20 mg orally) and/or CBD (800 mg orally) to 60 healthy volunteers. The healthy volunteers were randomly allocated to four treatment groups stratified by their COMT genotype (one third each per group) receiving either $\Delta 9$ THC/placebo, $\mathrm{CBD} /$ placebo, $\Delta 9-\mathrm{THC} / \mathrm{CBD}$, or placebo/ placebo. Psychopathological changes were assessed by the
Positive and Negative Syndrome Scale (PANSS), the Assessment of hallucinogenic states (APZ) and the Betts Questionnaire upon Mental Imagery (QMI). Adverse events where monitored throughout the trial in accordance with good clinical practise.

During the 4 study visits the data collected included blood and CSF samples for routine and safety reason as well as pharmacokinetic analyses, exploratory biomarkers, genetic analysis, urine analysis; imaging data from fMRI; electrophysiological data, neuropsychological and clinical tests, neurological and medial examination.

Results: Here we focus our interest on psychopathological and neuropsychological parameters. We observed significantly higher PANSS total as well as positive scores in the 49-THC/placebo (PANSS total: $\mathrm{MV} \pm \mathrm{SD}$ : $46.3 \pm 11.14$; PANSS positive score: $\mathrm{MV} \pm \mathrm{SD}: 12.3 \pm 4.28$ ) as well as the $\triangle 9-\mathrm{THC} / \mathrm{CBD}$ condition (PANSS total: $\mathrm{MV} \pm \mathrm{SD}$ : $49.3 \pm 13.5$; PANSS positive score: $\mathrm{MV} \pm \mathrm{SD}: 12.6 \pm 3.92$ ) compared to the $\mathrm{CBD} /$ placebo (PANSS total: $\mathrm{MV} \pm \mathrm{SD}$ : $32.3 \pm 2.712$; PANSS positive score: $\mathrm{MV} \pm \mathrm{SD}: 7.9 \pm 0.99$ ) or placebo/placebo (PANSS total: MV \pm SD: $32.1 \pm 3.17$; PANSS positive score: $\mathrm{MV} \pm \mathrm{SD}: 7.5 \pm 0.74)$ groups. Furthermore, we found significantly higher APZ scores in the $\triangle 9-\mathrm{THC} /$ placebo (APZ-OSE: $\mathrm{MV} \pm \mathrm{SD}: 2.5 \pm 2.2$; APZ-AIA: $\mathrm{MV} \pm \mathrm{SD}$ : $3.3 \pm 3.5$ ) as well as the $\Delta 9$-THC/CBD condition (APZ-OSE: $\mathrm{MV} \pm \mathrm{SD}: 2.7 \pm 2.3$; APZ-AIA: $\mathrm{MV} \pm \mathrm{SD}: 4.9 \pm 3.8)$ compared to the $\mathrm{CBD} /$ placebo (APZ-OSE: $\mathrm{MV} \pm \mathrm{SD}: 0.13 \pm 0.35$; APZ-AIA: $\mathrm{MV} \pm$ SD:. $0.27 \pm 0.70$ ) or placebo/placebo (APZOSE: MV \pm SD: $0.14 \pm 0.36$; APZ-AIA: MV \pm SD: $0.21 \pm 0.43$ ) groups for the APZ categories Oceanic Boundlessness (OSE) and Dread of Ego Dissolution (AIA).

There was no influence of both CBD and $\triangle 9$-THC on mental imagery as assessed by the QMI. We found no significant influence of the COMT genotype on pro-psychotic effects of the investigated cannabinoids. Furthermore, we found no clear correlation of the ratio of $\Delta 9$-THC/CBD serum levels as well as no correlation of the serum levels of $\triangle 9$-THC and CBD alone with psychopathology. Noteworthy, in the $\Delta 9$ THC/placebo condition nausea $(N=5 / 15)$ and vomiting $(N=3 / 15)$ was a prominent side-effect when administering the compound as single dose.

Conclusions: In our study, we found no protective action of CBD on $\triangle 9$-THC's psychotomimetic effects at the dosages used. CBD itself did not reveal any psychotic symptoms or side-effects. Contrary to to a previous study by Leweke et al. (2000) there was no influence of both CBD and $\Delta 9$-THC on mental imagery. While $\Delta 9$-THC is approved as an antinausea compound with a maximum daily dosage of $20 \mathrm{mg}$, we interestingly observed for $\Delta 9$-THC nausea and vomiting in our healthy subjects. Consistent with a recent study by Tunbridge et al. (2015) there was no influence of the COMT genotype on psychopathology or mental imagery. These results provide further support for previously described observations on CBD influence on $\triangle 9$-THC's effects while CBD showed sufficient plasma concentrations when $\Delta 9$ THC levels reached a peak-point.

Keywords: Cannabidiol, THC, Psychopathology, Healthy Subjects.

Disclosure: Nothing to disclose. 
W203. Genome-Wide Significant Associations of BSNIP1 Endophenotypes With Common SNPs

Ney Alliey-Rodriguez ${ }^{\star}$, Tamar A Grey, Madeline Klinger, Rebecca Shafee, Steve McCarroll, Jeffrey Bishop, Matcheri Keshavan, Neeraj Tandon, Jaya Padmanabhan, Godfrey Pearlson, Brett Clementz, Peter Buckley, John Sweeney, Elena Ivleva, Carol Tamminga, Sarah Keedy, Elliot Gershon

University of Chicago, Chicago, Illinois, United States

Background: The Bipolar and Schizophrenia Network of Intermediate Phenotypes (BSNIP) is an ongoing NIMHfunded study aimed to identify biomarker-based classification of psychoses, with clinical, imaging and neurophysiological phenotypes, consistent with Research Domain Criteria. We ran genome-wide association analyses (GWAS) with clinical and endophenotype variables from the BSNIP1 phenotypic database.

Methods: 667 phenotypes were examined in 1,117 unrelated individuals (561 males, 556 females; 754 cases and 361 controls) from the BSNIP1 study. They were genotyped using the Illumina PsychChip followed by imputation using the 1000 Genomes reference panel. After filtering for common SNPs 4,322,238 markers were used in GWAS using PLINK regression analysis, controlling for population stratification with PCA. For imputed data, the significance threshold for a GWAS is $1 \mathrm{E}-08$.

Results: 75 SNP markers had significant association with 27 different phenotypes; 40 of these markers are located within 17 genes, and the other 35 are intergenic. Because of Linkage Disequilibrium, only 34 of these 75 markers represent truly independent genome-wide significant associations. The most significant associations were found for "Number of Hospitalizations" with rs73536304 an intronic SNP in CDH7 (Cadherin7, $P=7.14 \mathrm{E}-12$ ), Freesurfer-derived structural MRI "Right Ventral Diencephalon volume" with rs79543758, an intronic SNP in LRP1B (LDL receptor related protein 1B, $P=1.24 \mathrm{E}-11$ ), and volume of "Inferior horn of the left lateral ventricle" with rs80262609, an intronic SNP in NRXN1 (Neurexin1, $P=1.29 \mathrm{E}-11$ ). Other significant associations were found with other brain structures and EEG-based neurophysiological variables.

Conclusions: The biological understanding of endophenotypes is enhanced by significant genetic associations. CDH7 had previously been associated with Bipolar Disorder in GWAS, and in our study with a clinical marker of disease severity for the first time. Structural variants (CNVs) disrupting NRXN1 gene had been associated with a wide spectrum of brain disorders including Schizophrenia, but this is the first report of an NRXN1 common variant associated with a brain region implicated in schizophrenia (hippocampus and other medial temporal structures form the floor of the left inferior horn).

Keywords: Dimensions of Psychosis, Brain Imaging, Imaging Genetics, Psychiatric Genetics, Endophenotypes.

Disclosure: Nothing to disclose.
W204. Brain Structure Biomarkers in the Psychosis Biotypes: Findings From the Bipolar-Schizophrenia Network for Intermediate Phenotypes (B-SNIP)

Elena Ivleva*, Brett Clementz, Anthony Dutcher, Sara Arnold, Haekyung Jeon-Slaughter, Sina Aslan, Bradley Witte, Gaurav Poudyal, Hanzhang Lu, Shashwath Meda, Godfrey Pearlson, John Sweeney, Matcheri Keshavan, Carol Tamminga

University of Texas Southwestern Medical Center, Dallas, Texas, United States

Background: Current definitions of psychotic disorders lack biological validity, motivating search for alternative biomarker-driven disease entities. Building on experimental constructs-Biotypes-previously developed from a battery of cognitive and neurophysiologic measures (Clementz et al., 2015), here we examine brain anatomy characteristics as independent validators for the Biotypes, alongside conventional diagnoses, testing whether gray matter density (GMD) measures depict unique brain structure characteristics across Biotypes, and whether these biomarkers better discriminate biologically-based Biotype constructs than the symptomdriven diagnoses.

Methods: Whole brain GMD measures were examined in psychosis probands, their first-degree relatives, and healthy subjects from the B-SNIP sample, initially organized by Biotype, and then by conventional diagnosis $(n=1,409) .3$ Tesla T1-weighted MPRAGE or IR-SPGR images were merged across sites and analyzed using optimized voxelbased morphometry/DARTEL, with subsequent subject-level regional GMD characterization, distribution and predictor analyses.

Results: Probands grouped by Biotype vs. healthy showed a step-wise pattern of GMD reductions: Biotype1, extensive and diffusely distributed GMD loss, with the largest effect sizes in frontal, anterior/middle cingulate, and temporal regions; Biotype2, intermediate and more localized reductions, with the largest effects in insula and fronto-temporal regions; Biotype3, small reductions localized to anterior limbic regions. Relatives grouped by their respective probands' Biotype showed regionally distinct GMD characteristics, with primarily anterior (fronto-temporal) reductions in Biotype1; posterior (temporo-parietal, cerebellar) GMD loss in Biotype2; and normal GMD in Biotype3. When organized by conventional diagnosis, probands with schizophrenia and schizoaffective disorder vs. healthy showed overlapping GMD reductions, with the largest effect sizes in fronto-temporal and parietal regions, while psychotic bipolar probands had small reductions, primarily in frontal regions. GMD changes in relatives by diagnosis followed regional patterns observed in probands, albeit less extensive. Biotypes showed strong between-group separation with broader regional GMD differences and higher effect sizes, whereas the three diagnoses poorly separated from each other. Biotype was the strongest predictor for whole brain GMD change, especially in probands, whereas conventional diagnoses lacked the ability to predict such GMD alterations. Conclusions: The GMD biomarkers depict unique brain structure alterations within each Biotype consistent with their cognitive and sensorimotor profiles: Biotype1, poor cognitive and sensorimotor function, and the largest GMD 
loss; Biotype2: poor cognitive function, exaggerated sensorimotor reactivity, and an intermediate in magnitude and distribution GMD loss; Biotype3: near normal cognition and sensorimotor reactivity, and minimal GMD loss. Broad, overlapping GMD reductions in the fronto-temporal and parietal regions observed in both Biotypel and 2 are consistent with poor cognitive function found in these Biotype constructs. GMD reductions in frontal as well as posterior (temporo-occipital) sensory cortices observed in Biotype 2 can represent an anatomical substrate for exaggerated sensorimotor reactivity characteristic for this Biotype. In that, altered cell packaging and synaptic alterations in regional inhibitory circuitries (e.g., NMDA-mediated GABAergic inhibitory interneuron-pyramidal cell assemblies in temporal cortex), along with diminished inhibitory frontal effects onto primary sensory cortices, may result in exaggerated neural activity captured by EEG-based sensorimotor discriminant function in our Biotype 2 construct. In addition, biologically-driven Biotype groups show stronger between-group discrimination and predictive function for GMD alterations than conventional symptom-based diagnoses. The Biotype constructs provide proof of concept that structural brain characteristics, along with an overall biomarker battery, can re-classify psychotic individuals and their relatives into groups that are neurobiologically distinctive and appear biologically meaningful, but are currently 'hidden' under biologically nonspecific clinical diagnoses. Biomarker-based search for novel disease entities may be highly advantageous for understanding neurobiological architecture of complex brain disorders, providing paths for individualized biomarker-based diagnosis and treatment.

Keywords: Biotype, PSYCHOSIs, Biological Marker, Gray Matter Density, Voxel-Based Morphometry.

Disclosure: Nothing to disclose.

\section{W205. 7-Tesla MRI Reveals Regional Hippocampal Deficits in Childhood-Onset Schizophrenia}

Dale Zhou*, Siyuan Liu, Diane Broadnax, Rebecca Berman, Judith Rapoport, Adam Thomas

National Institute of Mental Health, Bethesda, Maryland, United States

Background: Childhood-onset schizophrenia (COS) is a rare and severe form of the disorder occurring before age 13 . Previous work has established decreased hippocampal volume in both COS and adult-onset schizophrenia (AOS) patients. However, studies using 1.5- and 3-Tesla MRI have limited regional sensitivity due to lower image resolution, and reliable segmentation tools often classify individual hippocampal regions into diluted subfields. In the present study, we use 7-Tesla imaging and a novel segmentation technique to investigate regional alterations within the hippocampus in COS. We hypothesized that COS patients would show decreased volume in the dentate gyrus and in $\mathrm{CA} 3$, in accordance with the proposed glutamate-mediated plasticity deficit model of schizophrenia. In this model, abnormal glutamate signaling in the dentate and CA3 may relate to deficits in plasticity, which in turn could contribute to psychotic symptoms and impaired cognition.
Methods: Subjects were recruited nationally and matched for age and sex. We acquired $0.7 \mathrm{~mm}$ isotropic MPRAGE scans and $0.5 \mathrm{~mm}$ isotropic T2*-weighted scans using a Siemens 7Tesla Magnetom MRI scanner. Hippocampal subfields were automatically segmented using FreeSurfer (v6.0 beta, Apr $2016)$ in COS patients $(n=13$, mean age $=19.8$ years, 8 female), their unaffected siblings $(n=13$, age $=19.3,8$ female), and healthy controls $(n=13$, age $=20.5,8$ female $)$. Analysis of covariance was used to compare volume differences between groups with covariates including age, sex, and total cortical gray matter volume. Pairwise group comparisons were calculated using Tukey's HSD and Cohen's d was calculated using adjusted means and pooled standard deviation.

Results: We found that patients with COS had significant differences in subfield volumes that were localized to the parasubiculum, CA1, dentate gyrus, and CA4. For the current sample, COS patients had smaller volume of the dentate gyrus granule cell layer compared to controls (left hemisphere: $p=0.018$, Cohen's $\mathrm{d}=0.64$ ). Patients also exhibited reduced volumes in the parasubiculum (right: $p=$ 0.043, $\mathrm{d}=0.71$ ), CA1 (right: $p=0.039, \mathrm{~d}=0.77$ ), and CA4 (left: $p=0.05, \mathrm{~d}=0.59$ ) compared to controls. There were neither significant differences between patients and siblings, nor between siblings and controls.

Conclusions: Volume reductions within the parasubiculum, $\mathrm{CA} 1$, dentate gyrus, and CA4 in COS patients reveal regionspecific vulnerabilities. These results intersect with those of larger 1.5-Tesla imaging consortia for AOS (finding volume reductions in all hippocampal subfields compared to controls) but exhibit greater effect sizes, underlining the benefits of new segmentation methods on 7-Tesla images and possibly more striking abnormalities for very early onset disorders. A recent multi-site study utilizing the novel FreeSurfer v6.0 beta segmentation technique on 3-Tesla images found selective volume deficits of only CA1 in AOS compared to controls, differing from the more widespread pattern of volume deficits we observed in COS. However, among that AOS sample, the more chronic and ill AOS patients presented volume reductions in all subfields, suggesting severity and onset of illness contribute to the extent of regional vulnerabilities. On the one hand, our findings suggest a more general involvement of hippocampal subfields in schizophrenia than can be explained by only the glutamate-mediated plasticity deficit model of schizophrenia, but on the other hand display a more specific localization of marked volume loss than found in larger AOS studies. Ongoing analyses are examining hippocampal shape differences using deformation morphometry and Advanced Normalization Tools, as well as investigating clinical correlates of these anatomical measures.

Keywords: Hippocampal Subfields, 7-Tesla, Hippocampal Volume, Childhood-Onset Schizophrenia.

Disclosure: Nothing to disclose.

\section{W206. Cortical Oscillations in Juvenile Ferrets Following Maternal Immune Activation During Pregnancy}

Yuhui Li, John Gilmore, Flavio Frohlich*

University of North Carolina at Chapel Hill, Chapel Hill, North Carolina, United States 
Background: Neuronal synchronization and oscillations represent fundamental mechanisms underlying normal brain function. In schizophrenia, brain rhythms are disrupted in both sensory-evoked and resting-state activity, indicating that aberrant neural oscillations may have the potential to be a disease or risk marker. However, as schizophrenia is a neurodevelopmental disorder in which early pre- or postnatal events contribute to its pathogenesis, it still remains obscure whether abnormal oscillations are present in early development, or whether they can only be observed as the clinical symptoms start to emerge in early adulthood. Recent studies found that decreased amplitude and synchrony of cortical oscillations in adolescence underlie the pathology of schizophrenia, but it is still unknown when in the course of development these alterations arise.

Methods: Here, we adapted an existing prenatal maternalimmune activation paradigm5, which models features of schizophrenia, and applied it to the ferret (Mustela putorius furo). We injected polyinosinic: polycytidylic acid (poly I:C, $10 \mathrm{mg} / \mathrm{kg}$, intraperitoneal) into pregnant ferrets at the gestational age 30 (E30, full gestation $\sim 41$ days). We confirmed the occurrence of immune activation by an increase of body temperature, as well as increases of serum cytokine levels. We then implanted multi-electrode arrays in the primary visual cortex (area 17) in the offspring and recorded the spontaneous and light-evoked unit activity and local field potential (LFP) before and after eye-opening (postnatal day $22 \sim 29$ and day $35 \sim 47$, respectively) in the freely moving animal. The results were compared to data from age-matched control animals. Eight animals (four before eye-opening and four after) with maternal immune activation and nineteen control animals (eight before eyeopening and eleven after) were used in this study.

Results: Overall, the spontaneous oscillation amplitude was increased after eye-opening compared to before eye-opening, especially in the higher frequency range (gamma band, 30$100 \mathrm{~Hz}$, power increased by $923.5 \%$, student t-test, $p<$ $0.001)$. These gamma oscillations were decreased in amplitude in the poly I:C group compared to the control in both age groups (before eye-opening, power decreased by $73.9 \%$, $p<0.001$; after eye-opening, power decreased by $88.6 \%$, $p<0.001)$. Furthermore, phase-amplitude coupling (PAC) of the gamma oscillation amplitude to the phase of lower frequency oscillations (delta, theta and alpha) was significantly decreased in the poly I:C group in both age groups (before eye-opening, PAC value decreased by $49.0 \%-59.3 \%$ for phase of the variant of lower frequency oscillations, $p<$ 0.01 , after eye-opening, decreased by $23.6 \%-54.6 \%, p<0.01$ ). Whole-field light flashes induced a peak in the gamma band of the LFP and the amplitude of this visual response was decreased in the poly I:C group (before eye-opening, power decreased by $63.0 \%, p<0.001$; after eye-opening, power decreased by $82.6 \%, p<0.001)$. Finally, in control animals, cortical oscillations were entrained by visual stimuli with frequency up to $30 \mathrm{~Hz}$ after eye-opening. This effect was decreased in the poly I:C group for higher frequencies $(>$ $10 \mathrm{~Hz}$, decreased by $10.1 \%-60.1 \%, p<0.01)$.

Conclusions: Our results show that the amplitude and synchrony of cortical oscillations in early development is altered after maternal immune activation in pregnancy. The results suggest that the functional interaction between neuronal circuits by synchronization of electrical activity patterns is impaired from a very early stage in postnatal development after an environmental insult that models risk for schizophrenia. A parallel study is investigating the behavioral outcome in the ferrets with maternal immune activation. The long-term goal is to leverage these findings into novel brain stimulation treatments that aim to correct the observed aberrant neuronal activity pattern to restore physiological development of cortical circuits.

Keywords: Schizophrenia, Neurodevelopment, Ferret.

Disclosure: Pulvinar Neuro: Ownership, Self. Research reported in this publication was supported in part by the National Institute of Mental Health under Award Number R01MH101547 (FF). The content is solely the responsibility of the authors and does not necessarily represent the official views of the National Institutes of Health. This work was also partially supported by UNC Psychiatry, UNC School of Medicine (FF), and the Foundation of Hope (YL).

\section{W207. Reduced DISC1 in Astrocytes Impairs Cognitive Behaviors in Mice}

Chantelle Terrillion*, Joshua Crawford, Alexey Shevelkin, Sun-Hong Kim, Daisuke Fukudome, Akira Sawa, Atsushi Kamiya, Mikhail Pletnikov

Johns Hopkins University School of Medicine, Baltimore, Maryland, United States

Background: DISC1 has been identified as a genetic risk factor associated with major psychiatric disorders, including schizophrenia, and is expressed in both neurons and astrocytes. Neuronal DISC1 in the hippocampus and the prefrontal cortex has been implicated in cognitive function. However, the role of DISC1 in astrocytes, which have also been shown to play a role in normal cognition, has not been evaluated. We hypothesized that decreased expression of DISC1 in astrocytes in the hippocampus or prefrontal cortex would impair cognitive behavior in mice.

Methods: Mice were injected with the vector AAV1-GFAP:: GFP-miR30-DISC1 (Disc1 KD) in the hippocampus or prefrontal cortex (PFC) to knock down DISC1 in astrocytes, or the scrambled control vector AAV1-GFAP::GFP-mir30Ctrl (Ctrl). 2 weeks following injections, mice were tested in several cognitive behaviors, including novel object recognition, social interaction, Barnes maze and trace fear conditioning. Astrocyte morphology was assessed in the mouse brain following behavioral experiments.

Results: DISC1 KD in hippocampal astrocytes did not alter locomotor activity or anxiety related behavior but significantly decreased social preference and reduced preference for a novel mouse in the social interaction test. Additionally, DISC1 KD in hippocampal astrocytes impaired performance in the Barnes maze and reduced cue-dependent freezing in in trace fear conditioning. While DISC1 KD in PFC astrocytes also impaired performance in the Barnes maze, no significant effects of this KD were observed on cuedependent freezing in trace fear conditioning. We found that DISC1 KD reduced filopodia length and branch level in transduced astrocytes.

Conclusions: Reduction of DISC1 expression in brain astrocytes leads to brain region-dependent deficiencies in complex cognitive tasks. Reduction of filopodia length and 
arborization in astrocytes following DISC1 KD suggests that astrocytic dysfunction likely contributes to the cognitive deficits. Further understanding of the role DISC1 has in astrocyte function in relation to cognitive behaviors will allow us to improve treatment of cognitive symptoms in patients with major psychiatric disorders.

Keywords: Psychiatric Disorders, Cognitive Function, DISC1, Astrocytes.

Disclosure: Nothing to disclose.

\section{W208. Cognitive Functioning in First Episode Psychosis: Comparison of a Two-Year Coordinated Specialty Care Program to Community Care}

Nina Schooler*, Anzalee Khan, Richard Keefe, Patricia Marcy, Delbert Robinson, John Kane

State University of New York Downstate Medical Center, Washington, District of Columbia, United States

Background: Significant cognitive impairment is already seen in first episode psychosis (FEP) and the degree of cognitive impairment is recognized as an important moderator of long-term outcomes. An important question is whether specialized FEP treatment can improve cognitive functioning, which has been relatively resistant to change. The NIMH sponsored RAISE-ETP study provided an opportunity to compare NAVIGATE, a coordinated specialty care intervention designed for FEP, to Community Care in a randomized clinical trial.

Methods: The radnomized clinical trial (RCT) was conducted at thirty-four sites in the US. Seventeen sites were randomly assigned to provide NAVIGATE and 17 to provide Community Care. Four hundred four consenting participants who were 15 to 45 years old, were experiencing a first episode of psychosis and had not received more than 6 months of antipsychotic medication entered the study. Treatment and assessment continued for up to two years. The Brief Assessment of Cognition in Schizophrenia (BACS) was administered by trained on-site research assistants at baseline, one and two years. Age and sex adjusted T-scores for the BACS Composite and six sub-tests (Verbal Memory, Digit Sequencing, Verbal Fluency, Token Motor, Symbol Coding and Tower of London) were calculated at baseline, month 12 and 24. Generalized estimating equations (GEEs) were applied to compare interventions with respect to changes in cognition between baseline and months 12 and 24 and adjusting for within and between site variation.

Results: The NAVIGATE and Community Care groups included 221 and 181 participants, respectively of the 223 and 181 in the study. At Month 12, 62.7\% of the baseline sample completed the BACS, and at Month 24, 43.8\% of the baseline sample did so. The GEE models showed significant interaction effects. The interaction between Group and Visit was a statistically significant predictor of change in: Verbal Memory, Verbal Fluency, Symbol Coding, Tower of London and the Cognitive composite ( $p<0.001$ for all subtests). Further examination of the parameter estimates showed that for the NAVIGATE group, there was significant improvement at Month 12 and Month 24 for Verbal Memory $(p=0.002, \quad p=0.003$, respectively), Digit Sequencing $(p=0.012, \quad p=0.020$, respectively), Tower of London
( $p=0.001, p=0.002$, respectively) and the Cognitive Composite $(p=0.001, p<0.001$, respectively). For the Community Care group, there was a significant improvement at Month 12 and Month 24 for the Cognitive Composite $(p=0.036$, $p=0.20$, respectively); only the Tower of London showed significant difference from Baseline to Month 24 for the Community Care group $(p<0.001)$.

Conclusions: The results of this analysis suggest that NAVIGATE resulted in greater improvement in cognitive functioning in both an overall score and for specific components than did Community Care. NAVIGATE is a multi-component intervention; each intervention was guided by a manual and supervision by a central team. NAVIGATE includes psychopharmacological treatment with antipsychotics and other psychotropic medications using an internet based decision support system, an individual psychotherapy called Individual Resiliency Training, family psychoeducation and supported employment and education. The role of individual components cannot be readily determined and further analyses will evaluate the effect of moderator variables such as duration of untreated psychosis. To date, pharmacologic treatments to improve cognition have not demonstrated efficacy. Cognitive remediation strategies, notably Cognitive Enhancement Therapy pioneered by Gerard Hogarty, has shown efficacy in FEP patients but to the best of our knowledge, this is the first RCT to show an effect of a broad FEP focused intervention on cognitive functioning.

Keywords: First-Episode Psychosis, Cognition, Randomized Clinical Trial.

Disclosure: Nothing to disclose.

W209. Rapid Augmentation of Antipsychotic Drugs by Sodium Nitroprusside (SNP): Behavioral Assessment and Effect on Brain Dopaminergic Transmission in Rats

Torgny Svensson*, Joep Titulaer, Anna Malmerfelt, Monica Marcus

Karolinska Institutet, Stockholm, Sweden

Background: In recent years a single injection of the nitric oxide donor sodium nitroprusside (SNP) has been found to induce a rapid (within 4 hours) and sustained (several weeks) antipsychotic effect in treatment-resistant schizophrenic patients on a stable antipsychotic medication. Previous preclinical studies had shown that a single injection of SNP can produce a prolonged block of the psychotomimetic effects of phencyclidine or ketamine that lasts for at least a week. A recent study in normal adult rats (Aghajanian, ACNP 2015) propose that the antipsychotic activity of a single exposure to SNP may be explained by rapid and persisting changes in brain synaptic function and morphology, including enhancements in excitatory postsynaptic current (EPSC) responses and spine morphology in layer $\mathrm{V}$ pyramidal cells in medial prefrontal cortex (mPFC) brain slices. Here we have studied the antipsychotic-like effect of SNP in rats using behavioral techniques, both when given alone and in combination with a sub-effective dose of risperidone. Correlative biochemical studies of regional dopamine release in brain were also performed. 
Methods: We used the conditioned avoidance response (CAR) test to investigate the antipsychotic-like efficacy, since this preclinical behavioral assay has shown a very high predictive validity to identify drugs with clinical antipsychotic activity, and in vivo microdialysis in freely moving animals to measure neurotransmitter efflux in the mPFC and the nucleus accumbens (NAc), respectively.

Results: Risperidone $0.25 \mathrm{mg} / \mathrm{kg}$ i.p. given alone, showed a $20 \%$ suppression of CAR, which is far below the degree of CAR suppression required to indicate a significant clinical antipsychotic effect in schizophrenia, which is $70-80 \%$. Importantly, addition of SNP $1,1.5$ and $2 \mathrm{mg} / \mathrm{kg}$ i.p. to risperidone dramatically enhanced the antipsychotic-like effect of risperidone to 67,86 and $100 \%$ suppression of $\mathrm{CAR}$, respectively. However, addition of the highest dose of $\mathrm{SNP}$, i.e. $2 \mathrm{mg} / \mathrm{kg}$, resulted in escape failures which could indicate risk of non-specific side effects. Interestingly, SNP $2 \mathrm{mg} / \mathrm{kg}$ given alone generated a small, $11 \%$, but significant suppression of CAR. In the NAc, addition of SNP $1 \mathrm{mg} / \mathrm{kg}$ did not enhance the risperidone-induced dopamine output. The effect on dopamine output in the mPFC is still under investigation and the definite results will be presented at the meeting.

Conclusions: The present results fully support the clinical observation that a single injection of SNP can very rapidly and even dramatically augment the clinical efficacy of antipsychotic drugs in schizophrenia, albeit within a relatively narrow dose-range. That a single injection of SNP alone was not found to exert any clinically relevant suppression of CAR makes it similar to other drugs that both clinically and preclinically have been shown to augment the antipsychotic effect of various neuroleptics. Clearly, the major difference and advantage of SNP over these compounds is the long-lasting augmentation generated by single exposure to the NO-donor. Needless to say our present data do not support any antipsychotic activity of SNP alone as proposed recently from preclinical data (c.f. above), Although the antipsychotic-like effect of drugs as assessed by the CAR-test in rats is primarily localized in the ventral striatum, the fact that add-on SNP did not change DA efflux in this region may indicate that the site of the augmentation rather resides in the prefrontal cortex, as has previously been shown with other compounds that augment antipsychotic drugs.

Keywords: Schizophrenia, Nitric Oxide Donor, Rapid Antipsychotic Augmentation, Behavioral Analysis, Biochemistry. Disclosure: Nothing to disclose.

\section{W210. Hippocampal Glutamate and Functional Connectivity as Biomarkers of Treatment Response to Antipsychotic Medication: A Longitudinal Imaging Study in Unmedicated Patients Before and After Treatment}

Adrienne Lahti*, David White, Nina Kraguljac, Meredith Reid

University of Alabama at Birmingham, Birmingham, Alabama, United States

Background: Approximately $30 \%$ of patients with schizophrenia do not improve with antipsychotic drug (APD) and
$60 \%$ show sub-optimal response. Our prior studies have showed that hippocampal regional cerebral blood flow $(\mathrm{rCBF})$ is elevated in unmedicated patients and that $\mathrm{rCBF}$ reduction over the first week of treatment is correlation with subsequent good treatment response (Lahti, 2009). To characterize the pathophysiology of this pattern, we conducted a longitudinal study where hippocampal neurochemistry and function were measured while patients were unmedicated and after a 6-week course of APD.

Methods: We enrolled 34 unmedicated (minimum of 10 days) patients with psychosis. They were scanned before and after 6 weeks of APD treatment with risperidone. Based on a median split of treatment response [defined as the percent change on the Brief Psychiatric Rating Scale (BPRS) psychosis score between week 6 and baseline], we categorized patients as good (TR) $(n=17)$ or poor (TPR) $(n=17)$ treatment responders. Matched healthy controls (HC) $(n=23)$ were scanned twice, 6 weeks apart. Resting state fMRI and magnetic resonance imaging (MRS) (PRESS sequence) of the left (L) hippocampus were obtained at both time points. fMRI preprocessing and analyses were conducted using the CONN toolbox in SPM8. The first eigenvariate of the blood oxygen level-dependent time series from a L hippocampal seed region, defined by the AAL atlas, was extracted and correlated with the time series from each of fourteen individual network masks (Shirer, 2012), providing a $\mathrm{z}$-score for hippocampus to network connectivity for each network, for each participant, at each time point. MRS data were quantified in the time domain, incorporating prior knowledge from in vitro and in vivo metabolite spectra using jMRui. N-acetyl-aspartate, choline, and glutamate+ glutamine $(\mathrm{Glx})$, referenced to creatine were quantified for each participant at each time point. Two separate analyses were performed to analyze MRS and hippocampus to network connectivity. Generalized linear mixed effects models with an identity link were employed to estimate the association between the dependent variable(s) (MRS metabolite values, or hippocampus to network connectivity values), Group (HC, TR and TPR), and Time (baseline and week 6). The generalized linear mixed model allowed us to control for Group-level intercepts accounting for repeated measures and nested values in the case of metabolites with common reference values $(\mathrm{Cr}) /$ connectivity values. A number of additional analyses were performed to explore the relationship between altered neurochemical and functional connectivity patterns observed in patients.

Results: At baseline, Glx levels were significantly higher in TR compared to HC $(p<0.01)$. After 6 weeks of treatment, TR, but not TPR, showed a significant decrease in Glx levels $(p<0.02)$. At baseline, the functional connectivity between the $\mathrm{L}$ hippocampus and the right executive network was significantly greater in TPR compared to HC. In TR, despite changes in Glx, no change in hippocampus functional connectivity from baseline to week six was observed. Likewise, in TNR, hippocampal Glx did not explain a significant proportion of the variance in the pattern of abnormal functional connectivity from the left hippocampus to the right executive control network.

Conclusions: This data suggest that connectivity abnormalities unrelated to Glx levels could play a role in poor treatment response whereas glutamatergic plasticity might contribute to good treatment response. The lack of 
relationship between altered glutamate levels and functional connectivity patterns in TR and TPR suggest independent pathophysiological mechanisms. These data indicate that prior to treatment hippocampal Glx and functional connectivity are measures that could be leveraged to predict treatment response in first episode psychosis patients.

Lahti et al., Neuropsychopharmacology, 2009

Shirer et al., Cerebral Cortex, 2012

Keywords: Psychosis, Antipsychotic Medication, Glutamate, Functional Connectivity, Hippocampus.

Disclosure: Janssen, Inc.: Provided medication for study, Self; Janssen, Inc: Grant, Self.

\section{W211. Cognition in Psychosis: A Four-Cluster Solution Extension With Resting State Functional Connectivity}

Julie McCarthy*, Justin T. Baker, Dost Ongur, Lesley Norris, Richard Juelich, Kathryn E. Lewandowski

Harvard Medical School, Belmont, Massachusetts, United States

Background: Variation in cognitive ability is evident across individuals with psychosis, yet a clear cognitive profile of strengths and weaknesses has not been established. Using cluster analysis allows for individuals to be grouped in a data-driven approach rather than by predefined diagnostic grouping criteria. A four-cluster solution has been identified in prior research examining cognitive profiles in psychosis. However, cluster solutions are dependent upon the measures used to generate them, and the relationship between these cognitive clusters and biological measures is unclear. We aimed to: a) extend a transdiagnostic cluster analysis using a different battery of cognitive measures in a separate sample and $b$ ) assess associations between clusters and fMRI resting state networks.

Methods: Individuals with psychosis $(n=120)$ and healthy controls $(n=31)$ completed the MATRICS Battery to assess cognitive ability, clinical and functioning measures, and an fMRI scan to examine resting state connectivity (RSFC). A K-means cluster approach and canonical discriminant function analysis were used to analyze the cluster structure of the MATRICS data. Mean fMRI BOLD time courses were computed for surface ROIs and correlated across all pairwise interactions, and group level ANOVAs were performed on individual interregional correlation values. Differences across clusters were compared by diagnosis and clinical symptom measures, community functioning, and network connectivity. Results: A four-cluster solution provided adequate fit, and similar to our previous report - yielded a 'neuropsychologically normal' cluster, a globally and significantly impaired cluster, and two clusters of mixed cognitive profiles. Clusters differed on several clinical variables; diagnoses were distributed amongst all clusters, although not evenly. In terms of RSFC, Clusters 2, 3 and 4 showed network disconnectivity compared to the neuropsychologicallynormal cluster (Cluster 1) and healthy controls, particularly in fronto-parietal control regions. All patient clusters, including Cluster 1, differed from healthy controls in default mode connectivity.

Conclusions: This replication in an independent sample using a different but related cognitive battery suggests that cognitive clusters identify meaningful groupings and are not simply a function of the measures used to derive them. Clusters were associated with RSFC in networks associated with relevant cognitive processes; regardless of cognitive profile, patient status was associated with abnormalities in default mode connectivity. Identification of groups of patients who share similar neurocognitive profiles may help pinpoint relevant clinical and neural abnormalities underlying these traits.

Keywords: Cognition, Psychosis, Resting State Functional Connectivity.

Disclosure: Nothing to disclose.

\section{W212. Genetic Associations With Cognitive Performance in Psychotic Disorders From the Bipolar-Schizophrenia Network on Intermediate Phenotypes Consortium}

Jeffrey Bishop ${ }^{*}$, Lauren Mills, Scot Hill, Ney AllieyRodriguez, James Reilly, Pranav Nanda, Jaya

Padmanabhan, Neeraj Tandon, Rebecca Shafee, Steve McCarroll, Steven Hyman, Richard Keefe, Godfrey Pearlson, Brett Clementz, Carol Tamminga, Matcheri Keshavan, Elliot Gershon, John Sweeney

University of Minnesota, Minneapolis, Minnesota, United States

Background: Cognitive deficits are known to be present and familial in schizophrenia and bipolar disorder. Understanding the genetic contributors to cognitive impairments in psychotic disorders as well as healthy controls can contribute to our understanding of disease pathophysiology and its behavioral consequences.

Methods: The Brief Assessment of Cognition in Schizophrenia (BACS) was administered to 1059 participants (schizophrenia $N=299$, schizoaffective disorder $N=179$, psychotic bipolar disorder $N=236$, and controls $N=345$ ) in the Bipolar-Schizophrenia Network on Intermediate Phenotypes (B-SNIP) study. Participants were 15-65 years of age, clinically stable outpatients (if probands), and without significant neurological disorder, or recent substance abuse or dependence. Participants were genotyped using the Illumina PsychChip followed by imputation using the 1000 Genomes reference panel resulting in 4,322,238 SNP markers. A mixed modeling GWAS approach (EMMAX) was performed using BACS total composite $\mathrm{Z}$ scores examined as a quantitative trait phenotype in relation to genetic data while controlling for genetically-driven ancestry measures, age, and sex. Probands and controls were grouped together for primary analyses and then also stratified by the top two genetically-derived ancestry groups.

Results: When all participants were examined together, the most prominent region associated with BACS performance was the SFMBT1 locus on chromosome 3 (lowest $p=1.110 \mathrm{E}$ $-7 ; N=10$ SNPs $p<1.0 \mathrm{E}-5)$. Analyses stratified by ancestry also revealed a robust association of SFMBT1 in participants of predominantly Caucasian ancestry (lowest $p=2.410 \mathrm{E}-7$ ). Robust associations with the LCO101928516 locus on chromosome 6 (lowest $p=4.6 \times 10 \mathrm{E}-8 ; \quad N=18 \quad$ SNPs $p<1 \times 10 \mathrm{E}-5)$ were identified in participants of predominantly African ancestry. Additionally there were $N=30$ genes or defined genetic loci harboring SNPs with suggestive 
$(p<1 \mathrm{E}-5)$ associations with BACS performance. Notable trends in top associations include genes previously associated with risk for schizophrenia or bipolar disorder (CDH12, DNM3, ITIH1, ITIH4, LRFN5, NMBR, NTRK3); brain structure, white matter, or development (INPP5D, LRFN5, NTRK3); and cognition (AKAP6, TNFSF10).

Conclusions: We identified SNPs in genes potentially associated with neuropsychological performance as measured by the BACS. The most robust association was in participants of predominantly African ancestry at a gene locus on chromosome 6 (LCO101928516) that is to date not well characterized for molecular function or relationships with brain-related phenotypes. Additionally, many SNPs at the SFMBT1 locus on chromosome 3 also appear to be associated with cognitive performance in our study sample. This gene is related to expression factors related to histone and chromatin regulation. Other genes harboring SNPs with suggestive associations include those previously associated with risk for psychotic disorders, brain structure or development, and cognition. These data suggest that cognitive impairments in psychotic disorders may be influenced by genes also known to be risk factors for psychiatric disorders, but also other genes related to brain processes or epigenetic gene regulation. These findings as well as novel loci identified herein require validation and will be further investigated in the BSNIP2 study.

Keywords: Genetics, Cognition, Bipolar Disorder, Schizophrenia.

Disclosure: Nothing to disclose.

\section{W213. Microglial Pruning of Dendritic Spines in the Prefrontal Cortex During Adolescence}

Allyson Mallya, Hui-Dong Wang, Tuula Ritakari, Brad Grueter, Ariel Deutch*

Vanderbilt University Medical Center, Nashville, Tennessee, United States

Background: Aberrant developmental synaptic elimination during adolescence has been suggested to contribute to both the reduced spine density of pyramidal cells (PCs) in the prefrontal cortex (PFC) and the cognitive deficits in schizophrenia. Recent data indicates that microglia are critically involved in synaptic pruning during early postnatal periods1-3. However, the role of microglia in the structural and functional maturation of the PFC, which is characterized by a protracted postnatal development with a later maturation than other cortices 4,5 , has not yet been examined. We therefore determined if microglia contribute to the developmentally-specific pruning of dendritic spines in the PFC, with a focus on the periadolescent period.

Methods: Equal numbers of male and female SpragueDawley rats, weaned at 21 days of age, were used as subjects. Randomly selected Layer 5 PCs from the prelimbic area of the PFC of one hemisphere were intracellularly filled with the fluorescent dye Lucifer Yellow for dendritic spine density measurements in rats sacrificed on postnatal days 24 and 30 (a time of increasing PFC PC spine density), 35 (posited peak spine density), and 39 and 50 (decreasing spine density). The contralateral hemisphere was used to quantitatively determine immune-histochemical colocalization of microglia (identified by Ibal expression) and PFC PC dendritic spines (revealed by PSD-95-immunoreactivity) at these five periadolescent ages.

Results: Dendritic spine density on L5 PFC PCs peaked between P30 and P35. We observed a significant increase in Iba1 and PSD-95 colocalization in the PFC at P39 relative to P24 and P30, consistent with active microglial pruning of dendritic spines on PFC PCs. We also noted a suggestion of sex differences in the timing of the increase in microglial engulfment of spines: males displayed a non-significant trend $(p=0.06)$ toward an increase at P39 relative to earlier time points, while females showed no such trend.

Conclusions: Our data suggests that the developmentallyspecific pruning of dendritic spines on PCs in the PFC occurs during adolescence, substantially later than in other areas of the brain; current efforts are examining if the presynaptic components of synapses are also engulfed by microglia during adolescence.

These studies serve as a foundation for understanding the processes governing developmental PFC synaptic elimination, which is thought to be disrupted in schizophrenia and other disorders. The age at which we observe a sharp increase in microglial sculpting of PFC neurons is thought to correspond to early adolescence. Prodromal symptoms emerge in human adolescents and subsequently may evolve into a psychotic episode. An exaggeration of synaptic pruning in the PFC by microglia supports the hypothesis of Feinberg6 that a deranged enhancement of synaptic elimination is a key pathophysiological feature of schizophrenia. Our work may guide the identification of pharmacological targets aimed at early intervention to attenuate or prevent the cortical pathology and emergence of cognitive dysfunction in schizophrenia. In future experiments we will treat mice that conditionally express the diphtheria toxin (DT) receptor in microglia with DT to ablate microglia during critical pruning periods and assess in the adult the structural and functional consequences of adolescent microglial ablation, including the effects on spine density and on PFC-dependent behaviors (for example working memory) that are disrupted in schizophrenia.

Keywords: Schizophrenia, Synaptic Aberrations, Prefrontal Cortex, Microglia, Adolescent Development, Cortical Circuit Development.

Disclosure: Nothing to disclose.

\section{W214. Comparison of Omega-3 Versus Placebo Supplementation of Risperidone for the Treatment of Early Phase Psychosis}

Delbert Robinson*, Philip Szeszko, Juan Gallego, Majnu John, Anil Malhotra

Hofstra Northwell School of Medicine, Glen Oaks, New York, United States

Background: Omega- 3 fatty acids as supplements or primary treatments for psychotic disorders have been of considerable interest for over a decade but the overall results of studies with omega-3 treatments for psychotic disorders have been inconsistent. Our main study attempted to determine which patients would benefit by omega-3 supplementation of antipsychotic treatment. In this report, we present the 
clinical outcomes of the treatment trial component of the study.

Methods: Inclusion criteria for subjects were: (1) current DSM-IV-defined diagnosis of schizophrenia, schizophreniform, schizoaffective disorder or bipolar disorder with psychosis; (2) did not meet DSM-IV criteria for a current substance-induced psychotic disorder, a psychotic disorder due to a general medical condition, delusional disorder, brief psychotic disorder, shared psychotic disorder; (3) current positive symptoms rated $\geq 4$ (moderate) on one or more of these BPRS items: conceptual disorganization, grandiosity, hallucinatory behavior, unusual thought content; (4) in a early phase of illness as defined by having taken antipsychotic medications for a cumulative lifetime period of 2 years or less, (5) age 15 to 40; (6) competent and willing to sign informed consent; and (7) for women, negative pregnancy test and agreement to use a medically accepted birth control method. Exclusion criteria were: (1) serious neurological or endocrine disorder or any medical condition or treatment known to affect the brain; (2) any medical condition which requires treatment with a medication with psychotropic effects; (3) significant risk of suicidal or homicidal behavior; (4) cognitive or language limitations, or any other factor that would preclude subjects providing informed consent; (5) medical contraindications to treatment with risperidone, omega-3 supplements (e.g. bleeding disorder, seafood allergies) or placebo capsules (e.g. allergies to capsule components); (6) lack of response to a prior adequate trial of risperidone; 7) currently taking omega-3 supplements.

The trial lasted 16 weeks; the primary outcome measure was the BPRS total score. All subjects received treatment with risperidone; the target dose was $3 \mathrm{mg}$ daily but doses up to $6 \mathrm{mg}$ per day were permitted. Concomitant allowed medications included benztropine mesylate, lorazepam, propranolol, zolpidem and rozerem. Subjects were randomly assigned on a 1:1 basis to treatment with omega-3 or placebo. Each omega- 3 capsule contained $370 \mathrm{mg}$ EPA and $200 \mathrm{mg}$ DHA as well as $2 \mathrm{mg} / \mathrm{g}$ Tocopherol. The placebo was a soybean/corn blend. Subjects took one capsule twice a day.

Results: The sample included 50 subjects; 36 (72\%) were men. The mean age of subjects assigned to placebo was 22.4 $(\mathrm{SD}=5.3)$ years and $23.1(\mathrm{SD}=5.3)$ years for those assigned to omega-3. Four had bipolar disorder; the rest non-affective psychotic disorders. The mean lifetime days of antipsychotic treatment prior to study entry was 28.1 (95\% CI: 9.4, 46.8) and the median number of days was 6.5 . $76 \%$ of subjects had taken antipsychotics for 14 days or less in their lifetime.

The mean length of time in controlled treatment was 11.36 $(\mathrm{SD}=6.61)$ weeks with placebo and $11.16(\mathrm{SD}=6.72)$ weeks with omega-3 $(p=0.92)$. Longitudinal analyses of risperidone daily dose (averaged separately for each treatment week) revealed a effect of time $(\mathrm{F}=2.39, \mathrm{df}=16,465$, $p=0.0019)$ but no treatment by time interaction or effect of time. Longitudinal analyses of lorazepam daily dose (averaged over each treatment week) revealed a treatment by time interaction $(\mathrm{F}=3.00, \mathrm{df}=16,390, p<0.0001)$. Lorazepam average dose was significantly lower in the placebo condition at week $1(p<0.0007)$ and week $2(p=0.0012)$ and at a trend level at week $0(p=0.0977)$ and week $3(p=0.0582)$. Given the difference in lorazepam prescription, average lorazepam dose per week was included in the analysis model of BPRS scores over time. This analysis revealed a significant effect of treatment condition $(\mathrm{F}=3.90, \mathrm{df}=1,296, p=0.0493)$ favoring omega-3 treatment. Least square mean estimates of baseline BPRS scores were 41.6403 (SE = 1.4368) for omega-3 and $42.3797(\mathrm{SE}=1.5559)$ for placebo treatment; corresponding week 16 scores were $22.5868(\mathrm{SE}=2.1744)$ and 27.2235 ( $\mathrm{SE}=2.4397$ ). Secondary analyses of BPRS factors revealed a trend $(p=0.0541)$ difference in the hostilityuncooperativeness factor favoring omega- 3 treatment but no difference on positive, negative or depression/anxiety factors. Conclusions: Our data suggest that omega-3 supplementation of antipsychotic treatment with early phase patients may be clinically useful, especially given the favorable side effect profile of omega-3 supplements. Prior omega-3 studies in psychotic disorders have often focused on positive symptom outcomes. Our data suggest that future studies should examine potential beneficial effects in other domains as well. Keywords: Omega-3 Fatty Acid, Early Psychosis, Risperidone.

Disclosure: Otsuka: Consultant, Grant Support, Self; Innovative Science Solutions: Consultant, Self; Costello Medical, Consultant, Self.

\section{W215. The Efficacy and Safety of Lurasidone in Adolescent Patients With Schizophrenia: A 6-Week, Double-Blind, Placebo-Controlled, Multicenter Study}

Christoph U. Correll, Robert Goldman, Robert Silva, Josephine Cucchiaro, Ling Deng, Antony Loebel*

Sunovion Pharmaceuticals, Inc., Fort Lee, New Jersey, United States

Background: Several atypical antipsychotic agents are approved by the US Food and Drug Administration to treat adolescent patients with schizophrenia. However, antipsychotic-associated weight gain and metabolic dysfunction exemplify the need to identify additional evidence-based treatments that are both efficacious and well tolerated in this vulnerable patient population. Lurasidone is an atypical antipsychotic agent that has demonstrated efficacy in the treatment of adults with schizophrenia in the dose range of $40-160 \mathrm{mg} / \mathrm{d}$. The objective of this randomized, double-blind, placebo-controlled study was to evaluate the acute efficacy and safety of lurasidone in adolescent patients with schizophrenia.

Methods: Adolescents (13-17 years old) diagnosed with schizophrenia (DSM-IV-TR criteria; Positive and Negative Syndrome Scale [PANSS] total score $\geq 70$ and Clinical Global Impression-Severity score $\geq 4$ ) were randomly assigned to 6 weeks of double-blind treatment with fixed-dose lurasidone $40 \mathrm{mg} / \mathrm{d}, 80 \mathrm{mg} / \mathrm{d}$, or placebo. Changes from baseline to week 6 in PANSS total and subscale (positive, negative, general psychopathology, excitability) scores were evaluated using mixed-model repeated-measures analysis. Treatment response was defined as $\geq 20 \%$ reduction from baseline in PANSS total score at week 6 (with 30 points subtracted from each score to account for the scale range) and was analyzed using a logistic regression model with last observation carried forward (LOCF) approach. The number needed to treat (NNT) to attain one additional responder was calculated as the reciprocal of the difference in response rates for lurasidone and placebo. 
Results: A total of 326 patients (mean age, 15.4 years) were randomized and received lurasidone $40 \mathrm{mg} / \mathrm{d} \quad(N=108)$, $80 \mathrm{mg} / \mathrm{d}(N=106)$, or placebo $(N=112)$. The PANSS total score at week 6 demonstrated a placebo-adjusted, leastsquares (LS) mean improvement of $-8.0 \quad(P<0.001$; effect size, 0.51$)$ for the $40 \mathrm{mg} / \mathrm{d}$ group and $-7.7(P<0.001$; effect size, 0.48 ) for the $80 \mathrm{mg} / \mathrm{d}$ group. Placebo-adjusted LS mean change for lurasidone $40 \mathrm{mg} / \mathrm{d}$ and $80 \mathrm{mg} / \mathrm{d}$, respectively, was -3.2 $(P<0.001$; effect size, 0.62$)$ and $-3.2(P<0.001$; effect size, 0.60$)$ on the PANSS positive subscale, -1.7 $(P=0.011$; effect size, 0.41$)$ and $-1.6(P=0.022$; effect size, $0.35)$ on the PANSS negative subscale, $-2.8(P=0.012$; effect size, 0.38$)$ and $-2.8(P=0.011$; effect size, 0.37$)$ on the PANSS general psychopathology subscale, and $-1.1(P=0.016$; effect size, 0.36$)$ and $-1.8(P<0.001$; effect size, 0.53$)$ on the PANSS excitability subscale. The proportion of patients classified as treatment responders at week 6 (LOCF) was $42.0 \%$ in the placebo group, $63.9 \%$ in the lurasidone $40 \mathrm{mg} / \mathrm{d}$ group $(P<0.001 ; \mathrm{NNT}=5)$, and $65.1 \%$ in the lurasidone $80 \mathrm{mg} / \mathrm{d}$ group $(P<0.001 ; \mathrm{NNT}=5)$. The most common adverse events (incidence $\geq 5 \%$ in either lurasidone group and at least twice the rate of placebo) for lurasidone $40 \mathrm{mg} / \mathrm{d}$, $80 \mathrm{mg} / \mathrm{d}$, and placebo, respectively, were nausea $(12.7 \%$, $14.4 \%, 2.7 \%)$, somnolence $(9.1 \%, 11.5 \%, 5.4 \%)$, akathisia (9.1\%, 8.7\%, $1.8 \%)$, vomiting $(8.2 \%, 6.7 \%, 1.8 \%)$, and sedation $(5.5 \%, 1.9 \%, 1.8 \%)$. The rate of study discontinuation due to adverse events was higher in placebo-treated versus lurasidone-treated patients $(8.0 \%$ vs $3.7 \%$, respectively). LS mean change in weight at week 6 for the lurasidone $40 \mathrm{mg} / \mathrm{d}, 80 \mathrm{mg} / \mathrm{d}$, and placebo groups was $0.17 \mathrm{~kg}, 0.49 \mathrm{~kg}$, and $0.05 \mathrm{~kg}$, respectively. Median change at week 6 (LOCF) in total cholesterol was $-4.0 \mathrm{mg} / \mathrm{dL},-2.0 \mathrm{mg} /$ $\mathrm{dL}$, and $-7.0 \mathrm{mg} / \mathrm{dL}$, respectively; in triglycerides was $-2.0 \mathrm{mg} /$ $\mathrm{dL}, 7.0 \mathrm{mg} / \mathrm{dL}$, and $-2.0 \mathrm{mg} / \mathrm{dL}$, respectively; and in glucose was $0.0 \mathrm{mg} / \mathrm{dL}, 1.0 \mathrm{mg} / \mathrm{dL}$, and $0.0 \mathrm{mg} / \mathrm{dL}$, respectively.

Conclusions: In this double-blind, placebo-controlled study of adolescent patients, lurasidone $(40 \mathrm{mg} / \mathrm{d}$ and $80 \mathrm{mg} / \mathrm{d}$ ) demonstrated statistically significant efficacy and clinically meaningful improvement across a wide spectrum of symptoms associated with schizophrenia. Lurasidone treatment was also generally well tolerated and associated with minimal changes in weight and metabolic parameters.

ClinicalTrials.gov identifier: NCT01911429.

Keywords: Schizophrenia, Lurasidone, Adolescence, Psychopharmacology.

Disclosure: Sunovion Pharmaceuticals Inc.: Employee, Self.

W216. Relationship Between Untreated Psychosis and Intrinsic Corticostriatal Connectivity in Patients With First-Episode Schizophrenia

Deepak Sarpal*, Delbert Robinson, Todd Lencz, Miklos Argyelan, Katherine Karlsgodt, Juan Gallego, Majnu John, John Kane, Philip Szeszko, Anil Malhotra

Hofstra Northwell School of Medicine, Glen Oaks, New York, United States

Background: Patients with first-episode psychosis on average experience psychotic symptoms for over two years prior to initiation of treatment. Increased duration of untreated psychosis (DUP) is associated with worsened clinical outcomes; however, evidence compiled from numerous studies suggests that longer DUP does not result in structural brain abnormalities. To date, few studies have examined the relationship between DUP and functional neuroimaging measures. In the present study we used a seedbased resting-state functional connectivity approach to examine the impact of DUP on cortcostriatal circuitry.

Methods: We examined patients with first-episode schizophrenia who underwent resting state scanning prior to entering 12 weeks of prospective treatment with secondgeneration antipsychotic drugs. DUP was quantified based on interviews with patients and their families. Patients were followed during treatment and response to treatment was defined, a priori, based on minimal psychotic symptom items of the Brief Psychiatric rating Scale. We generated functional connectivity maps from 6 regions of interest (ROIs) within the striatum in each hemisphere. Group level analyses were performed for each ROI, independently, with log transformed DUP as a covariate. Significance was defined at $p<0.05$, cluster corrected. Post-hoc analyses were performed to test whether significant results these group level analyses mediate the relationship between DUP and treatment response.

Results: We included 85 patients in our analyses (mean age 21 ; 61 males, 24 females) who had a mean DUP of 102 weeks ( $\mathrm{SD}=77$; median $=35$ weeks $)$. We found that increased DUP correlated with overall decreased functional connectivity between striatal nodes and frontal and parietal regions, some of which are within the central executive network. In posthoc analyses, a primary explanatory factor within these significant connections showed a mediation effect between DUP and treatment response.

Conclusions: Our results indicate that variation in corticostriatal circuitry may play a role in the relationship between increasing DUP and worsened response to treatment. Future prospective studies will be necessary to examine causal relationships DUP, striatal connectivity, and negative clinical outcomes.

Keywords: First Episode Schizophrenia, Resting State Functional Connectivity, Treatment Response, Recent Onset Psychosis, Cortico-Striatal Circuit.

Disclosure: Nothing to disclose.

\section{W217. Visual Contrast Sensitivity Deficits in Clinical High Risk and Early Psychosis Suggest Magnocellular Pathway Dysfunction That is Associated With Symptoms, Neurocognition and Global Functioning}

Kristin Cadenhead*, Manju Ilapakurti, Karen Dobkins

University of California, San Diego, La Jolla, California, United States

Background: Patients with schizophrenia, their first degree relatives and those with schizotypal personality disorder exhibit deficits in visual information processing that have been linked to cognitive deficits and social functioning. The visual information processing deficits have been hypothesized to occur primarily via the magnocellular subcortical pathways or dorsal processing stream. One means of parsing the integrity of the subcortical magnocellular and parvocellular pathways function is by using visual contrast sensitivities (VCS). Specifically, VCS for near threshold luminance 
(black/white) stimuli is known to be governed primarily by the magnocellular channel, while VCS for near threshold chromatic (red/green) stimuli is governed by the parvocellular channel.

Methods: VCS for luminance and chromatic stimuli (counterphase-reversing sinusoidal gratings, $1.22 \mathrm{c} /$ degree, $8.3 \mathrm{~Hz}$ ) was assessed in subjects at Clinical High Risk (CHR) for psychosis $(N=68)$, patients diagnosed with First Episode (FE) psychosis $(N=34)$, and Healthy Comparison $(\mathrm{HC})$ subjects $(N=63)$.

Results: Individuals in the early phase of psychosis exhibited reduced VCS in the luminance condition $\mathrm{F}(2,166)=3.42$, $p<0.05)$ when compared to $\mathrm{HC}$ population, suggesting deficits in magnocellular pathway function. Post hoc analysis demonstrated significant differences $(p<.05)$ between CHR and FE subjects when compared to controls. These luminance VCS deficits were not accounted for by antipsychotic use in the FE sample but the $16 \mathrm{CHR}$ subjects who were receiving an antipsychotic had more deficits than the CHR subjects not receiving antipsychotics $(p<0.05)$. There were also significant group by gender interaction effects $(\mathrm{F}[5,163\}=4.3, p<0.001)$ in the luminance condition due to deficits in FE Males $(p<0.01)$ as well as CHR Females $(p<0.01)$ vs Male and Female HCs respectively.

Impaired VCS in the Luminance condition was associated with greater levels of thought disorder in both CHR and FE groups while delusional symptoms were significantly associated with deficits in the FE sample. Neurocognitive deficits in the domains of verbal learning and memory, executive functioning and working memory were all associated with poor performance in the VCS paradigm in both CHR and FE samples. Finally, poor functioning was significantly correlated with deficits in the luminance condition in the FE sample.

Conclusions: Individuals in the early phase of psychosis demonstrate deficits in VCS that are most evident in the luminance condition, suggesting greater magnocellular pathway dysfunction. These VCS deficits are associated with thought disorder, delusional content, neurocognitive deficits and poor global functioning, implicating a central role of early visual information processing in multiple deficits of psychotic illness. Antipsychotic use in the CHR sample was associated with greater VCS deficits. It is unclear whether the antipsychotics caused the VCS deficits or whether those CHR subjects on antipsychotics or more symptomatic and perhaps at greater risk for psychosis.

Keywords: Schizophrenia Prodrome, First-Episode Psychosis, Visual Contrast Sensitivity, Visual Information Processing.

Disclosure: Nothing to disclose.

\section{W218. Sleep Disturbance is Associated With Higher Cerebrospinal Fluid Norepinephrine and Blood Pressure in Combat Veterans With Repetitive Blast Mild Traumatic Brain Injury and Posttraumatic Stress Disorder}

Elaine Peskind ${ }^{\star}$, Rebecca Hendrickson, Garth Terry, Murray Raskind, Gail Li

VA Puget Sound Health Care System, Seattle, Washington, United States

Background: Release of norepinephrine (NE) from diffuse locus ceruleus projections to neocortex and limbic brain areas increases arousal and attention to novel environmental stimuli. During normal sleep, brain NE release is reduced, and is very low or absent during "normal dreaming" REM sleep. In Veterans with posttraumatic stress disorder (PTSD) and in preclinical models of mild traumatic brain injury (mTBI), brain noradrenergic activity is elevated, particularly at night. These elevations may contribute to sleep disturbance in PTSD and in mTBI.

Methods: Forty-one Operation Enduring Freedom/Operation Iraqi Freedom/Operation New Dawn (OEF/OIF/OND) Veterans who experienced one or more blast concussion mTBIs (mean mTBIs =20) during Afghanistan and/or Iraq deployment underwent lumbar puncture for measurement of cerebrospinal fluid (CSF) NE concentrations. Most Veterans met criteria for full or subsyndromal PTSD. Sleep disturbances were quantified with the Pittsburgh Sleep Quality Index (PSQI), PTSD symptoms were quantified with the PTSD Checklist-Military (PCL-M), depression was quantified with the Patient Health Questionnaire-9 (PHQ-9), postconcussive symptoms were quantified with the Neurobehavioral Symptom Inventory (NSI), and blood pressure (BP) was quantified with a manual sphygmomanometer.

Results: Veterans with moderate or severe sleep disturbance had higher CSF NE $(150 \pm 90$ vs. $69 \pm 16 \mathrm{pg} / \mathrm{ml}), p<0.01)$; higher systolic BP $(129 \pm 13$ vs. $113 \pm 10 \mathrm{mmHg}, p<0.01)$; higher diastolic BP $(82 \pm 12$ vs. $74 \pm 8 \mathrm{mmHg}, p=0.02)$; higher PCL-M hyperarousal $(18 \pm 5$ vs. $10 \pm 3, p<0.01)$ symptom cluster scores; higher PHQ-9 scores (13 \pm 8 vs. $4 \pm$ $5)$; and higher NSI postconcussive symptom scores $(35 \pm 16$ vs. $18 \pm 13$ ) than did Veterans with no or mild sleep disturbance.

Conclusions: These results demonstrate a high prevalence of PTSD in OEF/OIF/OND Veterans who have experienced blast concussion mTBIs. In such Veterans, troublesome sleep disturbance is associated with higher indices (CSF NE) of brain noradrenergic activity and co-regulated peripheral sympathetic nervous system activity (BP) as well as daytime hyperarousal symptoms. Although these associations do not indicate direction causality, the efficacy of anti-adrenergic medications for PTSD sleep disturbance and daytime hyperarousal suggest that brain noradrenergic hyperactivity contributes to the pathophysiology of PTSD comorbid with mTBI.

Keywords: Mild Traumatic Brain Injury, PTSD, Norepinephrine, Cerebrospinal Fluid.

Disclosure: Nothing to disclose.

\section{W219. Characterizing Sleep Spindles in 11,630 Individuals From the National Sleep Research Resource}

Shaun Purcell*, Dara Manoach, Charmaine Damenuele, Brian Cade, Sara Mariani, Roy Cox, Richa Saxena, Jen Pan, Jordan Smoller, Susan Redline, Robert Stickgold

Harvard Medical School, Boston, Massachusetts, United States

Background: Sleep spindle activity, an EEG signature of nonREM sleep, reflects a heritable set of traits, implicated in both sleep regulation and normal cognitive functioning, that may also be biomarkers of neuropsychiatric disease risk. As a 
prelude to future human molecular genetic studies on this phenomenon, we detected and characterized spindles in 11,630 individuals aged 4 to 97 from the US National Sleep Research Resource, on whom we combined polysomnography, demographic and medical data, in order to describe fundamental features and epidemiological distributions.

Methods: We applied automated signal-processing and spindle detection to just under 30,000 hours of stage 2 nonrapid eye movement (N2) sleep.

Results: While spindle phenotypes demonstrated high testretest reliabilities and stability over time, they showed distinct developmental trajectories across the life course, which varied for spindles of different frequencies. Spindles also demonstrated age- and frequency-dependent dynamics across the course of the night. Leveraging a subset of related individuals, we demonstrated that genetic factors significantly contribute to the variation and covariation of spindle and spectral sleep phenotypes. We also identified previously unappreciated correlates of spindle activity that could impact genetic and other studies, including racial differences and a confounding effect of body mass index, mediated by increased cardiac interference in the EEG signal. Finally, we attempted to untangle the impact that various factors have on spindle amplitude as opposed to spindle density (number per minute), highlighting some critical measurement issues.

Conclusions: Taken together, our findings have the potential to inform future studies that aim to better understand the genetic architecture of sleep spindles and their relation to behavioral and health outcomes including neuropsychiatric disease.

Keywords: Sleep Spindles, EEG, Heritability, Epidemiology. Disclosure: Nothing to disclose.

\section{W220. Consequent Sleep After One-Night Deprivation Alters Cortical Grey Matter Volume and Reduces Cerebrospinal Fluid Space}

Sukru Demiral ${ }^{\star}$, Dardo Tomasi, Corinde Wiers, Elsa Lindgren, Gregg Miller, Lori Talagala, Ehsan Shokri Kojori, Gene-Jack Wang, Helene Benveniste, Nora Volkow

National Institute on Alcohol Abuse and Alcoholism, Bethesda, Maryland, United States

Background: Sleep deprivation is known to have a detrimental effect on cognitive performance (Killgore, 2010) and can temporarily influence brain microstructure, leading to changes in white matter between rested and sleep deprived states (Elvsashagen et.al., 2015). Preclinical studies have shown that cerebrospinal fluid (CSF) transport into the brain-wide glymphatic pathway comprising peri-vascular compartment and brain parenchyma including grey mater (GM) leading to clearance of waste products from the interstitial fluid (ISF) space in particular during deep wave sleep (Xie et.al., 2014). While these studies are intriguing in regards to the role of sleep, it is largely unknown whether or not glymphatic system dynamics during sleep observed in rodent brain are mirrored in the live human brain. Accordingly, in this pilot study, we examined CSF and GM volume changes in healthy volunteers during sleep and the awake state.

Methods: Ten healthy subjects ( 7 males, 3 females; ages 31 53) visited the clinic on two nights; one night they were allowed to sleep, and the other night they were kept awake under supervision of a nurse. Sessions were done in randomized order. During the night, electroencephalography (EEG) recording was used to assess the quality of sleep and sleep deprivation. Structural Magnetic Resonance Imaging (sMRI) scans were performed in a $3 \mathrm{~T}$ Siemens Prisma scanner with a 32-channel RF coil in the following mornings at 9AM. Participants stayed awake in the scanner after the rested night, but were instructed to fall sleep after the sleepdeprived night. An MRI-compatible video camera was used to monitor sleep during MRI. Physiological signals (respiration and cardiac) were recorded using a BIOPAC system. High resolution T1 (3d MPRAGE, TR $=2400 \mathrm{~ms}$, TE $=2.14$ $\mathrm{ms}, \mathrm{TI}=1000 \mathrm{~ms}$, Flip Angle $=8 \mathrm{deg}, 0.7 \mathrm{~mm}$ isotropic) and T2 $(3 \mathrm{~d}$ T2-SPACE, TR $=3200 \mathrm{~ms}, \mathrm{TE}=565 \mathrm{~ms}$, Flip Angle $=$ variable, $0.7 \mathrm{~mm}$ isotropic) images were entered into Freesurfer program to create volumetric parcellations for each session separately. CSF volume was calculated by subtracting the whole brain volume (including grey and white matter, and cerebellum) from the estimated intracranial volume (ICV). Ventricle volumes were also calculated. $\mathrm{H} 2 \mathrm{O}$ levels in the grey matter in the precuneus was measured with MR spectroscopy.

Results: Paired t-tests were performed to compare brain volumetric measures during sleep versus rested wakefulness. Sleep deprivation relative to rested wakefulness showed a significant decrease in the CSF volume $(\sim 20,000 \mathrm{~mm} 3$, $p<.05)$ and a significant increase in grey matter volume $(\sim 8,000 \mathrm{~mm} 3, p<.05)$. However, there were no differences in total ventricle size. White matter volume and GM H2O increased during sleep compared to rested wakefulness at trend levels $(p=.1)$. There was also a negative correlation between the CSF volume and GM H2O in the sleep session $(\mathrm{r}=-.54, P=.066)$.

Conclusions: Our preliminary results indicate that sleep (sleep-deprived) results in cortical and CSF volume changes in addition to increases in GM H2O supporting the concept that ISF space volume may also increase during sleep in humans. CSF increases in ISF space during sleep might not only be driven by cellular shrinkage and other microscale changes, but may also be related to GM flexibility and spatial distribution constraints imposed by macro scale changes.

Keywords: Brain Structure, Sleep, CSF.

Disclosure: Nothing to disclose.

\section{W221. Central Amygdala Corticotropin Releasing Factor Neurons Encode and Modulate Binge Drinking and Relapse}

Dennis Sparta*, James Irving, Colin Maehler, Kasey Girven

University of Maryland School of Medicine, Baltimore, Maryland, United States

Background: Alcohol abuse costs the U.S. approximately $\$ 223.5$ billion yearly; binge drinking accounts for approximately three-quarters of that. Therefore, binge drinking is a 
severe public health problem. Corticotropin releasing factor (CRF) neurons in the central amygdala (CeA) are thought to modulate binge drinking, but the precise circuit mechanisms remain poorly understood.

Methods: In our first set of experiments, we examined the effects of repeated photostimulation of CeA CRF neurons on binge drinking. Next, we acutely pharmacogenetically inhibited CeA CRF neurons immediately before the start of a binge drinking session. In a final set of experiments, we combined optogenetics with in vivo electrophysiology to identify and record from CeA CRF neurons in freely moving mice during a binge drinking session.

Results: We found that repeated optogenetic stimulation of CeA CRF neurons did not affect acute binge drinking. However, it did increase binge drinking during context reinstatement sessions. Acute pharmacogenetic inhibition of CeA CRF neurons did significantly reduce binge drinking compared to controls. Finally, we were able to identify and record from putative $\mathrm{CeA} \mathrm{CRF}$ neurons in vivo during a binge drinking task. We found that putative CeA CRF neurons exhibited a net increase of firing in response to licks of ethanol. Furthermore, these neurons became more active during the session as the mouse consumed more ethanol.

Conclusions: Our data indicates that CeA CRF neurons play a complex and critical role in binge drinking. Thus, activity of CeA CRF neurons may be involved in the creation of longterm impulse to consume ethanol

Keywords: Optogenetics, Binge Drinking, CorticotropinReleasing Factor (CRF), Central Amygdala, Electrophysiology.

Disclosure: Nothing to disclose.

\section{W222. Striatal Fast-Spiking Interneuron Encoding of Actions}

Bradley Roberts, Michael White, Mary Patton, Rong Chen, Brian Mathur*

University of Maryland School of Medicine, Baltimore, Maryland, United States

Background: Habits are encoded by the dorsolateral striatum, which is enriched in parvalbumin-expressing GABAergic fast-spiking interneurons (FSIs). Despite the powerful, feedforward inhibition of striatal medium spiny projection neurons (MSNs) that FSIs provide, exactly how FSIs activate to sculpt actions remains unknown.

Methods: To address this issue and to overcome the inherent limitation of in vivo electrophysiological recordings FSIs, which represent less than $1 \%$ of all striatal neurons, we employ in vivo calcium imaging of GCaMP6f in FSIs in awake, behaving mice in an open field.

Results: We found that FSIs synchronously activate just prior to action initiation to encode action velocity dynamics. Supporting a critical role for FSI synchrony, we show that synchronous optogenetic activation of FSIs has little impact on actions, while optogenetic induction of asynchronous FSI activity engenders hyperkinetic motor activity.

Conclusions: These findings suggest a causal role for FSI synchrony in action selection, and that FSIs may be critical for appropriately timing MSN activity for the refinement of well-learned actions such as habits.
Keywords: Dorsal Striatum, GABAergic Interneurons, Calcium Imaging, Electrophysiology, Synchrony.

Disclosure: Nothing to disclose.

\section{W223. Adolescent Intermittent Alcohol Exposure Enhances the Reinforcing Properties of Alcohol in the Central Amygdala During Adulthood in Male Wistar Rats}

Christopher Knight*, Robert Waeiss, Philip Johnson, Gerald Deehan, Richard Bell, William McBride, Sheketha Hauser, Zachary Rodd

Indiana University School of Medicine, Indianapolis, Indiana, United States

Background: The biological consequences of adolescent alcohol consumption on adult responsiveness to drugs of abuse are poorly understood. In humans, early onset of alcohol consumption is associated with increased risk of alcoholism and other drug dependency during adulthood. The central amygdala (CeA) has been shown to mediate anxiety, alcohol consumption, and alcohol dependency/ withdrawal. Recent data from our laboratory has indicated that $\mathrm{EtOH}$ is simultaneously rewarding and anxiolytic in the $\mathrm{CeA}$. The current experiment was conducted to determine the effects of adolescent EtOH exposure (binge) on the reinforcing properties of ethanol in the CeA during adulthood.

Methods: The adolescent intermittent ethanol (AIE) exposure model was used to produce consistent ethanol exposure in adolescent male Wistar rats. Briefly, the AIE regimen consisted of single doses of $5 \mathrm{~g} / \mathrm{kg} \mathrm{EtOH}(20 \% \mathrm{v} / \mathrm{v}$ by intragastric gavage) in a 2-day on, 2- day off sequence such that the animals received 16 intragastric ethanol treatments from PND25-55. Control rats were given similar water gavage treatment. On PND90, animals were implanted with a guide cannula aimed at the CeA. One week later, rats were allowed to self-administer aCSF, 100, 150, or $200 \mathrm{mg} \% \mathrm{EtOH}$ directly into the CeA. All rats were allowed to self-administer the assigned EtOH concentration for 4 sessions, and then were exposed to aCSF substitution (extinction) during sessions 5 and 6 . The original infusate was returned during the 7th session (reinstatement).

Results: Adult naïve male Wistar rats readily selfadministered $200 \mathrm{mg} \%$ into the CeA. In contrast AIE Wistar rats self-administered 100, 150, and $200 \mathrm{mg} \% \mathrm{EtOH}$ into the $\mathrm{CeA}$ in a dose-dependent manner. Specifically, control and AIE responded for aCSF comparably (about 8 infusions/ session). The number of infusions received per session was only significantly different in control rats given $200 \mathrm{mg} \%$ $\mathrm{EtOH}$ to self-administer (28 infusions/session). In contrast, AIE Wistar rats responded significantly more for 100, 150, and $200 \mathrm{mg} \%$ (18, 49, and 39 infusions/session, respectively). Thus, the data indicated a leftward and upward shift to the dose-response curve for EtOH self-administration into the CeA produced by AIE treatment.

Conclusions: The data indicate that exposure to 'binge-like' ethanol consumption during adolescence produced persistent alterations within the CeA that enhanced the reinforcing properties of ethanol during adulthood. Previous research in our laboratory has indicated that the nociceptin system 
mediates ethanol reinforcement in the CeA. Therefore, it is possible that AIE produced persistent alterations in the nociceptin system in the CeA. Future research will be conducted to determine the biological basis for the alteration in ethanol reinforcement in the CeA produced by adolescent ethanol exposure.

Keywords: Adolescent Alcohol Exposure, Central Amygdala, Intracranial Self-Administration, Reward.

Disclosure: Nothing to disclose.

\section{W224. Adolescent Ethanol or Nicotine Exposure Enhances the Neurochemical Response to Nicotine Within the Mesolimbic Dopamine System During Adulthood}

Gerald Deehan, Jr, Christopher Knight, R. Aaron Waiess, Richard Bell, William McBride, Sheketha Hauser, Zachary Rodd*

Indiana University School of Medicine, Indianapolis, Indiana, United States

Background: Early onset of drug use is associated with an increase rate of drug addiction during adulthood. Individuals that consume alcohol during adolescence also initiate other drug use during this time period, including nicotine usage. The co-morbidity of alcohol and nicotine use/dependence is pervasive throughout life. Recent data from our laboratory has indicated that adolescent alcohol consumption enhances the reinforcing properties of alcohol within the posterior VTA and the neurochemical response to ethanol within the mesolimbic dopamine system. The current experiments examined the effects of alcohol or nicotine exposure during adolescence on the ability of nicotine microinjected into the posterior VTA to stimulate dopamine (DA) release in the nucleus accumbens shell (AcbSh) during adulthood.

Methods: Alcohol preferring $(\mathrm{P})$ rats were given access to alcohol during post-natal day (PND) 30-50 (voluntary consumption averaged $5.8 \mathrm{~g} / \mathrm{kg} /$ day). Animals were withheld from drug exposure until after PND90. After that time point, nicotine $(0,1,10$ or $50 \mu \mathrm{M})$ was microinjected (5 sec infusion-15 sec timeout, 30 infusions) into the posterior VTA and microdialysis samples were collected from the AcbSh. A similar experiment examined the effects of adolescent nicotine exposure on the response to nicotine within the mesolimbic dopamine system during adulthood. Briefly, Wistar male rats were administered 0 or $0.5 \mathrm{mg} / \mathrm{kg}$ nicotine during post-natal day 30-55. After PND 90, nicotine was microinjected directly into the posterior VTA (100 or $200 \mu \mathrm{M})$ and DA levels were recorded in the AcbSh.

Results: In P rats that consumed ethanol during adolescence there was a leftward and upward shift to the dose-response curve for the ability of nicotine microinjected into the posterior VTA to stimulate DA release in the AcbSh. Specifically, microinjection of $1 \mu \mathrm{M}$ nicotine into the posterior VTA stimulated (>50\%) DA release in the AcbSh (this effect was not observed in non-drinking $\mathrm{P}$ rats). Further, microinjection of $50 \mu \mathrm{M}$ nicotine into the posterior VTA of adolescent drinking $P$ rats invoked a release of DA in the AcbSh that far exceed that observed in adolescent nondrinking P rats (194\% versus 78\%, respectively). Adolescent treatment in Wistar rats also enhanced adult responsiveness to nicotine within this system. In adolescent naïve Wistar rats, microinjections of 100 or $200 \mu \mathrm{M}$ nicotine into the posterior VTA stimulated a similar increase in DA release in the AcbSh (50-60\%). In contrast, microinjections of these nicotine concentrations into the posterior VTA in Wistar rats treated with nicotine during adolescence evoked a much larger response (187 and 234\%, respectively).

Conclusions: The data indicate that adolescent exposure to ethanol or nicotine enhances the adult response to nicotine. The enhanced stimulatory response to nicotine within the drug reward pathway may be part of the biological sequela caused by adolescent drug use which promotes/facilitate adult drug use. Further, the data indicate that there is crossreactivity between alcohol and nicotine in producing neuroadaptations that enhance the effects of the other compound during adulthood.

Keywords: Adolescence, Nicotine, Alcohol.

Disclosure: Nothing to disclose.

\section{W225. Presentation of Excitatory Conditioned Cues Result in the Activation of GABA Interneurons Within the Basolateral Amygdala}

Sheketha Hauser*, Gerald A. Deehan, Jr, Christopher P. Knight, Robert A. Waeiss, William J. McBride, Zachary A. Rodd

Indiana University School of Medicine, Indianapolis, Indiana, United States

Background: Conditioned cues can enhance (excitatory) or suppress (inhibitory) EtOH-seeking. Presentation of excitatory conditioned cue (CS+) in a non-drug paired environment results in an increase in dopamine and glutamate levels in the basolateral amygdala (BLA). In contrast, presentation of an inhibitory condition cue (CS-) in a non-drug paired environment reduces dopamine and glutamate levels in the BLA. Neuronal activity is increased in the BLA by presentation of a CS+ in a non-drug paired environment, but there is no significant alteration produced by presentation of a CS- or a neutral condition cue (CSO). Pharmacological silencing of the BLA blocks the enhancement of EtOH-seeking produced by presentation of the CS+.

Methods: The current experiments were conducted to determine the characteristics of neurons in the BLA activated by presentation of a CS+. The involvement of glutamatergic projection neurons containing $\mathrm{mGlu} 2 / 3$ receptors and GABAergic interneurons within the BLA in mediating the actions of CS+ was assessed. Briefly, rats were allowed to selfadminister $\mathrm{EtOH}$ daily under operant conditions for 10 weeks in the presence of an odor cue (CS+). Rats were then subjected to extinction training for 7 days in the presence of another odor cue (CS-). Rats were exposed to an additional odor cue (CSO) in a neutral environment during both CS+ and CS- exposure. On test day, animals were placed into a non-drug paired environment and were exposed to the CS+, CS-, or CS0 for $20 \mathrm{~min}$. Brain slices were then dual labelled for cfos and mGlu2/3 or cfos and GABA.

Results: The data indicated that the; 1 ) overall number of mGlu2/3containing neurons in the BLA was equal for all 3 conditioned cues, 2) the number of dual labeled cfos and mGlu2/3 neurons were also equivalent, but 3 ) there was a 
significant more non-mGlu2/3 receptor neurons activated by presentation of the CS+ compared to all other groups. In contrast, here was a significant effect of CS+ on both total number of cFos+ neurons and dual labelled cFos $+/ \mathrm{GABA}+$ neurons.

Conclusions: Therefore, the data indicate that presentation of the CS+ activates GABA interneurons in the BLA, and these neurons may mediate the effects of excitatory conditioned cues on drug-seeking.

Keywords: GABAergic Interneurons, Basolateral Amygdala, Conditioned Cues, Ethanol-Seeking, Alcohol Preferring P Rats.

Disclosure: Nothing to disclose.

\section{W226. Alcohol-Induced Place Preference in Humans is Robust and Reproducible}

Emma Childs*, Joseph A Lutz, Jessica DeArcangelis

University of Illinois at Chicago, Chicago, Illinois, United States

Background: Conditioned place preference has long been used to measure rewarding drug effects in rodents. Recently, we have translated the paradigm to humans using analogous procedures and outcome measures. The primary aim of this experiment was to replicate the findings from our prior place conditioning study with alcohol at a second site to demonstrate reliability of this new human laboratory model. Methods: Healthy male and female non-dependent alcohol drinkers participated in the experiment. They completed six conditioning sessions, 3 with $0.8 \mathrm{~g} / \mathrm{kg}$ alcohol (ALC) and 3 with a non-alcoholic control beverage (noALC). One group of participants (PAIRED) always received ALC in one distinct room and noALC in a separate distinct room. Another group (UNPAIRED) received ALC and noALC in both rooms. We measured the change in time spent in the rooms during $10 \mathrm{~min}$ alcohol-free room exploration tests conducted before and after all conditioning sessions had been completed.

Results: At the post-conditioning room exploration test, individuals in the PAIRED group exhibited a significant increase in the percentage time spent in the room paired with alcohol administration while individuals in the UNPAIRED group did not $(p<0.05)$.

Conclusions: These findings replicate those of our prior study of alcohol place conditioning indicating that this new human model is robust and reproducible at a different site. The use of this new paradigm will advance our understanding of the pre-clinical model and will also be a useful tool to study the role of conditioned associations in drugtaking and the development of alcohol use disorder. In particular, an exciting application of this model will be to evaluate potential therapeutic approaches to target drug-cue associations in the treatment of drug and alcohol addiction. Keywords: Alcohol, Conditioned Place Preference, Humans. Disclosure: Nothing to disclose.
W227. Does Decision-Making Modify the Association Between Parental Education and Cannabis use?

\section{Catalina Lopez-Quintero*, Samuel Hawes, Raul Gonzalez}

Florida International University, Miami, Florida, United States

Background: Population and clinical based studies have documented that childhood disadvantage predicts cannabis use and cannabis use related problems (Fothergill et al., 2016; Bowes et al., 2012). Furthermore, deficiencies in executive function (EF) predict drug use onset (Peeters, et al., 2015; Tarter et al., 2003) and moderate the relationship between amount of cannabis use and development of cannabis-related problems (Gonzalez et al., 2015). However, the extent to which neurocognitive processes and socio-economic factors interact to influence cannabis use and cannabis-related problems has not been well established. In this study, we assessed the role of decision-making as a potential moderator of the relationship between socio-economic factors and cannabis use. The a priori hypothesis guiding this study is that among adolescents, those with deficits in decisionmaking performance and whose parents achieved the lowest level of education will have a greater likelihood of reported cannabis use and more cannabis use related-symptoms.

Methods: We analyzed baseline data from 313 Latino adolescents (ages 14-17 ), who completed an assessment that included measures of demographics, medical history, mental health, substance use, and neurocognitive functioning, as part of an on-going longitudinal study that assesses decision-making and episodic memory in trajectories to cannabis addiction (R01DA031176, PI: Raul Gonzalez). Decision-making was assessed with the Iowa Gambling Task (IGT) and parental education was used as a proxy measure for socio-economic status. Descriptive statistics were carried out to characterize the sample, and multivariable-adjusted logistic regression models were used to assess the association between parental education and lifetime cannabis use, cannabis use in the month prior to the assessment, and endorsement of at leats one DSM-IV symptom for a cannabis use disorder in the six-months prior to assessment among lifetime cannabis users. Subgroup differences in these associations, based on tertiles of IGT performance, were tesded by including an IGT tertile* parental education interaction term in the models.

Results: About 8 in 10 study participants reported using cannabis in their lifetimes, and 5 in 10 in the month prior to the assessment. Among lifetime cannabis users, about half (47.6\%) had experienced at least one DSM-IV symptom for a cannabis use disorder in the six-months prior to assessment, and more than one-quarter $(28.5 \%)$ met criteria for a cannabis use disorder in the six-months prior to assessment. Results of the logistic regression models indicated that compared to adolescents with highly educated parents, those whose parents had the lowest levels of education were more likely to report ever having used cannabis $(\mathrm{AOR}=2.19$; $p<0.05)$. No association was found between parental education and cannabis use in the month prior to the assessment or occurrence of a cannabis use disorder symptoms in the six-months prior to assessment. However, these findings appear to be influenced by performance in 
decision-making. Specifically, among study participants with an IGT score in the lowest tertile, the likelihood of cannabis use in the month prior to the assessment $(A O R=5.42$; $p<0.02$ ) was significantly greater among those whose parents reported the lowest level of education, relative to those with highly educated parents. Among study participants with an IGT score in the lowest tertile, the odds of ever having used cannabis $(\mathrm{AOR}=10.9 ; p<0.05)$, and experiencing at least one cannabis use related-symptom in the sixmonths prior to the assessment $(\mathrm{AOR}=27.2 ; p<0.01)$ were significantly greater among those whose parents achieved an intermediate level of education compared to those with highly educated parents.

Conclusions: Our findings suggest that decision-making modifies the association between parental education and cannabis use, such that relative to adolescents whose parents had the highest level of education and with a high performance in the decision-making task, those with poor decision-making and intermediate or low parental education levels exhibit greater odds of cannabis use and cannabis userelated symptoms. Longitudinal studies with better assessments of socio-economic status and cannabis use and decision-making trajectories are needed to understand the mechanisms linking neurocognitive processes, socioeconomic factors, and drug use trajectories among adolescents.

Keywords: Cannabis, Decision Making, SocioEconomic Status.

Disclosure: Nothing to disclose.

\section{W228. Neuronal Mechanisms Supporting Memory System Bias Following Withdrawal From Cocaine}

Eric Harvey, Nick Angelillis, Adam Gold, Pamela Kennedy*

University of California, Los Angeles, Los Angeles, California, United States

Background: Background: Transcriptional changes throughout brain circuits that regulate motivation and reward have been largely implicated in the cognitive and behavioral adaptations associated with excessive drug use and relapse. However, less is known about how drugs of abuse affect learning and memory processes more broadly - which may contribute to inefficiencies in current treatment strategies. Decades of research in the field of learning and memory suggest that the brain is composed of multiple memory systems. Among these systems is the hippocampus (HC), which encodes information flexibly and supports behaviors that can be rapidly updated. Another system is the dorsolateral striatum (DLS), which encodes information inflexibly and supports behaviors that are "hard-wired" (habits). Evidence suggests that both systems process information concurrently, but compete for control over behavioral selection across experience (Packard \& McGaugh, 1996; McDonald \& White, 2002). Deficits in tasks requiring the use of HC-dependent memory processing predict poor treatment outcomes in cocaine users and preference towards the use of DLS-dependent learning strategies has been associated with addictive drug use (Aharonovich et al., 2006; Bohbot et al., 2013). The neural mechanisms supporting the dissociable effects of drugs of abuse on memory systems in the brain remain poorly understood. Here we investigated the molecular and behavioral effects of prior cocaine exposure in a dual-solution plus maze task that can be solved using either HC-dependent or DLS-dependent learning strategies.

Methods: To determine the extent to which withdrawal from repeated cocaine exposure biases competition between memory systems, adult male Long-Evans rats were given daily injections of cocaine $(20 \mathrm{mg} / \mathrm{kg} \cdot 14$ days $)$ or saline. Following a 3-week drug free period rats were trained and tested in a dual-solution plus maze task (Packard \& McGaugh, 1996). In daily sessions rats learned to approach a consistently baited goal arm from the same start arm. Probe trials in which rats were started from the opposite start arm were administered to test whether rats adopted an HCdependent spatial strategy or a DLS-dependent response strategy to solve the task. The DLS and HC were collected to assess differential regulation of gene transcription associated with learning strategy.

Results: Consistent with previous findings, saline treated rats adopted a "cognitive", HC-dependent learning strategy early in training. In contrast, repeated cocaine treatment (replicated with self-administration) biased rats towards the immediate use of a "stimulus response/habit" learning strategy dependent on the DLS. This memory system bias was associated with increased and decreased expression of the transcription factor C/EBP beta and other plasticity genes in the DLS and HC, respectively. We further report that treatment with the kappa opioid receptor antagonist, JDTic, throughout withdrawal supports the use of HC-dependent learning strategies in cocaine-exposed animals. Investigations into epigenetic mechanisms mediating the effects of prior cocaine exposure on transcriptional processes and memory system bias are underway and will be presented.

Conclusions: Discussion: These findings suggest that prior cocaine exposure may cause persistent changes in plasticity and gene regulation in the DLS and $\mathrm{HC}$, which may promote the capture of new learning by the DLS and support the pervasive use of an inflexible habit memory system (DLS) in lieu of a more flexible, easily updated memory system (HC). We hypothesize that this shift is in part mediated through epigenetic regulation of gene inducibility in both the DLS and $\mathrm{HC}$.

Keywords: Cocaine Addiction, Gene Expression, Kappa Opioid Receptor.

Disclosure: Nothing to disclose.

\section{W229. Pharmacogenetics of Naltrexone for Alcoholism: Human Laboratory and Neuroimaging Findings in Individuals of East Asian Descent}

Lara Ray*, ReJoyce Green, Dara Ghahremani, Kent Hutchison, Taylor Rohrbaugh, Karen Miotto

University of California, Los Angeles, California, United States

Background: Pharmacogenetic studies have advanced the gene coding for $\mu$-opioid receptors (OPRM1) as a potential moderator of responses to naltrexone in alcohol use disorder (AUD) samples. The most widely studied variant of the 
OPRM1 gene is the Asn40Asp single nucleotide polymorphism (SNP), a functional mutation thought to affect receptor activity such that the Asp40 allele binds $\beta$-endorphin three times stronger than the Asn 40 allele. Studies have found that Asp40 carriers have a stronger striatal dopamine response to intravenous alcohol administration and report stronger subjective feelings of alcohol reward. Findings from the COMBINE Study demonstrated that when treated with Medication Management alone and naltrexone, $87.1 \%$ of Asp40 carriers had a good clinical outcome, compared with only $54.8 \%$ of Asn 40 homozygotes. While these findings are promising, studies have also highlighted allele frequency imbalance as a function of ethnicity such that the Asp 40 allele frequency is approximately $20 \%$ in Caucasians, $5 \%$ in individuals of African Ancestry, and as high as 50\% among individuals of East Asian descent.

Methods: To extend pharmacogenetics of naltrexone, this study randomized heavy drinkers $(N=87$, average age 27.2) of East Asian descent, each of whom completed two doubleblinded, counterbalanced intravenous alcohol administration sessions, one after taking NTX (50 mg/day) and one after taking matched placebo for five days. A subset of MRIeligible participants $(N=56)$ also completed a functional neuroimaging (fMRI) task examining cue-induced craving for alcohol in each medication condition.

Results: Preliminary mixed model analyses of the alcohol administration paradigm confirmed that, as expected, naltrexone attenuated the urge to drink alcohol across rising levels of $\mathrm{BrAC}$, as compared to placebo $(\beta=-0.217, \mathrm{SE}=0.11$, $p<0.05)$. Preliminary analyses of the fMRI cue-reactivity (i.e., taste cues) paradigm, indicated that naltrexone was associated with attenuated activation of the amygdala, bilateral post-central gyrus, and left frontal pole during the presentation of alcohol vs. control taste cues, and as compared to placebo (uncorrected $p<0.001$ ). Analyses are underway to test the effects of Asn40Asp SNP of the OPRM1 gene as a moderator of responses to naltrexone in the human laboratory and in the neuroimaging paradigms, including gene dose-response models.

Conclusions: These analyses will elucidate the pharmacogenetic effects of the Asn40Asp SNP of the OPRM1 gene on biobehavioral and neural markers of response to naltrexone in individuals of Asian descent, an ethnic group most likely to express the positive predictive allele (Asp40). These findings seek to inform personalized medicine approaches for AUD and to address health disparities by advancing pharmacogenetic studies in ethnic minority groups.

Keywords: Alcohol, Naltrexone, Pharmacogenetics.

Disclosure: Nothing to disclose.

\section{W230. A Dual Mechanism for Regulation of GLT-1 Expression by Cocaine}

Ronald Kim, Emily Williams, Marian SepulvedaOrengo, Kati Healey, Kathryn Reissner*

University of North Carolina at Chapel Hill, Chapel Hill, North Carolina, United States

Background: Drug seeking after withdrawal is associated with alterations in glutamatergic signaling within the nucleus accumbens (NAc), including decreased glutamate uptake.
The astroglial glutamate transporter, GLT-1, accounts for the majority of glutamate clearance from the synapse and plays an important role in the modulation of glutamatergic signaling. Drugs of abuse, including cocaine, significantly decrease GLT-1 protein expression in the NAc, and pharmacological restoration of GLT-1 can reduce cocaine seeking in the rat self-administration and reinstatement model. Interestingly, the magnitude of cocaine-induced GLT-1 suppression is a function of both degree of cocaine exposure and length of withdrawal. However, the mechanism of GLT-1 protein regulation by cocaine is completely unknown. Because modulation of GLT-1 is a seminal feature of cocaine seeking behavior, we chose to investigate the mechanism by which cocaine self-administration can influence GLT-1 protein and mRNA expression, using two commonly employed behavioral paradigms.

Methods: We utilized both the short-access self-administration and extinction, as well as the long-access incubation behavioral models to assess GLT-1 expression. Male Sprague-Dawley rats were trained to self-administer i.v. cocaine $(0.75 \mathrm{mg} / \mathrm{kg} / \mathrm{inf})$ or saline for either 2 or 6 hours per day for 10 days, followed by either 3 weeks of extinction training ( $2 \mathrm{hrs} /$ day) or 45 days of forced abstinence. Rats were rapidly decapitated 24 hours following the last extinction/withdrawal day, and tissue was harvested from the NAc, prelimbic cortex (PL) and basolateral nucleus of the amygdala (BLA). In the first study, gene expression was then examined using qRT-PCR and gene specific primers for GLT-1 splice variants (GLT-1a and GLT-1b). In the second study, an antibody against 5-methylcytosine was used to perform methylated DNA immunoprecipitation (MeDIP), to measure GLT-1 gene methylation. The amount of GLT-1 methylation was then quantified using quantitative PCR and primers specific to GLT-1.

Results: No differences were found in GLT-1a or GLT-1b mRNA levels between cocaine and saline rats in the shortaccess self-administration and extinction model in any of the 3 brain regions analyzed. In contrast, in the NAc of longaccess cocaine incubated rats, a significant decrease was found in GLT-1a mRNA, however no difference was found in GLT-1b. A similar decrease in GLT-1a specifically was observed in the BLA. In the PL of cocaine incubated rats, we observed a trend toward a decrease in GLT-1a, and a statistically significant decrease in GLT-1b gene expression. To further investigate the downregulation of GLT-1 mRNA in the NAc, we assessed levels of GLT-1 gene methylation following protracted withdrawal from long-access selfadministration of cocaine or saline. Long access-cocaine self-administration and withdrawal resulted in a several-fold increase in GLT-1 gene methylation over levels from rats that self-administered saline. Current studies are designed to assess whether GLT-1 gene methylation is observed immediately after long-access self-administration, or whether the incubation period is required.

Conclusions: We have found that GLT-1 protein and mRNA levels in the NAc are differentially regulated based on cocaine exposure and withdrawal time, indicating multiple mechanisms of regulation. While short-access self-administration and extinction is sufficient to suppress protein levels, a significant decrease in GLT-1 mRNA is observed in the NAc only after long access cocaine self-administration and withdrawal. Furthermore, withdrawal after long-access 
cocaine self-administration induces specific changes in GLT-1 gene expression that differ among the GLT-1 splice variants. The decrease in GLT-1 mRNA in the NAc is associated with hypermethylation of the GLT-1 gene, suggesting transcriptional suppression responsible for the observed decrease in GLT-1 mRNA which is engaged following protracted cocaine exposure and withdrawal. Future studies will examine additional epigenetic factors that may also contribute to the observed decreases in GLT-1 mRNA after long access cocaine self-administration and withdrawal, as well as the mechanism(s) for reduction in GLT-1 protein expression independent of gene expression following short access self-administration and extinction.

Keywords: Cocaine, GLT-1, DNA Methylation.

Disclosure: Nothing to disclose.

\section{W231. Progesterone for Postpartum Smoking Relapse Prevention}

Ariadna Forray*, Kathryn Gilstad-Hayden, Mahmet Sofuoglu, Kimberly Yonkers

Yale University School of Medicine, New Haven, Connecticut, United States

Background: Smoking is the leading cause of preventable mortality, and maternal smoking is associated with poor pregnancy outcomes and increased infant morbidity and mortality. Close to half of women who were smokers prior to conception are able to quit smoking in pregnancy. Unfortunately, over $50 \%$ relapse within the first few weeks after delivery and $70-80 \%$ relapse within a year. While this decrease in smoking is attributed to a woman's motivation to adopt healthy behaviors in pregnancy, biological factors may also play a role. Progesterone modulates multiple brain functions implicated in the pathogenesis of drug addiction, and production in pregnancy increases by a factor of 8 and drops to perimenopausal levels after delivery. In preclinical studies, progesterone diminishes nicotine self-administration and inhibits nicotinic receptors, including the $\alpha 4 \beta 2$ subunit, implicated in nicotine addiction. Human studies, although limited, show that exogenous administration of progesterone attenuates both craving for cigarettes and the subjective rewarding effects of smoking in recently abstinent female smokers. The current study tested the efficacy of postpartum progesterone replacement in preventing relapse to smoking in postpartum women with a history of pre-pregnancy smoking.

Methods: This was an 8-week, double-blind, parallel, randomized, placebo-controlled pilot trial. We recruited 41 pregnant women who smoked cigarettes during the 6 months before pregnancy and achieved abstinence by 32 weeks of gestation. Participants were randomized to receive either oral micronized progesterone (200 mg twice daily) or placebo following delivery for 8 weeks. The primary outcome was the 7 - day point prevalence abstinence at week 8 . Continuous abstinence for weeks 1 through 8 was examined as a secondary outcome. Abstinence was determined by selfreport via Timeline Followback at weekly visits, confirmed by an expired carbon monoxide level of $<8 \mathrm{ppm}$. Differences in abstinence rates were analyzed using logistic regression models adjusted for stratifying variables, age ( $\leq 25$ vs. $>25$ years) and pre-quit smoking level ( $\leq 10$ vs. $>10$ cigarettes/ day). Cox proportional hazards regression was used to compare the hazard of relapse, adjusted for age and pre-quit smoking level.

Results: The average age for participants was 26 years, $63 \%$ were black, $7 \%$ white, $27 \%$ Hispanic, and $2 \%$ Native American. Women taking progesterone were twice as likely to be abstinent during week 8 compared to women taking a placebo, but this difference did not reach statistical significance $(\mathrm{OR}=2.1,95 \% \mathrm{CI}=0.58,0.42, p=0.26)$. Similarly, the progesterone group was 1.7 times more likely, but not statistically significantly so, than the placebo group to remain abstinent throughout the 8 -week treatment period $(\mathrm{OR}=1.67,95 \% \mathrm{CI}=0.42,6.54, p=0.47)$. The median time to relapse for the placebo group was 38 days, while the median time to relapse for the progesterone group was 52 days. The progesterone group had a $28 \%$ delay in time to relapse (Hazard Ratio $=0.72$ ) compared to the placebo group, but this difference was not significant $(p=0.45)$.

Conclusions: These preliminary findings support the promise of progesterone treatment in postpartum smokers and could constitute a therapeutic breakthrough. If these preliminary findings can be evaluated and replicated in a larger study with sufficient power, this may constitute an acceptable and safe smoking relapse prevention strategy for use during lactation.

Keywords: Prenatal Smoking, Progesterone, Relapse Prevention.

Disclosure: Nothing to disclose.

\section{W232. Naltrexone Moderates the Relationship Between Cue-Induced Craving and Subjective Response to Methamphetamine in Individuals With Methamphetamine Use Disorder}

\section{Daniel Roche*, Matthew Worley, Lara Ray \\ University of California, Los Angeles, Los Angeles, California, United States}

Background: There are currently no approved pharmacological treatments for methamphetamine (MA) use disorder. Increases in drug craving are often proximal to and preceding drug use, and accordingly, MA craving level is highly predictive of relapse. Thus, a reduction in craving may be a primary marker of medication efficacy in the treatment of MA use disorder. Recent findings from our laboratory suggest that naltrexone reduces MA cue-induced craving and alters the acute subjective effects of MA, thereby suggesting that naltrexone may be a promising pharmacotherapy for the disorder. Yet, the importance of subjective response to MA as a marker of medication efficacy has been debated, and the relationship between acute increases in MA craving and subsequent subjective MA effects has not been investigated. Therefore, the objective of this randomized, double-blind, placebo-controlled, and within-subject human laboratory study was to examine 1) the predictive relationship between cue-induced craving and subsequent acute subjective response to MA and 2) whether naltrexone moderated this association in a sample of 30 non-treatment-seeking individuals who met criteria for MA use disorder. 
Methods: Participants completed two separate four-day medication regimens (naltrexone or placebo, in a randomized, counterbalanced order) separated by a 7-14 day washout period between each condition. In order to minimize adverse events, participants were titrated on naltrexone from $25 \mathrm{mg}$ on day one to $50 \mathrm{mg}$ on days two through four. On day four of each medication regimen, participants completed a cue-reactivity paradigm followed by intravenous (IV) MA administration [two $15 \mathrm{mg}$ (5 ml) infusions]. Methamphetamine craving was assessed after the cue-reactivity paradigm using the Methamphetamine Urge Questionnaire. Subjective response to MA was assessed during IV MA infusion with the Drug Effects Questionnaire (DEQ), which included 10 total items, such as "How much do you feel any drug effects? (Feel)," "How good are the drug effects you are feeling right now? (Good)," "How high are you? (High)," and "How stimulated do you feel right now? (Stimulated)." After completion of the IV infusion, participants remained in the UCLA CTRC for an overnight stay and were discharged the following morning.

Results: Cue-induced craving for MA was significantly $(p<$ 0.05 ) and positively associated with post-infusion DEQ scores for nearly all assessed subjective effects, including "Feel", "High", "Good", "Stimulated", "Would Like More Drug", and "Would Like Drug Access," "Anxious," and "Depressed." Given the significant association between cueinduced craving and subjective effects of MA, we next examined whether this relationship was moderated by medication condition (i.e., naltrexone vs. placebo). Statistically significant cue-induced craving $\mathrm{x}$ medication condition interactions were observed for "Feel" $(b=-0.74, p<0.001)$, "High" ( $b=-0.37, p<0.001)$, "Good" $(b=-0.51, p<$ $0.001)$, and "Stimulated" $(b=-0.74, p<0.01)$. These interactions revealed that the positive association between cue-induced craving and subjective MA response was significantly reduced when receiving naltrexone compared with placebo. In the placebo condition, greater cue-induced craving significantly predicted greater ratings of "Feel", "High", and Good", but in the naltrexone condition these effects were no longer statistically significant. Greater cueinduced craving significantly predicted greater ratings of "Stimulated" in both medication conditions, but this effect was significantly attenuated in active naltrexone compared to placebo.

Conclusions: In sum, the present findings suggested that greater levels of cue-induced craving were associated with larger subjective responses to MA in terms of both positive and aversive subjective effects. Most importantly, additional analyses indicated that naltrexone moderated the association between cue-induced craving and those subjective effects that were predominantly positive or reward-related. These results may have elucidated a behavioral mechanism by which naltrexone is efficacious in treating MA use disorder. Craving level is highly and reliably predictive of relapse in MA use disorder. The current findings suggest that this may be due to individuals experiencing greater subjective MA effects after craving-induced relapse, which could increase the likelihood of a single lapse turning into full relapse. However, naltrexone was predominantly effective in reducing the positive or rewarding effects of MA in individuals who experienced high levels of cue-induced craving. Thus, naltrexone may be particularly effective in treating MA- dependent individuals who are susceptible to drug-cueinduced craving by breaking the link between craving level and the rewarding acute effects of MA. Future studies should further study the relationship between craving level and the acute effects of MA in real-world situations in treatment seeking populations and also examine whether naltrexone moderates the association between craving and subjective response in other drug use disorders.

Keywords: Methamphetamine, Naltrexone, Craving, Subjective Response.

Disclosure: Nothing to disclose.

\section{W233. The Role of the Gut Microbiome in Phenotypes Associated With Alcohol and Other Addictive Disorders: A Systematic Review}

Sofia Bouhlal*, Jamie E. Temko, Mehdi Farokhnia, Mary R. Lee, John Cryan, Lorenzo Leggio

National Institute on Alcohol Abuse and Alcoholism, Bethesda, Maryland, United States

Background: There is evidence for bidirectional signaling pathways between the gut and the central nervous system. Indeed, the gut-brain axis seems to play a role on a variety of physiological as well as pathological behaviors. An increasing body of research suggests that the gut microbiome may be involved in the pathophysiology of a wide range of neuropsychiatric disorders, including autism, anxiety and depression. Research on other neuropsychiatry areas is however in its infancy. The goal of this systematic review was to examine the existing preclinical and clinical literature on the role of the gut microbiome in eating disorders, obesity, alcohol and substance Use Disorders (ASUDs).

Methods: We conducted a systematic review on clinical and preclinical literature for original research articles. As part of the inclusion criteria, this systematic review specifically focused on outcomes linking the gut microbiota to behaviors (e.g., craving) related to the disease under investigation. We used Medline, Embase and Web of Science for the search.

Results: The review resulted in no findings for substance use disorder or obesity. A total of 10 papers were identified and selected for the other disorders studies here, i.e. alcohol use disorders (AUD) $(n=3)$ and eating disorders $(n=7)$. With respect to AUD, alcohol dependent patients seem to have an increased intestinal permeability associated with altered gut microbial composition and activity as well as decreased craving for alcohol after 3 weeks of abstinence. There was also evidence linking changes in gut microbiota in alcoholdependent patients with peripheral markers of inflammation. On the other hand, there is no work that has investigated whether these changes persist past short-term abstinence and whether they predict relapse. There are also few studies on the link between the gut microbiome and behavioral outcomes in eating disorders. A limited literature has found elevated levels of autoantibodies against neuropeptides involved in appetite, weight and emotion regulation in patients with anorexia and bulimia. For instance, the $\mathrm{ClpB}$ protein produced by $\mathrm{E}$. Coli in the gut, has the same structure as the satiety hormone melanotropin. Antibodies against this gut protein, can cross-react with the endogenous satiety hormone. This cross-reaction (molecular mimicry) 
can result in the altered signaling of melanotropin and therefore, altered eating behavior. Thus, although the link to food craving is less clear, the autoantibodies signaling via the gut-brain pathway impacts behavior and plays a role in the pathophysiology of eating disorders.

Conclusions: This systematic review supports the preliminary notion that the gut microbiome may play a role in behaviors associated with AUD and eating disorders and also highlights the need for additional work to uncover the role of the gut microbiome in these disorders. This systematic review also serves as the background and rationale for an upcoming clinical study our group has developed. The study will investigate the relationship between gut microbiome composition and activity with alcohol drinking vs. alcohol abstinence in a well-controlled experimental inpatient study design. The relationship between gut microbiome and cueinduced craving will also be measured at baseline and at the end of treatment.

Keywords: Addiction, Alcohol and Substance Use Disorders, Microbiota-Gut-Brain Axis, Behaviors.

Disclosure: Nothing to disclose.

\section{W234. Role of the Agranular Insular Cortex in the Reconsolidation of Cocaine-Associated Contextual Memories and Drug-Context Induced Reinstatement}

Amy Arguello*, Rong Wang, Carey Lyons, Jessica Higginbotham, Matthew Hodges, Rita Fuchs

Michigan State University, East Lansing, Michigan, United States

Background: Drug addiction is a chronic relapsing disorder characterized by compulsive drug-seeking and drug-taking behavior, which can be elicited by exposure to a previously drug-associated environmental context. Re-exposure to drug-associated contexts can also initiate the retrieval and destabilization of context-response-cocaine associative memories, which necessitates their subsequent reconsolidation into long-term memory stores for continued stimulus control over behavior. Thus, interference with the reconsolidation of maladaptive drug-related memories may represent a novel target for relapse prevention. The functional integrity of the agranular insular cortex (AgrI) is necessary for explicit cueinduced reinstatement of cocaine-seeking behavior and for the reconsolidation of amphetamine-conditioned place preference memories. However, the contribution of this brain region to cocaine context-induced drug-seeking behavior remains to be determined. Therefore, the objectives of the present study were to determine whether the functional integrity of the AgrI is necessary for drug context-induced reinstatement of cocaine-seeking behavior and for the reconsolidation of context-response-cocaine associative memories that contribute to this phenomenon.

Methods: In order to evaluate the role of the AgrI in the expression of drug context-induced drug-seeking behavior in experiment 1, rats were trained to lever press for un-signaled cocaine infusions in a distinct context, followed by extinction training in a different context. Rats then received bilateral microinfusions of vehicle or a cocktail of the GABAA/B agonists baclofen+ muscimol (BM; 1.0 and $0.1 \mathrm{mM}$, respectively) into the AgrI or the overlying jaw region of the somatosensory cortex (SST, anatomical control region) immediately before a test of drug-seeking behavior (i.e. non-reinforced lever presses) in the previously cocainepaired context.

In order to examine the involvement of the AgrI in the reconsolidation of memories that underlie drug contextinduced reinstatement of drug-seeking behavior, rats received vehicle or BM microinfusions into the Agrl after brief re-exposure (15 minutes) to the previously drug-paired context, to reactivate contextual cocaine memories and initiate their reconsolidation. Drug-seeking behavior was assessed in the previously cocaine-paired context $72 \mathrm{hrs}$ later. The possible rate altering effects of intra-Agrl BM administration were also examined in a subset of rats. To this end, rats received vehicle or $\mathrm{BM}$ microinfusions into the AgrI immediately before a test of locomotor activity in a novel context.

Results: In experiment 1, BM-induced neural inactivation of the Agrl attenuated the expression of cocaine-seeking behavior in the previously cocaine-paired context relative to vehicle treatment, with no effects on locomotor activity in a novel context. Furthermore, BM-induced neural inactivation of the SSJ anatomical control region failed to alter drug context-induced reinstatement of drug-seeking behavior relative to vehicle treatment. In experiment 2, BM-induced inactivation of the Agrl following cocaine memory reactivation failed to alter subsequent reinstatement of cocaineseeking behavior relative to vehicle treatment.

Conclusions: The AgrI plays requisite roles in the expression of both drug context- and explicit conditioned-stimulusinduced drug-seeking behavior. Based on these findings and extant literature, the AgrI may encode the motivational effects of drug-associated stimuli, associated reward anticipation, or computations required for the reversal in cocaineseeking behavior upon cue exposure following extinction training. In contrast, the Agrl does not appear to play a critical role in the reconsolidation of contextual memories under the current experimental parameters. Interestingly, a previous study has shown that protein synthesis in the AgrI is necessary for the reconsolidation of amphetamineconditioned place preference memories. Thus, the Agrl may play a differential role in amphetamine and cocaine memory reconsolidation. However, it is more likely that the Agrl, similar to the nucleus accumbens, plays a more important role in the reconsolidation of Pavlovian, relative to instrumental, contextual memories.

Keywords: Memory Reconsolidation, Reinstatement, Cocaine Seeking.

Disclosure: Nothing to disclose.

W235. The Effect of Social Support on Quality of Life is Moderated by Oxytocin Genotype in Alcohol Use Disorder

Tiffany Love*, James Cranford, Robert Zucker, Kirk Brower

University of Michigan, Salt Lake City, Utah, United States

Background: There is a strong link between an individual's social environment and reported quality of life. Individuals 
with strong social ties tend to exhibit lower levels of psychological distress, depression, and anxiety. The degree to which an individual feels satisfied with their interpersonal relationships is generally positively associated with wellbeing and overall life satisfaction. This has particular link between social support and well-being has been widely demonstrated in a variety of control and psychiatric populations, including in persons diagnosed with Alcohol Use Disorder (AUD). Interestingly, there is a great deal of interindividual variability in the extent to which social support is beneficial to overall health. The underlying neurobiological origin for such variability is not currently known, however, some evidence suggests that variations in functioning within the oxytocin system may influence the degree to which the favorable effects of social interaction are felt. To explore this possibility, we evaluated oxytocin genotype, social support, and quality of life in a group of patients with DSM-IV alcohol dependence.

Methods: Blood was taken and DNA extracted from a sample of 288 patients admitted into treatment programs in Poland for alcohol dependence. Single nucleotide polymorphisms (SNPs) located near the oxytocin gene (OXT) were genotyped. Results for OXT SNP rs2740210 are given below. Social support was gauged using the Medical Outcomes Study Social Support Survey (MOSSS). Quality of life was evaluated using the Short Form Health Survey (SF36). The SF36 is a multidimensional 36-item healthrelated quality of life instrument which provides scores across 8 health domains (e.g., general health, physical functioning, general mental health).

Results: As expected, social support scores were positively correlated with all of the 8 quality of life domains measured by the SF36 (General health: $r=0.202, p=0.001$; general mental health: $r=0.371, p<0.001$; physical functioning: $\mathrm{r}=0.153, p=0.012$; social functioning: $\mathrm{r}=0.308, p<0.001$; vitality: $\mathrm{r}=0.388, p<0.001$; bodily pain: $\mathrm{r}=0.185, p=0.002$; role limitations due to physical problems: $r=0.181$, $p=0.003$; role limitations due to emotional problems: $\mathrm{r}=0.172, p=0.005)$.

Interestingly, using moderated multiple regression, we found OXT genotype significantly influenced the relationship between social support and general health perceptions $(p=0.016)$. While $\mathrm{C}$ carriers displayed the predicted positive relationships between social support and general health (CC, $r=0.156$; CA, $r=.319$ ), A homozygotes failed to exhibit such a relationship (AA, $\mathrm{r}=-0.282)$.

Conclusions: Discussion:

Our data are in line with previous reports which suggest that individual perceptions of well-being and quality of life are positively related to the degree of social support individuals believe to have in their life. Interestingly, we find that OXT genetic disposition influences the extent to which such beneficial effects of social support are felt in an alcoholdependent population. These findings may help to explain variability in the relationship between social support and well-being, and could perhaps inform clinical interventions for this population.

Keywords: Oxytocin, Social Support, Alcohol Use Disorder. Disclosure: R21 AA016104 and Fogarty International Center/NIDA International Substance Abuse Research Program Grant D43-TW05818.
W236. DRD2 Methylation and Regional Gray Matter Volumes in Young Adult Offspring From Families at Ultra-High Risk for Alcohol Dependence

Shirley Hill*, Vinod Sharma, Jessica O'Brien, Brian Holmes, Bobby Jones

Western Psychiatric Institute and Clinic, Pittsburgh, Pennsylvania, United States

Background: Deficits in social cognition seen in alcohol dependent $(\mathrm{AD})$ patients may be linked to prefrontal cortex (PFC) dysfunction. Family history of $\mathrm{AD}$ and fetal alcohol exposure may be independent predictors of brain morphology. We hypothesized that we would find: (1) a decrease in gray matter in high-risk (HR) offspring vs. low-risk (LR) controls in facial recognition and social cognition regions; that (2) DRD2 methylation differences would be seen by familial risk status; and (3) a significant association between DNA methylation of the DRD2 receptor gene and volume of regions with familial related reduction of volume would be seen.

Methods: MRI scans (3 T) from $43 \mathrm{HR}$ offspring (27.4+ 3.6 years) and 45 controls ( $24.5+4.1$ years) provided whole brain (WB) and region of interest (ROI) analyses. VBM8 toolbox was used for the WB analysis (threshold $p<0.005$; cluster $=$ 100 voxels); the MarsBaR ROI toolbox provided ROIs for SPSS analyses. Pyrosequencing of $\mathrm{CpG}$ sites within the DRD2 gene was performed.

Results: Controlling for prenatal exposure to alcohol, drugs and cigarettes, ICV, age and any previous personal substance use disorder, significant familial risk group differences were seen with HR males showing reduced volume of the Left Inferior Temporal $(\mathrm{F}=14.84, \mathrm{df}=1,29, p=0.001)$, Left Fusiform $(\mathrm{F}=4.57, \mathrm{df}=1,24.97, p=0.042)$ and Left Insula $(\mathrm{F}=4.44, \mathrm{df}=1,37, p=0.042)$ regions relative to $\mathrm{LR}$ males. Methylation was significantly increased in HR offspring $(p=0.003)$ and negatively related to gray matter volumes: fusiform (L) $(\mathrm{r}=-.370, p=0.001)$, insula $(\mathrm{r}=-.323$, $p=0.006)$ and inferior temporal regions $(\mathrm{r}=-.316$, $p=0.007)$.

Conclusions: Discussion:

Regions previously linked to facial affect perception and social cognition show lesser gray matter volumes in individuals at ultra high-risk for developing $\mathrm{AD}$ suggesting that neural underpinnings of social cognitive impairment may be premorbid risk factors. Methylation differences in the DRD2 gene by familial risk suggest that gene expression differences may play a role in the regional volumetric differences observed.

Keywords: Methylation, Alcohol Dependence, Familial Risk, DRD2, Gray Matter Volumes.

Disclosure: Nothing to disclose. 
W237. Preliminary Assessment of Inflammatory Gene Expression in Individuals With Cocaine Use Disorder and Anhedonia

Sarwar Khan, Gabriel Fries, Elena Dyukova, Consuelo Walss-Bass, Scott Lane, Joy Schmitz, Margaret Wardle*

University of Texas Health Science Center at Houston, Houston, Texas, United States

Background: Cocaine use disorder (CUD) affects almost 1 million people in the United States and creates a substantial health care burden. Current CUD treatments show variable efficacy, so further investigation is needed into individual differences that may affect treatment outcomes. One such individual difference is the presence vs. absence of anhedonia, a state of decreased pleasure capacity that is associated with poorer CUD treatment outcomes. Inflammatory processes can potentially produce anhedonic symptoms, and inflammatory processes are generally increased in CUD. We hypothesized that individuals with CUD and high levels of anhedonia would show differential expression of genes in inflammatory pathways, compared to individuals with CUD and low levels of anhedonia.

Methods: Individuals with CUD $(N=24)$ were recruited from the greater Houston area. Exclusion criteria were: potentially inflammatory medical conditions (except high blood pressure), psychotic disorders, pregnancy, menopause or oral contraceptive use. Anhedonia was measured with the Snaith-Hamilton Pleasure Scale (SHAPS), and a median split was used to produce high and low anhedonia groups (each $n=12$ ). Groups were matched on demographics, substance use, other psychiatric diagnoses, and high blood pressure. RNA was extracted from peripheral blood mononuclear cells in heparin-anticoagulated blood samples. Following assessment of quality and integrity, biotinylated RNA samples were loaded onto a HumanHT-12 v4 Expression BeadChip (Illumina) and scanned with the Illumina iScan reader. Differential gene expression was assessed using Genome Studio. We conducted a stringent analysis with a false discovery rate (FDR) correction of $1 \%$ and alpha of 0.05 , and an exploratory analysis of genes with uncorrected $p$ values $<0.01$. Any genes identified were subjected to Ingenuity Pathway Analysis (IPA) to identify major altered pathways and networks.

Results: No significantly differentially expressed genes (DEGs) were present with FDR correction, but 57 DEGs were indicated by uncorrected $p$-values $<0.01$. IPA found several networks with FDR-significant enrichment based on these DEGs, with the top differentially expressed networks being inflammatory response and cell death and survival. Consistent with these results, expression of several genes in the identified inflammatory response network significantly correlated with SHAPS scores. Additionally, canonical pathways related to immune processes such as phagosome maturation and B Cell development were significantly enriched.

Conclusions: This preliminary study indicates that inflammatory processes are potentially altered in individuals with high anhedonia and CUD. In the event this result is confirmed in larger studies, anti-inflammatory adjunctive therapies should be investigated to address treatment resistance in anhedonic individuals with CUD.
Keywords: Cocaine Addiction, Inflammation, Anhedonia, Gene Expression.

Disclosure: Nothing to disclose.

\section{W238. Glutamate Receptor Homeostasis in the Medial Prefrontal to Nucleus Accumbens Circuit is Associated With Individual Differences in Motor Impulsivity}

Noelle Anastasio*, Brionna Davis-Reyes

University of Texas Medical Branch of Galveston, Galveston, Texas, United States

Background: Impulsivity is a complex, multifaceted trait broadly defined as action without sufficient foresight. High inherent motor impulsivity (e.g., difficulty in withholding a response) may increase the likelihood that drug use escalates into dependence and increase the risk of relapse. The medial prefrontal cortex (mPFC) and nucleus accumbens (NAc) are two brain regions critical to decision making and goaldirected behavior. The mPFC exerts top-down control over the NAc and it is possible that attenuated glutamatergic neurotransmission through the ionotropic N-methyl-Daspartate (NMDA) and $\alpha$-amino-3-hydroxy-5-methyl-4-isoxazolepropionic acid (AMPA) receptors within this circuit governs high inherent impulsivity. The composition of NMDA and AMPA receptors critically regulates their function; the NMDA receptor is composed of GluN1 and GluN2A-D subunits while the AMPA receptor is composed of GluA1-4 subunits. The research objective guiding this experiment was to test the hypothesis that a homeostatic imbalance in the mPFC excitatory output to the NAc (as evidenced by a shift in the protein expression profile of the ionotropic NMDA and AMPA receptors within the $\mathrm{mPFC}$ and NAc, respectively) underlies high inherent motor impulsivity.

Methods: Outbred male Sprague Dawley rats were identified as high (HI) or low (LI) impulsive using the novel one-choice serial reaction time (1-CSRT) task in which nose-pokes after presentation of a visual stimulus resulted in food pellet delivery. Nose-pokes before presentation of the visual stimulus (i.e., premature responses) indexed motor impulsivity. The upper and lower quartile of animals were identified as HI or LI rats, respectively. Following HI or LI phenotypic identification, mPFC and NAc synaptosomal protein was extracted to assess expression of NMDA receptor and AMPA receptor subunits via immunoblot.

Results: Performance on the 1-CSRT task was rapidly acquired and the HI/LI phenotype was stable in that HI rats made significantly more premature responses than LI rats across 70 days of training $(p<0.001)$. In the mPFC, HI rats had lower GluN1 and GluN2A, but higher GluN2B synaptosomal protein expression vs. LI rats $(p<0.05)$. HI rats also expressed lower NAc synaptosomal GluA1 and GluA2 protein levels vs. LI rats $(p<0.05)$.

Conclusions: Taken together, these preliminary data suggest that compensatory downstream homeostatic mechanisms within the NAc, (putatively) occur in response to long-term glutamatergic receptor neurotransmission dysfunction from the $\mathrm{mPFC}$ and provide support to the hypothesis that glutamate hypofunction in the mPFC to NAc circuit underlies high inherent impulsivity. Determining how these 
receptors regulate glutamatergic balance within the $\mathrm{mPFC}$ to NAc circuit as it relates to impulsivity will lead to a better understanding of the biological mechanisms underlying high inherent impulsivity and advance the design of targeted therapeutics for disorders such as drug dependence and relapse.

Keywords: Impulsivity, NMDA Receptor, AMPA Receptors, Prefrontal Cortex, Nucleus Accumbens.

Disclosure: Nothing to disclose.

\section{W239. Mining the (5-HT) 5-HT2A Receptor (5-HT2AR) and 5-HT2CR Complex}

Kathryn Cunningham*, John Allen, Lisa A. Elferink, Scott R. Gilbertson, F. Gerard Moeller, Noelle C. Anastasio

University of Texas Medical Branch, Galveston, Texas, United States

Background: The serotonin (5-HT) 5-HT2A receptor (5HT2AR) and 5-HT2CR play important roles in behavior and physiology. We have recently demonstrated that knockdown of 5-HT2CR following microinjection of 5-HT2CR shRNA into the rat medial prefrontal cortex (mPFC) evokes a behavioral phenotype characterized by increased motor impulsivity and elevated reactivity to cues associated with cocaine self-administration. The 5-HT2CR knockdown in mPFC also resulted in upregulation of 5-HT2AR protein in the $\mathrm{mPFC}$ and a leftward shift in potency of systemic M100907 to suppress motor impulsivity, suggesting a functional disruption of local 5-HT2AR:5-HT2CR balance. Furthermore, co-immunoprecipitation studies suggested that a protein: protein interaction exists between 5-HT2AR and 5-HT2CR in mPFC. Here, we employed a multi-prong approach ex vivo and in vitro to test the hypothesis that the cortical 5-HT2AR:5-HT2CR regulation of motor impulsivity is achieved at the single neuron level through the formation of a 5-HT2AR:5-HT2CR protein complex.

Methods: We employed double-label immunohistochemistry, the Duolink proximity ligation assay (PLA), and luciferase complementation assay (LCA) technologies in either live cells and/or rat brain, as well as the in silico direct coupling analysis (DCA). In the LCA, two complementary $\mathrm{N}$ - (NLuc) and C-terminus (CLuc) components, which have no activity on their own, are fused to the two receptor proteins of interest, respectively. In the presence of the substrate D-luciferin, association of the two proteins brings the inactive luciferase fragments into close proximity to reconstitute the enzyme activity. We are co-expressing 5HT2AR-NLuc (or CLuc) and 5-HT2CR-CLuc (or NLuc), expressed on the $\mathrm{N}$-or $\mathrm{C}$-terminus in mammalian cells to demonstrate the formation of a protein: protein interaction between the 5-HT2AR and 5-HT2CR in live cells.

Results: In immunohistochemical analyses, we found that 5HT2AR and 5-HT2CR protein co-localized within the same cells in rat mPFC. In the PLA (Duolink), we found that native, unmodified proteins are in close proximity $(<45 \mathrm{~nm})$ in mPFC. In the DCA (which examines the co-evolution of residues in over 100 species), we identified candidate pairs of amino acid residues that are predicted to be in direct functional contact, most notably between the extracellular
$\mathrm{N}$-terminus domains of the two proteins. Co-expression of 5HT2AR-CLuc plus 5-HT2CR-NLuc in live cells demonstrated the formation of a protein: protein complex following the administration of luciferin.

Conclusions: Our findings suggest that 5-HT2AR:5-HT2CR protein interaction may provide a new neurobiological mechanism underlying behavior and a possible target for novel pharmacotherapeutics, such as heterobivalent ligands. Keywords: 5-HT2A Receptor, Serotonin 5-HT2C Receptor, Protein Complexes.

Disclosure: Nothing to disclose.

\section{W240. Role of Gadd45b in DNA Demethylation and Cocaine Action}

Faraz Sultan, Guan-En Graham, Lauren Silverwood, Jeremy Day*

University of Alabama at Birmingham, Birmingham, Alabama, United States

Background: Epigenetic mechanisms are central regulators of the function and information storage capacity of neuronal systems. Methylation of cytosine nucleobases in DNA is a multifunctional epigenetic regulatory modification capable of exerting powerful control gene. In the brain, activitydependent changes in DNA methylation are critical for synaptic plasticity and memory formation, and have been implicated in a broad range of neuropsychiatric disease states, including drug addiction. However, although activityrelated DNA demethylation requires the Gadd45 (Growth arrest and DNA-damage-inducible) protein family, very little is known about how DNA demethylation regulates the function of brain reward circuits or the role that Gadd45 family members play in behavioral responses to drugs of abuse.

Methods: Here, we combined unbiased genome-wide transcriptional profiling, pharmacological tools, and CRISPR/dCas9 transcriptional activation with traditional knockout and behavioral approaches in rodent model systems (both in vitro and in vivo) to dissect the role of Gadd45 family members in dopamine-dependent epigenetic regulation.

Results: We show that acute cocaine administration induced upregulation of Gadd45b mRNA in rat nucleus accumbens, but did not alter expression of other methylation-related transcripts. Similarly, acute dopamine treatment in striatal neuron cultures increased expression of Gadd45b and Gadd45g mRNA. This effect was mimicked by the Drd1 agonist SKF38393, suggesting upregulation in Drd1containing neurons. In vitro, CRISPR-targeted transcriptional activation of either Gadd45b or Gadd45g with a dCas9-VP64 fusion construct was capable of unsilencing a methylated reporter gene, suggesting a mechanistic link between Gadd45 induction and DNA demethylation. Finally, we show that both dopamine treatment (in vitro) and cocaine administration (in vivo) induce DNA demethylation, and that Gadd45b-/- mice exhibited impaired conditioned place preference for cocaine.

Conclusions: These results suggest that striatal Gadd45b functions as a dopamine-dependent immediate early gene to coordinate demethylation of DNA at downstream target 
$\overline{S 612}$

genes, and that this action is important for cocaine-related behavioral plasticity.

Keywords: Epigenetics, Addiction, DNA Methylation, $\mathrm{Nu}-$ cleus Accumbens.

Disclosure: Nothing to disclose.

\section{W241. Nicotine-Induced Synaptic Plasticity in the Orbitofrontal Cortex Differs Between Adolescent and Adult Mice}

Pavel Ortinski, Luyi Zhou, Jill Turner*

University of South Carolina, Columbia, South Carolina, United States

Background: More than $90 \%$ of adult smokers report their first use of tobacco before the age of 18. Smoking during adolescence increases the vulnerability to addiction and decreases the rate of successful quitting. Previous clinical studies have shown that adolescent rodents exhibited enhanced reward to nicotine. Considering that enormous neurodevelopment occurs during adolescence, nicotine may differentially affect the brain at these two stages.

Methods: We assess the impact of nicotine on long-term synaptic plasticity in the orbital frontal cortex (OFC), a brain region highly involved with impulse control, of adolescent and adult mice. To do this, we utilized long-term potentiation protocols to evaluate nicotine-induced circuit plasticity in the OFC during adolescence and early adulthood.

Results: We found that in adolescent mice, a high-frequency tetanic stimulation protocol could successfully induce longterm potentiation (LTP) in the OFC. Upon nicotine application, the same stimulus paradigm induced sustained long-term depression (LTD) in a neuregulin-erbB4 dependent manner. However, in adult mice, both the LTP and LTD effects were absent at baseline or during nicotine application. Conclusions: The highly vulnerable synaptic plasticity in OFC during adolescence may underline higher sensitivity to nicotine as well as increased susceptibiity to addiction and other co-morbid disorders, such as schizophrenia. These data will be discussed in the context of how nicotine differently affect the adolescent and adult brain and what potential consequences this may have for nicotine dependence

Keywords: Nicotine Dependence, Neuregulin-3, Adolescence, Schizophrenia, Synaptic Aberrations, Long Term Potentiation.

Disclosure: Nothing to disclose.

\section{W242. Neurobehavioral Mechanisms of Choices for Cannabis and Snack Food in Cannabis Smokers}

Gillinder Bedi*, Xuejun Hao, Anna Konova, Giovanni Paci, Mel Win Khaw, Evan Herrmann, Paul Glimcher, Margaret Haney

Columbia University Medical Center, New York, New York, United States

Background: A fundamental question about Substance Use Disorders concerns the neurobehavioral mechanisms of choices about drugs; that is, how and why do decisions to use drugs continue, often despite abundant negative consequences? In a previous study (Bedi et al, 2015), we assessed the neural substrates of decisions to smoke vs. decline cannabis in regular cannabis smokers, finding that choices for cannabis were associated with a distributed pattern of brain activation including striatum, insula, anterior and posterior cingulate, and dorsolateral prefrontal cortex. Despite these promising initial findings, numerous questions remain about the ways in which the human brain processes choices to use drugs. For instance, our first study did not allow disambiguation of specific cognitive processes guiding drug-related choice. Of particular importance is the process by which the brain quantitatively tracks value attributed to drugs during decision-making, given that changes in valuation processes likely contribute critically to the maintenance of Substance Use Disorders. A second question concerns the degree to which neural processing of drug-related decisions is distinct from processing of decisions about other types of reinforcers. The primary aim of the present study was thus to characterize neural encoding of value for cannabis and a natural reinforcer (snack food) in regular cannabis smokers. We also aimed to assess the effects of cues (cannabis, snack, neutral) on these processes.

Methods: Healthy, non-treatment-seeking participants reporting cannabis use ( $\geq 2$ 'joints' $\geq 4$ days per week; verified by urine toxicology) complete a six-day inpatient protocol. Following an initial day comprising two sessions in which they sample 6 snacks $(50-100 \mathrm{kcal}$, snacks chosen by participants) and 6 standardized puffs of cannabis $(5.6 \%$ THC), they complete 4 choice conditions in counterbalanced order: 1. Cannabis vs. money after neutral cues; 2 . Cannabis vs. money after cannabis cues; 3. Snacks vs. money after neutral cues; and 4. Snacks vs. money after snack cues. On each choice day, participants view visual cues prior to completing a behavioral economic task, repeatedly choosing between 0 to 6 cannabis puffs (or snacks) and a range of monetary options $(\$ 0.25$ - $\$ 30.00)$. This behavioral task serves to estimate an individualized indifference point (monetary equivalent) for 3 puffs of cannabis/snacks to be used as a fixed reference option for that day's fMRI choice task. Participants subsequently undergo multisensory cues prior to completing an fMRI scan in which they chose repeatedly between 0-6 cannabis puffs/snacks and their estimated monetary value for 3 puffs/snacks. This approach aims to ensure that participants vary their choices in the scanner between cannabis/snacks and money under each condition. Participants rate the strength of their decision on a 5-point scale, which is used as a measure of the perceived value of the cannabis/snack option relative to the fixed monetary amount (Hare et al, 2009). Following the scan, one choice from the behavioral task and a second from the fMRI task are randomly selected for implementation in the afternoon. fMRI data analysis involved two-factor (Cannabis vs Snack; Neutral vs Active Cues) within subjects GLMs including a parametric regressor for value attributed to cannabis/snacks, with value operationalized as strength of decision ratings (Hare et al, 2009). False Discovery Rate (FDR) correction was applied to a ventromedial Prefrontal Cortex (vmPFC) ROI, based on previous studies reliably showing value encoding in vmPFC (Levy and Glimcher, 2012). 
Results: Data collection is ongoing. To date, 16 volunteers (2 F, 14M; 3 White, 11 AA, 2 Mixed) have completed the study. They reported using cannabis an average of $6.5( \pm 1.0)$ days per week, smoking $4.4( \pm 1.8)$ 'blunts' per day. Initial analysis of data from the behavioral economic task indicates substantial individual variability in estimated indifference points for puffs/snacks across conditions, supporting the need to individualize monetary alternatives for the fMRI tasks. There were no differences in the proportion of choices for cannabis/snacks or money in the fMRI task as a function of the type of reinforcer (cannabis vs snacks), cues (neutral vs active), or the interaction between them, suggesting that the individualized monetary reference option functioned as intended. Preliminary fMRI analysis showed that behaviorally-estimated values for the cannabis options correlated with activation in a region of vmPFC overlapping with that observed to encode value signals in previous studies, but a similar pattern was not observed in the snack choices. Direct comparison between vmPFC value encoding for cannabis versus snack decisions revealed significant differences, such that value encoding in vmPFC was significantly greater for cannabis than for snack food. There was no main effect of cue type or interaction between cue and reinforcer type on value signals in vmPFC.

Conclusions: These findings provide initial support for the hypothesis that neural encoding of valuation signals for cannabis is intact, while value encoding for a non-drug reinforcer is disrupted, in regular cannabis smokers. Preliminary results are thus consistent with models identifying dysregulated valuation of drug relative to alternative reinforcers as a fundamental driver of addiction.

Keywords: Cannabis, Decision Making, Functional MRI (fMRI).

Disclosure: Nothing to disclose.

\section{W243. Sustained Opioid Antagonism Modulates Striatal Sensitivity to Baby Schema in Opioid Dependent Women}

An-Li Wang*, Steve Lowen, Igor Elman, Zhenhao Shi, Victoria Fairchild, Alexander Bouril, Ruben Gur, Daniel Langleben

University of Pennsylvania, Philadelphia, Pennsylvania, United States

Background: Opioid addiction is a growing global problem affecting up to 5 million individuals in the US alone. Observational studies in humans and primates suggest that opioid addiction is associated with deficits in pro-social behaviors including caregiving. The relevance of neurobiology of attachment to opioid dependence is supported by the notion that opioids and social attachments share neural mechanisms that converge on the mesocorticolimbic approach and reward pathway. Caring for the young is a fundamental social phenomenon that may be released by typical external appearance characteristic of juvenile animals and humans that are perceived as "cute". Baby schema (Kindchenschema) is a set of juvenile physical features that is perceived as "cute", releases the motivation for caregiving and activates the ventral striatum, a key component of the brain reward pathway. Preclinical research points to specific effects of exogenous opioid modulators (agonists and antagonists) on social cognition that vary with species, dose, prior socialization, opioid exposure and gender. Naltrexone is a competitive opioid antagonist blocking primarily $\mathrm{mu}$ opioid receptors, that is used to prevent relapse to opioids in detoxified opioid addicted patients. Although opioid antagonist treatment has been gaining acceptance as an alternative opioid maintenance treatment of opioid addiction, its effect on social cognition such as caregiving remains unknown. We hypothesized that XRNTX will enhance the brain AMRI and behavioral effects of baby schema in detoxified individuals with opioid use disorder.

Methods: Forty-seven opioid dependent patients and 25 controls underwent two functional magnetic resonance imaging sessions, approximately ten days apart, while viewing infant portraits with high, unmanipulated and low baby schema content and rating them for cuteness. Immediately after the first session, patients received an injection of extended-release naltrexone, while controls received no treatment.

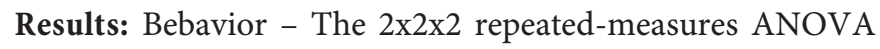
revealed a marginal significant main effect of Group ( $\mathrm{F}(1$, $42)=3.90, p=0.06$ ) with greater $\Delta$ Rating (i.e. RatingHigh RatingLow) in the patient group (0.71 \pm 0.06 vs. $0.53 \pm 0.07)$, but no main effects of Session $(\mathrm{F}(1,42)=0.00, p=0.99)$ or Gender $(\mathrm{F}(1,42)=2.00, p=0.17)$. There were no interactions (Session $\mathrm{x}$ Group: $\mathrm{F}(1,42)=0.56, p=0.46$; Session $\mathrm{x}$ Gender: $\mathrm{F}(1,42)=0.00, p=0.95$; Group x Gender: $\mathrm{F}(1,42)=0.00$, $p=0.97$; Session $\mathrm{x}$ Group $\mathrm{x}$ Gender: $\mathrm{F}(1,42)=0.56, p=0.46)$. Self-reported craving was significantly reduced (F $(1,24)=45.76, p<0.001)$ after the injection (i.e. OnXRNTX), but there was no gender difference (F $(1,24)=3.15, p=0.09)$. On-XRNTX, naltrexone plasma levels were $2.86 \pm 1.41 \mathrm{ng} / \mathrm{ml}$ and 6-beta-naltrexol levels were $7.31 \pm 4.40 \mathrm{ng} / \mathrm{ml}$. There was no gender difference in the naltrexone $(\mathrm{t}=1.049, p=0.308)$ or 6 -beta-naltrexol $(\mathrm{t}=1.627, p=0.121)$ plasma levels.

Imaging - Whole brain analysis of variance showed gender by group by session interaction in the ventral striatum. Brain responses increased in female patients and decreased in male patients across sessions, while the pattern was reversed in the controls.

Conclusions: We found that extended release naltrexone treatment in detoxified opioid dependent patients modulates the function of the brain network that supports hedonic processing and social cognition. These preliminary findings set the stage for future controlled studies of the effects of opioid modulators on the brain and behavioral correlates of social cognition. Since opioid agonists and antagonists are widely used, these observations may have clinical implications for both medical and non-medical use of opioids.

Keywords: BOLD Imaging, Opioid Dependence, Social Cognition, Baby Schema, Opioid Antagonist Treatment.

Disclosure: Nothing to disclose. 
W244. Parvalbumin Expressing Interneurons in the Nucleus Accumbens are Modulated by Calcium Permeable AMPA Receptors

Dipanwita Ghose, Sahana Nagabhushan Kalburgi, Carrie Grueter, Brad Grueter*

Vanderbilt University Medical Center, Nashville, Tennessee, United States

Background: The nucleus accumbens (NAc) core, a key brain region involved in reward-related behavior, is comprised of parallel output loops (D1 and D2 dopamine receptor expressing medium spiny neurons (MSNs)) whose activity is governed by excitatory drive and by, often overlooked, inhibitory interneuronal microcircuitry. Inhibition of local projection neurons within networks are known to be regulated by the GABAergic parvalbumin expressing fast spiking interneurons (PV-INs). Relative to excitatory synaptic transmission onto NAc MSNs, little is known regarding excitatory synaptic transmission onto NAc PVINs. We propose that activity of PV-INs gates excitatory (glutamatergic) drive within the NAc to correctly process reward-related behaviors. Our results indicate PV-INs functionally express calcium permeable AMPA receptors that are capable of inducing long term depression (LTD) of excitatory postsynaptic currents (EPSCs). Furthermore, intra-NAc block of calcium permeable AMPA receptors enhances locomotor activity and sensitivity to cocaine.

Methods: Male mice ages 6-10 weeks were utilized and experiments were performed in accordance with Vanderbilt University IACUC approved protocols. Targeted whole-cell patch-clamp recordings of EPSCs were obtained from PVINs and D1 or D2 dopamine expressing MSNs in NAc core slices. PV-INs were labelled with D1tdTomato by crossing PV-cre mice with Ai9 mice, a cre dependent Rosa26 tdTomato reporter line. In acute slices from D1-tdTomato bacterial artificial chromosome mice, MSNs were identified as D1-tdtomato positive (D1-MSN) or negative (D2-MSN). Excitatory afferents were stimulated at the border between the NAc core and cortex dorsal to the anterior commissure. All recordings were done in presence of picrotoxin $(50 \mu \mathrm{M})$ to block GABAA receptor mediated inhibitory synaptic currents. For experiments examining LTD, recordings from control cells were interleaved with recordings from cells undergoing experimental manipulations including bath application of agonists/antagonists. For behavioral studies, AAV-hSyn-DIO-hM3D(Gq)-mCherry and AAV-hSyn-DIO$\mathrm{hM} 4 \mathrm{D}(\mathrm{Gi})$-mCherry were injected into the NAc core 3 weeks prior to assessment. Behavioral analysis consists of locomotor response to cocaine. Intra-NAc drug infusions and intraperitoneal $\mathrm{CNO}$ injections were performed 30 minutes prior to cocaine injections. In vivo experiments were performed blind to drug or virus expression. Comparisons between different experimental manipulations were made using a two-tailed Student's t-test with $p<0.05$ considered significant.

Results: Here we find that $\mathrm{CNO}$ exposure from mice expressing hM4D(Gi) or hM3D(Gq) in PV-INs in the NAc has no effect on locomotor activity. However, when stimulated with $20 \mathrm{mg} / \mathrm{kg}$ cocaine, the PV-hM4D(Gi) mice exhibited a decrease in locomotor response while the PV$\mathrm{hM} 3 \mathrm{D}(\mathrm{Gq})$ mice showed an increase relative to PV-eGFP mice. We next investigated synaptic properties of PV-INs in the NAc. Our results show that NAc PV-INs have similar presynaptic properties to D1 and D2 MSNs, while excitatory synapses at PV-INs have a greater AMPAR/NMDAR ratio. Most notably, in contrast to D1 or D2 MSNs, PV-INs express robust AMPAR rectification at positive holding potentials. Investigation of synaptic plasticity mechanisms in the PVINs revealed that, similar to indirect pathway MSNs in the NAc, low frequency stimulation (LFS: $10 \mathrm{~Hz}, 5 \mathrm{~min}$ ) produces robust LTD in the PV-INs. Importantly, the mechanisms underlying this LFS-LTD are distinct from that reported in the D2 MSNs. LFS-LTD is absent at D1 MSNs and dependent upon mGluR5/CB1R/TRPV1 receptor function at excitatory synapses onto D2 MSNs. Unlike D2 MSNs, LFS-LTD at excitatory synapses onto PV-INs is independent of mGluRs and NMDARs. Furthermore, LFS-LTD at PV-IN excitatory synapses is sensitive to NASPM, a blocker of calcium permeable AMPA receptors, and when infused into the NAc, leads to enhanced locomotor activity and greater sensitivity to cocaine. Lastly, LFS failed to elicit LTD at PVIN excitatory synapses in slices from mice treated with cocaine.

Conclusions: The results of this study demonstrate that PVINs bi-directionally regulate NAc-dependent behavioral outcomes and undergo activity-dependent synaptic plasticity. Specifically, excitatory input into the NAc can have differential effects depending on the neuronal target. LFS at $10 \mathrm{~Hz}$ has no effect on D1 MSNs, elicits mGluR5/CB1R/ TRPV1-dependent LTD at D2 MSN synapses and CPAMPAR-dependent plasticity at PV-INs. Thus, integration of excitatory input in the NAc core can be cell-type specific. LTD of excitatory synapses onto PV-INs could attenuate feed-forward inhibition, thus alleviating a break on MSN output. Considering these neuronal subtypes receive similar excitatory inputs, we suggest that differential integration of excitatory drive onto PV-INS gates MSN output maintaining normal physiological circuit dynamics. Disruption of this feedforward mechanism may be a key component underlying NAc-dependent disease states.

Keywords: Nucleus Accumbens, Parvalbumin Interneurons, Synaptic Plasticity.

Disclosure: Nothing to disclose.

W245. ADHD, Smoking Withdrawal, and Resting State Functional Connectivity: Results of a fMRI Study With Methylphenidate Challenge

Maggie Sweitzer*, Scott Kollins, Rachel Kozink, Matt Hallyburton, Joseph English, Merideth Addicott, Jason Oliver, Francis McClernon

Duke University Medical Center, Durham, North Carolina, United States

Background: Tobacco smoking and ADHD are highly comorbid disorders, but little is currently known about neurobiological mechanisms underlying this comorbidity. A growing fMRI literature has revealed aberrant patterns of resting state functional connectivity ( $\mathrm{rsFC}$ ) among adults with ADHD across multiple brain networks, including the default mode (DMN), executive control (ECN), and salience networks (SN). These networks have also been implicated in 
nicotine dependence and are impacted by smoking withdrawal, but whether withdrawal-induced changes in rsFC may be exacerbated among smokers with ADHD compared to those without is unknown. Here, we examined changes in rsFC during smoking withdrawal compared with satiety among adult daily smokers with $(n=17)$ and without $(n=21)$ ADHD. Furthermore, we investigated the role of dopaminergic neurotransmission in these changes by examining the effects of methylphenidate (MPH).

Methods: Participants completed a resting state fMRI scan under each of 3 conditions: (a) smoking as usual+ placebo; (b) $24 \mathrm{hr}$ smoking abstinence+ placebo and (c) $24 \mathrm{hr}$ smoking abstinence+ MPH. Order of scans was randomized and placebo versus MPH conditions were double-blinded. RsFC analyses were conducted in CONN using the following spherical seed regions of interest: medial prefrontal cortex (mPFC), right and left dorsolateral prefrontal cortex (DLPFC), and dorsal anterior cingulate cortex (dACC) to probe DMN, ECN, and SN, respectively. Group differences were evaluated in SPM8 using a 2 (group) X 3 (condition) ANOVA, with cluster significance threshold of $P<.05$, FDR corrected.

Results: Across conditions, smokers with ADHD exhibited lower connectivity strength than controls between the dACC seed and right DLPFC and right inferior parietal lobule; and between the right DLPFC seed and the dACC/mid-cingulate, right inferior parietal lobule, right superior frontal gyrus, and right insula. In addition, significant group by abstinence condition interactions were observed for connectivity between $\mathrm{MPFC}$ and left insula/putamen and between right DLPFC and occipital cortex/cuneus. These interactions were characterized by a pattern of greater reductions in connectivity during abstinence compared with satiation among ADHD smokers relative to controls. The same pattern of group by drug (MPH versus placebo) interaction was observed between $\mathrm{mPFC}$ and left caudate/thalamus and between right DLPFC and occipital cortex/cuneus.

Conclusions: Overall, these results suggest that smokers with ADHD exhibit weaker rsFC strength between several regions involved in ECN and salience networks, and connectivity between these and other key regions is further attenuated during smoking withdrawal. Abstinence-induced decreases in connectivity between executive control and visual attention areas were reversed by $\mathrm{MPH}$ in ADHD smokers, suggesting dopaminergic involvement. These effects highlight a potential neurobiological mechanism underlying vulnerability to nicotine dependence and relapse among smokers with ADHD and may help to explain high rates of comorbidity.

Keywords: ADHD, Tobacco Smoking, Methylphenidate, Nicotine, Resting State Functional Connectivity.

Disclosure: Akili Interactive: Consulting and Research Support, Self; Alcobra: Consulting and Research Support, Self; Atentiv: Consulting, Self; Ironshore: Consulting and Research Support, Self; Medgenics: Research Support, Self; Arbor: Research Support, Self; Neos: Research Support, Self; Neurovance: Consulting and Research Support, Self.
W246. Opioid and Dopamine Receptor Activation Generates Reactive Oxygen via JNK/PRDX6 Signaling

Benjamin Land ${ }^{\star}$, Selena Schattauer, Lauren Burgeno, Jennifer Steger, Kathryn Reichard, Paul Phillips, Charles Chavkin

University of Washington, Seattle, Washington, United States

Background: Inactivation of opioid receptors limits therapeutic efficacy, and GPCR desensitization involves both arrestin-dependent and independent mechanisms. While arrestin-dependent inactivation has been well described, less is understood about arrestin-independent inactivation. Both the mu opioid receptor (MOR) and kappa opioid receptor (KOR) undergo arrestin-independent inactivation that requires activity of c-Jun N-terminal kinase (JNK), but the target and mechanism of JNK-mediated inactivation has remained elusive. A proteomic screen of receptor interacting proteins affected by JNK identified peroxiredoxin 6 (PRDX6), a dual-function enzyme that has separately regulated glutathione peroxidase and phospholipase A2 (PLA2) activity. In these experiments, we test whether activation of MOR, KOR and D2DR by a variety of ligands generates reactive oxygen species (ROS) by the PLA2-activity of PRDX6. We additionally test whether inhibition of PRDX6 by the drug MJ33 can block inactivation of MOR, KOR and D2DR in vivo. Finally, we test whether ROS production can produce a "cross-inactivation" of a nonopioid GPCR target, the dopamine D2 receptor (DRD2), using fast scan cyclic voltammetry.

Methods: In vitro studies- KOR, MOR, or DRD2 transfected HEK 293 cells were pretreated with vehicle, SP610025 (JNK inhibitor), MJ33 (PRDX6 inhibitor) or $\mathrm{N}$-acetyl cysteine (NAC, anti-oxidant). Cells were then incubated with the KOR antagonists norBNI or naloxone, MOR agonists morphine or fentanyl, or DRD2 agonist quinpirole. Cells undergoing ROS imaging were then incubated with CellRox Green, and all cells were subsequently prepared for imaging. In vivo studies- Wild-type mice were pretreated with MJ33 at various time-points. For KOR studies, $1 \mathrm{hr}$ after MJ33 administration animals were treated with norBNI, and 3 days later tail-flick analgesia was performed using U50,488, a KOR agonist. Analgesia was measured $30 \mathrm{~min}$ after baseline/ U50,488 administration. For MOR studies, $2 \mathrm{hr}$ after MJ33 administration animals were treated with morphine or fentanyl and tail-flick analgesia was performed in $30 \mathrm{~min}$ intervals for $3 \mathrm{hr}$ post-injection. A second injection of morphine or fentanyl was then given and tail-flick analgesia was again performed for 2.5 more hours to generate tolerance curves. For DRD2 studies, $1 \mathrm{hr}$ after MJ33 administration, animals were treated with quinpirole/saline in the home cage. Two hr later, animals received a second dose of quinpirole and then locomotor activity was assessed. Voltammetry studies- Animals were pretreated with MJ33/ saline $1 \mathrm{hr}$ prior to norBNI/saline. Coronal brain slices containing striatum were then prepared for DA in vitro voltammetry. Quinpirole dose-response curves were generated.

Results: Treatment of KOR/MOR-HEK 293 cells by norBNI/ morphine increased association of PRDX6 with the receptor complex, and this effect was reversed by pretreatment of the 
5616

JNK inhibitor SP610025. Likewise, treatment of KOR/MORHEK 293 cells by norBNI/morphine increased ROS and this was blocked by pretreatment of SP6, MJ33, or NAC. Interestingly, neither naloxone nor fentanyl induced ROS production. Further, in vivo pain assays demonstrated that the long-lasting antagonist effect of norBNI was attenuated by pretreatment of MJ33 or NAC, suggesting that PRDX6ROS production is required for long-term KOR inactivation by norBNI. Similarly, MJ33 prevented acute analgesic tolerance to morphine, again suggesting that PRDX6 signaling is required for MOR desensitization. To see if this phenomenon was a more general characteristic of inhibitory GPCRs, quinpirole was also shown to increase ROS in DRD2-expressing HEK 293 cells, and MJ33 abrogated quinpirole locomotor tolerance. Finally, using dopamine slice voltammetry, we show that norBNI was able to reduce the potency of quinpirole, suggesting a mechanism of ROSbased, cross-inactivation.

Conclusions: These results identify a novel signaling pathway that links GPCR activation to ROS production via PRDX6. Additional experiments have determined that the likely substrate for ROS is a palmitoylation site on Goi, and once irreversibly depalmitoylated by ROS, the G protein is "locked" on the receptor, preventing further signaling. Thus, the locked receptor complex has to be degraded and replaced before signaling is able to resume. Local ROS production by one GPCR may also affect nearby GPCRs, as shown by the ability of norBNI to inhibit quinpirole inhibition of dopamine release. Finally, the results suggest that PRDX6 inhibitors may be promising therapeutic targets to prevent opioid tolerance and dose escalation during pain management.

Keywords: GPCR, Morphine, Opioid Tolerance, Kappa Opioid Receptor, Dopamine.

Disclosure: Nothing to disclose.

\section{W247. Striatal BOLD Responses to VTA Optogenetic Stimulation During Inhibition of Locus Coeruleus Noradrenergic Neurons}

Natalie Zahr*, Eric Peterson, Dongjin Kwon, Amy Collins, Alexia Thomas, Xiomara Perez, Tanuja Bordia, Edith Sullivan, Adolf Pfefferbaum

Stanford University, Menlo Park, California, United States

Background: Optogenetics combined with functional MRI (i.e., optofMRI) permits whole brain evaluation of bloodoxygen level dependent (BOLD) responses to localized, cell specific stimulation. Optogenetic activation of dopamine neurons in the midbrain of awake, restrained animals drives striatal BOLD activity. Dexmedetomidine (Dex) is a highly selective agonist able to induce sedation by decreasing activity of noradrenergic neurons in the locus coeruleus. Systemic administration of Dex can decrease extracellular dopamine levels in the ventral striatum. Nevertheless, Dex is useful for fMRI because it induces a neural state similar to natural sleep, causes muscular relaxation, and relative to volatile anesthetics, results in less suppression of cortical burst firing and more localized functional activity. In this study, optogenetic fMRI was used to locally manipulate and globally visualize neural activity related to stimulation of dopamine neurons in the presence of Dex.

Methods: One male and 5 female Long-Evans tyrosine hydroxylase (TH)-Cre rats purchased from the RRRC (http://www.rrrc.us/) weighed $250-400 \mathrm{~g}$ at the time of surgery. Rats were unilaterally injected with a cre-inducible recombinant AAV vector expressing ChR2 (AAV5.EF1a. DIO.hChR2(H134R)-eYFP.WPRE.hGH, UNC vector core) and implanted with a $300 \mu \mathrm{m} 0.37 \mathrm{NA}$ optical fiber (Zirconium ferrule, Doric Lenses) in the right ventral tegmental area (VTA). The virus was allowed to express for a minimum of 4 weeks before experiments were initiated.

A Master-8 stimulus generator (AMPI, Israel) driving a $200 \mathrm{~mW}, 473 \mathrm{~nm}$ blue laser (optoEngine LLC) controlled duration and frequency of stimulation. Midbrain dopaminergic neurons were activated with $6 \mathrm{~mW}$ power $(84.85 \mathrm{~mW} /$ $\mathrm{mm} 2$ light intensity at the fiber tip). Rats were individually placed in a plexiglass cylinder: following $20 \mathrm{~min}$ of habituation, behavior was live-scored for alternating periods of 2 min OFF and 2 min ON laser stimulation (30 pulses @ 20 $\mathrm{HZ}, 10 \mathrm{~ms}$ duration). Two experimenters determined the number of $360 \mathrm{o}$ contralateral turns and exploratory rearing. Scores were averaged and summed for $10 \mathrm{~min}$ laser OFF and 10 min laser ON periods.

Animals were then used in optofMRI experiments using a similar laser stimulation protocol. For imaging experiments, animals were anesthetized with $0.15-0.2 \mathrm{mg} / \mathrm{kg}$ Dex (i.p.) and maintained, as necessary, with $0.10-0.15 \mathrm{mg} / \mathrm{kg} / \mathrm{hr}$ Dex (i.p.) via infusion pump. Imaging was performed on a $7 \mathrm{~T}$ Bruker system using a custom-made $2.5 \mathrm{~cm}$ single-channel surface coil and included a gradient echo EPI (flip angle $42^{\circ}$, TR $600 \mathrm{~ms}$, TE $15 \mathrm{~ms}$, resolution $0.5 \times 0.5 \mathrm{~mm}$, slice thickness $1 \mathrm{~mm}$, repetitions 780, scan time $7.8 \mathrm{~min}$ ). An event-related pseudorandom stimulus sequence - specifically, a maximum length (m-sequence) design - allowed simultaneous but uncorrelated presentation of two stimuli, such that both optogenetic and a "positive control" visual stimulus were applied.

fMRI processing was performed using AFNI, CMTK, and ANTS. For each fMRI run, spike values were removed by 3dDespike. Motion between volumes was corrected by aligning each volume to the base volume with the minimum outlier fraction using 3 dvolreg. fMRI distortion was corrected by applying each fMRI run (forward) with corresponding pepolar fMRI (backward) to CMTK's epiunwarp; the unwarped fMRI run was mapped into atlas space by registering mean fMRI volume and a Paxinos-Watson (PW) atlas using ANTS. Activation maps for optogenetic or visual stimulations were obtained by solving least square linear regressions for block stimulus patterns and motion statistics using $3 \mathrm{dDeconvolve.} \mathrm{For} \mathrm{detrending,} \mathrm{polynomials} \mathrm{up} \mathrm{to} \mathrm{3rd}$ degree were included in the design matrix. For the group activation map, a single design matrix was constructed by stacking each fMRI run with motion statistics and polynomials up to 3 . Using PW-rat parcellations aligned with the atlas, statistics for each ROI (i.e., striatum and superior colliculus) were produced.

Results: For behavior, paired t-tests demonstrated that with laser ON relative to OFF there was an increase in counterclockwise rotations $(\mathrm{t}(5)=5.54, p=.0062)$ and an increase in exploratory rearing $(\mathrm{t}(5)=3.72, p=.0137)$ in the 6 animals. Group $(n=6)$ concatenated AFNI analysis t-statistic map of 
the opto-stimulated fMRI data was masked with the PW atlas for analysis of left and right striatum and superior colliculus. Visual stimulation resulted in significant BOLD activity in both left $(\mathrm{t}(5)=24.83, p<.001)$ and right $(\mathrm{t}(5)=21.05$, $p<.001)$ superior colliculi. With optogenetic stimulation of VTA, mean BOLD activity was significant in the right $(\mathrm{t}$ $(5)=5.04, p<.005)$ but not left $(\mathrm{t}(5)=0.18$, n.s.) striatum.

Conclusions: optofMRI is a cutting edge technology that permits whole brain visualization of responses to cell-specific stimulation. While a previous study demonstrated robust and reliable BOLD responses in striatum to optogenetic stimulation of midbrain dopamine neurons in awake animals, the current findings establish the presence of BOLD activations in striatum in the presence of Dex. These results suggest the striatal BOLD response to direct optogenetic stimulation of midbrain dopamine neurons is resilient to the dopaminergic effects of Dex. Future work aimed at determining the sensitivity of the dopamine stimulated BOLD response to acute and chronic effects of alcohol will provide a better understanding of the interactions of alcohol with the dopaminergic system.

Keywords: Optogenetics, Functional MRI (fMRI), Dopamine, Noradrenergic System.

Disclosure: Nothing to disclose.

\section{W248. The Effects of Synthetic Psychoactive Cathinones on Lethality and Temperature}

Frank Hall*, Dawn Muskiewicz, Yasir Saber, Federico Resendiz-Gutierrez, Taylor Osting, Omar Issa

University of Toledo, Toledo, Ohio, United States

Background: Synthetic psychoactive cathinones (SPCs) are drugs with psychostimulant and entactogenic properties similar to methamphetamine (METH) and 3,4-methylenedioxymethamphetamine (MDMA). Abuse of SPCs is a substantial public health problem associated with adverse events, emergency room admissions, and lethal overdoses, although there is uncertainty as to whether all drugs in this class share similar risks, and whether those risks are greater than those associated with METH or MDMA. Hyperthermia is an important factor in the lethal and neurotoxic effects of METH and MDMA, but its importance for the adverse effects of SPCs is not known. In a recent study, we found that the SPC methylone (2-methyamino-1-[3,4-methylenedioxyphenyl]propan-1-one) had an LD50 that was slightly lower than that observed for METH or MDMA. Lethality has not been examined for most other SPCs.

Methods: LD50 studies were performed for the SPCs cathinone, methcathinone, methylenedioxypyrovalerone (MDPV), and mephedrone, as well as MDMA and METH. Subjects were adult male and female C57BL/6 J mice. After acclimatization to a test chamber and establishment of baseline temperature for 1 hour, subjects were injected with $0-120 \mathrm{mg} / \mathrm{kg}$ (base) IP of a test drug; 6 doses were tested for each drug; $N=5$ male and $N=5$ female subjects per condition. Temperature was measured at 20 minute intervals for 2 hours. Behavioral observations (e.g. hyperlocomotion, seizure, 5-HT behavioral syndrome, etc.) were also made for each interval.
Results: LD50 values for METH and MDMA were similar to previously reported values, 84.1 and $92.2 \mathrm{mg} / \mathrm{kg}$ respectively. Pronounced locomotor activity was observed at the lowest doses, and replaced by stereotypy at higher doses. Slight hyperthermia was observed at some doses. At high doses convulsions and death occurred within minutes. The LD50 for mephedrone was $105.4 \mathrm{mg} / \mathrm{kg}$; convulsions and death similarly occurred within minutes at the highest dose. By contrast, neither lethality nor convulsions were observed for MDPV, cathinone, or methcathinone at doses up to $120 \mathrm{mg} /$ $\mathrm{kg}$ IP. Moreover, in contrast to METH and MDMA, pronounced dose-dependent hypothermia was observed for mephedrone, MDPV, cathinone and methcathinone - a reduction of $7-8^{\circ} \mathrm{C}$ for some doses.

Conclusions: None of the SPCs studied here (MDPV, mephedrone, cathinone or methcathinone) had LD50 values that were lower than METH or MDMA. Similar to METH and MDMA, the highest dose of mephedrone induced lethality that was associated with convulsions. However, unlike those drugs, mephedrone reduced body temperature. Even more pronounced hypothermia was observed with cathinone, methcathinone and MDPV, but was not associated with lethality. These data indicate that: (1) not all SPCs exhibit greater lethality than METH and MDMA (at least under the conditions studied here); (2) SPCs differ substantially in their effects on thermoregulation; (3) as we have previously found for methylone, effects of SPCs on temperature appear to be independent of effects on lethality. Keywords: Synthetic Psychoactive Cathinones, Lethality, Temperature Regulation.

Disclosure: Nothing to disclose.

\section{W249. Opioid Receptor Activation Recruits PRDX6 and Depalmitoylates Goi to Block Signaling}

\section{Charles Chavkin*, Selena Schattauer, Jamie Kuhar, Shao En Ong}

\section{University of Washington, Seattle, Washington,} United States

Background: Both the mu opioid receptor (MOR) and kappa opioid receptor (KOR) undergo arrestin-independent inactivation that requires c-Jun $\mathrm{N}$-terminal kinase (JNK), but the target and mechanism of JNK-mediated receptor inactivation has been elusive. To identify the target of JNK, we first established that this was a membrane delimited process and determined that the JNK-target was a component of the receptor complex. We then used iterative SILAC proteomics with KOR and Goi as bait to identify interacting proteins whose association was affected by JNK. We found that JNK activation increased the opioid receptor binding of both Goi and peroxiredoxin 6 (PRDX6), a dual-function enzyme that has separately regulated glutathione peroxidase and phospholipase A2 (PLA2) activity. We subsequently determined PRDX6 increases opioid receptor binding of Gai by oxidizing the $\mathrm{N}$-terminal cysteine residue responsible for the thioester linkage between Goi and palmitic acid. PRDX6induced depalmitoylation of Goi disrupts agonist stimulated GDP exchange and G protein activation. The nature of this reaction suggested that it may also induce heterologous desensitization, and we also determined that KOR activation 
of JNK/PRDX6 promoted "cross-inactivation" of the coexpressed dopamine D2 receptor.

Methods: JNK kinase assay- Spinal cord membranes were incubated with JNK $1 \alpha 1$ in the presence or absence of ATP for $30 \mathrm{~min}$ and then assayed for [35 S]GTP $\gamma \mathrm{S}$ binding. Membranes were then incubated with DAMGO, U69,593, GTP $\gamma \mathrm{S}$, or buffer for $1 \mathrm{hr}$ in the presence of [35S]GTP $\gamma \mathrm{S}$. Bound [35 S]GTP $\gamma \mathrm{S}$ was separated from free [35 S]GTP $\gamma \mathrm{S}$ by rapid filtration using a Brandel cell harvester onto GF/B filters and measured using a liquid scintillation counter. SILAC proteomics- HEK293 cells stably expressing mycKOR and FLAG-Goi3 were grown in SILAC media, treated $5.5 \mathrm{hr}$ with vehicle or norBNI, and solubilized in $1 \%$ digitonin immunoprecipitation buffer. KOR interacting or Goi interacting proteins were immunoprecipitated with anti-myc or anti-FLAG agarose respectively, trypsinized and analyzed by mass spectrometry. Non-specific interactions were identified by immunoprecipitation in the presence of excess myc or FLAG peptide. Palmitoylation assay- HEK293 cells stably expressing mycKOR and FLAG-Goi3 were treated with vehicle or the PRDX6 inhibitor MJ33 prior to $5.5 \mathrm{hr}$ treatment with norBNI or vehicle, solubilized in $1 \%$ digitonin immunoprecipitation buffer supplemented with $\mathrm{N}$-ethylmaleimide, and immunoprecipitated with anti-myc agarose. Unbound proteins were then immunoprecipitated with anti-FLAG agarose. Both of these fractions were then assayed using the acyl-biotin-exchange method; palmitate groups were cleaved by incubation with hydroxylamine, and the freed cysteines then biotinylated. Streptavidin binding in western blot was then used to quantify biotinylation of Goi. ERK assay- HEK293 cells stably expressing mycKOR and HA-D2DR were pretreated with naloxone, norBNI or SP610025 and norBNI 3-5 hr prior to treatment with quinpirole, homogenized in lysis buffer, and analyzed for phospho-ERK and total ERK using western analysis.

Results: An in vitro JNK kinase assay using spinal cord membranes confirmed that addition of phospho-JNK inhibited both KOR and MOR signaling, suggesting that the substrate of JNK was part of the receptor complex. Using SILAC proteomics on myc-KOR, we found that Goi was selectively enriched after norBNI treatment. This was

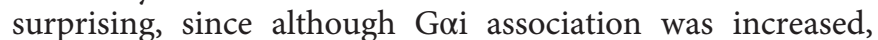
Goi signaling was completely blocked by norBNI. We verified this increased KOR-Gai association using coimmunoprecipitation. We next used SILAC proteomics on FLAG-Goi, and found that PRDX6 association was selectively increased after norBNI treatment. The PLA2 function of PRDX6 can generate reactive oxygen species (ROS), which can then permanently oxidize cysteine residues, disrupting the reversible palmitoylation of Goi that is required for G Protein signaling. We demonstrated that after norBNI treatment, there was a decrease in the palmitoylation of Goi that was blocked by MJ33, an inhibitor of PRDX6 PLA2 activity. Finally, using ERK1/2 phosphorylation as a readout of dopamine D2 receptor activity, we showed that norBNI activation of JNK/ PRDX6 reduced phospho-ERK stimulation by the D2 agonist quinpirole. These results suggest that PRDX6 can act as a cross-modulator of GPCR receptor function.

Conclusions: These results identify a novel mechanism linking GPCR activation to PRDX6 and subsequent depalmitoylation of the Goi. Additional experiments have determined that ROS are generated by both norBNI and morphine on KOR and MOR, respectively. Once irreversibly depalmitoylated by ROS, G Protein binding affinity increases and becomes stably "locked" on the opioid receptor, thus jamming and preventing further signaling. The long duration of action of selective kappa opioid receptor antagonist suggests that the complex does not spontaneously regenerate and recovery requires new receptor synthesis. Local ROS production by one GPCR affects nearby GPCRs, as shown by the ability of norBNI to inhibit quinpirole activation of ERK in an MJ33-dependent manner.

Supported by T32DA07278 (SSS) and P01 DA035764 (CC). Keywords: Morphine, Opioid Tolerance, Dopamine D2 Receptor, Kappa Opioid Receptor.

Disclosure: Nothing to disclose.

\section{W250. Increased Expression of Proenkephalin and Prodynorphin Mrnas in the Nucleus Accumbens of Compulsive Methamphetamine Taking Rats}

Jean Lud Cadet*, Irina Krasnova, Donna Walther

National Institute on Drug Abuse, Baltimore, Maryland, United States

Background: Methamphetamine (METH) use disorder is associated with deficits in executive function, memory formation, and other cognitive disturbances. These neuropsychological observations are thought to be secondary to pathological abnormalities observed in the brain of some METH addicts. Indeed, METH-addicted individuals who abuse large amounts of the drug have been shown to suffer from structural and functional abnormalities in various brain regions. These, in turn, might be relevant to the high risk of relapse to METH use reported even after prolonged periods of abstinence. These high rates of recidivism also suggest that there may be long-lasting molecular events that drive the clinical course of METH addiction that manifests in compulsive drug-taking behaviors and recurrent binge episodes. Depending on drug availability, binges can last days to weeks.

Methods: In the present study, we have used genome-wide approaches to measure potential long-term transcriptional alterations in the nucleus accumbens (NAc) of METH selfadministering rats. We used rats of divergent phenotypes generated by footshocks as adverse consequences to distinguish rats that continue to take the drug compulsively (shock-resistant, SR) from those that become abstinent (shock-sensitive, SS) in the presence of increasing footshocks. Outbred male Sprague-Dawley rats (Charles River, Raleigh, NC), weighing 350-400 g were used in these experiments. Rats were group-housed in pairs and habituated for 1 week before surgery. Rats had access to food and water ad libitum. Rats were anesthetized with a ketamine/ xylasine mixture (50 and $5 \mathrm{mg} / \mathrm{kg}$, i.p., respectively) and were inserted with catheters into the jugular vein. After surgery, rats were individually housed for an additional week and maintained under a reversed 12 - $\mathrm{h}$ light/dark cycle. Catheters were flushed with gentamicin (Butler Schein; $5 \mathrm{mg} / \mathrm{ml}$ ) every 2 days after surgery. All animal procedures were conducted according to the NIH Guide for the Care and Use of Laboratory Animals and were approved by the Animal Care and Use Committee of the NIDA Intramural Research 
Program (IRP). For self-administration training, rats were brought to the self-administration room and placed in Med Associates self-administration chambers where they remained throughout the duration of the experiment. Rats were trained to self-administer METH $(0.1 \mathrm{mg} / \mathrm{kg} /$ injection, i.v.) or saline on a FR1 schedule for twenty daily 9- $\mathrm{h}$ sessions. These consisted of three 3-hr sessions per day separated by $30 \mathrm{~min}$ breaks. Each chamber was equipped with two levers, with presses on the active lever resulting in reward deliveries paired to a 5- second tone-light cue. Presses on the inactive lever had no programmed consequences. Rats were trained in 4 cycles of 5 days of METH selfadministration with 2 week-end days off. Time off allowed for animals not to suffer excessive weight loss which is a known consequence of METH administration in rats. During training, all rats escalated their intake of the drug. Following METH SA training, we added the punishment contingency wherein $50 \%$ of the active lever-presses resulted in mild footshocks of $5 \mathrm{msec}$ duration through electrified grids. During this phase, rats continued METH self-administration every day (9- $\mathrm{h}$ a day) on the FR-1 reinforcement schedule with tone-light cues for 10 additional sessions. The initial foot-shock session was set at $0.18 \mathrm{~mA}$ and was progressively increased to $0.36 \mathrm{~mA}$ by increments of $0.06 \mathrm{~mA}$. Rats that self-administered saline were not subjected to punishment. Footshocks split the rats into SR and SS phenotypes. Animals were euthanized 2 hours after the last shock sessions and NAc tissues were dissected and processed to measure mRNA levels.

Results: To investigate potential molecular bases for the divergent phenotype, we performed a whole rat transcriptome study using Affymetrix rat arrays that cover more than 24,000 coding transcripts. The array experiments revealed that there were 23 differentially expressed genes between the resistant and sensitive rats, with 14 up- and 9 downregulated transcripts. Ingenuity pathway analysis revealed that these transcripts belong in a network of genes involved in cell signaling, behavior, and disorders of the basal ganglia. These genes included proenkephalin (PENK) and prodynorphin (PDYN), among others.

Conclusions: Because PDYN and PENK are expressed in dopamine D1- and D2-containing NAc neurons, respectively, these findings suggest that mechanisms that impact both cell types may play a role in the regulation of compulsive METH taking by rats. Therapeutic interventions that target these pathways may be of benefit in the treatment of METH addiction.

Keywords: Methamphetamine, Addiction, Proenkephalin, Prodynorphin, Nucleus Accumbens.

Disclosure: Nothing to disclose.

\section{W251. Resting Connectivity in Insular Sub Regions is Differentially Modulated by Nicotine Abstinence}

John Fedota*, Allison Matous, Kimberly Slater, Betty Jo Salmeron, Hong Gu, Thomas Ross, Elliot Stein

National Institute on Drug Abuse, Baltimore, Maryland, United States

Background: Nicotine dependence remains a large public health problem with individual recidivism rates exceeding
$90 \%$. In part, poor understanding of the neurobiological mechanisms of dependence contributes to such high relapse rates. One potential tool to characterize information processing in the human CNS is resting state functional connectivity (rsFC). The insula, which plays a central role in detecting internal bodily states and salient external stimuli has been implicated as a key hub for nicotine dependence along with its related neurocircuitry. The insula is a primary node of the salience network (SN), a large-scale brain network associated with switching between internal and external attentional focus via modulation of connections with the default mode network $(\mathrm{DMN})$ and the anticorrelated executive control network (ECN). The strength of such switching has been shown to be modulated by both state (acute nicotine administration) and trait (nicotine dependence) manipulations. Prior evidence in smokers also shows increased connectivity between the insula and DMN during nicotine withdrawal. However, the down regulated connection predicted between insula and ECN in withdrawn smokers has not been previously demonstrated. One reason for this lack of empirical evidence may be the use of a single insular seed when quantifying rsFC changes associated with nicotine abstinence. Recent results describe functionally distinct insular sub regions within the right hemisphere that show distinct patterns of network connectivity with the rest of the brain. The goal of the current investigation is to better characterize the effects of nicotine abstinence on rsFC among these insular sub regions and large-scale brain networks implicated in endogenous and exogenous attentional processing.

Methods: Resting-state BOLD data was collected from 17 cessation treatment-seeking smokers on two occasionsduring baseline smoking and again following $\sim 48 \mathrm{hrs}$ of full, biochemically assessed nicotine abstinence-to characterize changes in network connectivity during withdrawal. Based on prior evidence, three right hemisphere insular sub regions of interest (dorsal anterior, ventral anterior, and posterior) were identified and served as seeds in an analysis examining the resting connectivity between each of these ROIs and voxels within a priori group ICA-defined SN, ECN, or DMN. Results: Participant abstinence was verified physiologically, subjectively, and cognitively. Across all measures, a significant difference was observed between baseline and abstinent scans. All three insular sub region seeds showed changes in rsFC as a function of nicotine abstinence. Dorsal and posterior insular connections to other nodes of the SN were increased, showing stronger correlations during abstinence. A similar pattern was observed between the dorsal insula and a node within the DMN. In contrast, ventral insular connections to a node within the ECN was reduced during nicotine abstinence, with a positive ventral insulaECN correlation during baseline was replaced with a negative dorsal insula-ECN correlation during abstinence. Conclusions: Compared with smoking as usual, nicotine abstinence reduces rsFC between ventral anterior insula and the ECN, a large-scale network associated with attentional allocation. This is notable given links between ventral insula activation and focal attention capture. In contrast to the decline in ventral insula connectivity, both dorsal and posterior insular seeds showed increased connectivity during abstinence. Based on the dorsal insula's role in cognitive control and the posterior insula's role in interoceptive 
processing, these findings suggest further biasing of attention during abstinence towards internally generated signals like craving. Together, the current results suggest that nicotine abstinence disrupts network connectivity of smokers, and that acute nicotine abstinence may impact exogenous attentional allocation in addition to enhancing interoceptive processing as previously described. The isolation of specific circuits between insular sub regions and the ECN, SN, and DMN that are modulated by nicotine abstinence is an important foundational step in the identification of a brainbased biomarker of nicotine addiction and serves to more strongly link existing theoretical and empirical evidence of changes in rsFC associated with nicotine abstinence.

Keywords: Nicotine Addiction, Human Neuroimaging, Insula Connectivity.

Disclosure: Acknowledgement: Supported by the Intramural Research Program of the NIH/NIDA and FDA grant NDA13001-001-00000 to EAS.

\section{W252. Neural Correlates of Simulated Drug Choice in Individuals With Current and Remitted Cocaine Use Disorder}

Scott Moeller*, Anna Zilverstand, Anna Konova, Prantik Kundu, Muhammad Parvaz, Keren Bachi, Nelly Alia-Klein, Rita Goldstein

Icahn School of Medicine at Mount Sinai, New York, New York, United States

Background: An important question in clinical addiction research concerns how to effectively model drug-seeking behavior along the full addiction spectrum (i.e., spanning active users to long-term abstainers). Active users often participate in ecologically valid drug-choice paradigms, during which they - especially those with more severe addiction - often choose receiving drug infusions over alternative rewards (e.g., money, chocolate). In contrast, ethical considerations typically prohibit drug administration tasks in abstaining individuals; instead, tasks that assess choice antecedents, such as drug cue-reactivity or attention bias, are used. Although these tasks can also distinguish subgroups of drug users, they are limited in capturing the choice aspects of drug administration studies, which might be underpinned by distinct neural substrates (e.g., those involved in computing value). A paradigm is needed that measures drug-choice, but that can be used in abstaining individuals. Here, we investigated value-based decisionmaking for drug stimuli, rather than actual drugs, in individuals with current or remitted cocaine use disorder (CUD). To do so, we developed a new fMRI simulated drugchoice task inspired by the neuroeconomics of simple choice. Methods: Forty individuals with a DSM-IV history of CUD (19 current: median 3 days abstinent; 21 remitted: median 365 days abstinent) and 29 matched controls viewed, in an event-related manner, images depicting food (e.g., hamburger), threat (e.g., pointed gun), or cocaine (e.g., pipe) (75 trials per category). On each trial, participants indicated their choice (Strong No, No, Yes, or Strong Yes) to view these images or a neutral reference image (wicker basket). Scanning occurred shortly before lunch on a Siemens $3 \mathrm{~T}$ Skyra, using Human Connectome Project (HCP) acquisition parameters. Behavior was analyzed with 3 (Picture: food, threat, cocaine) $\times 3$ (Group: active CUD, remitted CUD, control) mixed ANOVA. The fMRI data were modeled trialby-trial from stimulus onset until response ( $\max 2.5 \mathrm{sec}$ ), and were analyzed with a parallel 3 (Picture) $\times 3$ (Group) flexible factorial model in SPM8. Significance was set at $p<0.05$ cluster-corrected, with a search threshold of $p<0.001$ uncorrected. In addition to these full models, and following prior drug-choice studies, we conducted a priori analyses on the cocaine $>$ food image contrast, for both brain and behavior.

Results: Behavioral analyses revealed main effects of Picture (food $>$ cocaine $>$ threat) and Group (active CUD $>$ remitted CUD $>$ control) (both $p<0.001$ ). Importantly, the Picture $\times$ Group interaction also reached significance $(p<0.001)$, driven by group differences in choice for cocaine versus food images (active CUD $>$ remitted CUD $=$ control). Across all CUD, higher cocaine $>$ food choice correlated with higher baseline cocaine craving $(p<0.001)$. Analysis of the fMRI data revealed significant main effects of Group, but not Picture. For Group, controls had more activation than all CUD, but especially active CUD, across all picture types in bilateral posterior brain regions (e.g., lingual gyrus, cerebellum, and precuneus); active CUD additionally had lower activations than the other groups in temporal, parietal, and midcingulate cortices. Although interactions in the full model were not significant, the SPM ANOVA on the a priori cocaine $>$ food contrast revealed that both CUD groups had higher activations than controls in the anterior cingulate cortex (ACC) [Brodmann Area (BA) 32)] and the right inferior frontal gyrus (IFG) (BA 44, 45) (both pcluster $<0.034)$. Finally, independently of these betweengroup analyses, higher cocaine $>$ food activation in the subgenual ACC extending into the medial orbitofrontal cortex (OFC)/rostral ACC (BA 11, 24, 32, 47) correlated with lower cocaine $>$ food choice in all participants (pcluster $<0.003)$. Together, self-reported craving and cocaine $>$ food subgenual ACC activation explained $51.2 \%$ of the variance in cocaine $>$ food choice in all CUD $(p<0.001)$. These effects were not explained by depression or cigarette smoking, which differed among groups.

Conclusions: Paralleling drug administration studies, individuals with CUD, and especially active CUD, made more choices to view cocaine versus food images than controls, which correlated with higher cocaine craving. The fMRI data revealed that CUD diagnosis, rather than active use per se, was associated with overall higher activation to the cocaine versus food images in regions typically associated with salience and cognitive conflict (ACC), and response inhibition (IFG). Thus, in the current CUD sample (which included participants in remission for months or years), the act of deciding whether to view cocaine images appeared to invoke executive self-control processes. Higher activations in these regions, perhaps when combined with higher activation in another region classically implicated in implicated in value (including aversive) processing (subgenual ACC), may explain the fewer choices for cocaine than food in CUD. Withdrawal symptoms, which might modulate the value of cocaine and food, could also be involved. Future studies can evaluate whether task behavior and associated brain activations can prospectively predict drug use and clinical outcomes in CUD. With minor modifications, this 
task can be translated to other addictions and even other psychopathologies similarly marked by impaired response inhibition and salience attribution (iRISA).

Keywords: Cocaine Addiction, Functional MRI (fMRI), Reward-Based Decision-Making, Craving, Choice.

Disclosure: Nothing to disclose.

\section{W253. Nicotine Alters Orbitofrontal Cortex Activity and} Cue Salience During Associative Learning

\section{Nicholas Simon*, Bita Moghaddam \\ University of Memphis, Pittsburgh, Pennsylvania, United States}

Background: Nicotine enhances the motivational salience of environmental cues, engendering ongoing seeking of nicotine and other drugs of abuse. Therefore, it is of interest to elucidate how nicotine alters neuronal cue processing to facilitate development of circuit-specific interventions for addiction. Here, we assessed the acute effects of nicotine on task-relevant neuronal activity in orbitofrontal cortex (OFC), which encodes information about outcome expectancy during cues and reward anticipation.

Methods: We recorded single unit activity from 385 units in OFC across 7 sessions of Pavlovian conditioning. Each session consisted of 50 cue/reward pairings, followed by subcutaneous administration of $0.2 \mathrm{mg} / \mathrm{kg}$ nicotine, then an additional 50 trials. Phasic responses to cue initiation, outcome anticipation, and reward delivery were compared before and after nicotine exposure.

Results: Rats quickly acquired the relationship between cue and reward, as assessed by goal-tracking behavior during the cue, reaching peak performance on session 5 . Nicotine initially reduced goal-tracking, but this effect was eliminated on session 5 . The percentage of units selective for all taskrelated events increased across sessions as rats acquired the task. Interestingly, a subset of OFC neurons selective for cue onset were disengaged by nicotine, no longer demonstrating changes in firing rate at cue onset. This suggests that nicotine may reduce OFC-driven outcome representation during cues, perhaps shifting motivational focus from impending outcomes to the cue itself. Nicotine did not cause any substantial difference in activity of reward- or outcome anticipation-selective units. In a separate group of rats, nicotine caused an increase in sign-tracking behavior, reflective of increased salience being attributed to cues.

Conclusions: Nicotine attenuated cue-evoked responding in certain OFC neurons, while sparing neuronal responding to other task-related events. Nicotine also increased signtracking behavior during cue presentation, suggesting that patterns of OFC activity evoked by reward-predictive cues are related to nicotine-induced cue salience.

Keywords: Nicotine, Electrophysiology, Orbitofrontal Cortex, Conditioned Cue.

Disclosure: Nothing to disclose.
W254. Deep TMS of the Insula Using the H-Coil Modulates Dopamine Release: A Crossover [11C] PHNO-PET Pilot Trial in Healthy Humans

Saima Malik*, Mark Jacobs, Sang Soo Cho, Isabelle Boileau, Daniel Blumberger, Markus Heilig, Alan Wilson, Zafiris Daskalakis, Antonio Strafella, Abraham Zangen, Bernard Le Foll

Centre for Addiction and Mental Health, Toronto, Canada

Background: Modulating the function of the insular cortex could be a novel therapeutic strategy to treat addiction to a variety of drugs of abuse as this region has been implicated in mediating drug reward and addictive processes. The recent advent of the $\mathrm{H}$-coil has permitted the targeting of deeper brain structures which was not previously feasible. The goal of this study was to bilaterally target the insular region using the H-coil with repetitive Transcranial Magnetic Stimulation (rTMS) and subsequently measure changes in dopamine levels using Positron Emission Tomography (PET) with [11C]-(+)-propyl-hexahydro-naphtho-oxazin (PHNO).

Methods: This was a within-subject, crossover, blinded and sham-controlled pilot study. Eight healthy, right-handed subjects, aged 19-45, participated in the investigation, and seven were included in the final analysis. All subjects underwent 3 PHNO-PET scans preceded by rTMS (sham, $1 \mathrm{~Hz}$ or $10 \mathrm{~Hz}$ ), on 3 separate days.

Results: Low frequency rTMS $(1 \mathrm{~Hz})$, targeting the insular cortex, significantly decreased dopamine levels in the substantia nigra, sensorimotor striatum and associative striatum.

Conclusions: Replicating this study in tobacco smokers or alcoholics would be a logical follow-up to assess whether $\mathrm{H}$-coil stimulation of the bilateral insula can be employed as a treatment option for addiction.

Keywords: Insula, PET, Dopamine, rTMS.

Disclosure: Nothing to disclose.

W255. Melanocortin-4 Receptors (MC4Rs) in Amygdala Mediate Thermal Hyperalgesia in Alcohol-Dependent Rats

Nicholas Gilpin*, Emily Roltsch, Renata Impastato

Louisiana State University Health Sciences Center, New Orleans, Louisiana, United States

Background: Humans with Alcohol Use Disorder (AUD) are more sensitive to painful stimuli during withdrawal (WD), raising the possibility that humans drink alcohol to relieve pain, and that chronic alcohol drinking worsens pain outcomes. Alcohol-dependent rats exhibit increases in nociceptive sensitivity (i.e., allodynia, hyperalgesia) during alcohol WD. Brain melanocortin-4 receptors (MC4Rs) have been implicated in nociception in rodent models, especially in relation to morphine analgesia, since antagonism of brain MC4Rs potentiates the analgesic effects of morphine. In these studies, we aimed to explore: [1] the effects of alcohol WD on thermal nociception in alcohol-dependent rats, [2] the effects of chronic alcohol on expression of MC4R and its endogenous agonist in relevant brain regions, and [3] the 
ability of acute alcohol and MC4R antagonists to rescue thermal hyperalgesia in alcohol-dependent rats.

Methods: In Experiments 1 and 2, male Wistar rats were exposed to chronic intermittent (14 hrs/day) alcohol vapor or ambient air for eight weeks, sacrificed 6-8 hours into WD, and brains were processed for either alpha-melanocytestimulating hormone (a-MSH) immunoreactivity (Experiment 1) or quantification of MC4R expression using Western blot analysis (Experiment 1). In Experiments 3-6, male Wistar rats were tested for baseline thermal nociception using the Hargreaves method, then exposed to either chronic intermittent alcohol vapor (alcohol-dependent rats) or ambient air (alcohol-naïve controls), and tested for thermal nociception during alcohol withdrawal. After dependence induction (and surgery where appropriate), rats were tested for the effects of acute alcohol drinking and acute fixed-dose alcohol (Experiment 3), intra-ventricular infusion of endogenous (AgRP) and synthetic (HS014) MC4R antagonists (Experiment 4), intra-nasal HS014 administration (Experiment 5), and intraamygdala infusions of HS014 (Experiment 6) on alcohol withdrawal-induced thermal hyperalgesia.

Results: Alcohol-dependent rats exhibited higher MC4R expression and lower a-MSH Immunoreactivity in central amygdala (CeA) during withdrawal, relative to alcohol-naïve controls. Alcohol withdrawal-induced thermal hyperalgesia was abolished by 1) 30 minutes of voluntary oral alcohol selfadministration immediately prior to Hargreaves tests, 2) bolus alcohol injections, 3) intra-ventricular AgRP infusion, 4) intra-ventricular HS014 infusion, 5) intra-nasal HS014, and 6) intra-CeA infusion of HS014.

Conclusions: Our results implicate brain MC4R systems in thermal hyperalgesia in alcohol-dependent rats. MC4R antagonists reduced thermal hyperalgesia in alcoholwithdrawn rats, in agreement with past results showing that MC4R antagonism abolishes hyperalgesia during morphine $\mathrm{WD}$, and also prevents development of tolerance to the analgesic effects of chronic morphine. Intra-amygdala HS014 also reduced withdrawal hyperalgesia, in agreement with past results showing that intra-amygdala MC4R antagonists reduce nociception in rodents. The Western blot and immunohistochemistry data suggest that alcohol withdrawal may increase expression of the endogenous MC4R ligand, a-MSH, in rat $\mathrm{CeA}$, resulting in counteradaptive reductions in MC4R expression in CeA, but this counteradaptation may not be sufficient to rescue behavioral pathology, which would explain the ability of MC4R antagonist treatments to reduce thermal hyperalgesia in alcohol-withdrawn rats.

Keywords: Pain, Alcohol dependence, Melanocortin, Amygdala.

Disclosure: Nothing to disclose.

\section{W256. Reinforcing Effects of "Bath Salts" Mixtures: Studies With MDPV, Methylone, and Caffeine}

Gregory Collins*, Brenda Gannon, Kayla Galindo, Kenner Rice

University of Texas Health Science Center at San Antonio, San Antonio, Texas, United States

Background: Designer drugs of abuse have become a serious public health problem worldwide. One such class of drugs is designer stimulants, which are often marketed as "bath salts", and purported to be "safe" and "legal" alternatives to drugs such as cocaine and methamphetamine, despite their being associated with high rates of abuse, toxicity, and death. Although a wide variety of preparations exist, bath salts most often contain synthetic derivatives of cathinone. In the US, the most common synthetic cathinones are MDPV (an inhibitor of monoamine transporters) and methylone (a substrate of monoamine transporters); however, many products have been found to contain mixtures of two or more synthetic cathinones, or mixtures of a synthetic cathinone and caffeine. Thus, bath salts should be thought of as drug mixtures. The current studies used IV selfadministration to quantify the reinforcing effects of MDPV, methylone, and caffeine administered alone, and to determine how the reinforcing effects of these drugs are changed when administered as binary, bath salts mixtures.

Methods: Adult male Sprague Dawley rats were trained to self-administer either $0.032 \mathrm{mg} / \mathrm{kg} / \mathrm{inf}$ MDPV or $0.32 \mathrm{mg} / \mathrm{kg} /$ inf methylone under a fixed ratio schedule of reinforcement. All rats were subsequently transitioned to a progressive ratio (PR) schedule in order to quantify the reinforcing effectiveness of MDPV, methylone, and caffeine, administered alone, and as binary mixtures ( $n=12$ per pair of drugs). Drug mixtures were based on the concept of dose equivalence, with each pair of drugs evaluated at three ratios (3:1, 1:1, and $1: 3)$ relative to the mean ED50 for each drug. Dose addition analyses were used to calculate predicted, additive effect levels for each pair of doses for each combination of drugs using the Emax, ED50, and slope parameters derived from dose-response curves obtained for each drug alone in individual rats.

Results: When administered alone, MDPV, methylone and caffeine each maintained responding in a dose dependent manner with MDPV being the most effective reinforcer $(E \max =25.9$ infusions $)$, followed by methylone $($ Emax $=$ 16.3 infusions), and caffeine (Emax $=9.3$ infusions). Although high levels of responding were maintained by each of the bath salts mixtures, the nature of the interaction varied depending upon the constituent drugs. For combinations containing a cathinone and caffeine (MDPV+caffeine and methylone+caffeine), the interactions were generally additive, however, when mixed at a 3:1 ratio of their ED50s the reinforcing effects of MDPV and caffeine were found to be supra-additive. Unlike with combinations of cathinones and caffeine, when MDPV and methylone were combined (at 3:1, 1:1, or 1:3) a significant departure from additivity was observed in the sub-additive direction. Although not a stated goal of the current studies, toxicities were observed during sessions in which larger dose pairs of MDPV and methylone were available for self-administration.

Conclusions: Bath salts preparations often contain multiple pharmacologically active compounds, however, little is known about the nature of the interactions among bath salts constituents. These studies suggest that the reinforcing effects of drug mixtures containing a cathinone (MDPV or methylone) and caffeine are additive in nature, however, supra-additive interactions can be observed. Interestingly, these studies also suggest that sub-additive interactions can occur when MDPV and methylone are combined. Although sub-additive interactions are unusual for two drugs with similar mechanisms of action, such an interaction might be 
due to slight differences in their mechanisms of action (inhibitor vs. substrate) and/or differences in their selectivity for dopamine over serotonin transporters. It is also possible that the self-administration of MDPV+methylone mixtures was limited by an interaction between the adverse or toxic effects of these drugs rather than a decrease in their reinforcing effects, per se. Future studies will further investigate interactions exist between these drugs for other abuse-related and toxic effects.

Keywords: Self-Administration, Bath Salts, Caffeine.

Disclosure: Nothing to disclose.

\section{W257. Role of Ventral Tegmental Area SGK1 Phosphorylation and Activity in Drug-Associated Behaviors}

Marie Doyle, Vedrana Bali, Sophia Kaska, Sarah Cooper, Michelle Mazei-Robison*

Michigan State University, East Lansing, Michigan, United States

Background: Drugs of abuse potently modulate the mesolimbic dopamine system and changes in cellular activity and gene regulation within the ventral tegmental area (VTA) contribute to behavioral outputs that characterize drug seeking and abuse. Previous work from our lab has shown that serum- and glucocorticoid-regulated kinase 1 (SGK1) phosphorylation and catalytic activity are increased by chronic but not acute administration of cocaine and morphine. Increased phosphorylation of SGK1 and its substrate $\mathrm{N}$-myc downstream regulated gene (NDRG) are observed 1 hour, but not 24 hours, post-injection. However, the functional significance of these changes in drug-related behaviors remains unclear. Thus, our lab has created viral constructs that mimic or prevent phosphorylation at specific SGK1 residues, such as Ser78, whose phosphorylation is increased by drugs of abuse, as well as the canonical Ser422 and Thr256 sites. We evaluate voluntary drug intake using a morphine two-bottle choice task, and drug reward via conditioned place preference (CPP) for both cocaine and morphine. In addition to examining the effect of altered VTA SGK1 activity during drug exposure, we also test whether VTA SGK1 activity is changed following abstinence and drug re-exposure.

Methods: To assess changes in SGK1 and NDRG phosphorylation induced by chronic drug exposure, adult male and female c57bl6 mice were injected daily with saline or drug (morphine or cocaine, $20 \mathrm{mg} / \mathrm{kg}$ ) for 7 days. To determine changes in SGK1 signaling following drug abstinence and reexposure, mice were injected with saline, cocaine, or morphine for 7 days and were then subjected to 6 days of abstinence before receiving a challenge dose of saline or drug in either the drug paired, or a novel environment. Finally, changes in SGK1 or NDRG phosphorylation were assessed following re-exposure to saline- or cocaine-paired chambers in mice with established cocaine CPP. In all biochemical studies, mice were sacrificed within 1 hour of drug and/or environment exposure, VTA and nucleus accumbens (NAc) were microdissected, and samples were processed for western blot analysis using established procedures. For drug environment studies, locomotor activity and/or CPP were assessed to allow correlation of biochemical findings with behavioral results. In order to determine whether VTA SGK1 activity or phosphorylation mediates drug-related behavior, herpes simplex virus (HSV) constructs were used to overexpress an SGK1 kinase-dead mutant (K127Q), mutants that mimic or prevent Ser78 phosphorylation (S78D, S78A), and mutants that mimic or prevent phosphorylation as the canonical SGK1 regulatory sites Thr256 and Ser422 (T256DS422D, T256A-S422A). Vectors were stereotaxically injected into the VTA using established coordinates and procedures and behavioral analysis began 1 day following surgery. To assess morphine consumption, we used a two-bottle choice paradigm. Following acclimation to bottles filled with just water, singly-housed mice were given bottles filled with $0.2 \%$ sucrose and $0.05 \mathrm{mg} / \mathrm{ml}$ morphine sulfate or $0.01 \mathrm{mg} / \mathrm{ml}$ quinine sulfate, which were measured daily for 4 days. For $\mathrm{CPP}$, mice underwent twice-daily conditioning for two days, receiving saline in one chamber and cocaine $(7.5 \mathrm{mg} / \mathrm{kg})$ in the opposite chamber. A 20 min post-test was conducted the following day and time spent in the paired - unpaired chamber was calculated.

Results: As expected, we found that chronic morphine or cocaine increased SGK1 and NDRG phosphorylation in the VTA. Surprisingly, significant increases in VTA SGK1 phosphorylation were also observed in mice with previous drug experience that received saline, but not drug, on challenge day. This effect was not observed in the NAc, or in the VTA of animals that received chronic injections of drug in their home-cage and challenge injections in the locomotor chambers. Our preliminary data suggest that this increase may be a function of drug reward, as we observed a positive correlation between VTA pSGK1 levels and cocaine CPP score in mice that were returned to the previously cocainepaired chamber but received a saline injection. Further, VTA SGK1 activity and phosphorylation appear to affect drug intake. We found that decreasing total VTA SGK1 activity via HSV-mediated overexpression of a SGK1 kinase-dead mutant $\mathrm{K} 127 \mathrm{Q}$ decreased voluntary morphine intake in a two-bottle choice test. A similar decrease was observed when changes in SGK1 S78 phosphorylation were blocked by HSVmediated overexpression of an S78A mutant. Together, these data suggest that VTA SGK1 activity and phosphorylation are altered both during chronic drug exposure and following drug abstinence and re-exposure to drug-associated environment, and that changes in SGK1 activity and phosphorylation are sufficient to alter drug reward.

Conclusions: Surprisingly, significant increases in SGK1 phosphorylation were observed in mice with previous drug experience that received saline, but not drug, on challenge day. In contrast, this effect was not observed in mice that received a saline challenge in a novel environment, suggesting that SGK1 activity is not only increased during chronic exposure to drugs, but may be increased in response to environment-induced anticipation of reward. Future studies will utilize viral vectors to determine whether changes in VTA SGK1 signaling influence drug seeking or craving. We have established that decreasing VTA SGK1 catalytic activity, or S78 phosphorylation, is sufficient to decrease voluntary intake of morphine, and future studies will determine whether increasing activity and phosphorylation can similarly increase drug intake or reward. In total, the goal of these studies is to characterize the role of VTA SGK1 activity in 
drug-related behaviors to better understand the neuroadaptations that contribute to drug addiction and the potential of SGK1 activity as a novel target for treatment.

Keywords: Ventral Tegmental Area (VTA), Cocaine, Morphine, CPP.

Disclosure: Nothing to disclose.

\section{W258. THC-Induced Effects on Cognition and Cannabinoid Self-Administration in Old-World Monkeys}

\section{William John, Thomas Martin, Michael Nader*}

Wake Forest School of Medicine, Winston-Salem, North Carolina, United States

Background: Marijuana (Cannabis sativa) is the most commonly abused illicit drug in the United States, with an estimated 4.2 million people that meet DSM criteria for dependence (SAMHSA, 2013). As a result, there is a need for more research to better understand the pharmacological, environmental, and biological determinants of cannabis use in order to develop more effective treatment strategies for cannabis use disorder. The present study utilized animal models of marijuana abuse to examine two behavioral consequences: reinforcing effects of cannabinoid agonists and the long-term effects of these compounds on cognition in Old World monkeys (rhesus monkeys). Cannabis-related impairments to cognitive function may represent novel therapeutic targets for cannabis-use disorder; however, the exact nature, persistence, and reversibility of those deficits remain unclear. A major obstacle in animal models has been the difficulty in demonstrating the reinforcing effects of $\Delta 9$ tetrahydrocannabinol (THC), the main psychoactive component of marijuana, by self-administration (SA) procedures in animals. Reinforcing effects of THC have only been established in New World primates (squirrel monkeys). Thus, an objective of the present study was to establish the conditions necessary for intravenous SA of THC and the cannabinoid receptor (CBR) agonist CP55,940 in rhesus monkeys, a species more closely related to humans than squirrel monkeys.

Methods: For these studies, adult male rhesus monkeys $(N=6)$ responded in the mornings on tasks designed to assess different cognitive domains using CANTAB touchscreens and were then transported to another chamber where responding was maintained under a fixed-ratio (FR) 10 schedule of food presentation. These same monkeys, plus three additional animals $(N=9)$, were also studied in models of cannabinoid self-administration. The effects of CP55,940 and THC were first examined on FR10 food-maintained responding and body temperature prior to giving them access to self-administer each drug. THC $(0.01-0.3 \mathrm{mg} / \mathrm{kg})$ was also examined on several cognitive baselines (delayed match-to-sample, DMS, compound discrimination, CD, compound discrimination and reversal, CDR, and intradimensional/extradimensional, ID/ED, set-shifting). Next, monkeys were administered THC daily until tolerance developed to rate-decreasing effects on FR10 foodmaintained responding. The effects of THC on cognition (22 hrs after THC treatment) was studied, along with redetermining the THC dose-response curve.
Results: Both THC and CP55,940 dose-dependently decreased food-maintained responding, with CP55,940 being 15-times more potent than THC. CP55,940 (0.001-3.0 $\mu \mathrm{g} / \mathrm{kg})$ functioned as a reinforcer in three monkeys, while THC $(0.01-10 \mu \mathrm{g} / \mathrm{kg})$ did not have reinforcing effects in any subject. There was an inverse relationship between CP55,940 reinforcement and sensitivity to decreases in food-maintained responding. THC (0.01-0 .3 mg/kg) dosedependent decreases in working memory, CD learning and ED set shifting. During chronic treatment, THC (1.0-2.0 mg/ $\mathrm{kg}$, s.c.), tolerance developed to the effects on foodmaintained responding. When THC SA was reexamined, it functioned as a reinforcer in three monkeys. Chronic THC produced persistent residual impairment (22 hours after administration) to working memory when the cognitive load was high (i.e., long delays) but not to discrimination and reversal learning or attentional set-shifting performance. Chronic THC treatment resulted in tolerance to the acute impairments in compound discrimination learning and working memory. During abstinence, there was recovery of impairments within 2 weeks.

Conclusions: These data indicate that chronic THC-induced disruptions in cognitive performance depended on the cognitive domain, cognitive load, and time of assessment following THC administration. Tolerance developed more rapidly to the rate-decreasing effects on FR10 foodmaintained responding than cognitive performance and this tolerance was associated with increases in THC selfadministration in some monkeys. Understanding individual differences in vulnerability to THC SA and long-term effects on cognition may lead to novel treatment strategies for marijuana abuse.

Keywords: Marijuana, Cognition, Drug Abuse, Animal Models, Cannabinoids.

Disclosure: Nothing to disclose.

\section{W259. Effect of Cigarette Smoking on Neuroinflammation: A [11C]DAA-1106 Positron Emission Tomography Study}

Arthur Brody*, Hubert Robert, Lizette Garcia, Ryutaro Enoki, Kyoji Okita, Edythe London, Erika Nurmi, Lauren Seaman, Mark Mandelkern

University of California, San Diego, San Diego, California, United States

Background: Microglia respond swiftly to damage or infection in the brain by transforming into an activated phenotype and performing inflammatory functions. Cigarette smoking is known to impair normal bodily inflammatory functions, such as wound healing, while also inducing certain aspects of the inflammatory response. Over the past several years, the radioligand $\mathrm{N}$-(2,5-dimethoxybenzyl)-N(5-fluoro-2-phenoxyphenyl) acetamide labeled with carbon11 (abbreviated as [11C]DAA1106) has emerged as a reliable second-generation radiotracer for positron emission tomography (PET) scanning for examining levels of activated microglia (a marker for neuroinflammation) in brain. We sought to determine the effect of cigarette smoking on this marker for neuroinflammation. 
Methods: Fifty-one participants (35 smokers in the satiated state and 16 non-smokers) underwent [11C]DAA1106 PET scanning (370 MBq bolus injection) of the brain, along with magnetic resonance imaging (MRI) to assist in localization of regions on PET scans. During PET scanning, two $5 \mathrm{ml}$ blood samples were drawn - one for determination of plasma nicotine level and the other for determination of genotype for a single-nucleotide polymorphism (rs6971), which identifies low-, mixed-, and high- affinity binders of the radiotracer (as is standard for this type of research). Automated brain volumes of interest (VOIs) were determined on MRI using FSL software and transferred to coregistered PET scans, with whole brain being the primary VOI. Standardized uptake value (SUV), defined as average VOI activity concentration $(\mathrm{Bq} / \mathrm{mL})$ for $20-40 \mathrm{~min}$ postinjection divided by the injected activity per unit body weight $(\mathrm{Bq} / \mathrm{kg})$, was determined for the whole brain and other VOIs. For SUV values, analysis of covariance (ANCOVA) was performed, with group (smoker vs. non-smoker) as a factor and genotype (mixed or high affinity) as a nuisance covariate. Six scans were excluded from the analysis because of technical problems $(n=2)$ or participants being lowaffinity binders $(n=4)$.

Results: Smokers and non-smokers differed on whole brain SUV values (ANCOVA; F[1,42] $=7.0 ; p=0.01$ ) due to smokers having $16 \%$ lower values than non-smokers. For completeness, ANCOVAs for automated subcortical VOIs were also performed, and 13 of 14 regions also exhibited significant group differences due to smokers having lower SUV values. There were no significant group differences for the injected dose of radiotracer or body weight. Within the smoker group, there were no significant correlations between plasma nicotine levels and [11C]DAA1106 SUV values.

Conclusions: Smokers have less binding of [11C]DAA1106 globally than nonsmokers, which suggests impaired microglial activation. Study findings are consistent with much prior research demonstrating that smokers have impaired inflammatory function compared to non-smokers. Study findings are also supportive of prior research demonstrating that constituents of tobacco smoke other than nicotine affect inflammatory processes.

Keywords: Tobacco, Smoking, Neuroinflammation, Positron Emission Tomography, Nicotine.

Disclosure: Nothing to disclose.

\section{W260. Decreased Functional Connectivity in Women After Naltrexone and Bupropion Combination Therapy}

Gene-Jack Wang*, Jizheng Zhao, Dardo Tomasi, Ehsan Shokri Kojori, Ruiliang Wang, Corinde E. Wiers, Elisabeth C. Caparelli, Nora D. Volkow

National Institute on Alcohol Abuse and Alcoholism, Bethesda, Maryland, United States

Background: The control of body weight involves a complex and distributed neural to regulate metabolic needs that relies on reward/motivation, learning/memory and emotional/ mood processes. Dopamine and endogenous opioids play critical roles in the control of eating behavior. Our prior study using food related cues and fMRI showed that a combination of naltrexone sustained release (SR) $32 \mathrm{mg}$ and bupropion SR $360 \mathrm{mg}$ (NB) therapy blunted the hypothalamic reactivity to food cues while enhancing the activation of regions involved with self-control and internal awareness in women. However, the effects of $\mathrm{NB}$ on the resting-state communications between these regions is largely unknown. Functional connectivity density mapping (FCDM) has been used to assess the spatial distribution of brain connectivity hubs, whereas seed-voxel correlation approaches have been used to identify connectivity patterns relative to specific brain regions in the resting-state. Here we used FCDM to guide seed-voxel correlation analyses to evaluate the effects of NB on brain functional connectivity (FC).

Methods: Dutch behavior and eating questionnaire (DBEQ) was obtained in healthy women $(n=37,18-45$ years old). The NB and placebo groups underwent $4 \mathrm{~T} \mathrm{MRI} \mathrm{(eyes} \mathrm{open)}$ before and after 4 weeks' treatment either with NB $(n=17)$ or with placebo $(n=20)$ with BMI 27-40. In each image visit, we conducted a 5 - min resting-state fMRI scan (T2* weighted single shot gradient echo-planar imaging sequence in a $4 \mathrm{~T}$ MRI scanner, $\mathrm{TR} / \mathrm{TE}=1600 / 20 \mathrm{~ms}, 4 \mathrm{~mm}$ slice thickness, $1 \mathrm{~mm}$ gap, 33 coronal slices, 64x64 matrix size, $3.1 \times 3.1 \mathrm{~mm}$ resolution and 90॰flip angle) under 15-17 hour fasting conditions. The images were realigned, normalized to the MNI space, $0.01-0.1 \mathrm{~Hz}$ band-pass filtered, and time points with excessive motion (framewise displacement $>$ $0.5 \mathrm{~mm}$ ) were removed. Nuisance signal fluctuations in white matter, cerebrospinal fluid and the whole brain, as well as 6 motion parameters (obtained from realignment) and their temporal derivatives were removed from data. Local and global functional connectivity densities (FCD) were then computed for each image time series using the standard correlation threshold $(\mathrm{r}=0.6)$. The $\mathrm{FC}$ of the brain regions showing significant group effects on FCD were subsequently assessed using seed-voxel correlation analyses. Statistical significance was based on family-wise error (FWE) corrections for multiple comparisons at the cluster level (PFWE $<$ 0.05 ) using a minimum cluster size of $\mathrm{k}=50$ and a cluster forming threshold of $P<0.001$.

Results: No significant differences were found between NB and placebo groups in demographic characteristics and DBEQ scores. There were no significant group differences on FCD at baseline. After 1-month treatment, the NB showed lower FCD than the placebo group in right precuneus (both global and local FCD) and left middle frontal gyrus (local FCD). The seed-voxel correlation analysis for the precuneus seed did not show significant group differences at baseline. However, after NB treatment the right precuneus seed demonstrated lower negative FC with the cingulate gyrus, insula, left superior parietal lobule, left precuneus, right superior occipital gyrus, left cuneus, right precentral gyrus, and left superior frontal gyrus for the NB group than for the placebo group. The right precuneus also showed lower positive connectivity with dorsolateral prefrontal and superior parietal cortices for the NB group than for the placebo group and there was a significant treatment by group interaction effect in the parietal cortex.

Conclusions: The marked decreases in FCD in the precuneus in the NB group suggests that combined drug treatment affects the brain functional connectivity in obese and overweight women. In particular, NB therapy reduced the FC of the parietal cortex with limbic regions involved in saliency and reward processing, and with prefrontal regions 
involved in the control of eating behavior. The FC changes after the NB administration might help to better understand neural mechanisms mediating the therapeutic benefits of NB in obesity.

Keywords: Functional MRI (fMRI), Naltrexone, Resting State Functional Connectivity, Bupropion.

Disclosure: Orexigen Therapeutics Inc: Lecture Fees and Research Funding, Self.

\section{W261. Selective Activation of $(\alpha 4) 3(\beta 2) 2 \mathrm{nAChRs}$ Reduces Ethanol Consumption Without Affecting Ethanol Intoxication}

Jingyi Wang*, Jon Lindstrom, Theodore Kamenecka, Paul Kenny, Robert Messing

The University of Texas at Austin, Austin, Texas, United States

Background: Tobacco and alcohol are highly co-abused substances. The FDA-approved smoking cessation aid varenicline (Chantix ${ }^{\circ}$ ), a selective partial agonist of $\alpha 4 \beta 2$ nicotinic acetylcholine receptors (nAChRs), also reduces alcohol consumption in humans and rodents. $\alpha 4 \beta 2 \mathrm{nAChRs}$ can assemble in two stoichiometries: $(\alpha 4) 3(\beta 2) 2$ and $(\alpha 4) 2$ $(\beta 2) 3$. We found that varenicline partially activates and fully desensitizes both stoichiometric forms in vitro. It is unknown whether varenicline modulates one or both stoichiometries to reduce alcohol consumption.

Methods: The small molecule 3-[3-(3-pyridinyl)-1,2,4-oxadiazol-5-yl]benzonitrile (NS9283) selectively enhances the sensitivity of $(\alpha 4) 3(\beta 2) 2 \mathrm{nAChRs}$ for ACh. However, NS9283 itself does not directly activate or inhibit activation of nAChRs, like positive allosteric modulators. Here we used NS9283 to increase function of $(\alpha 4) 3(\beta 2) 2$ nAChRs and investigated their role in ethanol consumption, using C57BL/ $6 \mathrm{~J}$ mice in an intermittent access 2-bottle choice procedure. Moreover, NS9283 potentiated activation and desensitization of $(\alpha 4) 3(\beta 2) 2 \mathrm{nAChRs}$ by varenicline in vitro. To confirm that the effect of NS9283 on ethanol consumption was through its action at $(\alpha 4) 3(\beta 2) 2 \mathrm{nAChRs}$, we tested whether subthreshold doses of NS9283 and varenicline synergize to reduce ethanol drinking. Since varenicline increases hypnotic effect of ethanol, we also evaluated whether NS9283 affected the ethanol-induced loss of the righting reflex (LORR). We also studied the effect of NS9283 on ethanol metabolism in mice.

Results: We found that administration of $10 \mathrm{mg} / \mathrm{kg}$ NS9283 significantly reduced ethanol intake in male mice by $29 \%$ during the first three hours of drinking. This decrease was similar to that produced by $2 \mathrm{mg} / \mathrm{kg}$ varenicline. However, NS9283 did not alter ethanol consumption in female mice. This suggests that endogenous cholinergic signaling by $(\alpha 4) 3$ $(\beta 2) 2 \mathrm{nAChRs}$ regulates ethanol consumption in a sex dependent manner. In addition, we found that while $2.5 \mathrm{mg} /$ $\mathrm{kg}$ NS9283 and $0.1 \mathrm{mg} / \mathrm{kg}$ varenicline had no effect, in combination at those doses, they significantly reduced ethanol consumption. There was no difference in duration of LORR between NS9283 $(10 \mathrm{mg} / \mathrm{kg})$ and vehicle treated male mice after administration of $3.6 \mathrm{~g} / \mathrm{kg}$ ethanol. Like varenicline, NS9283 did not alter clearance of ethanol.
Conclusions: These results indicate that modulating the activity of $(\alpha 4) 3(\beta 2) 2 \mathrm{nAChRs}$ contributes to the ability of varenicline to reduce ethanol consumption, but not to the enhancement of ethanol intoxication by varenicline. Positive allosteric modulators selective for $(\alpha 4) 3(\beta 2) 2 \mathrm{nAChRs}$ could prove to be new therapeutics, with less side effects than varenicline, for the treatment of excessive drinking.

Keywords: Alcohol Consumption, Varenicline, Nicotinic Acetylcholine Receptors, Positive Allosteric Modulators. Disclosure: Nothing to disclose.

\section{W262. A 12-Week, Multi-Center, Double-Blind, Randomized, Placebo-Controlled, Phase-2, Dose Selection Study of Lorcaserin Hydrochloride for Smoking Cessation: Preliminary Report}

Charles Wilcox*, My-Linh Tong, Nader Oskooilar, Judy Morrissey, Don De Francisco, Mellissa Henry, Lynn Badgett, Daniel Grosz

Pharmacology Research Institute, Newport Beach, California, United States

Background: Tobacco use is the leading cause of preventable illness and death around the world. Belviq [lorcaserin hydrochloride $(\mathrm{HCl})]$ is a selective serotonin (5-HT2C) receptor agonist which decreases food intake and body weight through its actions in the central nervous system. Lorcaserin has been independently investigated by two different academic investigators demonstrating the ability to inhibit nicotine self-administration in rats. The potential combined benefits of smoking cessation, and prevention of associated weight gain, would address two significant challenges in the fight against this leading cause of preventable illness and early death. Here we are reporting on the Phase- 2 results, solely from our research center, where our primary objective was to evaluate the efficacy of lorcaserin $\mathrm{HCl}$ in smoking cessation.

Methods: This was a randomized, double-blind, twelve-week [dosing], placebo-controlled, parallel-group dose selection study. Seventy-five (75) male and female smokers, ages 18to-65 inclusive, smoking ten (10) or more cigarettes per day for at least the past year, who were motivated to stop smoking, were screened for entry into the trial. Fifty-nine (59) eligible smokers were randomly assigned to one of three treatment groups: lorcaserin $10 \mathrm{mg}$ once daily; lorcaserin $10 \mathrm{mg}$ twice daily, or placebo in a 3:3:2 ratio. Randomized patients were instructed to take the study medication for two weeks before attempting to quit on Study Day 15, a.k.a. "Target Quit Date." The primary efficacy endpoint was based on the end-expiratory carbon monoxide- (CO-) confirmed Continuous Abstinence Rate (CAR) from study weeks 9-12, defined as zero reported smoking via Nicotine Use Inventory (NUI) with exhaled $\mathrm{CO}$ measurement $\leq 10 \mathrm{ppm}$. As is customary in these trials, and ideally in practice, all of the participants also received ten minutes of smoking cessation counseling during clinic visits at Day -1 and weekly through Week 12.

Results: Our initial "top-line" sustained cessation-related results demonstrated a $33.3 \%$ quit rate for high dose [20 mg] lorcaserin, versus $9.5 \%$ for low dose [10 mg] lorcaserin and $21.4 \%$ for placebo. None of our initial results demonstrated 
any statistically significant findings. We also looked at several baseline variables previously demonstrating some predictive utility, including but not limited to: age-startedsmoking, (other) smokers in household, number of years smoking, number of lifetime quit attempts, baseline body mass index (BMI) and baseline Fagerstrom score. Atypically, and perhaps in part because of our small sample size(s), none of the aforementioned baseline variables demonstrated any statistically significant correlation with cessation-related success. Our revised statistical analyses, based on less stringent "success criteria" produced statistically significant results. Using either $\mathrm{NUI}=0$ or $\mathrm{CO} \leq 10 \mathrm{ppm}$, high dose lorcaserin demonstrated significantly better efficacy when compared to placebo $(p<0.02)$ as well as low dose lorcaserin $(p<0.005)$. When we looked at "harm/risk reduction" as defined by NUI values of $\leq 5$ and a CO value of $\leq 10 \mathrm{ppm}$, hereto lorcaserin $20 \mathrm{mg}$ was significantly more efficacious than placebo $(p<0.02)$, as well as low dose lorcaserin $(p<$ $0.01)$. Additionally, when we analyzed for NUI $\leq 5$ or $\mathrm{CO} \leq$ $10 \mathrm{ppm}$ at Week 12/Endpoint, high dose lorcaserin again demonstrated statistically significant superiority over placebo $(p<0.01)$ and low dose lorcaserin $(p<0.01)$. Finally, we also analyzed baseline versus endpoint BMI values comparing successful quitters and non-quitters, as well as by treatment group assignments, and there were no statistically significant findings.

Conclusions: Lorcaserin $10 \mathrm{mg}$ once daily and twice daily did not demonstrate statistically significant results when analyzed vis-à-vis the pre-specified protocol criteria. However, when re-analyzed utilizing our redefined "harm reduction" criteria, high dose lorcaserin was statistically significantly superior to both placebo and low dose lorcaserin at multiple time points, including Endpoint/Week 12. High dose lorcaserin appears to have clinical and commercial potential as an efficacious smoking cessation agent - without associated weight gain - and, we believe, warrants further investigation.

Keywords: Smoking Cessation, Clinical Trials, Harm Reduction, Nicotine Addiction, Nicotine Dependence.

Disclosure: Nothing to disclose.

W263. Differential Regulation of Mood and Motivation by Separate Accumbens Outputs in Cocaine SelfAdminstrating Rats

\section{Amy Loriaux*, David Self}

University of Texas Southwestern Medical Center, Dallas, Illinois, United States

Background: Cocaine users often cite negative mood as a key factor behind relapse. It is not fully understood whether the same circuitry implicated in this enhancement in drug seeking is also involved in the subsequent decreases in mood seen in chronic drug users. The nucleus accumbens shell (NAcSh), a region implicated in drug addiction, is known for its ability to convert both rewarding and aversive affective information into appropriate motivational output. It is unknown by which outputs the NAcSh accomplishes this and whether chronic cocaine use alters these pathways. The NAcSh projects primarily to the ventral pallidum (VP) and the ventral tegmental area (VTA). Recent work suggests differential regulation of mood by these pathways. However, the role of these pathways in mediating addiction is currently debated.

Methods: In the present study, we used a target-specific optogenetic approach to selectively activate NAcSh projections to either the VTA or VP in male Sprague-Dawley rats. We tested the hypotheses that increased activity in the NAcSh-VTA pathway would increase, while NAc-VP pathway activation would decrease, mood and motivation for cocaine. Rats were bilaterally injected with either AAV2hSyn-hChR2(H134)-EYFP or control virus into the NAcSh, and implanted with optic fibers in the terminal regions in the VTA or VP. Rats were trained to self-administer intravenous cocaine $(0.5 \mathrm{mg} / \mathrm{kg} /$ infusion, i.v.) $4 \mathrm{~h} /$ day for 3 weeks. We measured the effect of laser stimulation of each pathway (30 min pretreatment, $10 \mathrm{sec} / \mathrm{min}, 20 \mathrm{~Hz}, 10-12 \mathrm{~mW}$ at fiber tip) on motivation for cocaine as assessed by 1) performance on a progressive ratio (PR) schedule of cocaine reinforcement, 2) drug-paired lever presses under extinction and 3) cocaine-primed reinstatement. We measured behavioral despair and anhedonia with the forced swim and sucrose preference tests, respectively. All methods were approved by the Institutional Animal Care and Use Committee of the University of Texas Southwestern Medical Center.

Results: Optogenetic stimulation of NAcSh-VP terminals, while having no effect on mood, significantly decreased the motivation for cocaine as indicated by a $44 \%$ drop in breakpoint and $64 \%$ drop in the number of days to extinguish. Conversely, NAcSh-VTA stimulation induced anhedonia, as indicated by $33 \%$ lower sucrose preference scores, while producing a $72 \%$ increase in cocaine seeking during reinstatement.

Conclusions: These findings suggest that separate but overlapping pathways in the ventral striatum may mediate the changes in mood and motivation accompanying cocaine addiction. They further suggest that, in its implication in both mood and cocaine seeking, the pathway from the NAcSh to the VTA may mediate some of the negative reinforcing properties of drugs that promote relapse. Finally, targeting the NAcSh-VP pathway may provide therapeutic benefits in treating cocaine craving without affecting mood. Keywords: Cocaine Seeking, Depression, Nucleus Accumbens Shell, Ventral Pallidum, Ventral Tegmental Area (VTA).

Disclosure: Nothing to disclose.

\section{W264. Menthol Disrupts Nicotine's Psychostimulant Properties in an Age and Sex-Dependent Manner}

Yann Mineur*, Benjamin Fait, David Thompson, Mose Tenna, Marina Picciotto

Yale University School of Medicine, New Haven, Connecticut, United States

Background: Flavoring agents such as menthol can make nicotine and tobacco products less aversive and menthol smokers have a harder time quitting, along with a significantly higher rate of relapse. Adolescents, are more likely to use menthol cigarettes than adults, and are at much higher risk for transition to life-long smoking than counterparts using non-menthol cigarettes. Thus, in addition to 
understanding how the flavoring and soothing effect of menthol could contribute to greater intake of tobacco products and therefore higher exposure to nicotine, it is important to identify mechanisms that underlie the behavioral changes induced by menthol-laced nicotinic products, including the potential alteration of nicotine's psychostimulant properties.

Methods: The psychostimulant effects of nicotine in mice can be reliably and sensitively evaluated by recording home cage locomotor activity during a 10- day drinking paradigm. This paradigm allows mice to titer their nicotine intake over the circadian period and avoids the hyperlocomotion induced by passive injection of the drug. Nicotine $(200 \bullet \mathrm{g} /$ $\mathrm{ml})$ and menthol $(10 \mu \mathrm{g} / \mathrm{ml} \mathrm{L}$-menthol) were mixed in a $2 \%$ saccharin water solution were provided to male and female C57BL/6 J, adolescent (PND 24-35) and adult (PND> 90 days) mice. In order to determine whether $\beta 2$-containing nicotinic acetylcholine receptors ( $\beta 2 \mathrm{nAChRs})$, critical for nicotine-induced hyperlocomotion and reinforcement, contributed to behavioral responses to menthol, this drinking regimen was also tested in $\beta 2 \mathrm{KO}$ adult mice.

Nicotine intake was tested throughout behavioral testing and cotinine blood levels were tested following the exposure paradigm.

Results: There was an overall interaction between treatment and age, and treatment and sex in liquid consumption, and further analyses revealed that adult male mice drank less when given nicotine, while the groups given menthol with or without nicotine drank more. Adult female mice consumed significantly less liquid when given menthol alone or with nicotine, but this decrease was not observed in $\beta 2 \mathrm{KO}$ females. Liquid consumption of the combined nicotine and menthol solution was also significantly decreased in adolescent female mice. Interestingly, blood cotinine levels in adult males did not vary with nicotine consumption, and was equivalent between in mice that received nicotine alone or with menthol.

Adult male mice showed a significant decrease in locomotion during the active phase (nights) in response to menthol, without or without nicotine. This response to menthol was not detected in adult female mice or adolescent mice of either sex. Adult male mice also showed locomotor bursts during the inactive phase of the light cycle in response to nicotine exposure that was reversed by menthol. Menthol alone increased locomotion in female $\beta 2 \mathrm{KO}$ mice but had no effect on locomotion or bursts of activity in male mice.

Conclusions: This dataset confirms that menthol can influence behavioral responses to nicotine, independently of effects on intake. Menthol increases nicotine intake in adult male mice despite decreasing the psychostimulant effect. Further, the response to menthol differs significantly by age and sex, suggesting that human studies comparing adolescent menthol smokers and adult menthol smokers of both sexes are warranted.

Keywords: Menthol, Nicotine, Animal Model, Psychostimulation, Addiction.

Disclosure: Nothing to disclose.
W265. Nadph Oxidase Isoform 2 is Involved in the Alcoholism Vulnerability of Adult Rats Exposed to Ethanol in Utero

Marcela Contreras, Carolina Goic, Daniel Rodriguez, Erwin De La Fuente, Paola Haeger*

Universidad Catolica del Norte, Coquimbo, Chile

Background: Ethanol intake during pregnancy may generate severe effects in the cognitive development of the offspring. Children with prenatal ethanol exposure may exhibit morphological and functional alterations, which are called by the generic term "fetal alcohol spectrum disorders" (FASD). Particularly, prenatal ethanol exposure in both human as well as animal models increases the vulnerability to abuse alcohol and other addicted substances.

Ethanol exposure in utero increases oxidative stress in developing organs, including the brain. Indeed, even a brief exposure to ethanol during gestation can produce perdurable imbalance between the levels of intracellular reactive oxygen species (ROS) and brain antioxidants that can be correlated with cognitive deficits. Interestingly, antioxidants treatment, during mother ethanol ingestion, has been repeatedly shown to improve behavioral deficits in rodent models of FASD. However, the impact of the general antioxidant treatment in the adult age of the exposed offspring, and the specific ROSdependent mechanism, has still not been fully studied. We are testing the role of NADPH oxidase, isoform 2 (NOX2) (postsynaptic superoxide generator) on ethanol seeking behavior of animal exposed to ethanol in utero.

Methods: We developed a rat model of FASD, which has been evaluated at adult age (p70). Male rats were analyzed by behavioral tests such as voluntary ethanol consumption through free choice paradigm and ethanol dependent contextual place preference. Apocynin (5 mM) and gp91 ds-tat (100 nmoles intra VTA) were used to inhibit NOX 2 in vivo. NOX2 expression was analyzed by western blot using commercial antibody for gp91phox, a membrane subunit of NOX2.

Results: These animals consumed around 25\% more ethanol than control rats, during one day of freechoice alcohol test; they spent significantly more time in the ethanolpaired compartment in the conditioning place preference test. Interestingly, in vivo inhibition of NOX2 using apocynin, from 33 to 70 days old, or inhibition of NOX2 into ventral tegmental area (VTA) rescued the altered behaviors. Moreover, the expression of gp91phox is significantly reduced in prefrontal cortex, but is unaltered in VTA or hippocampus. Conclusions: We are currently performing studies to understand the specific mechanism by which NOX2 is contributing to increase vulnerability to alcohol consumption and seeking behavior.

Keywords: Addiction, Fetal Programming, Oxidative Stress. Disclosure: Funded by FONDECYT grant $N^{\circ} 1140855$. 


\section{W266. Defining the Role of Corticotropin Releasing Factor Binding Protein in Alcohol Consumption}

Carolina Haass-Koffler*, Andrea Henry, Gerd Melkus, Jeffrey Simms, Mohammed Naemmuddin, Carsten Nielsen, Amy Lasek, Molly Magill, Melanie Schwandt, Reza Momenan, Colin Hodgkinson, Selena Bartlett, Robert Swift, Antonello Bonci, Lorenzo Leggio

Brown University, Providence, Rhode Island, United States

Background: The corticotropin releasing factor (CRF) exerts its effects by acting on its receptors and on the binding protein (CRFBP), and has been implicated in alcohol use disorder (AUD). Therefore, identification of the exact contribution of each protein that mediates CRF effects is necessary to design effective therapeutic strategies for AUD. Methods: A series of in vitro and in vivo experiments across different species were performed to define the biological discrete role of CRFBP in AUD. All animal procedures were approved by the Ernest Gallo and Clinical Center Institutional Animal Care and Use Committee, were in accordance with NIH guidelines for the Humane Care and Use of Laboratory Animals and were conducted in agreement with the Guide for the Care and Use of Laboratory Animals, National Research Council, 1996. Human subject data were collected under screening and assessment NIAAA protocols approved by the appropriate NIH IRBs. All participants provided written consent before participating.

Results: First, to establish the CRFBP role in receptor signaling, we developed a novel chimeric cell-based assay and showed that that CFRBP full length (FL) can stably be expressed on the plasma membrane. We discovered that only CRFBP $(10 \mathrm{kD})$ fragment is able to potentiate CRFintracellular $\mathrm{Ca} 2+$ release. We provide evidence that CRHBP gene loss increased ethanol consumption in mice. Then, we demonstrate that selective reduction of CRHBP expression in the center nucleus of the amygdala (CeA) decreases ethanol consumption in ethanol-dependent rats. CRFBP amygdalar downregulation, however, does not attenuate yohimbineinduced ethanol self-administration. This effect was associated with decreased hemodynamic brain activity in the CRFBP downregulated CeA and increased hemodynamic activity in the caudate putamen during yohimbine administration. Finally, in alcohol-dependent patients, genetic variants related to the $\operatorname{CRFBP}(10 \mathrm{kD})$ fragment were associated with greater risk for alcoholism and anxiety, while other genetic variants were associated with reduced risk for anxiety.

Conclusions: Taken together, our data provides evidence that CRFBP may possess both inhibitory and excitatory roles and may represent a novel pharmacological target for the treatment of AUD.

Keywords: Corticotropin-Releasing Factor (CRF), Chimeric Protein, CRHBP Knockout Mice, shRNA, Human Genetic. Disclosure: Nothing to disclose.
W267. History of Psychiatric Illness Among Young Adults Confers Risk for a More Severe Cannabis Use Presentation

Randi Schuster*, Emily Nip, Jodi Gilman, Madeleine Fontaine, Ailish Hanly, Anne Eden Evins

Harvard Medical School, Boston, Massachusetts, United States

Background: Rates of cannabis use may be disproportionately represented among young adults with psychiatric illness (1-4), potentially due to bidirectional relationships between cannabis use and mental illness (5-9). However, the literature on co-morbidity among young adults is small and mainly confined to clinical samples. Given increasing cannabis use among young adults (10), it is critical to understand whether psychiatric illness increases risk of problematic cannabis during young adulthood.

Methods: A community sample of 64 young adults, aged 1825 years, who reported using cannabis at least weekly was assessed at baseline, when they were using cannabis as usual, and weekly for four consecutive weeks. Participants were randomly assigned to 30 days of cannabis abstinence, confirmed by frequent quantitative assays of urine cannabis metabolite concentration $(n=39 ; 61.0 \%)$ or monitoring without an abstinence requirement $(n=25 ; 39.0 \%)$.

Twenty-eight participants (43.8\%) had at least one current or lifetime Axis I disorder, assessed with the Structured Clinical Interview for DSM-IV (SCID-IV). Cannabis use dependence was determined at baseline with scores $\geq 12$ on the Cannabis Use Disorder Identification Test - Revised (11). Age of initiation, amount and frequency of cannabis use was assessed at baseline using a modified, 90-day Timeline Follow-Back method (12). Among those randomized to abstinence, cannabis withdrawal was assessed weekly using the Cannabis Withdrawal Scale (13).

Results: A MANOVA was conducted in the full sample, which revealed a significant difference in cannabis use severity (dependent variables: dependence, age of initiation, frequency and quantity of past 90 day use) based on history of psychiatric illness $(\mathrm{F}(4,59)=3.48, p=0.013$; Wilk's $\lambda=$ 0.81 ). Follow-up analyses revealed that among weekly or more frequent cannabis users, those with a psychiatric illness were more likely to have cannabis dependence $(\chi 2(1, N=$ $64)=6.02, p=0.01 ; 60.5 \%$ and $52.7 \%$ with cannabis dependence among those with and without psychiatric illness, respectively) and use more frequently in the past three months $(\mathrm{t}(62)=-3.57, p=0.0007$; with psychiatric illness: $\mathrm{M}=65.4$ days, $\mathrm{SD}=22.5$; without psychiatric illness: $\mathrm{M}=45.5$ days, $\mathrm{SD}=21.9)$. Psychiatric illness did not predict amount of cannabis consumed in the past three months and age of initiation ( $p$ 's $>0.15$ ).

A multilevel mixed-effects regression with a random effect for intercept was fit to the data to examine if cannabis withdrawal symptoms across 30 days of abstinence varied by history of psychiatric illness. The main effects for group and time ( $\mathrm{p}$ 's $=0.08)$, suggested that there was a trend for those with psychiatric illness to have greater withdrawal symptoms and for withdrawal symptoms to decline across 30 days of abstinence. There were significant linear, quadratic and cubic interactions between group and time on withdrawal symptoms $\quad(B=28.35, \quad p=0.007 ; \quad B=-12.55, \quad p=0.005$; 
$\mathrm{B}=1.30, p=0.006$, respectively). In the first week of abstinence, the median linear decrease in number of withdrawal symptoms was greater for those without (Med = $-5.0, \mathrm{IQR}=-19.0,1.5)$ than those with psychiatric illness $(\mathrm{Med}=0, \mathrm{IQR}=-19.0,12)$. However, the median linear decrease in number of withdrawal symptoms over 30 days of abstinence was greater for those with (Med =-7.0, IQR = $31.0,14.0)$ than without history of psychiatric illness $(\mathrm{Med}=-2.5, \mathrm{IQR}=-23.0,4.5)$.

Conclusions: Results suggest that a history of psychiatric illness is a risk factor for more problematic cannabis use among young adults, consistent with findings among adults.
A novel finding in this investigation is that those with a history of psychiatric illness have greater withdrawal and later recovery of withdrawal symptoms, a mechanism that may underlie heavier cannabis use. That is, these youth may be more likely to use heavily to minimize aversive physiological and psychological symptoms that arise in early abstinence. As frequent use during this developmental period is the best predictor of poorer longer term outcomes $(10,14)$, targeting vulnerability factors to delay onset of use is a promising strategy for reducing drug-related harms.

Keywords: Cannabis, Young Adults, Withdrawal.

Disclosure: Nothing to disclose. 